DAVID BÖHM

ALEXANDER GROSSMANN

MICHAEL REICHE

ANTONIA SCHRADER

Open-Access-Publikationsworkflow für akademische Bücher

Ein Handbuch für Hochschulen und Universitäten 

DAVID BÖHM

ALEXANDER GROSSMANN

MICHAEL REICHE

ANTONIA SCHRADER

\section{Open-Access-Publikationsworkflow für akademische Bücher}

Ein Handbuch für Hochschulen und Universitäten 


\title{
Über die Autoren
}

David Böhm ist wissenschaftlicher Mitarbeiter im Forschungsprojekt und übernahm neben der inhaltlichen Umsetzung der Projektziele, v a. gestalterische und technische Aufgaben. Nach seiner Berufsausbildung zum Mediengestalter Digital/Print absolvierte er den Bachelorstudiengang Buch- und Medienproduktion der HTWK Leipzig.

Alexander Grossmann ist Initiator und Leiter des Forschungsprojektes und seit 2013 Professor für Verlagsmanagement und Projektmanagement in Medienunternehmen (Buchhandel und Verlage) an der HTWK Leipzig. Vor dieser Tätigkeit war er über 12 Jahre in leitenden Positionen in verschiedenen Wissenschaftsverlagen. Im Projekt war er v. a. für die betriebswirtschaftlichen Betrachtungen sowie die nachhaltige Verstetigung des Workflows verantwortlich.

Michael Reiche ist Initiator und Leiter des Forschungsprojektes und seit 2008 Professor für Verfahrenstechnik der Medienvorstufe an der HTWK Leipzig. Innerhalb des Projektes verantwortete er v. a. die Entwicklung des Workflow-Modells und unterstützte die praktische und technikorientierte Durchführung dieses.

Antonia Schrader ist wissenschaftliche Mitarbeiterin im Forschungsprojekt und übernahm neben der inhaltlichen Umsetzung der Projektziele, v. a. koordinative und operative Aufgaben. Sie ist Absolventin des Masterstudiengangs Medienmanagement und des Bachelorstudiengangs Buch- und Medienproduktion der HTWK Leipzig.

\section{Bibliografische Information der Deutschen Nationalbibliothek}

Die Deutsche Nationalbibliothek verzeichnet diese Publikation in der Deutschen Nationalbibliografie. Detaillierte bibliografische Daten sind im Internet unter http://dnb.de abrufbar.

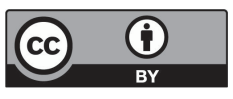

Der Text dieses Werks ist unter der Creative-Commons-Lizenz CC BY 4.0 International veröffentlicht. Den Vertragstext der Lizenz finden Sie unter https:// creativecommons.org/licenses/by/4.0/. Die Abbildungen sind von dieser Lizenz ausgenommen, hier liegt das Urheberrecht beim jeweiligen Rechteinhaber.

\section{Die Online-Version dieser Publikation ist abrufbar unter \\ http://doi.org/10.33968/9783966270175-00 \\ Das Addendum dieser Publikation ist abrufbar unter \\ http://doi.org/10.33968/9783966270175-11}

(C) 2020 David Böhm, Alexander Grossmann, Michael Reiche, Antonia Schrader

\author{
Herausgeber \\ Open-Access-Hochschulverlag \\ Karl-Liebknecht-Str. 132 \\ 04277 Leipzig \\ Deutschland \\ Gedruckt auf säurefreiem Papier \\ ISBN (Hardcover) 978-3-96627-015-1 \\ ISBN (Softcover) 978-3-96627-016-8 \\ ISBN (ePub) 978-3-96627-018-2 \\ ISBN (PDF) 978-3-96627-017-5
}

Hochschule für Technik, Wirtschaft und Kultur Leipzig

Druck und Bindung in Deutschland und den Niederlanden 


\section{Kurzfassung}

Die zeitnahe, transparente und nachhaltige Verbreitung nachprüfbarer wissenschaftlicher Ergebnisse ist eine der wesentlichen Anforderungen an die wissenschaftliche Kommunikation und Infrastruktur. Open Access, also die offene und kostenfreie Nutzung von wissenschaftlicher Literatur, ist hierfür die Grundvoraussetzung. Hochschulen und Universitäten sind in der Regel die Institutionen, an denen Wissenschaftler neue Forschungsergebnisse erzeugen und zur Veröffentlichung als Buch vorbereiten. Neben klassischen Wissenschaftsverlagen veröffentlichen daher immer mehr Hochschulverlage wissenschaftliche Publikationen.

Das vorliegende Handbuch beschreibt einen nachhaltigen, allgemeingültigen State-of-the-Art-Workflow zur Herstellung und Distribution von akademischen Büchern, der es Hochschulen und Universitäten ermöglicht, bei weitest möglicher Verbreitung, Sichtbarkeit und Zugänglichkeit eigene Forschungsarbeiten und Graduierungsschriften in digitaler Form im Open Access und als gedrucktes Buch zu veröffentlichen. Dieses Workflow-Modell wird anhand ausgewählter Fallbeispiele als Proof of Concept demonstriert und spiegelt den aktuellen Stand der derzeit im Verlagsbereich technischen und wirtschaftlichen Möglichkeiten wider. Anhand der Fallbeispiele wurden zudem der Zeit-, Kosten- und Personalaufwand erfasst, sodass anderen Hochschulen und Universitäten Anhaltspunkte für nötige Investitionen bei der Gründung und dem Betrieb eigener OA-Hochschulverlage gegeben werden.

\section{Abstract}

The need for an immediate, transparent and sustainable dissemination of current research has become a prerequisite for the further development of science today. Making research results freely available online as Open Access is considered to achieve the widest possible dissemination worldwide. Everyone can access anywhere Open Access publications without any restrictions or paywalls. Meanwhile more and more academic institutions and universities have already started their own presses to publish books Open Access.

In the present manual a sustainable and ideal workflow for producing and publishing academic books is presented. That workflow enables universities to publish their publications both as Open Access and printed books in a state-of-theart way and without any restrictions regarding the license, the variety of formats, print run etc. As proof of concepts we have processed different use cases which demonstrates the current status of the technically and economically possibilities in the publishing sector. Furthermore, data on time, costs and personnel resources were determined, which can be used by other universities and academic institutions to indicate the necessary investments for founding and operation of their own Open Access university presses. 



\section{Danksagung}

Wir möchten uns ganz herzlich bei allen Unterstützern bedanken, welche die Durchführung des Forschungsprojektes unterstützten und zur Erstellung des vorliegenden Werkes beitrugen. Insbesondere danken wir allen Personen, die bereit waren, in Workshops, in Interviews und vor Ort in den Verlagen, den Bibliotheken und bei den Dienstleistern, ihr Wissen mit uns zu teilen, mit uns zu diskutieren und damit an der Erstellung des Workflow-Modells beizutragen. Weiterhin gebührt all jenen Studierenden Dank, die im Rahmen ihrer akademischen Ausbildung in den Studiengängen Buch- und Medienproduktion und Medienmanagement an der HTWK Leipzig das Projekt unterstützten, indem sie bspw. Manuskripte ausgezeichnet haben und für das Gelingen von Workshops sorgten. Auch den verschiedenen Dienstleistern im In- und Ausland gebührt Dank für die Bereitschaft, sich auf Neues einzulassen. Und, selbstverständlich, soll dem Bundesministerium für Bildung und Forschung gedankt werden, welches das Projekt gefördert, begleitet und damit ein Zeichen für eine neue, offenere Wissenschaftskommunikation gesetzt hat.

Leipzig, Februar 2020

Die Autoren 



\section{Vorwort}

Die den Menschen umgebende Umwelt ist komplex und mehrdeutig. Diese Aussage gilt für alle Lebensbereiche; so auch für den beruflichen Alltag, vor allem vor dem Hintergrund der zunehmenden Automatisierung der Arbeitswelt und der aktuellen Digitalisierungsinitiativen. Die Open-Access-Strategie selbst entspringt den neuen Möglichkeiten der Digitalisierung und setzt eine etablierte digitale Infrastruktur voraus. Sie bettet sich gleichsam in die sich ständig wandelnde digitale Welt ein, weiß deren aktuelle Möglichkeiten zu nutzen und muss deren meist temporär anzusetzende Limitierungen kennen und respektieren. Sie profitiert aber vor allem von Demokratisierung der Mittel als Resultat der flächendeckenden Verfügbarkeit von Rechentechnik: Wo Produzenten und Konsumenten als Akteure der Medienwelt zuvor trennscharf nebeneinander auftraten, existiert nun jene neue Gattung der Prosumenten. Man eignet sich im disruptiven Modus Handeln und Hinterfragen die seit den Zeiten Gutenbergs immer weiter verfeinerte Infrastruktur zur Manifestation des Weltwissens in Büchern - bestehend aus dem Verlagswesen, dem Buchhandel und dem Bibliothekswesen - an, um dieser eine eigene, neue, vermeintlich schnellere und effizientere digitale entgegenzustellen.

Diese Situation lässt sich demnach so beschreiben, dass die etablierte Infrastruktur weiterhin besteht und bemüht ist, den laufenden Wandel ihrer Bedingungen aufzunehmen, während sich parallel dazu eine zweite etabliert, welche sicherlich die Erfolge der bestehenden Infrastruktur zu ihrem Vorteil zu nutzen weiß, zugleich aber eben auch neue Geschäftsfelder und neue Geschäftsprozesse schafft.

Dass dies zur Verunsicherung in einer etablierten Branche führt, muss nicht verwundern, hat doch die Vereindeutigung seit der Zeit der Druckerverleger Gutenberg'scher Prägung zu einer immer feineren Arbeitsteilung und zur Schaffung von spezialisierten Rollenmodellen geführt, deren Effizienz das Geschäftsmodell der Verlage erst erfolgreich werden ließ. Berufsausbildungen und Studiengänge schufen und schaffen genau die hierfür notwendigen Fähigkeiten und Fertigkeiten, bestimmen aber auch, trotz permanenter Aktualisierungsbemühungen, die Leistungsgrenzen des Systems. Die Durchsetzung der Gesellschaft mit moderner und mobiler Informationstechnik, der kaum mehr reglementierte Zugriff auf diese, schließlich die angesprochene Demokratisierung der Mittel: All das hat zur Verbreitung und zur Nutzung von Informationen durch jede Person, unabhängig von Zeit und Ort, geführt. Es ist mittlerweile eher die Regel als die Ausnahme, dass sich Fachfremde mit Hilfe neuer Technik die notwendigen Kompetenzen in alten Märkten und Geschäftsfeldern aneignen, diese umgestalten und vereinnahmen. Dieser Prozess der disruptiven Transformation begegnet uns in vielen Situationen des Alltags, genauso wie in zahlreichen Bereichen der Medienlandschaft. Ein Beispiel sind Enzyklopädien und lexikalische Verlage, deren Produkte, Wertschöpfungsketten und Geschäftsmodelle bis in die 2000er Jahre über 1 Jahrhundert stetig und wirtschaftlich erfolgreich existierten. Abgelöst wurden sie in einem disruptiven Wandel innerhalb kurzer Zeit durch die Internet-Plattform Wikipedia. Statt eines 
geschlossenen Kreises von Redakteuren arbeitet nun jeder, der möchte, an den Inhalten mit, die kostenlos und frei zugänglich jedermann zur Verfügung stehen und eine Weiternutzung erlauben.

Ambiguität ist einerseits eine Treiberin von Innovationen, weil sie Gleichzeitigkeit und Parallelität von Denkmustern und Strukturen zulässt, andererseits erhöht sie die Komplexität. Open Access (OA) ist ein gutes Beispiel für Ambiguität. Wissenschaftliche Ergebnisse sicht- und dann auch nutzbar zu machen ist in allen Disziplinen unerlässlich für die Förderung des Fortschritts. Das bedingt vor allem in den Natur- und Lebenswissenschaften ein hohes Veröffentlichungstempo, welches die traditionellen Abläufe in der Gutenberg'sch geprägten Infrastruktur kaum noch halten können. Digitale Plattformen erlauben die Veröffentlichung von wissenschaftlichen Texten ohne die Zeitverzögerung eines Herstellungsprozesses physischer Produkte, der hin zum Rezipienten eine gewisse Logistikleistung zu erbringen hat. Diese veränderte Situation bringt nun neue Abläufe und Rollenmodelle hervor, welche die etablierten umgehen bzw. assimilieren; sie befinden sich derzeit in ihrer Erprobungsphase und verheißen noch wenig Verlässlichkeit. Nichtsdestoweniger werden sie ausprobiert, benutzt, verändert, verbessert, was zu einer großen Heterogenität und damit Verunsicherung im Feld führt. Effektivität wird dabei zwar oft erreicht, die Effizienz in der Regel jedoch nicht bewertet; auch, weil für die beobachtete Ambiguität (noch) keine Methoden hierzu entwickelt wurden. Eine Strategie des Umgangs mit Ambiguität kann der Versuch sein, Eindeutigkeit herzustellen bzw. wiederherzustellen und damit die Komplexität so weit wie möglich zu reduzieren. Wenn man unter dem Begriff des Modellierens die Schaffung einer abstrakten (und möglichst vereinfachten) Vorstellung eines komplexen Sachverhaltes verstehen will, bietet die Methode einen Ansatz für den Umgang mit der Problematik.

Diese Überlegungen waren die Ausgangslage für die vorliegende Veröffentlichung; zugleich formen sie die Grundlage der methodischen Vorgehensweise zur Erfassung eines medienneutralen, allgemeingültigen Workflows zur Veröffentlichung von wissenschaftlichen Arbeiten als Monografien in OA. Die hier veröffentlichten Ergebnisse lassen sich ohne weiteres grundsätzlich auch auf alle anderen Formen wissenschaftlicher Publikationen als Buch erweitern, wie bspw. herausgegebene Werke bzw. Sammelbände, Konferenz-Proceedings oder Lehrbücher. Das vorliegende Handbuch kann daher als Transferkonzept mit konkreten Handlungsanweisungen für andere Hochschulen und Universitäten dienen, die einen OAHochschulverlag etablieren wollen. Daneben soll das in diesem Projekt vorgestellte Konzept allgemein die Sichtbarkeit und Akzeptanz von OA als anerkannte und von Kollegen und Mitarbeitern dieser Einrichtungen unterstützte Form des offenen und freien Zugangs zu wissenschaftlichen Inhalten und Informationen stärken. 


\section{Inhaltsverzeichnis}

Abbildungsverzeichnis .......................................................................... XII

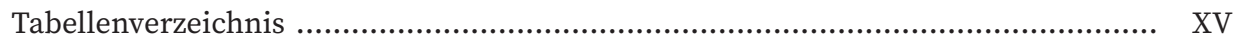

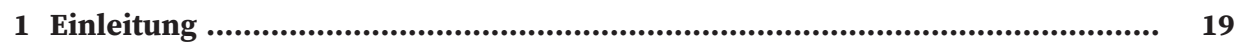

2 Modell und Modellierung ............................................................................... 33

2.1 Theoretischer Ansatz ...................................................................... 34

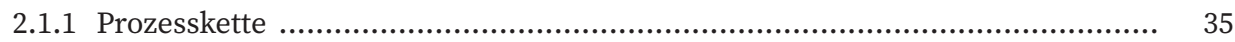

2.1.2 Prozessbegriff ................................................................................... 36

2.1.3 Prozessklassifikation ....................................................................... 38

2.1.4 Interaktionen ................................................................................. $\quad 39$

2.1.5 Abgrenzung und Benennung von Prozessen ........................................... 41

2.2 Merkmale des Workflow-Modells .............................................................. 42

2.3 Ist-Analyse .................................................................................. 44

2.4 Die Bedeutung der Rollen in einem Workflow ............................................. 48

3 Open-Access-Publikationsworkflow für akademische Bücher ....................... 53

3.1 Beschreibung des Workflow-Modells ..................................................... 53

3.1.1 Die Nomenklatur des Workflow-Modells .............................................. 54

3.1.2 Mega-Prozesse ............................................................................. 54

3.1.3 Null-Prozesse ...................................................................................... 55

3.1.4 Das Workflow-Modell nach Prozessgruppen .......................................... 57

3.1.4.1 Prozessgruppen der inhaltsorientierten Prozesse .................................. 57

3.1.4.2 Prozessgruppen der produktionsorientierten Prozesse ........................... 60

3.1.4.3 Prozessgruppen der managementorientierten Prozesse ........................... 62

3.2 Beschreibung der Workflow-Rollen .......................................................... 64

3.3 Qualitätsrichtlinien ..................................................................... 74

3.4 Prozesskennblätter ..................................................................... 76

4 Anwendung des Publikationsworkflow-Modells ............................................ 237

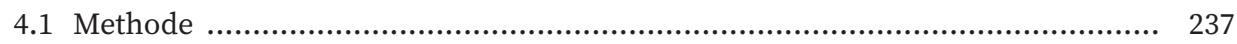

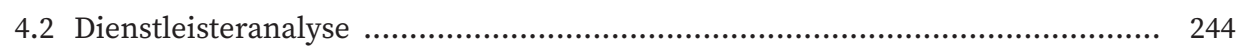

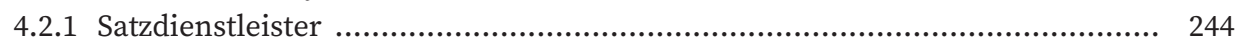

4.2.2 Druck- und Vertriebsdienstleister .................................................... 247

4.2.3 Veröffentlichungsplattformen für OA-Publikationen .............................. 250

4.2.4 Metadaten-Aggregatoren ..................................................................... 253

4.2.5 Dienstleister für Fach- und Sprachlektorat ........................................... 255

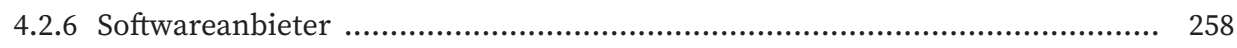




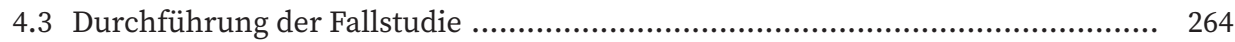

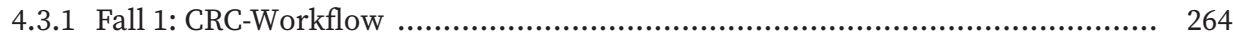

4.3.2 Fall 2: XML-first-Workflow in Fremdleistung .......................................... 285

4.3.3 Fall 3: XML-first-Workflow in Fremd- und Eigenleistung .......................... 301

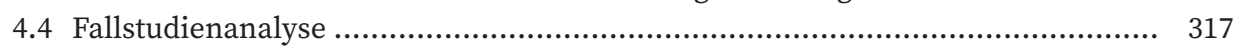

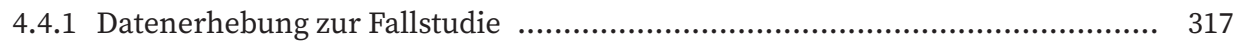

4.4.1.1 Übersicht zu den Workflow-Rollen .................................................. 317

4.4.1.2 Zeitlicher Ablauf der Publikationsworkflows ....................................... 320

4.4.1.3 Arbeitsaufwand der Publikationsworkflows ......................................... 329

4.4.1.4 Kostenaufwand der Publikationsworkflows ........................................ 331

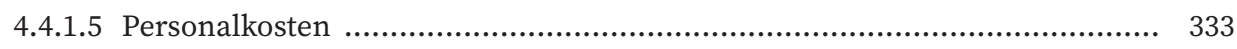

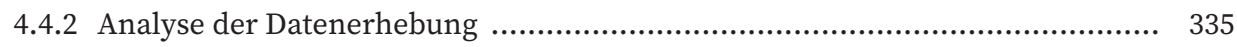

4.4.2.1 Analyse der Workflow-Rollen ......................................................... 336

4.4.2.2 Analyse des zeitlichen Ablaufs ........................................................ 336

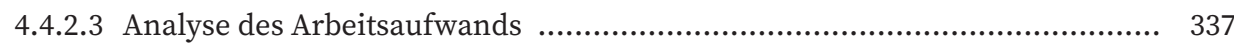

4.4.2.4 Analyse des Kostenaufwands ............................................................. 343

4.4.2.5 Analyse der Personalkosten ......................................................... 348

4.4.2.6 Analyse der Dienstleister ............................................................. 349

4.4.3 Diskussion der Ergebnisse ............................................................. 359

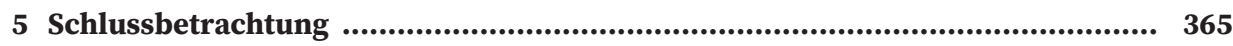

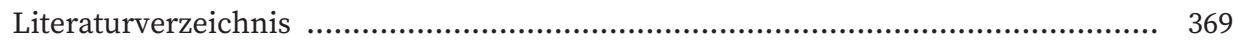

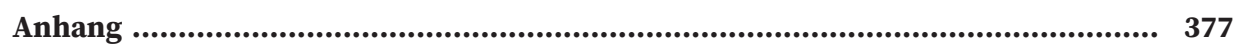

Anhang A Qualitätsrichtlinien .................................................................. 379

A.1 Qualitätsrichtlinien für inhaltsorientierte Prozesse .................................. 379

A.2 Qualitätsrichtlinien für produktionsorientierte Prozesse ............................ 382

A.3 Qualitätsrichtlinien für managementorientierte Prozesse ............................ 386

Anhang B Checklisten ......................................................................... 389

Anhang C Ladenpreis-Kalkulation ................................................................. 415

Anhang D Datenerhebung ....................................................................... 423

D.1 Arbeitsaufwand der Publikationsworkflows je Prozess ............................... 423

D.2 Kostenaufwand für Null-Prozesse ..................................................... 428

Anhang E Weitere Dokumente ........................................................................ 431

E.1 Informationsblatt für Autoren und Gutachter .......................................... 432

E.2 Bibliografische Angaben zu den Werken aus der Fallstudie ......................... 435 


\section{Abbildungsverzeichnis}

Abb. 1 Modell der Herleitung einer Monografie aus der niedrigen

Abstraktionsstufe

Abb. 2 Modellierung auf Prozessebene ......................................................... 38

Abb. 3 Verkettung von Prozessen ........................................................... 40

Abb. 4 Kaskadierung des Abstraktionslevels bzw. Prozessebenen .................... 41

Abb. 5 Das Prozesskennblatt ................................................................. 47

Abb. 6 Open-Access-Publikationsworkflow für akademische Bücher ................ 53

Abb. 7 Schema zur Bildung der Strukturnamen der Prozesse .......................... 54

Abb. 8 Mega-Prozesse des Workflow-Modells ................................................. 55

Abb. 9 Prozessgruppen der inhaltsorientierten Prozesse ............................. 57

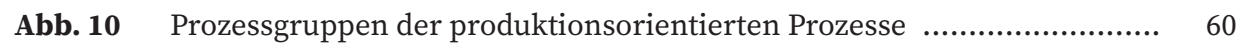

Abb. 11 Prozessgruppen der managementorientierten Prozesse ....................... 62

Abb. 12 Übersicht zur Ausführung der Workflow-Rollen in der Fallstudie ......... 318

Abb. 13 Gantt-Diagramm zum zeitlichen Ablauf von Fall 1 (S. 1/2) ..................... 321

Abb. 14 Gantt-Diagramm zum zeitlichen Ablauf von Fall 1 (S. 2/2) ..................... 322

Abb. 15 Gantt-Diagramm zum zeitlichen Ablauf von Fall 2 (S. 1/3) ..................... 323

Abb. 16 Gantt-Diagramm zum zeitlichen Ablauf von Fall 2 (S. 2/3) ..................... 324

Abb. 17 Gantt-Diagramm zum zeitlichen Ablauf von Fall 2 (S. 3/3) ..................... 325

Abb. 18 Gantt-Diagramm zum zeitlichen Ablauf von Fall 3 (S. 1/3) .................... 326

Abb. 19 Gantt-Diagramm zum zeitlichen Ablauf von Fall 3 (S. 2/3) ..................... 327

Abb. 20 Gantt-Diagramm zum zeitlichen Ablauf von Fall 3 (S. 3/3) ..................... 328

Abb. 21 Netzdiagramm zum Arbeitsaufwand der Publikationsworkflows ........... 331

Abb. 22 Prozentualer Verteilung des Arbeitsaufwands der Publikationsworkflows pro Prozessgruppe ..................................................................... 338

Abb. 23 Prozentuale Verteilung der Gesamtherstellungskosten der Publikationworkflows ................................................................. 344

Abb. 24 Netzdiagramm zur qualitativen Analyse der Satzdienstleister ................ 354

Abb. 25 Reklamation: Elemente des Buchrückens ragen auf die U1 des Buches (Fall 2)

Abb. 26 Sichtbare Schwankungen bei der Positionierung des Umschlags innerhalb einer Auflage (Fall 2)

Abb. 27 Netzdiagramm zur qualitativen Analyse der Druck- und

Vertriebsdienstleister .............................................................. 359

Abb. 28 Checkliste für die Publikationsfähigkeit des Werkes (S. 1/4) $\quad$.................. 390

Abb. 29 Checkliste für die Publikationsfähigkeit des Werkes (S. 2/4) $\quad$.................. 391

Abb. 30 Checkliste für die Publikationsfähigkeit des Werkes (S. 3/4) $\quad$.................. 392

Abb. 31 Checkliste für die Publikationsfähigkeit des Werkes (S. 4/4) $\quad$................... 393

Abb. 32 Richtlinie für die Produktfreigabe (S. 1/4) ........................................ 394 
Abb. 33 Richtlinie für die Produktfreigabe (S. 2/4) ......................................... 395

Abb. 34 Richtlinie für die Produktfreigabe (S. 3/4) ......................................... 396

Abb. 35 Richtlinie für die Produktfreigabe (S. 4/4) ...................................... 397

Abb. 36 Richtlinie für die Barrierefreiheit beim EPUB (S. 1/2) ......................... 398

Abb. 37 Richtlinie für die Barrierefreiheit beim EPUB (S. 2/2) ........................... 399

Abb. 38 Richtlinie für gedruckte Produkte ............................................. 400

Abb. 39 Autoren-Checkliste für die benötigten Informationen zum

Abb. 40 Autoren-Checkliste für die benötigten Informationen zum

Abb. 41 Autoren-Checkliste für die benötigten Informationen zum

Abb. 42 Autoren-Checkliste für die benötigten Informationen zum Vertragsabschluss (S. 4/4) .......................................................... 404

Abb. 43 Mustervertrag für Hochschulpublikationen (S. 1/4) ............................ 405

Abb. 44 Mustervertrag für Hochschulpublikationen (S. 2/4) ............................ 406

Abb. 45 Mustervertrag für Hochschulpublikationen (S. 3/4) ............................. 407

Abb. 46 Mustervertrag für Hochschulpublikationen (S. 4/4) ............................ 408

Abb. 47 Einverständniserklärung für das elektronische Publizieren auf dem Publikationsserver der HTWK Leipzig (S. 1/2) ................................... 409

Abb. 48 Einverständniserklärung für das elektronische Publizieren auf dem Publikationsserver der HTWK Leipzig (S. 2/2) ................................. 410

Abb. 49 Richtlinie für die Bildung von DOIs ............................................... 411

Abb. 50 Richtlinie für die Erstellung von Metadaten (S. 1/2) ........................... 412

Abb. 51 Richtlinie für die Erstellung von Metadaten (S. 2/2) ............................. 413

Abb. 52 Schema für eine progressive (Ladenpreis)-Kalkulation ........................ 415

Abb. 53 Informationsblatt für Autoren und Gutachter (S. 1/3) ......................... 432

Abb. 54 Informationsblatt für Autoren und Gutachter (S. 2/3) ......................... 433

Abb. 55 Informationsblatt für Autoren und Gutachter (S. 3/3) ......................... 434 


\section{Tabellenverzeichnis}

Tab. 1 Gebühren für die Publikationen von Buchkapiteln oder Monografien ausgewählter Fachverlage weltweit ............................................. 23

Tab. 2 Übersicht zu möglichen Null-Prozessen ........................................... 56

Tab. 3 Legende der verwendeten BPMN-Elemente in den Prozessabläufen ....... 77

Tab. 4 Übersicht der beauftragten Satzdienstleister der Fallstudie .................. 245

Tab. 5 Übersicht der angefragten Satzdienstleister, die nicht für die Fallstudie beauftragt wurden ..................................................................... 247

Tab. 6 Übersicht der beauftragten Druck- und Vertriebsdienstleister der Fallstudie 248

Tab. 7 Übersicht der angefragten Druck- und Vertriebsdienstleister, die nicht für die Fallstudie beauftragt wurden ............................................ 249

Tab. 8 Übersicht zu den OA-Veröffentlichungsplattformen der Fallstudie .......... 250

Tab. 9 Übersicht der Metadaten-Aggregatoren der Fallstudie .......................... 253

Tab. 10 Übersicht zu Dienstleister für Fach- und Sprachlektorat ....................... 255

Tab. 11 Übersicht der recherchierten Anbieter von Plagiatssoftware .................. 258

Tab. 12 Übersicht zu Anbietern von Content-Management-Systemen ................ 261

Tab. 13 Übersicht zu Anbietern von Customer-Relationship-ManagementSystemen ....................................................................... 262

Tab. 14 Arbeitsaufwand der Publikationsworkflows pro Prozessgruppe ............. 330

Tab. 15 Gesamt-Kostenaufwand der Publikationsworkflows ............................ 332

Tab. 16 Kostenaufwand der Publikationsworkflows für die Prozessgruppe P-CiP . 332

Tab. 17 Kostenaufwand der Publikationsworkflows für die Prozessgruppe P-GP .. 332

Tab. 18 Kostenaufwand der Publikationsworkflows für Prozess M-PVe-100 ....... 333

Tab. 19 Kostenaufwand der Publikationsworkflows für Prozess M-Pv-200 .......... 333

Tab. 20 Berechnung der Plankapazität einer Vollzeitstelle $(40 \mathrm{~h}) \quad$....................... 334

Tab. 21 Beispielhafte Berechnung der anfallenden Personalkosten einer Vollzeitstelle $(40 \mathrm{~h})$................................................................ 335

Tab. 22 Dauer der Publikationsworkflows ............................................... 337

Tab. 23 Kosten pro Seite ................................................................... 345

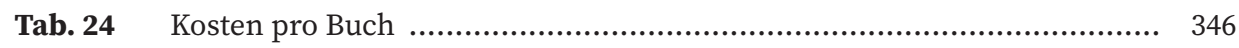

Tab. 25 Kosten für Prozess M-Pv-200 ........................................................ 347

Tab. 26 Summe der Personalkosten der Publikationworkflows ........................ 348

Tab. 27 Summe der Personalkosten, inkl. Druckkosten für Fall 2.2 und 3.2 ......... 349

Tab. 28 Qualitative Analyse von Dienstleister A ......................................... 350

Tab. 29 Qualitative Analyse von Dienstleister B ......................................... 351

Tab. 30 Qualitative Analyse von Dienstleister C ......................................... 352

Tab. 31 Bewertungsskala der beauftragten Satzdienstleister für die Fallstudie ..... 353

Tab. 32 Qualitative Analyse von Dienstleister E ......................................... 355 
Tab. 33 Qualitative Analyse von Dienstleister F ........................................ 357

Tab. 34 Qualitative Analyse von Dienstleister G ...................................... 357

Tab. 35 Qualitative Analyse von Dienstleister H ...................................... 358

Tab. 36 Bewertungsskala der beauftragten Satzdienstleister für die Fallstudie ..... 358

Tab. 37 Richtlinie für die Konzept-Annahme ............................................... 379

Tab. 38 Richtlinie für die Content-Vereinnahmung .................................. 380

Tab. 39 Richtlinie für die Plagiatsprüfung .............................................. 381

Tab. 40 Richtlinie für die Wahl des Peer-Review-Verfahrens ........................... 381

Tab. 41 Richtlinie für die Evaluation der Publikationsfähigkeit ...................... 381

Tab. 42 Richtlinie für die Publikationsannahme ..................................... 382

Tab. 43 Richtlinie für das Fachkorrektorat ................................................ 382

Tab. 44 Richtlinie für das Sprachlektorat .............................................. 382

Tab. 45 Richtlinie für das technische Copy Editing ................................... 383

Tab. 46 Cover- und Umschlag-Gestaltungsrichtlinie ................................. 383

Tab. 47 Richtlinie für die Produktfreigabe ............................................. 384

Tab. 48 Richtlinie für die Produktumsetzung ............................................ 384

Tab. 49 Richtlinie für gedruckte Produkte ................................................ 384

Tab. 50 Richtlinie für Pflichtexemplare ................................................ 385

Tab. 51 Richtlinie für die Wahl der primären OA-Veröffentlichungsplattform ...... 385

Tab. 52 Richtlinie für die Wahl weiterer Veröffentlichungsplattformen ............. 385

Tab. 53 Richtlinie für die Wahl des Langzeitarchivs .................................... 386

Tab. 54 Richtlinie für den Vertragsabschluss \& die Buchvorankündigung .......... 386

Tab. 55 Richtlinie für die Vertragsunterzeichnung .................................... 386

Tab. 56 Richtlinie für die Wahl des Produktionswegs ................................. 386

Tab. 57 Richtlinie für die Bildung von DOIs ........................................... 387

Tab. 58 Richtlinie zur Preisstrategie ........................................................ 387

Tab. 59 Richtlinie für die Erstellung von Metadaten .................................. 387

Tab. 60 Richtlinie für die Verbreitung von Metadaten .................................. 387

Tab. 61 Richtlinie zur internen Archivierung ............................................. 388

Tab. 62 Erläuterung der Ladenpreis-Kalkulation in der Fallstudie .................... 416

Tab. 63 Erwartete Herstellungskosten der Publikationsworkflows .................... 417

Tab. 64 Erwartete Vertriebskosten für die Ladenpreis-Kalkulation .................... 418

Tab. 65 Ladenpreis-Kalkulation für die Hardcover der Fallstudie ...................... 419

Tab. 66 Ladenpreis-Kalkulation für die Softcover der Fallstudie ...................... 420

Tab. 67 Ladenpreis-Kalkulation für den Fortdruck eines Hardcovers der

Tab. 68 Festgelegte Ladenpreise in der Fallstudie ......................................... 422

Tab. 69 Arbeitsaufwand der Publikationsworkflows der I-Ka-Prozesse ............... 423

Tab. 70 Arbeitsaufwand der Publikationsworkflows der I-Cv-Prozesse ............... 423

Tab. 71 Arbeitsaufwand der Publikationsworkflows der I-Qe-Prozesse ............... 424

Tab. 72 Arbeitsaufwand der Publikationsworkflows der I-Ca-Prozesse ............... 424

Tab. 73 Arbeitsaufwand der Publikationsworkflows der P-Ca-Prozesse .............. 424

Tab. 74 Arbeitsaufwand der Publikationsworkflows der P-CUe-Prozesse ............. 425 
Tab. 75 Arbeitsaufwand der Publikationsworkflows der P-CiP-Prozesse ............. 425

Tab. 76 Arbeitsaufwand der Publikationsworkflows der P-GP-Prozesse .............. 426

Tab. 77 Arbeitsaufwand der Publikationsworkflows der P-EP-Prozesse .............. 426

Tab. 78 Arbeitsaufwand der Publikationsworkflows der P-PFs-Prozesse ............. 426

Tab. 79 Arbeitsaufwand der Publikationsworkflows der P-PVe-Prozesse ............. 427

Tab. 80 Arbeitsaufwand der Publikationsworkflows der M-Pv-Prozesse ............. 427

Tab. 81 Arbeitsaufwand der Publikationsworkflows der M-MM-Prozesse ........... 427

Tab. 82 Arbeitsaufwand der Publikationsworkflows der M-Bb-Prozesse ............. 428

Tab. 83 Kostenaufwand des Null-Prozesses Software beschaffen ...................... 428

Tab. 84 Kostenaufwand des Null-Prozesses Mitgliedschaften schließen .............. 429

Tab. 85 Kostenaufwand des Null-Prozesses ISBN-Stamm erwerben .................. 430

Tab. 86 Kostenaufwand des Null-Prozesses Außendarstellung pflegen ............... 430 



\section{Einleitung}

Die zeitnahe, transparente und nachhaltige Verbreitung nachprüfbarer wissenschaftlicher Ergebnisse ist eine wesentliche Anforderung an heutige und zukünftige Kommunikationsformen in der Wissenschaft und an die dafür erforderliche Infrastruktur. ${ }^{1}$ OA stellt hierbei die Grundvoraussetzung für einen offenen und ungehinderten Zugang zu den Ergebnissen aktueller Forschung und neuesten wissenschaftlichen Informationen durch Rezipienten weltweit dar. ${ }^{2}$ Die weitreichende Bedeutung von $\mathrm{OA}$ für die rasche und freie Verbreitung von Forschungsergebnissen sowie der Einfluss auf ökonomische und gesellschaftliche Faktoren wurden ausführlich in einer umfangreichen Studie zusammengefasst. ${ }^{3}$ Zahlreiche Veröffentlichungen belegen außerdem, dass Forschungsarbeiten, die als OA veröffentlicht wurden, häufiger genutzt und zitiert werden (Impact). Diese Form der Veröffentlichung wissenschaftlicher Publikationen ermöglicht es darüber hinaus, neue, offenere und transparentere Verfahren zur Qualitätssicherung einzuführen und zu nutzen (Open Peer Review). ${ }^{4}$ OA-Plattformen verändern die wissenschaftliche Erkenntnisproduktion, soziale Medien und Wissenschaftsblogs die Wissenschaftskommunikation. Digitale Publikationsformen stellen die etablierten Veröffentlichungsweisen in vielen Bereichen der modernen Forschung heute vor Probleme. ${ }^{5}$

Während OA in Naturwissenschaften, Technik und Medizin (engl. STM) seit 2002 zunehmende Verbreitung erfährt und heute einen Anteil von etwa $15 \%$ der jährlich neu (in der Regel digital) veröffentlichten, ca. 2 Mio. Fachartikel in diesen Bereichen aufweist ${ }^{6}$, ist der Anteil in den Geistes- und Sozialwissenschaften sowie in den Rechtswissenschaften deutlich geringer ${ }^{7}$. Diese Statistik korreliert mit der Beobachtung, dass in diesen wissenschaftlichen Disziplinen die Publikation von Forschungsergebnissen als Monografie oder Sammelband in (gedruckter) Buchform überwiegt, im Gegensatz zu den STM-Bereichen.

OA bei wissenschaftlicher Fachliteratur umfasst alle frei zugängliche Beiträge in elektronischen Fachzeitschriften und Büchern oder Sammelwerken, Vorabveröffentlichungen von Manuskripten (Preprints) oder die Online-Fassungen von bereits veröffentlichten Fachbeiträgen als Postprint. Physisch können diese digitalen Werke von Forschern oder Institutionen auf Servern von Verlagen, universitä-

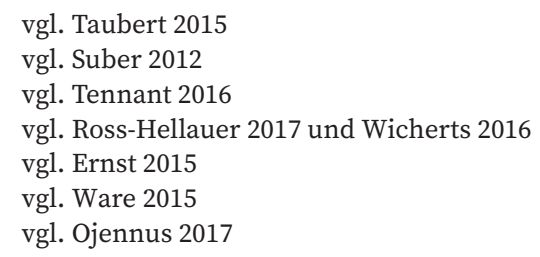


ren oder institutionellen Archiven, fachbezogenen Servern oder privaten Websites frei zur Verfügung gestellt werden. Dabei muss neben der Zugänglichkeit durch ein geeignetes digitales Format (z. B. PDF oder XML) auch die Auffindbarkeit durch entsprechende Metadaten sichergestellt sein. Eine zentrale Forderung der Berliner Erklärung für OA ist außerdem die Sicherung und Langzeitarchivierung der Beiträge. ${ }^{8}$ Auch schließt OA das Zugänglichmachen von wissenschaftlichen Primär- und Metadaten, Quellentexten und von digitalen Reproduktionen ein.

Der Anteil der OA-Veröffentlichungen in Fachzeitschriften oder bei einzelnen Beiträgen in Sammelwerken übersteigt bei Weitem die Zahl an OA-Büchern. Ende 2019 erscheinen weltweit 14.035 reine OA-Fachzeitschriften mit regelmäßiger Veröffentlichung, die im Directory of Open Access Journals (DOAJ) gelistet sind. Knapp viereinhalb Millionen Fachartikel in Zeitschriften sind derzeit als OA frei verfügbar. Dagegen beträgt die Zahl der als OA publizierten Bücher weltweit derzeit nur 22.191 Titel. International geben 327 kommerzielle Verlage und Universitätsverlage (engl. Presses) OA-Bücher heraus.

Die Leistungsmerkmale zur Erstellung eines OA-Buches erweitern in vielen Punkten die Anforderungen an die Publikation eines gedruckten Fachbuches. Darunter fallen u. a. die Lizenzierung als Creative-Commons-Lizenz (CC-Lizenz), die Vergabe eines Document Object Identifiers (DOI), eine Langzeitarchivierung oder die Aufnahme in das Directory of Open Access Books (DOAB). Letzteres Verzeichnis arbeitet analog zum bereits seit 2003 im Bereich von wissenschaftlichen Fachartikeln etablierten Verzeichnis der OA-Fachzeitschriften, dem DOAJ ${ }^{9}$ in Übereinstimmung mit den Kriterien zur Förderung durch Forschungsförderer in Europa (OAPEN - Open Access Publishing in European Networks) und die Weitergabe der Meta- und Katalogdaten an Aggregatoren sowie an die Deutsche Nationalbibliothek (DNB). Bei der Erfüllung dieser Anforderungen gibt es erhebliche Unterschiede zwischen den Anbietern, Verlagen oder University Presses. Daher sind nach einer 2015 durchgeführten Studie nur etwa mehr als $50 \%$ der als OA erschienenen wissenschaftlichen Bücher in den Katalogen von Hochschul- und Universitätsbibliotheken auffindbar ${ }^{10}$, was ein ernstzunehmendes Problem für $\mathrm{OA}$ bei Büchern darstellt. Insbesondere fehlt bei vielen OA-Büchern der Eintrag in OAPEN und das $D O A B$ als Grundvoraussetzung für eine umfassendere Sichtbarkeit und Verbreitung der Werke. ${ }^{11}$

Auch in den technischen Voraussetzungen für OA gibt es gravierende Unterschiede. Die medienneutrale digitale Aufbereitung von Artikeln in elektronischen Fachzeitschriften als Voraussetzung für OA wurde seit Anfang der 2000er Jahre zunehmend standardisiert. ${ }^{12}$ Diese Standardisierung beinhaltet die Erfassung und Auszeichnung der Volltexte in XML in JATS/NLM zur medienneutralen Datenhal-

vgl. Max-Planck-Gesellschaft 2019

vgl. Olijhoek 2015

vgl. Publishers Communication Group 2015

vgl. Snijder 2017

vgl. Journal of Pharmacology \& Pharmacotherapeutics 2010 
tung, Erstellung einer PDF-Instanz zur Verbreitung und Zugänglichmachung sowie einer einheitlichen Metadatenstruktur, die v. a. von Verlagen und den wichtigsten internationalen Repositorien im STM-Bereich, wie z. B. PubMed Central (PMC) ${ }^{13}$, sukzessive übernommen wurde. Im Gegensatz dazu liegen viele, auch von Fachverlagen als OA veröffentlichte Bücher derzeit nur als PDF-Dokument vor, da eine Aufbereitung in Volltext-XML in der Vorstufe nicht erfolgt ist. Außerdem verwenden die Verlage unterschiedliche Dokumenttypdefinitionen (engl. DTD), bzw. Schemata zur Auszeichnung von Inhalten und Metadaten. Nur wenige Fachverlage haben bislang ihre Produktion von E-Books, und damit auch die der OA-Werke, bereits vollständig auf eine Inhaltsaufbereitung in XML-BITS ${ }^{14}$ umgestellt, die kapitelweise ausgezeichnete Metadaten aufweist (z. B. Elsevier [NL]), De Gruyter [D]). Dieser Zustand erschwert die Aufnahme von OA-Büchern in Kataloge und Verzeichnisse und damit deren ungehinderte Verbreitung als zentrale Forderung von OA. Außerdem ist die Rezeption des digitalen Buches auf ein einziges, proprietäres Datenformat (PDF) eingeschränkt, was die Anforderungen der freien Portierung auf andere (mobile) Lesegeräte und die Langzeitarchivierung erschwert. Schließlich fehlt bei den meisten heute (digital) verfügbaren Fachbüchern eine Schnittstelle zu offenen Forschungsdaten, wie es die Forderung nach Transparenz und Reproduzierbarkeit als wesentlicher Bestandteil guter wissenschaftlicher Praxis verlangt. ${ }^{15}$

Nach wie vor spielen Monografien eine wichtige Rolle in der Wissenschaftskommunikation, v. a. in den Geistes- und Sozialwissenschaften wie auch den Rechtswissenschaften. Die Verfügbarkeit dieser wissenschaftlichen Literatur ist die Voraussetzung dafür, anschließende Forschung durchführen und neue Forschungsergebnisse veröffentlichen zu können. Im Laufe der 1990er Jahre führten kontinuierliche drastische Preissteigerungen nahezu aller regelmäßig erscheinenden wissenschaftlichen Fachzeitschriften zur sogenannten Zeitschriftenkrise (engl. serial crisis). ${ }^{16}$ So betrug etwa im Jahr 2004 der Anteil der Zeitschriften am Budget aller Institutionen der American Research Libraries (ARL) bereits über $75 \%$ des durchschnittlichen Beschaffungsetats dieser Bibliotheken. ${ }^{17}$ Diese Entwicklung ging damit in erheblichen Maße zu Lasten der nicht-periodischen Publikationen und führte insgesamt zu einem Rückgang der Anschaffungen von Fachbüchern. ${ }^{18}$ Die Einbußen bei den Verkäufen von Monografien bewirkten eine zunehmende Destabilisierung der akademischen Verlage und schufen Barrieren für Nachwuchsforscher, auch durch die geringe Auswahl an Publikationskanälen. ${ }^{19}$ Diese Ent-

vgl. PubMed Central 2019

vgl. BIT 2019

vgl. McKiernan 2016

vgl. u. a. Chan 2004; Harnad et al. 2004; Douglas 1990; Fisher 2008; Houghton 2001; Tananbaum 2003; Rose-Wiles 2011

vgl. Sperr 2006

vgl. Sperr 2006

vgl. Ferwerda 2017 
wicklung gefährdet den eingangs beschriebenen Stellenwert von Monografien in der Wissenschaftskommunikation.

Einen Ausweg aus dieser Krise weist das OA-Modell: Nach der Budapest Open Access Initiative (BOAI) wird der Begriff OA wie folgt definiert:

Open Access meint, dass [= Peer-Review-Fachliteratur] kostenfrei und öffentlich im Internet zugänglich sein sollte, so dass Interessenten die Volltexte lesen, herunterladen, kopieren, verteilen, drucken, in ihnen suchen, auf sie verweisen und sie auch sonst auf jede denkbare legale Weise nutzen können, ohne finanzielle, gesetzliche oder technische Barrieren jenseits von denen, die mit dem Internetzugang selbst verbunden sind. In allen Fragen des Wiederabdrucks und der Verteilung und in allen Fragen des Copyrights generell sollte die einzige Einschränkung darin bestehen, den Autoren die Kontrolle über ihre Arbeit zu belassen und deren Recht zu sichern, dass ihre Arbeit angemessen anerkannt und zitiert wird. ${ }^{20}$

Während der ungehinderte und kostenlose Zugang ein wesenhaftes Merkmal von $\mathrm{OA}$ ist, sind die Regelungen zur Weiter- und Nachnutzung der Inhalte unterschiedlich und in einer CC-Lizenz festgelegt. ${ }^{21}$ Das Fehlen einer solchen Lizenz oder eine Verfügbarmachung von Fachbüchern erst nach einer bestimmten Zeit (1 bis 3 Jahre) ohne konkrete Erläuterung der Möglichkeiten zur Nachnutzung stellt eine Grauzone dar, die nicht im Sinne der Definition der BOAI den Anforderungen an OA entspricht, aber von manchen Autoren oder Organisationen als eine Form des Green $O A$ bezeichnet wird. Da diese Nomenklatur oft zu Irritationen oder Unsicherheit bei Autoren führt, gehen wir in dieser Arbeit stets auf OA im Sinne der BOAI ein, die in der Literatur meist als Gold $O A$ bezeichnet wird.

Die Verbreitung von Monografien unterstützt OA auf zwei Arten: Einerseits werden durch eine digitale, im Internet frei verfügbare Fassung des Werkes Zugang und Auffindbarkeit erleichtert; andererseits besteht mit OA die Möglichkeit, neue Geschäftsmodelle in Anlehnung an die bereits länger bestehenden Formen bei wissenschaftlichen Zeitschriften zu entwickeln. Während bei Fachzeitschriften in der Regel Gebühren in Form von Article Processing Charges (APC) verlangt werden ${ }^{22}$, die vom Autor, seiner Hochschule oder einer Institution zur Forschungsförderung (DFG, FFW, Wellcome) an einen Verlag bezahlt werden müssen $^{23}$, werden diese Gebühren bei Monografien als Book Processing Charges (BPC) bezeichnet. In beiden Fällen wird die Gebühr nur bei einer Annahme und Veröffentlichung des Werkes erhoben, so dass präziser von einer Article/Book Publication Charge gesprochen werden müsste. Die Gebühren für die Veröffentlichung einzelner Beiträge als Gold OA in Fachzeitschriften sind inzwischen stark preisdifferenziert und liegen je nach Zeitschrift zwischen einigen Hundert Euro bis zu mehre-

20 vgl. Budapest Open Access Initiative (deutsche Übersetzung) (2019)

21 vgl. Creative Commons (2019)

22 vgl. Solomon 2016

23 vgl. Jahn, et al. 2016 
ren Tausend Euro pro Artikel; im Mittel betrug die von Institutionen zwischen 2005 und 2015 pro Fachbeitrag bezahlte APC $1.298 € .{ }^{24}$ Die Gebühren für die Publikation eines wissenschaftlichen Buches als OA liegen im Durchschnitt deutlich höher und werden entweder auf Basis einzelner Kapitel oder des ganzen Buches berechnet. Je nach Verlag schwankt die BPC sehr stark: zwischen unter $1.000 €$ bis über $15.000 €$ für eine einzelne OA-Buchproduktion ${ }^{25}$, ohne dass der Preis nachweisbar mit dem Leistungsangebot korreliert. In Tabelle 1 sind die BPC ausgewählter Verlage für eine Monografie nach Verlag und der CC-Lizenz dargestellt. ${ }^{26}$

Tab. 1 Gebühren für die Publikationen von Buchkapiteln oder Monografien ausgewählter Fachverlage weltweit ${ }^{27}$

\begin{tabular}{|c|c|c|c|}
\hline Publisher & $\begin{array}{l}\text { No. } \\
\text { of OA } \\
\text { books }\end{array}$ & BPC & License \\
\hline De Gruyter & 388 & 10000 EUR fixed price for monographs and textbooks & CC-BY-NC-ND \\
\hline Springer & 349 & $\begin{array}{l}17000 \text { USD per Monograph or Edit Volume } \\
2600 \text { USD per Chapter }\end{array}$ & CC-BY \\
\hline MDPI AG & 311 & $\begin{array}{l}\text { Monographs: from } 4000 \mathrm{CHF} \text { for accepted manuscript, } \\
\text { Edition Chpaters: from } 600 \mathrm{CHF} \text { for accepted manuscirpt } \\
\text { Both depending on word count }\end{array}$ & CC-BY \\
\hline \multirow{2}{*}{ Brill } & \multirow{2}{*}{237} & $\begin{array}{l}990 \text { EUR per chapter (up to } 35 \text { pages) } \\
8500 \text { EUR per book (up to } 350 \text { pages) } \\
14 \text { EUR per extra page }\end{array}$ & $\mathrm{CC}-\mathrm{BY}-\mathrm{NC}$ \\
\hline & & $\begin{array}{l}2590 \text { EUR per chapter (up to } 35 \text { pages) } \\
18500 \text { EUR per book (up to } 350 \text { pages) } \\
28 \text { EUR per extra page }\end{array}$ & CC-BY \\
\hline $\begin{array}{l}\text { Böhlau Publishing } \\
\text { House }\end{array}$ & 221 & no BPC found & no license found \\
\hline Bloomsbury Academic & 146 & $\begin{array}{l}\text { up to } 75000 \text { words: } 6500 \mathrm{GBP} \\
\text { from } 75000 \text { to } 90000 \text { words: } 7000 \mathrm{GBP} \\
\text { from } 90000 \text { to } 105000 \text { words: } 7500 \mathrm{GBP} \\
\text { from } 105000 \text { words and over: } 8500 \mathrm{GBP}\end{array}$ & CC-BY-NC-ND \\
\hline CNRS Éditions & 87 & $\begin{array}{l}\text { no BPC found } \\
\text { Freemium Solution via Open Edition }\end{array}$ & other license \\
\hline Palgrave Mcmillan & 81 & 11000 GBP for a monograph & $\mathrm{CC}-\mathrm{BY}$ \\
\hline $\begin{array}{l}\text { transcript Publishing } \\
\text { House }\end{array}$ & 79 & no BPC found & CC-BY-NC-ND \\
\hline Routledge & 33 & $10000 \mathrm{GBP}+$ taxes for monographs & CC-BY-NC-ND \\
\hline
\end{tabular}

In die Analyse dieser sehr stark variierenden Preisstruktur geht einerseits die Frage nach den tatsächlichen Kosten einer Monografie für einen Verlag ein, andererseits die Unsicherheit, ob OA die Verkäufe der gedruckten Fassung verringert oder 
sogar kannibalisiert. Zur Frage des wirtschaftlichen Aufwands einer wissenschaftlichen Buchpublikation ermittelte eine Studie von 2016 für den Verlag Kosten (ohne Overhead) zwischen 22.000 bis 34.000 \$ pro Buch. ${ }^{28}$ Diese Kosten erscheinen jedoch bei genauerer Betrachtung sehr hoch und decken sich nicht mit den Kosten, die bei einer progressiven Kalkulation eines Fachbuches (Bottom-Up-Rechnung) für sämtliche direkten und indirekten Kosten zum Deckungsbeitrag 2 (Gross Margin) beitragen. Diese liegen in der Regel deutlich unter den oben genannten Werten. Einer der Verfasser hat im Rahmen einer vor kurzem erschienenen Studie mit demselben Ansatz die Kosten für die Publikation eines wissenschaftlichen Fachaufsatzes berechnet und kommt je nach Wahl der Anbieter beim gleichen Leistungsumfang auf eine APC zwischen 200 und 1.000 \$, wobei hier hauptsächlich eine hohe Ablehnungsquote im Begutachtungsverfahren (Peer Review) die Gesamtkosten nach oben treibt ${ }^{29}$. Insofern war es auch eine Aufgabe der im vorliegenden Werk beschriebenen Untersuchung, eine Bestätigung der Kostenkalkulation für Monografien vorzunehmen, indem sowohl der Personalaufwand im Verlag als auch alle Kosten für externe Dienstleistungen und Services vollumfänglich berücksichtigt werden. Diese Kosten wurden über Fallbeispiele, also konkrete Buchveröffentlichungen, genau erfasst und durch Umrechnung des Zeitaufwands schließlich die tatsächlichen Aufwendungen für verschiedene Arten von Monografien ermittelt (vgl. Kapitel 4.4).

Kosten für ein einzelnes OA-Fachbuch von $1.000 €$ oder mehr stellen oft ein ernstzunehmendes Hindernis für Wissenschaftler dar, da nicht alle Forschungsförderer und nur wenige Institutionen in Europa eigene OA-Publikationsfonds für Bücher eingerichtet haben (Beispiel: Universität Zürich) oder diese Kosten aus den Etats der Bibliotheken kaum in nennenswertem Umfang bereitgestellt werden können. ${ }^{30}$ In den deutschsprachigen Ländern unterstützen derzeit lediglich die Volkswagen-Stiftung und der Austrian Science Fund bzw. die Universität Konstanz sowie die Akademie der Schönen Künste, Wien, Autoren bei der Finanzierung der BPC für OA-Fachbücher. Einer Veröffentlichung eines Fachbuchs als OA sind damit sowohl durch das Fehlen einer flächendeckenden Förderung, als auch durch die hohen Kosten der BPC momentan in Deutschland sehr hohe Hürden gesetzt. Im Unterschied zum drastischen Rückgang und zur Substitution gedruckter wissenschaftlicher Zeitschriften durch die Einführung digitaler Fassungen seit Ende der 1990er Jahre werden daher die Verkäufe gedruckter Monografien durch eine frei verfügbare digitale Fassung im Durchschnitt noch nicht beeinträchtigt. ${ }^{31}$ Dieses hybride Geschäftsmodell, also die Verfügbarmachung der digitalen Fassung als OA und Verkauf des gedruckten Buches in der verlagsüblichen Form, führt entgegen der verbreiteten Annahme also nicht zu einer Substitution oder gar Kannibalisierung der Printverkäufe von neu veröffentlichten Monografien. Während OA auf

28 vgl. Maron et al. 2016

29 vgl. Grossmann, Brembs n. d.

30 vgl. Nature 2019

31 vgl. Snijder 2014 
den Verkauf gedruckter Exemplare der sogenannten Frontlist eines Verlages keinen Einfluss hat, konnte in einer Studie ein negativer Effekt auf die Backlist, also bereits vor einigen Jahren erschienenen Werken gefunden werden. ${ }^{32}$ Hier sind die Abverkäufe allerdings ohnehin gering im Vergleich zum Jahr der Erstveröffentlichung, so dass aus Sicht eines Verlages durch OA nur die Long-Tail-Verkäufe beeinträchtigt würden, nicht aber die substantiellen Verläufe der Frontlist.

Trotz der Vorteile von OA auch für die Verbreitung und Auffindbarkeit von Monografien ist ihr Anteil an der Zahl der insgesamt publizierten wissenschaftlichen Bücher in den letzten Jahren nur langsam angestiegen. Ende 2019 waren insgesamt 22.191 wissenschaftliche Bücher von 327 Verlagen weltweit im DOAB aufgelistet $^{33}$. Zum Vergleich: Jährlich werden etwa 86.000 neue Fachbücher weltweit publiziert. ${ }^{34}$ Ein Grund für die relativ geringe Zunahme an OA-Monografien könnte der von vielen Fachverlagen verlangte BPC für die Veröffentlichung eines Buches sein. In Anbetracht der eingangs beschriebenen hohen Preise für die Veröffentlichung eines Fachbuches als Gold $O A$ muss auch nach der Finanzierbarkeit gefragt werden. Für Autoren, die OA veröffentlichen wollen, bieten Bibliotheken und die Deutsche Forschungsgemeinschaft (DFG) seit einigen Jahren Publikationsfonds an, die zusätzliche Mittel für die Veröffentlichung von Fachzeitschriftenbeiträgen als OA bereitstellen. Für Monografien gibt es in Deutschland eine vergleichbare, einheitliche Förderung, jedoch nicht in der Qualität wie etwa seit 2018 in der Schweiz: Dort fördert der Schweizer Nationalfonds (SNF) über eine transparente, modular aufgebaute Förderrichtlinie OA für Buchpublikationen mit Pauschalen und festen Obergrenzen. ${ }^{35}$ Ähnliche Fördermaßnahmen für OA-Bücher gibt es inzwischen auch in Österreich, nachdem zuvor die dortige Förderung von Fachbüchern über einen sogenannten Druckkostenzuschuss komplett abgeschafft wurde. Weltweit gab es 2017 insgesamt 18 Förderorganisationen, die Beihilfen zur Publikation von OA-Büchern oder Buchkapiteln vergeben. ${ }^{36}$

Eine wesentliche Bedeutung bei der Publikation wissenschaftlicher Ergebnisse kommt der Qualitätssicherung zu. Das gilt bei Fachzeitschriften genauso wie bei Fachbüchern. Seit Jahrzehnten haben Fachverlage weltweit Abläufe, Workflows und Standards entwickelt, die im Wesentlichen unverändert bis heute angewandt werden, wenn es um eine hohe Qualität der veröffentlichten Forschungsarbeiten geht. Davon ausgehend ist sofort klar, dass OA per se keinen Einfluss darauf hat, welche Qualität eine Fachpublikation hat, da diese stets durch den zugrundeliegenden Workflow und die Standards zur Qualitätssicherung im Verlag festgelegt wird, nicht durch das Geschäftsmodell. (Gold) OA ist in dieser wirtschaftlichen Betrachtungsweise lediglich ein alternatives Geschäftsmodell in der Verlagsbranche, dem kein verändertes Arbeitsprinzip in den Fachverlagen bei der Qualitätssicherung

\footnotetext{
32 vgl. Snijder 2014

33 vgl. Directory of Open Access Books 2019

34 vgl. Grimme 2016

35 vgl. SNF 2018

36 vgl. Ferwerda 2017
} 
zugrunde liegt. Die in der Presse oder in der Literatur leider immer wieder anzutreffenden gegenteiligen Behauptungen sind damit falsch; tatsächlich resultieren diese oft aus einer unzureichenden Kenntnis fundamentaler Prozesse der Verlagsindustrie und der Abläufe in Fachverlagen.

Folgende Aspekte kennzeichnen den Qualitätsstandard einer Fachveröffentlichung, also auch einer Monografie:

- Peer Review: die Begutachtung des eingereichten Werkes durch einen oder mehrere unabhängige Fachgutachter vor der Veröffentlichung sowie die Umsetzung der von den Gutachtern empfohlenen oder geforderten Korrekturen oder Ergänzungen. Bei Monografien, die in Reihen erscheinen, wird diese Aufgabe oft von den Herausgebern der Reihe oder einem Herausgebergremium (Editorial Board) übernommen.

- Lektorat: die Prüfung des von den Gutachtern freigegebenen Werks auf formale Stimmigkeit und Konsistenz, welche heute oft als Copy Editing bezeichnet wird und sich aufgrund der Komplexität der inhaltlichen Materie vom klassischen Lektorat, z. B. in Belletristik-Verlagen, grundsätzlich unterscheidet.

- Projektmanagement: die Planung, Koordination und Durchführung des gesamten Veröffentlichungsprozesses; von der Beratung des Autors vor Einreichen des Manuskriptes und der Disposition von Verlagsdienstleistungen bis hin zur Produktion der gedruckten und digitalen Fassung sowie der Anbindung an die Vertriebskanäle des Buchhandels.

- Verarbeitung und Ausstattung: die gedruckte Fassung soll im Hinblick auf Haltbarkeit, Langlebigkeit und Gebrauchsfertigkeit den Anforderungen an eine archivierbare Publikation entsprechen.

- Digitale Verbreitung: neben der gedruckten Fassung ist heute die elektronische Fassung einer Fachveröffentlichung, unabhängig von $\mathrm{OA}$, zwingend erforderlich, um eine schnelle Verbreitung, Weitergabe und Volltext- Recherche zu ermöglichen. Hierfür sind einerseits Metadaten zur Katalogisierung und für Suchmaschinen erforderlich, die sich an etablierten Standards orientieren (JATS, BITS); anderseits sollte der Volltext der Publikation in mehreren digitalen Formaten vorliegen, wie bspw. als PDF, EPUB und $\mathrm{MOBI}^{37}$, um die Lesbarkeit auf verschiedenen Endgeräten (Desktop, Tablet, Smartphone, E-Book-Reader) zu gewährleisten.

- Indizierung in Datenbanken: die wichtigsten Datenbanken für digitale Publikationen im Bereich OA sind OAPEN und DOAB, hinzu kommen weitere Repositorien von Institutionen oder Fachorganisationen. Außerdem ist die Anlage eines DOIs auf Buch- und Kapitelebene erforderlich, um die Auffindbarkeit weiter zu erhöhen.

- Langzeitarchivierung: die Monografien müssen unabhängig vom Verlag auch im Falle einer Geschäftsaufgabe oder Schließung dieses Verlages oder der tech- 
nischen Infrastruktur digital verfügbar bleiben. Dazu werden von Fachverlagen seit Jahren die Plattformen CLOCKSS und PORTICO genutzt, die auch eine Langzeitarchivierung von Büchern ermöglichen.

- Nutzungsdaten und Altmetrics: die Zahl der Zugriffe auf den Volltext soll auf einer tagesaktuellen Basis transparent für Autoren und Nutzer zusammen mit der digitalen Fassung der Monografie offen sichtbar sein. Zusätzlich sollen die Zitierungen der Monografie oder einzelner Kapitel in anderen Arbeiten gezählt und angezeigt werden. Darüber hinaus spielen heute Erwähnungen in sozialen Netzwerken, Blogs oder auf Webseiten eine deutlich höhere Rolle als noch vor einigen Jahren (Alternative Article [Book] Level Metrics = Altmetrics). Tagesaktuelle Daten darüber werden von Anbietern wie bspw. Altmetric bereitgestellt und erlauben einen einfachen und transparenten Überblick über den akademischen Diskurs und den Impact in der Community oder der Gesellschaft bereits unmittelbar nach Erscheinen einer Monografie.

- Marketing: wie jedes Buch benötigt auch ein wissenschaftliches Werk Marketing in Form geeigneter Maßnahmen. Das sind heute nicht mehr die klassischen Buchanzeigen in gedruckten Fachmagazinen oder Katalogen der Verlage, sondern Kampagnen in sozialen Netzwerken oder zielgerichtete Endkundenwerbung in anderen digitalen Medien. Auch die Integration in Aggregationsnetzwerke wie ResearchGate oder ScienceOpen erhöht die Sichtbarkeit und damit Nutzung der Werke, insbesondere im Kontext von kuratierten Listen (Collections). ${ }^{38}$

- Berücksichtigung der Förderrichtlinien: da in der Regel von einer Förderung der Monografie als OA ausgegangen wird, sind die dafür vom Förderer oder der Forschungsorganisation verlangten Voraussetzungen zu erfüllen (Compliance). Insbesondere sind der Förderer und das Förderkennzeichen in den Metadaten zu veröffentlichen (nicht nur im Volltext).

Eine weitere Darstellung vieler wichtiger Qualitätsstandards für OA-Monografien und -Sammelbände hat die Arbeitsgemeinschaft der Universitätsverlage entwickelt. ${ }^{39}$ Vorgenannte Aspekte und Prozesse zur Qualitätssicherung von Fachbüchern wurden in der vorliegenden Untersuchung analysiert und bei der Entwicklung des hier beschriebenen Workflow-Modells berücksichtigt.

Hochschulen und Universitäten stellen in der Regel die Institutionen dar, an denen Wissenschaftler neue Forschungsergebnisse erzeugen und zur Veröffentlichung als Buch vorbereiten. Aus den oben beschriebenen Gründen wird jedoch nur ein Bruchteil davon als OA publiziert und wenn, dann oft unter erheblichen (technischen oder lizenztechnischen) Einschränkungen hinsichtlich Format oder Metadaten. ${ }^{40}$ Das kann sich negativ auf die Sichtbarkeit und Auffindbarkeit von 
Veröffentlichungen, z. B. in institutionellen Repositorien, auswirken. Außerdem fehlt, was die ökonomischen und technischen Grundlagen betrifft, an den Institutionen und Hochschulen in der Regel Know-how aus der Verlagsbranche. Ein naheliegendes Ziel kann also die Befähigung dieser Einrichtungen sein, in einem kosten- und personaleffizienten Workflow ihre Publikationen als OA selbst zu veröffentlichen. Damit wäre eine deutliche Verbesserung hinsichtlich der eingangs beschriebenen Problemstellung aus ökonomischer wie aus technischer Sicht in absehbarer Zeit nachhaltig und unabhängig vom Forschungsgebiet möglich.

Eine Alternative zu klassischen Wissenschaftsverlagen können daher Universitätsverlage oder von Hochschulen und Institutionen selbst betriebene Verlagseinrichtungen sein. Universitätsnahe Fachverlage oder Eigenverlage der Universitäten gibt es seit etlichen Jahrzehnten. Jedoch gerieten auch diese Verlage mit den schrumpfenden Beschaffungsetats spätestens seit den 1990er Jahren stärker unter Druck, genau wie andere klein- und mittelständische Fachverlage. Die Zeitschriftenkrise führte jedoch nicht ursächlich zu einem Niedergang der Universitätsverlage, wie oft vermutet wird. ${ }^{41}$ Die Landschaft derartiger Universitätsverlage ist, hinsichtlich ihrer Entstehungsgeschichte oder Anbindung an die Hochschule, an Fachgesellschaften oder externe Verlage als Partner, sehr heterogen. Ebenfalls sehr unterschiedlich ist das angebotene Leistungsspektrum. In vielen Fällen konzentrieren sich diese Verlage auf bestimmte Fachbereiche oder bilden nur einen Teilbereich der weiter oben beschriebenen Merkmale zur Qualitätssicherung ab. OA wurde in diesen Verlagen anfangs eher als Nebenprodukt aufgefasst, der in der Regel auf die Ablage einer PDF-Datei auf einem institutseigenen Server oder anderen Repositorien beschränkt war. ${ }^{42}$ Mittlerweile bieten die meisten, aber längst nicht alle Universitätsverlage Gold $O A$ an. ${ }^{43}$ Nur in wenigen Fällen wurde ein (fachspezifischer) Verlag mit direkter Anbindung an eine Universität bzw. Fachgesellschaft systematisch und ohne externe Partner oder wirtschaftliche Kooperationen aufgebaut und hinsichtlich des angewandten Workflows dokumentiert, wie bspw. der Verlag Language Science Press in Berlin. ${ }^{44}$ Ende 2019 gab es in Deutschland von Aachen bis Würzburg 38 Universitätsverlage, die seit ihrer Gründung insgesamt zwischen 3 und 2.680 Bücher oder Buchwerke publiziert haben. ${ }^{45}$

Ziel der hier beschriebenen Untersuchung war es daher, einen nachhaltigen, allgemeingültigen State-of-the-Art-Workflow zur Herstellung und Verbreitung von OA-Monografien zu entwickeln, der es Hochschulen und Universitäten ermöglicht, ohne die eingangs beschriebenen Einschränkungen hinsichtlich technischem Leistungsumfang und bei größtmöglicher Verbreitung, Sichtbarkeit und Zugänglichkeit eigene Forschungsarbeiten und Graduierungsschriften in digitaler Form als OA und als gedrucktes Buch zu veröffentlichen. Dieser Workflow soll den Stand des

vgl. Jones et al. 2014

vgl. Hamann und Hürlimann 2018

vgl. Schüller-Zwierlein 2019

vgl. Nordhoff 2018

vgl. Schüller-Zwierlein 2019 
derzeit im Verlagsbereich technisch und wirtschaftlich Möglichen erreichen und dazu dienen, Studierende und Mitarbeiter, wo möglich, aktiv in dessen Umsetzung und stetige Weiterentwicklung einzubeziehen.

Der Workflow soll anhand ausgewählter Fallbeispiele als Proof of Concept demonstriert und abschließend angewendet in Form eines konkreten Prototyps eines OA-Hochschulverlags verstetigt werden, um die Nachhaltigkeit des Konzeptes zu demonstrieren. Das vorliegende Handbuch dient dazu, den beschriebenen Workflow so präzise und umfassend zu dokumentieren, dass eine leichte Übertragbarkeit an andere Institutionen und Hochschulen im Rahmen eines offenen Wissenstransfers gegeben ist.

Es soll die Forschungsfrage beantwortet werden, ob und wie es möglich ist, ein Workflow-Modell zu entwickeln, das idealtypisch, d. h. allgemeingültig durchführbar, personaleffizient und nachhaltig ist. Darüber hinaus sollen folgende Faktoren bei der Entwicklung des OA-Hochschulverlages als Modellverlag beachtet und umgesetzt werden:

- medienneutrale Aufbereitung von Texten, Formeln, Grafiken und Abbildungen in XML zur uneingeschränkten Weitergabe von Inhalten,

- alle Werke sind in den gängigen elektronischen Formaten (PDF, EPUB, MOBI) als OA verfügbar, außerdem in gedruckter Form als Soft- und Hardcover-Ausgabe,

- Verwendung der Industriestandards aus der Verlags- und Buchhandelsbranche zur Herstellung, Produktion, Katalogisierung und Verbreitung von

(Fach-)Büchern,

- Erfüllung bestehender OA-Policies und Förderrichtlinien (z. B. DFG, MPG, Wellcome, FFW) für OA,

- Schnittstelle zu offenen Forschungsdaten (Open Data),

- Qualitätssicherung durch Peer-Review-Verfahren,

- kostenneutrale Umsetzung von OA-Buchprojekten als Voraussetzung für Nachhaltigkeit durch Einbindung in studentische Projektarbeit im Curriculum,

- maximale Sichtbarkeit und Verbreitung der OA-Buchprojekte,

- leichte Integration des Modellverlages in öffentliche Institutionen, etwa Hochschulen und Universitäten bzw. deren Bibliotheken, zum nachhaltigen Transfer des Konzeptes.

Daraus ergeben sich folgende grundlegende Anforderungen an einen idealtypischen Workflow:

- vollständige, medienneutrale Erfassung des Inhalts (Text, Grafiken, Abbildungen, Referenzen, Verweise) in XML,

- Auszeichnung der Metadaten in BITS als NLM/JATS-kompatibler Standard im Buchbereich,

- Langzeitarchivierung (z. B. CLOCKSS oder PORTICO),

- Katalogeinträge in $O A P E N$ und $D O A B$, 
- Vergabe von ISBN und e-ISBN für alle Ausgabearten (Hardcover, Softcover, EPUB, ePDF, MOBI),

- kapitelweise Zuordnung eines DOIs, der bei Crossref registriert ist,

- Qualitätssicherung durch ein (offenes) Peer-Review-Verfahren,

- Kompatibilität mit vorhandenen OA-Policies und Richtlinien der Forschungsförderer in Europa (DFG, MPG, Wellcome, FWF u. a.),

- Anbindung an soziale Netzwerke und Open-Science-Plattformen,

- Indizierung der Metadaten in einer disziplinübergreifenden FachinformationsDatenbank,

- Schnittstelle zu offenen Forschungsdaten (Open Data).

Um diese Anforderungen in ein Modell umzusetzen und dieses auf Konsistenz und Anwendbarkeit zu prüfen, müssen die nachfolgend beschriebenen Phasen durchgeführt werden:

- Phase 1: Die Analyse und Modellierung eines medienneutralen Workflows zur Veröffentlichung wissenschaftlicher Hochschularbeiten als OA unter Einbeziehung aller klassischen und digitalen Distributionswege.

- Phase 2: Die Ableitung von Workflows für verschiedene Anwendungsszenarien in Form einer Fallstudie mit ausgewählten Manuskripten und deren Abarbeitung.

- Phase 3: Die Implementierung eines Pilotprojekts in Form eines nachhaltig wirtschaftenden Hochschulverlags zur regelmäßigen Veröffentlichung von Forschungs- oder Graduierungsarbeiten in gedruckter und digitaler Form.

In einer ersten Phase müssen die für die Erstellung eines abstrakten WorkflowModells notwendigen Informationen zusammengetragen werden. Dies geschieht im ersten Schritt semistrukturiert. Durch eine umfassende Literaturrecherche werden die im Feld vorhandenen Standards und etablierten Workflows bzw. BestPractice-Anwendungen analysiert und auf Adaptierbarkeit für das zu erstellende Modell untersucht. Zusätzlich werden weitere Informationen durch andere Methoden, wie Umfragen und Experteninterviews erhoben. Dabei ist es wichtig, alle Stakeholder, d.h., bereits bestehende Hochschulverlage, Hochschulbibliotheken, Wissenschaftsverlage und Dienstleister einzubeziehen, um die verschiedenen Sichten auf den gesamten Ablauf im Modell abbilden zu können. Hauptsächliches Ziel ist dabei, das derzeitige Leistungsspektrum, die Vertriebswege, Bearbeitungszeiten und Kosten vergleichbar zusammenzustellen. Aus diesem Informationspool wird das Workflow-Modell erstellt und in mehreren Iterationsschleifen gemeinsam mit den Stakeholdern, z. B. in Form von Workshops, vervollständigt und verfeinert.

Hat das Modell einen bestimmten Grad der Vollständigkeit erreicht, wird in einer zweiten Phase mit der praktischen Erprobung begonnen. Hierfür werden reale Manuskripte von Graduierungsarbeiten aus verschiedenen Fachgebieten und in unterschiedlichen Komplexitätsgraden in Form einer Fallstudie als OA-Mono- 
grafien hergestellt. Dabei sollen verschiedene konkrete Workflows, die von dem abstrakten Workflow-Modell abgeleitet werden, getestet werden. Diese Workflows sollen zwischen den extremen Polen der kompletten Eigenfertigung und dem Outsourcing aller Produktionsprozesse aufgestellt und durchgeführt werden. Dabei sind die Veröffentlichungen grundsätzlich OA digital sowie als gedrucktes Buch in unterschiedlichen Ausgabeformaten (Softcover, Hardcover, Hardcover mit Schutzumschlag und Fadenheftung) herzustellen. Die Erfahrungen aus der praktischen Erprobung fließen in das Workflow-Modell zurück. Als weiteres Ergebnis wird neben den Publikationen ein Manual zur Anwendung des Workflows an einer Hochschule erstellt. Dieses berücksichtigt neben den technischen Anforderungen auch alle wirtschaftlichen und rechtlichen Aspekte, die eine Verstetigung des Workflows im Sinne der Nachhaltigkeit des Konzeptes bei Umsetzung an einer Hochschule oder Universität erfordert.

In Phase 3 wird anhand der in Phase 2 verifizierten Workflow-Modells ein OA-Hochschulverlag als Modellverlag gegründet und aufgebaut, der in der Praxis die erarbeiteten Workflows verstetigt und für die Veröffentlichung neuer OABücher in der Zukunft sorgt. Dieser Hochschulverlag dient gleichzeitig als Prototyp für andere OA-Hochschulverlage. Ziel ist es, dadurch einen transparenten Wissenstransfer zu ermöglichen und Hilfestellungen zur Portierung und Weiternutzung des Workflows an anderen Standorten zu geben. Die konkrete Arbeitsweise des OA-Hochschulverlages und die einzelnen Schritte zum Transfer des Konzeptes an andere Einrichtungen werden in Form eines Handbuchs (Transfer-Tutorial) vollständig dokumentiert.

In den folgenden Kapiteln wird die angewandte Methodik (vgl. Kapitel 2) und das daraus abgeleitete Workflow-Modell zur Publikation von OA-Monografien an Hochschulen und Universitäten (vgl. Kapitel 3) allgemeingültig vorgestellt. Kapitel 4 beschreibt die Anwendung des Modells auf konkret definierte Fallbeispiele in Form einer Fallstudie. Die in diesem Zusammenhang erhobenen Daten werden anschließend im Hinblick auf eine Empfehlung für Nutzer an Hochschulen und Institutionen verglichen und diskutiert (vgl. Kapitel 4.4). Nach der Zusammenfassung (vgl. Kapitel 5) wird eine ausführliche Darstellung von Qualitätsrichtlinien und konkreter Checklisten zur Umsetzung des Workflows im Anhang gegeben. 



\section{Modell und Modellierung}

Ein Modell dient in erster Linie der diskussions- und anschlussfähigen Darstellung eines komplexen Sachverhalts. Eine eindeutige Kommunikation über einen Gegenstand kann grundsätzlich nur dann stattfinden, wenn die Kommunikationspartner sich einer vereinbarten Symbolmenge - das kann eine natürliche oder artifizielle Sprache sein, aber auch ein abstrakter Zeichenvorrat - bedienen, um mittels dieser die Kommunikationsinhalte auszutauschen: Dies geschieht, indem der Sender die Inhalte codiert und der Empfänger aufgrund der Vereinbarungen schließlich in der Lage ist, diese zu dekodieren. Gelingt dies nicht, sind Kommunikationsstörungen bis hin zum vollständigen Informationsverlust die Folge.

In dem heterogenen Umfeld der neu entstehenden Hochschulverlage ist die Kommunikation über Aufbau und Abläufe derselben ebenso verschieden wie deren Konzepte und Geschäftsmodelle. Es soll daher versucht werden, diese Kommunikation dadurch zu vereinheitlichen und zu unterstützen, indem ein abstraktes Modell aller Prozesse in einem Workflow zur Herstellung und Veröffentlichungen von OA-Monografien bereitgestellt wird. Bevor dieser eigentliche Modellierungsprozess beschrieben wird, sollen nachfolgend die Anforderungen an dieses Modell aufgeklärt und die Vorgehensweise bei der Modellbildung diskutiert werden.

Versteht man unter dem Begriff des Modellierens die Schaffung einer abstrakten (und möglichst vereinfachten) Vorstellung eines komplexen Sachverhaltes, lässt sich damit ein Ansatz entwickeln, das intendierte Problem zu lösen. Vor der Aufgabenstellung der Formulierung eines abstrakten (Workflow-)Modells für die Veröffentlichung von OA-Monografien ist es unerlässlich, auch zwei politelische ${ }^{46}$ Ziele zu formulieren:

Einerseits ist ein hoher Grad an Eindeutigkeit zu modellieren, damit sowohl die Komplexität gemindert wird, als auch die Anforderungen an Standardisierung und Automatisierung erfüllt und somit deren Umsetzung in die Praxis gesichert werden. Andererseits ist eine möglichst hohe Ambiguitätstoleranz herzustellen, um die Übertragbarkeit angesichts der heterogenen Einsatzbedingungen zu gewährleisten, was aber wiederum Komplexität in das Modell einbringt. In diesem Spannungsfeld ist der Modellierungsprozess durchzuführen. Üblicherweise wird als Modellierung ein Prozess verstanden, der dem Zweck dient, ein Abbild bzw. Modell des Ist-Zustandes des jeweiligen Betrachtungsfeldes zu erstellen. Dafür werden verschiedene Methoden eingesetzt: So etwa zur Analyse bestehender Pro-

46 Politelie ist ein Merkmal von Problemstellungen, zu deren Lösung nicht nur ein einziges Ziel, sondern mehrere, manchmal auch sich widersprechende Ziele verfolgt werden müssen. 
duktionsumgebungen die Technik der teilnehmenden Beobachtung, zumeist in Verbindung mit verschiedenen Interviewtechniken. Auf einem höheren Abstraktionslevel, dem der Referenzmodellierung, kann z. B. in unternehmensübergreifenden Arbeitsgruppen eine branchenweite Analyse vorgenommen werden. Ein erfolgreiches Beispiel für diesen Ansatz in der Verlagsbranche ist die Berliner Werkstatt Herstellung. ${ }^{47}$

Konsequenterweise entsteht im Ergebnis der Analyse ein in erster Näherung statisches Modell. Für die Zielstellung, ein Objektfeld über einen zeitlich konstanten Abschnitt diskutieren zu können, wird dieser Arbeitsschritt als Verständnisgewinnungsprozess verstanden.

Aus dem resultierenden Verständnisgewinnungsmodell lässt sich ein Vorgehensmodell ableiten. Dieses beschreibt die Schritte, die zur Erstellung eines Referenzmodells notwendig sind. Der Prozess startet mit der Einordnung des Modells hinsichtlich der Problemstellung. Dabei ist zwischen den Polstellen des reinen technischen Anwendungssystems und einer betriebswirtschaftlichen bzw. organisatorischen Anwendung zu unterscheiden. Beide besitzen eigene Modellierungsalgorithmen und Notationen. Der Modellansatz für einen allgemeingültigen Workflow zur Herstellung von OA-Monografien zielt vornehmlich auf die technische Beschreibung der Arbeitsabläufe ab, zugleich kann er nicht vollständig von der betriebswirtschaftlichen Betrachtungsebene abgekoppelt werden. Daher soll bei der Modellierung die technologische Sicht dominieren, d. h. es soll versucht werden, für die Modellbeschreibung eine möglichst abstrakte Notation anzuwenden. Weiterhin soll das Objektfeld und damit der Gültigkeitsbereich eingeschränkt werden. Hierbei ist auf höchster Ebene die Domäne der Hochschulverlage anzunehmen.

\subsection{Theoretischer Ansatz}

Um die zu erwartende Komplexität beim Modellierungsprozess zu bewältigen, ist es notwendig, diese konsequent auf dem jeweiligen Abstraktionslevel zu diskutieren. Dadurch wird vermieden, dass Detailprobleme die jeweilige Sicht verstellen. Ein geeigneter Abstraktionsansatz ist dabei, dass aus der Systemtheorie abgeleitete Black-Box-Modell. Kurz gesagt kann in der Box, die als ein offenes System im Sinne der Systemtheorie und weiterhin als eine triviale Maschine nach der Definition von v. Foerster ${ }^{48}$ angesehen werden soll, die Komplexität der Abläufe verborgen werden. Letztlich ist diese dann nur durch die Interaktionen des Systems mit der Umwelt für einen Beobachter im Luhmann'schen Sinne ${ }^{49}$ beobachtbar. Auf der höchsten Abstraktionsebene lässt sich das Modell also sehr einfach durch eine Box

47 In der Berliner Werkstatt Herstellung hatten sich Hersteller und Branchenexperten zusammengetan, um idealtypische Standardprozesse und Standardworkflows für die Herstellungsprozesse in den Verlagen zu definieren.

48 vgl. von Foerster 1993

49 vgl. Luhmann et al. 2008 
beschreiben: Diese soll alle möglichen Trajektorien zwischen dem Input und dem Output umfassen, die an der Eingangsseite durch das zu veröffentliche Manuskript und auf der Ausgabeseite durch die (mehrkanalig) erfolgte Veröffentlichung dargestellt werden (vgl. Abbildung 1).

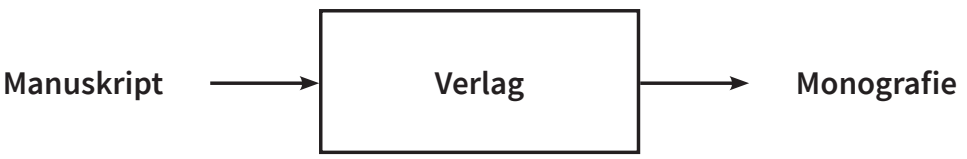

Abb. 1 Modell der Herleitung einer Monografie aus der niedrigen Abstraktionsstufe

Dieser Abstraktionsgrad ist für das zu erreichende Ziel zu niedrig. Eine sinnvolle Abstraktion lässt sich aber auf der Prozessebene vornehmen. Wie ein Prozess definiert werden kann, wird später dargestellt. Da das Modell einen allgemeingültigen und anpassbaren Publikationsworkflow für OA-Bücher wiedergeben soll, ist zunächst der Begriff „Workflow“ zu definieren.

\subsubsection{Prozesskette}

Für den Begriff „Workflow“ existieren verschiedene Definitionen. Die deutsche Übersetzung „Arbeitsvorgangskette“ transportiert die semantische Bedeutung allerdings nur ungenügend. In der Herkunftssprache besteht das Wort aus zwei separaten Begriffen, denen jeweils eine eigene Bedeutung zukommt. So ist im Begriff „Work“ die Summe aller Verrichtungen subsummiert, die notwendig sind, um ein Produkt mit all seinen vom Kunden vorgegebenen Eigenschaften herzustellen. In diesem Zusammenhang wird auch definiert, dass sich eine Verrichtung (auch Funktion oder Teilprozess) durch eine Wertschöpfung oder zumindest eine Eigenschaftsänderung an einem Produkt oder Teilprodukt erkennen lässt. Dies erleichtert die Abgrenzung einer solchen Verrichtung.

Der Teilbegriff „Flow“ leistet dann die Ordnung der als „Work“ definierten Verrichtungen in eine sinnvolle Reihenfolge. Diese muss noch nicht optimal sein: Im Ansatz genügt die Erreichung von Effektivität, d.h. die Reihenfolge muss zunächst nur sicherstellen, dass technologisch das gewünschte Ergebnis erreicht werden kann. Die Effizienz eines Workflows kann durch Optimierungen auch anschließend verbessert werden.

Die Flussmetapher lässt sich auf die drei hauptsächlichen Flüsse in einem Unternehmen anwenden. Der einer Betrachtung am ehesten zugängliche ist der Produktfluss: Er beschreibt den Weg des Produktes über eine gegebene Ausrüstungsstruktur ${ }^{50}$, konkret von den Ausgangsmaterialien über die im Workflow ent-

50 d. h., über alle im betrachteten Unternehmen zur Verfügung stehenden Maschinen, Anlagen und informationstechnische Infrastrukturen 
stehenden Teilprodukte bis hin zum Endprodukt mit allen vom Kunden vorgegebenen Eigenschaften. Bei der Betrachtung des Produktflusses geht es also um die jeweiligen Veränderungen des Produktes an den Arbeitsstationen und damit um die Wertschöpfung.

Als ein weiterer Fluss muss der Energiefluss beobachtet werden: In einer modernen Produktion existieren kaum noch Verrichtungen, die keine Energie verbrauchen. Um die Komplexität von Workflow-Beschreibungen zu mindern, bietet sich hier die Vereinfachung an: So wird das Vorhandensein der notwendigen Energie an den Arbeitsstationen als vorausgesetzt angenommen. Wie später detailliert beschrieben, wird die Sicherstellung der Energieversorgung in die Peripherie delegiert und muss nicht im Workflow-Modell betrachtet werden.

Der dritte hauptsächliche Fluss in einer Produktionsumgebung ist der Informationsfluss: Auch, wenn auf den ersten Blick der Produktfluss als der wichtigste für ein Workflow-Modell erscheint, wird er bei tatsächlich über den Informationsfluss, d. h., über die Modellierung der Metadaten, modelliert. Der Produktfluss ist in den meisten Umgebungen als nahezu linear anzunehmen (Ausnahmen bilden komplexe Fertigungsunternehmen im Maschinen-, Anlagen- und Fahrzeugbau), während der Informationsfluss ein darüber liegendes, engmaschiges Informationsnetzwerk aufspannt.

Dies bedingt eine kennzeichnende Eigenschaft moderner Produktionssysteme: Durch die zunehmende und teilweise bereits abgeschlossene Vernetzung der Arbeitsstationen eilt der Informationsfluss dem Produktfluss voraus. D. h., dass in dem Moment, in dem das Material eine Arbeitsstation erreicht, die notwendigen Informationen zur zu anstehenden Verrichtung bereits vorhanden sind und das Arbeitsmittel schon vorbereitet bzw. gerüstet und ohne Zeitverlust produktionsbereit ist. Dieser vorauseilende Informationsfluss enthält alle notwendigen Informationen (Metadaten) für die Produktion; er beschreibt also alle Eigenschaftstransformationen bis zur Realisierung des Endproduktes. Darum ist es konsequent, wenn ein Workflow-Modell den Informationsfluss modelliert.

\subsubsection{Prozessbegriff}

Um eine wirksame Prozessdefinition im Sinne der Systemtheorie vorzunehmen, sind zweiphilosophische Leistungen notwendig: Einerseits muss sich das, was später ein „Prozess“ genannt werden soll, von seiner Umgebung, also vom „Nichtprozess“, abgrenzen lassen, so dass dessen Input- und Outputbeziehungen beobachtet werden können. In einer Prozessumgebung bietet es sich an, die Abgrenzung durch den Verrichtungszweck vorzunehmen. So kann bspw. die gezielte Beschichtung eines Substrats mit Farbmitteln (das wir als „drucken“ bezeichnen können) auf einer Maschine stattfinden, die genau für diesen Zweck konstruiert wurde (hier also die Druckmaschine) und die in der Regel keine andere Funktionalität anbietet. Diese Maschine grenzt sich schon durch ihre Außenverkleidung sichtbar und damit vorstellbar von der Umwelt ab; sie wird, verkürzt gesagt, mit unbedruckten Bogen beschickt und produziert bedruckte Bogen. Hier fällt die Abgren- 
zung leicht. Problematischer ist dieser Vorgang, wenn es sich um Transformationen nicht-gegenständlicher (Teil-)Produkte handelt. So finden z. B. Satz- und Bildbearbeitungsprozesse auf derselben „Maschine“, einem Computer, statt. Die Verrichtungszwecke sind aber klar zu differenzieren: Das eine Mal werden Texte transformiert, das andere Mal (digitale) Bilder; in der Regel unter Verwendung je unterschiedlicher Applikationen oder Programme. In Softwareumgebungen sind aber auch Anwendungen vorstellbar, die mehrere Transformationen zulassen, z. B. im Zusammenhang mit hoch automatisierten Satzprozessen oder bei Inlinefertigung. Dann ist umso sorgfältiger der singuläre Verrichtungszweck zu bestimmen, damit der Prozess eindeutig abgegrenzt werden kann.

Die zweite philosophische Leistung ist die Benennung des abgegrenzten Prozesses. Hier kann zumeist auf ein vorhandenes, normal- oder (idealerweise) formalsprachliches Reservoir von Begriffen zurückgegriffen werden. Bei der verwendeten Terminologie ist weiterhin zu beachten, dass sie lokalisierbar sein muss, sich also für die Übersetzung in fremde Sprachen eignet. Um das Abstraktionslevel bei der Benennung zu erhöhen, kann eine Nomenklatur für die Prozessbenennung eingeführt werden.

Eine Nomenklatur ist eine Sammlung von Benennungen oder Begriffen in einer Domäne, für die sie ein kontrollierbares Vokabular darstellt. Aus Modellsicht werden an die Nomenklatur keine erhöhten Bedingungen gestellt. Sie muss jedoch skalierbar sein und es empfiehlt sich, sie „sprechend“ anzulegen, um die Akzeptanz zu erhöhen. D. h., Prozesse, die Strukturen zugeordnet werden, können über den Strukturnamen, oder - praxisnäher - über ein Kürzel des Strukturnamens eingeordnet werden, was sowohl beim Einpflegen in ein elektronisches WorkflowManagement-System als auch in der interpersonellen Kommunikation zu ein(ein)deutigen Ergebnissen führt. Für das Workflow-Modell wurde eine sprechende Nomenklatur entwickelt, die in Kapitel 3.1.1 erläutert wird.

Ist ein Prozess abgegrenzt und benannt, können dessen Interaktionen mit der Umwelt mittels eines Luhmann'schen Beobachters beobachtet werden. Diese Methodik bringt die Größe Zeit ein, da Beobachtungen in der Regel nicht einen diskreten Zeitpunkt, sondern einen Zeitraum betreffen. Beobachtet werden kann, wie sich auf der Input- und auf der Outputseite eines Prozesses die Größen ändern. So werden bspw. bei einem Prozess, der „Orthografiekorrektur“ genannt wurde, die vom Autor gelieferten, unkorrigierten Texte, einer Transformation in dann korrigierte Texte unterzogen. Diese Transformation ist auch eine Wertschöpfung, da die korrigierten Texte einen höheren Wert im Sinne der Gesamtwertschöpfung bei einer Buchherstellung besitzen. Es lässt sich also beobachten, wie die unkorrigierten Texte auf der Inputseite des Prozesses ihren Zustand ändern - „verbraucht“ werden -, während auf der Outputseite ein Teilprodukt „erscheint“, das zumindest einer Anforderung des Kunden an das (Buch)Produkt genügt, nämlich der, orthografisch und grammatisch korrekt zu sein. 


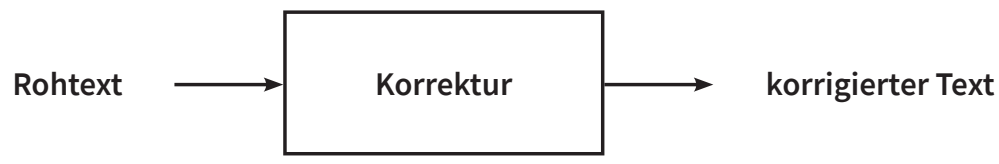

Abb. 2 Modellierung auf Prozessebene

Es ist also beobachtbar, dass sich der Zustand des Produktes über den Prozess geändert hat, analog zur Zustandsänderung des Prozesses selbst: Dieser hatte zu Beginn einen eindeutigen Anfangszustand, der dadurch beschrieben wurde, dass ein zu korrigierendes Manuskript vorlag. Da der Verrichtungszweck des Prozesses darin besteht, Texte zu korrigieren, war dieser Anfangszustand geeignet, den Prozess zu starten. Dies wiederum heißt, dass es eines Ereignisses bedarf, bei gegebenem Anfangszustand einen Prozess in Gang zu setzen. Dieses Ereignis kann von außen auf den Prozess einwirken oder auch in einer automatisierten Umgebung aus einer Kombination von Zuständen generiert werden. Im Beispielfall hat der Korrektor das zu korrigierende Manuskript in seinem Postfach entdeckt und daraufhin selbständig den Korrekturprozess begonnen. Dieser hat eine bestimmte Zeit gedauert, welche durch den Umfang des Manuskriptes, die Fehlerrate und die Arbeitsgeschwindigkeit des Korrektors bestimmt wurde. Der Prozess hat also Zeit „verbraucht“. Endlich hat der Korrektor den Prozess abgeschlossen und den gewünschten Endzustand hergestellt: das korrigierte Manuskript liegt vor.

Es ist also zu beobachten, dass der Prozess wie auch das zu transformierende Material seine jeweiligen Zustände ändert, wobei Zeit konsumiert wird und es bestimmter Ereignisse bedarf, diese zu kontrollieren. Diese Beobachtungen lassen sich verallgemeinern; hin auf eine Arbeitsdefinition für den Prozessbegriff im Rahmen des Modellierungsprozesses: Ein Prozess soll also als ein diskretes kybernetisches System verstanden werden, dessen Grenzen der Verrichtungszweck vorgibt. $\mathrm{Zu}$ beschreiben ist er durch seine Input-Output-Beziehungen. Der Prozess besitzt einen eindeutigen Anfangszustand: Er wird durch ein Ereignis initiiert und transformiert dann (unter Verbrauch von Energie) eine definierte Inputmenge in eine (möglichst ebenfalls definierte und voraussagbare) Outputmenge. Nachdem er seinen Endzweck erreicht hat, geht der Prozess in seinen Endzustand über.

\subsubsection{Prozessklassifikation}

Prozesse lassen sich nach vielfältigen Kriterien näher beschreiben. Die wichtigsten dabei sind der Prozesstyp, die Spezifik und die Orientierung auf einen Beobachtungsgegenstand.In der Prozesstypologie werden Kernprozesse, Supportprozesse und Administrationsprozesse unterschieden. Kennzeichnendes Unterscheidungsmerkmal ist der Grad der Wertschöpfung eines Prozesses. So kann angenommen werden, dass Kernprozesse per definitionem wertschöpfend sind, d. h. nach dem Ablauf des Prozesses liegt ein transformiertes Halbfertigprodukt vor, das im Wert höher einzuschätzen ist als vor Prozessbeginn. In einem Fertigungsunternehmen 
sind alle Fertigungsprozesse als Kernprozesse zu betrachten. In der Konsumgüterproduktion ist die Wertschöpfung gut zu beobachten, in einem Hochschulverlag ist diese Beobachtung dagegen schwieriger. Durch Korrektur- oder Lektoratsprozesse wird eine Wertschöpfung am Text, durch die Bildbearbeitung eine Wertschöpfung am Bildmaterial vorgenommen. Diese ist in der Regel kaum quantitativ darstellbar.

Supportprozesse sind nicht direkt wertschöpfend, sichern aber die Wertschöpfungsprozesse ab. Beispiele hierfür sind etwa Instandhaltungsprozesse in der Fertigung oder die Sicherung der Funktionsfähigkeit der Informationstechnik im Verlag. Manchmal werden auch die Einkaufsprozesse in diese Kategorie eingeordnet.

Administrationsprozesse sind ebenfalls nicht wertschöpfend, eigentlich sogar wertmindernd. Sie sind aber für die Funktionsfähigkeit eines Unternehmens unerlässlich, seien es Leitungsprozesse oder Personalprozesse. Für die hier perspektivierte Modellierung der Abläufe im Hochschulverlag soll sich weitestgehend auf wertschöpfende (bzw. eigenschaftsändernde) Prozesse beschränkt werden. Wenn eine Verschränkung in andere Prozessebenen notwendig ist, soll diese aber ebenfalls modelliert werden können.

Weiterhin sind idealtypische und unternehmensspezifische Prozesse zu unterscheiden: Idealtypische Prozesse sind dahingehend beschreibbar, dass die Beschreibung nicht nur in den Workflows eines einzelnen Unternehmens anwendbar ist, sondern eine allgemeine Gültigkeit für eine Domäne besitzt. Unternehmensspezifische Prozesse haben einen - zumeist auf ein Unternehmen oder eine Unternehmensgruppe - eingeschränkten Geltungsbereich und markieren so in der Regel die Abgrenzung zwischen Wettbewerbern. Im zu schaffenden Modell sind sie diejenigen spezifischen Prozesse, die den Unterschied der Herstellung von OAMonografien gegenüber herkömmlichen Büchern darstellen. Ziel des Modellierungsprozesses soll es daher sein, dass diese so allgemeingültig modelliert werden, dass sie als idealtypisch angesehen und eingesetzt werden können.

\subsubsection{Interaktionen}

Im vorherigen Abschnitt wurde für den Workflow-Begriff die semantische Bedeutung des Teilbegriffes „Flow“ herausgearbeitet. Es sei erinnert, dass darunter die Bildung einer sinnvollen Abfolge der Arbeitsverrichtungen, welche die eben definierten Prozesse darstellen, verstanden werden soll. Wenn sich Prozesse gegen ihre Umwelt abgrenzen, kann diese Folgenbildung nur durch Interaktionen mit der Umwelt beschrieben werden. Hier lässt sich eine Eigenschaft des Black-BoxAnsatzes nutzen: So kann beobachtet werden, dass sich der Output eines Prozesses, im obigen Beispiel das korrigierte Manuskript, als Input für einen nachfolgenden Prozess darstellt. Im Beispielsfall könnte das der Satzprozess sein, in dem die korrigierten Texte in das Layout gesetzt werden. Prozesse lassen sich also durch ihre Input-Output-Beziehungen miteinander verketten. Die Kenntnis dieser simplen Eigenschaft ist die notwendige Voraussetzung dafür, Workflow-Modelle zu entwerfen. 


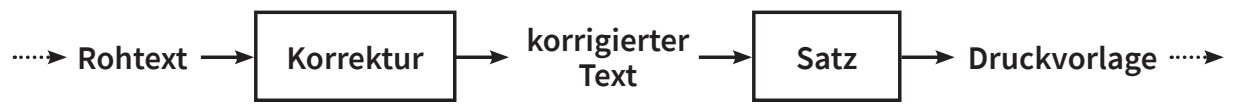

Abb. 3 Verkettung von Prozessen

Es ist zu beachten, dass sich Fertigungssysteme weitestgehend als Sequenz modellieren lassen, realistisch aber weniger Abfolgen, als vielmehr Netzwerke bilden. Daher sind für das Modell verschiedene Arten von Verzweigungen vorzusehen: Der Output eines Prozesses kann aufgeteilt werden und damit mehrere Nachfolgeprozesse bedienen ${ }^{51}$ bzw. können die Outputs mehrerer Prozesse vor einem Nachfolgeprozess zusammengeführt werden ${ }^{52}$. Letztendlich sind logische Verknüpfungen von Interaktionen über die Input-Output-Beziehungen möglich, d.h. über UND- und ODER-Verknüpfungen.

Das Abstraktionslevel des Modells wird also auf der Prozessebene festgelegt, so dass die Prozesse über ihre Inputs und Outputs im Sinne eines „Flows“ miteinander verknüpft werden können. Da sich sowohl der Input wie auch der Output eines Prozesses in der Regel zwar auf eine - meist beobachtbare - Eigenschaft beschränken, sind zumeist mehrere Metainformationen für die Ausführung notwendig. So wäre z. B. für einen Prozess „Bildbearbeitung“ neben Informationen zu den Speicherorten der zu bearbeitenden Bilder und ggf. Informationen zu den Transformationen, wie Beschnittgrößen, Tonwertkorrekturen oder Filteranwendungen notwendig, sondern auch Daten zum Auftrag, wie z. B. Termindaten, etc. D. h., wie auch der Output, besteht der Input aus einer Vielzahl von Metadaten, für die es zumeist genügt, sie als einen Vektor darzustellen ${ }^{53}$. Damit kommt, bei der Entscheidung, welche Metadaten als Input bzw. Output zu beachten sind, wieder Komplexität in das System. Bei der Entscheidung ist es also notwendig, strikt die gewählte Abstraktionsebene zu berücksichtigen. Viele Metainformationen, die vermeintlich zu diesem Abstraktionslevel gehören, sind übergeordneten (höheren) oder untergeordneten (tieferen) Abstraktionslevels zuzuordnen. Der oben angesprochene Energiefluss, der als Input für jedes technische System anzunehmen wäre, soll per definitionem nicht betrachtet werden. Das ist möglich, weil er einem höheren Abstraktionslevel zugehörig ist. Im konkreten Falle eines Hochschulverla-

51 Eine praktische Anwendung findet sich bei unterschiedlichen Fertigungsgeschwindigkeiten von Maschinen, indem der Ausstoß einer schnell fertigenden Maschine auf mehrere Maschinen mit einer geringeren Produktionsgeschwindigkeit (Engpassmaschinen) aufgeteilt wird, um die Gesamtproduktionsgeschwindigkeit aufrechterhalten zu können.

52 Hier wäre ein Montageprozess vorstellbar, bei dem Teilfertigprodukte aus verschiedenen vorgelagerten Prozessen zusammengefügt werden. Beispielhaft sei hier der Layoutprozess zu nennen, in dem die in vorangehenden Prozessen korrigierten Texte und bearbeiteten Bilder in das Buchlayout eingefügt werden.

53 In komplexeren Szenarien können auch mehrdimensionale Vektoren oder Matrizenzur Anwendung kommen. 
ges wäre das die Haustechnik, die dafür verantwortlich ist, dass die verwendeten Computer im Unternehmensnetzwerk einsatzbereit und erreichbar sind. Für das zu entwickelnde Modell sollen also nur diejenigen Metadaten extrahiert werden, welche auf der Prozessebene direkt verändert werden.

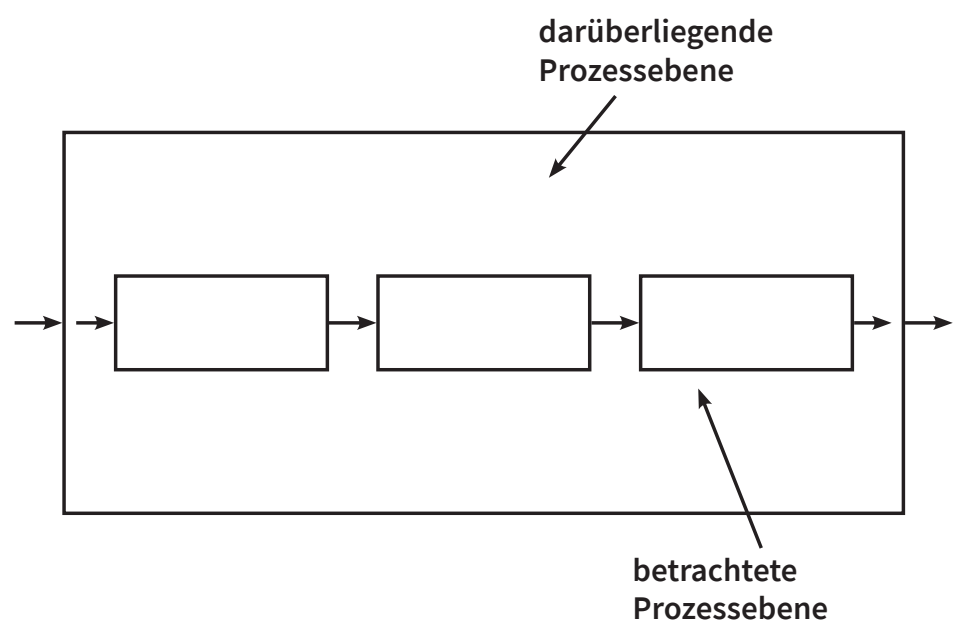

Abb. 4 Kaskadierung des Abstraktionslevels bzw. Prozessebenen

Es ist also sicherzustellen, dass nur all die Parameter betrachtet werden, welche auf dem gewählten Abstraktionslevel wirklich notwendig sind. Andere, wie z. B. die Energieversorgung der Prozesse, kann an eine darüber liegende Prozessebene, im angesprochenen Fall die Haustechnik, delegiert werden. Dadurch wird Komplexität reduziert. Die philosophische Arbeit des Abgrenzens und Benennens sowohl der Prozesse als auch aller relevanten Metainformationen im Rahmen einer IstAnalyse soll zur Erleichterung formalisiert werden. Zu diesem Zweck können Prozesskennblätter eingesetzt werden, die weiter unten im Kapitel erläutert werden.

\subsubsection{Abgrenzung und Benennung von Prozessen}

Für die Modellierung des Workflow-Modells und, zu diesem Zweck, zur Abgrenzung und Benennung der Prozesse für die Publikation von OA-Büchern, wurde sich - wie bereits in Kapitel 1 erläutert - zunächst auf Recherchen konzentriert.

Diese umfasst einerseits die Analyse der frei zugänglichen Webauftritte der kommerziellen und nichtkommerziellen Wissenschaftsverlage, auf denen Informationen für Autoren zum Publikationsablauf bereitgestellt werden. Andererseits wurden die Standardworkflow-Elemente der Berliner Werkstatt Herstellung als wichtige Datenbasis angesehen. Die Erkenntnisse der Berliner Werkstatt Herstellung sollen daher in das zu entwickelnde Workflow-Modell eingehen, insofern sie noch 
dem aktuellen Stand der Technik entsprechen und die Erfüllung der hier formulierten Zielstellung für das Workflow-Modell unterstützen.

Durch die Auswertung der aufgezählten Quellen konnten erste Prozesse für das Workflow-Modell formuliert werden. Durch Workshops ließen sich gemeinsam mit den Stakeholdern weitere Prozesse für die Publikation von OA-Monografien in akademischen Institutionen identifizieren und in das hier vorgestellte Workflow-Modell (vgl. Abbildung 6) modellieren.

\subsection{Merkmale des Workflow-Modells}

\section{Workflow-Varianten}

Im Rahmen der Modellierung wurde festgestellt, dass das Workflow-Modell, um den heterogenen Einsatzbedingungen in Hochschulverlagen entsprechen zu können, verschiedene, grundlegende Workflow-Varianten abbilden muss.

Graduierungs- und andere wissenschaftliche Arbeiten werden im Hochschulrahmen nicht originär für eine Veröffentlichung angefertigt, sondern entstehen in der Regel im Rahmen des Curriculums oder als Teil eines Forschungsprojektes. Dennoch entstehen so immer wieder veröffentlichungswerte Manuskripte. Diese sind zumeist mit einem Textverarbeitungssystem verfasst, z. B. Microsoft Word oder - in den naturwissenschaftlichen Disziplinen - mit TeX. Die Autoren dieser Arbeiten erstellen auf ihrem eigenen typografischen bzw. gestalterischen Niveau und mit den Mitteln und Limitierungen der eingesetzten Systeme mehr oder weniger druckreife Vorlagen. Zudem sind sie zumeist lediglich dazu verpflichtet, neben den Belegexemplaren ihrer Arbeit eine Version in einem geschlossenen digitalen Format (z. B. als PDF-Instanz) abzuliefern. Wenn diese Manuskripte als ausreichend angesehen und unverändert gedruckt werden, kann diese Workflow-Variante als Camera-Ready-Copy (kurz CRC) bzw. kamerafertig bezeichnet werden (vgl. Fall 1, Kapitel 4.3.1). Lektorats- bzw. Korrekturleistungen sind durch den Hochschulverlag nur in einem beschränkten Maße und auch nur unter Einbeziehung des Autors möglich, weil keine offenen Daten vorliegen. Soll das Manuskript in einer Reihe mit einem zugrundeliegenden Corporate Design erscheinen, sind die gestalterischen Möglichkeiten lediglich auf den Umschlag beschränkt. Zudem sind auf dieser Grundlage nur die digitale Veröffentlichung als PDF-Dokumente und ggf. der Druck der Monografie möglich. Andere Publikationsweisen, etwa als EBook im EPUB-Format, oder Zusatzangebote, wie Verlinkungen, können nicht realisiert werden. Sind CRC-Workflows für einen Hochschulverlag vorgesehen, sollten die Autoren dazu motiviert werden, ihre digitalen Manuskripte unter Verwendung von vorgefertigten Templates zu erstellen, die von der Hochschule zur Verfügung gestellt werden und die Einhaltung des Corporate Designs unterstützen. So kann zumindest in einem gewissen Maße gewährleistet und kontrolliert werden, dass die Veröffentlichungen formal und typografisch einheitlich sind.

Verbindliche Layouts und personaleffiziente Arbeit sind aber nur durch einen hohen Automatisierungsgrad des Publikationsworkflows zu erreichen. Rückgrat 
eines solchen automatisierten Workflows sind medienneutrale, zumeist auf der Basis von XML ausgezeichnete Manuskripte, welche durch Stylesheets in die speziellen Formate der verschiedenen Ausgabekanäle überführt werden. Die effizienteste Methode ist dabei die Nutzung sogenannter XML-first-Workflows, bei denen der Herstellungsprozess bereits mit einem medienneutral ausgezeichneten Manuskript beginnt. Es wird nicht ohne weiteres möglich sein, solche ausgezeichneten Manuskripte von den Autoren einzufordern. Um dies zu erreichen, bestehen zwei Möglichkeiten:

Zunächst kann man auch in diesem Zusammenhang die Autoren motivieren, Templates zu benutzen, welche allerdings nicht auf Typografie und Gestaltung abstellen, wie bei CRC-Workflows, sondern auf die logische Strukturierung der Manuskripte. Das können z. B. Word- oder InDesign-Instanzen sein, die sich in medienneutrale Strukturen exportieren lassen, welche dann die Eingabeformate der automatisierten Produktionsworkflows darstellen.

Darüber hinaus können die eingereichten Manuskripte unter der Kontrolle des Hochschulverlages ausgezeichnet werden, was sich wiederum in Eigen- (vgl. Fall 3.1, Kapitel 4.3.3) oder in Fremdleistung ${ }^{54}$ (vgl. u. a. Fall 2, Kapitel 4.3.2) realisieren lässt. Das Outsourcing von Dienstleistungen v. a. im Zusammenhang mit XML-Workflows wird im Moment auch von vielen etablierten Verlagen beabsichtigt oder bereits durchgeführt. Für einen Hochschulverlag, zumal in der Gründungsphase, sollten Outsourcing-Modelle zumindest in Erwägung gezogen werden, denn, wie bereits in Kapitel 1 erläutert, mangelt es bei einem Großteil der bereits existierenden Hochschulverlage an Kompetenzen sowie an personellen oder zeitlichen Ressourcen, um Publikationen selbst medienneutral umzusetzen. Wichtig ist bei der Entscheidung über ein Outsourcing die genaue Kenntnis der potentiell auszulagernden Prozesse. Auch hier soll das Workflow-Modell ansetzen und diese so feingranular beschreiben, dass es für verschiedene Fertigungstiefen ${ }^{55}$ anwendbar ist und als Kommunikations- und damit auch als verbindliche Verhandlungsgrundlage zwischen Hochschulverlag und Dienstleister fungieren kann.

Eine eher selten genutzte Workflow-Variante sind XML-last-Workflows. Bei diesen wird das Manuskript entweder als geschlossenes oder offenes Datenformat geliefert und aus diesen Daten dann die Druckvorlage für die Monografie erstellt. Anschließend werden die digitalen Formate aus dieser herausgezogen. Für einen Hochschulverlag ist diese Variante weniger effizient.

Die beiden zuletzt beschriebenen Workflow-Varianten werden auch als Single-Source-Multiple-Channel-Workflows beschrieben. D.h., dass die verschiede-

54 Darunter soll das Outsourcing von Prozessen an Dienstleistungsunternehmen verstanden werden. Welche Prozesse geeignet sind, um Satz- und Druckdienstleister als Rollenträger in den Publikationsworkflow einzubinden, wird durch die verschiedenen Anwendungsfälle in Kapitel 4.3 demonstriert.

55 Die Fertigungstiefe beschreibt das Verhältnis von Eigenfertigung und Fremdbezug und damit die vertikale Ausdehnung der Produktion. Eine hohe Fertigungstiefe haben Unternehmungen mit einem sehr hohen Eigenfertigungsanteil. 
nen Ausgabekanäle hier aus einer einzigen Datenquelle erzeugt werden, woraus sich mindestens zwei Vorteile ergeben: Erstens ist die Datenpflege sicherer und effizienter, da Änderungen lediglich in einer Quelle vorgenommen werden müssen und nicht in den verschiedenen Instanzen der Ausgabeformate; also in der Druckvorlage, im EPUB und in weiteren Formaten. Zweitens können die Formate aller Ausgabekanäle konsistent gehalten werden, indem sie on demand erzeugt werden; D. h., vorgehalten und dem Nutzer angeboten werden keine statischen Formate, sondern der Nutzer stößt die Erzeugung der Ausgabeformate erst an, wenn er Zugriff auf sie wünscht. Das Ausgabeformat über den gewählten Kanal wird damit erst zum Zeitpunkt des Bedarfes mit der aktuellen Datenversion für den anfordernden Nutzer erstellt. Darüber hinaus kann die Produktion der Ausgabe auch an die individuellen Bedürfnisse angepasst werden. Auf diese Weise lassen sich auch Anforderungen an die Barrierefreiheit umsetzen: So können etwa Dokumente für Sehgeschädigte in einem größeren Schriftgrad oder - bei Einschränkungen der Farbsehtüchtigkeit - in einer barrierefreien Farbenkombination ausgefertigt werden.

Eine personaleffiziente Infrastruktur für einen Hochschulverlag sollte daher mit einem Single-Source-Multiple-Channel-Workflow aufgebaut und das Workflow-Modell daher konsequent so modelliert werden, dass es derartige Workflows unterstützt.

\section{Mega-Prozesse}

Neben den Prozessen als solche enthält das Modell ein weiteres Klassifikationsmerkmal; die Unterteilung in Mega-Prozesse. Sie sollen einerseits der leichteren Orientierung im Modell dienen; andererseits wird erwartet, dass das Rollenmodell weitestgehend auf die Klassifikationsstufen abgestimmt und die abgeleiteten Stellen dann in den entsprechenden Stufen angelegt werden können (vgl. Kapitel 3.1.2).

\section{Null-Prozesse}

Zusätzlich müssen auch Prozesse betrachtet werden, die unternehmensspezifisch sind und somit zwar nicht direkt im Modell berücksichtigt werden können, jedoch das Verständnis des Workflow-Modells erleichtern. Sie liegen in der Verantwortung des Anwenders des Workflow-Modells und werden als Null-Prozesse definiert. Beispiele hierzu finden sich im Kapitel 3.1.3.

\subsection{Ist-Analyse}

Das Prozesskennblatt erfüllt im Modellierungsprozess zunächst zwei Funktionen: In der Startphase der Modellierung dient es der strukturierten Aufnahme von Informationen im Rahmen einer Ist-Analyse; nach deren Abschluss ist es die Grundlage für die Abstraktion und den Entwurf des Modells. Nach der Erstellung des Modells ermöglichen die Prozesskennblätter die Erweiterung der abstrakten Modellelemente. 


\section{Vorgehensweise}

Eine häufig angewendete Methode in der Ist-Analyse der Abläufe eines Unternehmens ist die top-down ablaufende, intellektuelle Analyse. Sie schöpft die notwendigen Informationen vorwiegend aus dem Studium von Dokumenten oder aus bestimmten Praktiken, etwa Expertenbefragungen. Häufiger Anwendung findet die Methode der teilnehmenden Beobachtung, bei welcher sich der Beobachter nahe oder idealerweise im zu analysierenden Arbeitsprozess befindet und die Informationen direkt aus den Informationsprozessen an der Wirkstelle ableiten kann. Beiden Ansätzen ist gemeinsam, dass eine große Menge an Daten anfällt, die im Beobachtungsprozess nicht sofort eingeschätzt und abstrahiert werden kann. Um den nachfolgenden Auswertungsprozess zu unterstützen, ist es sinnvoll, die Daten während des Beobachtungsprozesses in Prozesskennblättern strukturiert zu erfassen. Im Mittelpunkt der Beobachtung oder der Analyse steht ein einzelner Prozess, dessen Abgrenzung und idealerweise Benennung bereits erfolgt sind (1). Im Sinne einer systemtheoretischen Beobachtung werden seine vorgelagerten und nachfolgenden Prozesse identifiziert (2), die zu konsumierenden und zu produzierenden Materialien und Metadaten bestimmt und schließlich in das Formular eingetragen. Im Beobachtungs- bzw. Definitionsprozess muss dabei noch kein Augenmerk auf Vollständigkeit gelegt werden; der Analyseprozess ist iterativ angelegt und die Datenqualität wird in mehreren Schleifen verbessert. Der Zwang zur Formalisierung bei der Erfassung der Informationen im Prozesskennblatt reduziert aber bereits Komplexität. Für eine ungebundene, narrative Beschreibung des Prozesses steht aber ein Freitextfeld zur Verfügung (3).

In den meisten Anwendungsfällen wird eine sequentielle Vorgänger-Nachfolger-Beziehung der Prozesse angenommen werden können. Manchmal sind aber auch Prozessnetzwerke zu beschreiben, weil ein Prozess in der Praxis mehr als einen Vorgänger haben bzw. mit seinem Output mehr als einen Nachfolgeprozess bedienen kann. Auf dem Prozesskennblatt lassen sich die Vorgänger-NachfolgerBeziehungen mit bis zu 3 vorgelagerten bzw. nachfolgenden Prozessen erfassen. Im Ausführen der Prozesse zeigen diese unweigerlich ein Zeitverhalten, d. h. es fallen verschiedene Zeitenquantitäten an, die zu verschiedenen Zwecken nachgehalten werden müssen. Dies betrifft vor allem Rüst-, und Bearbeitungszeiten, die der Bearbeitungszweck des Prozesses einfordert. Auf der höchsten Abstraktionsstufe sind das die Zeiten, die vom Moment der Verfügbarkeit aller Inputs bis zur Bereitstellung des Outputs vergehen. Diese Spannen können einerseits in Zeiten für Teilprozesse, andererseits auch in Zeiten für verschiedene Ressourcen, etwa Personalressourcen (Arbeitszeit) und Maschinenressourcen (Maschinenzeiten) etc., unterteilt werden. In selten Fällen existieren Normzeiten, die für die zeitlichen Beschreibungen herangezogen werden können.

In den meisten Fällen lassen sich die Zeiten auch nur schwer quantifizieren. Um sicherzustellen, dass das Modell sie erfasst, wurden sie zunächst qualitativ in Form von Platzhaltern auf den verwendeten Prozesskennblättern dargestellt (4). Gleiches gilt für die Prozesskosten: Im Rahmen der Fallstudie wurden diese Größen quantifiziert, wenn dazu Informationen vorlagen. Zudem enthält das Prozess- 
kennblatt des hier beschriebenen Workflow-Modells Qualitätsrichtlinien (5), die als Hilfestellungen oder Festlegungen dienen, um das Workflow-Modell adaptieren zu können.

Jeder Prozess wird darüber hinaus durch eine Rolle oder unter der Verantwortung einer Rolle durchgeführt (6). Die am Prozess beteiligten Rollen werden ebenfalls im Kennblatt erfasst. Weiter unten wird der Rollenbegriff einführend besprochen. Die bisher diskutierten Angaben werden bei einer Ist-Aufnahme bzw. beim Prozessentwurf erfasst und iterativ im Entwurfsprozess geschärft. Wie oben beschrieben, ermöglicht das Prozesskennblatt nach der Modellierung - als Abstraktion der aufgenommenen Daten - die erweiternde, ausführliche Dokumentation des entworfenen Workflows. Während die abstrakte Darstellung auf die Prozessbezeichnung und die In- und Outputs reduziert wird, kann mit Hilfe des eindeutigen Struktur- bzw. Prozessnamens die ausführliche Beschreibung auf dem Prozesskennblatt referenziert werden. Um eine konsistente Dokumentation des Modells mittels der Prozesskennblätter zu garantieren, sollen diese nach Abschluss der Modellierung einem abschließenden Lektorat unterzogen werden. Die abstrakte modellhafte Darstellung und die Prozesskennblätter bilden die Kommunikationsbasis für das Workflow-Modell und sind daher immer gemeinsam zu verwenden. 


\section{COAHNerlag

(1)

PROZESS-NR.

PROZESSNAME

(2)

vorgelagerte Prozesse

nachgelagerte Prozesse

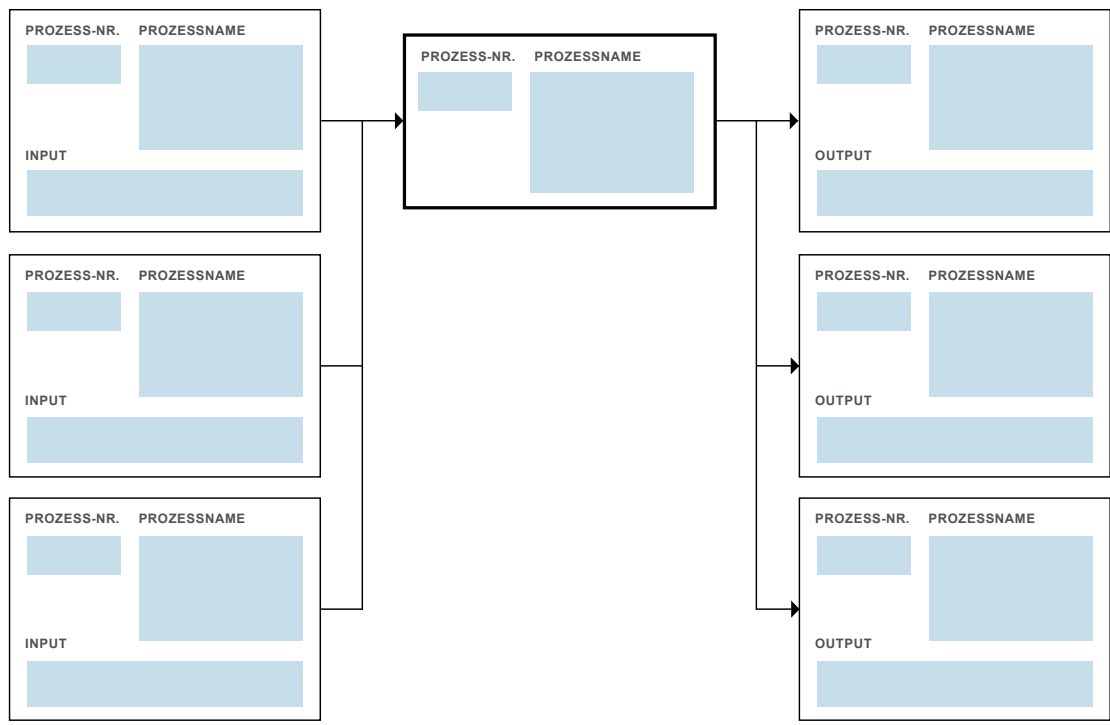

PROZESSBESCHREIBUNG (3)

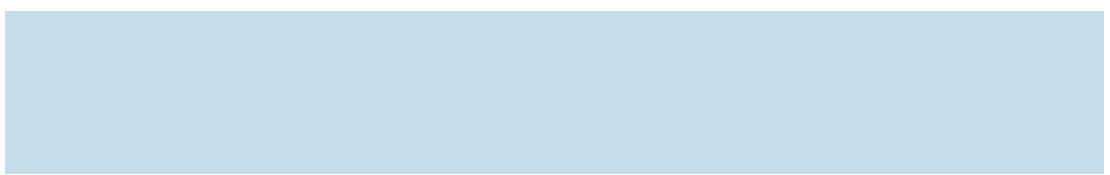

ROLLE (6)

RICHTLINIEN (5)

RESSOURCEN (4)
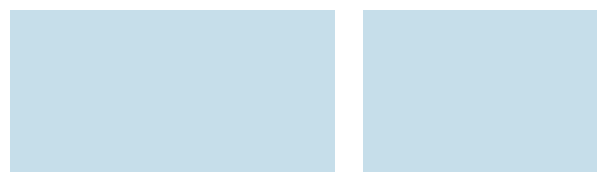

$\square$ KOSTEN

$\square$ ZEIT
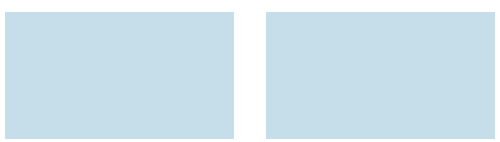

Abb. 5 Das Prozesskennblatt 


\subsection{Die Bedeutung der Rollen in einem Workflow}

Arbeitsteilige Prozesse sind dadurch gekennzeichnet, dass die einzelnen, spezialisierten Prozesse durch kompetente Mitarbeiter durchgeführt bzw. - bei hoch automatisierten Produktionen - überwacht und geführt werden. Diese Mitarbeiter besitzen in der Regel alle für die Ausführung von Prozessen notwendigen Fähigkeiten und Fertigkeiten, spielen also im Herstellungsprozess eine definierte Rolle. Damit kommt dem Rollenbegriff eine wichtige Bedeutung zu: Sieht man die Prozesse als Bestandteile der Ablauforganisation an, dann wird diese über die Rollen auf granularer Ebene mit der Organisation des Unternehmens verbunden. Die Rolle beschreibt im Zusammenhang mit der Prozessdefinition alle Anforderungen und Kompetenzen, welche an den jeweiligen Rollenträger gestellt werden, der die Rolle im Prozessablauf einnimmt. Dabei kann einerseits lediglich ein stark abgegrenztes Set an Fertigkeiten notwendig sein, wie z. B. bei der kurz getakteten Fließbandfertigung, oder aber ein umfangreiches, aus mehreren Kompetenzen bestehendes, wie beim Hersteller im Verlag oder beim Bibliothekar. Ein konsistentes Workflow-Modell ist ohne ein ebensolches Rollenmodell nicht vorstellbar. Ebenso, wie sich ändernde Anforderungen an die Fertigungsprozesse zu neuen Prozessdefinitionen führen, haben diese auch Einfluss auf die Rollen: Sie verändern sich, überkommende Rollen werden obsolet, neue entstehen. Im Sinne der Modellierung kann folgende Abhängigkeit von der Granularität der Prozessdefinition und vom Umfang der zugeordneten Rollen angenommen werden: Je niedriger der Abstraktionsgrad der Prozessbeschreibung ist, desto heterogener sind die Anforderungen an die Rollendefinition.

Dies soll kurz am Beispiel eines Herstellungsprozesses in einem Verlag erläutert werden. Definiert man diesen auf einem sehr niedrigen Abstraktionslevel, kann er hinreichend beschrieben werden anhand der Aufgabe der Transformation von eingehenden Manuskripten in druckfertige Daten. Konkret wird der Prozess an der Inputseite durch eingehende Rohtexte, nicht druckfertiges Bildmaterial usw. beaufschlagt; an der Outputseite werden druckfertige Vorlagen mit Qualitätsmerkmalen wie geeigneter Typografie, exakter Bildauflösung, korrekt zugeordneter Farbraumprofile etc. erwartet. Die zugeordnete Rolle des klassischen Herstellers erfordert daher alle Fähigkeiten und Fertigkeiten, die für diesen Transformationsprozess notwendig sind, d. h. mindestens Kompetenzen im Satz, in der Bildbearbeitung, im technischen Korrektorat sowie im Projektmanagement und der Autorenbetreuung. Heutzutage kommen noch weitere informationstechnische Anforderungen hinzu, wie die Beherrschung von Webtechnologien und mehrkanaligem Publizieren. Die aus den Anforderungen ableitbare Rollenbeschreibung stellt aktuell auf die klassischen Ausbildungsberufe des Mediengestalters oder die Abschlüsse von Publishing-Studiengängen ab; mit diesen Rollenbeschreibungen suchen Verlage neue Mitarbeiter. Gleiches lässt sich für die Rollenbeschreibung von Bibliotheksmitarbeitern sagen. Wird das Abstraktionslevel bei der Prozessdefinition erhöht, gliedert sich der klassische Herstellungsprozess in granulare Prozesse, wie Textbearbeitung/Satz, Bildbearbeitung, Projektma- 
nagement, Korrektorat etc. Damit werden die Inputs spezifischer, was die Anforderungen an die einzelnen Transformationsprozesse schärft. Das mindert insgesamt den Komplexitätsgrad, in der Konsequenz lassen sich die Prozesse so besser beherrschen. Gleichzeitig werden die Anforderungsprofile an die Rollen weniger komplex. Der Rollenträger für den Prozess Satz muss alle notwendigen Fähigkeiten und Fertigkeiten mitbringen, um mit den vorhandenen Satzwerkzeugen die Druckvorlagen nach den betrieblichen Anforderungen zu erstellen. Er muss aber nicht die Anforderungen aus dem Prozess Bildbearbeitung erfüllen oder das Buchprojekt insgesamt managen.

Da, wie oben ausgeführt, ein Modell für Herstellungsprozesse von OA-Monografien auf einer feingranularen Ebene aufgestellt werden soll, müssen auch die Rollen in einem adäquaten Abstraktionsgrad beschrieben werden. Grundsätzlich soll also gelten, dass im Modell lediglich die Anforderungen an die Rolle beschrieben werden, welche aus dem Prozess, dem die Rolle zugewiesen wird, erwachsen. Weiterhin muss diese Rolle konsistent benannt werden; wie für die Prozesse, sind auch für Rollen die beiden philosophischen Leistungen des Abgrenzens und des Bezeichnens zu erbringen.

In der betrieblichen Praxis wird allerdings weniger mit dem Rollenbegriff gearbeitet; geläufiger ist hier der personalwirtschaftliche Begriff der Stellen. In der Regel vereint eine Stelle unterschiedliche Rollen, so dass sich eine ausreichende Kapazitätsnachfrage für deren Schaffung ergibt. Erfordert ein Prozess genau eine Kapazität, die eine Stelle rechtfertigt, kann die Stelle der Rolle entsprechen. Ist die Kapazitätsanfrage gering bzw. fällt sie diskontinuierlich an, können die Kapazitätsanforderungen mehrerer Rollen zu einer Stelle gebündelt werden. Dies entspräche dann z. B. wieder der Rolle für einen Prozess auf einem niedrigeren Abstraktionslevel. In der betrieblichen Organisation werden Rollen also über den Stellenbegriff subsummiert. Für diesen werden üblicherweise Stellen- bzw. Arbeitsplatzbeschreibungen ausgearbeitet, welche beim Recruiting dafür sorgen sollen, dass entsprechend befähigte Personen ${ }^{56}$ gefunden werden, die die Stellen temporär besetzen und im Sinne des Workflow-Managements als Rollenträger im Zusammenhang mit den Prozessen auftreten. Damit in der späteren Praxis konsistente Stellenbeschreibungen erstellt werden können, ist es im Modellierungsprozess also wichtig, die Rolle als semantische Einheit scharf von der Stelle und dem Arbeitnehmer als Person zu trennen. Denn das Verhältnis zwischen der Rolle im Prozess und der Person, die die Rolle ausfüllt, kann verschieden implementiert werden. Folgende 3 Fälle sind zu unterscheiden:

1. Ein Prozess, dem eine Rolle zugeordnet ist, generiert genügend Kapazitätsnachfrage, so dass eine der Rolle entsprechende Stelle geschaffen werden

56 Das Skillset bzw. Kompetenzprofil einer Person soll alle Fähigkeiten und Fertigkeiten enthalten, welche diese durch Ausbildung, Weiterbildung und Erfahrung in den berufsbezogenen und sozialen Federn erworben hat. (vgl. Hesse/Schrader 2019) 
kann. Die Stellenbeschreibung stimmt dann vollständig mit den Anforderungen überein, die der Prozess an die Rolle stellt.

2. In einer Stelle können die Kapazitätsanforderungen mehrerer Rollen gebündelt werden. Dies geschieht, wenn

a) die Kapazitätsanforderungen aus einzelnen Prozessen zu gering sind oder

b) wenn die Kapazitätsanforderungen diskontinuierlich anfallen und eine Stelle nicht gleichmäßig ausgelastet werden kann.

3. Die Kapazitätsanforderungen aus einem Prozess sind so hoch, dass sie nicht von einer Stelle abgedeckt werden können. Somit werden mehrere Stellen geschaffen, die demAnforderungsprofil des Prozesses an die Rolle entsprechen. Dies geschieht bspw. auch bei Schichtarbeit oder Stellvertreterregelungen.

Abstrakt definiert die Rolle also die Summe aller Anforderungen, die ein Prozess an den Ausführenden stellt, um den Input in der gewünschten Zeit in einen entsprechenden Output zu transformieren. Je nach Komplexität des Prozesses bzw. abhängig von der Schärfe der Abgrenzung (Abstraktionslevel) kann dabei das Anforderungsprofil variieren. Der Erwerb der erforderlichen Fähigkeiten und Fertigkeiten bei den potentiellen Rollenträgern muss organisiert werden, damit sie bei Bedarf in ausreichendem Maß zur Verfügung stehen. Bisher wird dies im Rahmen der Lehr- und universitären Ausbildung gesichert. Berufsbilder bzw. Studiengänge sollen quantitativ wie qualitativ den Bedarf an Arbeitskräften decken. Dabei ist es notwendig, schnell auf den Wandel der Bedingungen zu reagieren. Grundständige Berufsbilder, wie Bibliothekar oder Mediengestalter mit den entsprechenden Studiengängen der Bibliothekswissenschaften und der Buch- und Medienproduktion, decken ein breites Spektrum von Fähigkeiten und Fertigkeiten in der jeweiligen Domäne ab und sichern mehr oder weniger gut deren Funktionsfähigkeiten. Die aktuelle Entwicklung der Dezentralisierung und Digitalisierung von Arbeit bedingt aber, dass sich die Kompetenzfelder in einem steigenden Maß überschneiden oder, anders ausgedrückt, die Abgrenzungen zwischen den Domänen unschärfer werden. Die Konsequenzen daraus sind einerseits die Überfrachtung der Lehrpläne in allen Ausbildungsformen und andererseits die zunehmende Zahl von spezialisierten Studiengängen an den Hochschulen. Diese wiederum führen zu heterogenen Kompetenzprofilen, die dem Markt zur Verfügung stehen. Wenn diese Profile auf unscharfe Rollenbeschreibungen treffen, ist die kompetente Besetzung der Rollen oder Stellen schwierig. Erhöhter Einarbeitungs- bzw. Schulungsaufwand ist die Folge. Unternehmen können hier kaum auf die Ausbildungsprofile in den Berufsund Hochschulen Einfluss nehmen, wohl aber auf die Rollendefinition im Sinne einer konsistenten Workflow-Definition; tatsächlich sollten sie diese Möglichkeit konsequent nutzen. Dies impliziert, dass die Rollenbeschreibungen sich nicht (mehr) ausschließlich an den bekannten Fähigkeiten und Fertigkeiten der etablierten Berufsbilder, sondern an den Notwendigkeiten der Unternehmensworkflows ausrichten müssen. Die Herstellung von OA-Veröffentlichungen generiert neue Anforderungen im Schnittmengenbereich von Verlag und Hochschulbibliothek. 
Daher sind im Zusammenhang mit dem Workflow-Modell neue Rollenbeschreibungen entstanden, welche in Kapitel 3.2 konkret für das hier entwickelte Workflow-Modell beschrieben werden.

Es bleibt hier zu konstatieren, dass in einem Hochschulverlag, der OA-Monografien verlegt, neue Stellen geschaffen werden müssen, welche die klassischen Kompetenzen von Herstellern und Bibliothekaren vereinen. Das Ziel kann jedoch nicht sein, dass jeder Rollenträger bzw. jede Person, die eine Stelle in diesem Verlag besetzt, alle hier notwendigen Anforderungen abdeckt. Tatsächlich ist dieser Anspruch nicht haltbar und würde zu einer weiteren Unschärfe der Ausbildungsprofile führen. Vielmehr bietet eine feingranulare Rollendefinition mit intelligenter Bündelung der Anforderungen, die zudem das eventuelle Outsourcen von speziellen bzw. diskontinuierlichen Bedarfen (Spitzen) beinhaltet, die Möglichkeit, die spezifischen Anforderungen eines Hochschulverlages richtig zu beschreiben. Aus diesen können präzise Stellenbeschreibungen abgeleitet und entstehende Hochschulverlage mit kompetentem Personal ausgestattet werden. 



\section{Open-Access-Publikationsworkflow für akademische Bücher}

Nachdem in den vorangegangenen Kapiteln erläutert wurde, aus welcher Motivation heraus das hier vorzustellende Workflow-Modell entstanden ist, wie es modelliert wurde und welche Anforderungen an die Entwicklung gestellt werden, erfolgt in diesem drittem Kapitel die konkrete Beschreibung der Merkmale des Modells.

\subsection{Beschreibung des Workflow-Modells}

Das Grundmodell ist als Wertschöpfungsketten-Modell angelegt und deriviert seine Notation entsprechend daraus. Abbildung 6 stellt das Überblicksmodell dar. Dessen einzelne Bestandteile werden im nachfolgenden Kapitel weitergehend diskutiert.

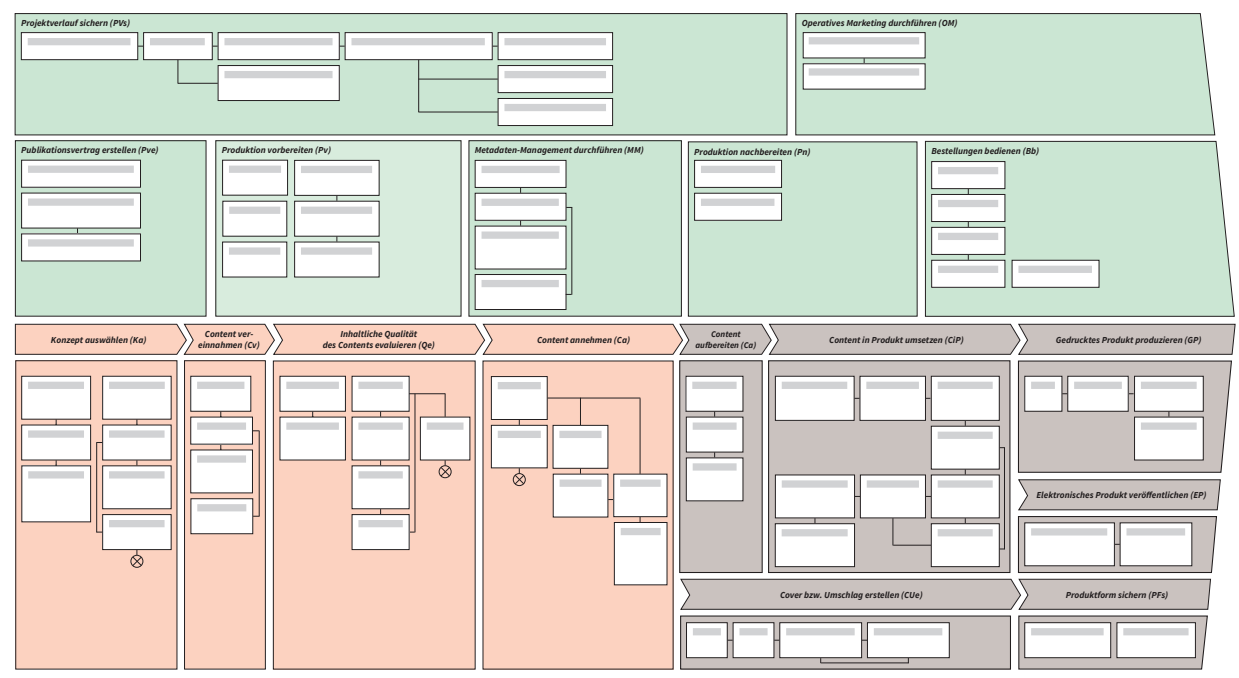

Abb. 6 Open-Access-Publikationsworkflow für akademische Bücher (veröffentlicht unter http://doi.org/10.14293/S2199-1006.1.SOR-.PPHCU45.v3) 


\subsubsection{Die Nomenklatur des Workflow-Modells}

Wie in Kapitel 2 erläutert, dient eine Nomenklatur der Orientierung in einem Modell. Nomenklaturen stellen in der Regel ein Kürzel des Strukturnamens dar und sollten skalierbar und „sprechend“ angelegt werden.

Diesem Anspruch entsprechend wurde die Nomenklatur für das hier beschriebene Workflow-Modell entwickelt. Der Strukturname ergibt sich aus den Kürzeln der Klassifikation von Mega-Prozess und Prozessgruppe, deren Bedeutung nachfolgend näher erläutert werden. Zum Zweck der Skalierbarkeit wurden die Prozessnamen statt mit Kürzeln mit Nummerierungen versehen. In der Regel werden aufeinanderfolgende Prozesse jeweils in Zehnerschritten nummeriert. Weisen Prozesse innerhalb einer Gruppe verschiedene Verrichtungszwecke auf, erfolgt die Nummerierung je 100er-Einheit. Diese Vorgehensweise macht das Modell nachhaltig und skalierbar. Da Workflows einer ständigen Veränderung unterworfen sind, ist eine Nomenklatur zu entwerfen, die es ermöglicht, in der Zukunft hinzukommende Prozesse in das Klassifikationsschema einordnen zu können. Daraus ergibt sich die Nomenklatur des Workflow-Modells, auf die nachfolgend referenziert wird.

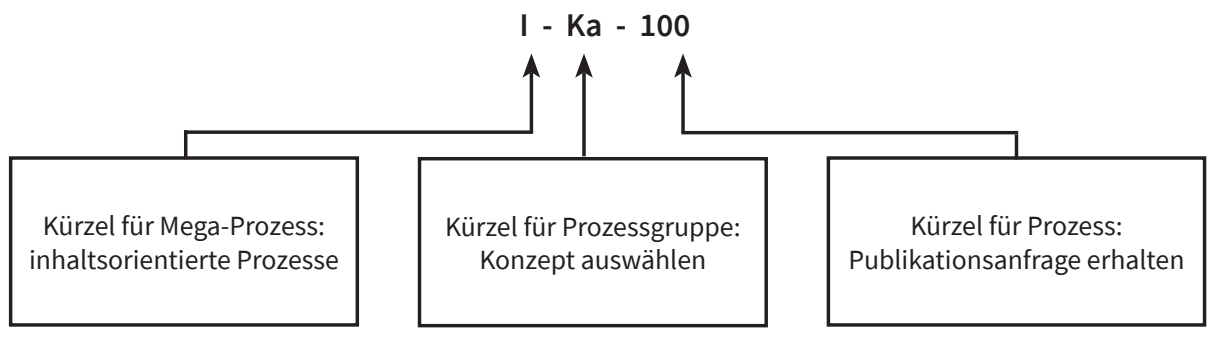

Abb. 7 Schema zur Bildung der Strukturnamen der Prozesse

\subsubsection{Mega-Prozesse}

Die Mega-Prozesse stellen ein Merkmal und die höchste Abstraktionsebene des Workflow-Modells dar. Sie lassen sich durch den Gegenstand, an dem die Wertschöpfung vollzogen wird, abgrenzen (vgl. Kapitel 2.2).

Hierzu zählt einerseits der zu veröffentlichte Inhalt - im weiteren Content genannt -, der akquiriert, ausgewählt, qualitätsgesichert und für die Veröffentlichung bzw. die Druckproduktion vorbereitet wird. Diese Prozesse werden im Modell als inhaltsorientiert (content oriented) bzw. I-Prozesse qualifiziert.

Ist die digitale Vorlage für die Veröffentlichung bzw. den Druck hergestellt, werden keine weiteren Veränderungen mehr am Inhalt vorgenommen. Das Dokument wird als Container betrachtet, der über die verschiedenen Kanäle bereitge- 
stellt werden muss. Die dafür notwendigen Prozesse werden als produktionsorientierte (production oriented) bzw. P-Prozesse bezeichnet.

Im Workflow-Modell wird davon ausgegangen, dass die inhalts- und produktorientierten Prozesse eine Sequenz bilden. Darüber hinaus wurden administrative, also managementorientierte bzw. M-Prozesse, beschrieben. Deren zeitliche Verortung erstreckt sich über den gesamten Workflow. Diese managementorientierten Prozesse können also grundsätzlich an jeder Stelle im Workflow begonnen werden, zugleich immer parallel zu den inhalts- und produktorientierten Prozessen verlaufen und gegebenenfalls in diese durchgreifen.

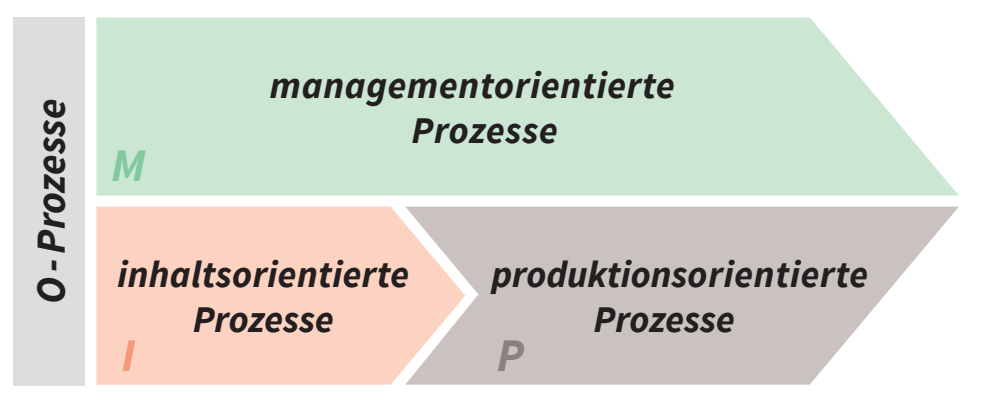

Abb. 8 Mega-Prozesse des Workflow-Modells

Neben den I-, P- und M-(Mega-)Prozessen werden Prozesse im Workflow-Modell in Prozessgruppen unterteilt. Vor der Erläuterung dieser sollen hier zunächst Beispiele für Null-Prozesse (vgl. Kapitel 2.2) angeführt werden.

\subsubsection{Null-Prozesse}

Null-Prozesse lassen sich von den idealtypischen Prozessen des Workflow-Modells durch ihre Eigenschaft abgrenzen, zwar nicht zur Wertschöpfung des Publikationsworkflows beizutragen, jedoch Voraussetzung für die operative Arbeit eines Hochschulverlages zu sein. Es sind initiierende, strategische Prozesse, die nicht oder äußerst selten wiederholt werden müssen und im Rahmen der Gründung des Verlages geklärt werden sollten. Für die Null-Prozesse wurden keine Prozesskennblätter erstellt. Tabelle 2 dient als Übersicht, welche Aktivitäten Null-Prozesse sein können. 
Tab. 2 Übersicht zu möglichen Null-Prozessen

Null-Prozess

Rechtsform für Verlag festlegen

\section{Beschreibung/Beispiele}

- Ist der Verlag eine Kapitalgesellschaft, ein eigenständiges Institut der Hochschule oder an die Hochschulbibliothek angegliedert?

\begin{tabular}{|c|c|c|}
\hline $\begin{array}{l}\text { Arbeitsplatzbeschreibungen } \\
\text { erstellen }\end{array}$ & $\begin{array}{l}- \\
-\end{array}$ & $\begin{array}{l}\text { Welche Tätigkeiten müssen wie durchgeführt } \\
\text { werden? } \\
\text { Welche Kompetenzen sind hierfür notwendig? }\end{array}$ \\
\hline $\begin{array}{l}\text { strategischen Marketing-Plan } \\
\text { erstellen und durchführen }\end{array}$ & $\begin{array}{l}- \\
- \\
- \\
-\end{array}$ & $\begin{array}{l}\text { Wie soll der Verlag innerhalb der Institution } \\
\text { positioniert sein? } \\
\text { Wie soll der Verlag innerhalb der } \\
\text { Publikationslandschaft positioniert sein? } \\
\text { Erstellung der Programmplanung } \\
\text { Soll sich einem Verbund, wie der } \\
\text { Arbeitsgemeinschaft der Universitätsverlage oder } \\
\text { Association of European University Press (AEUP), } \\
\text { angeschlossen werden? }\end{array}$ \\
\hline Außendarstellung pflegen & - & $\begin{array}{l}\text { Aufbau einer Verlagswebsite } \\
\text { Einrichtung offizieller Kontaktmöglichkeiten }\end{array}$ \\
\hline $\begin{array}{l}\text { Overhead-Kosten ermitteln und } \\
\text { Finanzierung klären }\end{array}$ & - & $\begin{array}{l}\text { Welche Kosten können nicht eindeutig dem } \\
\text { Publikationsworkflow zugeordnet werden? } \\
\text { Welche Kosten können nicht eindeutig dem } \\
\text { Publikationsworkflow zugeordnet werden? }\end{array}$ \\
\hline $\begin{array}{l}\text { Herausgebergremium festlegen } \\
\text { (optional) }\end{array}$ & - & $\begin{array}{l}\text { Wer übernimmt qualitätssichernde Prozesse im } \\
\text { Publikationsworkflow? }\end{array}$ \\
\hline Software beschaffen & - & $\begin{array}{l}\text { z. B. Adobe Cloud, XML-Editor, Plagiatsprüfungs- } \\
\text { Software, Projektmanagement-Software etc. }\end{array}$ \\
\hline ISBN-Stamm erwerben & - & Wie viele ISBNs werden benötigt? \\
\hline Mitgliedschaften schließen & $\begin{array}{l}- \\
- \\
- \\
-\end{array}$ & $\begin{array}{l}\text { für den Erwerb von DOIs (Crossref, Datacite) } \\
\text { für OA-Veröffentlichungsplattformen } \\
\text { für die Distribution von Metadaten } \\
\text { für die Distribution von gedruckten Büchern } \\
\text { für die Langzeitarchivierung }\end{array}$ \\
\hline Leistungsumfang festlegen & - & $\begin{array}{l}\text { Übernimmt der Verlag den Satz des Werkes oder } \\
\text { muss dies vom Autor übernommen werden? } \\
\text { Muss ein eigenes XML-Template entwickelt werden? }\end{array}$ \\
\hline $\begin{array}{l}\text { Qualitätsrichtlinien für relevante } \\
\text { Prozesse festlegen } 57\end{array}$ & - & $\begin{array}{l}\text { Erstellen einer Muster-Publikationsvertragsvorlage } \\
\text { Erstellen von Checklisten }\end{array}$ \\
\hline
\end{tabular}




\section{Null-Prozess}

Produktqualität bzw. -ausstattung definieren

\section{Beschreibung/Beispiele}

- Buchformat/e festlegen

- Innentypografie festlegen

- Richtlinien für Covergestaltung erstellen

- die Standardausstattung des gedruckten Produktes definieren

\subsubsection{Das Workflow-Modell nach Prozessgruppen}

Die Prozesse des Workflow-Modells lassen sich in 17 Prozessgruppen zusammenfassen. Die Abgrenzung ergibt sich aus dem Verrichtungszweck der ihnen zugeordneten Prozesse und bildet einen weiteren Teil der Nomenklatur (vgl. Kapitel 2) ab. Im Modell sind die Prozessgruppen als Überschriften erkenntlich und sollen im Folgenden erläutert werden.

\subsubsection{Prozessgruppen der inhaltsorientierten Prozesse}

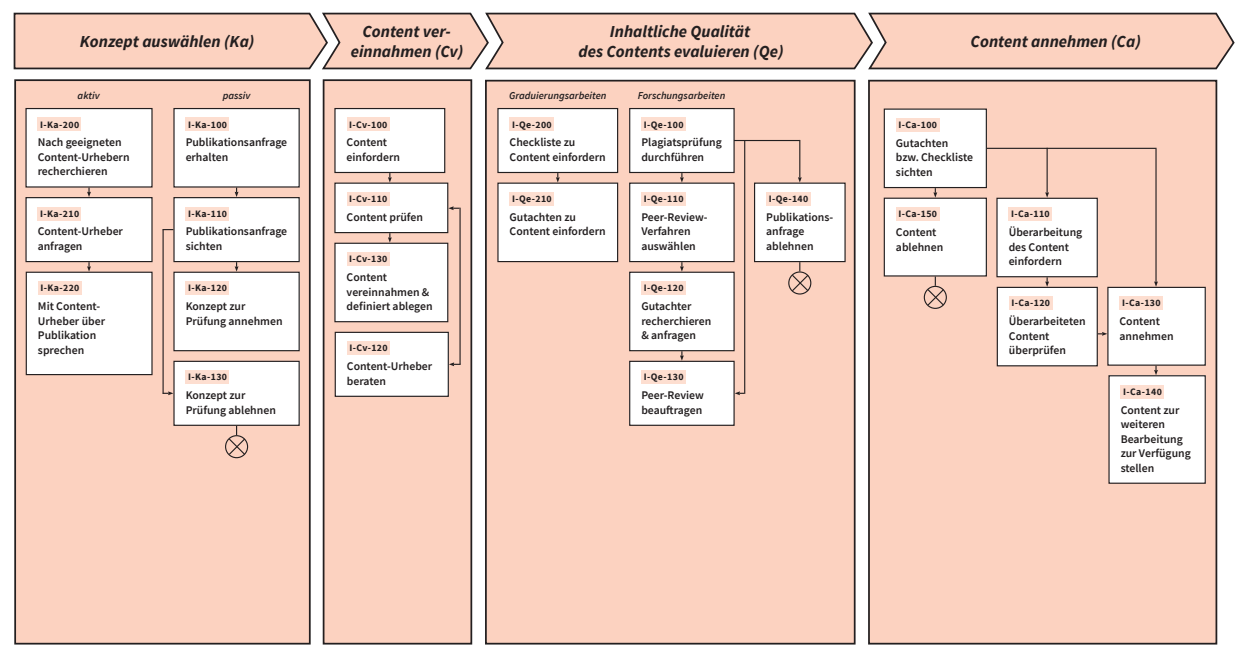

Abb. 9 Prozessgruppen der inhaltsorientierten Prozesse 


\section{I-Ka: Konzept auswählen}

Die Prozesse der Prozessgruppe I-Ka werden in der Regel den Beginn des Publikationsworkflows darstellen; sie beschreiben die notwendigen Verlagsaktivitäten zur Inhaltsbeschaffung. Dabei werden im Workflow-Modell zwei Möglichkeiten berücksichtigt, wie Content (als Konzept eines Werks oder als vollständiges Manuskript) für das Verlagsprogramm akquiriert wird.

Entweder bietet der Verlag interessierten Autoren Kontaktmöglichkeiten an, damit diese selbstständig ihren Inhalt beim Verlag einreichen können, wenn sie publizieren möchten, oder der Verlag wird selbst tätig und sucht nach geeigneten Inhalten bzw. Autoren für sein Verlagsprogramm. Diese Möglichkeiten sind im Modell durch die Bezeichnungen "passiv" und "aktiv“ gekennzeichnet. Hierbei schließen die zwei Möglichkeiten nicht per se einander aus, sondern können auch gleichzeitig im Verlag oder in einzelnen Projekten Anwendung finden.

\section{I-Cv: Content vereinnahmen}

Grundlage für die Prozessgruppe I-Cv ist das gleichnamige Standardworkflow-Element „3.4 Content vereinnahmen“ der Berliner Werkstatt Herstellung; es wurde mit nur leichten Änderungen in das Workflow-Modell übertragen. ${ }^{58}$ Die Prozesse dieser Prozessgruppe folgen auf die von Prozessgruppe I-Ka; sie umfassen Verlagsaktivitäten, die notwendig sind, um entscheiden zu können, ob der akquirierte Inhalt weitere Prozesse des Workflows durchlaufen wird. Sofern dies der Fall ist und es nicht zum Abbruch des Workflows an dieser Stelle kommt, folgen die Prozesse der Prozessgruppe I-Qe.

\section{I-Qe: Inhaltliche Qualität des Contents evaluieren}

Entsprechend seiner Benennung umfasst die Prozessgruppe I-Qe Prozesse zur Beurteilung der inhaltlichen Qualität des Contents. Ziel ist es, eine Einschätzung zur Publikationsfähigkeit hinsichtlich des fachlichen Inhalts zu erhalten und bei unzulänglicher Qualität den Publikationsworkflow abzubrechen.

Wie im Kapitel 2 erläutert wurde, werden im Hochschulrahmen üblicherweise Graduierungs- oder anderweitige wissenschaftliche Arbeiten verfasst, die im Rahmen des Curriculums oder eines Forschungsprojektes entstehen. Für die Prozesse der Prozessgruppe I-Qe ist diese Unterscheidung relevant: Im Falle eines Forschungsprojektes wurde, wenn das Manuskript dem Verlag vorliegt, die verschriftliche Forschungsarbeit (als Teilergebnis) in der Regel noch nicht unabhängig auf ihre wissenschaftliche Qualität geprüft. Daher ist es Aufgabe des Verlages, die Eignung der Arbeit für eine Veröffentlichung zu überprüfen. Als Standardverfahren hat sich hierfür das sogenannte Peer-Review etabliert: Hierbei werden je nach Verfahren ein oder mehrere, unabhängige Experten ${ }^{59}$ aus dem entsprechenden Fach-

58 vgl. Berliner Werkstatt Herstellung 2007, S. 116

59 Als Experten in diesem Kontext können durchaus auch Professoren, Fachreferenten oder Lehrbeauftragte der eigenen Institution betrachtet werden. 
gebiet damit beauftragt, den zur Veröffentlichung vorgeschlagenen Content zu bewerten. ${ }^{60}$ Das standardisierte Peer-Review wird im Workflow-Modell durch die Prozesse I-Qe-100 bis I-Qe-140 widergespiegelt.

Neben diesen Forschungsarbeiten publizieren Hochschulverlage auch schriftliche Graduierungsarbeiten von Absolventen der Hochschule, der sie angeschlossen sind. Solche Arbeiten entstehen zum Abschluss des Curriculums, um den entsprechenden akademischen Grad zu erlangen. Nach Abgabe der Arbeiten durch den Studierenden durchläuft diese eine interne Qualitätssicherung, die üblicherweise darin besteht, dass Hochschulprofessoren und ggf. weitere Gutachter ${ }^{61}$ einerseits die inhaltliche Qualität und andererseits die Korrektheit der wissenschaftliche Methodik zur Untersuchung des Forschungsgegenstandes beurteilen. Zur Standardisierung dieser Qualitätssicherung haben die akademischen Institutionen häufig Grundsätze zur Sicherung guter wissenschaftlicher Praxis formuliert, die den Gutachtern als Richtlinie der Beurteilung dient. Üblicherweise beurteilen und bewerten mindestens zwei, bei höherwertigen Abschlussarbeiten wie Doktorarbeiten mindestens drei Gutachter die Arbeit und erstellen Gutachten, bevor der akademische Grad verliehen wird. Diese Vorgehensweise entspricht dem standardisierten Peer-Review bei Forschungsarbeiten und ist bei Graduierungsarbeiten bereits abgeschlossen, bevor der Publikationsworkflow beginnt. Dennoch ist es empfehlenswert, dass ein Hochschulverlag die Publikationsfähigkeit einer Graduierungsarbeit selbst überprüft. Aus diesem Grund wurden die Prozesse I-Qe-200 und I-Qe-210 formuliert und der Prozessgruppe I-Qe zugeordnet.

\section{I-Ca: Content annehmen}

Die Prozesse der Prozessgruppe I-Ca beschreiben sowohl alle Aktivitäten, die eine Entscheidung herbeiführen, ob der akquirierte Content grundsätzlich zur Publikation geeignet ist (auf Grundlage der Ergebnisse aus den Prozessen der vorhergehenden Prozessgruppe I-Qe) als auch solche Aktivitäten, die die Qualität des Contents sichern sollen, bevor die technische Umsetzung des Contents erfolgt. Diese qualitätssichernden Prozesse sind für den weiteren Verlauf des Publikationsworkflows entscheidend, schließlich können sich inhaltliche Änderungen am Content, sogenannte Autorkorrekturen, zu Zeit- und dann auch zu Kostentreibern in den produktionsorientierten Prozessen auswachsen. Um einen effizienten Ablauf des Publikationsworkflows zu gewährleisten, sollte daher, nachdem die Prozesse der Prozessgruppe I-Ca abgeschlossen sind, zumindest aus Autorensicht ein inhaltlich fertiges Manuskript vorliegen.

60 vgl. Deutsche Zentralbibliothek für Medizin 2019

61 Weitere Gutachter können bspw. wissenschaftliche Mitarbeiter der Hochschule oder Mitarbeiter eines Unternehmens sein. Gerade in praxisorientierten Studiengängen ist es üblich, dass der Forschungsgegenstand der Abschlussarbeit eine Problematik eines Unternehmens ist, weswegen die Arbeit in Kooperation mit dem Unternehmen erstellt und dementsprechend auch geprüft wird. 


\subsubsection{Prozessgruppen der produktionsorientierten Prozesse}

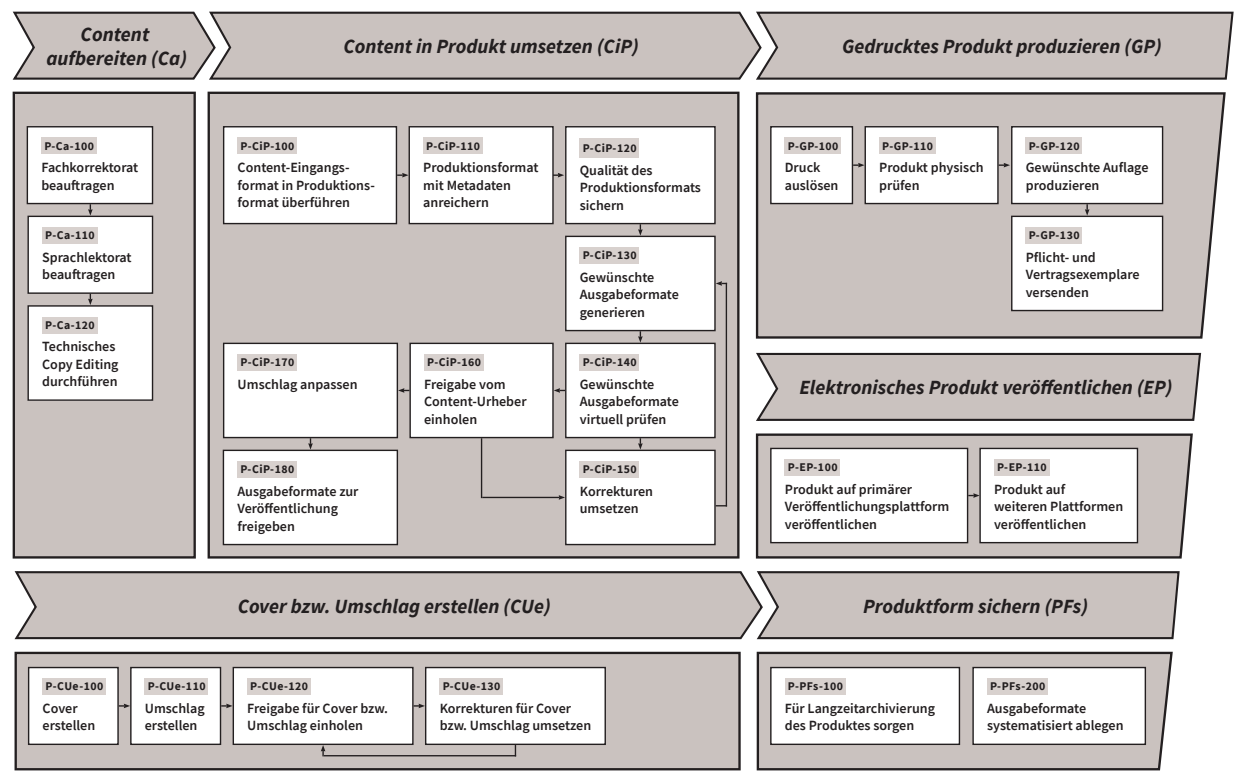

Abb. 10 Prozessgruppen der produktionsorientierten Prozesse

\section{P-Ca: Content aufbereiten}

Die Prozessgruppe P-Ca umfasst die Prozesse, die die Aufbereitung des Contents für die Durchführung der Produktion zum Ziel haben. Auch hier hat sich die Konzeption an einem Standardworkflow-Element der Berliner Werkstatt Herstellung orientiert, in dem der Prozess „3.5. Content aufbereiten“ definiert wird. ${ }^{62}$ In der Veröffentlichung der Berliner Werkstatt Herstellung sind dabei die Arbeitsschritte „Content technisch aufbereiten“ und „Content inhaltlich aufbereiten“ benannt. Diese Arbeitsschritte sind in die Definition der Prozesse der Prozessgruppe P-Ca eingeflossen (vgl. Prozess $\mathrm{P}$-Ca-100 und P-Ca-110 für "Content inhaltlich aufbereiten“ und Prozess $P$-Ca-120 für „Content technisch aufbereiten“).

\section{P-CUe: Cover bzw. Umschlag erstellen}

Die Prozessgruppe P-CUe umfasst Aktivitäten, die die Erstellung des Covers und sofern es auch gedruckte Produkte geben soll - des Umschlags für den jeweiligen Content zum Ziel haben. Das schließt auch Korrektur- und Freigabeschleifen ein, die durch den Richtungspfeil von Prozess P-CUe-130 zu Prozess P-CUe-120 symbol- 
isiert werden. Die frühzeitige Durchführung dieser Prozesse wird empfohlen, da zur Buchvorankündigung bzw. Distribution von Metadaten häufig bereits ein Cover verlangt wird.

\section{P-CiP: Content in Produkt umsetzen}

Kernstück der produktionsorientierten Prozesse sind die der Prozessgruppe P-CiP. Sie können im traditionellen Sinn auch als Satzprozesse verstanden werden. Auch hier waren für die Benennung der zugehörigen Prozesse die Ergebnisse der Berliner Werkstatt Herstellung Vorbild. ${ }^{63}$ Der Fokus lag hierbei auf der Abbildung von Single-Source-Multiple-Channel-Workflows, respektive eines XML-first-Workflows, der zum Zeitpunkt der Erstellung des vorliegenden Werkes den aktuellen Stand der Technik widerspiegelte. Um die Anpassungsfähigkeit des Modells sicherzustellen, wurde jedoch darauf verzichtet, XML in die Prozessnamen aufzunehmen. Schließlich ist es möglich, dass XML als Standard für die Aufbereitung von Content in medienneutrale Daten eines Tages langfristig durch eine andere Technologie abgelöst wird. Aufgrund der Anpassbarkeit des Workflow-Modells kann, indem Prozesse an dieser Stelle weggelassen werden (z. B. der Prozess P-CiP-100), auch die Workflow-Variante eines CRC-Workflow ${ }^{64}$ abgebildet werden. Wie sich dies in der Praxis darstellt, wird anhand der Fallstudie (vgl. Fall 1, Kapitel 4.3.1) deutlich.

\section{P-GP: Gedrucktes Produkt produzieren}

Entsprechend seiner Benennung umfasst die Prozessgruppe P-GP die Prozesse zur Herstellung des Produkts in den gewünschten gedruckten Ausgabeformaten Hardcover und/oder Softcover. Diese Gruppe umfasst also die Druckprozesse. Sofern lediglich eine Veröffentlichung des Produktes in elektronischen Publikationsformen geplant ist, kann das Modell durch Weglassen dieser Prozessgruppe entsprechend angepasst werden.

\section{P-EP: Elektronisches Produkt veröffentlichen}

Die Prozessgruppe P-EP beschreibt Prozesse, die die Veröffentlichung des Produktes in den gewünschten, elektronischen Publikationsformen, wie ePDF, EPUB und/ oder MOBI, zum Ziel haben.

\section{P-PFs: Produktform sichern}

Die Prozesse der Prozessgruppe P-PFs entsprechen inhaltlich dem gleichnamigen Standardworkflow-Element der Berliner Werkstatt Herstellung. ${ }^{65}$ Sie beschreiben die notwendigen Verlagsaktivitäten, um das publizierte Produkt in allen gewünschten Publikationsformen systematisch intern, aber auch unabhängig vom Verlag zu archivieren.

63 vgl. Berliner Werkstatt Herstellung 2007, S. 126

64 vgl. Kapitel 2.2

65 vgl. Berliner Werkstatt Herstellung 2007, S. 135 


\subsubsection{Prozessgruppen der managementorientierten Prozesse}

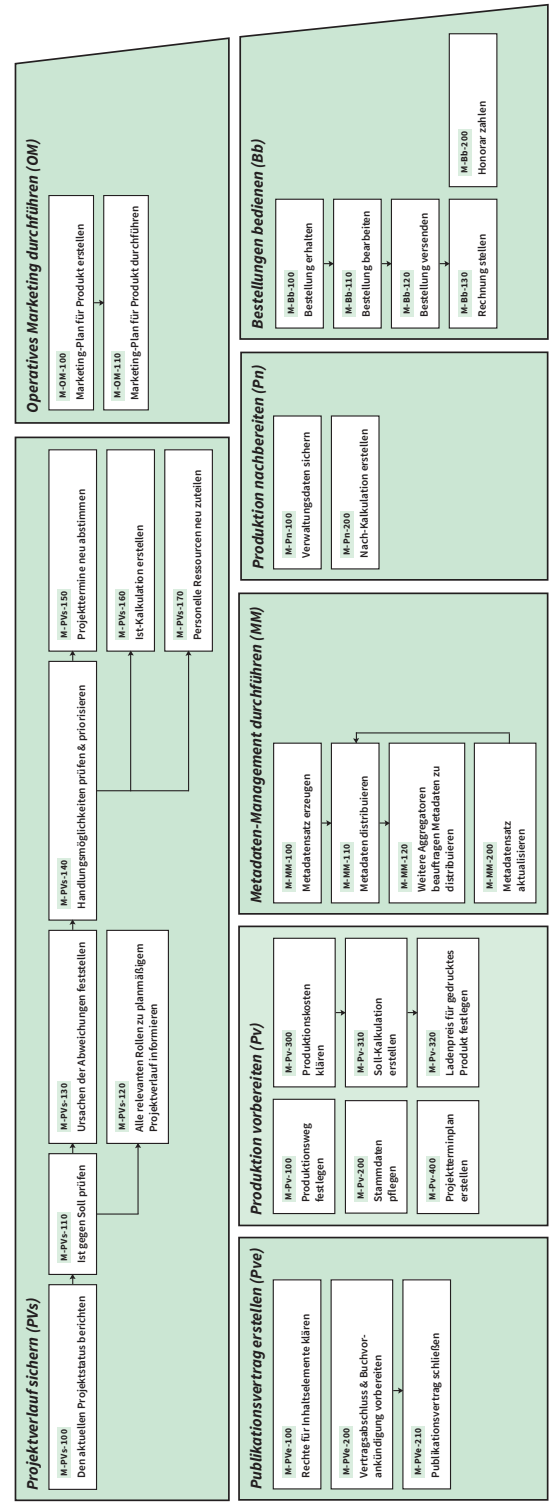

Abb. 11 Prozessgruppen der managementorientierten Prozesse 
M-OM: Operatives Marketing durchführen

Der Prozessgruppe M-OM zugeordnet sind Prozesse, die zur Bewerbung des Produktes beitragen. Ihr zentrales Ziel ist es, einen Marketingplan zu erstellen und umzusetzen (Prozesse M-OM-100 und M-OM-110). Über dessen Gestaltung entscheidet jede Institution selbst. Je nach Anwendung des Modells kann der Marketingplan auch sehr früh erstellt werden, so dass sich seine Umsetzung über den gesamten Publikationsworkflow erstreckt.

\section{M-PVe: Publikationsvertrag erstellen}

Die Prozessgruppe M-PVe umfasst die Prozesse, die die rechtliche Grundlage für die Publikation eines Werkes sicherstellen. Vor allem der Prozess M-PVe-210 ist hierbei ausschlaggebend und als Meilenstein im Publikationsworkflow zu qualifizieren. Die Prozesse $M-P V e-100$ und $M-P V e-200$ sollten idealerweise dem Prozess $M-P V e-210$ vorgelagert sein, um Mehrarbeit zu vermeiden und keinen Abbruch des Publikationsworkflows zu riskieren.

\section{M-Pv: Produktion vorbereiten}

Im Gegensatz zum Standardworkflow-Element Produktion vorbereiten der Berliner Werkstatt Herstellung ${ }^{66}$, das als Vorbild für die Prozesse der Prozessgruppe $M-P v$ verwendet wurde, ist diese Prozessgruppe den managementorientierten Prozessen zugeordnet. Grund dafür ist, dass die Prozesse dieser Gruppe nicht der Wertschöpfung des Produktes dienen, sondern für den effizienten Ablauf des Publikationsworkflows sorgen. Sie sind somit von den produktionsorientierten Prozessen abzugrenzen, auch, wenn sie wichtige Inputs für diese liefern. Wie alle managementorientierten Prozesse können auch die der Prozessgruppe $M-P v$ zu jedem Zeitpunkt im Workflow begonnen werden. Allerdings ist ihre Durchführung die Voraussetzung für die Prozesse der Gruppe $M-P V s$, weshalb sie vor deren Prozessen durchgeführt werden müssen.

Da es Anspruch der Entwicklung des Workflow-Modells war, ein hybrides Geschäftsmodell zu entwickeln, das auf die Veröffentlichung der digitalen und gedruckten Fassung eines Buches in der verlagsüblichen Form zielt (vgl. Kapitel 1), enthält diese Prozessgruppe den Prozess $M-P v$-320. Dieser ist relevant, wenn solch ein hybrides OA-Geschäftsmodell verfolgt wird.

\section{M-MM: Metadaten-Management durchführen}

Ihrer Benennung entsprechend schließt die Prozessgruppe $M-M M$ alle Prozesse ein, die die Erstellung und Distribution von Metadaten zum Produkt zum Zweck haben. Da die Verbreitung von Metadaten nachweislich die Sichtbarkeit von Werken erhöht, sollten diese Prozesse so früh wie möglich durchgeführt werden. 


\section{M-PVs: Projektverlauf sichern}

Um den effektiven und effizienten Ablauf des Publikationsworkflows zu garantieren, wurden die Prozesse der Gruppe M-PVs formuliert. Sie entsprechen Reporting-Prozessen in Unternehmen jeder Branche. Ihr Ziel ist es, generell den Informationsbedarf der hier beteiligten Personen oder hierfür relevanten Rollen zu decken, Transparenz zu schaffen sowie Entscheidungen vorzubereiten und zu kontrollieren. ${ }^{67}$

Nach Durchführung der vorgelagerten Prozesse der Gruppe $M-P v$, können die Prozesse der Gruppe $M-P V s$ erstmals durchgeführt werden. Um die Effizienz des Publikationsworkflows zu sichern, empfiehlt es sich, diese Prozesse in regelmäßigen Abständen zu wiederholen.

\section{M-Pn: Produktion nachbereiten}

Die Prozesse der Prozessgruppe M-Pn können, weil sie Input aus produktionsorientierten Prozessen benötigen, erst im Anschluss an diese erfolgen. Sie dienen der Nachbetrachtung des Publikationsworkflows. Bei ihrer Beschreibung wurde sich wieder an den Ergebnissen der Berliner Werkstatt Herstellung orientiert. ${ }^{68}$

\section{M-Bb: Bestellungen bedienen}

Auch die Prozesse der Prozessgruppe M-Bb können erst nach den produktionsorientierten Prozessen durchgeführt werden. Ihr Ziel ist es, die Bestellungen des nun auf dem Markt verfügbaren Produktes zu bedienen und dem Autor ggf. das vereinbarte Honorar zukommen zu lassen. Daher sind sie für den gesamten Produktlebenszyklus relevant und wiederholen sich - voraussichtlich - regelmäßig.

\subsection{Beschreibung der Workflow-Rollen}

Bevor die Prozesse des Modells mit Hilfe der Prozesskennblätter im Detail beschrieben werden, erfolgt an dieser Stelle zunächst die konkrete Rollenbeschreibung. Wie bereits in Kapitel 2.4 erläutert, werden hier lediglich die Benennung der jeweiligen Rolle sowie die Anforderungen an sie dargestellt, die sich aus dem Prozess, dem die Rolle zugewiesen wird, ergeben. Die Abgrenzung der Rollen ergibt sich aus dem Anforderungsprofil. Aufgrund der Komplexität einiger Prozesse kommt es zwischen einigen Rollen jedoch zu Überschneidungen ihrer Anforderungsprofile. Zur besseren Verständlichkeit werden daher, ergänzend $\mathrm{zu}$ den Anforderungsprofilen, jeweils Beispiele genannt, wer die Rolle einnehmen, d. $h$. wer Rollenträger sein kann. Es sei gesagt, dass diese Ergänzung keinen Anspruch auf Vollständigkeit erhebt.

67 vgl. Gründerszene 2019

68 vgl. Berliner Werkstatt Herstellung 2007, S. 133-137 


\section{Content-Lieferant}

\begin{tabular}{ll}
\hline Beschreibung & $\begin{array}{l}\text { Der Content-Lieferant trägt Inhalte, die er als für die Publikation } \\
\text { geeignet betrachtet, an den Hochschulverlag heran oder unterstützt } \\
\text { bei der Akquise von neuen Konzepten bzw. bei der } \\
\text { Programmplanung. }\end{array}$ \\
\hline Anforderungsprofil & - Gespür für die Qualität wissenschaftlicher Arbeiten \\
& - Kenntnisse zur Nachfrage von wissenschaftlichen Arbeiten \\
& (Marktkenntnis) \\
\hline Rollenträger (Beispiele) & - Hochschulabsolventen \\
& - Doktoranden \\
& - Professoren oder andere Hochschulmitarbeiter \\
& - Mitarbeiter der Hochschulbibliothek \\
& - Herausgebergremium \\
& - Doktorandenkolleg \\
\hline
\end{tabular}

\section{Content-Urheber}

\begin{tabular}{|c|c|}
\hline Beschreibung & $\begin{array}{l}\text { Der Content-Urheber ist für die Erstellung von Content (Konzept oder } \\
\text { Inhalt) verantwortlich. Diese Rolle kann der des Content-Lieferanten } \\
\text { entsprechen. Die Abgrenzung der zwei genannten Rollen ist jedoch } \\
\text { essentiell, da inhaltliche Änderungen am Content, wie im Prozess I- } \\
\text { Ca-110 beschrieben, nur vom Content-Urheber, nicht aber vom } \\
\text { Content-Lieferanten vorgenommen werden können. }\end{array}$ \\
\hline Anforderungsprofil & $\begin{array}{l}\text { - } \text { fundiertes inhaltliches Fachwissen } \\
\text { - } \text { grundlegende bis tiefgreifende Kenntnisse zur Erstellung einer } \\
\text { wissenschaftlichen Arbeit } \\
\text { - Kommunikationsfähigkeit } \\
\text { - grundlegendes Verständnis für Korrekturen und die Fähigkeit, } \\
\text { diese umzusetzen }\end{array}$ \\
\hline $\begin{array}{l}\text { Rollenträger } \\
\text { (Beispiele) }\end{array}$ & $\begin{array}{ll}- & \text { Wissenschaftler und Forscher } \\
- & \text { Absolventen } \\
- & \text { Studierenden }\end{array}$ \\
\hline
\end{tabular}




\section{Content-Abnehmer}

\begin{tabular}{ll}
\hline Beschreibung & Die Rolle des Content-Abnehmers ist für den Ablauf des \\
& Publikationsworkflows, insbesondere für die Kommunikation im \\
& Hochschulverlag, verantwortlich. Grundsätzlich wird hierfür keine \\
& Fachexpertise benötigt. \\
\hline Anforderungsprofil & - fundierte Marktkenntnis und die Fähigkeit, Trends zu erkennen \\
& - Verständnis von Methoden zur Qualitätssicherung und \\
& - Konzeptioneller Arbeit \\
& - Fähigkeiten im Projektmanagement \\
& - Kommunikationsfähigkeit \\
& - Managementfähigkeit \\
& - grundlegende Kenntnisse in der Produktion von OA-Büchern \\
& - grundlegende Kenntnisse im Bereich Typografie und Layout \\
& - grundlegende juristische Kenntnisse (Urheberrecht, \\
& Vertragsabschluss) \\
\hline Rollenträger (Beispiele) & Mitarbeiter des Hochschulverlags
\end{tabular}

\section{Marketing-Manager}

Beschreibung Der Marketing-Manager ist für die Bewerbung des zu erstellenden Produktes verantwortlich.

\begin{tabular}{ll}
\hline Anforderungsprofil & - fundierte Marktkenntnis \\
& $-\quad$ Kenntnisse und grundlegende bis tiefgreifende Kompetenzen \\
& - in der Durchführung von Marketingmaßnahmen (im Verlag) \\
& - Managementfähigkeit, insbesondere Kenntnisse und \\
& \\
\hline Rollenträger (Beispiele) & - Mitarbeiter des Hochschulverlags \\
& - Mitarbeiter der Öffentlichkeitsarbeit der Institution \\
& - studentische Hilfskraft \\
& - freie Marketingexperten \\
& - Dienstleister für Marketing \\
\hline
\end{tabular}




\section{Gutachter}

\begin{tabular}{|c|c|}
\hline Beschreibung & $\begin{array}{l}\text { Die Rolle Gutachter ist für die Überprüfung und Beurteilung des } \\
\text { Contents hinsichtlich seiner wissenschaftlichen Qualität zuständig. } \\
\text { Der Gutachter kann, ausgehend von seiner Fachexpertise, } \\
\text { einschätzen, ob der Content den grundlegenden Anforderungen an } \\
\text { Originalität und Aktualität der Forschung auf diesem Gebiet } \\
\text { entspricht. }\end{array}$ \\
\hline Anforderungsprofil & 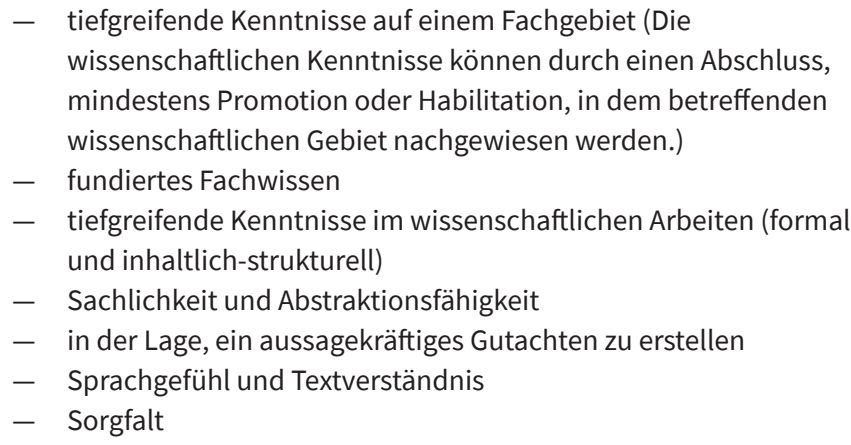 \\
\hline $\begin{array}{l}\text { Rollenträger } \\
\text { (Beispiele) }\end{array}$ & $\begin{array}{l}\text { - Professoren } \\
\text { - } \text { aktive Wissenschaftler } \\
\text { - } \text { Fachleute (als Nachweis der Befähigung zum wissenschaftlichen } \\
\text { Arbeiten kann eine Promotion oder Habilitation angesehen } \\
\text { werden) }\end{array}$ \\
\hline
\end{tabular}

\section{Content-Prüfer}

\begin{tabular}{ll}
\hline Beschreibung & Der Content-Prüfer verantwortet die Qualitätssicherung des zu \\
publizierenden Contents im Hochschulverlag. Die Rolle entspricht \\
einem Lektor in einem klassischen Wissenschaftsverlag. Sofern die \\
entsprechenden Kompetenzen vorhanden sind, können die Rollen \\
des Content-Prüfers und Gutachters auch von einer einzelnen \\
Person übernommen werden. \\
$-\quad$ tiefgreifende Kenntnisse im wissenschaftlichen Arbeiten (formal \\
Anforderungsprofil & und inhaltlich-strukturell) \\
- & tiefgreifende Kenntnisse der deutschen Sprache (Grammatik, \\
& Orthografie, Idiomatik) \\
- & Kommunikationsfähigkeit \\
- & Textverständnis ${ }^{69}$ \\
- & breite Allgemeinbildung
\end{tabular}


Content-Prüfer

\begin{tabular}{lll}
\hline & - & grundlegende bis tiefgreifende Kenntnisse zum Urheberrecht \\
& - & Kenntnisse im Bereich Typografie und Layout \\
& - & Sorgfalt \\
& - & Managementkompetenz \\
& - & Mitarbeiter des Hochschulverlags \\
Rollenträger & - & Externe Fachlektoren (freie Lektoren mit einem \\
& wissenschaftlichen Abschluss in dem betreffenden Fachbereich)
\end{tabular}

\section{Barrierefreiheit-Beauftragter}

\begin{tabular}{|c|c|}
\hline Beschreibung & $\begin{array}{l}\text { Der Barrierefreiheit-Beauftragte stellt im Hochschulverlag sicher, } \\
\text { dass mögliche Richtlinien für barrierefreie Bücher erfüllt werden. }\end{array}$ \\
\hline Anforderungsprofil & $\begin{array}{l}\text { - grundlegende bis tiefgreifende Kenntnisse der Kriterien für } \\
\text { barrierefreie Bücher } \\
\text { - Kommunikationsfähigkeit }\end{array}$ \\
\hline Rollenträger (Beispiele) & $\begin{array}{l}\text { - Barrierefreiheit- oder Gleichstellungsbeauftragter der } \\
\text { Institution } \\
\text { - } \quad \text { Mitarbeiter des Hochschulverlags } \\
\text { - } \\
\text { Mitarbeiter zentraler Einrichtungen, wie der Deutschen } \\
\text { Zentralbücherei für Blinde (DZB) }\end{array}$ \\
\hline
\end{tabular}

\section{Copy Editor}

\begin{tabular}{ll}
\hline Beschreibung & Der Copy Editor ist für die Qualitätssicherung des Contents \\
& $\begin{array}{l}\text { zuständig. Er ist dafür verantwortlich, den Content so } \\
\text { aufzubereiten, dass ein fachlich korrektes, in sich konsistentes und } \\
\text { sprachlich fehlerfreies Manuskript für die Produktion vorliegt. }{ }^{70}\end{array}$ \\
\hline Anforderungsprofil & - Muttersprachler in der den Content betreffenden Sprache \\
& - Sprachgefühl und Textverständnis \\
& - Sorgfalt \\
\hline Rollenträger (Beispiele) & - Kommunikationsfähigkeit \\
& - Dienstleister für Copy Editing \\
\hline
\end{tabular}




\section{Fachkorrektor}

\begin{tabular}{ll}
\hline Beschreibung & Der Fachkorrektor ist für die Durchführung des Fachkorrektorats \\
& verantwortlich (vgl. Prozess P-Ca-100). Er redigiert den Content nicht \\
& nur sprachlich, sondern achtet auch auf fachliche Aspekte, etwa auf \\
& die Einhaltung wissenschaftlicher Schreibweisen, die Richtigkeit \\
& zitierter Aussagen, folgerichtige Darstellungen von Thesen sowie auf \\
& korrekte Quellenangaben und Bildnachweise ${ }^{71}$. Entsprechend muss \\
& die Rolle des Fachkorrektors von einem Experten auf dem jeweiligen \\
& Fachgebiet eingenommen werden. \\
& $-\quad$ fundiertes inhaltliches Fachwissen \\
- & tiefgreifende Kenntnisse im wissenschaftlichen Arbeiten (formal \\
Anforderungsprofil & und inhaltlich-strukturell) \\
- & Sachlichkeit \\
- & tiefgreifende Kenntnisse der Sprache (Grammatik, Orthografie, \\
- & Idiomatik), in der der Content verfasst ist \\
- & Sprachgefühl und Textverständnis \\
- & Sorgfalt \\
\hline & Experte auf dem relevanten Fachgebiet \\
Rollenträger & wissenschaftlichen Abschluss in dem betreffenden Fachbereich) \\
\hline Beispiele) &
\end{tabular}

\section{Sprachlektor}

\begin{tabular}{|c|c|}
\hline Beschreibung & $\begin{array}{l}\text { Der Sprachlektor ist für die Durchführung des Sprachlektorats } \\
\text { verantwortlich (vgl. Prozess P-Ca-100). Er überprüft den Content auf } \\
\text { die korrekte Anwendung der Sprache. Dies umfasst bspw. die } \\
\text { Überprüfung von Rechtschreibung, Silbentrennung, Groß- und } \\
\text { Kleinschreibung, Grammatik und Satzbau. Ein Sprachlektorat ist vor } \\
\text { allem sinnvoll, wenn der Autor den Content nicht in seiner } \\
\text { Muttersprache verfasst hat. }\end{array}$ \\
\hline Anforderungsprofil & $\begin{array}{l}\text { - } \text { tiefgreifende Kenntnisse der Sprache, in der der Content verfasst } \\
\text { wurde (Grammatik, Orthografie, Idiomatik) } \\
\text { - } \text { grundlegende Kenntnisse der Anforderungen wissenschaftlicher } \\
\text { Texte }\end{array}$ \\
\hline $\begin{array}{l}\text { Rollenträger } \\
\text { (Beispiele) }\end{array}$ & $\begin{array}{l}\text { - } \text { professioneller Sprachlektor } \\
\text { - } \text { Muttersprachler mit Erfahrungen auf dem relevanten Fachgebiet } \\
\text { - }\end{array}$ \\
\hline
\end{tabular}




\section{Cover-Ersteller}

\begin{tabular}{|c|c|}
\hline Beschreibung & $\begin{array}{l}\text { Der Cover-Ersteller ist für die Erstellung von Cover und Umschlag } \\
\text { des Produktes zuständig. }\end{array}$ \\
\hline Anforderungsprofil & $\begin{array}{l}\text { - grundlegende bis tiefgreifende Kenntnisse von Typografie und } \\
\text { Layout sowie die Fähigkeit, diese mit geeigneter Software } \\
\text { umzusetzen } \\
\text { - Kreativität }\end{array}$ \\
\hline Rollenträger (Beispiele) & $\begin{array}{l}\text { - } \quad \text { Mitarbeiter des Hochschulverlags } \\
\text { - } \quad \text { Mitarbeiter der Öffentlichkeitsarbeit der Institution } \\
\text { - } \quad \text { freie Grafiker oder Verlagshersteller } \\
\text { - } \quad \text { Satzdienstleister }\end{array}$ \\
\hline
\end{tabular}

\section{Content-Umsetzer}

\begin{tabular}{ll}
\hline Beschreibung & Die Rolle Content-Umsetzer verantwortet die Umsetzung des \\
& $\begin{array}{l}\text { Contents in ein Produkt. Je nachdem, welchen Leistungsumfang } \\
\text { der Hochschulverlag anbieten möchte und welche Kompetenzen im } \\
\text { Hochschulverlag vorhanden sind, kann diese Rolle von einem } \\
\text { Satzdienstleister, einem Verlagsmitarbeiter oder vom Content- } \\
\text { Urheber selbst eingenommen werden. }\end{array}$ \\
\hline Anforderungsprofil & - grundlegende bis tiefgreifende Kompetenzen im crossmedialen \\
& - Publizieren \\
& - Kenhigkeiten im Projektmanagement \\
& - Mitarbeiter des Hochschulverlags \\
Rollenträger (Beispiele) & freie Verlagshersteller oder Grafiker \\
& - Satzdienstleister \\
\hline
\end{tabular}

\section{Metadaten-Manager}

Beschreibung

Der Metadaten-Manager ist im Hochschulverlag für die Erstellung und Distribution der Metadaten zum Content bzw. zum Produkt verantwortlich (betrifft v. a. die Prozesse M-MM-100 bis M-MM-200). Im Fokus stehen hier v. a. die Erstellung und die Verteilung der Metadaten an Metadaten-Aggregatoren, etwa an das Verzeichnis Lieferbarer Bücher (VLB) oder an den Großbuchhandel (vgl. Kapitel 4.2.4).

\begin{tabular}{|c|c|c|}
\hline Anforderungsprofil & - & $\begin{array}{l}\text { grundlegende bis tiefgreifende Kompetenzen im Metadaten- } \\
\text { Management } \\
\text { Affinität zu Technik }\end{array}$ \\
\hline
\end{tabular}


Metadaten-Manager

- grundlegende bis tiefgreifende Kompetenzen im

Bibliothekswesen

- Projektmanagement-Kompetenzen

\begin{tabular}{|c|c|c|}
\hline Rollenträger & - & Mitarbeiter des Hochschulverlags \\
\hline (Beispiele) & - & Mitarbeiter der Hochschulbibliothek \\
\hline & - & Dienstleister für das Metadaten-Management \\
\hline
\end{tabular}

\section{Metadaten-Aggregator}

\begin{tabular}{ll}
\hline Beschreibung & $\begin{array}{l}\text { Der Metadaten-Aggregator wird vom Metadaten-Manager } \\
\text { beauftragt, Metadaten zu distribuieren, da er über den Zugang zu } \\
\text { gewünschten Distributionskanälen verfügt. Diese Rolle kann } \\
\text { ausschließlich von Personen außerhalb des Hochschulverlags } \\
\text { eingenommen werden, etwa von Mitarbeitern des } \\
\text { Großbuchhandels. }\end{array}$ \\
\hline Anforderungsprofil & - verfügt über Zugang zu gewünschten Distributionskanälen \\
\hline Rollenträger (Beispiele) & - Dienstleister für das Metadaten-Management \\
& $-\quad$ Mitarbeiter des Großbuchhandels
\end{tabular}

\section{Content-Distributor}

\begin{tabular}{|c|c|}
\hline Beschreibung & $\begin{array}{l}\text { Die Rolle Content-Distributor verantwortet die Veröffentlichung und } \\
\text { Verbreitung der Produkte des Hochschulverlages. }\end{array}$ \\
\hline Anforderungsprofil & $\begin{array}{l}\text { - grundlegende bis tiefere Kenntnisse über Druckverfahren, } \\
\text { Auslieferung, Veröffentlichung, Buchhandel und Abwicklung } \\
\text { von Bestellvorgängen }\end{array}$ \\
\hline Rollenträger (Beispiele) & $\begin{array}{l}\text { - Mitarbeiter des Hochschulverlags } \\
\text { - } \quad \text { Dienstleister }\end{array}$ \\
\hline
\end{tabular}

\section{Content-Archivar}

Beschreibung Der Content-Archivar sorgt für das systematisierte Ablegen und

Archivieren aller Daten und Informationen zum Produkt.

\begin{aligned} \hline Anforderungsprofil & - Strukturiertheit \\ & - Grundlegende Kenntnisse im Archivarfach \end{aligned}

Rollenträger (Beispiele) — Mitarbeiter des Hochschulverlags 


\section{Projektmanager}

Beschreibung Die Rolle Projektmanager ist für die zeitliche Planung der

Produktion des Contents zuständig.

\begin{tabular}{ll}
\hline Anforderungsprofil & - Kommunikationsfähigkeit \\
& - Entscheidungsfähigkeit \\
& - Managementfähigkeit \\
& - Durchsetzungsstärke \\
& - Prundlegende bis tiefgreifende Kenntnisse im Personalwesen \\
& - Analysefähigkeit \\
& - die Fähigkeit, Effizienzpotenziale zu erkennen \\
& - geistige Flexibilität \\
& - Kenntnisse im Umgang mit Projektmanagement-Tools \\
\hline Rollenträger (Beispiele) & - Mitarbeiter des Hochschulverlags
\end{tabular}

\section{Controller}

\begin{tabular}{ll}
\hline Beschreibung & Die Rolle Controller koordiniert und steuert die Buchprojekte im \\
& Hochschulverlag. Zu diesem Zweck berichten andere Rollen \\
& relevante Kennzahlen zum laufenden Projekt an den Controller. \\
\hline Anforderungsprofil & - Kommunikationsfähigkeit \\
& - Entscheidungsfähigkeit \\
& - Zahlenaffinität \\
& - strategisches Denken \\
& - Prozess- und Zielorientierung \\
& - proaktives Handeln \\
& - Durchsetzungsstärke \\
& - Analysefähigkeit \\
& - die Fähigkeit, Effizienzpotenziale zu erkennen \\
& - geistige Flexibilität \\
& - Kenntnisse im Umgang mit Controlling-Tools \\
& - grundlegende bis tiefgreifende betriebswirtschaftliche \\
& \\
\hline Renntnisse & \\
\hline Rollenträger (Beispiele) & Mitarbeiter des Hochschulverlags
\end{tabular}




\section{Betriebswirt}

\begin{tabular}{|c|c|}
\hline Beschreibung & $\begin{array}{l}\text { Die Rolle Betriebswirt ist für die Kalkulation der Kosten für die } \\
\text { jeweiligen Buchprojekte verantwortlich }\end{array}$ \\
\hline Anforderungsprofil & $\begin{array}{ll}- & \text { Zahlenaffinität } \\
- & \text { grundlegende bis tiefgreifende kaufmännische Kenntnisse } \\
- & \text { grundlegende bis tiefgreifende Kenntnisse im Rechnungs-und } \\
\text { Steuerwesen }\end{array}$ \\
\hline Rollenträger (Beispiele) & $\begin{array}{ll}\text { - } & \text { Mitarbeiter des Hochschulverlags } \\
\text { - } & \text { Mitarbeiter der Finanzabteilung der Institution }\end{array}$ \\
\hline
\end{tabular}

\section{Drucker}

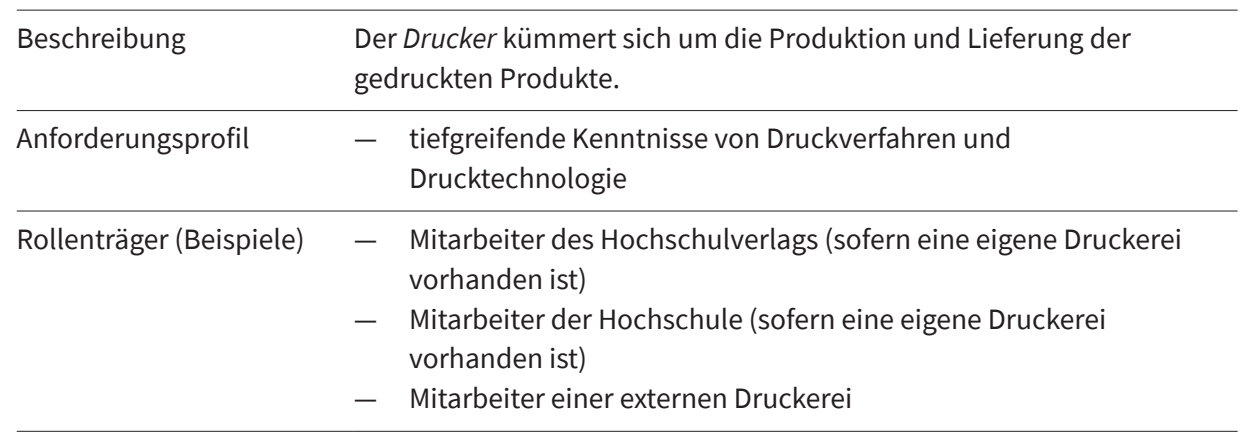

\section{Dienstleister (Metarolle)}

\begin{tabular}{ll}
\hline Beschreibung & $\begin{array}{l}\text { Die Rolle Dienstleister wird von demjenigen eingenommen, den der } \\
\text { Hochschulverlag mit der Umsetzung angebotener Services } \\
\text { beauftragt. }\end{array}$ \\
\hline Anforderungsprofil & - abhängig je nach Unternehmen \\
\hline Rollenträger (Beispiele) & - \\
\hline
\end{tabular}

\section{Kunde (Metarolle)}

Beschreibung Die Rolle des Kunden nehmen alle Personen ein, die die vom Hochschulverlag veröffentlichten Produkte erwerben. 


\subsection{Qualitätsrichtlinien}

Die Prozesskennblätter des hier vorgestellten Workflow-Modells enthalten für einige Prozesse den Verweis auf Qualitätsrichtlinien. Als Richtlinie werden hierbei Hilfestellungen oder Festlegungen verstanden, die Entscheidungen begründen oder erläutern, wie ein Prozess durchzuführen ist.

Im Sinne der Standardisierung des Publikationsworkflows empfiehlt es sich, vor dem Beginn der operativen Arbeit im Hochschulverlag Qualitätsrichtlinien festzulegen. Dabei bleibt es dem Anwender des Modells überlassen, wo er solche Richtlinien für relevant hält. Die Richtlinien für einen Prozess sollten so gestaltet werden, dass sie in jedem Projekt anwendbar sind. Weicht ein Projekt vom „Tagesgeschäft“ ab, sollte für diesen Einzelfall die Anwendbarkeit geprüft und die Erstellung fall- und prozessspezifischer Richtlinien erwogen werden. Das Prinzip der Richtlinie sollen im Folgenden vier Beispiele illustrieren.

\section{Beispiel 1:}

Der Publikationsworkflow beginnt mit der Auswahl oder Beschaffung von Konzepten. Um entscheiden zu können, ob eine Publikationsanfrage zur weiteren Prüfung angenommen werden kann (Prozess I-Ka-110), prüft der Content-Abnehmer die eingegangene Publikationsanfrage dahingehend, ob das Manuskript tendenziell in das Verlagsprogramm passt und ob die im Manuskript bearbeitete Forschungsfrage ein Alleinstellungsmerkmal aufweist bzw. originell ist. Bei der aktiven Suche nach Content (Prozess I-Ka-200) kann die Benotung der Graduierungsarbeit ein relevantes Auswahlkriterium sein oder auch, ob die Arbeit eine Auszeichnung oder Empfehlung, etwa durch die betreuenden Professoren, erhalten hat. Ausschlaggebend sind mitunter auch das Dateiformat oder der geschätzte Umfang des Manuskriptes. Die Richtlinie für die Konzept-Annahme könnte somit folgendermaßen aussehen:

\section{Richtlinie für die Konzept-Annahme}

\begin{tabular}{lcl}
\hline für Graduierungsarbeiten: & $\checkmark$ & Die schriftliche Arbeit wurde mit 1,0 bis 1,3 benotet. \\
& $\checkmark$ & Die Arbeit wurde ausgezeichnet. \\
& $\checkmark$ & Die Arbeit wurde für die Veröffentlichung empfohlen. \\
\hline für alle anderen Konzepte: & $\checkmark$ & Das Manuskript passt in das Verlagsprogramm. \\
& $\checkmark$ & Die Forschungsfrage weist ein Alleinstellungsmerkmal oder \\
& & eine gewisse Originalität auf.
\end{tabular}

\section{Beispiel 2:}

Es wird davon ausgegangen, dass zu Beginn des Prozesses $I-C v$ - 110 das vollständige Manuskript des Content-Urhebers vorliegt. Um entscheiden zu können, ob der Content für die weiteren Prozesse vereinnahmt wird, kann bspw. folgende Richtlinie festgesetzt werden: Der Hochschulverlag hat ein Template zur Gestaltung des Manuskriptes zur Verfügung gestellt. Die entsprechenden Formatvorlagen wurden im eingereichten Manuskript korrekt angewendet und zugewiesen. Zudem liegen 
die im Manuskript enthaltenen Abbildungen, Grafiken oder Fotos zur weiteren Verarbeitung als einzelne Daten in Druckqualität vor. An dieser Stelle empfiehlt es sich außerdem, eine Richtlinie zur Wahrung der Barrierefreiheit zu formulieren. Diese kann bspw. vorgeben, dass das Manuskript darauf geprüft wird, ob Alternativtexte für Abbildungen und ausführliche Beschreibungen für komplexere Grafiken (Diagramme oder schematische Darstellungen) vorhanden sind; außerdem, ob Informationen nicht nur über visuelle Merkmale wie Farben, Formen oder Positionen vermittelt werden (es sollte nicht heißen ,der rote Balken im Diagramm zeigt ...“ oder „,der Infokasten mit der grünen Umrandung erklärt ...“). ${ }^{74}$

\section{Richtlinie für die Content-Vereinnahmung}

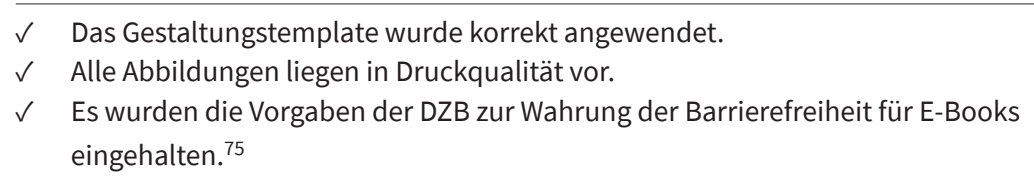

\section{Beispiel 3:}

Der managementorientierte Prozess $M-P v-100$ hat entscheidende Auswirkungen auf den weiteren Verlauf des Publikationsworkflows. Daher sollte hier dringend eine Richtlinie Anwendung finden, um entscheiden zu können, in welchen Ausgabeformaten der Content umgesetzt werden soll. Darin kann festgelegt werden, dass der Content ohne unverhältnismäßig hohen Aufwand medienneutral umsetzbar sein muss. Eine andere Richtlinie könnte lauten, dass sich der Produktionsweg nach der Finanzierung bzw. nach dem Projektbudget oder allein nach dem Wunsch des Content-Urhebers oder -Lieferanten richtet.

\section{Richtlinie für die Wahl des Produktionswegs}

$\checkmark$ Die medienneutrale Umsetzbarkeit des Contents ist möglich.

$\checkmark$ Die Wahl des Produktionswegs richtet sich nach dem Wunsch des Content-Urhebers oderLieferanten.

$\checkmark \quad$ Die Wahl des Produktionswegs richtet sich nach dem Budget bzw. nach den Finanzierungsmöglichkeiten.

\section{Beispiel 4:}

Um den Publikationsworkflow effizient zu gestalten, empfiehlt es sich außerdem, eine Richtlinie festzulegen, nach der entschieden wird, wann ein Sprachlektorat zu 
beauftragen ist (Prozess P-Ca-110). Ausschlaggebend für die Durchführung können bspw. folgende Punkte sein:

\section{Richtlinie für das Sprachlektorat}

$\checkmark$ Das Manuskript wurde von einem Nicht-Muttersprachler geschrieben.

$\checkmark$ Die Gutachter empfehlen die Durchführung eines Sprachlektorats am Manuskript.

$\checkmark \quad$ Aus den Gutachten bzw. Checklisten geht hervor, dass die Durchführung eines Sprachlektorats sinnvoll wäre.

$\checkmark$ Die Institution, in deren Namen publiziert wird, gibt vor immer ein Sprachlektorat durchzuführen.

An verschiedenen Stellen im Publikationsworkflow sind Richtlinien sinnvoll. Daher wurden vor Beginn der Fallstudie projektspezifische Richtlinien erstellt. Sie werden bei den entsprechenden Fällen ab Kapitel 4.3 beschrieben. Eine Übersicht zu diesen und weiteren möglichen Richtlinien ist außerdem im Anhang A zu finden.

\subsection{Prozesskennblätter}

Auf den nachfolgenden Seiten werden die Prozesse des Workflow-Modells im Detail mit Hilfe der Prozesskennblätter beschrieben. Dabei enthält die erste Seite des Blattes die narrative Prozessbeschreibung des jeweiligen Prozesses und eine Darstellung der vor- und nachgelagerten Prozesse mit ihren jeweiligen In- und Outputs zur besseren Orientierung im Modell. Zudem finden sich hier - sofern empfohlen - der Verweis auf eine Qualitätsrichtlinie zum Prozess sowie eine Auflistung der am Prozess beteiligten Rollen. Darüber hinaus werden, wie bereits in Kapitel 2.3 erläutert, die benötigten Ressourcen als Zeit- und Kostenvariable aufgeführt. Welche projektabhängigen, tatsächlichen Zeit- und Kostenaufwände für diese Variablen verbraucht wurden, ist durch die Fallstudie ermittelt worden. Sie werden in Kapitel 4.4 dargestellt.

Auf der zweiten Seite des Prozesskennblattes ist der Ablauf des Prozesses als Flussdiagramm modelliert. Dieser Prozessablauf ist die visuelle Darstellung der Prozessbeschreibung; er soll die Prozesse nachvollziehbarer machen.

Für die Darstellung der Flussdiagramme wurde sich am aktuellen Standard zur Geschäftsprozessmodellierung, BPMN 2.0 (Business Process Model and Notation 2.0 $)^{76}$ orientiert. Die folgenden Elemente aus dieser Modellierungssprache fanden bei der Beschreibung der Prozessabläufe Verwendung. 
Tab. 3 Legende der verwendeten BPMN-Elemente in den Prozessabläufen

\begin{tabular}{|c|c|}
\hline BPMN-Element & Bedeutung \\
\hline & $\begin{array}{l}\text { Symbol Sequenzfluss: } \\
\text { definiert die Abfolge der Ausführung von Aufgaben }{ }^{77}\end{array}$ \\
\hline & $\begin{array}{l}\text { Symbol Ereignis beginnt: } \\
\text { kennzeichnet den ersten Schritt in einem Prozess }{ }^{78}\end{array}$ \\
\hline & $\begin{array}{l}\text { Symbol Ereignis endet: } \\
\text { kennzeichnet den letzten Schritt in einem Prozess }{ }^{79}\end{array}$ \\
\hline \multirow[t]{3}{*}{ (x) } & $\begin{array}{l}\text { Symbol Abbruch: } \\
\text { kennzeichnet beim Endereignis, dass der Abbruch eines Prozesses } \\
\text { ausgelöst wurde }{ }^{80}\end{array}$ \\
\hline & $\begin{array}{l}\text { Symbol Aufgabe: } \\
\text { enthält eine Aktivität innerhalb eines Prozesses, die nicht weiter unterteilt } \\
\text { werden kann }{ }^{81}\end{array}$ \\
\hline & $\begin{array}{l}\text { Symbol Datenobjekt: } \\
\text { - } \quad \text { repräsentiert nach BPMN } 2.0 \text { „Informationen, die durch den Prozess } \\
\text { fließen, wie z. B. Dokumente, Emails, Briefe oder Datensätze“ } 82 \\
\text { - } \quad \text { stellt also „Datenanforderungen dar, von denen Aufgaben im } \\
\text { Geschäftsprozess abhängen“ } 83 \\
\text { - } \quad \text { wird in den Prozessabläufen als Symbol für eine Qualitätsrichtlinie } \\
\text { verwendet }\end{array}$ \\
\hline$\langle\boldsymbol{x}$ & $\begin{array}{l}\text { Gateway-Symbol Exklusiv: } \\
\text { - } \quad \text { repräsentiert nach BPMN } 2.0 \text { die Bewertung des Status „eines } \\
\text { Geschäftsprozesses in Bezug auf eine bestimmte Bedingung und } \\
\text { unterteilt den Fluss in Abhängigkeit davon in einen oder mehrere } \\
\text { einander gegenseitig ausschließende Pfade“84 } \\
\text { - } \\
\text { stellt (an diese Beschreibung angelehnt) in den Prozessabläufen einen } \\
\text { Entscheidungspunkt dar }\end{array}$ \\
\hline
\end{tabular}

77 vgl. Berliner BPM-Offensive 2019

78 vgl. Lucid Software Inc. (1) 2019

79 Lucid Software Inc. (1) 2019

80 Lucid Software Inc. (1) 2019

81 vgl. Lucid Software Inc. (1) 2019

82 Berliner BPM-Offensive 2019

83 Lucid Software Inc. (2) 2019

84 Lucid Software Inc. (2) 2019 


\section{Publikationsanfrage erhalten [I-Ka-100]}

\section{Prozessbeschreibung}

Der Content-Urheber oder Content-Lieferant schlägt dem Content-Abnehmer ein Konzept zur Publikation im Hochschulverlag vor.

Vor- und nachgelagerte Prozesse

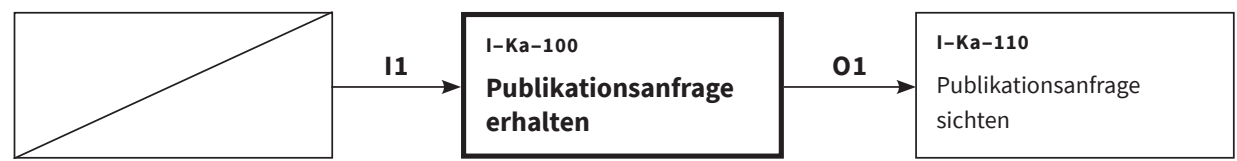

\section{Input}

- I1: Publikationsanfrage liegt vor

\section{Output}

- O1: eingegangene Publikationsanfrage

\section{Beteiligte Rollen}

- Content-Urheber

- Content-Lieferant

- Content-Abnehmer

\section{Ressourcen}

- Zeit: T-[I-Ka-100] 


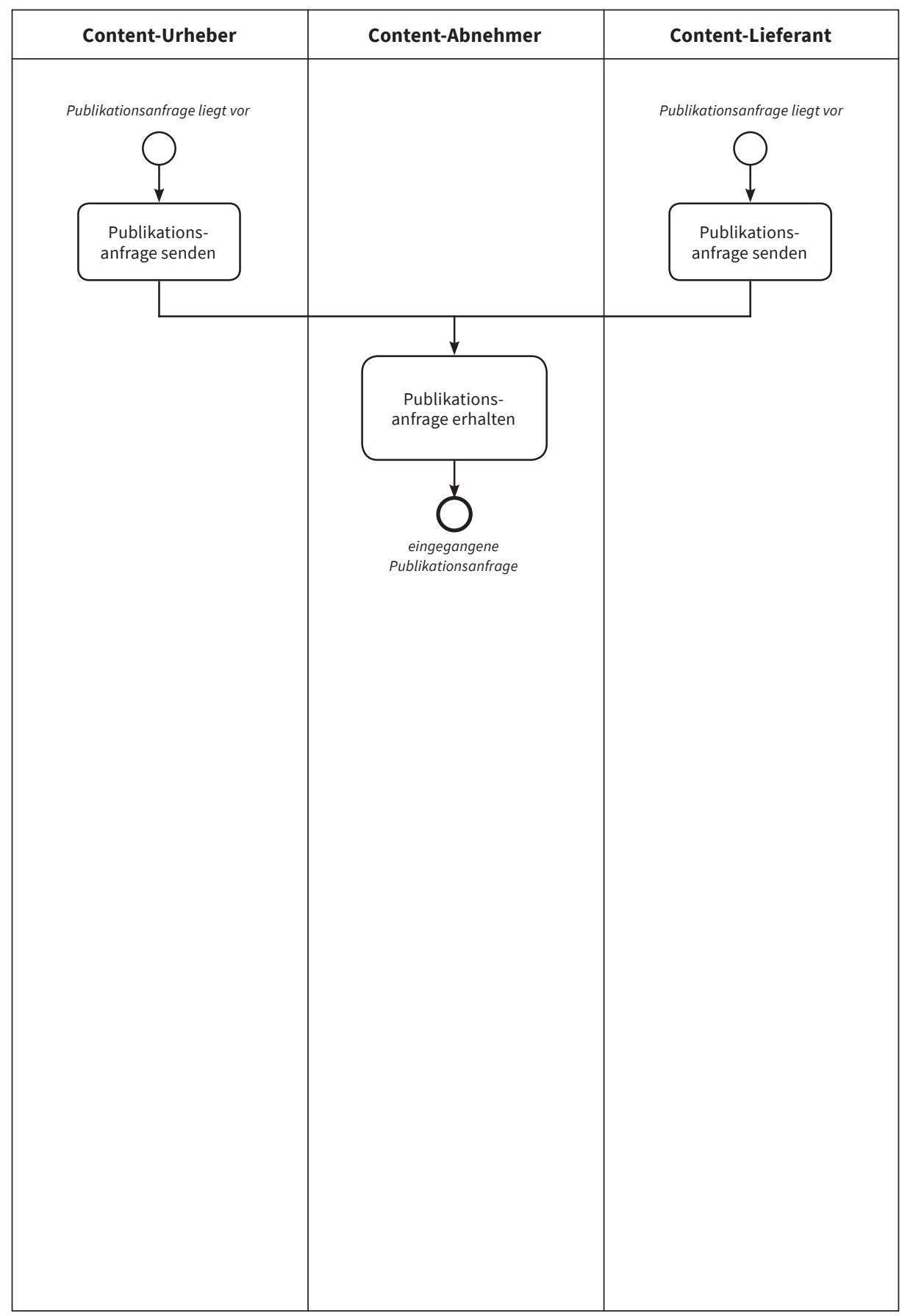




\section{Publikationsanfrage sichten [I-Ka-110]}

\section{Prozessbeschreibung}

Der Content-Abnehmer überprüft selbst, ob das Konzept die Richtlinie für die Konzept-Annahme erfüllt oder er leitet die Anfrage zur Überprüfung, bspw. an ein Gremium oder Beirat der Hochschule weiter.

Vor- und nachgelagerte Prozesse

\begin{tabular}{|c|c|c|c|c|}
\hline $\begin{array}{l}\text { I-Ka-100 } \\
\text { Publikationsanfrage } \\
\text { erhalten }\end{array}$ & $\stackrel{I 1}{\longrightarrow}$ & $\begin{array}{l}\text { I-Ka-110 } \\
\text { Publikationsanfrage } \\
\text { sichten }\end{array}$ & 01 & $\begin{array}{l}\text { I-Ka-120 } \\
\text { Konzept zur Prüfung } \\
\text { annehmen }\end{array}$ \\
\hline & & & 02 & $\begin{array}{l}\text { I-Ka-130 } \\
\text { Konzept zur Prüfung } \\
\text { ablehnen }\end{array}$ \\
\hline
\end{tabular}

\section{Input}

- I1: eingegangene Publikationsanfrage

\section{Output}

- O1: gesichtete Publikationsanfrage: Konzept erfüllt Richtlinie

- O2: gesichtete Publikationsanfrage: Konzept erfüllt nicht Richtlinie

\section{Qualitätsrichtlinie}

- Richtlinie für die Konzept-Annahme

\section{Beteiligte Rollen}

- Content-Abnehmer

\section{Ressourcen}

- Zeit: T-[I-Ka-110] 


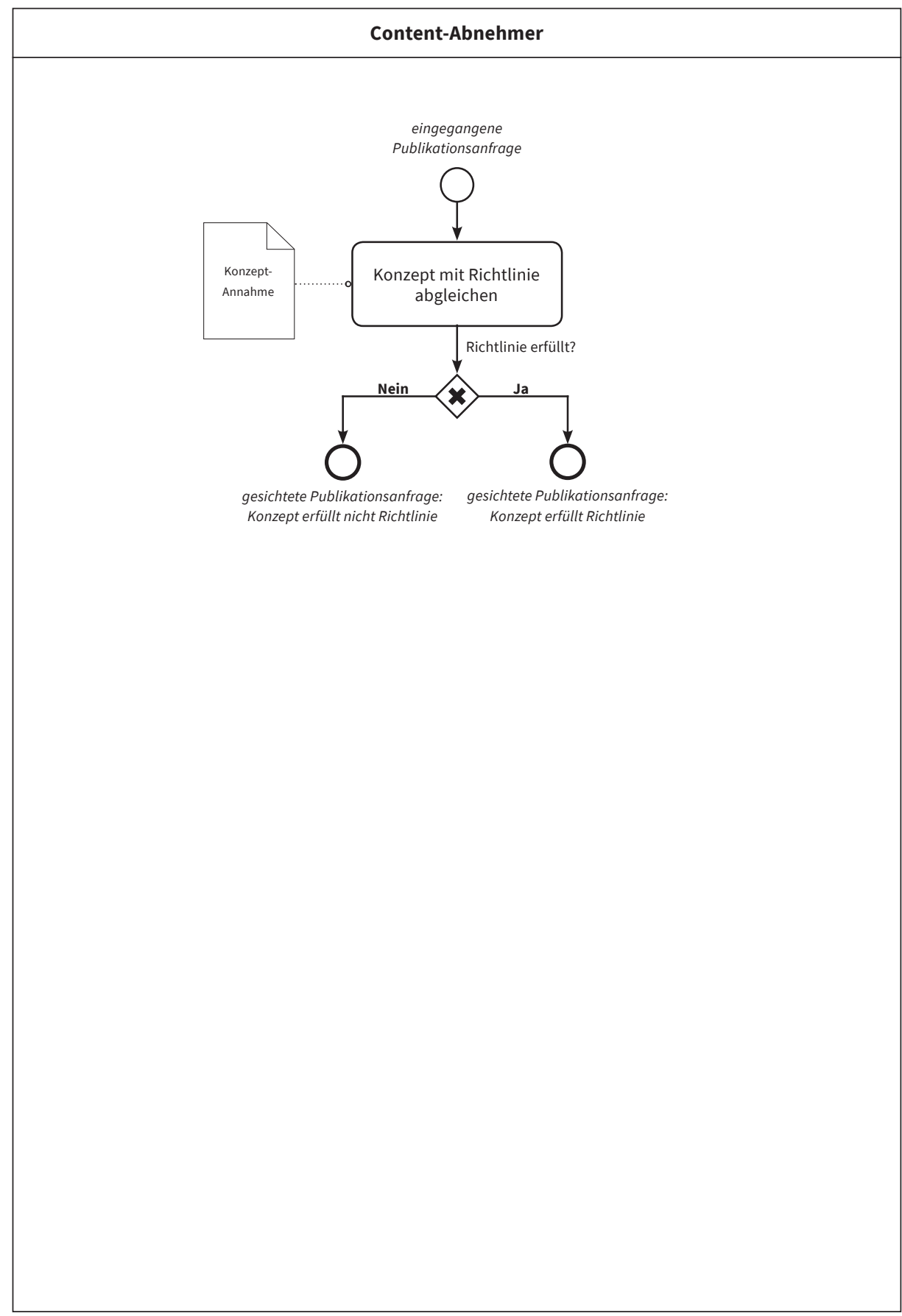




\section{Konzept zur Prüfung annehmen [I-Ka-120]}

\section{Prozessbeschreibung}

Entspricht das Konzept der Richtlinie, kann es zur weiteren Prüfung im Publikationsworkflow angenommen werden. Der Content-Abnehmer informiert den Content-Urheber oder Content-Lieferanten.

Vor- und nachgelagerte Prozesse

\begin{tabular}{|c|c|c|c|c|}
\hline $\begin{array}{l}\text { I-Ka-110 } \\
\text { Publikationsanfrage } \\
\text { sichten }\end{array}$ & I1 & $\begin{array}{l}\text { I-Ka-120 } \\
\text { Konzept zur Prüfung } \\
\text { annehmen }\end{array}$ & 01 & $\begin{array}{l}\text { I-Cv-100 } \\
\text { Content einfordern }\end{array}$ \\
\hline
\end{tabular}

\section{Input}

- I1: gesichtete Publikationsanfrage: Konzept erfüllt Richtlinie

\section{Output}

- O1: Konzept zur Prüfung angenommen

\section{Beteiligte Rollen}

- Content-Abnehmer

- Content-Urheber

- Content-Lieferant

\section{Ressourcen}

- Zeit: T-[I-Ka-120] 


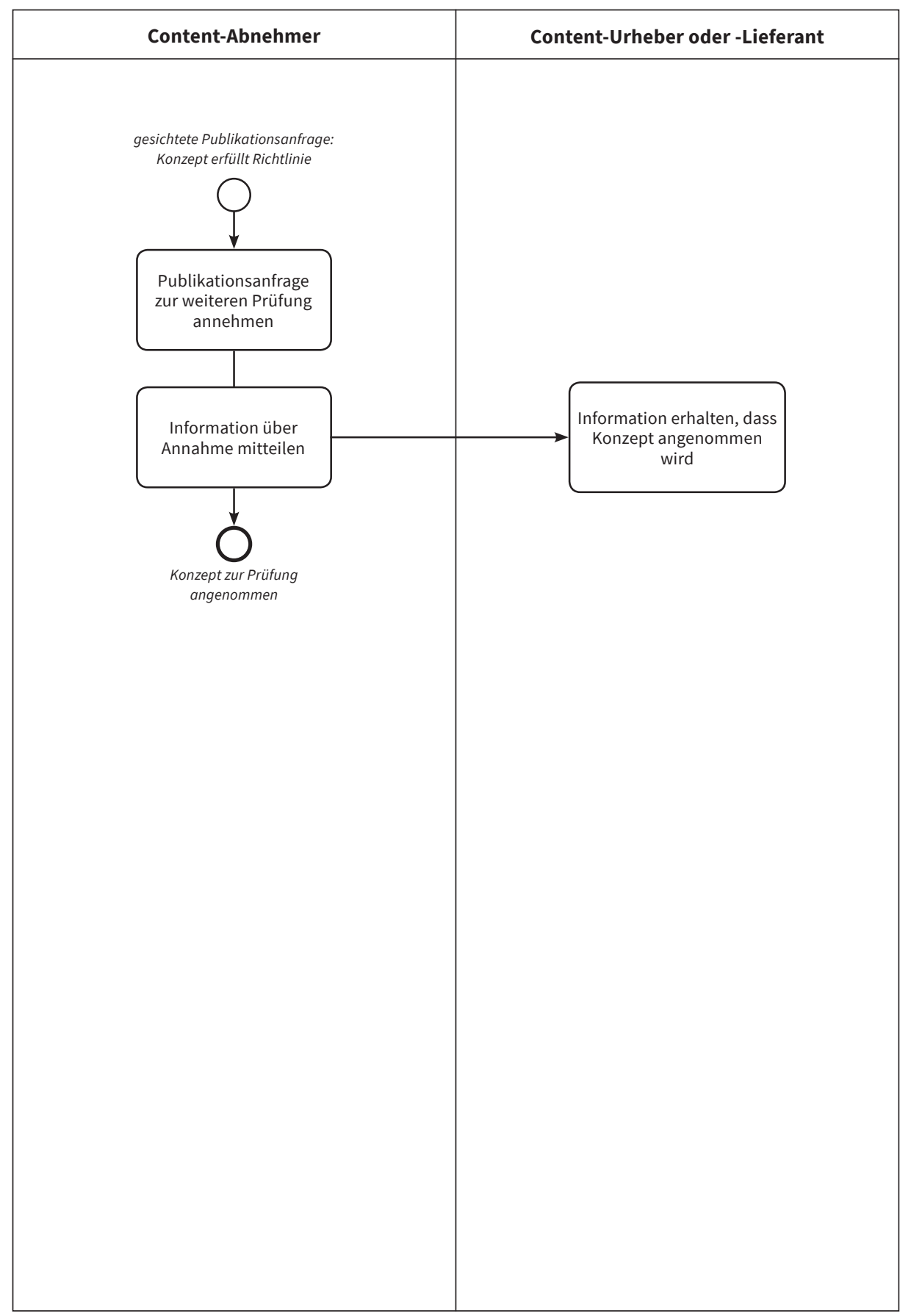




\section{Konzept zur Prüfung ablehnen [I-Ka-130]}

\section{Prozessbeschreibung}

Entspricht das Konzept nicht der Richtlinie, wird die Publikationsanfrage zur weiteren Prüfung im Publikationsworkflow abgelehnt. Der Content-Abnehmer informiert den Content-Urheber oder Content-Lieferanten.

Vor- und nachgelagerte Prozesse

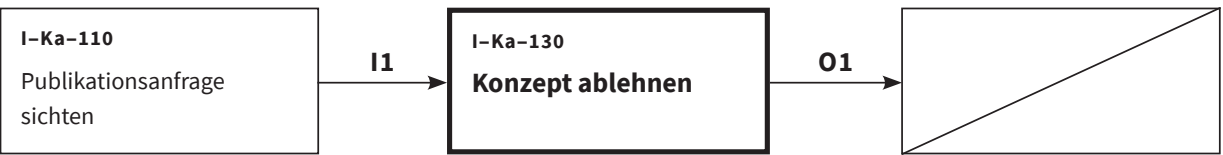

\section{Input}

- I1: gesichtete Publikationsanfrage: Konzept erfüllt nicht Richtlinie

\section{Output}

- O1: Konzept zur Prüfung abgelehnt

\section{Beteiligte Rollen}

- Content-Abnehmer

- Content-Urheber

- Content-Lieferant

\section{Ressourcen}

- Zeit: T-[I-Ka-130] 


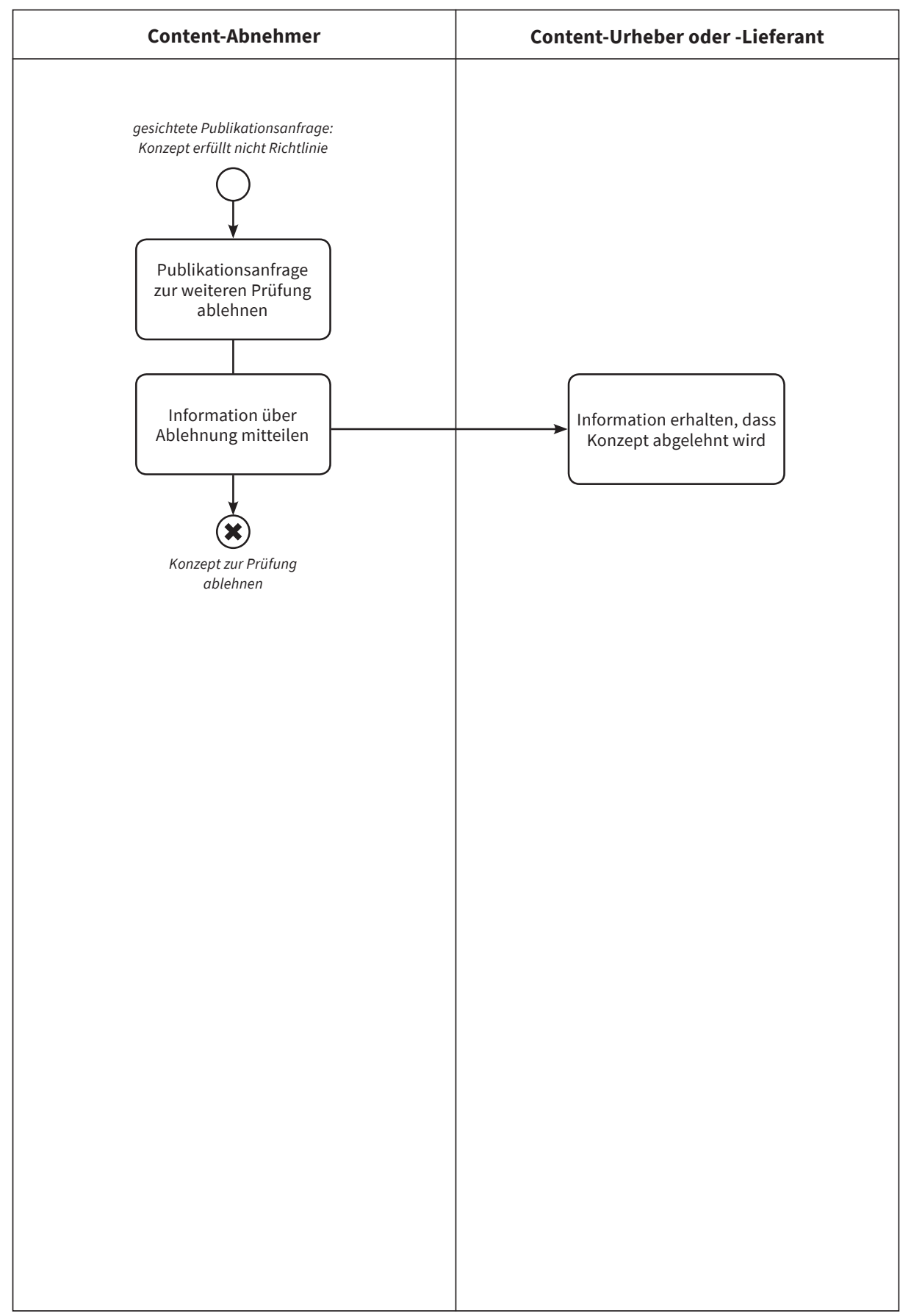




\section{Nach geeigneten Content-Urhebern recherchieren [I-Ka-200]}

\section{Prozessbeschreibung}

Der Content-Abnehmer recherchiert entsprechend der Richtlinie für die KonzeptAnnahme nach geeigneten Content-Urhebern, damit diese im Hochschulverlag publizieren oder in dessen Auftrag Content zur Publikation erstellen. Ggf. fragt er bei Content-Lieferanten nach Unterstützung an.

Vor- und nachgelagerte Prozesse

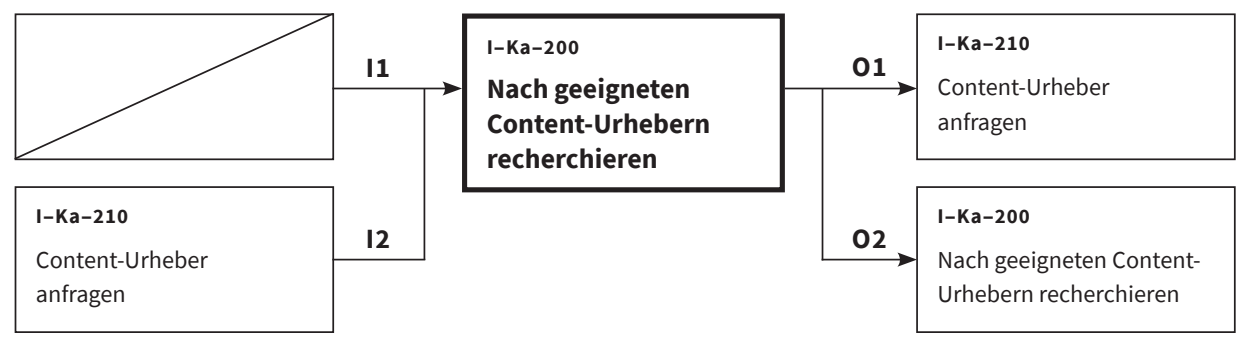

\section{Input}

- I1: Content zur Publikation gesucht

- I2: keinen geeigneten Content-Urheber gefunden, der Interesse hat

\section{Output}

- O1: geeigneten Content-Urheber gefunden

- O2: keinen geeigneten Content-Urheber gefunden

\section{Qualitätsrichtlinie}

- Richtlinie für die Konzept-Annahme

\section{Beteiligte Rollen}

- Content-Abnehmer

\section{Ressourcen}

- Zeit: T-[I-Ka-200] 


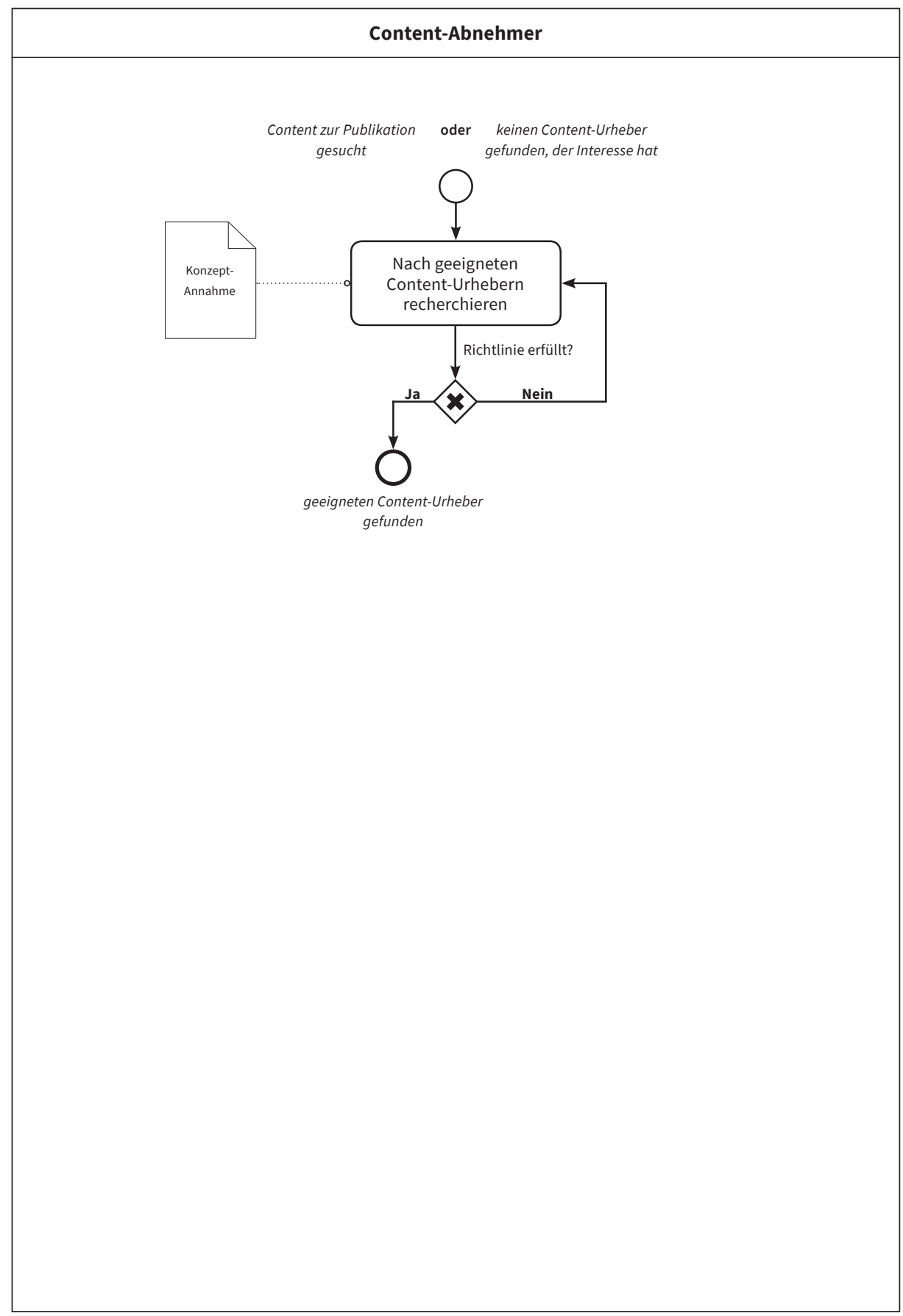




\section{Content-Urheber anfragen [I-Ka-210]}

\section{Prozessbeschreibung}

Der Content-Abnehmer fragt bei den geeigneten Content-Urhebern an, ob sie Content im Hochschulverlag publizieren möchten oder in dessen Auftrag Content zur Publikation erstellen würden. Mind. ein Content-Urheber hat Interesse. Sofern keiner der angefragten Content-Urheber Interesse hat, werden weitere recherchiert.

Vor- und nachgelagerte Prozesse

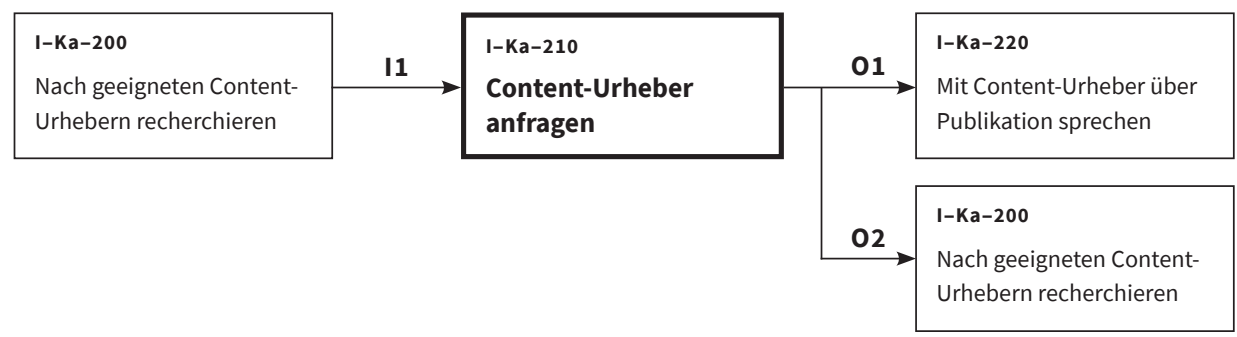

\section{Input}

- I1: geeigneten Content-Urheber gefunden

\section{Output}

- O1: mind. ein Content-Urheber hat Interesse

- O2: kein Content-Urheber hat Interesse

\section{Beteiligte Rollen}

- Content-Abnehmer

\section{Ressourcen}

- Zeit: T-[I-Ka-210] 


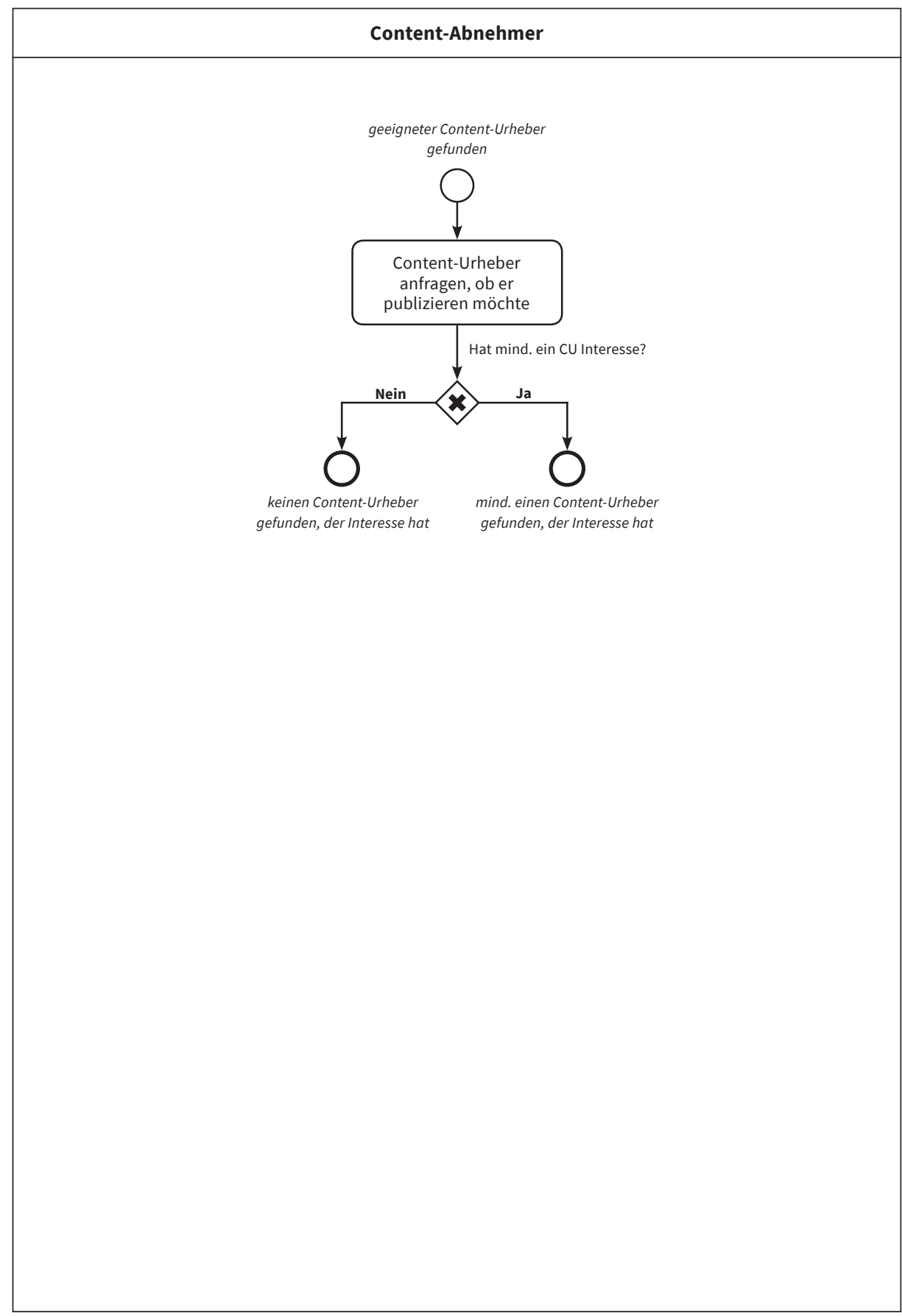




\section{Mit Content-Urheber über Publikation sprechen [I-Ka-220]}

\section{Prozessbeschreibung}

Der Content-Abnehmer bespricht mit dem Content-Urheber das Konzept der zu erstellenden Publikation. Der Content-Urheber ist einverstanden im Hochschulverlag zu publizieren.

Vor- und nachgelagerte Prozesse

\begin{tabular}{|c|c|c|c|c|}
\hline I-Ka-210 & & I-Ka-220 & & $\mathrm{I}-\mathrm{Cv}-100$ \\
\hline $\begin{array}{l}\text { Content-Urheber } \\
\text { anfragen }\end{array}$ & I1 & $\begin{array}{l}\text { Mit Content-Urheber } \\
\text { über Publikation } \\
\text { sprechen }\end{array}$ & 01 & Content einfordern \\
\hline
\end{tabular}

\section{Input}

- I1: Mind. ein Content-Urheber hat Interesse

\section{Output}

- O1: Publikationseinverständnis vom Content-Urheber

\section{Beteiligte Rollen}

- Content-Abnehmer

\section{Ressourcen}

- Zeit: T-[I-Ka-220] 


\section{Content-Abnehmer}

mind. einen Content-Urheber gefunden, der Interesse hat

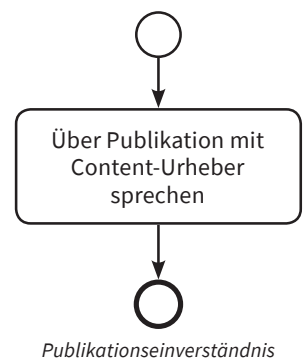

vom Content-Urheber 


\section{Content einfordern [I-Cv-100]}

\section{Prozessbeschreibung}

Der Content-Abnehmer fordert den benötigten Content vom Content-Urheber oder -Lieferanten ein. Dieser sendet den gewünschten Content an den Content-Abnehmer.

Vor- und nachgelagerte Prozesse

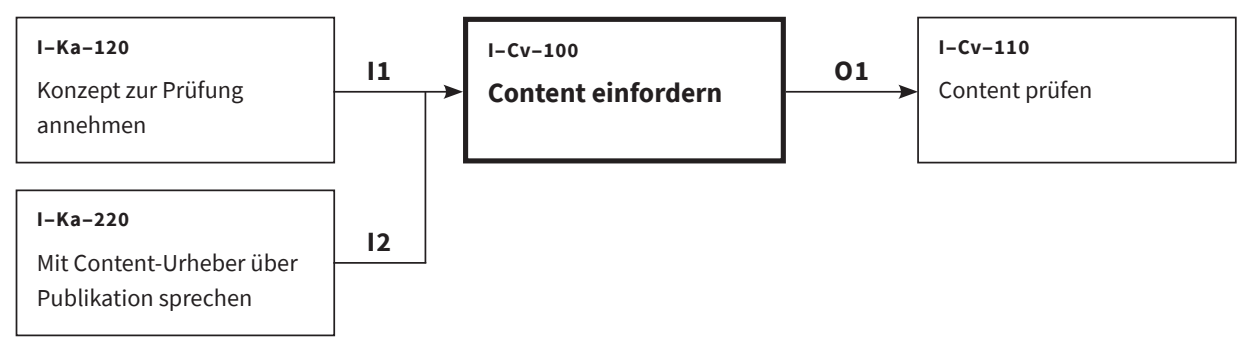

\section{Input}

- I1: Konzept zur Prüfung angenommen

- I2: Publikationseinverständnis vom Content-Urheber

\section{Output}

- O1: Content liegt vor

\section{Beteiligte Rollen}

- Content-Abnehmer

- Content-Urheber

- Content-Lieferant

\section{Ressourcen}

- Zeit: T-[I-Cv-100] 


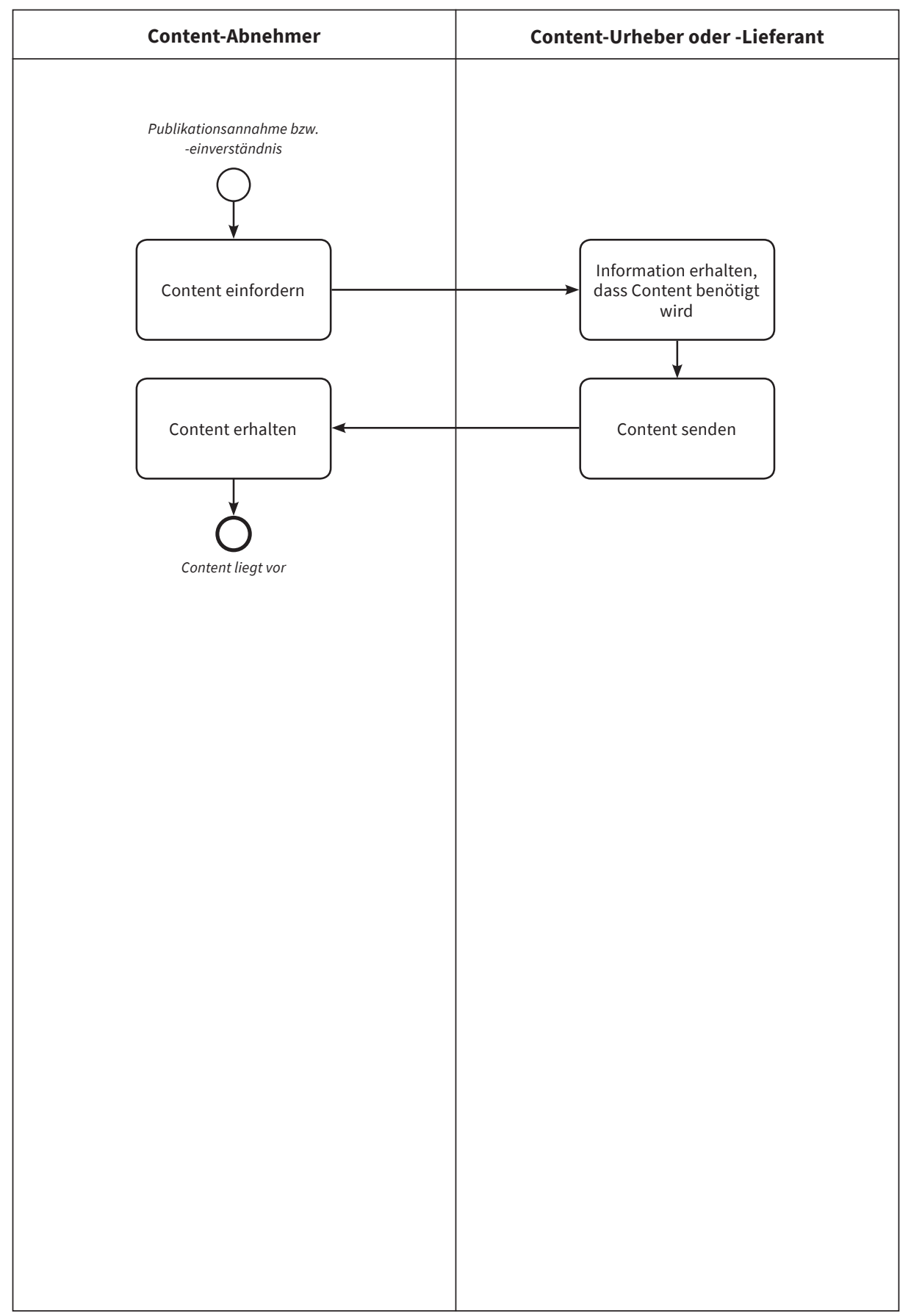




\section{Content prüfen [I-CV-110]}

\section{Prozessbeschreibung}

Der Content-Abnehmer überprüft den gelieferten Content entsprechend der Richtlinie für die Content-Vereinnahmung. Sofern zu der Richtlinie der Content-Vereinnahmung auch Richtlinien zur Sicherstellung der Barrierefreiheit zählen, prüft der Barrierefreiheit-Beauftragte den Content ebenfalls hinsichtlich dieser Richtlinie. Wird an dieser Stelle festgestellt, dass der Content Inhaltselemente (wie Abbildungen, Fotos, Grafiken) enthält, von denen der Content-Urheber nicht der Rechteinhaber ist, kann der Prozess $M-P V e-100$ bereits angestoßen werden.

Vor- und nachgelagerte Prozesse

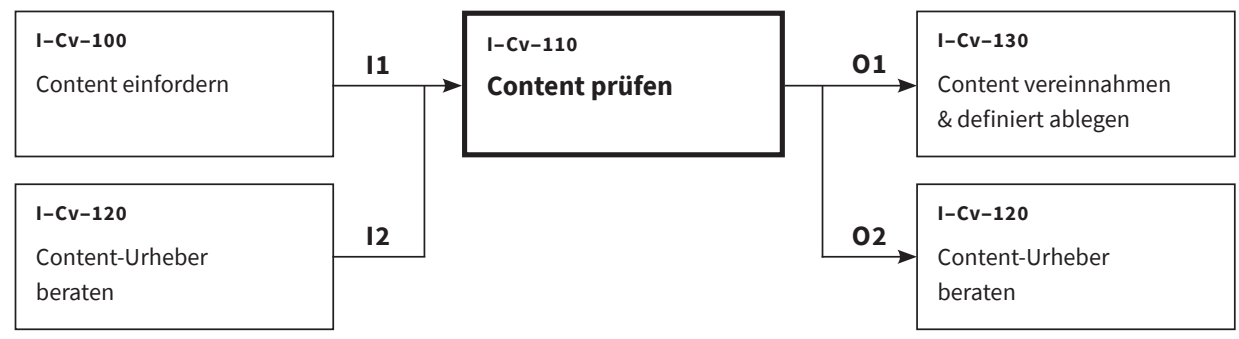

\section{Input}

- I1: Content liegt vor

- I2: überarbeiteten Content

\section{Output}

- O1: Content erfüllt Richtlinie

- O2: Content erfüllt Richtlinie nicht

\section{Qualitätsrichtlinie}

- Richtlinie für die Content-Vereinnahmung

\section{Beteiligte Rollen}

- Content-Abnehmer

- Barrierefreiheit-Beauftragter

\section{Ressourcen}

- Zeit: T-[I-Cv-110] 


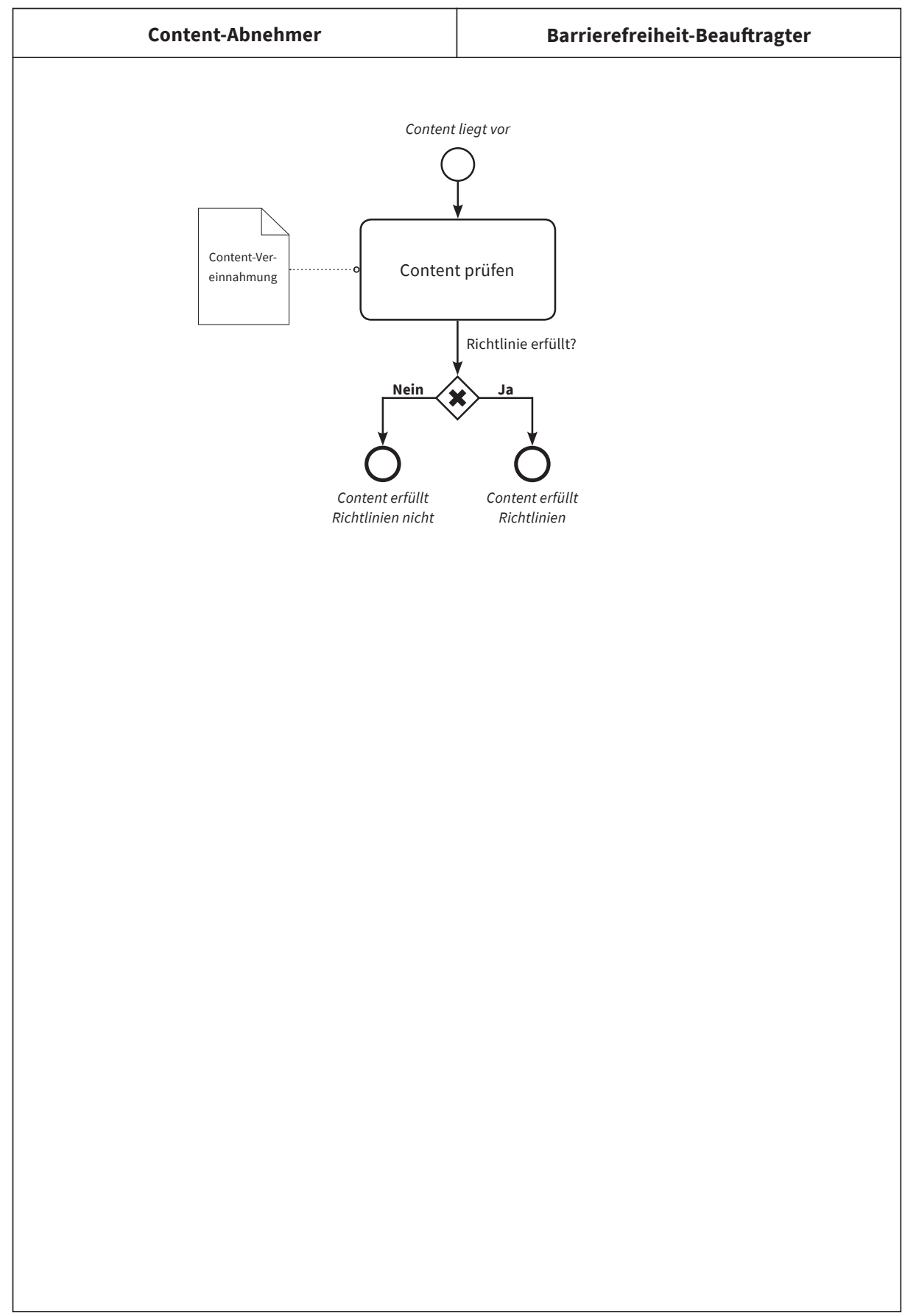




\section{Content-Urheber beraten [I-Cv-120]}

\section{Prozessbeschreibung}

Der Content erfüllt nicht die notwendige Richtlinie für die Content-Vereinnahmung bzw. die Barrierefreiheit. Der Content-Abnehmer und/oder der Barrierefreiheit-Beauftragte beraten den Content-Urheber bezüglich der notwendigen Korrekturen und fordern deren Umsetzung.

Vor- und nachgelagerte Prozesse

\begin{tabular}{|c|c|c|c|c|}
\hline $\begin{array}{l}\text { I-Cv-110 } \\
\text { Content prüfen }\end{array}$ & I1 & $\begin{array}{l}\text { I-Cv-120 } \\
\text { Content-Urheber } \\
\text { beraten }\end{array}$ & 01 & $\begin{array}{l}\text { I-Cv-110 } \\
\text { Content prüfen }\end{array}$ \\
\hline
\end{tabular}

\section{Input}

- I1: Content erfüllt Richtlinie nicht

\section{Output}

- O1: überarbeiteten Content

\section{Beteiligte Rollen}

- Content-Abnehmer

- Barrierefreiheit-Beauftragter

- Content-Urheber

\section{Ressourcen}

- Zeit: T-[I-Cv-120] 


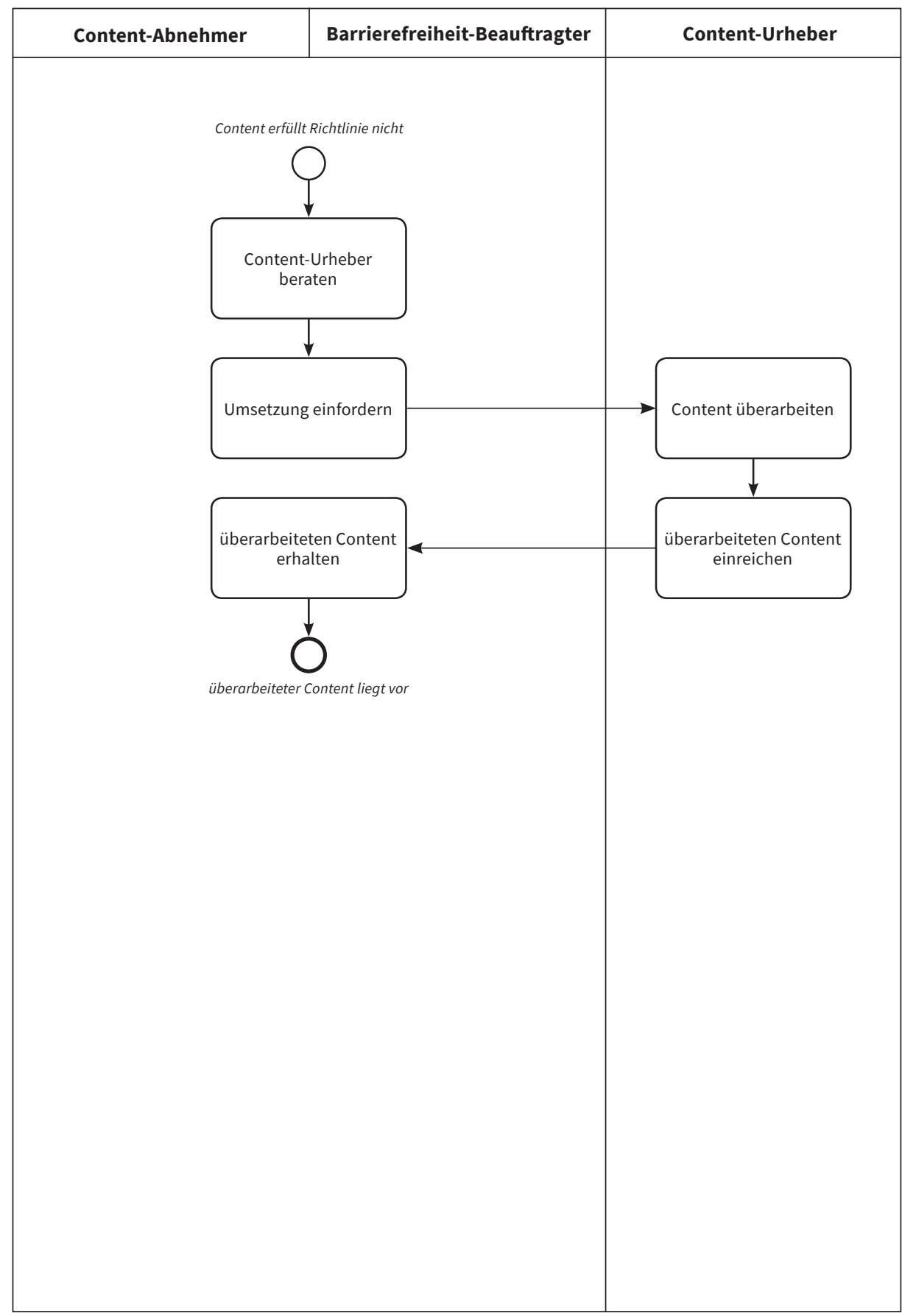




\section{Content vereinnahmen \& definiert ablegen [I-Cv-130]}

\section{Prozessbeschreibung}

Der Content-Abnehmer und ggf. der Barrierefreiheit-Beauftragter stellen fest, dass der Content die Richtlinie für die Content-Vereinnahmung erfüllt, sodass der Content vereinnahmt werden kann. Der Content-Abnehmer legt den Content so ab, dass alle relevanten Rollen Zugriff haben.

Vor- und nachgelagerte Prozesse

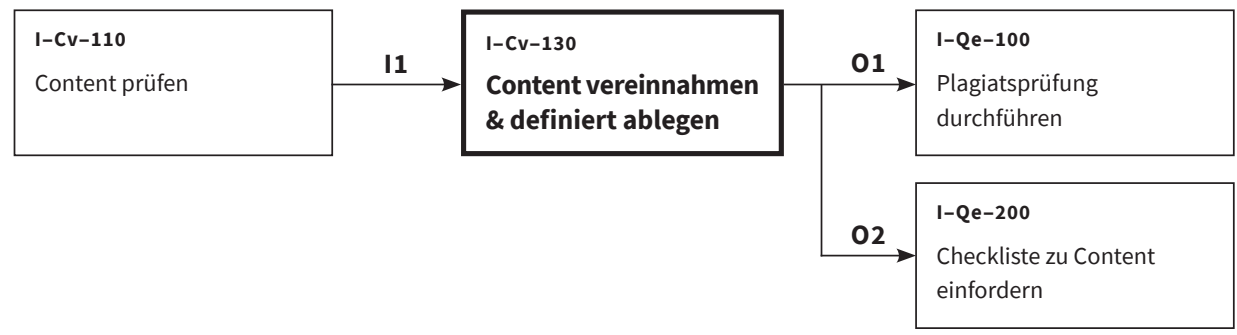

\section{Input}

- I1: Content erfüllt Richtlinie

\section{Output}

- O1: vereinnahmter und definiert abgelegter Content

- O2: vereinnahmter und definiert abgelegter Content

\section{Beteiligte Rollen}

- Content-Abnehmer

\section{Ressourcen}

- Zeit: T-[I-Cv-130] 


\section{Content-Abnehmer}

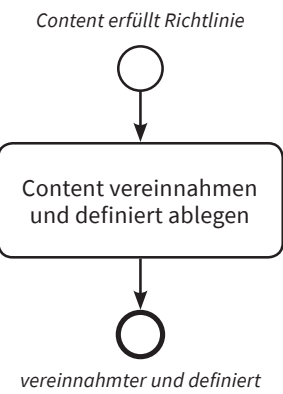

abgelegter Content 


\section{Plagiatsprüfung durchführen [I-Qe-100]}

\section{Prozessbeschreibung}

Der Content-Prüfer führt entsprechend der Richtlinie eine Plagiatsprüfung am Content durch und prüft ggf. kritisch die Ergebnisse.

Vor- und nachgelagerte Prozesse

\begin{tabular}{|c|c|c|c|c|}
\hline \multirow{2}{*}{$\begin{array}{l}\text { I-Cv-130 } \\
\text { Content vereinnahmen } \\
\text { \& definiert ablegen }\end{array}$} & \multirow[t]{2}{*}{ I1 } & \multirow{2}{*}{$\begin{array}{l}\text { I-Qe-100 } \\
\text { Plagiatsprüfung } \\
\text { durchführen }\end{array}$} & 01 & \\
\hline & & & & ablehnen \\
\hline & & & 02 & $\begin{array}{l}\text { I-Qe-110 } \\
\text { Peer-Review-Verfahren } \\
\text { auswählen }\end{array}$ \\
\hline
\end{tabular}

\section{Input}

- I1: vereinnahmter und definiert abgelegter Content

\section{Output}

- O1: nicht bestandene Plagiatsprüfung

- O2: bestandene Plagiatsprüfung

\section{Qualitätsrichtlinie}

- Richtlinie für die Plagiatsprüfung

\section{Beteiligte Rollen}

- Content-Prüfer

\section{Ressourcen}

- Zeit: T-[I-Qe-100]

- Kosten: K-[I-Qe-100] 


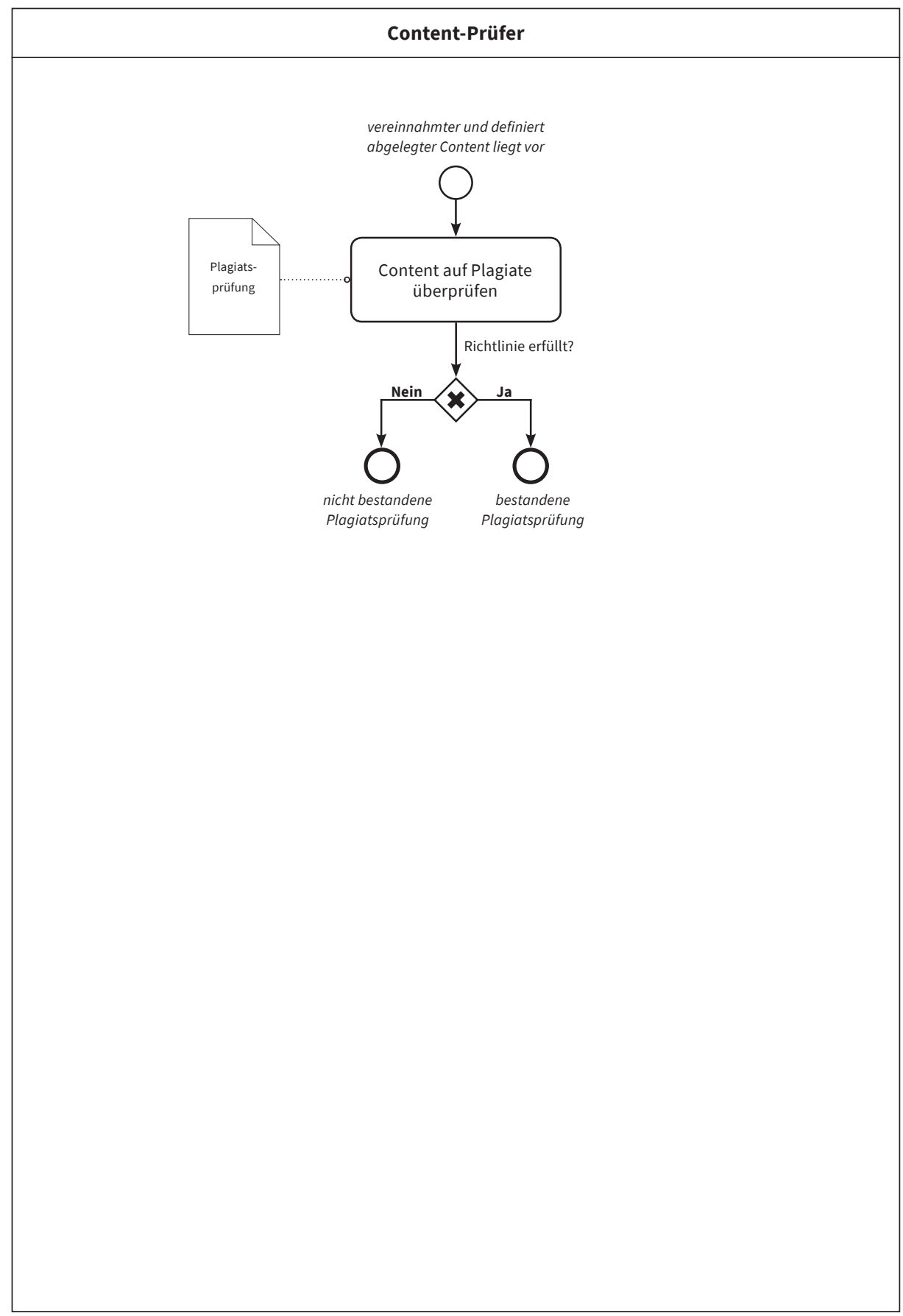




\section{Peer-Review-Verfahren auswählen [I-Qe-110]}

\section{Prozessbeschreibung}

Der Content erfüllt die Richtlinie für die Plagiatsprüfung und stellt kein Plagiat dar. Der Content-Prüfer wählt ein Peer-Review-Verfahren nach der Richtlinie für die Wahl des Peer-Review-Verfahrens aus.

Vor- und nachgelagerte Prozesse

\begin{tabular}{|c|c|c|c|c|}
\hline $\begin{array}{l}\text { I-Qe-100 } \\
\text { Plagiatsprüfung } \\
\text { durchführen }\end{array}$ & I1 & $\begin{array}{l}\text { I-Qe-110 } \\
\text { Peer-Review- } \\
\text { Verfahren auswählen }\end{array}$ & 01 & $\begin{array}{l}\mathbf{I}-\mathbf{Q} \mathbf{e - 1 2 0} \\
\text { Gutachter recherchieren } \\
\text { \& anfragen }\end{array}$ \\
\hline
\end{tabular}

\section{Input}

- I1: bestandene Plagiatsprüfung

\section{Output}

- O1: ausgewähltes Peer-Review-Verfahren

\section{Qualitätsrichtlinie}

— Richtlinie für die Wahl des Peer-Review-Verfahrens

\section{Beteiligte Rollen}

- Content-Prüfer

\section{Ressourcen}

- Zeit: T-[I-Qe-110] 


\section{Content-Prüfer}

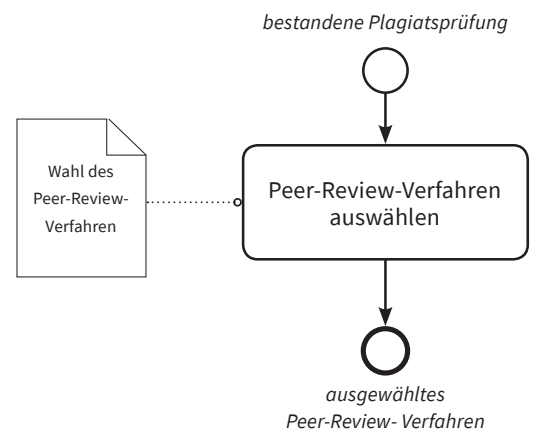




\section{Gutachter recherchieren \& anfragen [I-Qe-120]}

\section{Prozessbeschreibung}

Der Content-Prüfer recherchiert zu möglichen Gutachtern für das ausgewählte Peer-Review-Verfahren und fragt anschließend einen oder mehrere Gutachter an, ob sie ein Gutachten zum Content erstellen. Hierbei können sowohl externe Experten als auch Experten vor Ort (bspw. Hochschulprofessoren) in Frage kommen. Findet der Content-Prüfer keinen geeigneten Gutachter, recherchiert er weiter.

Vor- und nachgelagerte Prozesse

\begin{tabular}{|l|l|l|l|}
\cline { 2 - 3 } $\begin{array}{l}\text { I-Qe-110 } \\
\text { Peer-Review-Verfahren } \\
\text { auswählen }\end{array}$ & $\begin{array}{l}\text { I-Qe-120 } \\
\text { Gutachter } \\
\text { recherchieren } \\
\text { \& anfragen }\end{array}$ & $\mathbf{0 1}$ & $\begin{array}{l}\text { I-Qe-130 } \\
\text { Peer-Review } \\
\text { beauftragen }\end{array}$ \\
\cline { 2 - 4 }
\end{tabular}

\section{Input}

- I1: ausgewähltes Peer-Review-Verfahren

\section{Output}

- O1: Zusage der gewünschten Gutachter

\section{Beteiligte Rollen}

- Content-Prüfer

- Gutachter

\section{Ressourcen}

- Zeit: T-[I-Qe-120] 


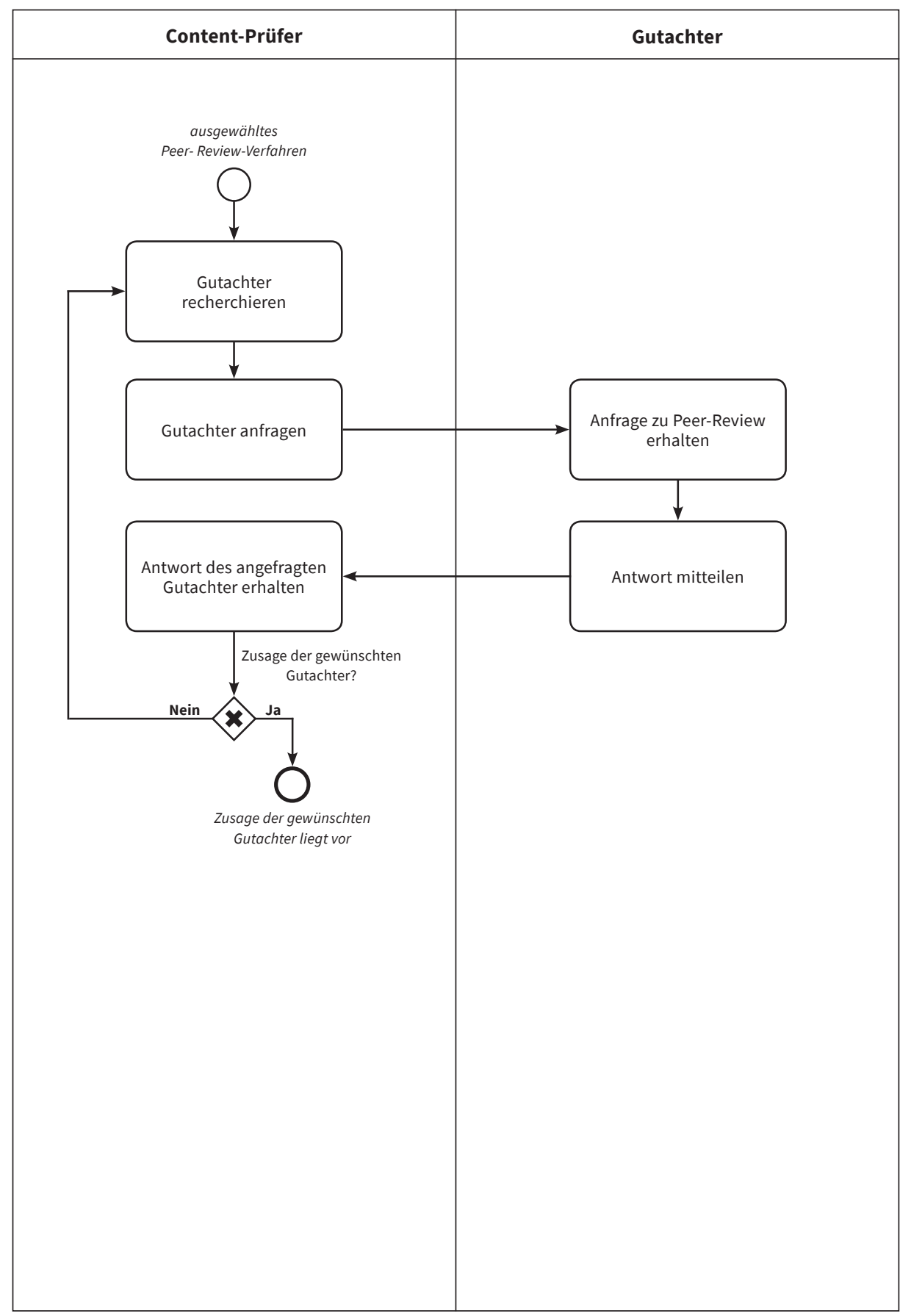




\section{Peer-Review beauftragen [I-Qe-130]}

\section{Prozessbeschreibung}

Der/die angefragte/n Gutachter ist/sind einverstanden ein Peer-Review am Content durchzuführen. Der Content-Prüfer beauftragt verbindlich die Durchführung, setzt eine Deadline und übermittelt den vereinnahmten Content des Content-Urhebers.

Vor- und nachgelagerte Prozesse

\begin{tabular}{|l|l|l|l|l|}
\hline $\begin{array}{l}\text { I-Qe-120 } \\
\text { Gutachter recherchieren } \\
\text { \& anfragen }\end{array}$ & $\mathbf{I 1} \longrightarrow \begin{array}{l}\text { I-Qe-130 } \\
\text { Peer-Review } \\
\text { beauftragen }\end{array}$ & $\mathbf{0 1}$ & $\begin{array}{l}\text { I-Ca-100 } \\
\text { Gutachten bzw. } \\
\text { Checkliste sichten }\end{array}$ \\
\cline { 2 - 3 }
\end{tabular}

\section{Input}

- I1: Zusage der gewünschten Gutachter

\section{Output}

- O1: Gutachten

\section{Beteiligte Rollen}

- Content-Prüfer

- Gutachter

\section{Ressourcen}

- Zeit: T-[I-Qe-130]

- Kosten: K-[I-Qe-130] 


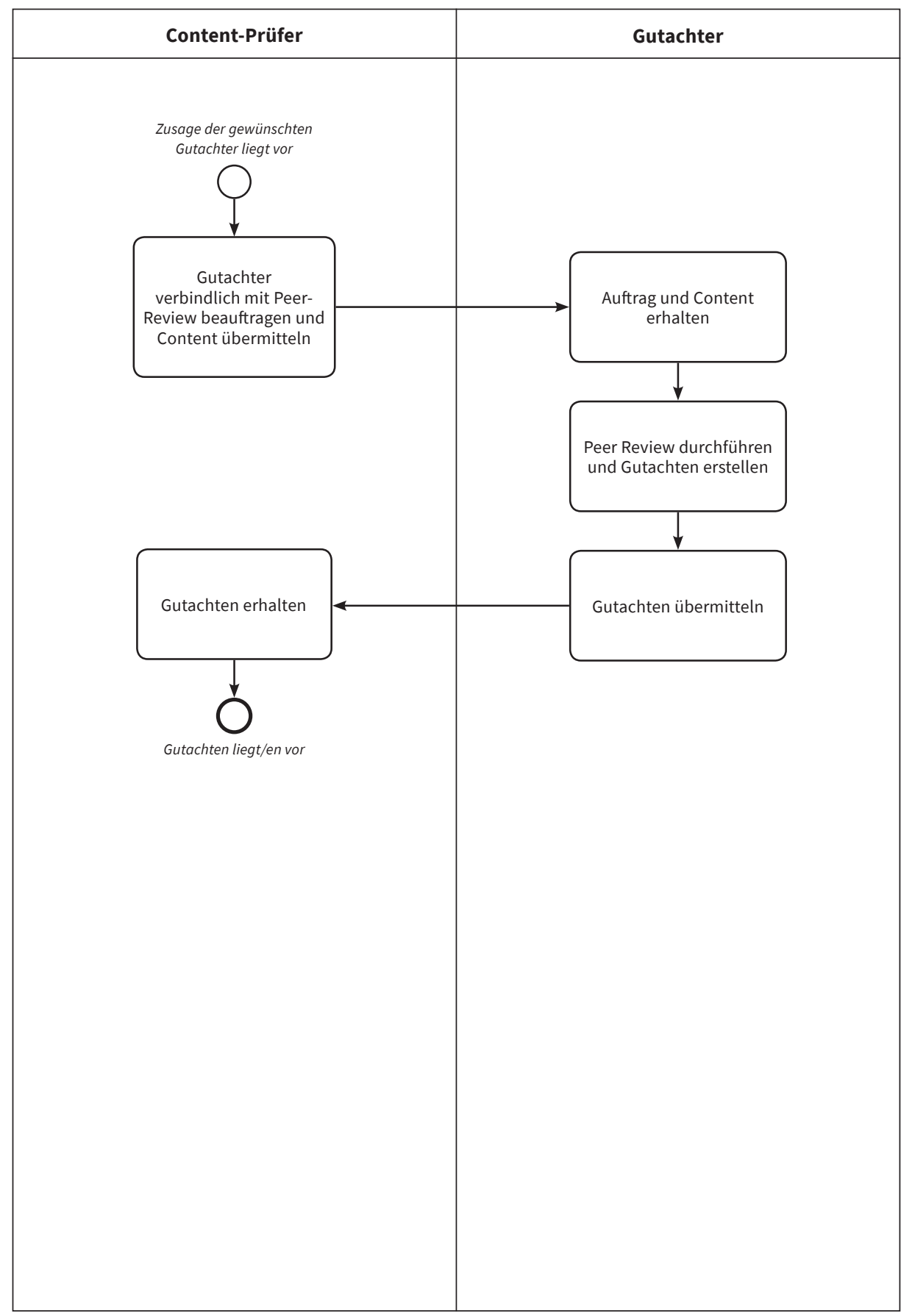




\section{Publikationsanfrage ablehnen [I-Qe-140]}

Prozessbeschreibung

Der Content stellt ein Plagiat nach der Richtlinie für die Plagiatsprüfung dar. Der Content-Prüfer informiert den Content-Urheber oder -Lieferanten über die finale Ablehnung der Publikationsanfrage.

Vor- und nachgelagerte Prozesse

\begin{tabular}{|l|l|l|l|}
\hline $\begin{array}{l}\text { I-Qe-100 } \\
\text { Plagiatsprüfung } \\
\text { durchführen }\end{array}$ & $\mathrm{I1} \longrightarrow \begin{array}{l}\mathrm{I}-\mathrm{Qe}-\mathbf{1 4 0} \\
\text { Publikationsanfrage } \\
\text { ablehnen }\end{array}$ \\
\cline { 2 - 3 }
\end{tabular}

\section{Input}

- I1: nicht bestandene Plagiatsprüfung

\section{Output}

- O1: abgelehnte Publikationsanfrage

\section{Beteiligte Rollen}

- Content-Prüfer

- Content-Urheber

- Content-Lieferant

\section{Ressourcen}

- Zeit: T-[I-Qe-140] 


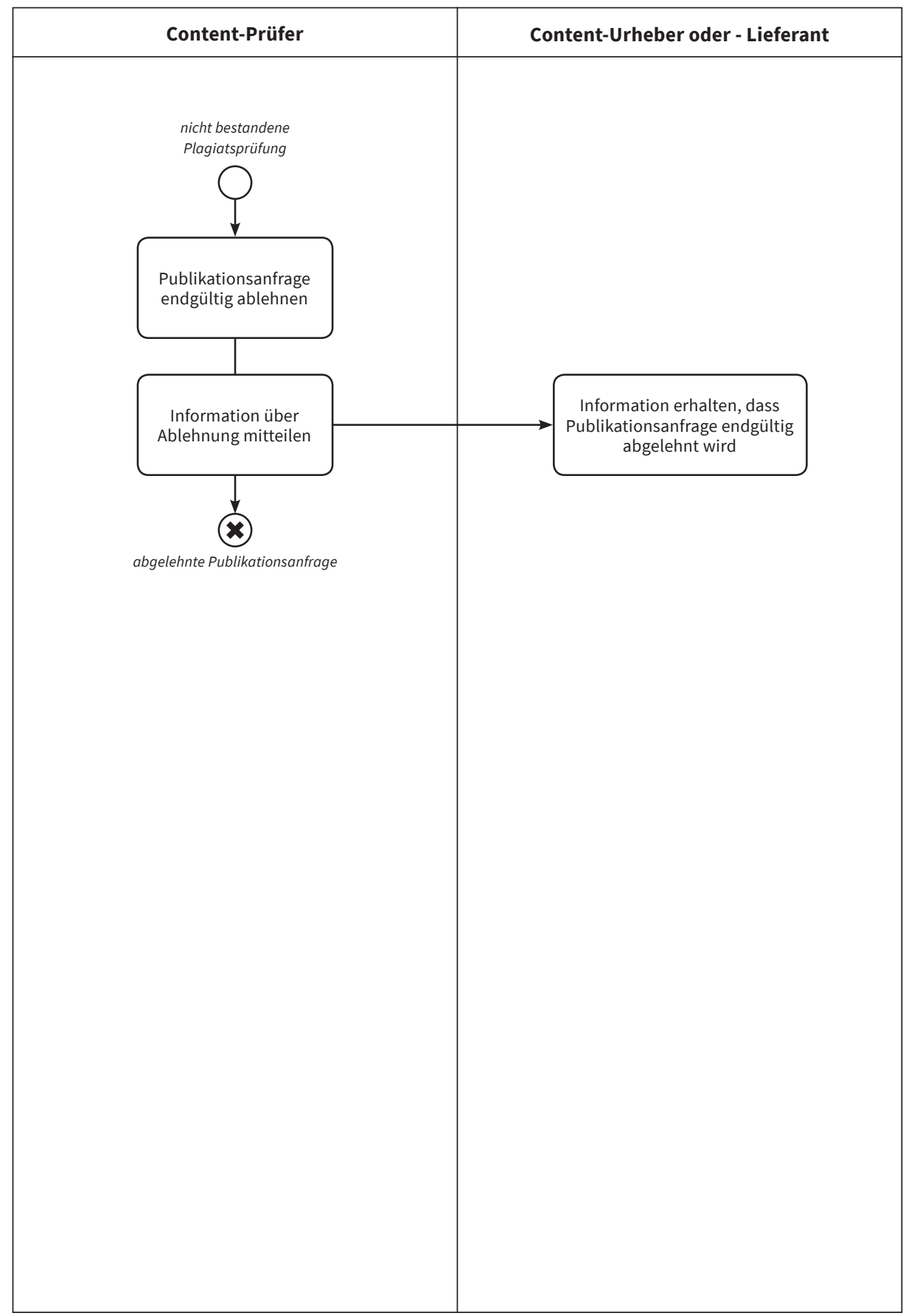




\section{Checkliste zu Content einfordern [I-Qe-200]}

\section{Prozessbeschreibung}

Zur Sicherung der Qualität bei Graduierungsarbeiten sollte der Content-Abnehmer die Gutachter der vorliegenden Graduierungsarbeit ermitteln und ihnen eine Checkliste zur Beurteilung der Publikationsfähigkeit zu senden. Der/die Gutachter füllen die Checkliste aus und senden sie an den Content-Abnehmer zurück.

Vor- und nachgelagerte Prozesse

\begin{tabular}{|l|l|l|l|}
\hline $\begin{array}{l}\text { I-Cv-130 } \\
\begin{array}{l}\text { Content vereinnahmen } \\
\text { \& definiert ablegen }\end{array}\end{array}$ & $\mathbf{I 1} \begin{array}{l}\text { I-Qe-200 } \\
\text { Checkliste zu Content } \\
\text { einfordern }\end{array}$ & $\mathbf{0 1}$ & $\begin{array}{l}\text { I-Ca-100 } \\
\text { Gutachten bzw. } \\
\text { Checkliste sichten }\end{array}$ \\
\cline { 2 - 3 }
\end{tabular}

\section{Input}

- I1: vereinnahmter und definiert abgelegter Content

\section{Output}

- O1: ausgefüllte Checkliste

\section{Qualitätsrichtlinie}

- Checkliste für die Evaluation der Publikationsfähigkeit

\section{Beteiligte Rollen}

- Content-Abnehmer

- Gutachter

\section{Ressourcen}

- Zeit: T-[I-Qe-200] 


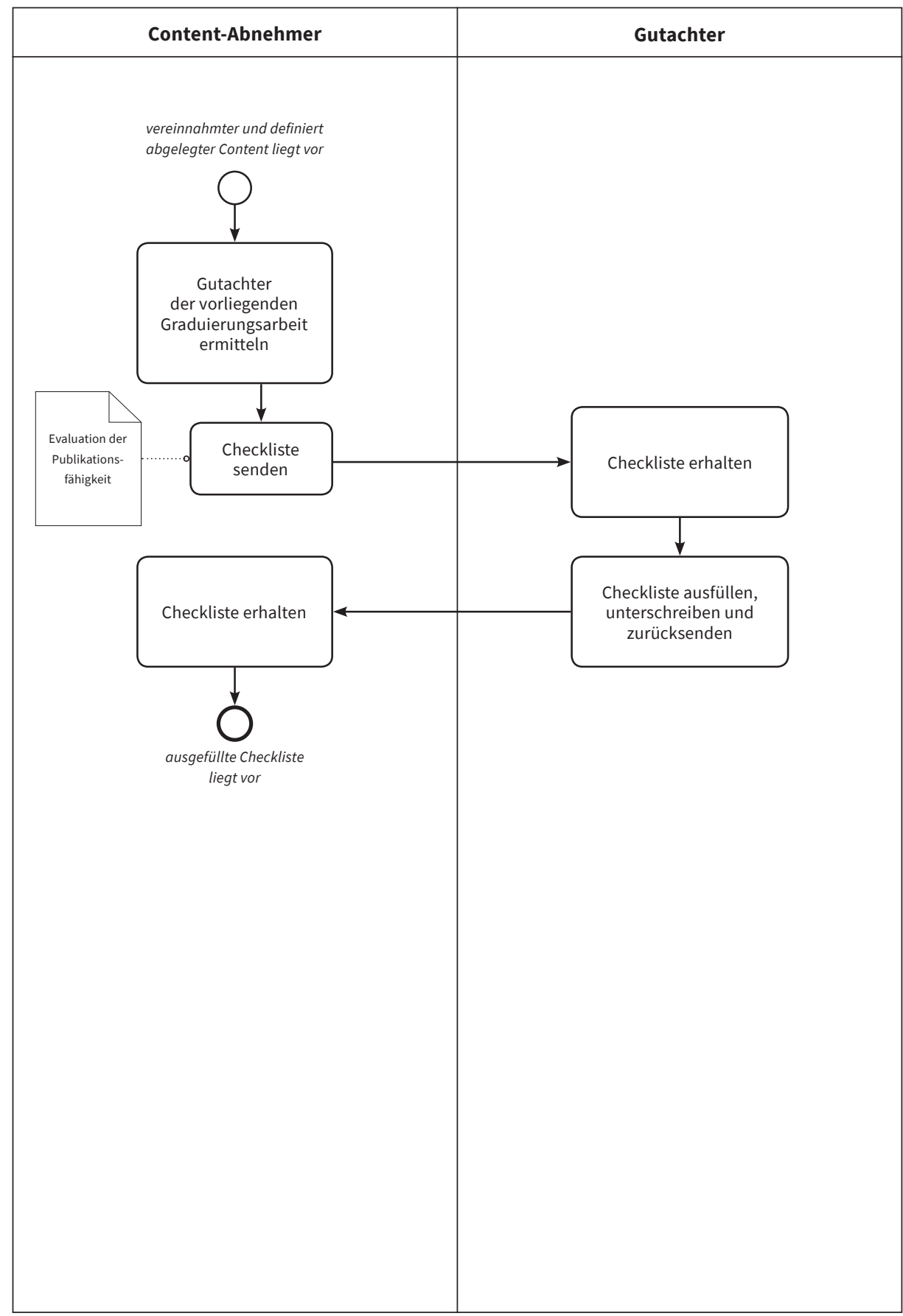




\section{Gutachten zu Content einfordern [I-Qe-210]}

\section{Prozessbeschreibung}

Sofern noch nicht geschehen, ermittelt der Content-Abnehmer den/die Gutachter der vorliegenden Graduierungsarbeit und fordert das/die erstelle/n Gutachten ein. Der/die Gutachter übermitteln diese/s.

Vor- und nachgelagerte Prozesse

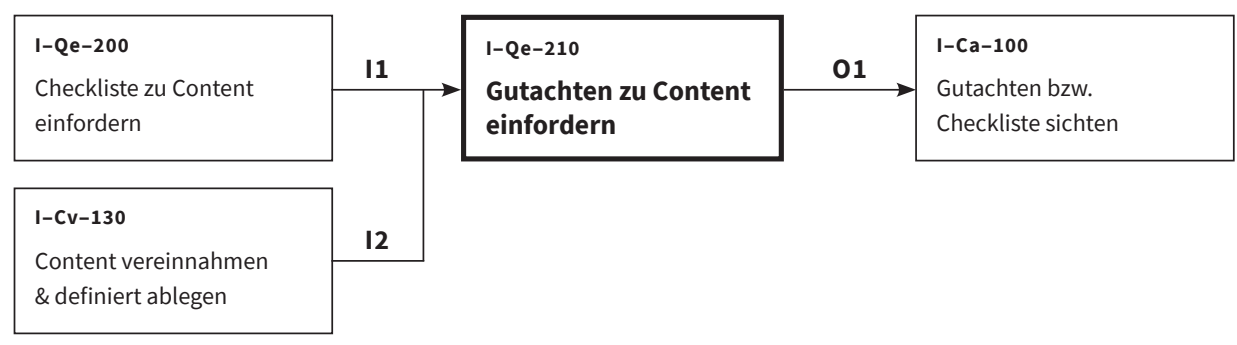

\section{Input}

- I1: Checkliste zur Publikationsfähigkeit

- I2: vereinnahmter und definiert abgelegter Content

\section{Output}

- O1: Gutachten

\section{Beteiligte Rollen}

- Content-Abnehmer

- Gutachter

\section{Ressourcen}

- Zeit: T-[I-Qe-210] 


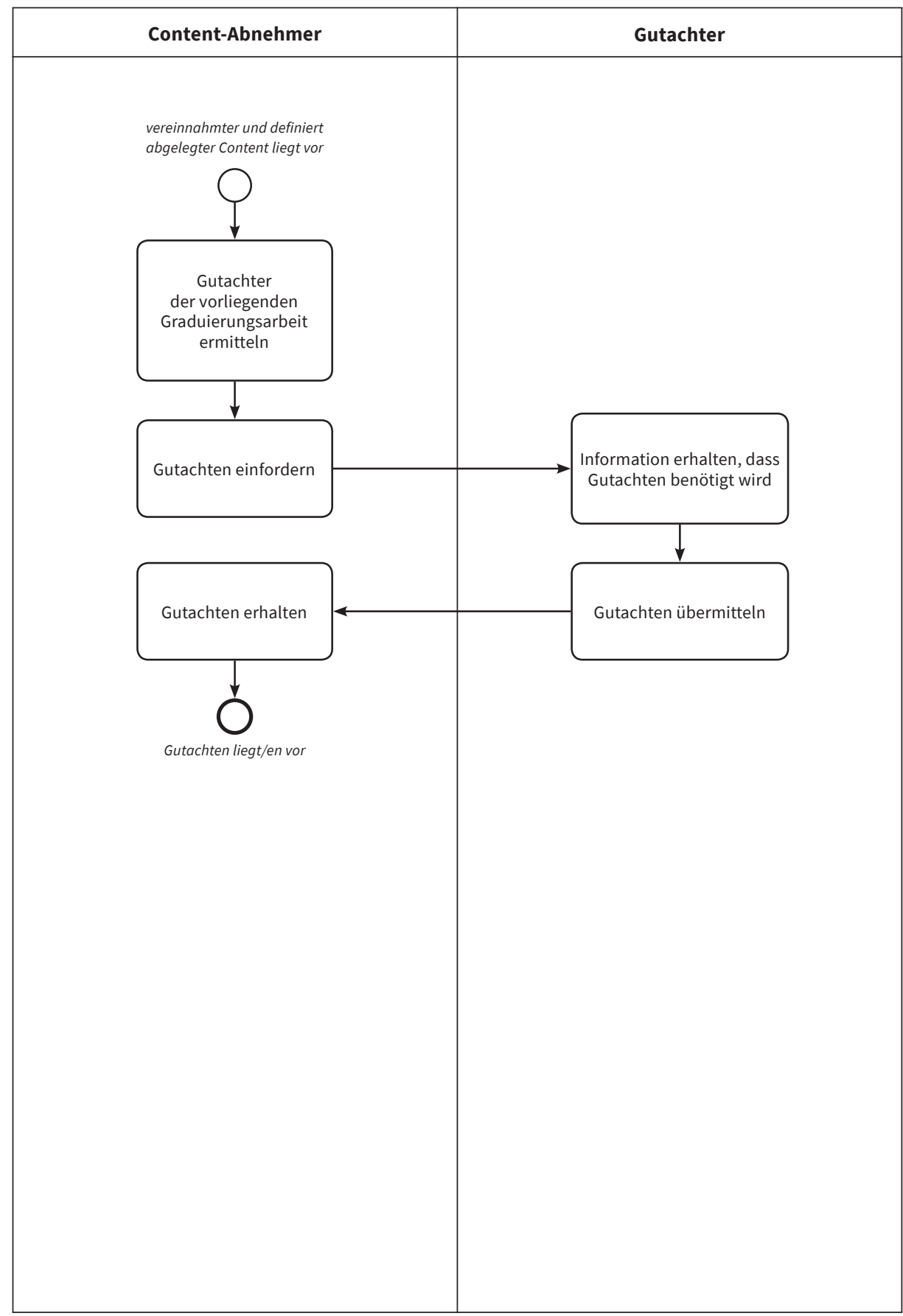




\section{Gutachten bzw. Checkliste sichten [I-Ca-100]}

\section{Prozessbeschreibung}

Der Content-Prüfer hat Gutachten und ggf. Checklisten zum Content erhalten. Er sichtet diese und entscheidet entsprechend der Richtlinie für die Publikationsannahme, ob der Content in der vorliegenden Form zur Publikation angenommen wird, ob der Content vor der Publikation überarbeitet werden sollte oder ob der Content für die Publikation abgelehnt wird.

Vor- und nachgelagerte Prozesse

\begin{tabular}{|c|c|c|c|c|}
\hline \multirow{2}{*}{$\begin{array}{l}\text { I-Qe-130 } \\
\text { Peer-Review } \\
\text { beauftragen }\end{array}$} & \multirow[t]{2}{*}{ I1 } & \multirow{2}{*}{$\begin{array}{l}\text { I-Ca-100 } \\
\text { Gutachten bzw. } \\
\text { Checkliste sichten }\end{array}$} & 01 & $\begin{array}{l}\text { I-Ca-130 } \\
\text { Content annehmen }\end{array}$ \\
\hline & & & & \\
\hline \multirow{2}{*}{$\begin{array}{l}\text { I-Qe-200 } \\
\text { Checkliste zu Content } \\
\text { einfordern }\end{array}$} & \multirow{2}{*}{12} & & \multirow{2}{*}{02} & I-Ca-110 \\
\hline & & & & Contents einfordern \\
\hline \multirow{2}{*}{$\begin{array}{l}\text { I-Qe-210 } \\
\text { Gutachten zu Content } \\
\text { einfordern }\end{array}$} & 13 & & \multirow[t]{2}{*}{03} & $\mathrm{I}-\mathrm{Ca}-150$ \\
\hline & & & & 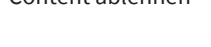 \\
\hline
\end{tabular}

\section{Input}

- I1 und I3: Gutachten

- I2: ausgefüllte Checkliste

\section{Output}

- O1: Entscheidung, anzunehmen

- O2: Entscheidung, um Überarbeitung zu bitten

- O3: Entscheidung, abzulehnen

\section{Qualitätsrichtlinie}

- Richtlinie für die Publikationsannahme

\section{Beteiligte Rollen}

- Content-Prüfer

\section{Ressourcen}

- Zeit: T-[I-Ca-100] 


\section{Content-Prüfer}

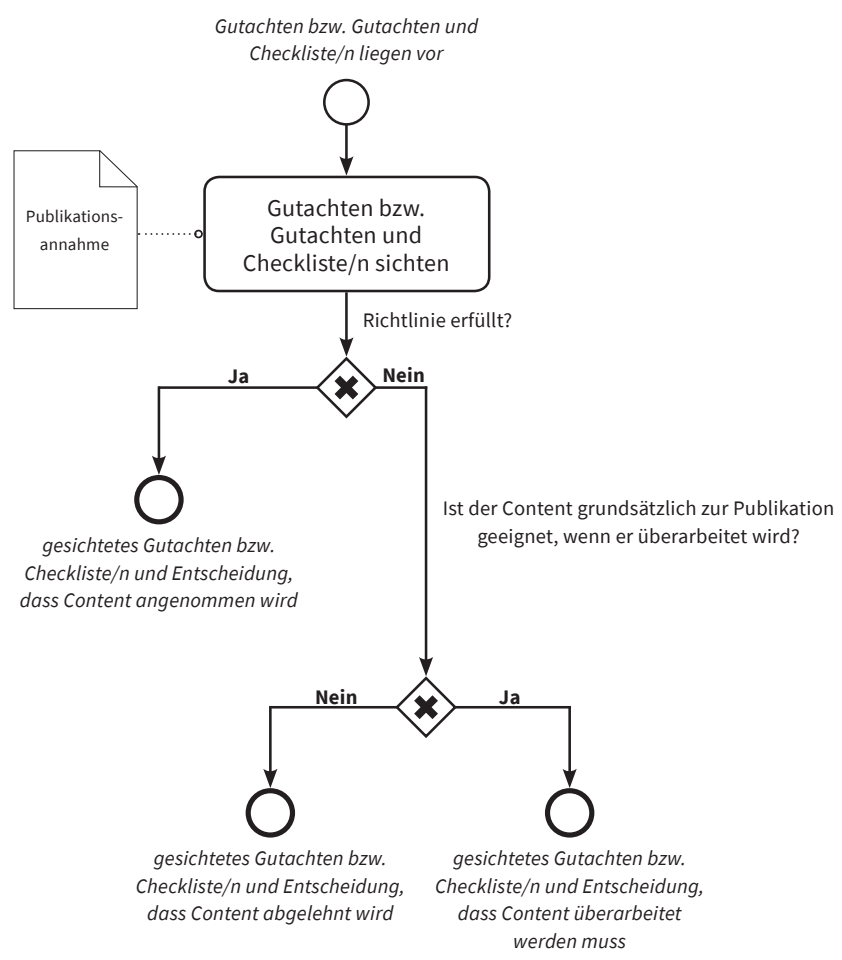




\section{Überarbeitung des Contents einfordern [I-Ca-110]}

\section{Prozessbeschreibung}

Die Sichtung der Gutachten bzw. der Checkliste/n zum Content ergab, dass noch Überarbeitungen am Content vorgenommen werden sollten, bevor dieser publiziert werden kann. Der Content-Prüfer informiert den Content-Urheber über diese Entscheidung und bittet ihn um die Überarbeitung der gewünschten Aspekte und Zusendung des überarbeiteten Contents.

Vor- und nachgelagerte Prozesse

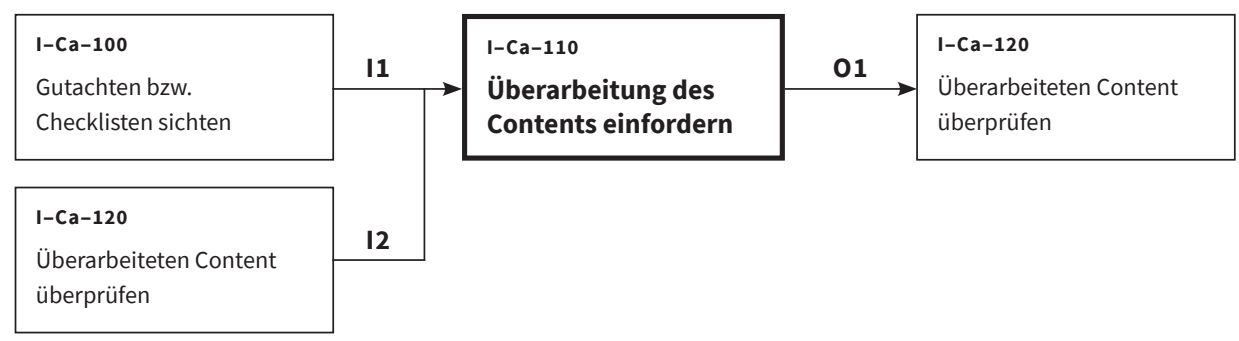

Input

- I1: Entscheidung, um Überarbeitung zu bitten

- I2: unzureichend überarbeiteter Content

\section{Output}

- O1: überarbeiteter Content

\section{Beteiligte Rollen}

- Content-Prüfer

- Content-Urheber

\section{Ressourcen}

- Zeit: T-[I-Ca-110] 


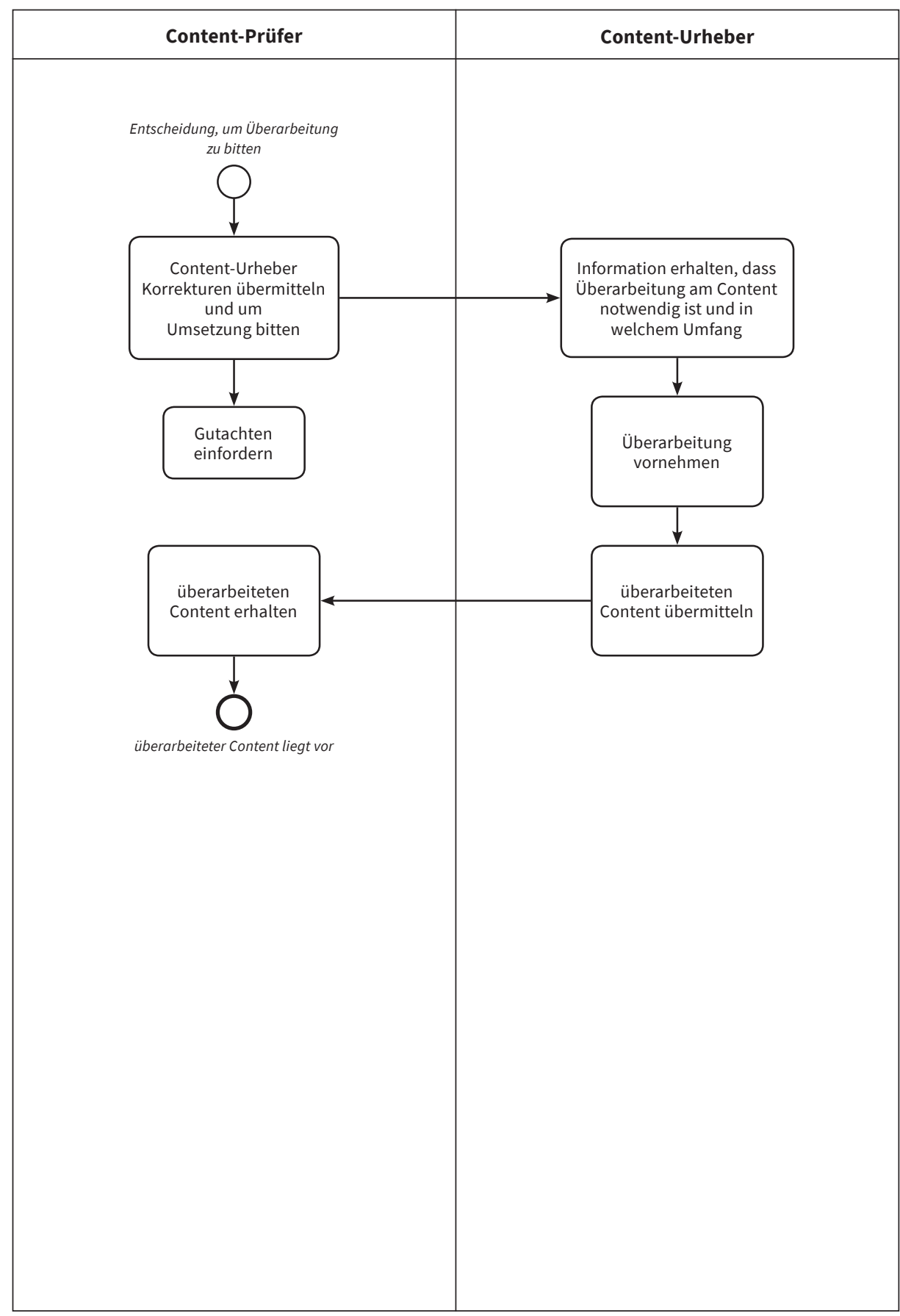




\section{Überarbeiteten Content überprüfen [I-Ca-120]}

\section{Prozessbeschreibung}

Der Content-Prüfer hat den überarbeiteten Content erhalten und überprüft, ob die Überarbeitungen ausreichend umgesetzt wurden. Ist er mit der Überarbeitung nicht zufrieden, liegt es in seinem Ermessen, ob er den Content zur Publikation ablehnt, oder ob er den Content-Urheber erneut auffordert den Content zu überarbeiten. Ist er mit dem überarbeiteten Content zufrieden, kann der Content zur Publikation im Hochschulverlag angenommen werden.

Vor- und nachgelagerte Prozesse

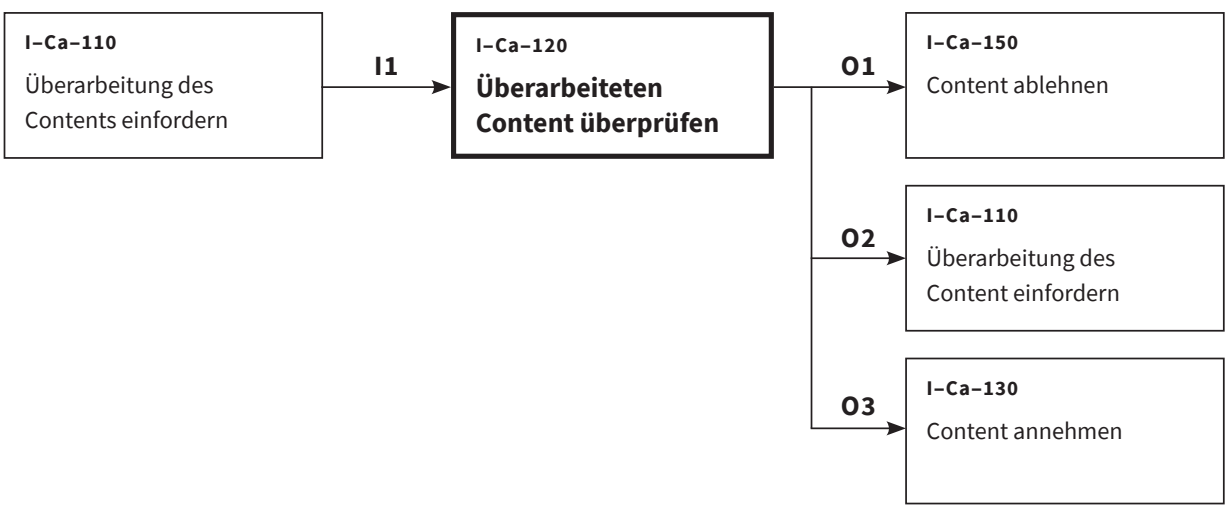

\section{Input}

- I1: überarbeiteter Content

\section{Output}

- O1: unzureichend überarbeiteter Content

- O2: unzureichend überarbeiteter Content

- O3: ausreichend überarbeiteter Content

\section{Beteiligte Rollen}

- Content-Prüfer

\section{Ressourcen}

- Zeit: T-[I-Ca-120] 


\section{Content-Prüfer}

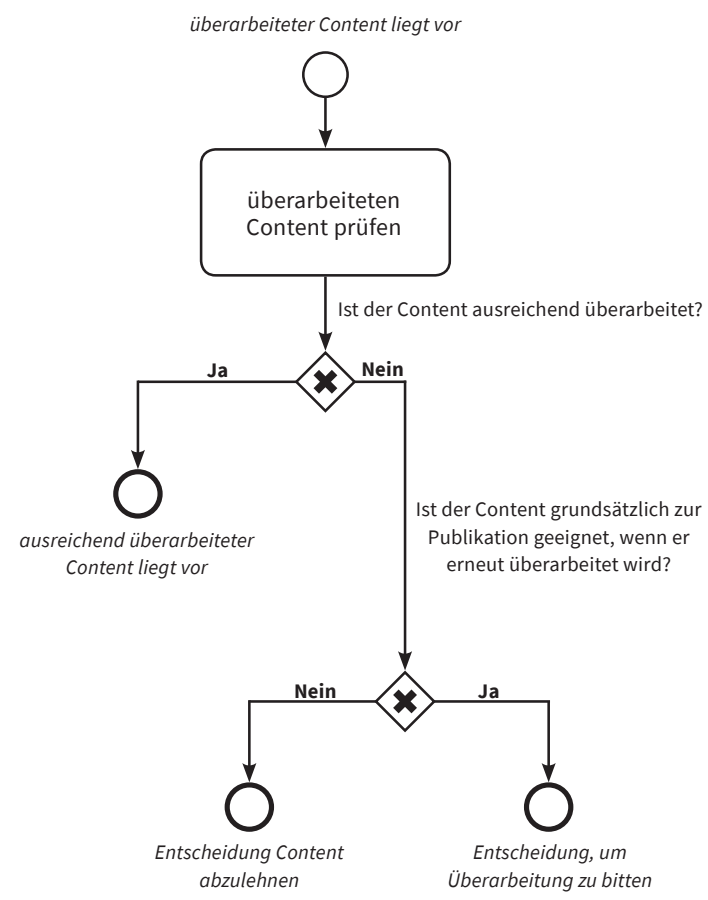




\section{Content annehmen [I-Ca-130]}

\section{Prozessbeschreibung}

Entweder hat die Sichtung der Gutachten bzw. der Checkliste/n ergeben, dass der Content in der vorliegenden Form publiziert werden kann oder der Content-Prüfer ist mit den Überarbeitungen des Content-Urhebers zufrieden. Er informiert den Content-Urheber und ggf. Content-Lieferanten über diese Entscheidung und über die weiteren Schritte im Publikationsworkflow.

Vor- und nachgelagerte Prozesse

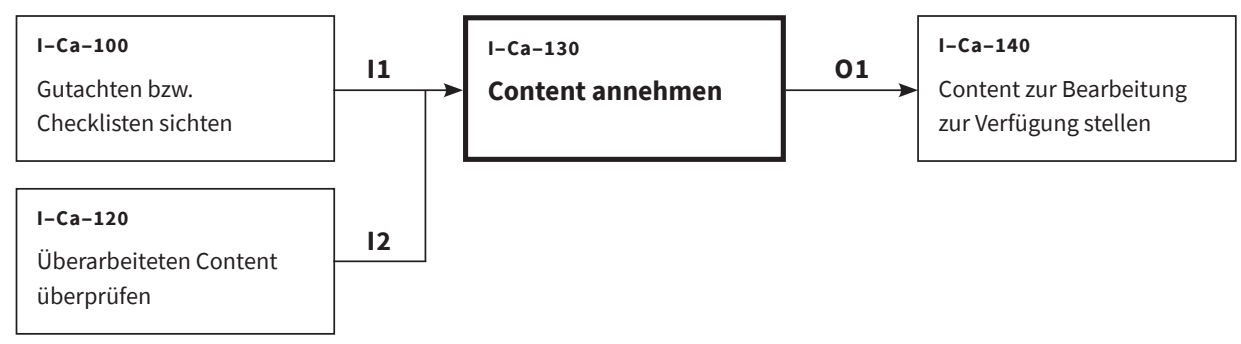

\section{Input}

- I1: Entscheidung, anzunehmen

- I2: ausreichend überarbeiteter Content

\section{Output}

- O1: angenommener Content

\section{Beteiligte Rollen}

- Content-Prüfer

- Content-Urheber

- Content-Lieferant

\section{Ressourcen}

- Zeit: T-[I-Ca-130] 


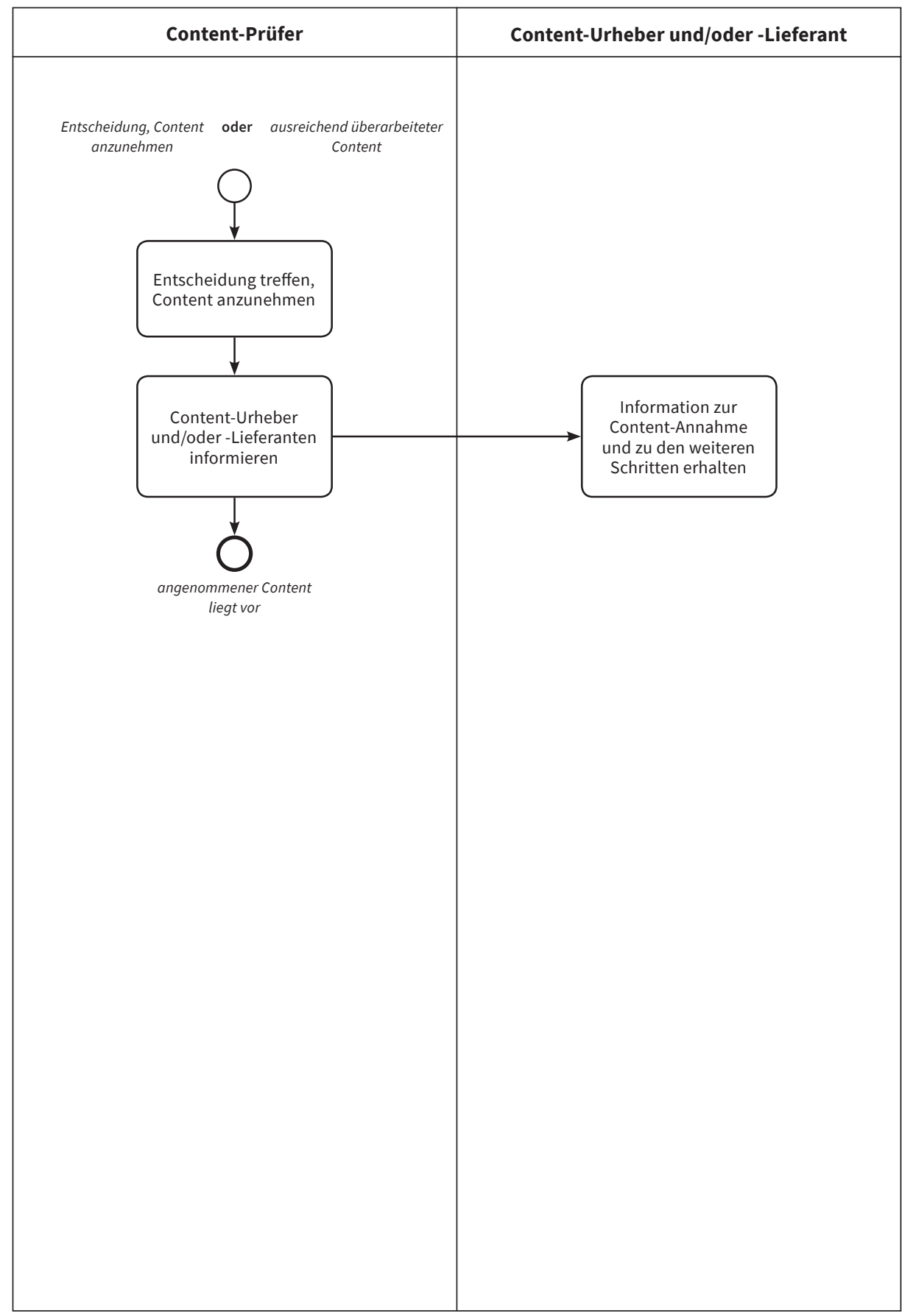




\section{Content zur weiteren Bearbeitung zur Verfügung stellen [I-Ca-140]}

\section{Prozessbeschreibung}

Nachdem der Content-Prüfer den Content zur Publikation angenommen hat, legt er alle benötigten Daten für alle relevanten Rollen ab, sodass sie zur weiteren Bearbeitung zur Verfügung stehen.

Vor- und nachgelagerte Prozesse

\begin{tabular}{|c|c|c|c|c|}
\hline \multirow[t]{3}{*}{$\begin{array}{l}\text { I-Ca-130 } \\
\text { Content annehmen }\end{array}$} & \multirow[t]{2}{*}{ I1 } & $\begin{array}{l}\text { I-Ca-140 } \\
\text { Content zur }\end{array}$ & 01 & $\begin{array}{l}\text { P-CUe-100 } \\
\text { Cover erstellen }\end{array}$ \\
\hline & & $\begin{array}{l}\text { Bearbeitung zur } \\
\text { Verfügung stellen }\end{array}$ & & \\
\hline & & & 02 & $\begin{array}{l}\text { P-Ca-100 } \\
\text { Fachkorrektorat } \\
\text { beauftragen }\end{array}$ \\
\hline
\end{tabular}

\section{Input}

- I1: angenommener Content

\section{Output}

- O1: zur weiteren Bearbeitung freigegebener Content

- O2: zur weiteren Bearbeitung freigegebener Content

\section{Beteiligte Rollen}

- Content-Prüfer

\section{Ressourcen}

- Zeit: T-[I-Ca-140] 


\section{Content-Prüfer}

angenommener Content

liegt vor

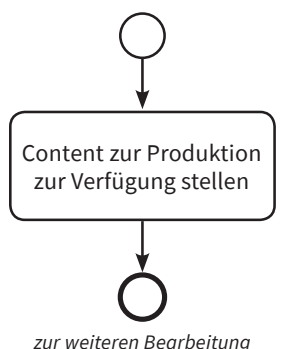

zur weiteren Bearbeitung

freigegebener Content 


\section{Content ablehnen [I-Ca-150]}

\section{Prozessbeschreibung}

Entweder die Sichtung der Gutachten bzw. der Checkliste/n hat ergeben, dass der Content nicht im Hochschulverlag publizieren werden soll oder der Content-Urheber hat den Content nicht ausreichend überarbeitet, sodass der Content-Prüfer ebenfalls zu dieser Entscheidung gelangt. Er informiert den Content-Urheber und ggf. Content-Lieferanten über diese Entscheidung.

Vor- und nachgelagerte Prozesse

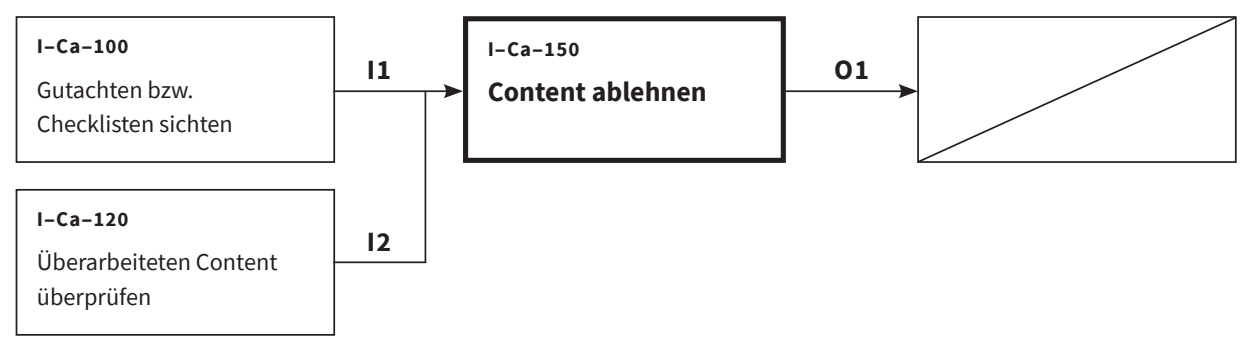

\section{Input}

- I1: Entscheidung, abzulehnen

- I2: unzureichend überarbeiteter Content

\section{Output}

- O1: abgelehnter Content

\section{Beteiligte Rollen}

- Content-Prüfer

- Content-Urheber

- Content-Lieferant

\section{Ressourcen}

- Zeit: T-[I-Ca-150] 


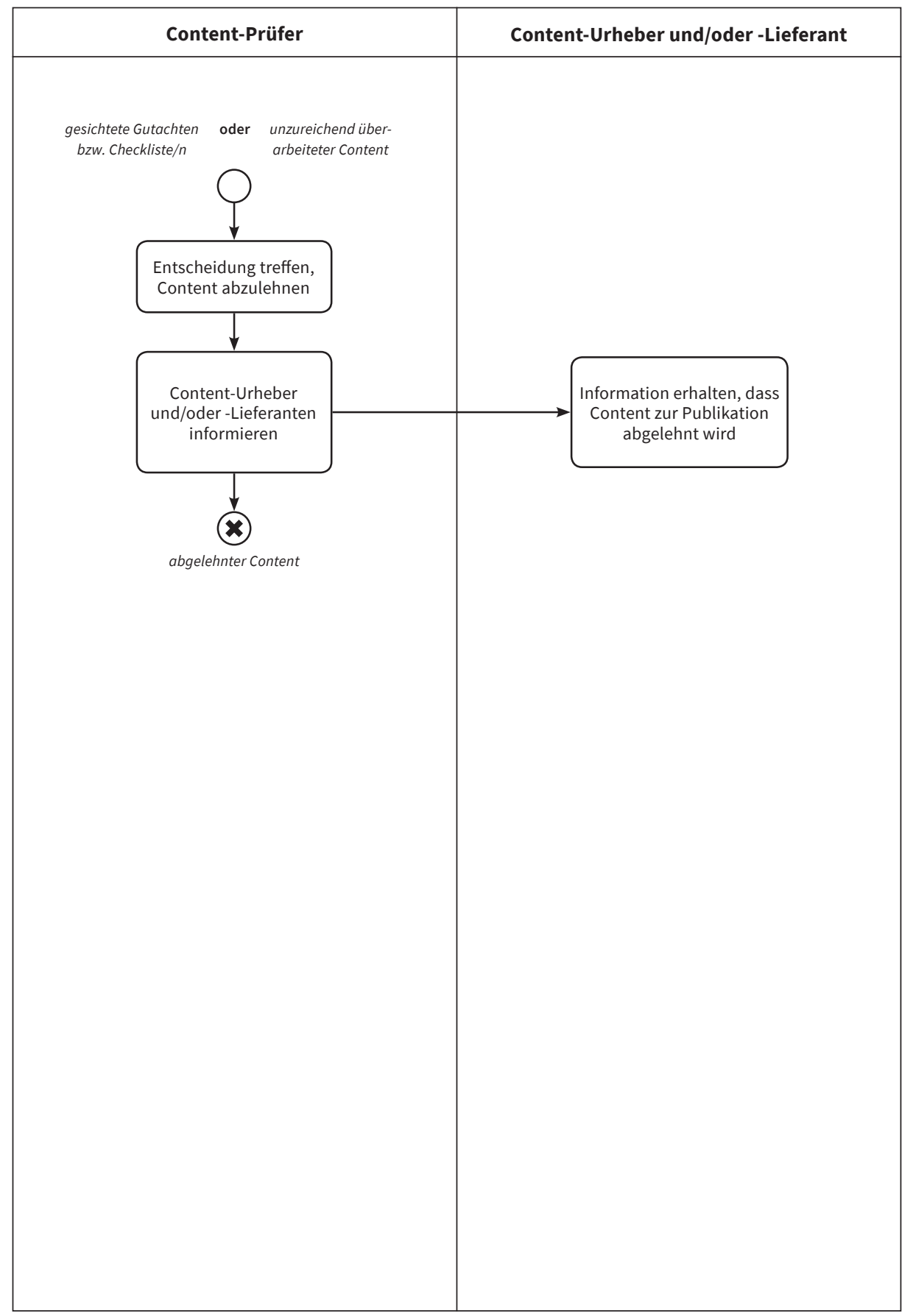




\section{Fachkorrektorat beauftragen [P-Ca-100]}

Prozessbeschreibung

Auf Grundlage der Richtlinie für das Fachkorrektorat, beauftragt der Copy Editor des Hochschulverlages einen Experten, um ein Fachkorrektorat am vorliegenden Content durchzuführen. Der Fachkorrektor übermittelt anschließend den Content zur weiteren Bearbeitung an den Copy Editor zurück.

Vor- und nachgelagerte Prozesse

\begin{tabular}{|l|l|l|l|}
\cline { 2 - 3 } $\begin{array}{l}\text { I-Ca-140 } \\
\text { Content zur Bearbeitung } \\
\text { zur Verfügung stellen }\end{array}$ & $\mathbf{I 1}$ & $\begin{array}{l}\text { P-Ca-100 } \\
\text { Fachkorrektorat } \\
\text { beauftragen }\end{array}$ & $\mathbf{0 1}$ \\
\cline { 2 - 3 } & $\longrightarrow \begin{array}{l}\text { P-Ca-110 } \\
\text { Sprachlektorat } \\
\text { beauftragen }\end{array}$ \\
\hline
\end{tabular}

\section{Input}

- I1: zur weiteren Bearbeitung freigegebener Content

\section{Output}

- O1: durch Fachkorrektorat bereinigter Content

\section{Qualitätsrichtlinie}

- Richtlinie für das Fachkorrektorat

\section{Beteiligte Rollen}

- Copy Editor

- Fachkorrektor

\section{Ressourcen}

- Zeit: T-[P-Ca-100]

- Kosten: K-[P-Ca-100] 


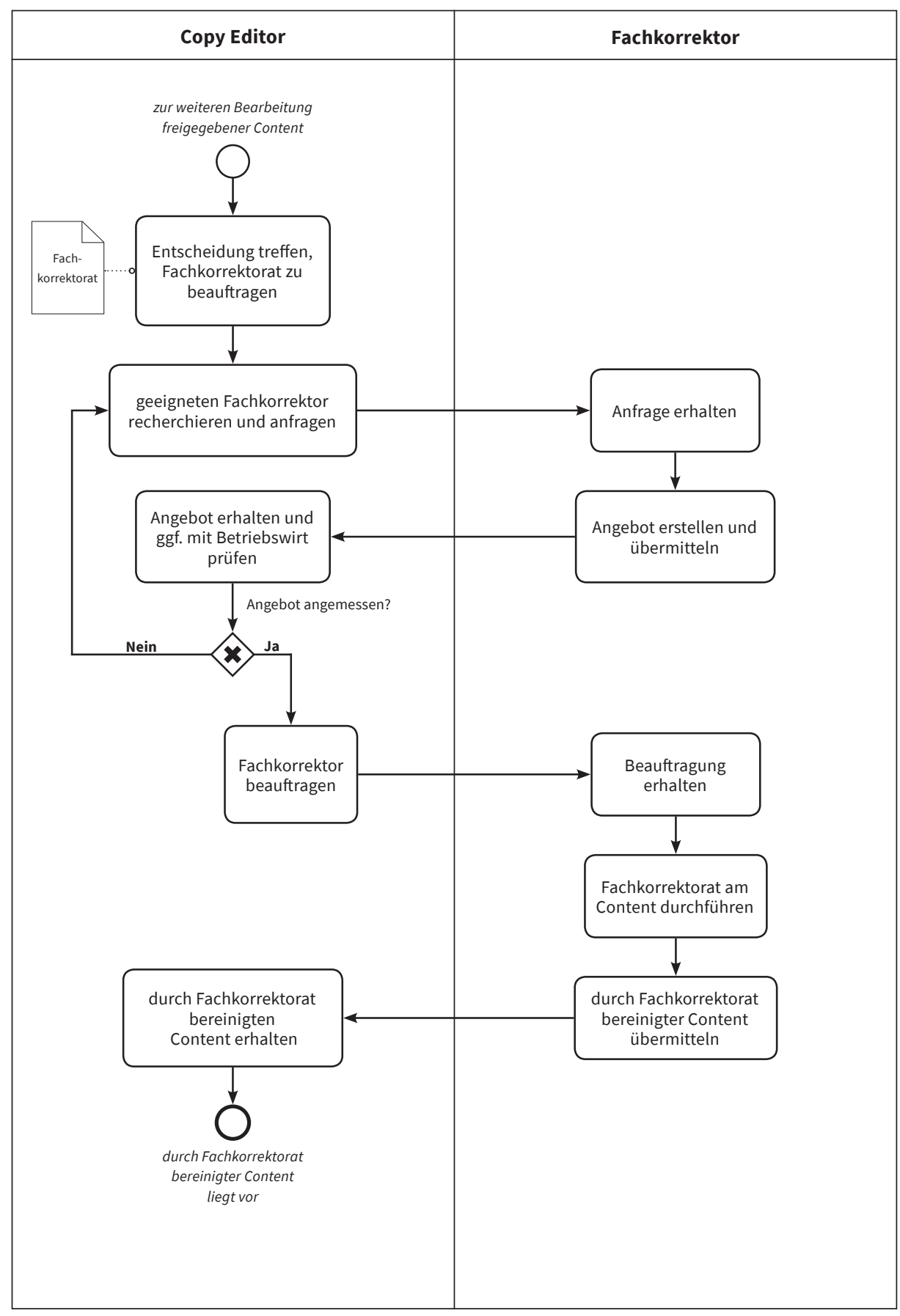




\section{Sprachlektorat beauftragen [P-Ca-110]}

\section{Prozessbeschreibung}

Auf Grundlage der Richtlinie für das Sprachlektorat, beauftragt der Copy Editor einen Experten, um am vorliegenden Content ein Sprachlektorat durchzuführen. Der Sprachlektor übermittelt nach Durchführung des Sprachlektorats, den Content zur weiteren Bearbeitung an den Copy Editor zurück.

Vor- und nachgelagerte Prozesse

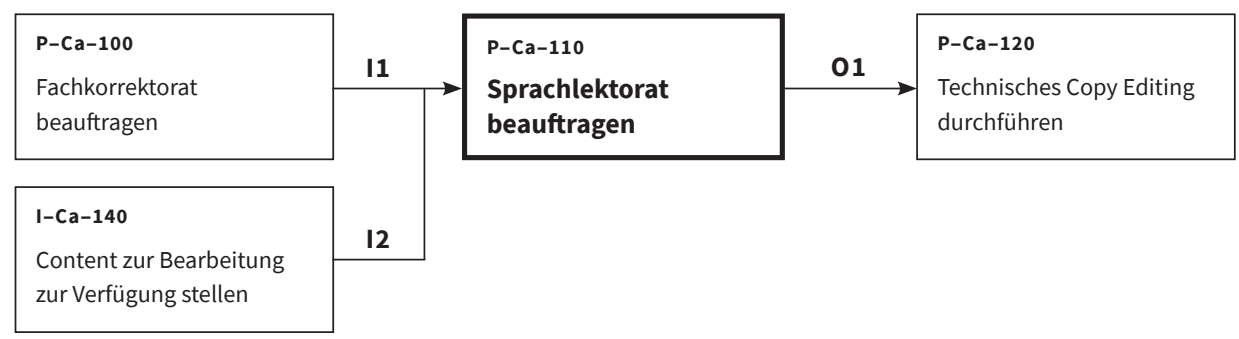

\section{Input}

- I1: durch Fachkorrektorat bereinigter Content

- I2: zur weiteren Bearbeitung freigegebener Content

\section{Output}

- O1: durch ggf. Fachkorrektorat und Sprachlektorat bereinigter Content

\section{Qualitätsrichtlinie}

- Richtlinie für das Sprachlektorat

\section{Beteiligte Rollen}

- Copy Editor

- Sprachlektor

\section{Ressourcen}

- Zeit: T-[P-Ca-110]

- Kosten: K-[P-Ca-110] 


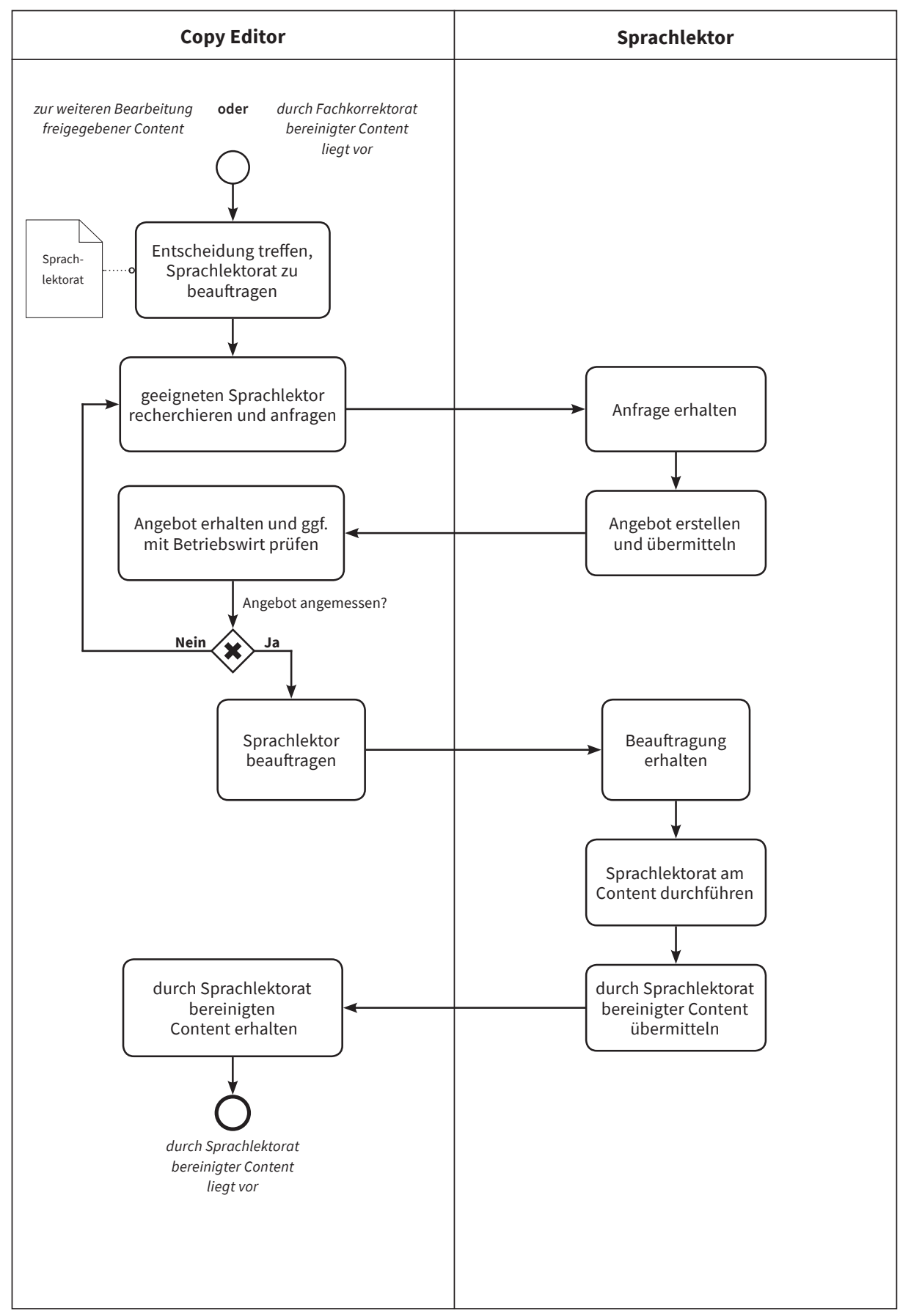




\section{Technisches Copy Editing durchführen [P-Ca-120]}

\section{Prozessbeschreibung}

Der Copy Editor führt am Werk das technische Copy Editing durch und nimmt ggf. Korrekturen vor. Anschließend liegt ein inhaltlich, finaler Content vor.

Vor- und nachgelagerte Prozesse

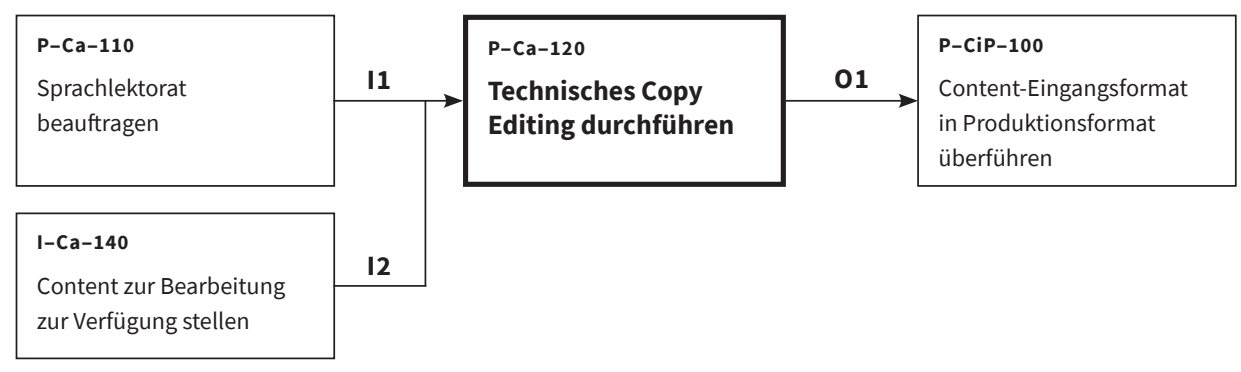

\section{Input}

- I1: durch Fachkorrektorat und Sprachlektorat bereinigter Content

- I2: zur weiteren Bearbeitung freigegebener Content

\section{Output}

- O1: inhaltlich finaler Content

\section{Qualitätsrichtlinie}

- Richtlinie für das technische Copy Editing

\section{Beteiligte Rollen}

- Copy Editor

\section{Ressourcen}

- Zeit: T-[P-Ca-120]

- Kosten: K-[P-Ca-120] 


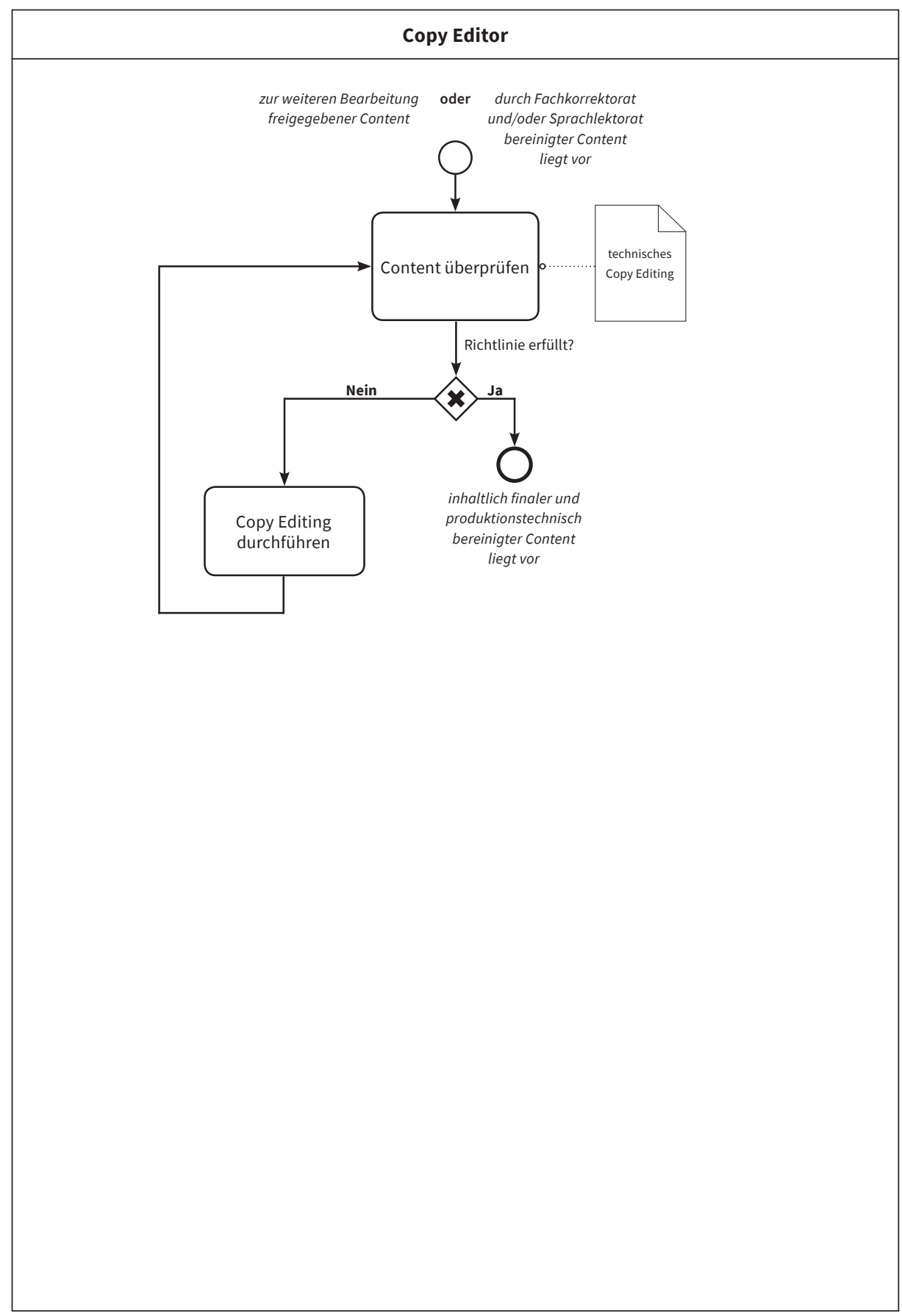




\section{Cover erstellen [P-CUe-100]}

\section{Prozessbeschreibung}

Der Cover-Ersteller erstellt entsprechend der Cover-Gestaltungsrichtlinie einen Entwurf für das Cover der zukünftigen Publikation.

Vor- und nachgelagerte Prozesse

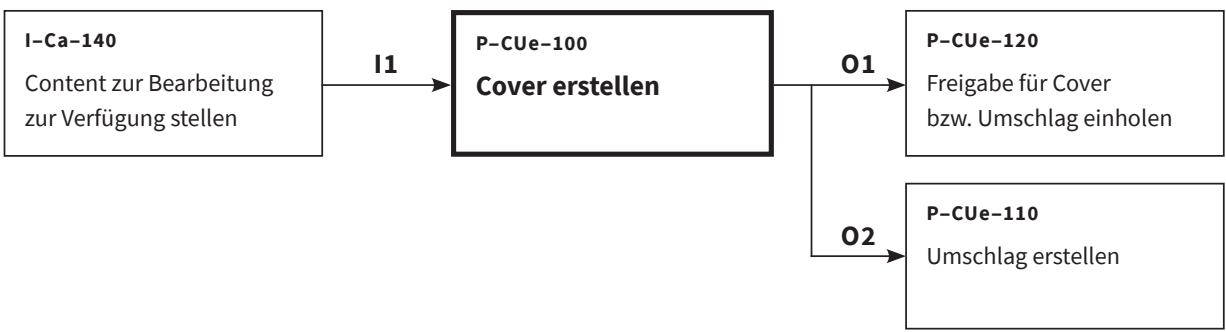

\section{Input}

- I1: zur weiteren Bearbeitung freigegebener Content

\section{Output}

- O1: Coverentwurf

- O2: Coverentwurf

\section{Qualitätsrichtlinie}

- Cover-Gestaltungsrichtlinie

\section{Beteiligte Rollen}

- Cover-Ersteller

\section{Ressourcen}

- Zeit: T-[P-CUe-100]

- Kosten: K-[P-CUe-100] 


\section{Cover-Ersteller}

zur weiteren Bearbeitung

freigegebener Content liegt vor

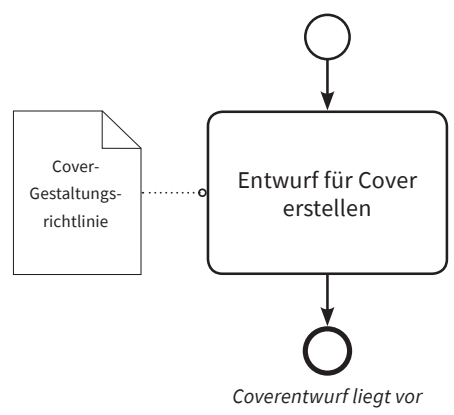




\section{Umschlag erstellen [P-CUe-110]}

\section{Prozessbeschreibung}

Sofern es ein gedrucktes Produkt geben soll, erstellt der Cover-Ersteller einen Entwurf für den Druckumschlag nach der Umschlag-Gestaltungsrichtlinie.

Vor- und nachgelagerte Prozesse

\begin{tabular}{|c|c|c|c|c|}
\hline $\begin{array}{l}\mathbf{P - C U e - 1 0 0} \\
\text { Cover erstellen }\end{array}$ & I1 & $\begin{array}{l}\text { P-CUe-110 } \\
\text { Umschlag erstellen }\end{array}$ & 01 & $\begin{array}{l}\text { P-CUe-120 } \\
\text { Freigabe für Cover } \\
\text { bzw. Umschlag einholen }\end{array}$ \\
\hline
\end{tabular}

\section{Input}

- I1: Coverentwurf

\section{Output}

- O1: Umschlagentwurf

\section{Qualitätsrichtlinie}

- Umschlag-Gestaltungsrichtlinie

\section{Beteiligte Rollen}

- Cover-Ersteller

\section{Ressourcen}

- Zeit: T-[P-CUe-110]

- Kosten: K-[P-CUe-110] 


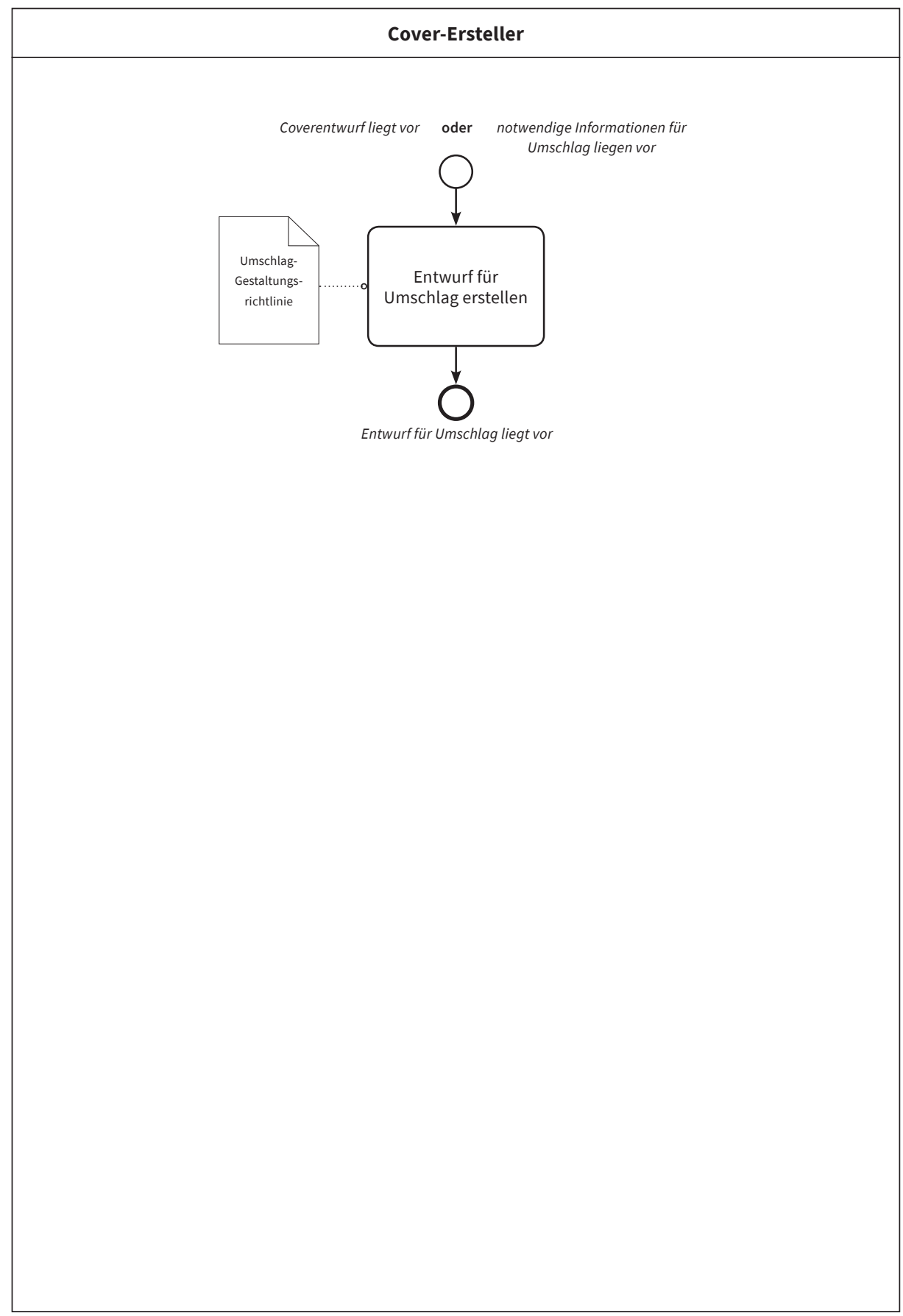




\section{Freigabe für Cover bzw. Umschlag einholen [P-CUe-120]}

\section{Prozessbeschreibung}

Der Cover-Ersteller sendet den ggf. bereits überarbeiteten Cover-bzw. Umschlagentwurf zur Freigabe an den Content-Urheber. Optional kann der Cover-Ersteller die Entwürfe zunächst zur Freigabe an den Content-Prüfer senden und dessen Korrekturen einarbeiten, bevor er die Entwürfe zur Freigabe an den Content-Urheber sendet. Der Content-Urheber sendet seine Korrekturen an den Cover-Ersteller zurück. Sofern der Content-Urheber das Cover selbst erstellt, nimmt er die Rolle des Cover-Erstellers ein und sendet seinen Entwurf an den Content-Prüfer zur Freigabe.

Vor- und nachgelagerte Prozesse

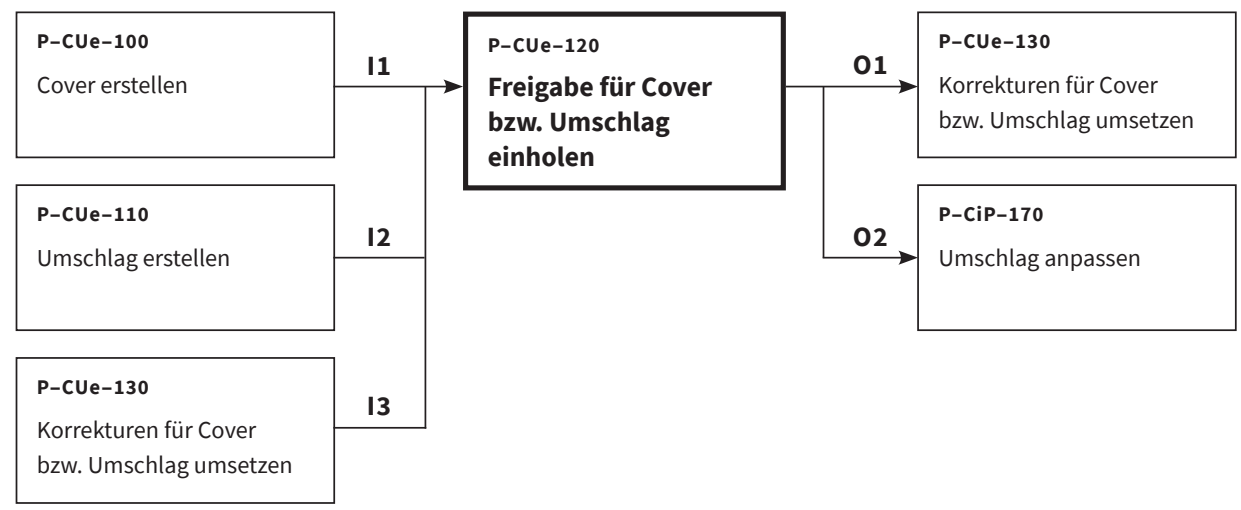

Input

- I1: Coverentwurf

- I2: Umschlagentwurf

- I3: überarbeiteter Cover- bzw. Umschlagsentwurf

\section{Output}

- O1: Korrekturen

- O2: freigegebener Umschlag

\section{Qualitätsrichtlinie}

- Richtlinie für die Produktfreigabe 


\section{Beteiligte Rollen}

- Cover-Ersteller

- Content-Urheber

- optional: Content-Prüfer

\section{Ressourcen}

- Zeit: T-[P-CUe-120]

- Kosten: K-[P-CUe-120] 


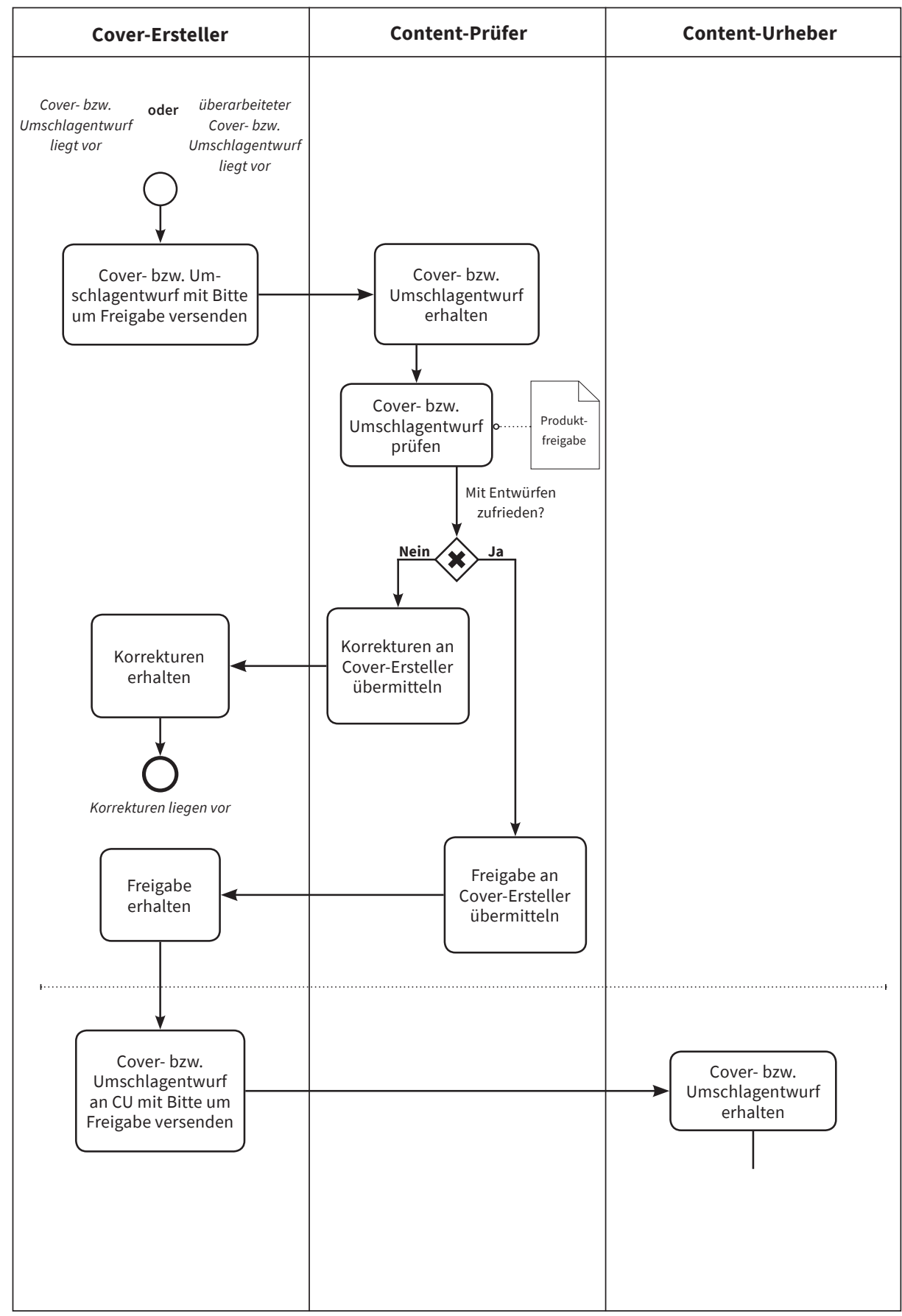




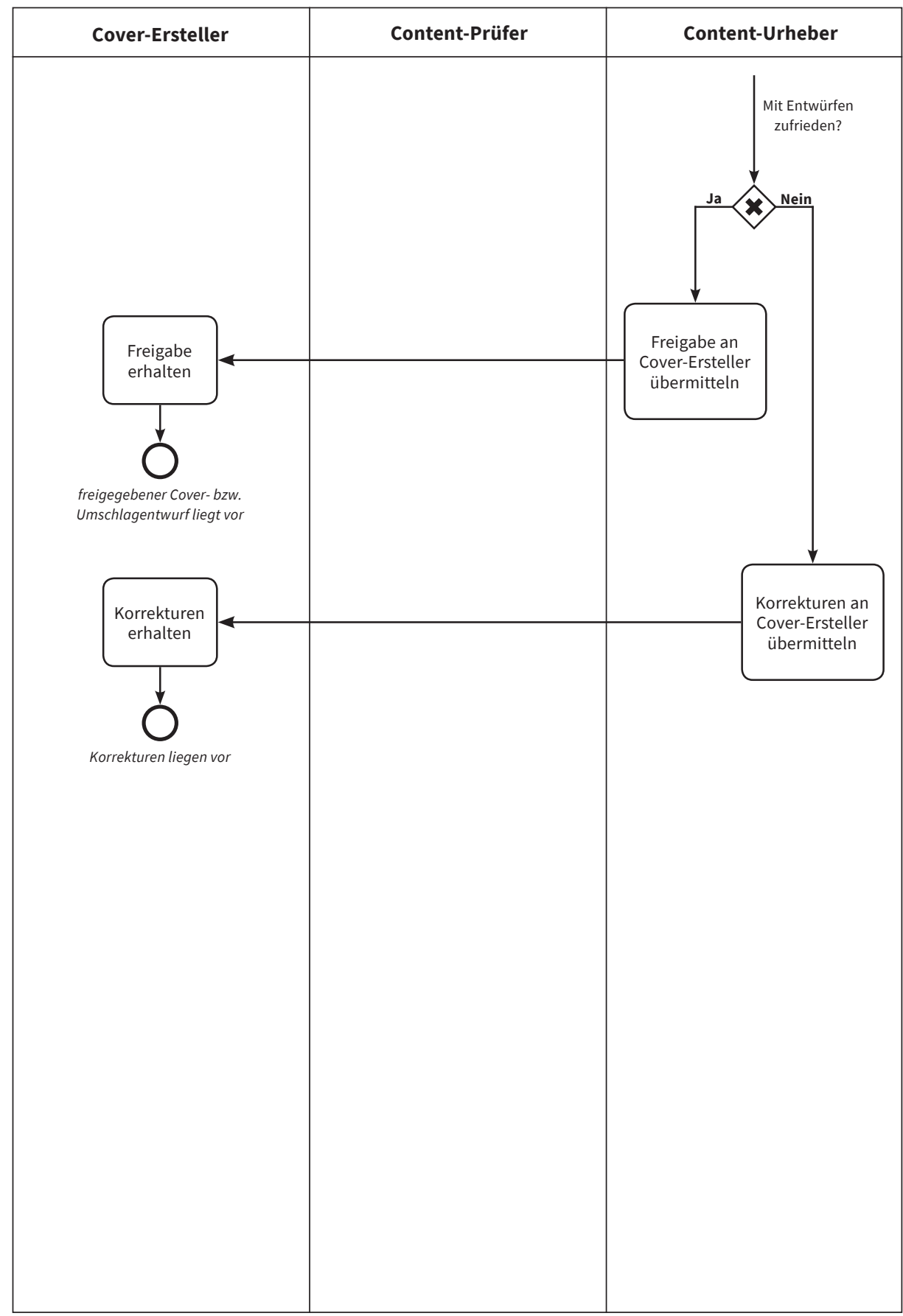




\section{Korrekturen für Cover bzw. Umschlag umsetzen [P-CUe-130]}

Prozessbeschreibung

Der Cover-Ersteller hat vom Content-Urheber und/oder Content-Prüfer Korrekturen zum Cover- bzw. Umschlagentwurf erhalten und setzt diese um.

Vor- und nachgelagerte Prozesse

\begin{tabular}{|c|c|c|c|c|}
\hline P-CUe-120 & & P-CUe-130 & & P-CUe-120 \\
\hline Freigabe für Cover bzw. & 11 & Korrekturen für & 01 & Freigabe für Cover bzw. \\
\hline Umschlag einholen & & $\begin{array}{l}\text { Cover bzw. Umschlag } \\
\text { umsetzen }\end{array}$ & & Umschlag einholen \\
\hline
\end{tabular}

\section{Input}

- I1: Korrekturen

\section{Output}

- O1: überarbeiteter Cover- bzw. Umschlagsentwurf

\section{Beteiligte Rollen}

- Cover-Ersteller

\section{Ressourcen}

- Zeit: T-[P-CUe-130]

- Kosten: K-[P-CUe-130] 


\section{Cover-Ersteller}

Korrekturen für Cover- bzw.

Umschlagentwurf erhalten

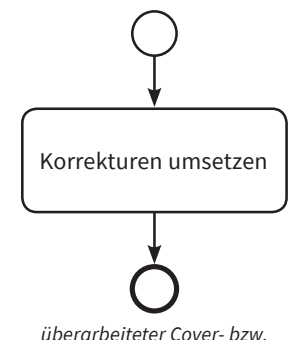

Umschlagentwurf liegt vor 


\section{Content-Eingangsformat in Produktionsformat überführen [P-CiP-100]}

\section{Prozessbeschreibung}

Der Content-Umsetzer erhält vom Copy Editor den inhaltlich finalen und produktionstechnisch bereinigten Content und überführt ihn in das Produktionsformat entsprechend der Richtlinie für die Produktumsetzung und sofern notwendig.

Vor- und nachgelagerte Prozesse

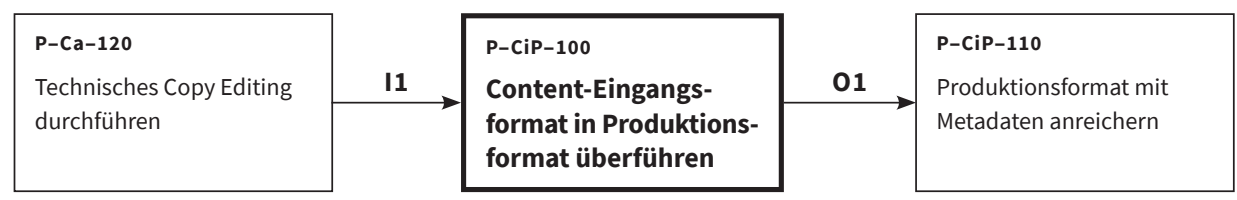

\section{Input}

- I1: inhaltlich finaler und produktionstechnisch bereinigter Content

\section{Output}

- O1: Content im Produktionsformat

\section{Qualitätsrichtlinie}

- Richtlinie für die Produktumsetzung

\section{Beteiligte Rollen}

- Content-Umsetzer

\section{Ressourcen}

- Zeit: T-[P-CiP-100]

- Kosten: K-[P-CiP-100] 


\section{Content-Umsetzer}

inhaltlich finaler und

produktionstechnisch bereinigter

Content liegt vor

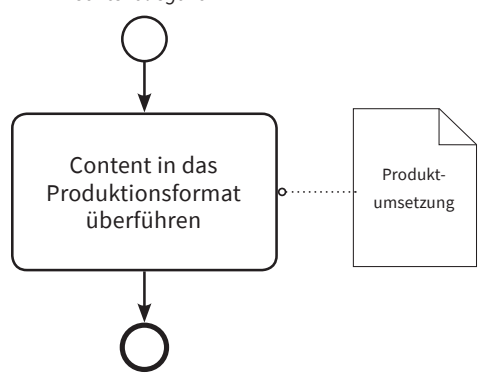

Content liegt im Produktions-

format vor 


\section{Produktionsformat mit Metadaten anreichern [P-CiP-110]}

\section{Prozessbeschreibung}

Der Content-Umsetzer reichert den Content im Produktionsformat mit Metadaten entsprechend der Richtlinie für die Produktumsetzung an.

Vor- und nachgelagerte Prozesse

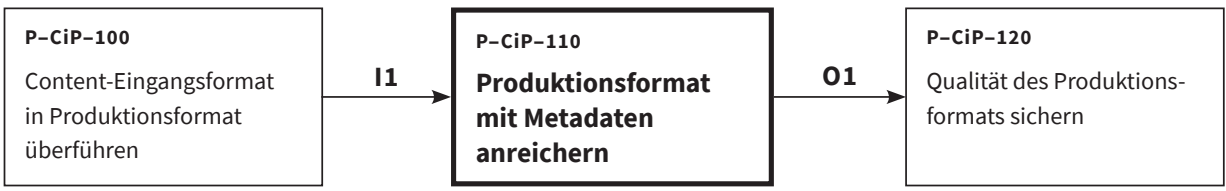

\section{Input}

- I1: Content im Produktionsformat

\section{Output}

- O1: angereicherter Content im Produktionsformat

\section{Qualitätsrichtlinie}

- Richtlinie für die Produktumsetzung

Beteiligte Rollen

- Content-Umsetzer

\section{Ressourcen}

- Zeit: T-[P-CiP-110]

- Kosten: K-[P-CiP-110] 


\section{Content-Umsetzer}

Content liegt im Produktions

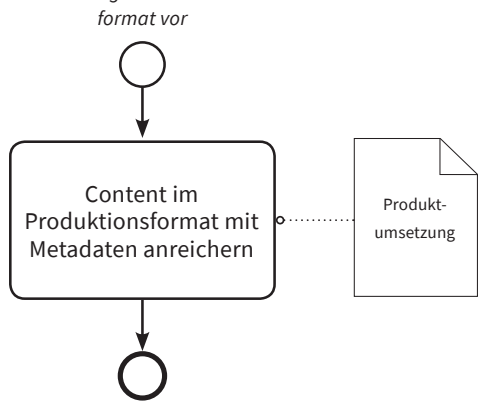

angereicherter Content im

Produktionsformat liegt vor 


\section{Qualität des Produktionsformats sichern [P-CiP-120]}

\section{Prozessbeschreibung}

Der Content-Umsetzer oder der Content-Prüfer überprüft das Produktionsformat nach der Richtlinie für die Produktumsetzung und beseitigt auftretende Fehler. Ggf. prüft der Barrierefreiheit-Beauftragte zusätzlich das Produktionsformat, um festzustellen, ob die Richtlinie eingehalten wurde. Sofern dies nicht der Fall ist, meldet er die gewünschten Korrekturen an den Content-Umsetzer zurück oder arbeitet sie selbst in das Produktionsformat ein.

Vor- und nachgelagerte Prozesse

\begin{tabular}{|l|l|l|l|}
\cline { 3 - 3 } $\begin{array}{l}\text { P-CiP-110 } \\
\text { Produktionsformat mit } \\
\text { Metadaten anreichern }\end{array}$ & $\mathbf{I 1} \longrightarrow \begin{array}{l}\text { P-CiP-120 } \\
\text { Qualität des } \\
\text { Produktionsformats } \\
\text { sichern }\end{array}$ & $\mathbf{0 1}$ & $\begin{array}{l}\text { P-CiP-130 } \\
\text { Gewünschte } \\
\text { Ausgabeformate } \\
\text { generieren }\end{array}$ \\
\cline { 2 - 4 }
\end{tabular}

\section{Input}

- I1: angereicherter Content im Produktionsformat

\section{Output}

- O1: qualitätsgesichertes Produktionsformat

\section{Qualitätsrichtlinie}

- Richtlinie für die Produktumsetzung

\section{Beteiligte Rollen}

- Content-Umsetzer

- Content-Prüfer

- Barrierefreiheit-Beauftragter

\section{Ressourcen}

- Zeit: T-[P-CiP-120]

- Kosten: K-[P-CiP-120] 


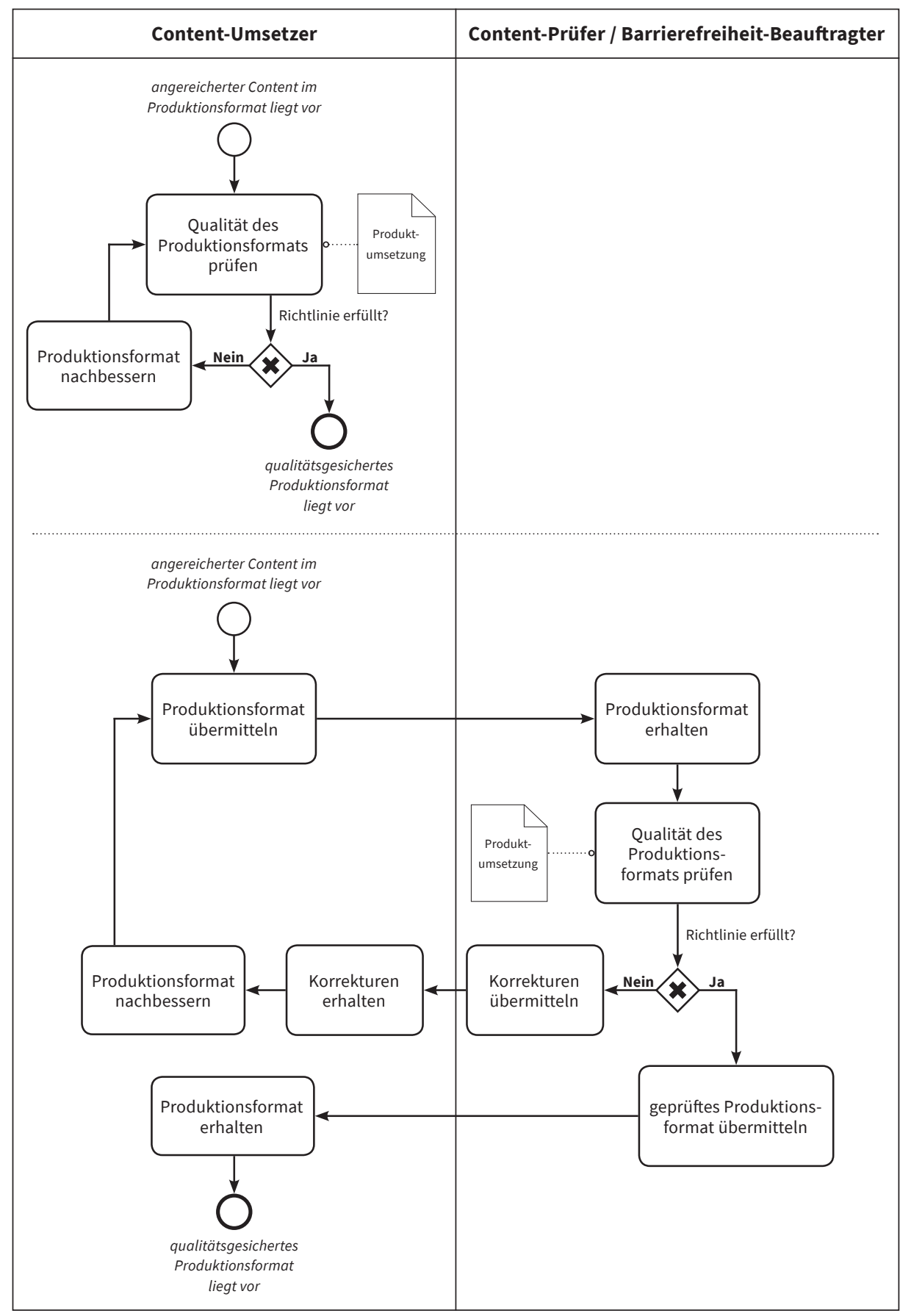




\section{Gewünschte Ausgabeformate generieren [P-CiP-130]}

\section{Prozessbeschreibung}

Der Content-Umsetzer generiert die gewünschten Ausgabeformate der Publikation und übermittelt sie an den Content-Prüfer. Je nach Absprache und Stand der Technik kann dies nur die PDF-Datei der Publikation oder darüber hinaus weitere übliche Ausgabeformate, wie EPUB und MOBI, sein.

Vor- und nachgelagerte Prozesse

\begin{tabular}{|c|c|c|c|c|}
\hline \multirow{2}{*}{$\begin{array}{l}\text { P-CiP-120 } \\
\text { Qualität des Produktions- } \\
\text { formats sichern }\end{array}$} & I1 & P-CiP-130 & 01 & \multirow{2}{*}{$\begin{array}{l}\text { P-CiP-140 } \\
\text { Gewünschte Ausgabe- } \\
\text { formate virtuell prüfen }\end{array}$} \\
\hline & & Ausgabeformate & \multirow{3}{*}{02} & \\
\hline \multirow{2}{*}{$\begin{array}{l}\text { P-CiP-150 } \\
\text { Korrekturen umsetzen }\end{array}$} & & & & P-CiP-160 \\
\hline & & & & $\begin{array}{l}\text { Freigabe vom Content- } \\
\text { Urheber einholen }\end{array}$ \\
\hline
\end{tabular}

\section{Input}

- I1: qualitätsgesichertes Produktionsformat

- I2: überarbeitetes Produktionsformat

\section{Output}

- O1: Publikation in den gewünschten Ausgabeformaten

- O2: Publikation in den gewünschten Ausgabeformaten

\section{Beteiligte Rollen}

- Content-Umsetzer

- Content-Prüfer

- Content-Urheber

\section{Ressourcen}

- Zeit: T-[P-CiP-130]

- Kosten: K-[P-CiP-130] 


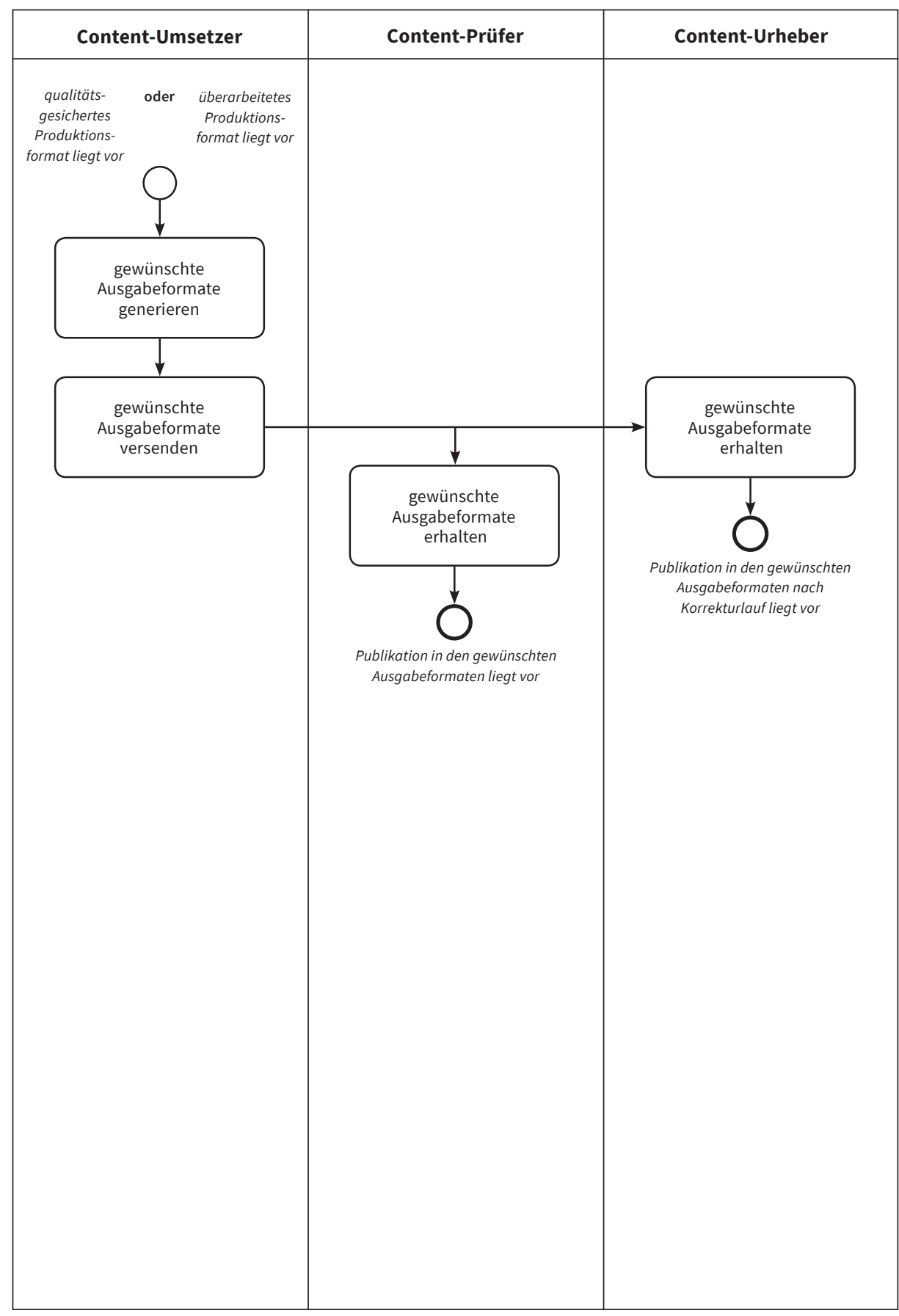




\section{Gewünschte Ausgabeformate virtuell prüfen [P-CiP-140]}

\section{Prozessbeschreibung}

Der Content-Umsetzer stellt dem Content-Prüfer die gewünschten Ausgabeformate der Publikation zur Verfügung. Dieser kontrolliert diese nach der Richtlinie für die Produktfreigabe. Wenn es Korrekturen vorzunehmen gibt, informiert er den Content-Umsetzer entsprechend. Falls es keine Korrekturen gibt, übermittelt der Content-Prüfer die gewünschten Ausgabeformate der Publikation zur Freigabe an den Content-Urheber.

Vor- und nachgelagerte Prozesse

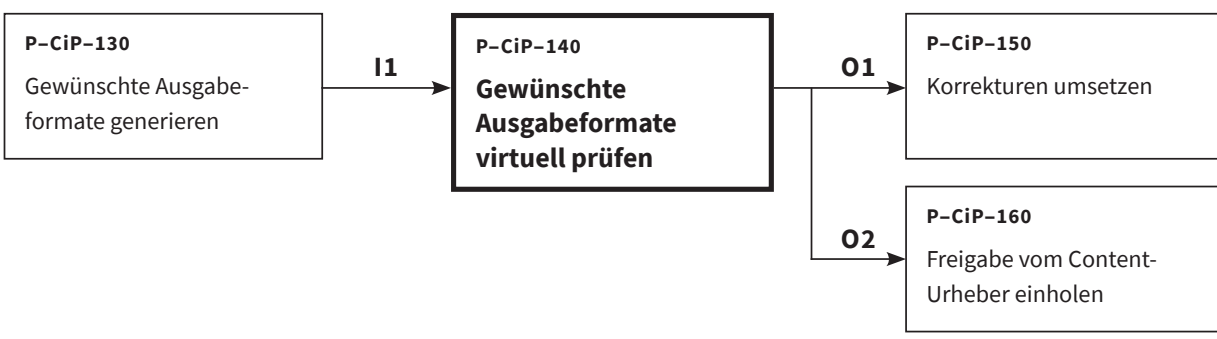

Input

- I1: Publikation in den gewünschten Ausgabeformaten

Output

- O1: Korrekturen

- O2: keine Korrekturen notwendig

Qualitätsrichtlinie

- Richtlinie für die Produktfreigabe

\section{Beteiligte Rollen}

- Content-Prüfer

- Content-Umsetzer

\section{Ressourcen}

- Zeit: T-[P-CiP-140]

- Kosten: K-[P-CiP-140] 


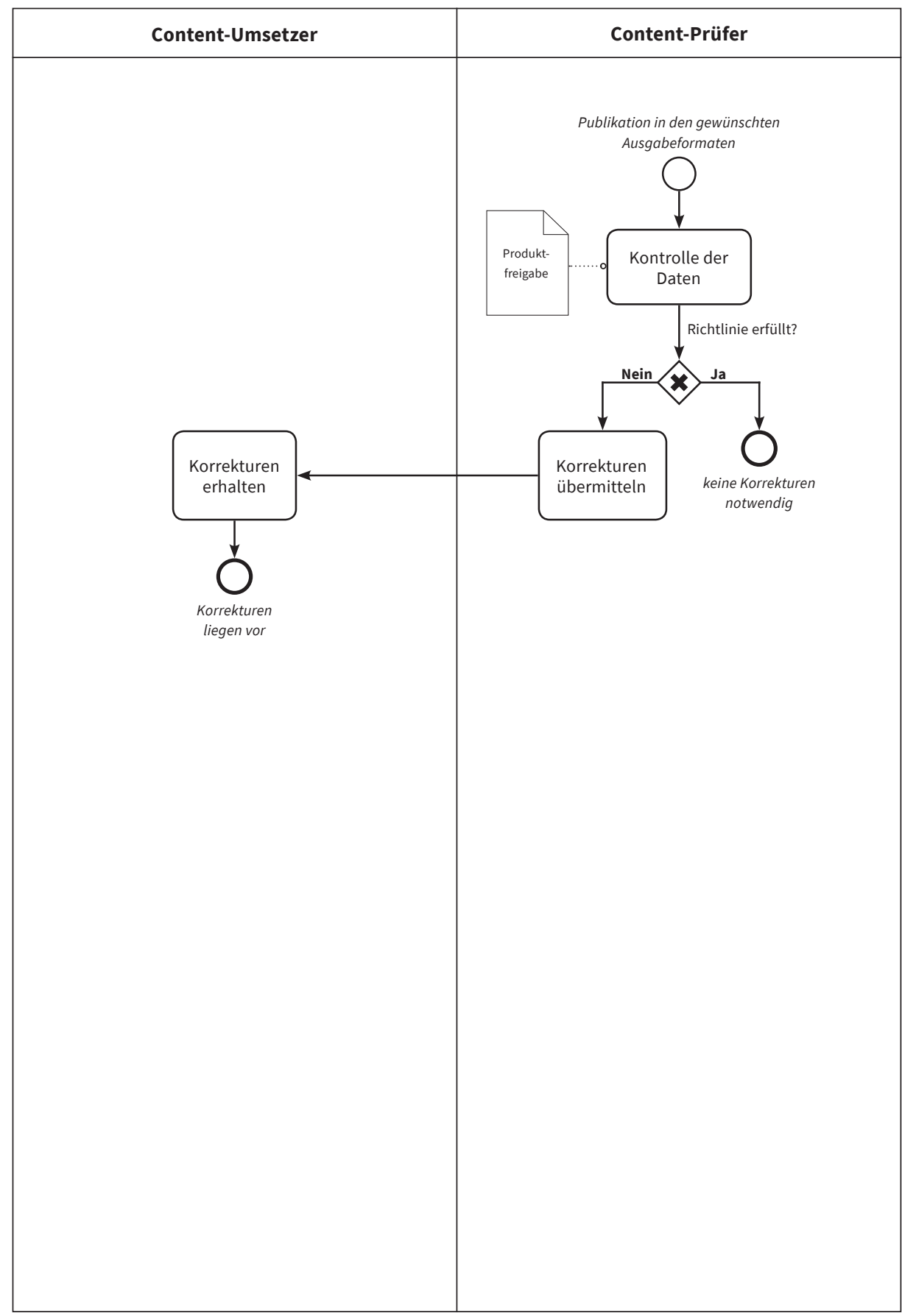




\section{Korrekturen umsetzen [P-CiP-150]}

\section{Prozessbeschreibung}

Der Content-Umsetzer erhält die Korrekturen des Content-Prüfers und/oder des Content-Urhebers. Er führt diese im Produktionsformat aus und generiert erneut die gewünschten Ausgabeformate der Publikation.

Vor- und nachgelagerte Prozesse

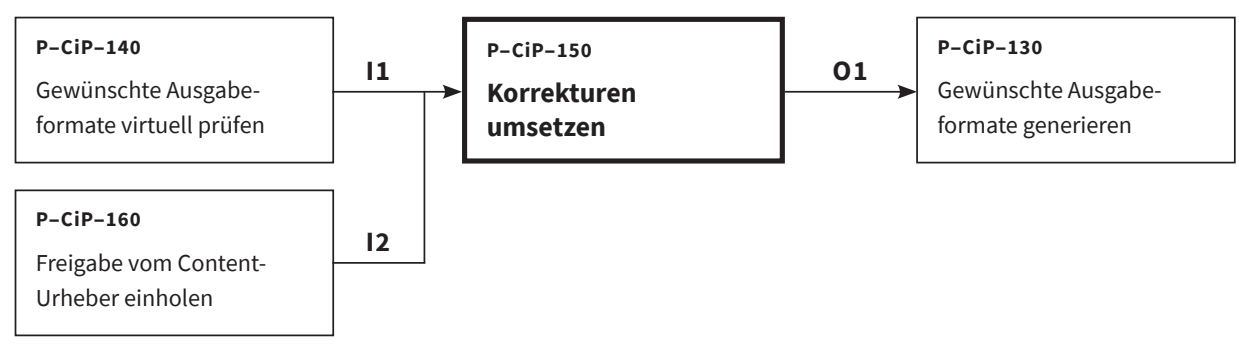

\section{Input}

- I1: Korrekturen des Content-Prüfers

- I2: Korrekturen des Content-Urheber

\section{Output}

- O1: überarbeitetes Produktionsformat

\section{Beteiligte Rollen}

- Content-Umsetzer

\section{Ressourcen}

- Zeit: T-[P-CiP-150]

- Kosten: K-[P-CiP-150] 


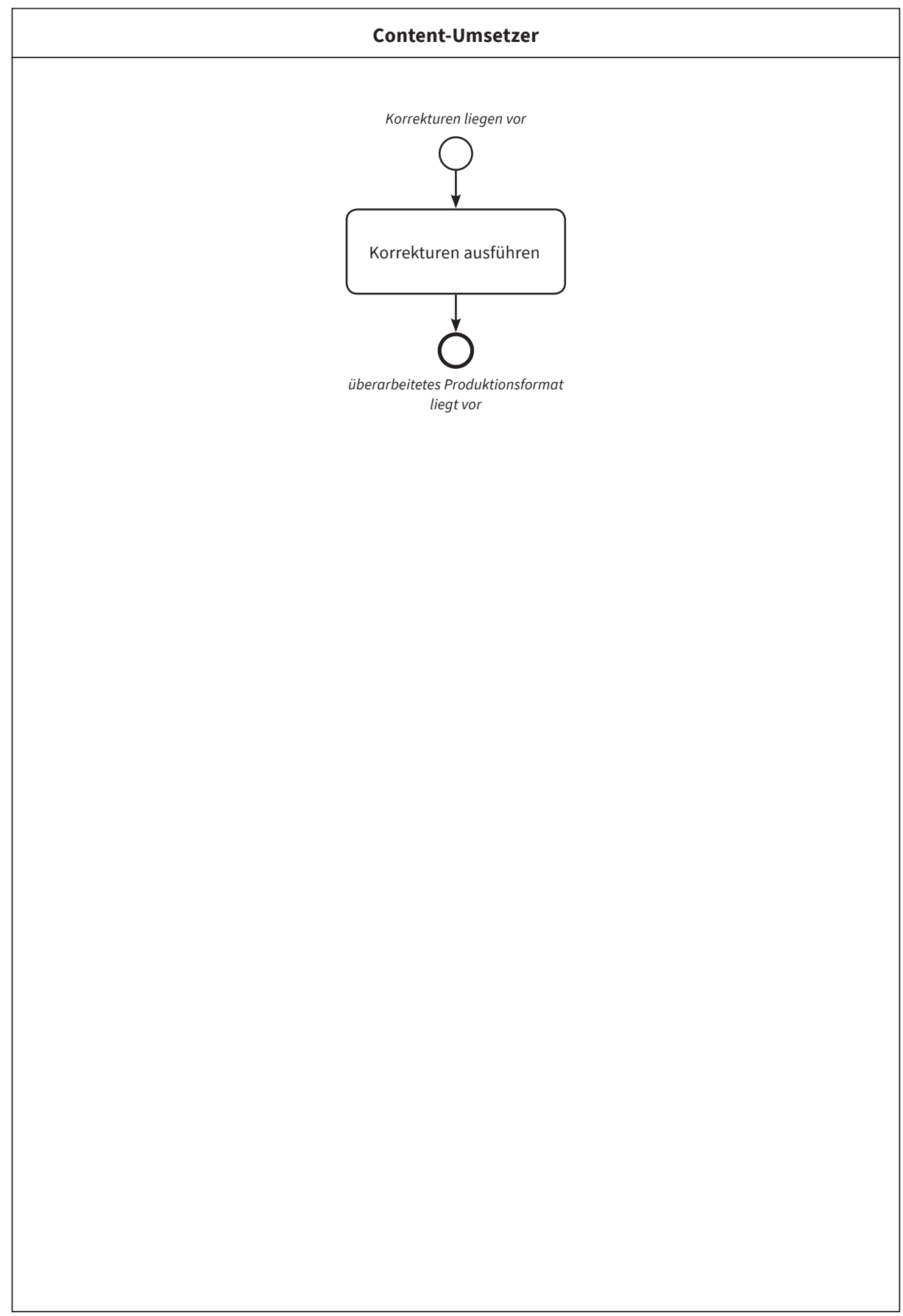




\section{Freigabe vom Content-Urheber einholen [P-CiP-160]}

\section{Prozessbeschreibung}

Der Content-Umsetzer oder Content-Prüfer übermitteln dem Content-Urheber die Publikation in den gewünschten Ausgabeformaten (üblicherweise nur die PDFDatei) zur Freigabe. Dieser kontrolliert die Daten und teilt entweder seine Korrekturwünsche mit oder erteilt die Freigabe.

Vor- und nachgelagerte Prozesse

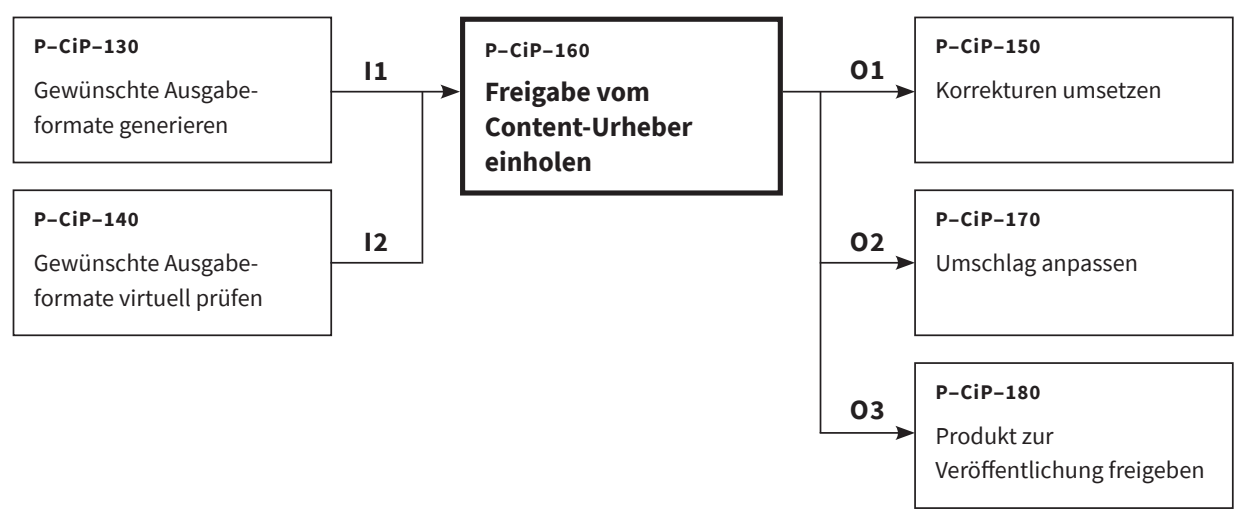

\section{Input}

- I1: überarbeitetes Produktionsformat

- I2: keine Korrekturen notwendig

\section{Output}

- O1: Korrekturen des Content-Urheber

- O2: finale und freigegebene Druck-Datei

- O3: Freigabe zur Publikation der gewünschten Ausgabeformate

\section{Beteiligte Rollen}

- Content-Urheber

- Content-Umsetzer

- Content-Prüfer

\section{Ressourcen}

- Zeit: T-[P-CiP-160]

- Kosten: K-[P-CiP-160] 


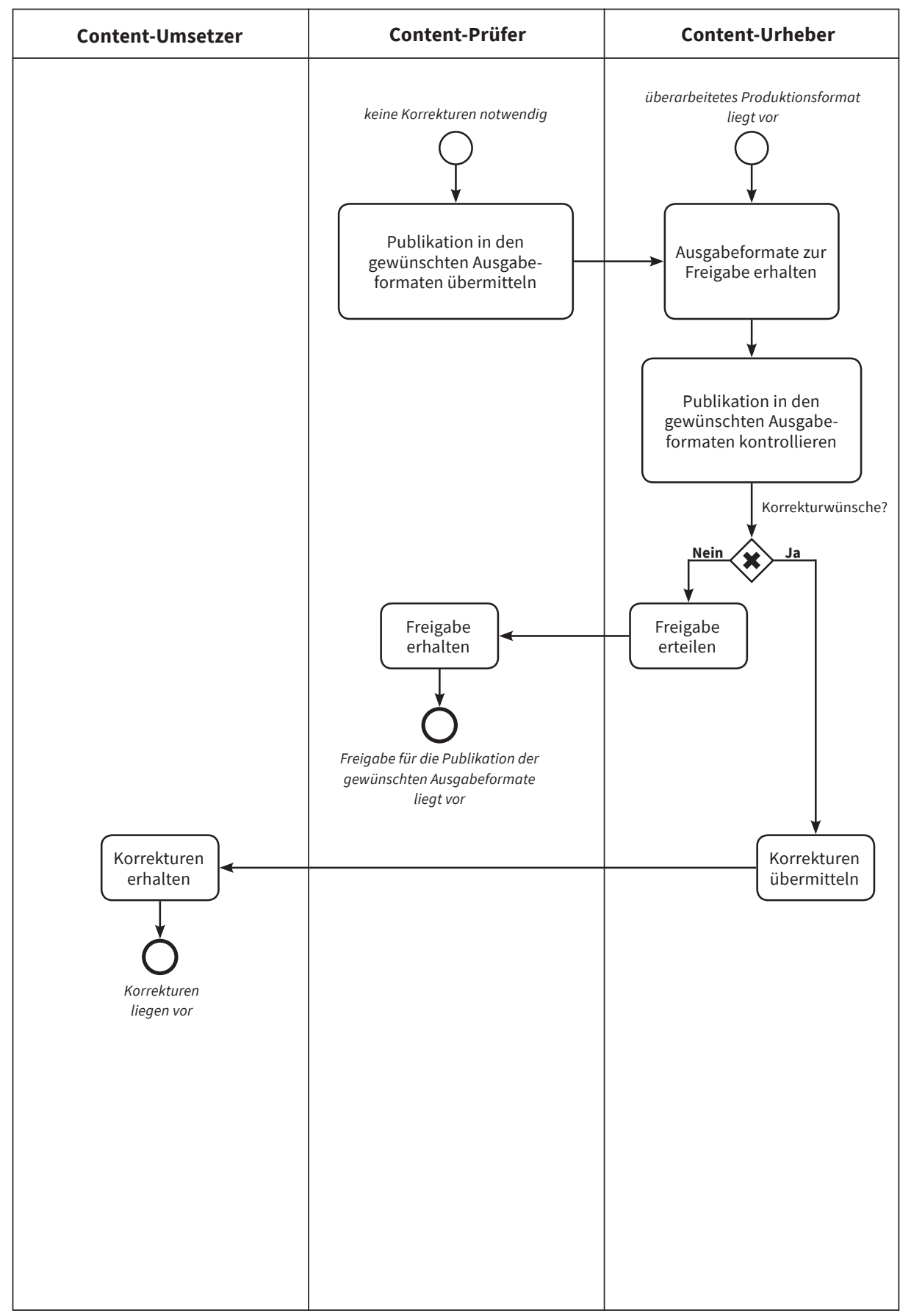




\section{Umschlag anpassen [P-CiP-170]}

\section{Prozessbeschreibung}

Der Cover-Ersteller berechnet auf Grundlage der finalen Druck-Datei die Rückenstärke für das gedruckte Produkt und passt dementsprechend den Rücken des Umschlags an. Anschließend wird der Umschlag für den Druck abgelegt.

Vor- und nachgelagerte Prozesse

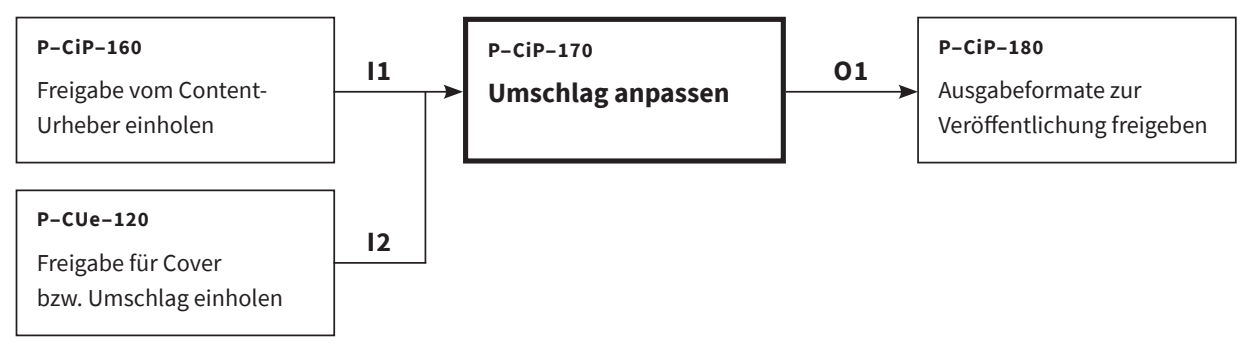

\section{Input}

- I1: finale und freigegebene Druck-Datei

- I2: freigegebener Umschlag

\section{Output}

- O1: angepasster Umschlag für das gedruckte Produkt

\section{Beteiligte Rollen}

- Cover-Ersteller

\section{Ressourcen}

- Zeit: T-[P-CiP-170]

- Kosten: K-[P-CiP-170] 


\section{Cover-Ersteller}

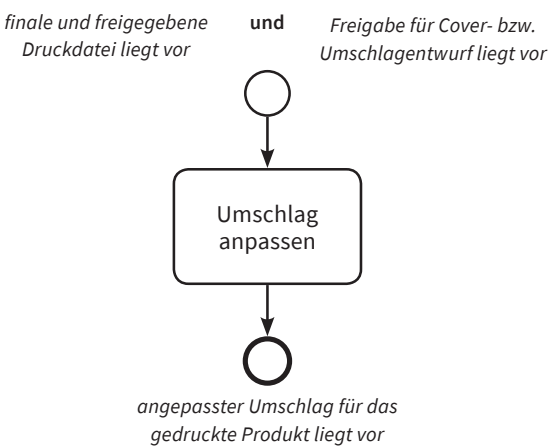




\section{Ausgabeformate zur Veröffentlichung freigeben [P-CiP-180]}

\section{Prozessbeschreibung}

Nachdem alle Korrekturen eingearbeitet worden sind, der Content-Urheber seine Freigabe gegeben hat, informiert der Content-Abnehmer alle relevanten Rollen hierüber und gibt die Ausgabeformate zur Veröffentlichung frei.

Vor- und nachgelagerte Prozesse

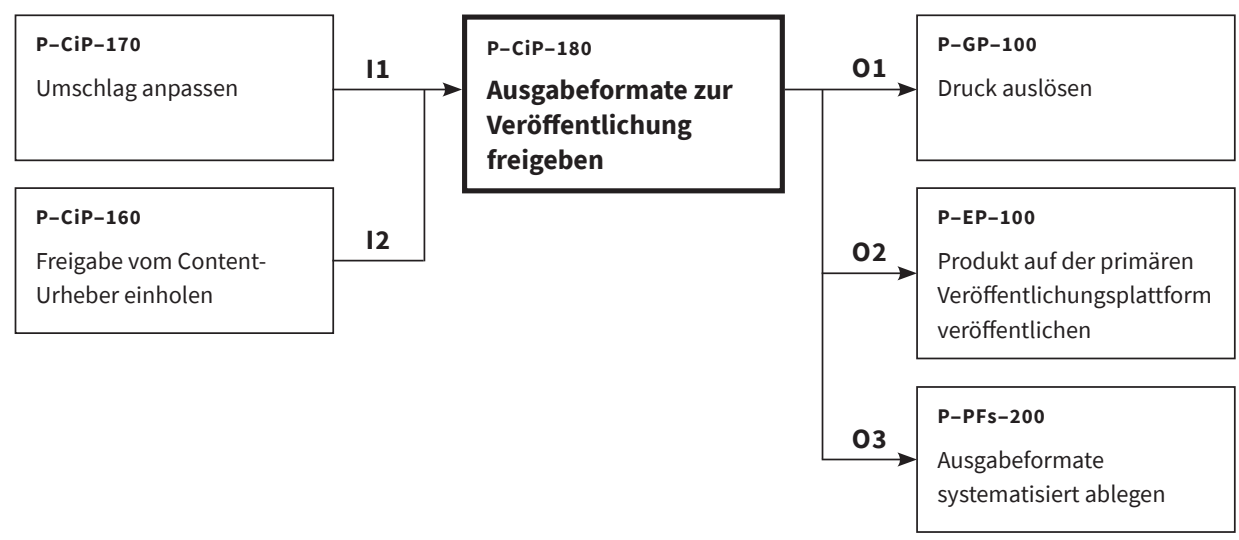

\section{Input}

- I1: angepasster Umschlag für das gedruckte Produkt

- I2: Freigabe zur Publikation der gewünschten Ausgabeformate

\section{Output}

- O1: finale Druckdaten

- O2: finale Daten der elektronischen Ausgabeformate

- O3: finale Daten der elektronischen Ausgabeformate

\section{Beteiligte Rollen}

- Content-Abnehmer

\section{Ressourcen}

- Zeit: T-[P-CiP-180] 


\section{Content-Abnehmer}

$\begin{aligned} & \text { Freigabe für die Publikation der } \\ & \text { gewünschten Ausgabeformate }\end{aligned}$
andoder $\quad \begin{array}{r}\text { angepster Umschlag für das } \\ \text { gedrodukt liegt vor }\end{array}$
gewünschten Ausgabeformate gedruckte Produkt liegt vor

liegt vor

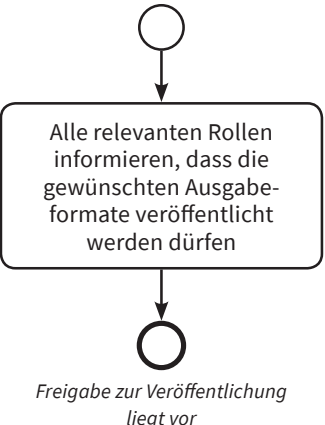




\section{Druck auslösen [P-GP-100]}

\section{Prozessbeschreibung}

Der Content-Distributor beauftragt den Drucker mit dem Druck der gewünschten Produkte und übermittelt die finalen Druckdaten für Umschlag und Inhalt. Der Drucker bestätigt den Erhalt des Auftrags und stellt ggf. Rückfragen zum Auftrag oder wann das Testexemplar oder die Auflage voraussichtlich geliefert wird.

Vor- und nachgelagerte Prozesse

\begin{tabular}{|l|l|l|l|}
\cline { 2 - 2 } $\begin{array}{l}\text { P-CiP-180 } \\
\text { Ausgabeformate zur } \\
\text { Veröffentlichung freigeben }\end{array}$ & $\mathbf{1 1} \begin{array}{l}\text { P-GP-100 } \\
\text { Druck auslösen }\end{array}$ & $\begin{array}{l}\text { P-GP-110 } \\
\text { Produkt physisch } \\
\text { prüfen }\end{array}$ \\
\cline { 2 - 3 }
\end{tabular}

\section{Input}

- I1: finale Druckdaten

\section{Output}

- O1: Bestätigung des Druckauftrags

\section{Beteiligte Rollen}

- Content-Distributor

- Drucker

\section{Ressourcen}

- Zeit: T-[P-GP-100] 


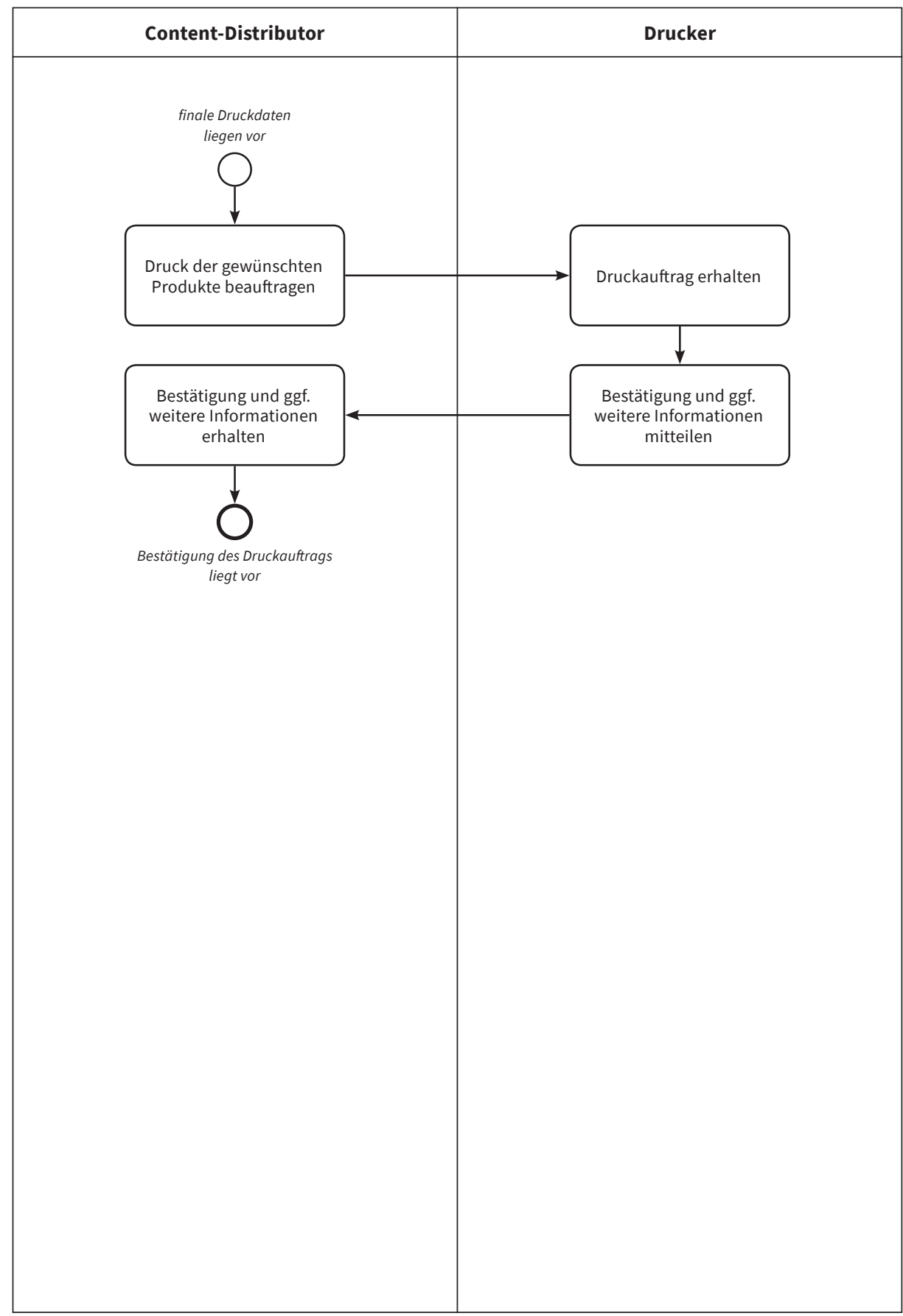




\section{Produkt physisch prüfen [P-GP-110]}

\section{Prozessbeschreibung}

Sofern vereinbart, erhält der Content-Distributor ein Testexemplar (Aushänger) des gedruckten Produktes vom Drucker, um die druck- und buchbinderische Qualität zu überprüfen, bevor die gesamte Auflage produziert wird. Sofern es Mängel diesbezüglich gibt, meldet er dies an den Drucker zurück, mit der Bitte sie nachzubessern. Anschließend wird die Freigabe die gesamte Auflage zu produzieren und zu liefern, erteilt. Falls kein Testexemplar geliefert wird, überprüft der ContentDistributor an dieser Stelle stichpunktartig die Qualität der Auflage, reklamiert bei groben Qualitätsmängeln die Produkte und bittet ggf. um Neudruck.

Vor- und nachgelagerte Prozesse

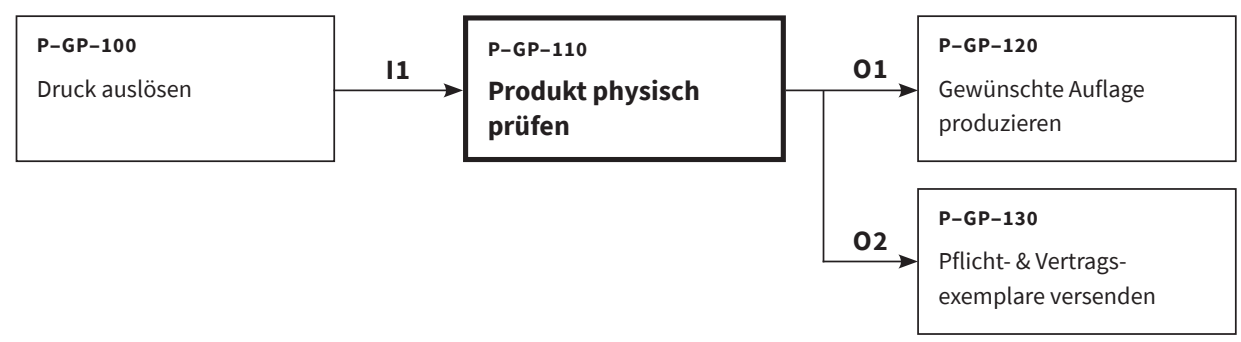

\section{Input}

- I1: Bestätigung des Druckauftrags

\section{Output}

- O1: Freigabe für gesamte Auflage oder Reklamation und Bitte um Neudruck

- O2: qualitätsgesicherte Auflage

\section{Qualitätsrichtlinie}

- Richtlinie für gedruckte Produkte

\section{Beteiligte Rollen}

- Drucker

- Content-Distributor

\section{Ressourcen}

- Zeit: T-[P-GP-110]

- Kosten: K-[P-GP-110] 


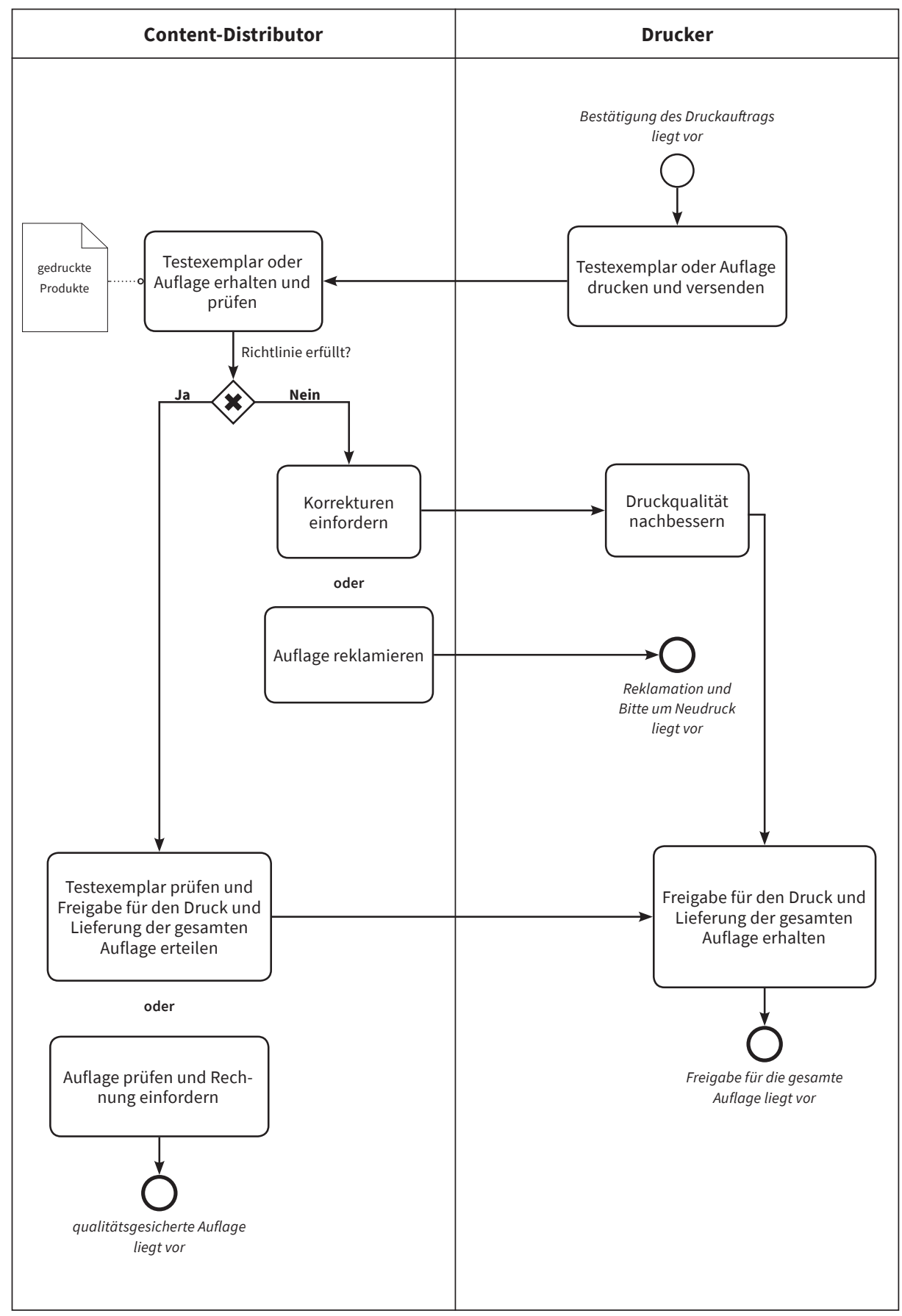




\section{Produkt physisch prüfen [P-GP-120]}

\section{Prozessbeschreibung}

Nachdem der Drucker vom Content-Distributor die Freigabe des Testexemplars oder die Bitte um Neudruck erhalten hat, produziert er die beauftragte Auflage und liefert sie an die genannte/n Lieferadresse/n.

Vor- und nachgelagerte Prozesse

\begin{tabular}{|c|c|c|c|c|}
\hline $\begin{array}{l}\text { P-GP-110 } \\
\text { Produkt physisch } \\
\text { prüfen }\end{array}$ & I1 & $\begin{array}{l}\text { P-GP-120 } \\
\text { Gewünschte Auflage } \\
\text { produzieren }\end{array}$ & 01 & $\begin{array}{l}\text { P-GP-130 } \\
\text { Pflicht- \& Vertrags- } \\
\text { exemplare versenden }\end{array}$ \\
\hline
\end{tabular}

\section{Input}

- I1: Freigabe für die gesamte Auflage oder Reklamation der Auflage und Bitte um Neudruck

\section{Output}

- O1: qualitätsgesicherte Auflage

\section{Beteiligte Rollen}

- Drucker

- Content-Distributor

\section{Ressourcen}

- Zeit: T-[P-GP-120]

- Kosten: K-[P-GP-120] 


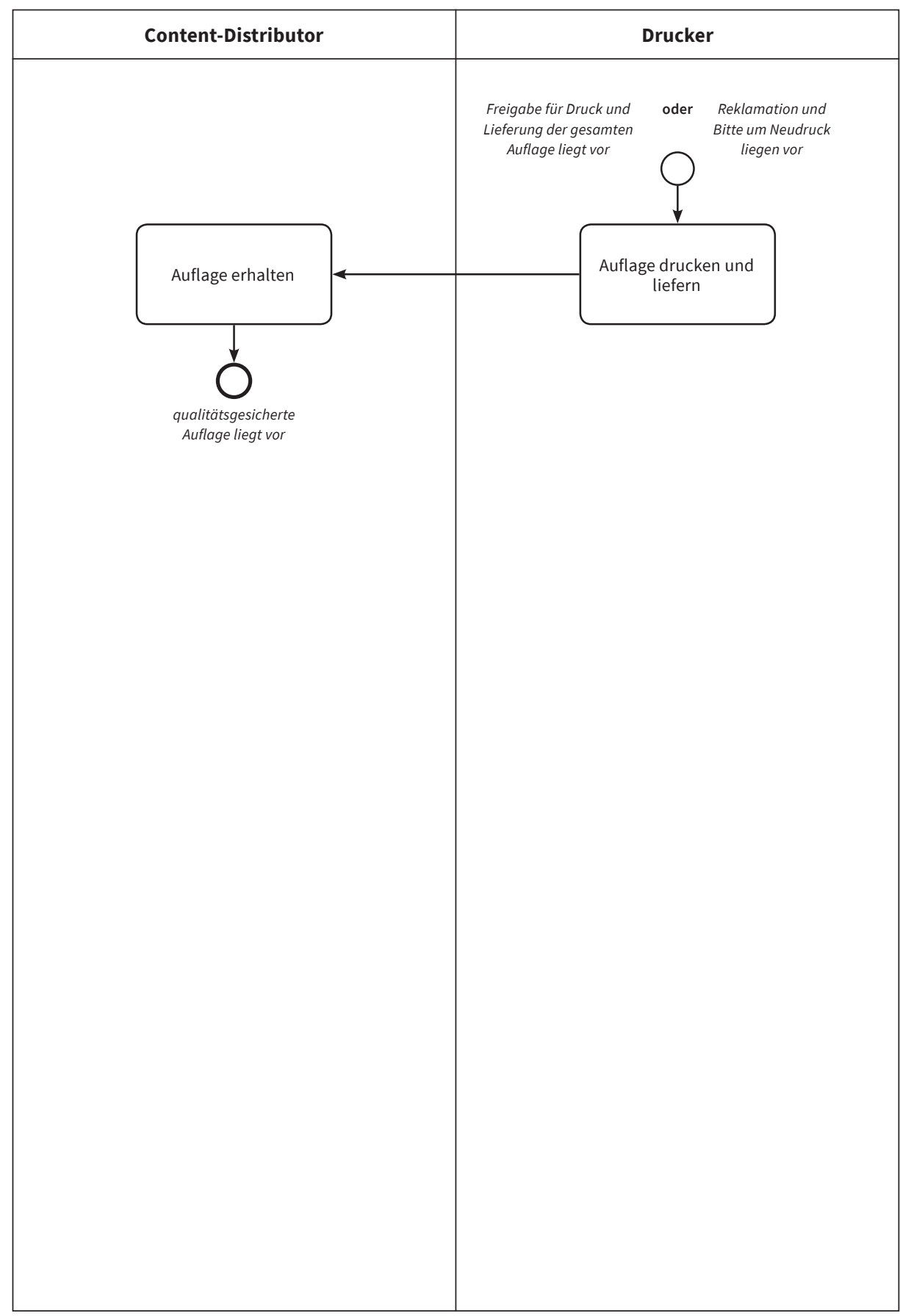




\section{Pflicht- und Vertragsexemplare versenden [P-GP-130]}

\section{Prozessbeschreibung}

Je nach Vereinbarung versendet der Drucker oder nach Erhalt der Auflage der Content-Distributor selbst die Pflicht- und Vertragsexemplare nach der festgelegten Richtlinie für Pflichtexemplare.

Vor- und nachgelagerte Prozesse

\begin{tabular}{|l|l|l|l|}
\hline $\begin{array}{l}\text { P-GP-120 } \\
\text { pewünschte Auflage }\end{array}$ & $\mathbf{I 1} \begin{array}{l}\text { P-GP-130 } \\
\text { Pflicht- \& Vertrags- } \\
\text { exemplare versenden }\end{array}$ \\
\cline { 2 - 3 }
\end{tabular}

\section{Input}

- I1: qualitätsgesicherte Auflage

\section{Output}

- O1: versendete Pflicht- und Vertragsexemplare

\section{Qualitätsrichtlinie}

- Richtlinie für Pflichtexemplare

Beteiligte Rollen

- Content-Distributor oder Drucker

\section{Ressourcen}

- Zeit: T-[P-GP-130]

- Kosten: K-[P-GP-130] 


\section{Content-Distributor oder Drucker}

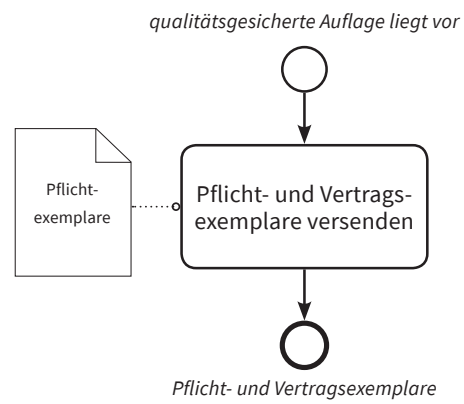

sind versendet 


\section{Produkt auf primärer Veröffentlichungsplattform veröffentlichen [P-EP-100]}

Prozessbeschreibung

Der Content-Distributor lädt das Produkt in den gewünschten, elektronischen Ausgabeformaten auf die primäre Veröffentlichungsplattform hoch, die durch die Richtlinie für die Wahl der OA-Veröffentlichungsplattform definiert worden ist.

Vor- und nachgelagerte Prozesse

\begin{tabular}{|c|c|c|c|c|}
\hline P-CiP-180 & & P-EP-100 & & P-EP-110 \\
\hline Ausgabeformate zur & I1 & Produkt auf primärer & 01 & Produkt auf \\
\hline Verottentlıchung freigeben & & $\begin{array}{l}\text { Veroffentlichungs- } \\
\text { plattform } \\
\text { veröffentlichen }\end{array}$ & & $\begin{array}{l}\text { weiteren Plattformen } \\
\text { veröffentlichen }\end{array}$ \\
\hline
\end{tabular}

\section{Input}

- I1: Freigabe zur Veröffentlichung

\section{Output}

- O1: elektronische Ausgabeformate der Publikation auf primärer Veröffenlichungsplattform veröffentlicht

\section{Qualitätsrichtlinie}

— Richtlinie für die Wahl der primären OA-Veröffentlichungsplattform

\section{Beteiligte Rollen}

- Content-Distributor

\section{Ressourcen}

- Zeit: T-[P-EP-100] 


\section{Content-Distributor}

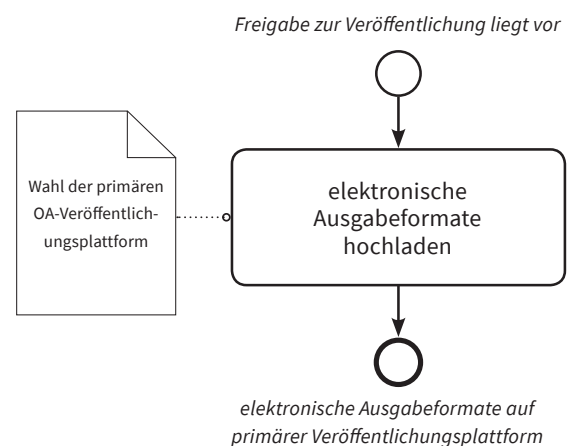

veröffentlicht 


\section{Produkt auf weiteren Plattform veröffentlichen [P-EP-110]}

\section{Prozessbeschreibung}

Der Content-Distributor lädt das Produkt in den gewünschten, elektronischen Ausgabeformaten auf weiteren Veröffentlichungsplattformen entsprechend der Richtlinie für die Wahl weiterer OA-Veröffentlichungsplattformen hoch, um die optimale Auffindbarkeit und Sichtbarkeit des Produktes zu gewährleisten.

Vor- und nachgelagerte Prozesse

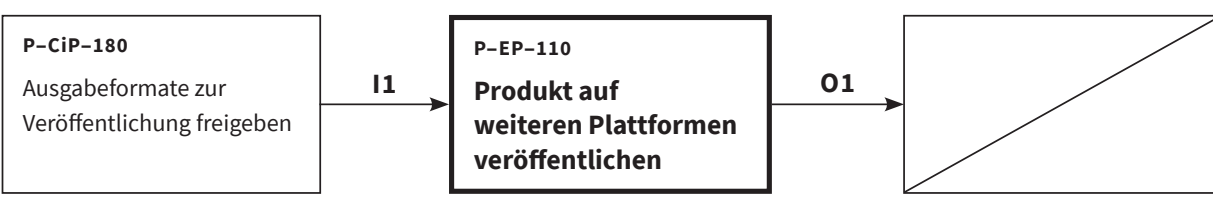

\section{Input}

- I1: Freigabe zur Veröffentlichung

\section{Output}

- O1: elektronische Ausgabeformate auf weiteren Veröffenlichungsplattform veröffentlicht

\section{Qualitätsrichtlinie}

- Richtlinie für die Wahl weiterer Veröffentlichungsplattformen

\section{Beteiligte Rollen}

- Content-Distributor

\section{Ressourcen}

- Zeit: T-[P-EP-110] 


\section{Content-Distributor}

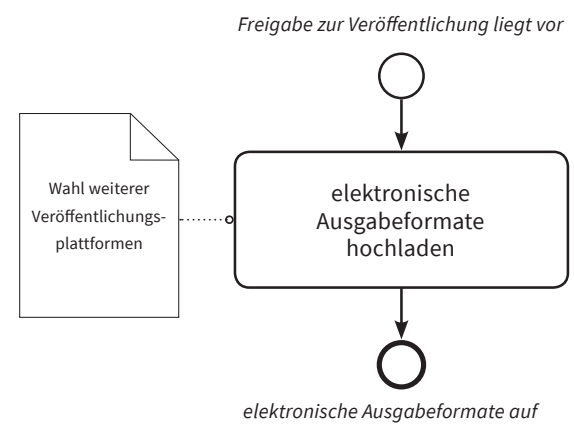

weiteren Veröffentlichungsplattform

$$
\text { veröffentlicht }
$$




\section{Für die Langzeitarchivierung des Produktes sorgen [P-PFs-100]}

\section{Prozessbeschreibung}

Der Content-Archivar sorgt für die Langzeitarchivierung aller oder ausgewählter Ausgabeformate der Publikation entsprechend der Richtlinie für die Wahl des Langzeitarchivs.

Vor- und nachgelagerte Prozesse

\begin{tabular}{|l|l|l|}
\hline $\begin{array}{l}\text { P-CiP-180 } \\
\text { Ausgabeformate zur } \\
\text { Veröffentlichung freigeben }\end{array}$ & $\mathbf{I 1}$ & $\begin{array}{l}\text { P-PFs-100 } \\
\text { Für Langzeit- } \\
\text { archivierung des } \\
\text { Produktes sorgen }\end{array}$ \\
\cline { 3 - 4 }
\end{tabular}

\section{Input}

- I1: Freigabe zur Veröffentlichung

\section{Output}

- O1: langzeitarchiviertes Produkt

\section{Qualitätsrichtlinie}

- Richtlinie für die Wahl des Langzeitarchivs

\section{Beteiligte Rollen}

- Content-Archivar

\section{Ressourcen}

- Zeit: T-[P-PFs-100]

- Kosten: K-[P-PFs-100] 


\section{Content-Archivar}

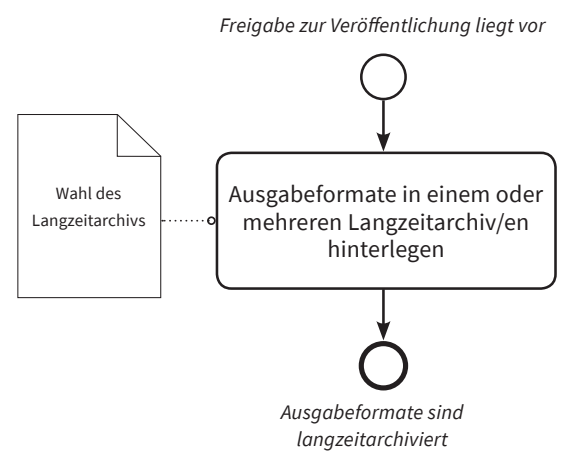




\section{Ausgabeformate systematisiert ablegen [P-PFs-200]}

\section{Prozessbeschreibung}

Der Content-Archivar legt alle Ausgabeformate der Publikation entsprechend der Richtlinie zur internen Archivierung ab.

Vor- und nachgelagerte Prozesse

\begin{tabular}{|l|l|l|l|}
\hline $\begin{array}{l}\text { P-CiP-180 } \\
\text { Ausgabeformate zur } \\
\text { Veröffentlichung freigeben }\end{array}$ & $\mathbf{I 1} \longrightarrow \begin{array}{l}\text { P-PFs-200 } \\
\text { Ausgabeformate } \\
\text { systematisiert ablegen }\end{array}$ \\
\cline { 2 - 3 }
\end{tabular}

\section{Input}

- I1: Freigabe zur Veröffentlichung

\section{Output}

- O1: intern archivierte Ausgabeformate

\section{Qualitätsrichtlinie}

- Richtlinie zur internen Archivierung

\section{Beteiligte Rollen}

- Content-Archivar

\section{Ressourcen}

- Zeit: T-[P-PFs-200] 


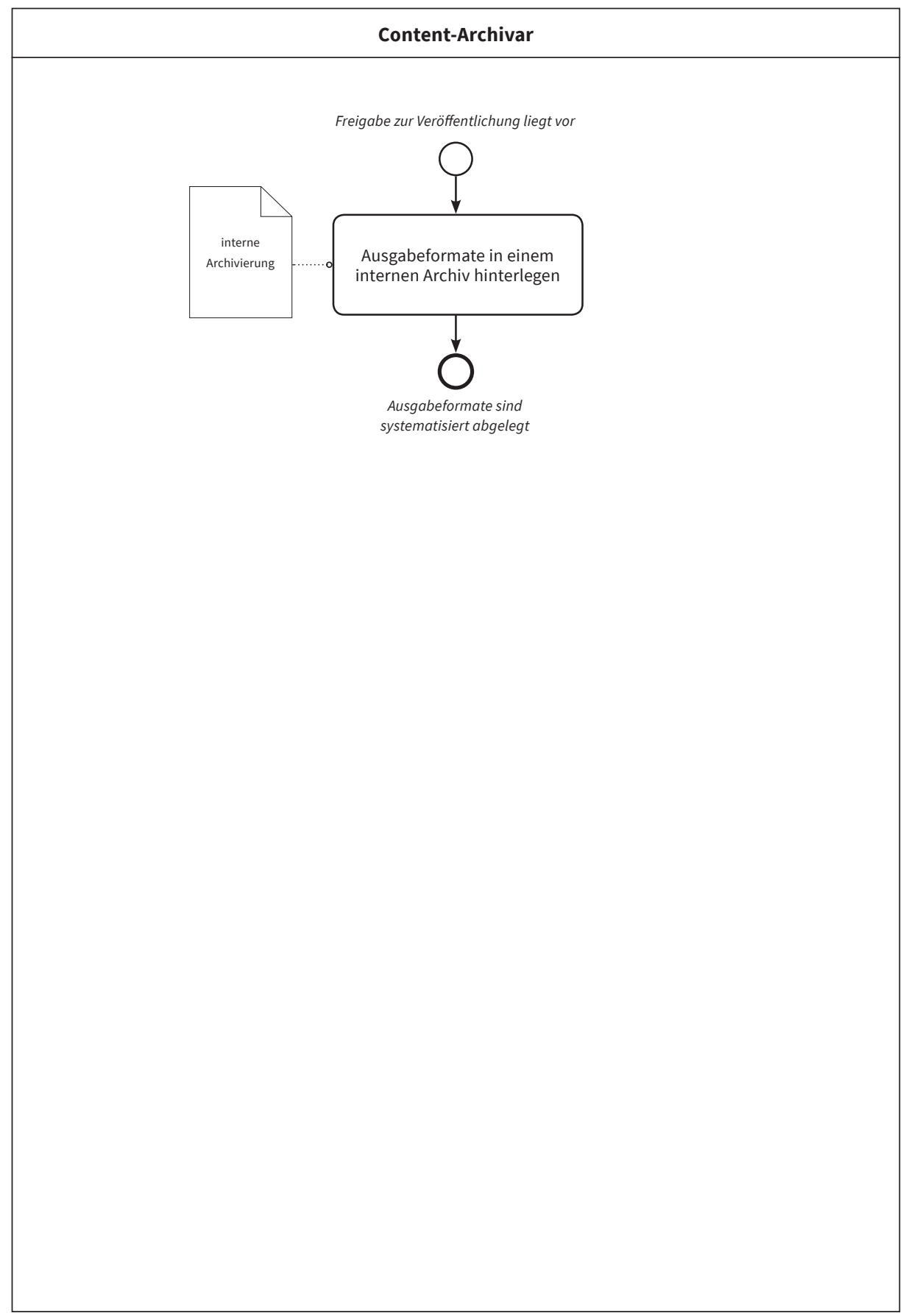




\section{Marketing-Plan für Produkt erstellen [M-OM-100]}

\section{Prozessbeschreibung}

Der Marketing-Manager erstellt - ggf. auf Grundlage der Informationen aus dem strategischen Marketing des Hochschulverlages - einen Marketing-Plan für die aktuelle Publikation. Dieser kann Termine und Maßnahmen für die Vorankündigung der Publikation, Maßnahmen zum Bewerben der Publikation nach Veröffentlichung u. v. w. enthalten.

Vor- und nachgelagerte Prozesse

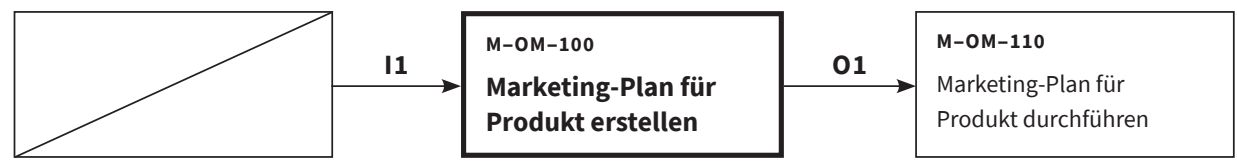

\section{Input}

- I1: Informationen vom strateg. Marketing-Plan

\section{Output}

- O1: operativer Marketing-Plan

\section{Beteiligte Rollen}

- Marketing-Manager

\section{Ressourcen}

- Zeit: T-[M-OM-100]

- Kosten: K-[M-OM-100] 


\section{Marketing-Manager}

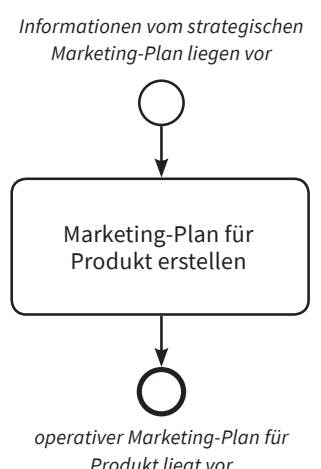




\section{Marketing-Plan für Produkt durchführen [M-OM-110]}

\section{Prozessbeschreibung}

Der Marketing-Manager führt die im Marketing-Plan festgelegten Maßnahmen zur Vorankündigung oder zur grundsätzlichen Bewerbung der Publikation entsprechend der festgelegten Termine durch.

Vor- und nachgelagerte Prozesse

\begin{tabular}{|l|l|l|}
\hline $\begin{array}{l}\text { M-OM-100 } \\
\text { Marketing-Plan für }\end{array}$ & $\mathbf{I 1} \longrightarrow \begin{array}{l}\text { M-0M-110 } \\
\text { Marketing-Plan für } \\
\text { Produkt erstellen }\end{array}$ \\
\cline { 1 - 2 }
\end{tabular}

\section{Input}

- I1: operativer Marketing-Plan

\section{Output}

- O1: durchgeführte Marketing-Maßnahmen

\section{Beteiligte Rollen}

- Marketing-Manager

\section{Ressourcen}

- Zeit: T-[M-OM-110]

- Kosten: K-[M-OM-110] 


\section{Marketing-Manager}

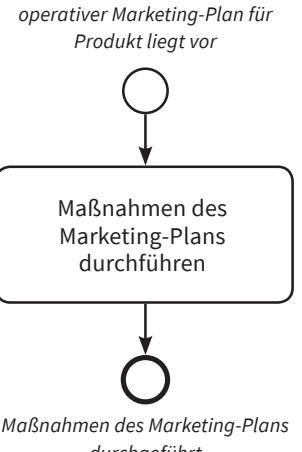

durchgeführt 


\section{Rechte für Inhaltselemente klären [M-PVe-100]}

\section{Prozessbeschreibung}

Der Content enthält Inhaltselemente (wie Abbildungen, Fotos, Grafiken), dessen Rechtinhaber der Content-Urheber nicht ist. Es muss daher vom Content-Abnehmer, Content-Lieferanten oder Content-Urheber selbst geprüft werden, ob diese Elemente im Rahmen der bevorstehenden Publikation im OA veröffentlicht werden dürfen. Dies kann beinhalten, dass bspw. Abbildungen dahingegen geprüft werden müssen, ob sie eine eigene geistige Schöpfung ihres Urhebers darstellen und somit sein Einverständnis zur Veröffentlichung benötigt wird.

Vor- und nachgelagerte Prozesse

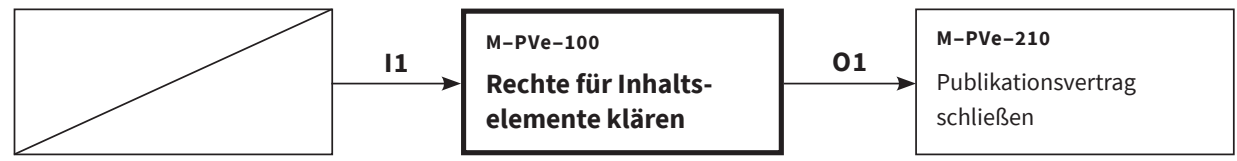

\section{Input}

- I1: es gibt Inhaltselemente im Content, dessen Rechteinhaber nicht der Content-Urheber ist

\section{Output}

- O1: Rechte für alle relevanten Inhaltselemente geklärt

\section{Beteiligte Rollen}

- Content-Abnehmer

- Content-Urheber oder -Lieferant

\section{Ressourcen}

- Zeit: T-[M-PVe-100]

- Kosten: K-[M-PVe-100] 


\section{Content-Abnehmer, Content-Urheber oder -Lieferant}

Erkenntnis, dass es

Inhaltselemente im Content gibt, dessen Rechteinhaber nicht der

Content-Urheber ist

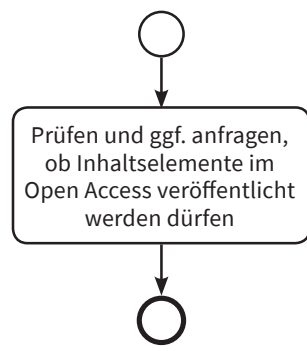

Rechte für alle relevanten

Inhaltselemente geklärt 


\section{Vertragsabschluss \& Buchvorankündigung vorbereiten [M-PVe-200]}

\section{Prozessbeschreibung}

Der Content-Abnehmer informiert den Content-Urheber entsprechend der Richtlinie für den Vertragsabschluss \& die Buchvorankündigung, welche Informationen hierfür vorliegen müssen. Der Content-Urheber übermittelt die geforderten Informationen an den Content-Abnehmer. Der Content-Abnehmer überprüft, ob alle benötigten Informationen für den Vertragsabschluss und für die Buchvorankündigung vorliegen.

Vor- und nachgelagerte Prozesse

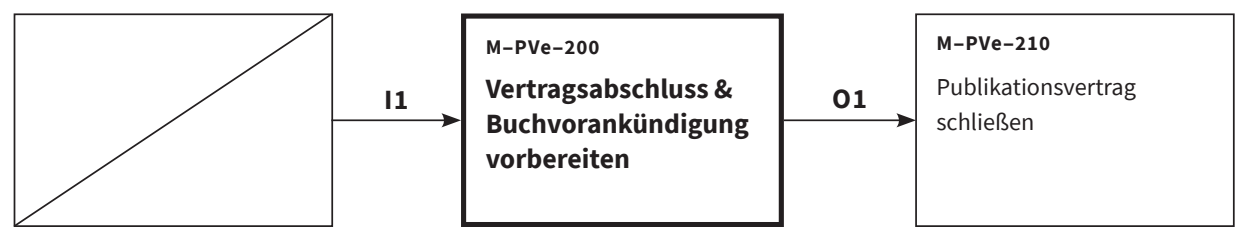

\section{Input}

- I1: Informationen zum Vertragsabschluss \& Buchvorankündigung benötigt

\section{Output}

- O1: vollständige Informationen zum Vertragsabschluss \& die Buchvorankündigung

\section{Qualitätsrichtlinie}

— Richtlinie für den Vertragsabschluss \& die Buchvorankündigung

\section{Beteiligte Rollen}

- Content-Abnehmer

- Content-Urheber

\section{Ressourcen}

- Zeit: T-[M-PVe-200] 


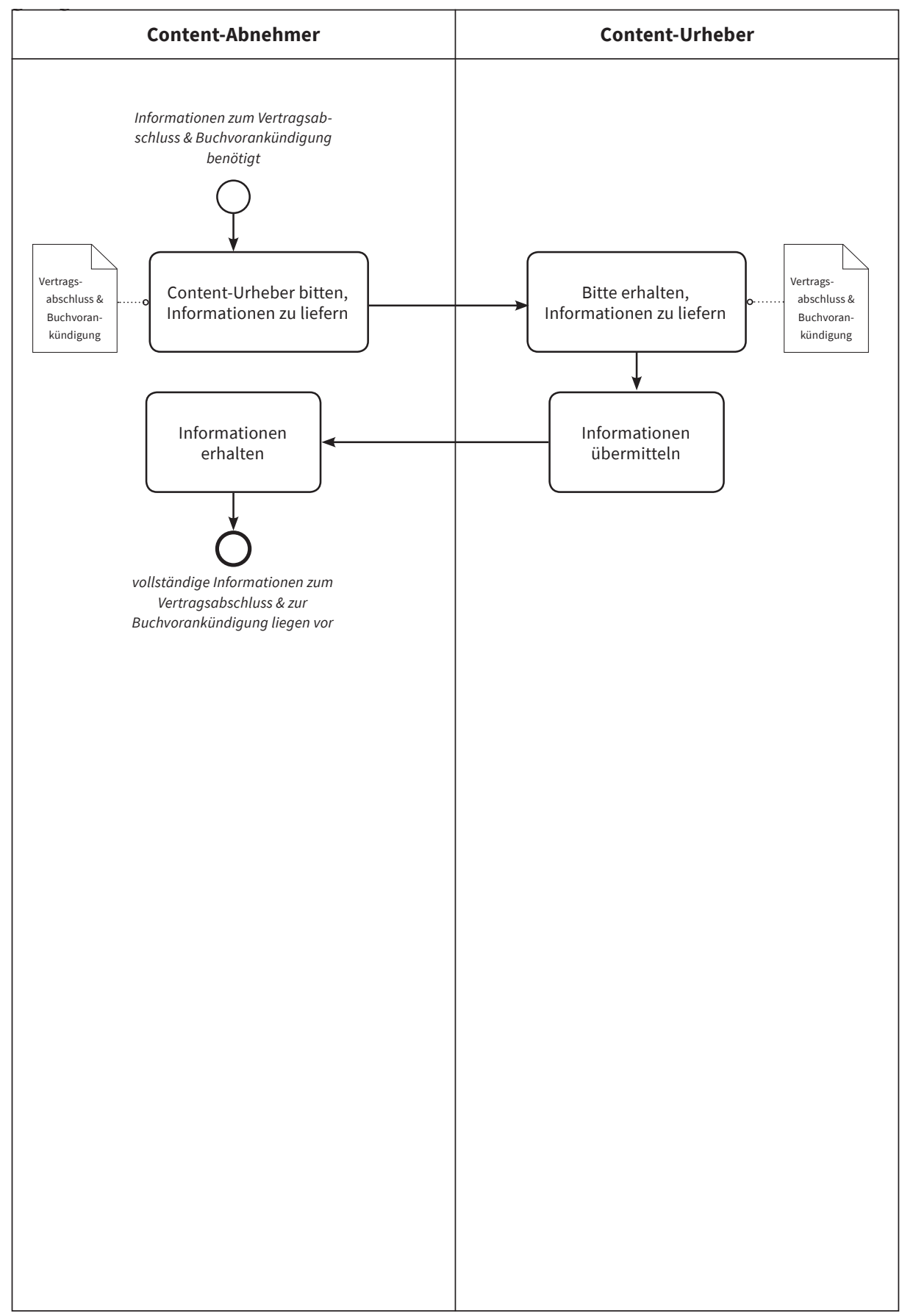




\section{Publikationsvertrag schließen [M-PVe-210]}

\section{Prozessbeschreibung}

Nachdem alle gewünschten Informationen zum Vertragsabschluss vorliegen, erstellt der Content-Abnehmer den Publikationsvertrag entsprechend der Richtlinie für die Vertragsunterzeichnung und übermittelt diesen an den Content-Urheber. Sofern beide Parteien mit dem Vertrag einverstanden sind, unterzeichnen sie diesen. Ggf. muss auch eine Einverständniserklärung für die Veröffentlichung auf einem Repositorium unterzeichnet werden.

Vor- und nachgelagerte Prozesse

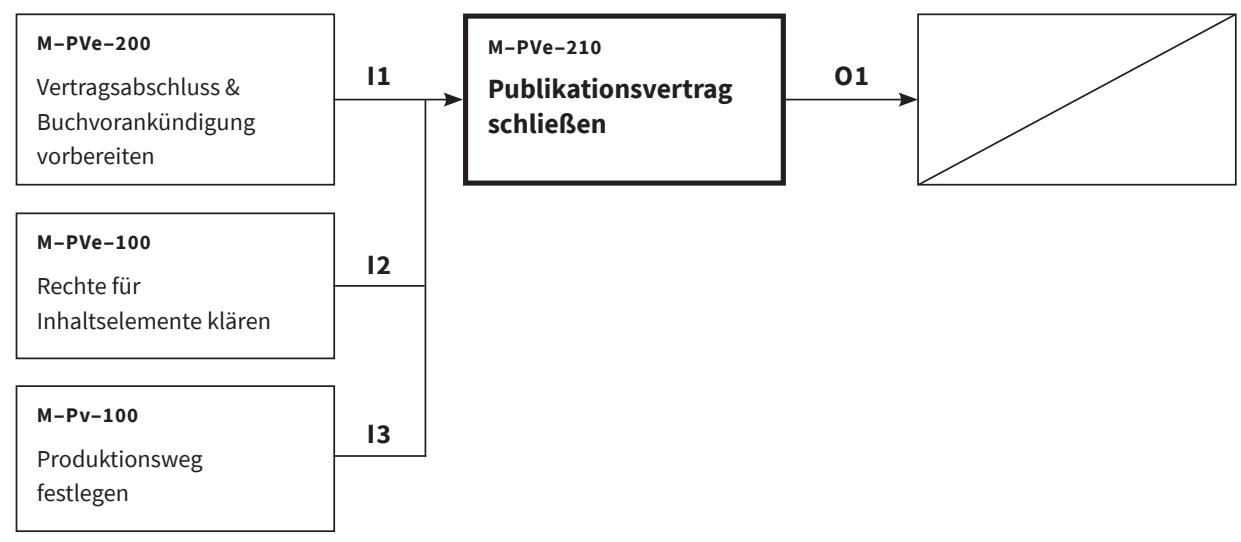

\section{Input}

- I1: vollständ. Informationen zum Vertragsabschluss \& Buchvorankündigung

- I2: Rechte für alle relevanten Inhaltselemente geklärt

- I3: Produktionsweg festgelegt

\section{Output}

- O1: geschlossener Publikationsvertrag

Qualitätsrichtlinie

- Richtlinie für die Vertragsunterzeichnung

\section{Beteiligte Rollen}

- Content-Abnehmer

- Content-Urheber 


\section{Ressourcen}

- Zeit: T-[M-PVe-210]

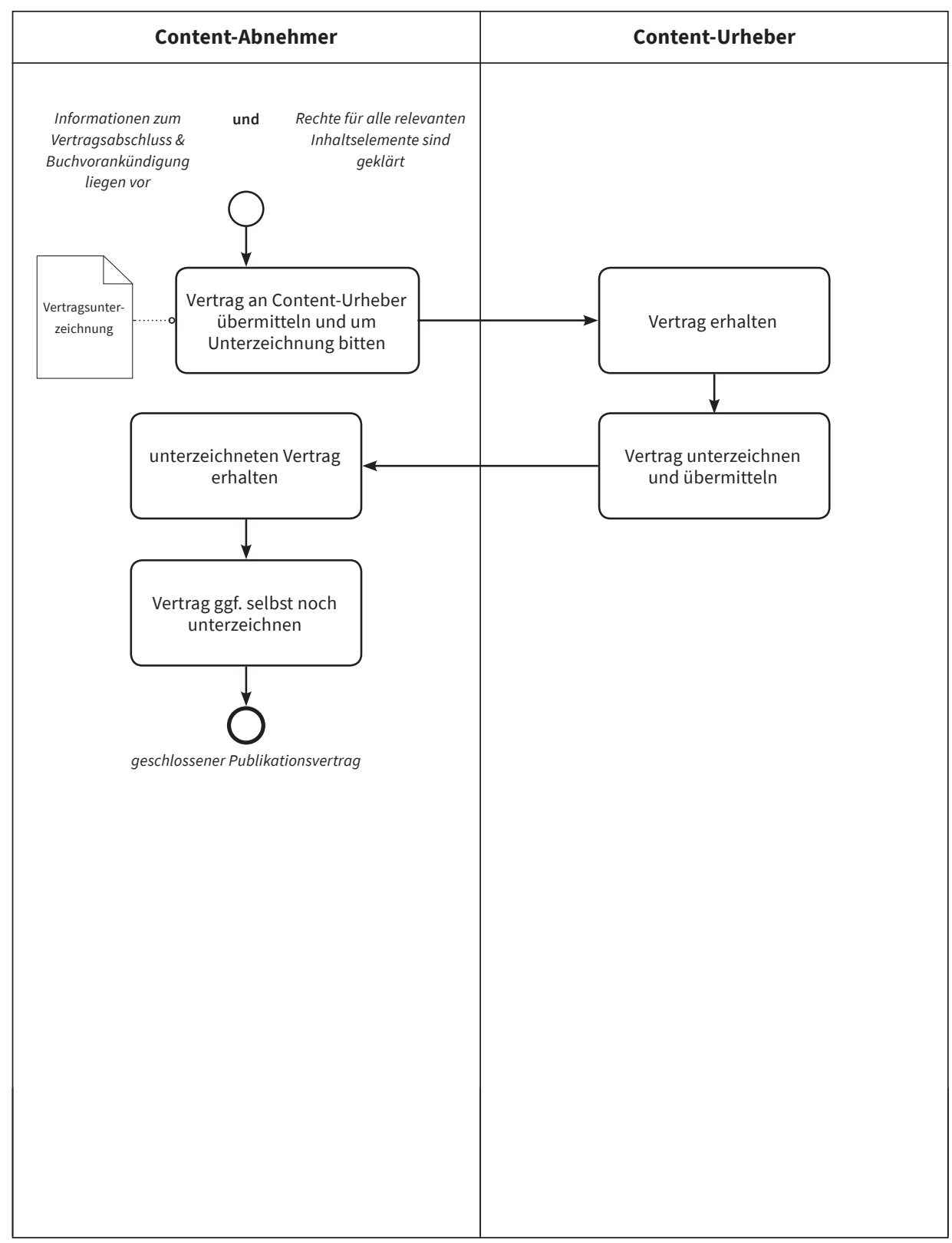




\section{Produktionsweg festlegen [M-Pv-100]}

\section{Prozessbeschreibung}

Der Content-Abnehmer entscheidet entsprechend der Richtlinie für die Wahl des Produktionswegs, in welchen Ausgabeformaten die Publikation erscheinen soll, sowie ob und wenn ja, welche Dienstleister mit der Durchführung, welcher Prozesse beauftragt werden.

Vor- und nachgelagerte Prozesse

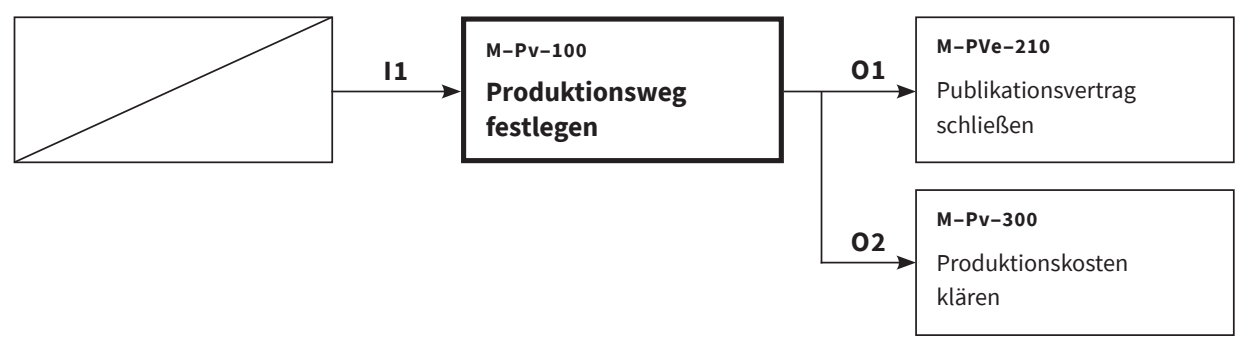

\section{Input}

- I1: relevante Informationen

\section{Output}

- O1: Produktionsweg festgelegt

- O2: Produktionsweg festgelegt

\section{Qualitätsrichtlinie}

- Richtlinie für die Wahl des Produktionswegs

\section{Beteiligte Rollen}

- Content-Abnehmer

\section{Ressourcen}

- Zeit: T-[M-Pv-100] 


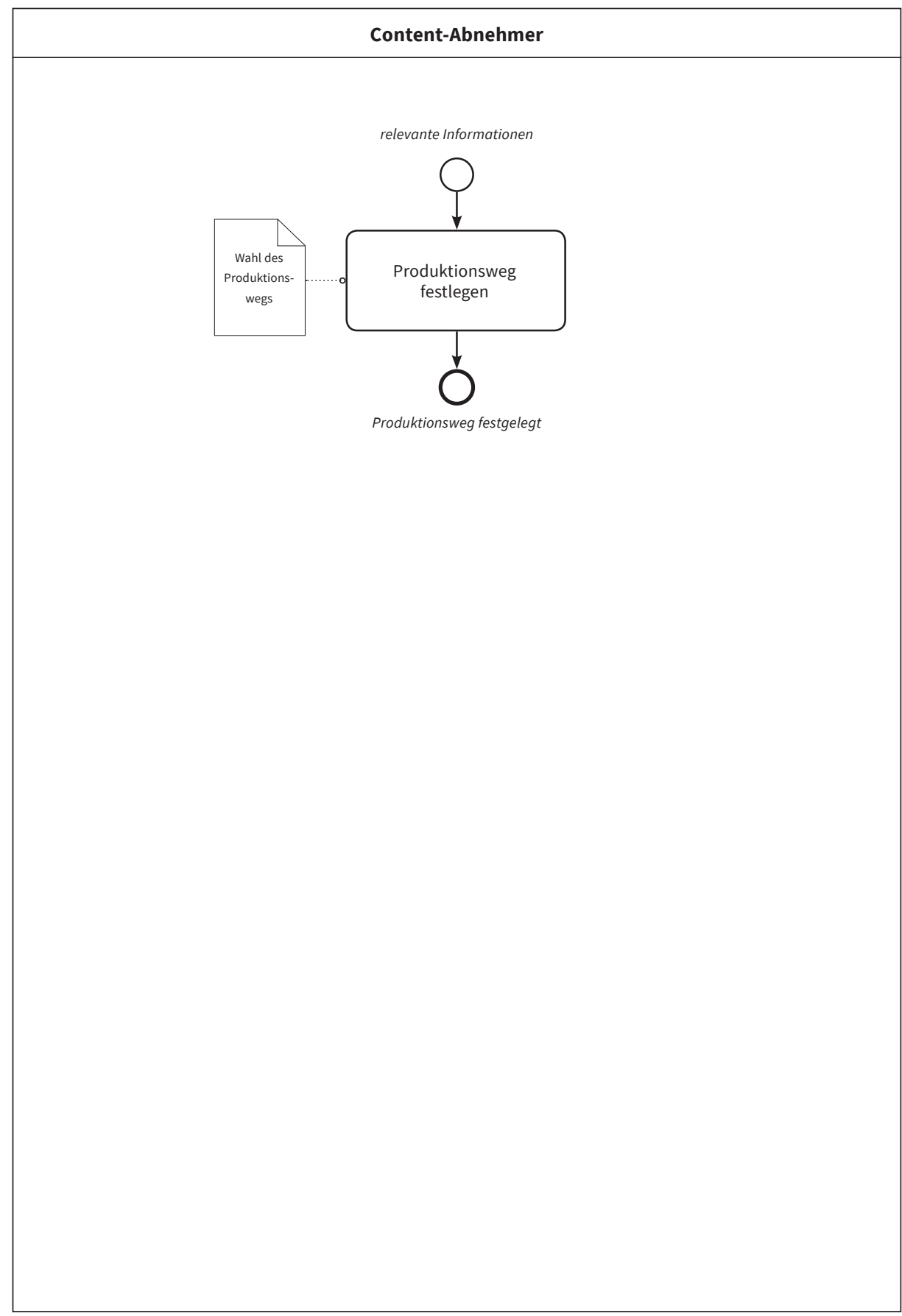




\section{Stammdaten pflegen [M-Pv-200]}

\section{Prozessbeschreibung}

Der Projektmanager erfasst alle relevanten Stammdaten, also Grundinformationen über die Publikation wie ISBN und DOI, Informationen zum Content-Urheber u. v. m., die für den weiteren Projektverlauf erforderlich sind.

Vor- und nachgelagerte Prozesse

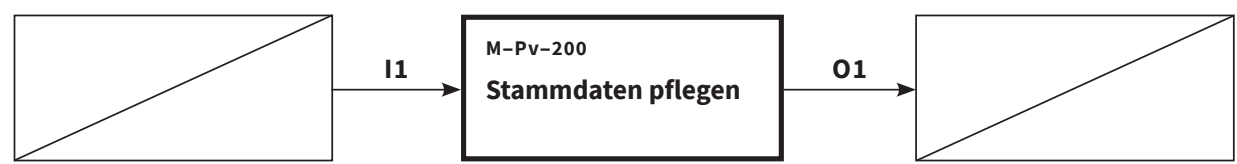

Input

- I1: Informationen, die bereits vorliegen

\section{Output}

- O1: gepflegte Stammdaten

\section{Qualitätsrichtlinie}

— Richtlinie für die Bildung von DOIs

Beteiligte Rollen

- Projektmanager

\section{Ressourcen}

- Zeit: T-[M-Pv-200] 


\section{Projektmanager}

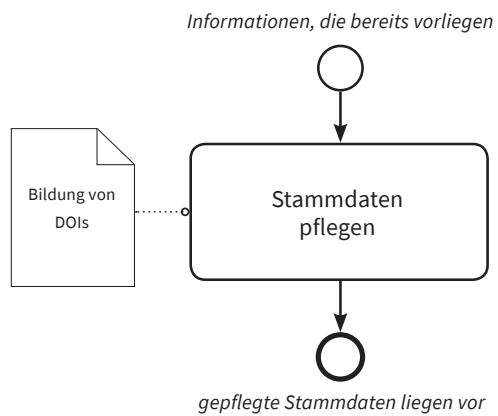




\section{Produktionskosten klären [M-Pv-300]}

\section{Prozessbeschreibung}

Der Betriebswirt informiert sich über die anfallenden Kosten für die Herstellung und Veröffentlichung der gewünschten Ausgabeformate der Publikation. Insbesondere müssen für Prozesse, die von Dienstleistern durchgeführt werden, Kostenvoranschläge für die gewünschten Dienstleistungen eingeholt werden.

Vor- und nachgelagerte Prozesse

\begin{tabular}{|l|l|l|l|l|}
\cline { 2 - 2 } $\begin{array}{l}\text { M-Pv-100 } \\
\text { Produktionsweg } \\
\text { festlegen }\end{array}$ & $\mathbf{I 1}$ & $\begin{array}{l}\text { M-Pv-300 } \\
\text { Produktionskosten } \\
\text { klären }\end{array}$ & $\mathbf{0 1}$ & $\begin{array}{l}\text { M-PV-310 } \\
\text { Soll-Kalkulation } \\
\text { erstellen }\end{array}$ \\
\cline { 2 - 3 } & &
\end{tabular}

\section{Input}

- I1: Produktionsweg festgelegt

\section{Output}

- O1: alle zu erwartende Kosten ermittelt

\section{Beteiligte Rollen}

- Betriebswirt

\section{Ressourcen}

- Zeit: T-[M-Pv-300] 


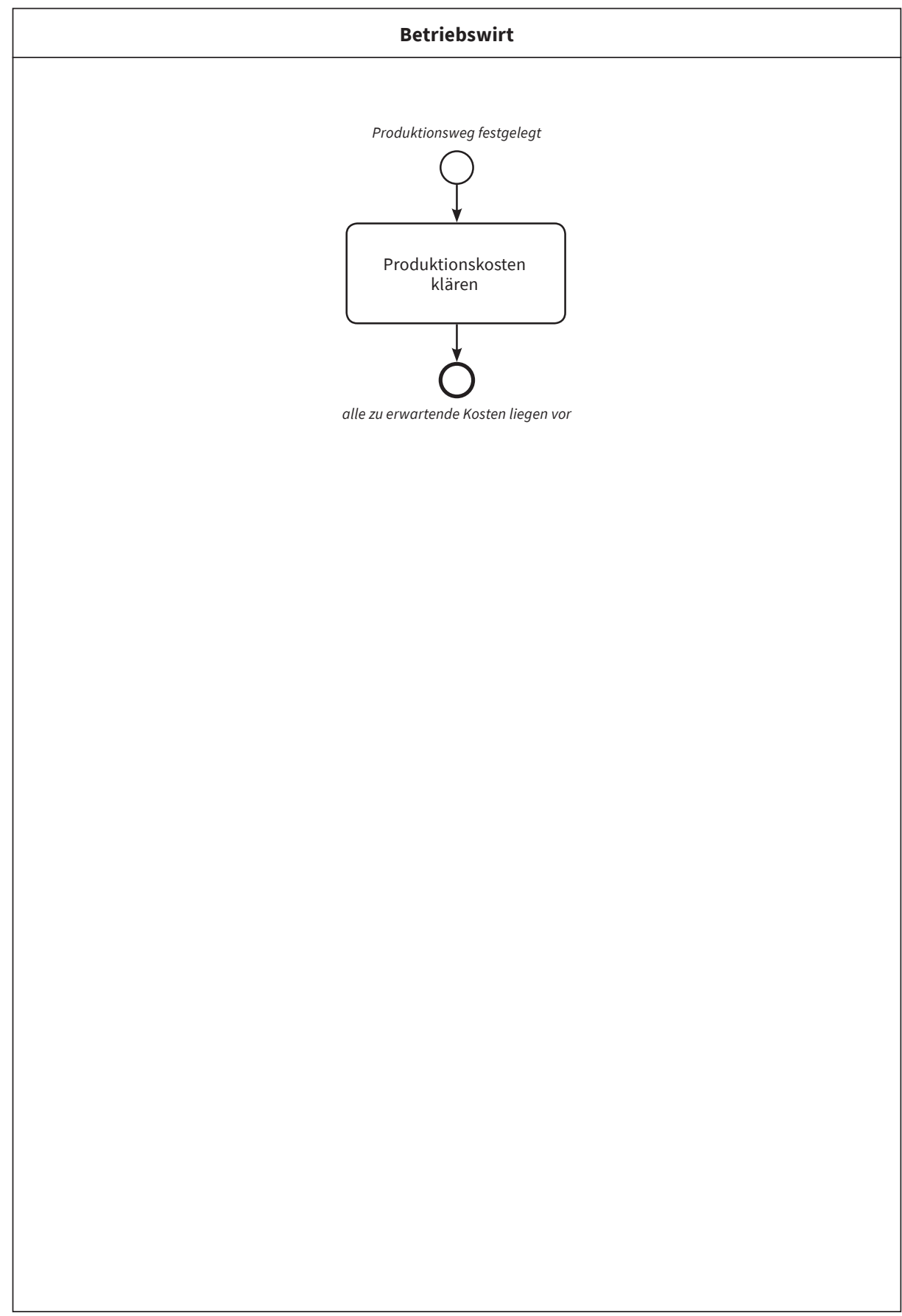




\section{Soll-Kalkulation erstellen [M-Pv-310]}

\section{Prozessbeschreibung}

Der Betriebswirt erstellt auf Basis der recherchierten Kosten eine Soll-Kalkulation für die Produktion und Veröffentlichung der Publikation in den gewünschten Ausgabeformaten.

Vor- und nachgelagerte Prozesse

\begin{tabular}{|c|c|c|c|c|}
\hline \multirow[t]{2}{*}{$\begin{array}{l}\text { M-Pv-300 } \\
\text { Produktionskosten } \\
\text { klären }\end{array}$} & $\stackrel{I 1}{\longrightarrow}$ & $\begin{array}{l}\text { M-Pv-310 } \\
\text { Soll-Kalkulation } \\
\text { erstellen }\end{array}$ & 01 & $\begin{array}{l}\text { M-PVs-160 } \\
\text { Ist-Kalkulation } \\
\text { erstellen }\end{array}$ \\
\hline & & & 02 & $\begin{array}{l}\text { M-Pv-320 } \\
\text { Ladenpreis für gedrucktes } \\
\text { Produkt festlegen }\end{array}$ \\
\hline
\end{tabular}

\section{Input}

- I1: alle zu erwartende Kosten ermittelt

\section{Output}

- O1: Soll-Kalkulation

- O2: Soll-Kalkulation

\section{Beteiligte Rollen}

- Betriebswirt

\section{Ressourcen}

- Zeit: T-[M-Pv-310] 


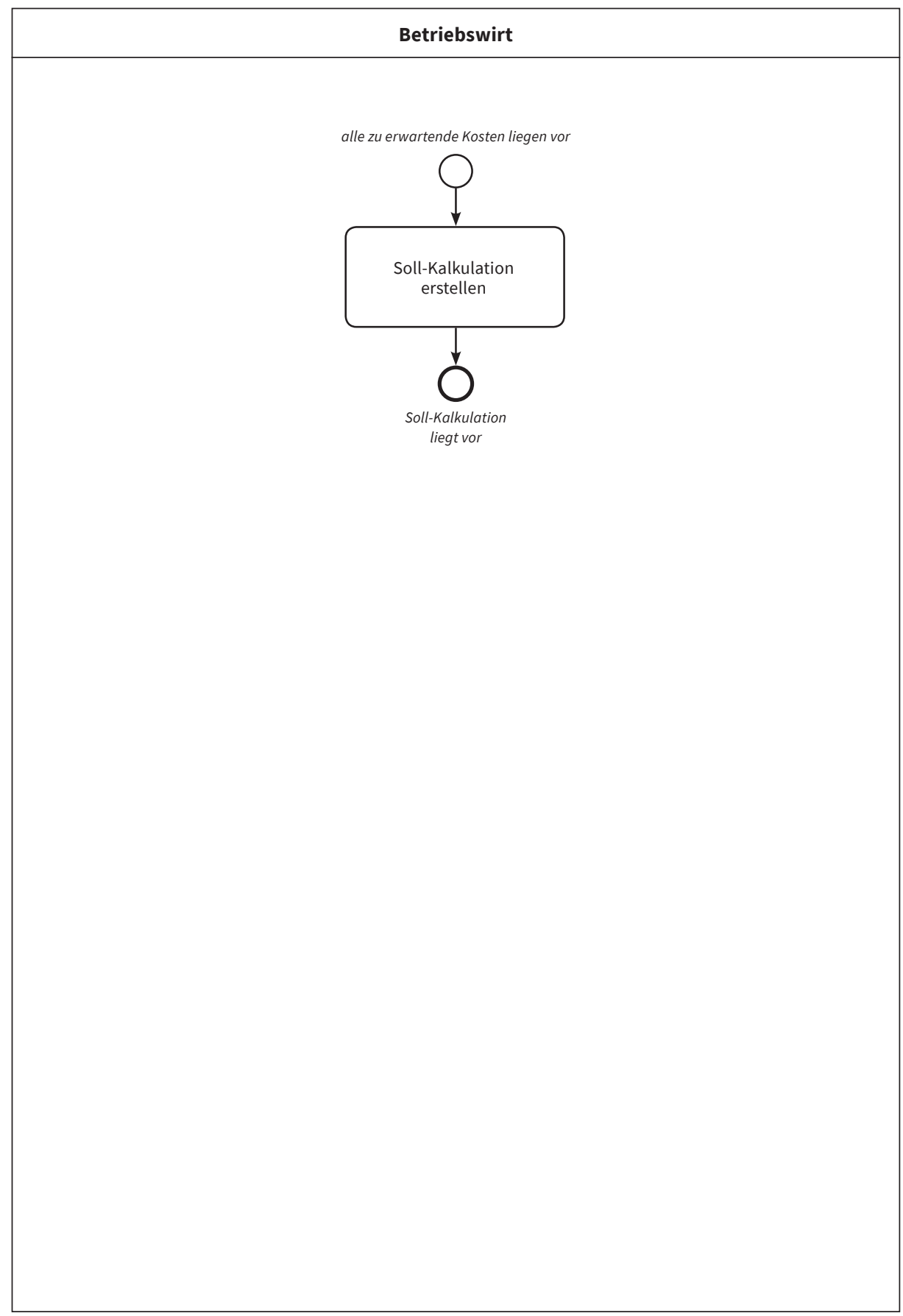




\section{Ladenpreis für gedrucktes Produkt festlegen [M-Pv-320]}

\section{Prozessbeschreibung}

Der Betriebswirt kalkuliert nach der Richtlinie zur Preisstrategie den Ladenpreis für das gedruckte Produkte und übermittelt diese an den Metadatenmanager.

Vor- und nachgelagerte Prozesse

\begin{tabular}{|l|l|l|}
\hline $\begin{array}{l}\text { M-Pv-310 } \\
\text { Soll-Kalkulation } \\
\text { erstellen }\end{array}$ & $\mathbf{I 1}$ \\
\cline { 2 - 3 } & $\begin{array}{l}\text { M-Pv-320 } \\
\text { Ladenpreis für } \\
\text { gedrucktes Produkt } \\
\text { festlegen }\end{array}$ \\
\cline { 2 - 3 }
\end{tabular}

\section{Input}

- I1: Soll-Kalkulation

\section{Output}

- O1: Ladenpreise für gedruckte Produkte

Qualitätsrichtlinie

- Richtlinie zur Preisstrategie

\section{Beteiligte Rollen}

- Betriebswirt

\section{Ressourcen}

- Zeit: T-[M-Pv-320] 


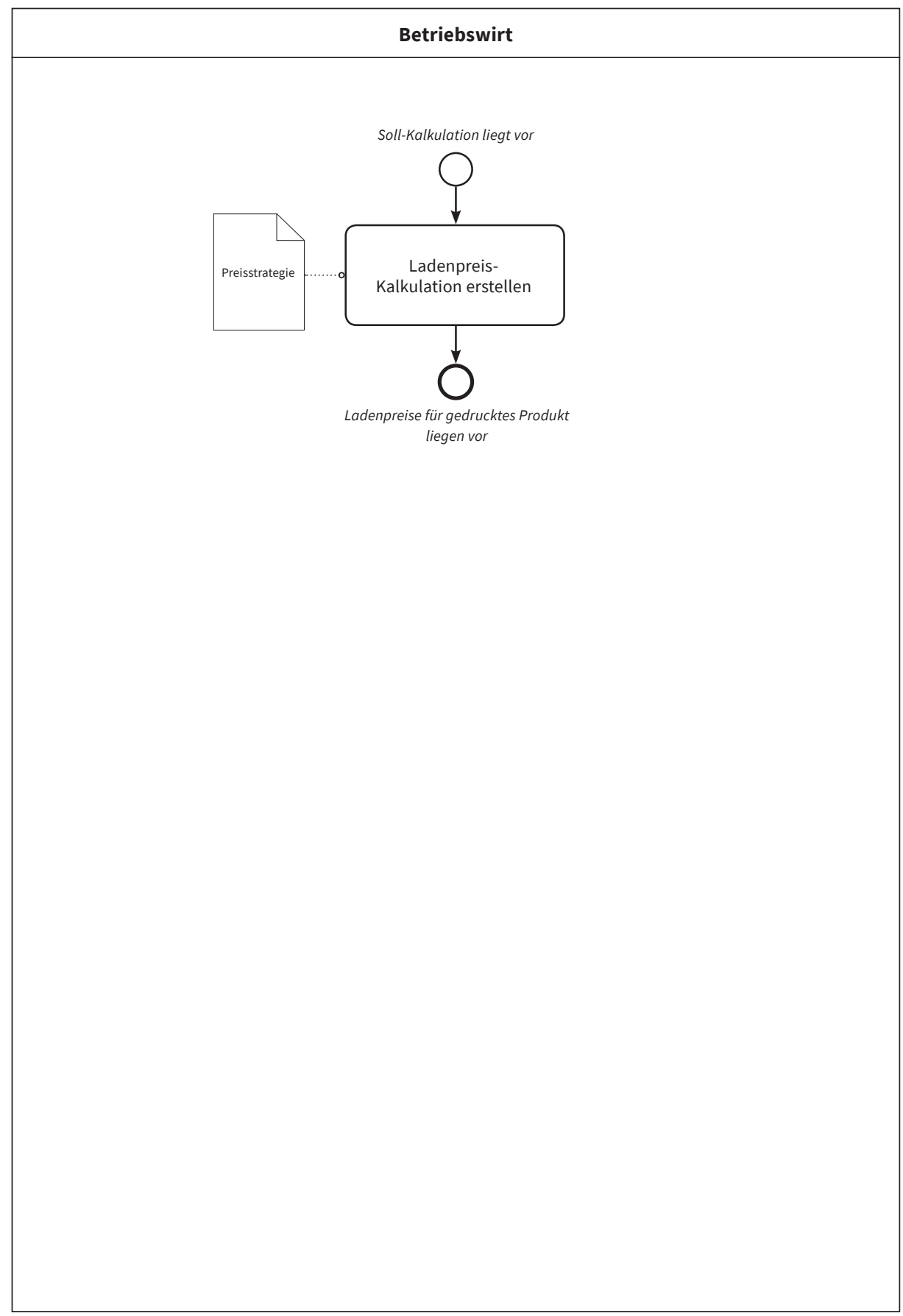




\section{Projektterminplan erstellen [M-Pv-400]}

\section{Prozessbeschreibung}

Der Projektmanager plant rückläufig vom festgelegten Erscheinungstermins der Publikation den Zeitraum zur Herstellung und Veröffentlichung und übermittelt diese an für alle relevanten Rollen.

Vor- und nachgelagerte Prozesse

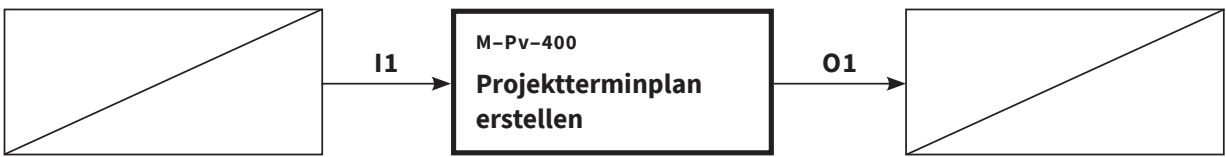

\section{Input}

- I1: Erscheinungstermin

\section{Output}

- O1: Soll-Projektterminplan

\section{Beteiligte Rollen}

- Projektmanager

\section{Ressourcen}

- Zeit: T-[M-Pv-400] 


\section{Projektmanager}

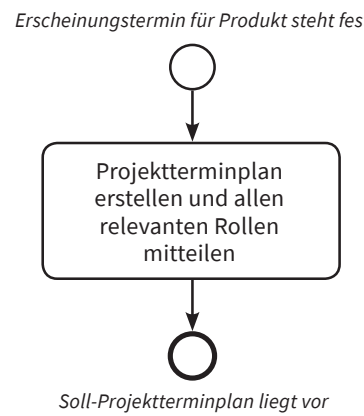




\section{Metadatensatz erzeugen [M-MM-100]}

\section{Prozessbeschreibung}

Der Metadaten-Manager erzeugt selbst oder unter zu Hilfenahme eines Tools, den Metadatensatz zur Publikation anhand der Richtlinie für die Erstellung von Metadaten. Dabei sollten die Anforderungen der Plattformen - wo der Metadatensatz eingepflegt werden soll - in der Richtlinie berücksichtigt werden.

Vor- und nachgelagerte Prozesse

\begin{tabular}{|l|l|l|l|l|l|l|}
\hline $\mathbf{I 1} \longrightarrow$ & $\begin{array}{l}\mathrm{M}-\mathrm{MM}-100 \\
\text { Metadatensatz } \\
\text { erzeugen }\end{array}$
\end{tabular}

\section{Input}

- I1: alle relevanten Informationen

\section{Output}

- O1: Metadatensatz

\section{Qualitätsrichtlinie}

- Richtlinie für die Erstellung von Metadaten

\section{Beteiligte Rollen}

- Metadaten-Manager

\section{Ressourcen}

- Zeit: T-[M-MM-100]

- Kosten: K-[M-MM-100] 


\section{Metadaten-Manager}

alle relevanten Informationen liegen vor

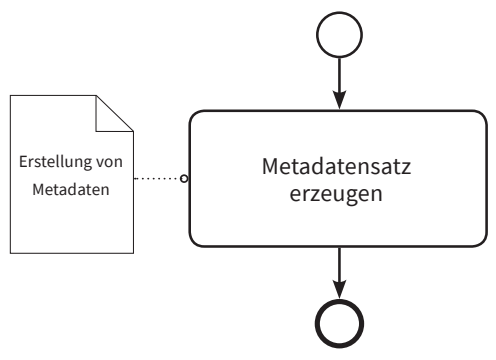

Metadatensatz liegt vor 


\section{Metadaten distribuieren [M-MM-110]}

\section{Prozessbeschreibung}

Der Metadaten-Manager selbst oder unter zu Hilfenahme eines Tools übermittelt den Metadatensatz zur Publikation an die Plattformen, Kataloge oder Verzeichnisse, die primär die Metadaten enthalten sollen.

Vor- und nachgelagerte Prozesse

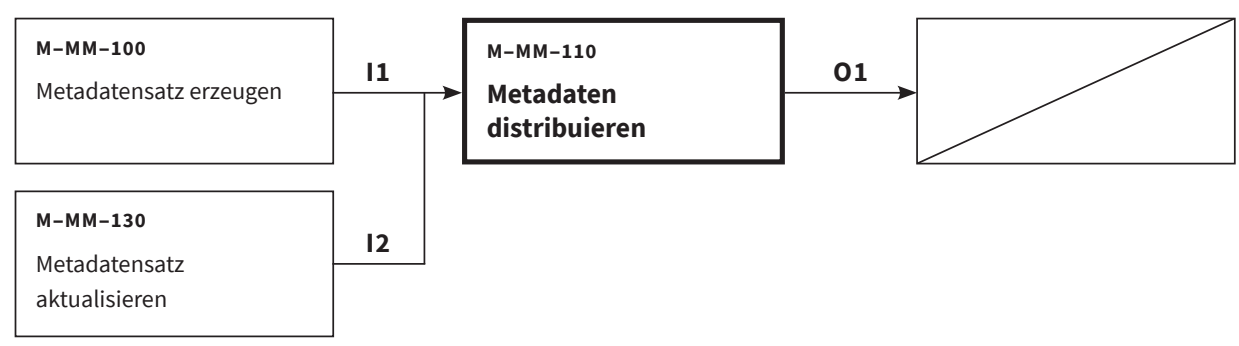

\section{Input}

- I1: Metadatensatz

- I2: aktualisierter Metadatensatz

\section{Output}

- O1: Auffindbarkeit und Sichtbarkeit der Publikation ist sichergestellt

\section{Qualitätsrichtlinie}

- Richtlinie für die Verbreitung von Metadaten

\section{Beteiligte Rollen}

- Metadaten-Manager

\section{Ressourcen}

- Zeit: T-[M-MM-110]

- Kosten: K-[M-MM-110] 


\section{Metadaten-Manager}

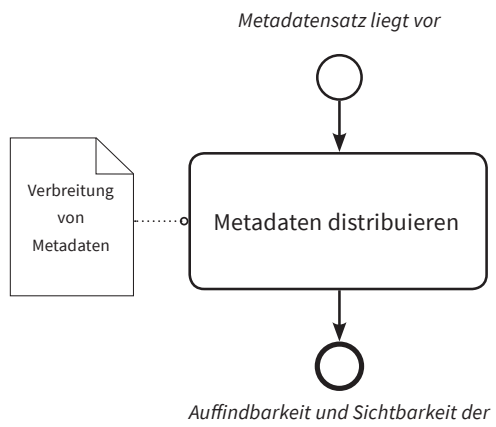

Publikation ist sichergestellt 


\section{Weitere Aggregatoren beauftragen Metadaten zu distribuieren [M-MM-120]}

\section{Prozessbeschreibung}

Der Metadaten-Manager beauftragt weitere Aggregatoren die Metadaten zur Publikation zu distribuieren. Die Aggregatoren distribuieren die Metadaten.

Vor- und nachgelagerte Prozesse

\begin{tabular}{|c|c|c|}
\hline$M-M M-100$ & & $M-M M-120$ \\
\hline Metadatensatz erzeugen & I1 & Weitere Aggregatoren \\
\hline
\end{tabular}

\section{Input}

- I1: Metadatensatz

\section{Output}

- O1: Auffindbarkeit und Sichtbarkeit der Publikation verbessert

\section{Beteiligte Rollen}

- Metadaten-Manager

- Metadaten-Aggregatoren

\section{Ressourcen}

- Zeit: T-[M-MM-120]

- Kosten: K-[M-MM-120] 


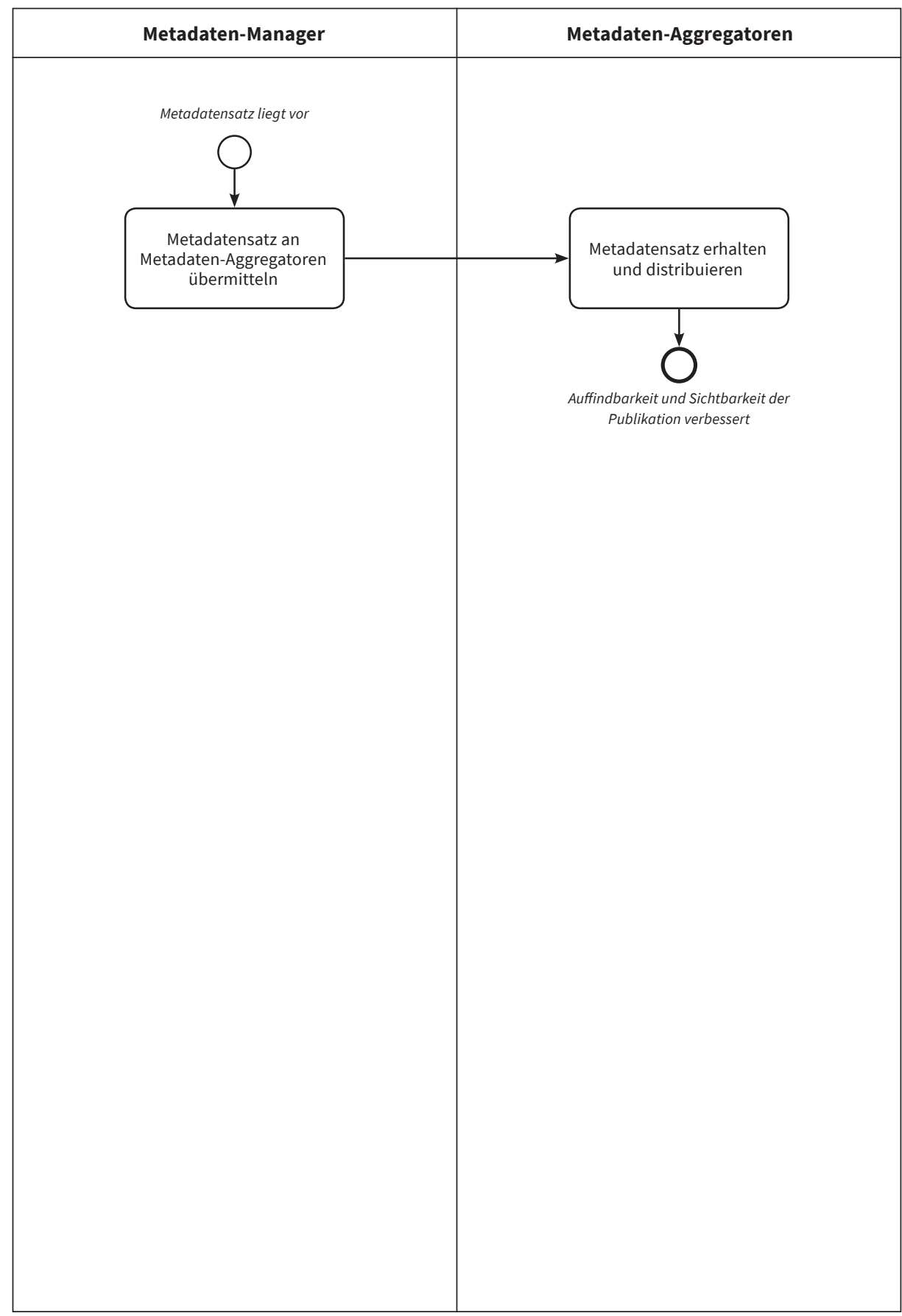




\section{Metadatensatz aktualisieren [M-MM-200]}

\section{Prozessbeschreibung}

Der Metadaten-Manager vervollständigt selbst oder unter zu Hilfenahme eines Tools, den Metadatensatz zum Produkt, sofern neue Informationen vorliegen, wie der finale Ladenpreis oder Seitenumfang, oder sich Änderungen, wie die primäre Veröffentlichungsplattform (URL), ergeben haben.

Vor- und nachgelagerte Prozesse

\begin{tabular}{|l|l|l|l|l|l|l|l|}
\hline $\mathbf{I 1}$ & $\begin{array}{l}\text { M-MM-200 } \\
\text { Metadatensatz } \\
\text { aktualisieren }\end{array}$
\end{tabular}

\section{Input}

- I1: Änderungen am Produkt oder der Veröffentlichungsplattform

\section{Output}

- O1: aktualisierter Metadatensatz

\section{Beteiligte Rollen}

- Metadaten-Manager

\section{Ressourcen}

- Zeit: T-[M-MM-200]

- Kosten: K-[M-MM-200] 


\section{Metadaten-Manager}

Änderungen an der Publikation oder der Veröffentlichungsplattform liegen vor

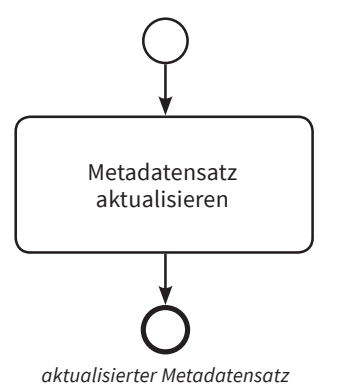




\section{Den aktuellen Projektstatus berichten [M-PVs-100]}

\section{Prozessbeschreibung}

Der Projektmanager, der Copy Editor und ggf. weitere relevante Rollen unterrichten in regelmäßigen Intervallen den Controller über den Projektverlauf hinsichtlich der Termine, Kosten und personellen Ressourcen, sodass der Controller auf Abweichungen im geplanten Projektverlauf reagieren kann.

Vor- und nachgelagerte Prozesse

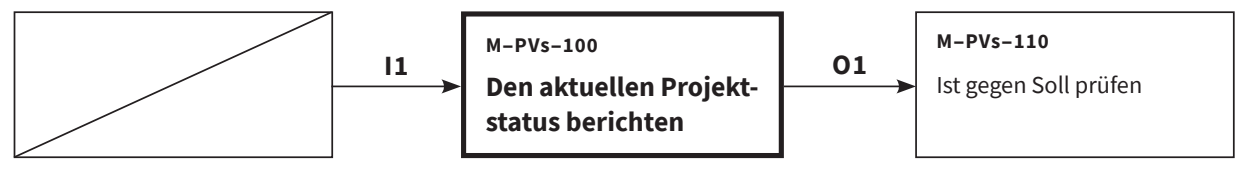

\section{Input}

- I1: Informationen von den relevanten Rollen

\section{Output}

- O1: aktueller Projektstatus

\section{Beteiligte Rollen}

- alle relevanten Rollen

- Controller

\section{Ressourcen}

- Zeit: T-[M-PVs-100] 


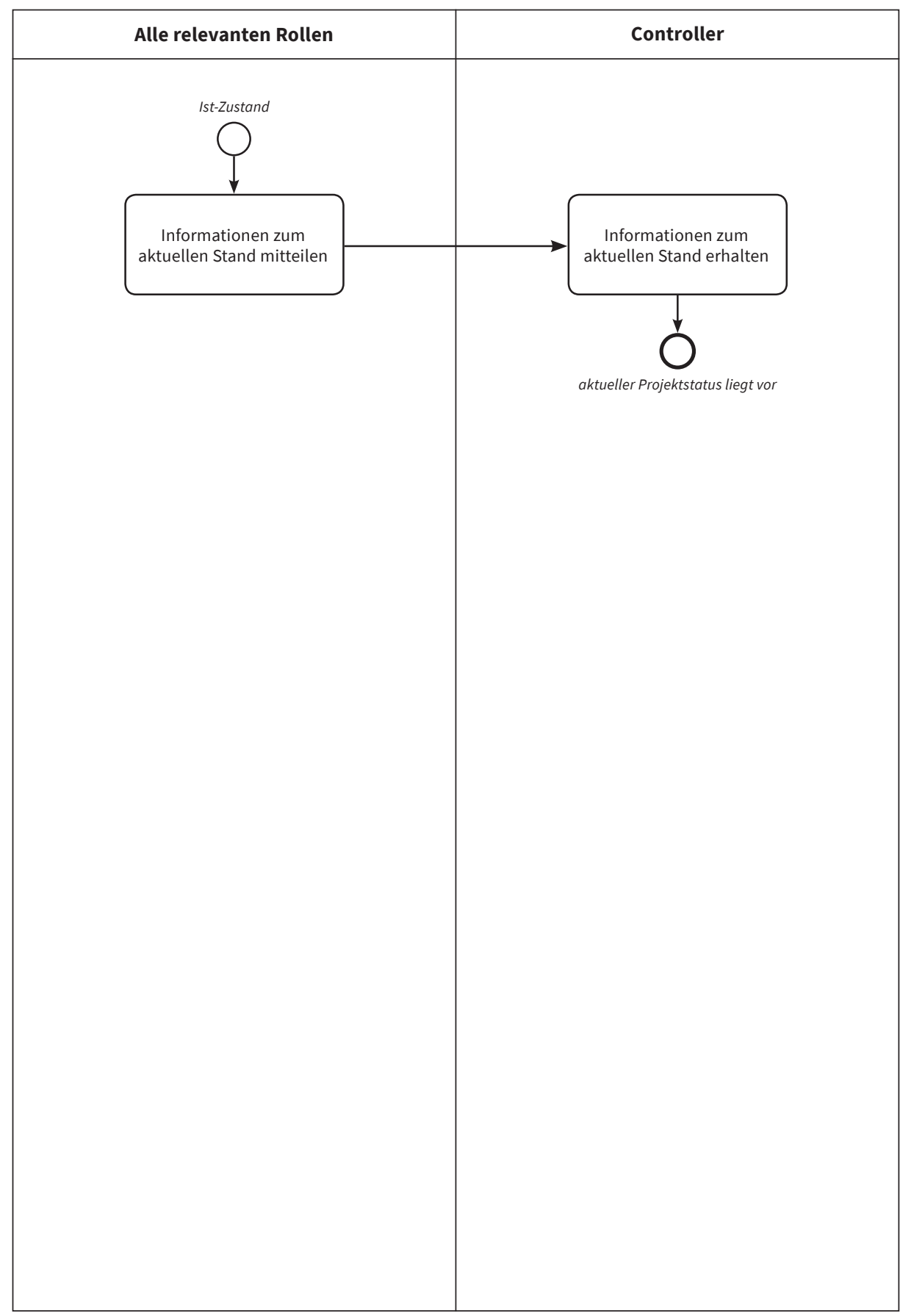




\section{Ist gegen Soll prüfen [M-PVs-110]}

\section{Prozessbeschreibung}

Der Controller erhält die Berichte aller relevanter Rollen zum Status des Projektverlaufs und vergleicht den aktuellen Stand mit dem Soll (zu Beginn des Projektes aufgestellter Terminplan, Soll-Kalkulation mit ggf. bereits vorhandenen Dienstleisterrechnungen, Aufgabenzuteilung auf die personellen Ressourcen (Mitarbeiter)). Sofern das Projekt hinsichtlich Termine, Kosten und personelle Ressourcen planmäßig verläuft, informiert er alle relevanten Rollen. Andernfalls ermittelt er die Ursachen der Abweichungen. Der Marketing-Manager sollte an dieser Stelle ebenfalls überprüfen, ob der Marketing-Plan planmäßig durchgeführt wird.

Vor- und nachgelagerte Prozesse

\begin{tabular}{|c|c|c|c|c|}
\hline $\begin{array}{l}\text { M-PVs-100 } \\
\text { Den aktuellen Projekt- } \\
\text { status berichten }\end{array}$ & I1 & $\begin{array}{l}\text { M-PVs-110 } \\
\text { Ist gegen Soll prüfen }\end{array}$ & 01 & $\begin{array}{l}\text { M-PVs-120 } \\
\text { Alle relevanten Rollen zu } \\
\text { planmäßigem Projekt- } \\
\text { verlauf informieren }\end{array}$ \\
\hline & & & 02 & $\begin{array}{l}\text { M-PVs-130 } \\
\text { Ursachen der } \\
\text { Abweichungen feststellen }\end{array}$ \\
\hline
\end{tabular}

\section{Input}

- I1: aktueller Projektstatus

\section{Output}

- O1: Info: keine Abweichungen festgestellt

- O2: Info: Abweichungen festgestellt

\section{Beteiligte Rollen}

- Controller

- Marketing-Manager

\section{Ressourcen}

- Zeit: T-[M-PVs-110] 


\section{Controller / Marketing-Manager}

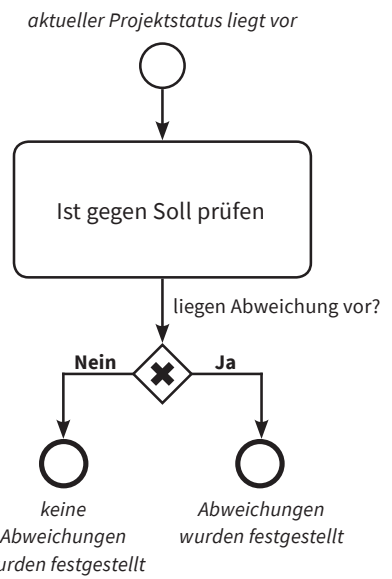




\section{Alle relevanten Rollen zu planmäßigem Projektverlauf informieren [M-PVs-120]}

\section{Prozessbeschreibung}

Nachdem der Controller den aktuellen Stand des Projektes mit dem Soll verglichen und feststellt hat, dass alles planmäßig verläuft, informiert er alle relevanten Rollen darüber.

Vor- und nachgelagerte Prozesse

\begin{tabular}{|l|l|}
\hline Ist gegen Soll prüfen & $\mathbf{I 1} \longrightarrow \begin{array}{l}\text { M-PVs-120 } \\
\text { Alle relevanten Rollen } \\
\text { zu planmäßigem } \\
\text { Projektverlauf } \\
\text { informieren }\end{array}$ \\
\cline { 2 - 4 }
\end{tabular}

\section{Input}

- I1: Info: keine Abweichungen festgestellt

\section{Output}

- O1: Info: planmäßiger Projektverlauf

\section{Beteiligte Rollen}

- alle relevanten Rollen

- Controller

\section{Ressourcen}

- Zeit: T-[M-PVs-120] 


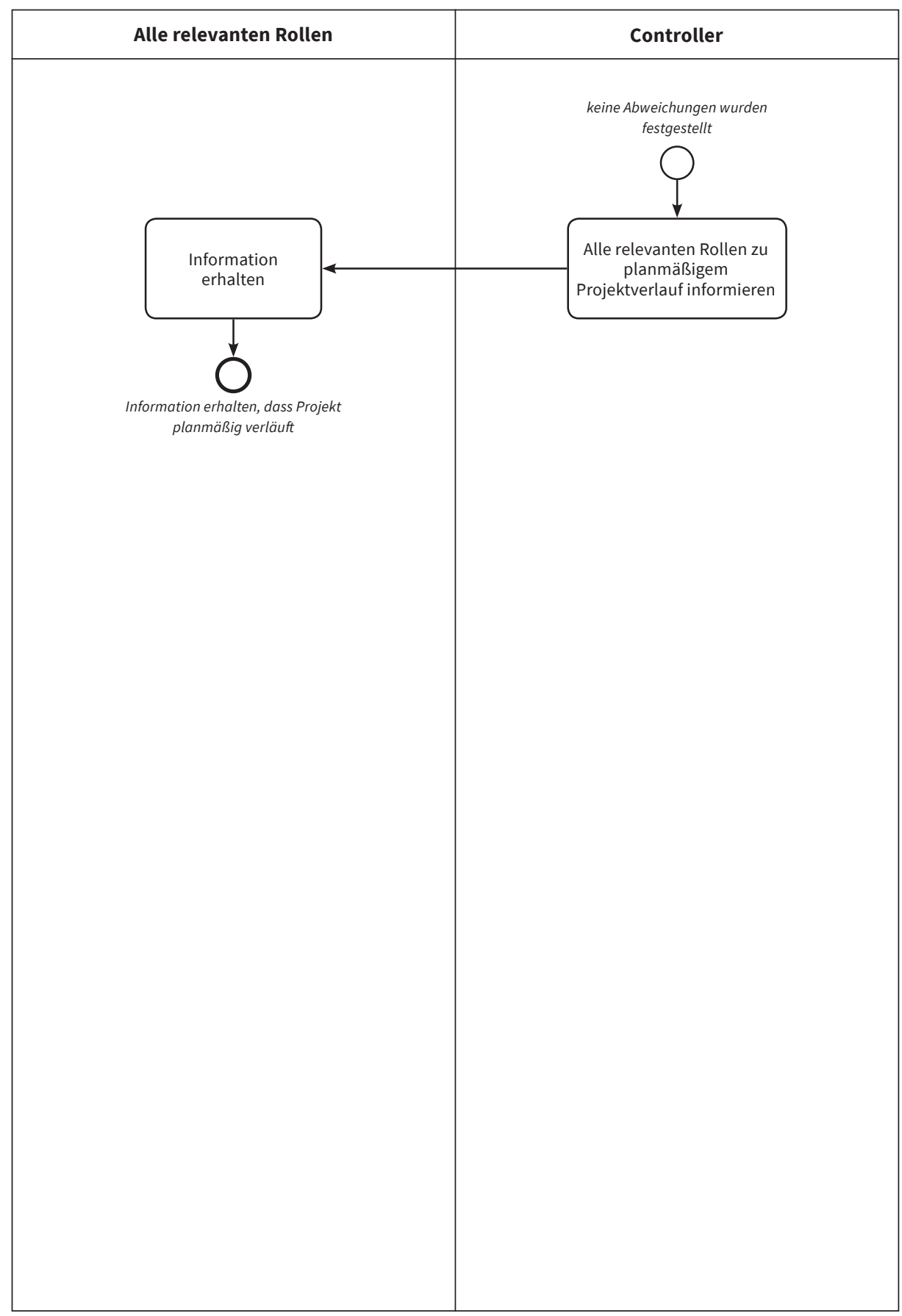




\section{Ursachen der Abweichungen feststellen [M-PVs-130]}

\section{Prozessbeschreibung}

Bei Abweichungen der Ist-Termine, der Ist-Kosten und/oder der Ist-Verfügbarkeit der personellen Ressourcen vom Soll sucht der Controller nach möglichen Ursachen für die Abweichungen. Der Marketing-Manager sollte an dieser Stelle ebenfalls die Ursachen für die Abweichung vom operativen Marketing-Plan feststellen.

Vor- und nachgelagerte Prozesse

\begin{tabular}{|l|l|l|l|l|}
\hline $\begin{array}{l}\text { M-PVs-110 } \\
\text { Ist gegen Soll prüfen }\end{array}$ & $\begin{array}{l}\text { M-PVs-130 } \\
\text { Ursachen der Abweich- } \\
\text { ungen feststellen }\end{array}$ & $\begin{array}{l}\text { M-PVs-140 } \\
\text { Handlungsmöglichkeiten } \\
\text { prüfen \& priorisieren }\end{array}$ \\
\cline { 2 - 3 }
\end{tabular}

\section{Input}

- I1: Abweichungen

\section{Output}

- O1: Ursachen für Abweichungen

\section{Beteiligte Rollen}

- Controller

- Marketing-Manager

\section{Ressourcen}

- Zeit: T-[M-PVs-130] 


\section{Controller / Marketing-Manager}

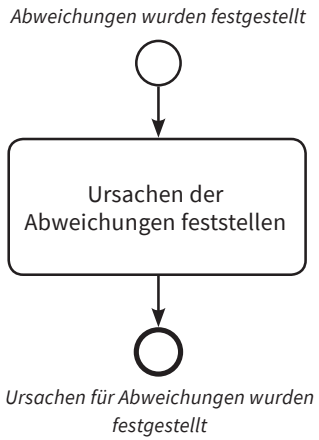




\section{Handlungsmöglichkeiten prüfen \& priorisieren [M-PVs-140]}

\section{Prozessbeschreibung}

Der Controller überprüft alle Handlungsmöglichkeiten, um den Abweichungen vom Projektterminplan, von der Soll-Kalkulation und von den geplanten personellen Ressourcen entgegenzuwirken und priorisiert diese. Der Marketing-Manager wird ebenfalls aktiv, um den Abweichungen vom Marketing-Plan entgegenzuwirken.

Vor- und nachgelagerte Prozesse

\begin{tabular}{|c|c|c|c|c|}
\hline \multirow{4}{*}{$\begin{array}{l}\text { M-PVs-130 } \\
\text { Ursachen der } \\
\text { Abweichungen feststellen }\end{array}$} & \multirow[t]{4}{*}{ I1 } & \multirow{4}{*}{$\begin{array}{l}\text { M-PVs-140 } \\
\text { Handlungsmöglich- } \\
\text { keiten prüfen \& } \\
\text { priorisieren }\end{array}$} & 01 & \\
\hline & & & & neu abstimmen \\
\hline & & & 02 & $\begin{array}{l}\text { M-PVs-160 } \\
\text { Ist-Kalkulation } \\
\text { erstellen }\end{array}$ \\
\hline & & & 03 & $\begin{array}{l}\text { M-PVs-170 } \\
\text { Personelle Ressourcen } \\
\text { neu zuteilen }\end{array}$ \\
\hline
\end{tabular}

\section{Input}

- I1: Ursachen für Abweichungen

\section{Output}

- O1: priorisierte Handlungsoptionen für Termine

- O2: priorisierte Handlungsoptionen für Kosten

- O3: priorisierte Handlungsoptionen für personelle Ressourcen

\section{Beteiligte Rollen}

- Controller

- Marketing-Manager

\section{Ressourcen}

- Zeit: T-[M-PVs-140] 


\section{Controller / Marketing-Manager}

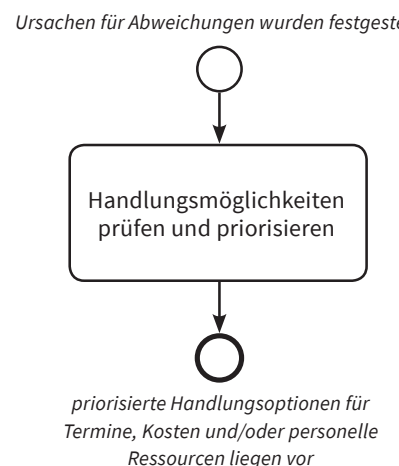




\section{Projekttermine neu abstimmen [M-PVs-150]}

\section{Prozessbeschreibung}

Der Controller informiert den Projektmanager, wie der Projektverlauf hinsichtlich der Termine, weiter verlaufen sollte. Der Projektmanager ändert darauf hin ggf. den Projektterminplan und informiert alle relevanten Rollen über die veränderten Termine.

Vor- und nachgelagerte Prozesse

\begin{tabular}{|l|l|l|l|}
\hline $\begin{array}{l}\text { M-PVs-140 } \\
\text { prüfen \& priorisieren }\end{array}$ & $\mathbf{I 1}$ & $\begin{array}{l}\text { M-PVs-150 } \\
\text { Projekttermine } \\
\text { neu abstimmen }\end{array}$ \\
\cline { 2 - 4 }
\end{tabular}

\section{Input}

- I1: priorisierte Handlungsoptionen für Termine

\section{Output}

- O1: Information zu neuen Terminen

\section{Beteiligte Rollen}

- Controller

- Projektmanager

\section{Ressourcen}

- Zeit: T-[M-PVs-150] 


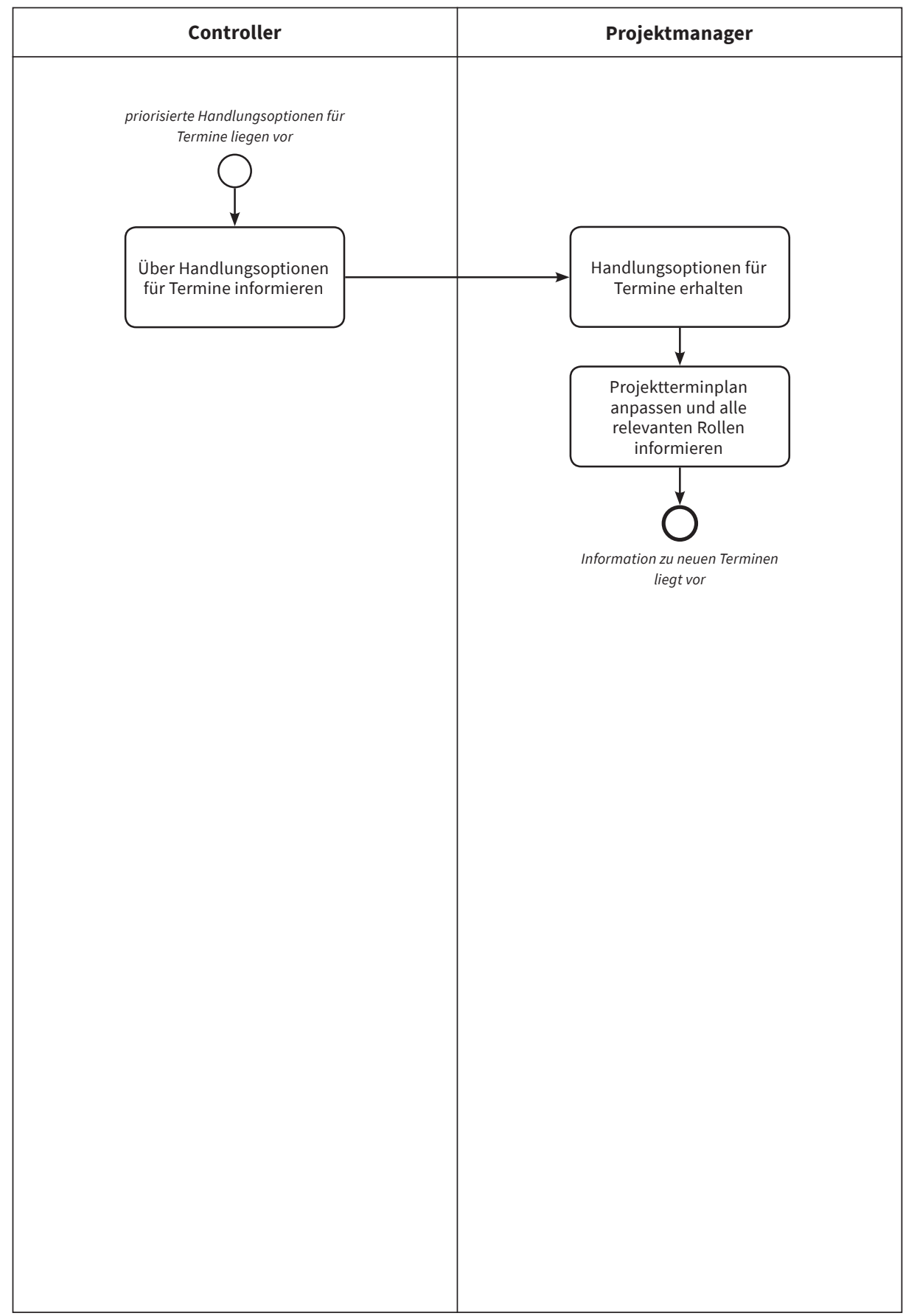




\section{Ist-Kalkulation erstellen [M-PVs-160]}

\section{Prozessbeschreibung}

Der Controller informiert den Betriebswirt, wie der Projektverlauf hinsichtlich der Kosten weiter verlaufen sollte, bzw. ob die Dienstleisterrechnungen, wie erwartet gestellt wurden. Der Betriebswirt erstellt dementsprechend eine Ist-Kalkulation.

Vor- und nachgelagerte Prozesse

\begin{tabular}{|c|c|c|c|c|}
\hline $\begin{array}{l}\text { M-PVs-140 } \\
\text { Handlungsmöglichkeiten } \\
\text { prüfen \& priorisieren }\end{array}$ & I1 & $\begin{array}{l}\text { M-PVs-160 } \\
\text { Ist-Kalkulation } \\
\text { erstellen }\end{array}$ & 01 & $\begin{array}{l}\text { M-Pn-200 } \\
\text { Nach-Kalkulation } \\
\text { erstellen }\end{array}$ \\
\hline
\end{tabular}

\section{Input}

- I1: priorisierte Handlungsoptionen für Kosten

\section{Output}

- O1: Ist-Kalkulation

\section{Beteiligte Rollen}

- Controller

- Betriebswirt

\section{Ressourcen}

- Zeit: T-[M-PVs-160] 


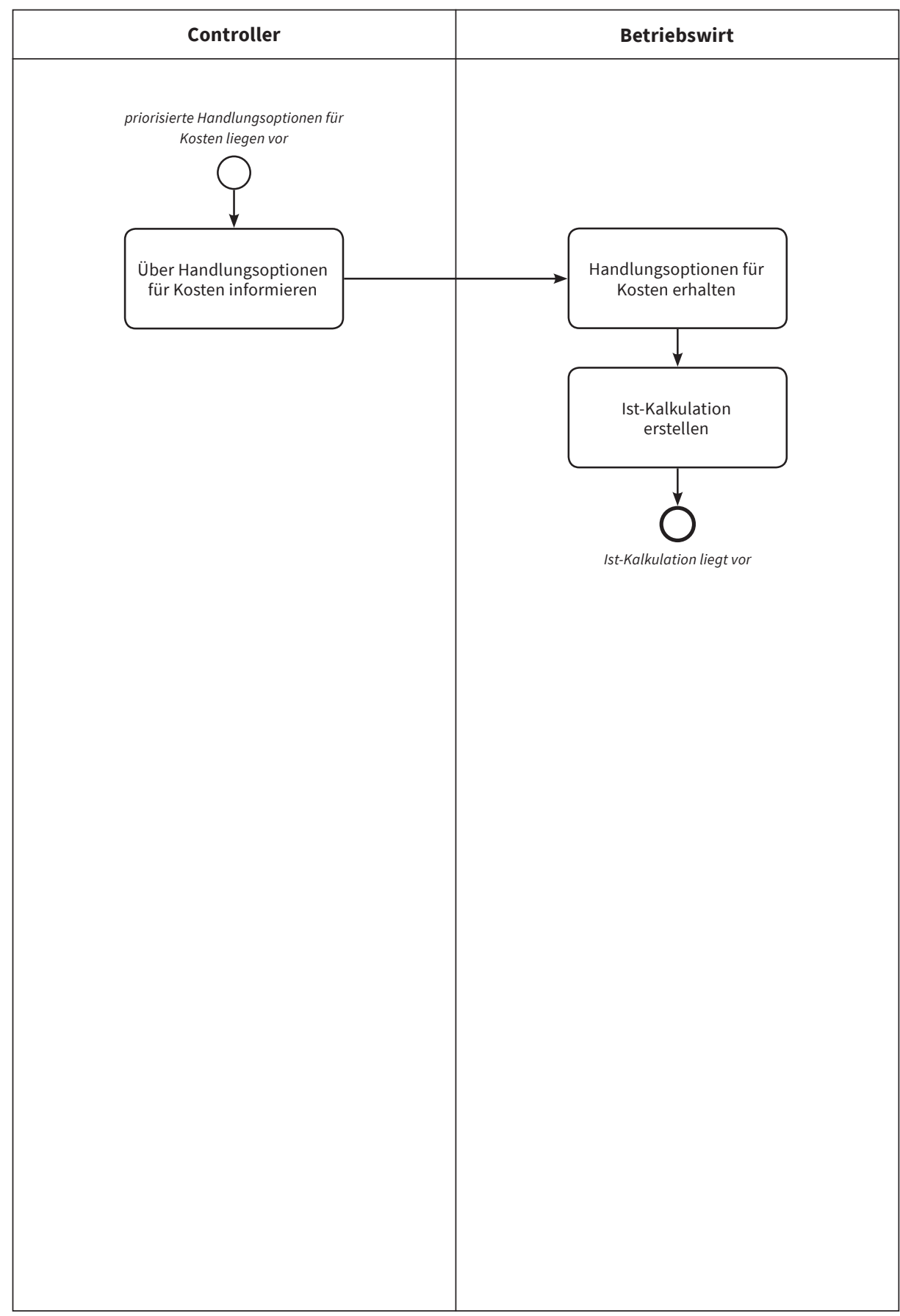




\section{Personelle Ressourcen neu zuteilen [M-PVs-170]}

Prozessbeschreibung

Der Controller informiert den Projektmanager, wie der Projektverlauf hinsichtlich der personellen Ressourcen weiter verlaufen sollte. Der Projektmanager ändert darauf hin ggf. die Verantwortlichkeiten von Mitarbeitern.

Vor- und nachgelagerte Prozesse

\begin{tabular}{|l|l|l|l|}
\hline $\begin{array}{l}\text { M-PVs-140 } \\
\text { Handlungsmöglichkeiten } \\
\text { prüfen \& priorisieren }\end{array}$ & $\mathbf{I 1} \longrightarrow \begin{array}{l}\text { M-PVs-170 } \\
\text { Personelle Ressourcen } \\
\text { neu zuteilen }\end{array}$ \\
\cline { 2 - 4 }
\end{tabular}

Input

- I1: priorisierte Handlungsoptionen für personelle Ressourcen

\section{Output}

- O1: Information zu neuen Verantwortlichkeiten

Beteiligte Rollen

- Controller

- Projektmanager

\section{Ressourcen}

- Zeit: T-[M-PVs-170] 


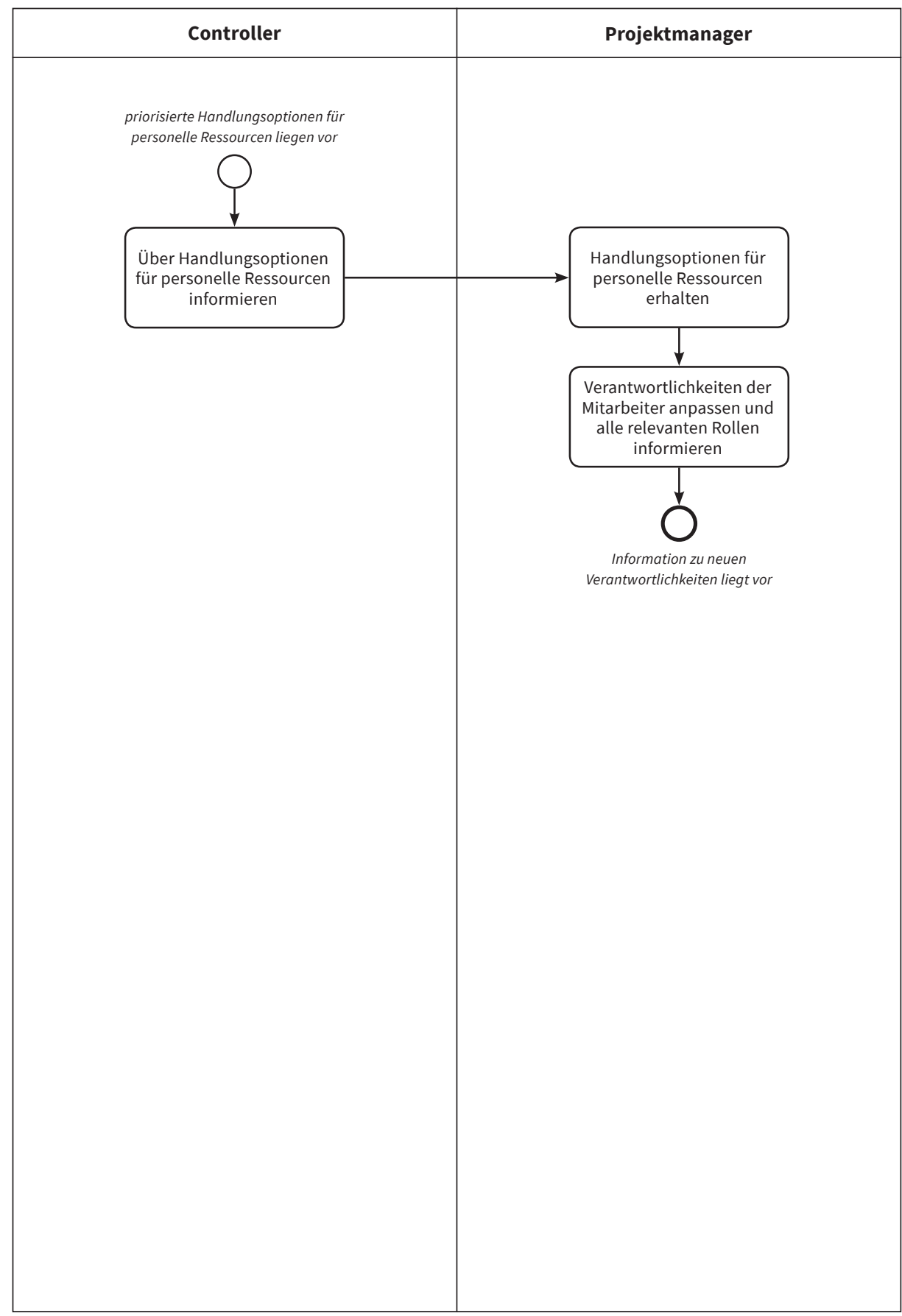




\section{Verwaltungsdaten sichern [M-Pn-100]}

\section{Prozessbeschreibung}

Der Content-Archivar erstellt aus den im Publikationsworkflow entstandenen Verwaltungsdaten einen Archivdatensatz. Den Datensatz legt der Content-Archivar systematisiert entsprechend der Richtlinie zur internen Archivierung ab.

Vor- und nachgelagerte Prozesse

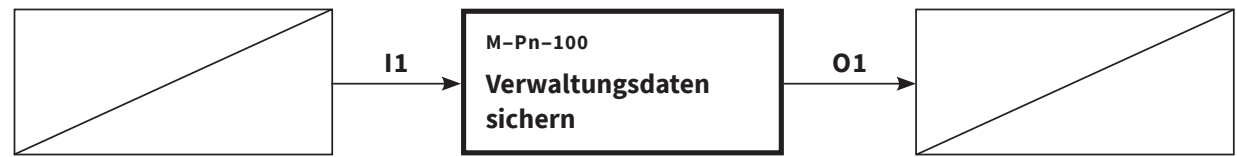

Input

- I1: vorhandene und relevante Verwaltungsdaten

\section{Output}

- O1: gesicherte Verwaltungsdaten in einem Archivdatensatz

\section{Qualitätsrichtlinie}

- Richtlinie für die interne Archivierung

\section{Beteiligte Rollen}

- Content-Archivar

\section{Ressourcen}

- Zeit: T-[M-Pn-100] 


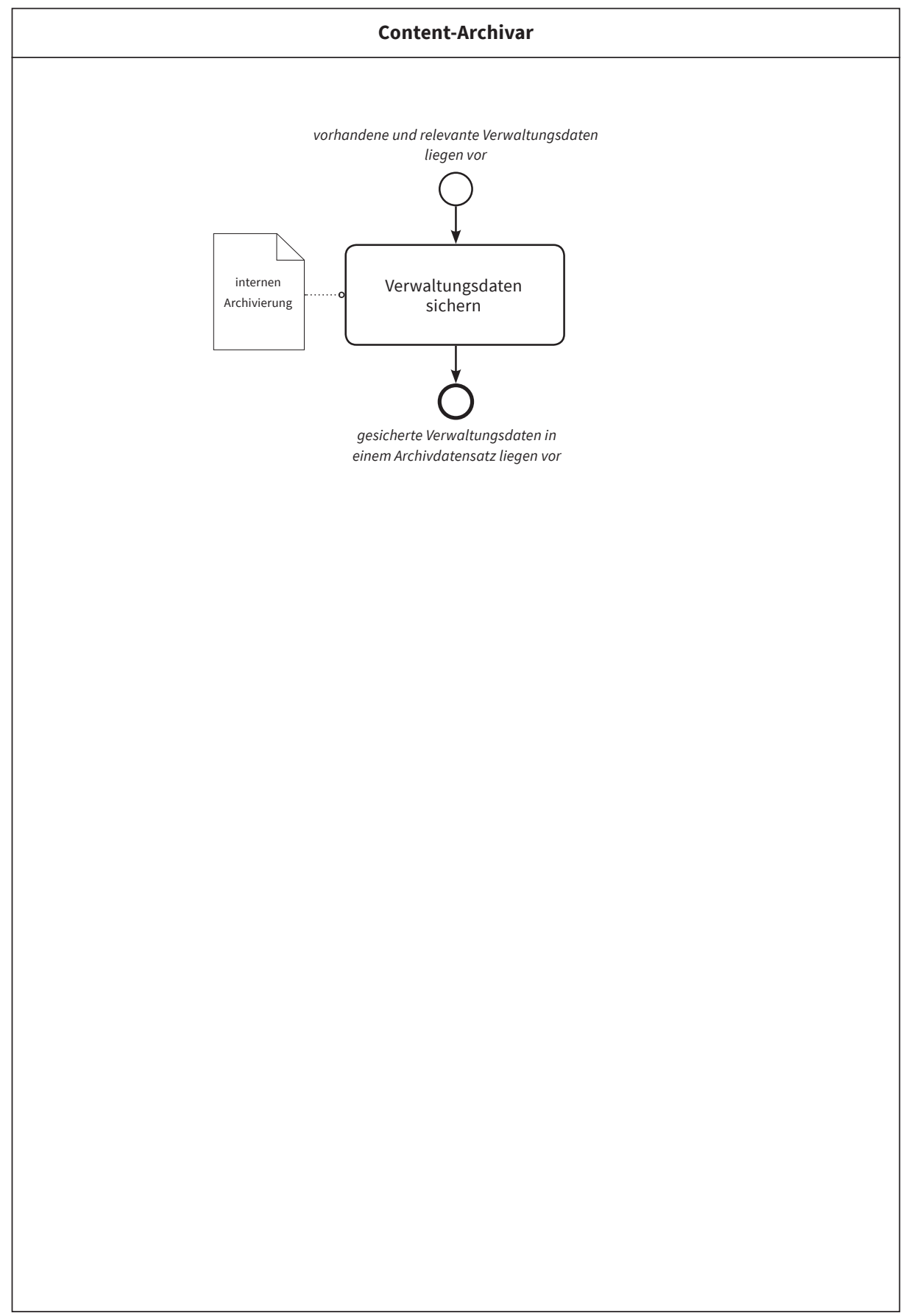




\section{Nach-Kalkulation erstellen [M-Pn-200]}

\section{Prozessbeschreibung}

Der Betriebswirt erstellt nach einem definierten Zeitraum bzw. nach dem Produktlebenszyklus des Produktes eine Nach-Kalkulation, um die Wirtschaftlichkeit des Produktes festzustellen und Handlungsoptionen für das nächste Projekt ableiten zu können. Hierbei muss das ggf. ausgezahlte Honorar berücksichtigt werden.

Vor- und nachgelagerte Prozesse

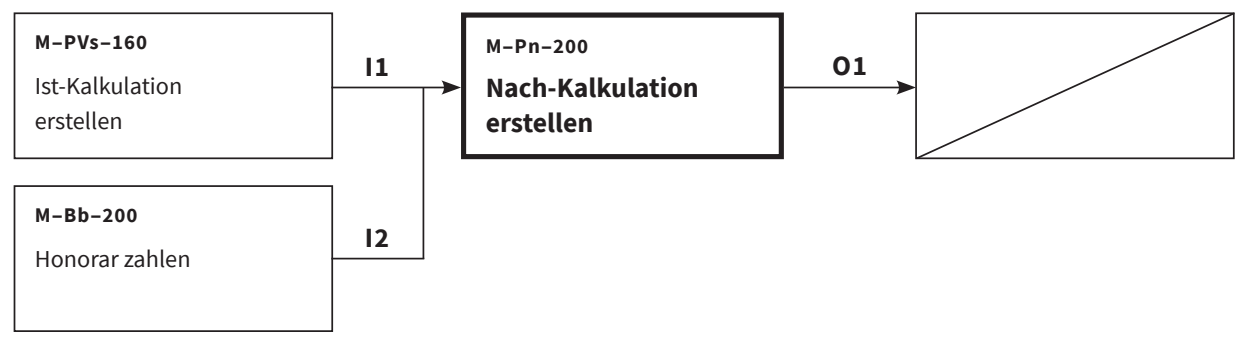

\section{Input}

- I1: Ist-Kalkulation

- I2: Honorar

\section{Output}

- O1: Nach-Kalkulation

\section{Beteiligte Rollen}

- Betriebswirt

\section{Ressourcen}

- Zeit: T-[M-Pn-200] 


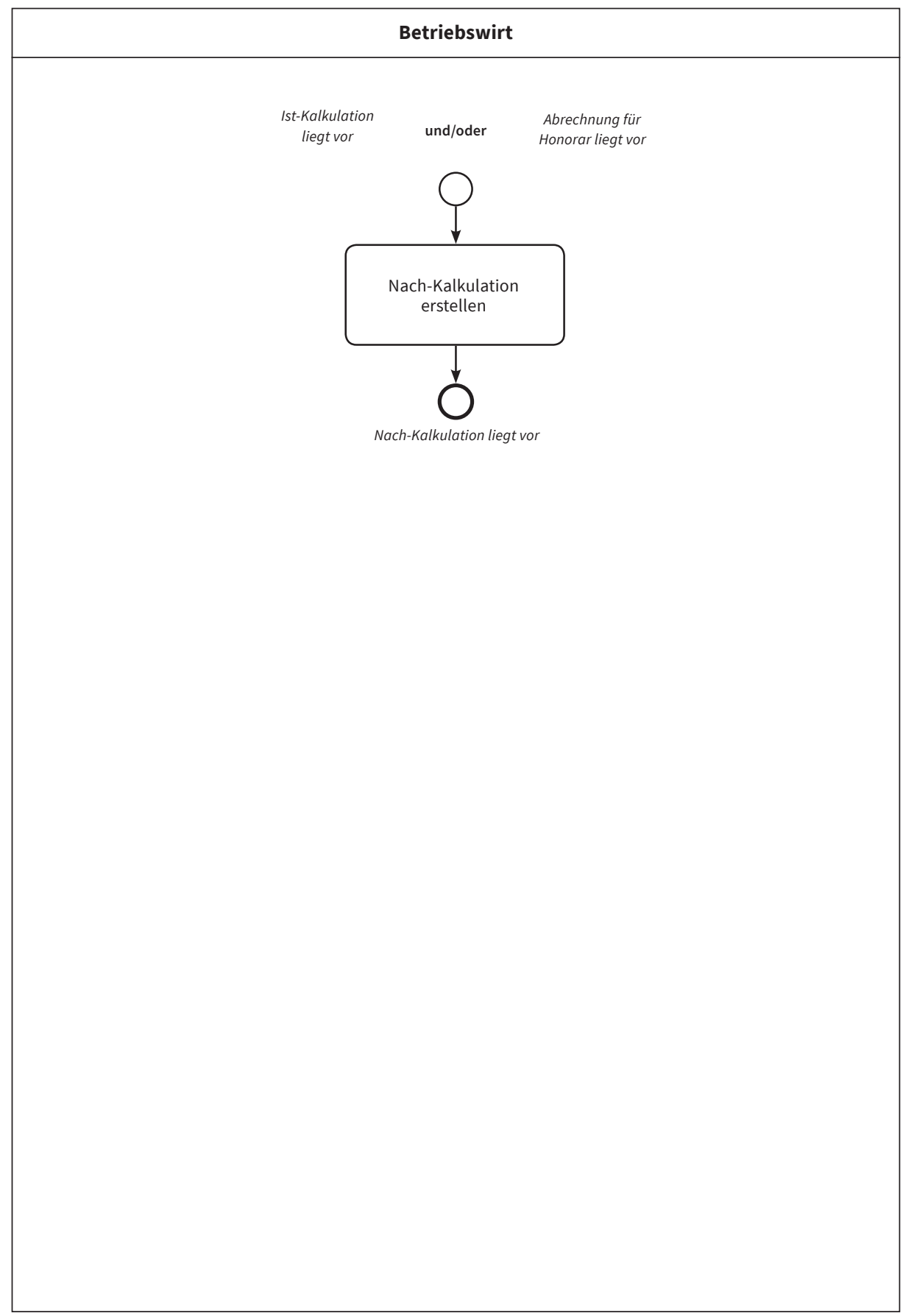




\section{Bestellung erhalten [M-Bb-100]}

\section{Prozessbeschreibung}

Der Content-Distributor oder der angeschlossene Dienstleister erhält eine Bestellung für ein gedrucktes Produkt.

Vor- und nachgelagerte Prozesse

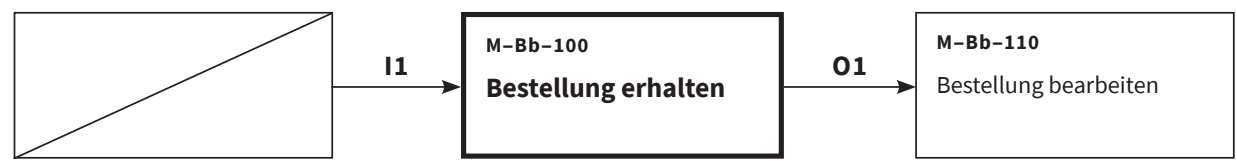

\section{Input}

- I1: Bestellung vom Kunden

\section{Output}

- O1: eingegangene Bestellung

\section{Beteiligte Rollen}

- Content-Distributor oder Dienstleister

- Kunde

\section{Ressourcen}

- Zeit: T-[M-Bb-100]

- Kosten: $\mathrm{K}-[\mathrm{M}-\mathrm{Bb}-100]$ 


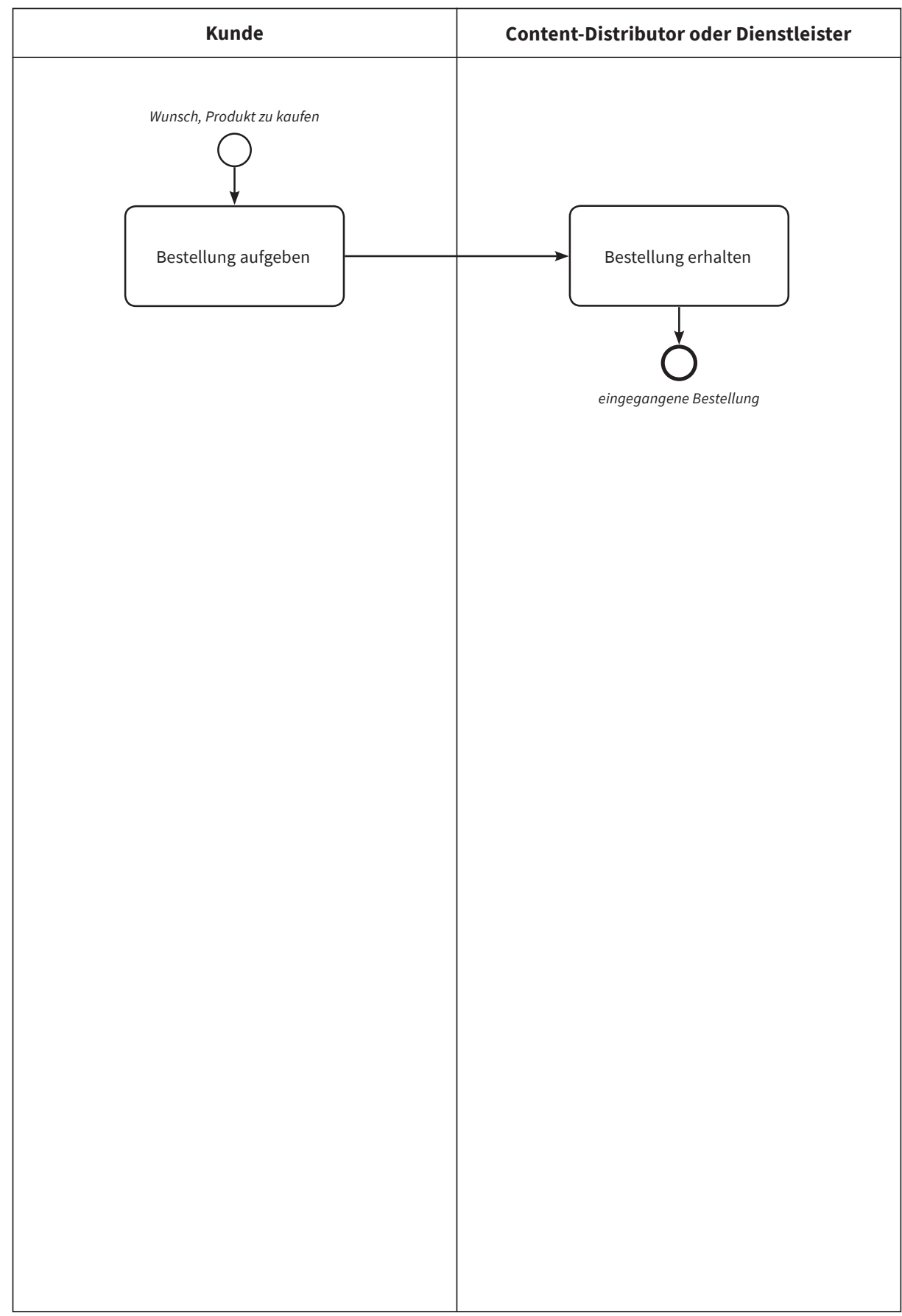




\section{Bestellung bearbeiten [M-Bb-110]}

\section{Prozessbeschreibung}

Der angeschlossene Dienstleister bearbeitet die Bestellung, indem er die bestellten Produkte produziert, er wird vom Content-Distributor damit beauftragt oder der Content-Distributor bedient selbst die Bestellung, da er bspw. die gedruckten Exemplare des Produktes im Hochschulverlag lagert.

Vor- und nachgelagerte Prozesse

\begin{tabular}{|c|c|c|c|c|}
\hline $\begin{array}{l}\mathbf{M - B b - 1 0 0} \\
\text { Bestellung erhalten }\end{array}$ & I1 & $\begin{array}{l}\text { M-Bb-110 } \\
\text { Bestellung bearbeiten }\end{array}$ & 01 & $\begin{array}{l}\mathbf{M}-\mathbf{B b}-\mathbf{1 2 0} \\
\text { Bestellung versenden }\end{array}$ \\
\hline
\end{tabular}

\section{Input}

- I1: eingegangene Bestellung

\section{Output}

- O1: bestellte Druckexemplare bereitstellen

\section{Beteiligte Rollen}

- Content-Distributor

- Dienstleister

\section{Ressourcen}

- Zeit: T-[M-Bb-110]

- Kosten: K-[M-Bb-110] 


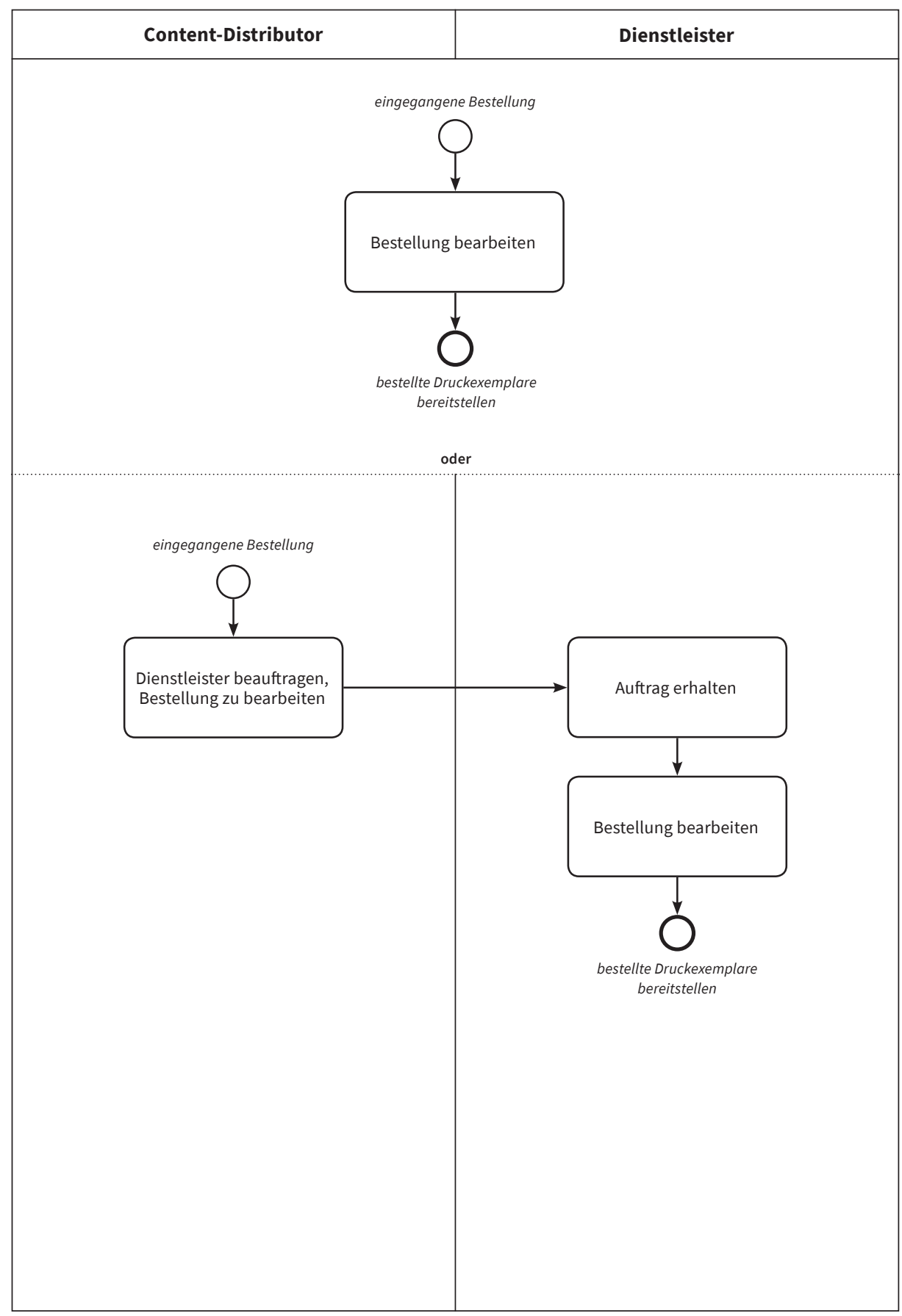




\section{Bestellung versenden [M-Bb-120]}

\section{Prozessbeschreibung}

Der Dienstleister versendet die bestellten Druckexemplare, je nach Absprache entweder direkt an den Kunden oder zunächst an den Content-Distributor, damit dieser die bestellten Druckexemplare selbst versendet.

Vor- und nachgelagerte Prozesse

\begin{tabular}{|c|c|c|c|c|}
\hline $\begin{array}{l}\mathbf{M - B b - 1 1 0} \\
\text { Bestellung bearbeiten }\end{array}$ & I1 & $\begin{array}{l}\text { M-Bb-120 } \\
\text { Bestellung versenden }\end{array}$ & 01 & $\begin{array}{l}\mathbf{M}-\mathbf{B b} \mathbf{- 1 3 0} \\
\text { Rechnung stellen }\end{array}$ \\
\hline
\end{tabular}

\section{Input}

- I1: bestellte Druckexemplare bereitstellen

\section{Output}

- O1: Bestellung bedient

\section{Beteiligte Rollen}

- Dienstleister

- Content-Distributor

- Kunde

\section{Ressourcen}

- Zeit: T-[M-Bb-120]

- Kosten: K-[M-Bb-120] 


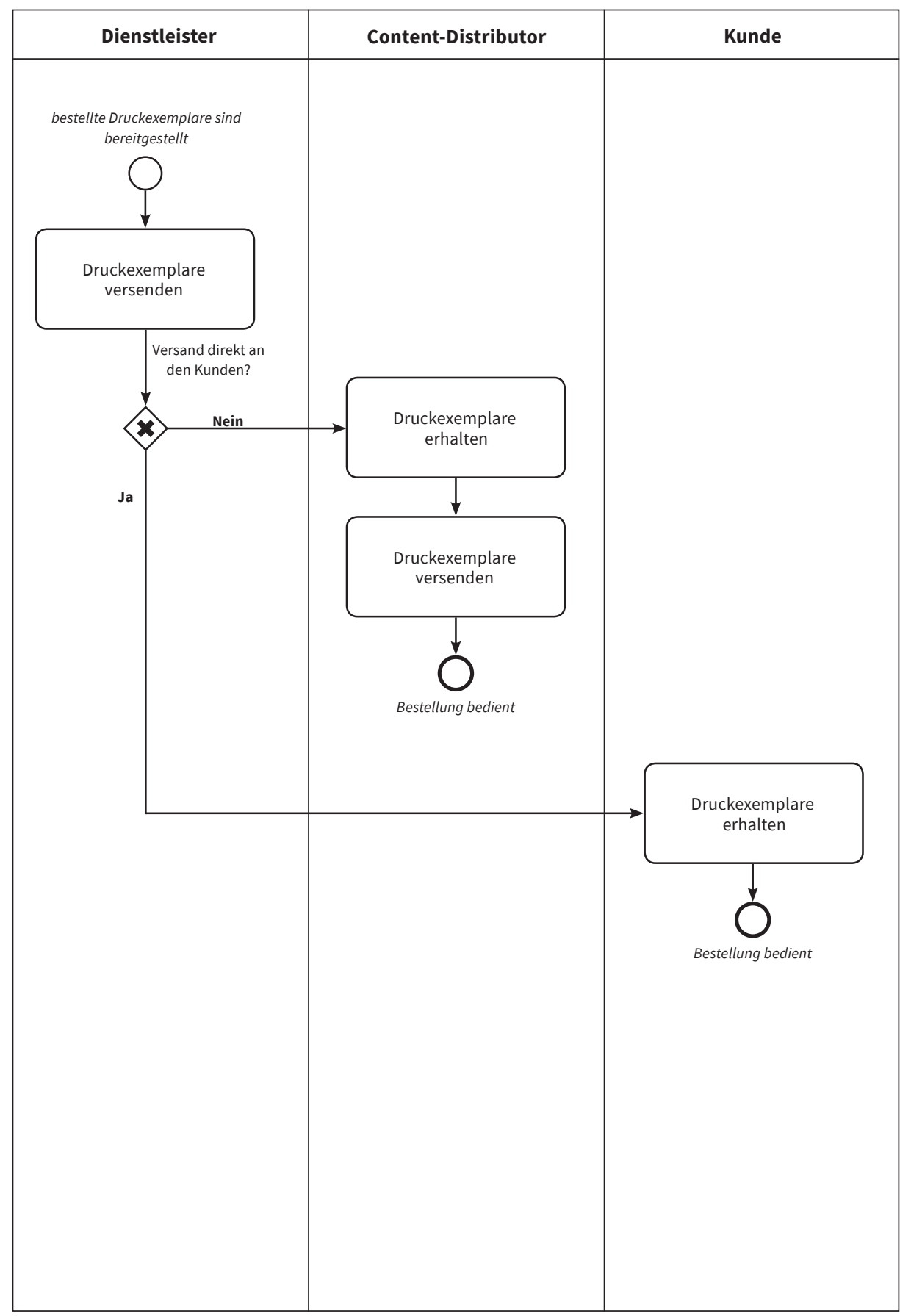




\section{Rechnung stellen [M-Bb-130]}

\section{Prozessbeschreibung}

Der Dienstleister oder Betriebswirt des Hochschulverlages erstellt die Rechnung für die bestellten Druckexemplare und übermittelt diese an den Kunden.

Vor- und nachgelagerte Prozesse

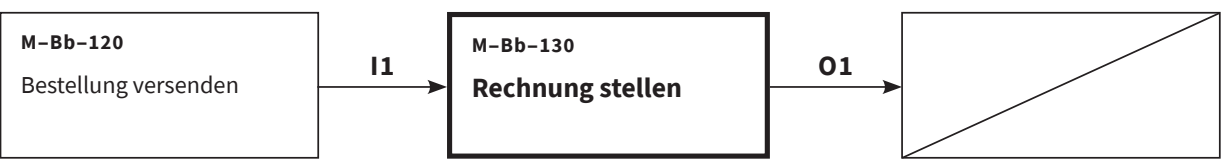

\section{Input}

- I1: Bestellung bedient

\section{Output}

- O1: Bestellung abgeschlossen

\section{Beteiligte Rollen}

- Dienstleister oder Betriebswirt

- Kunde

\section{Ressourcen}

- Zeit: T-[M-Bb-130]

- Kosten: $\mathrm{K}-[\mathrm{M}-\mathrm{Bb}-130]$ 


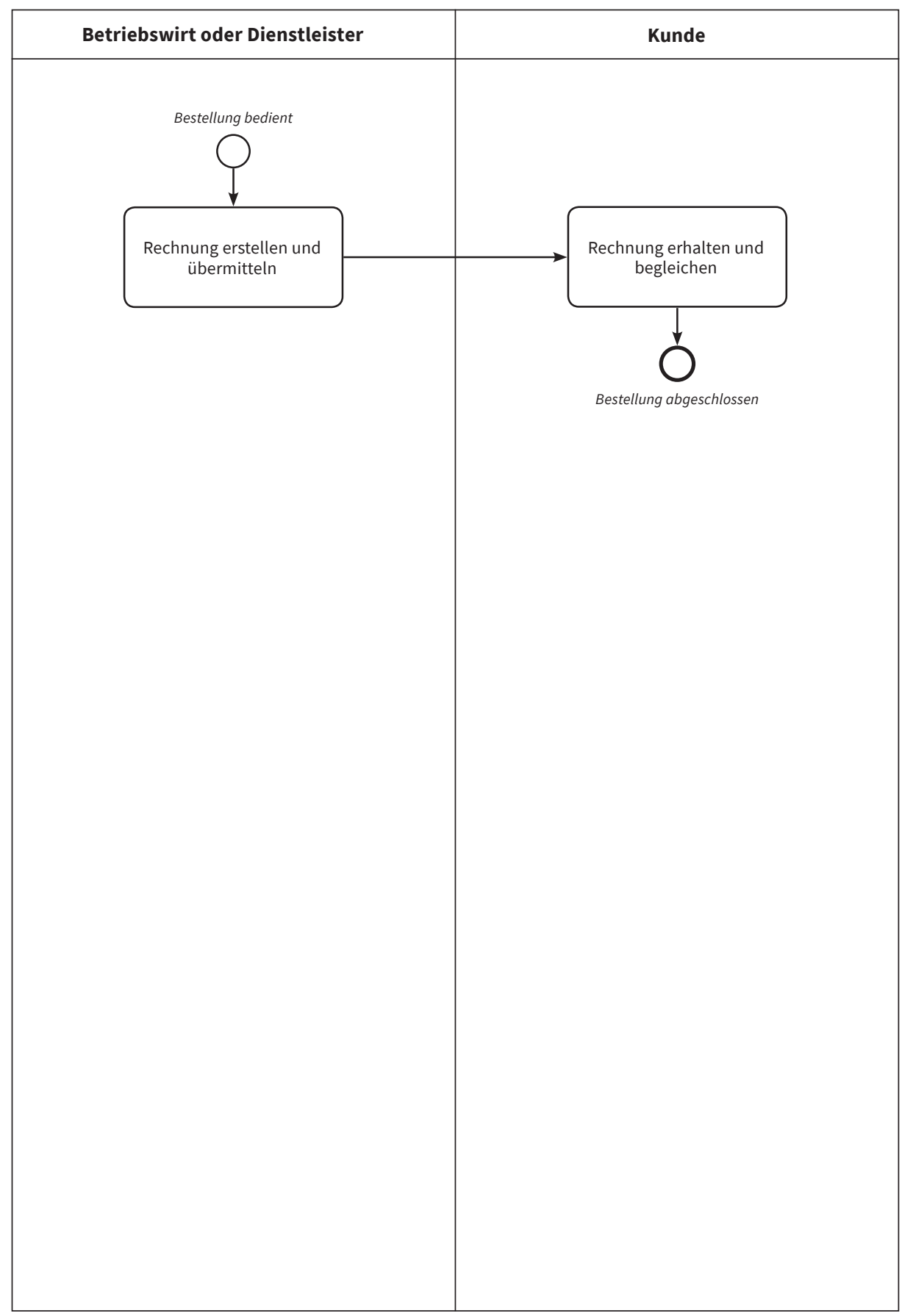




\section{Honorar auszahlen [M-Bb-200]}

\section{Prozessbeschreibung}

Der Betriebswirt sendet dem Content-Urheber in regelmäßigen Abständen eine Auflistung über die Anzahl der verkauften (gedruckten) Exemplare zu und zahlt das vereinbarte Autorenhonorar.

Vor- und nachgelagerte Prozesse

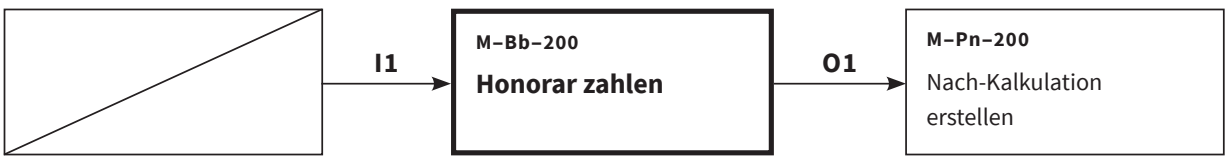

\section{Input}

- I1: Übersicht verkaufte Exemplare

\section{Output}

- O1: Honorar

\section{Beteiligte Rollen}

- Betriebswirt

- Content-Urheber

\section{Ressourcen}

- Zeit: T-[M-Bb-200]

- Kosten: K-[M-Bb-200] 


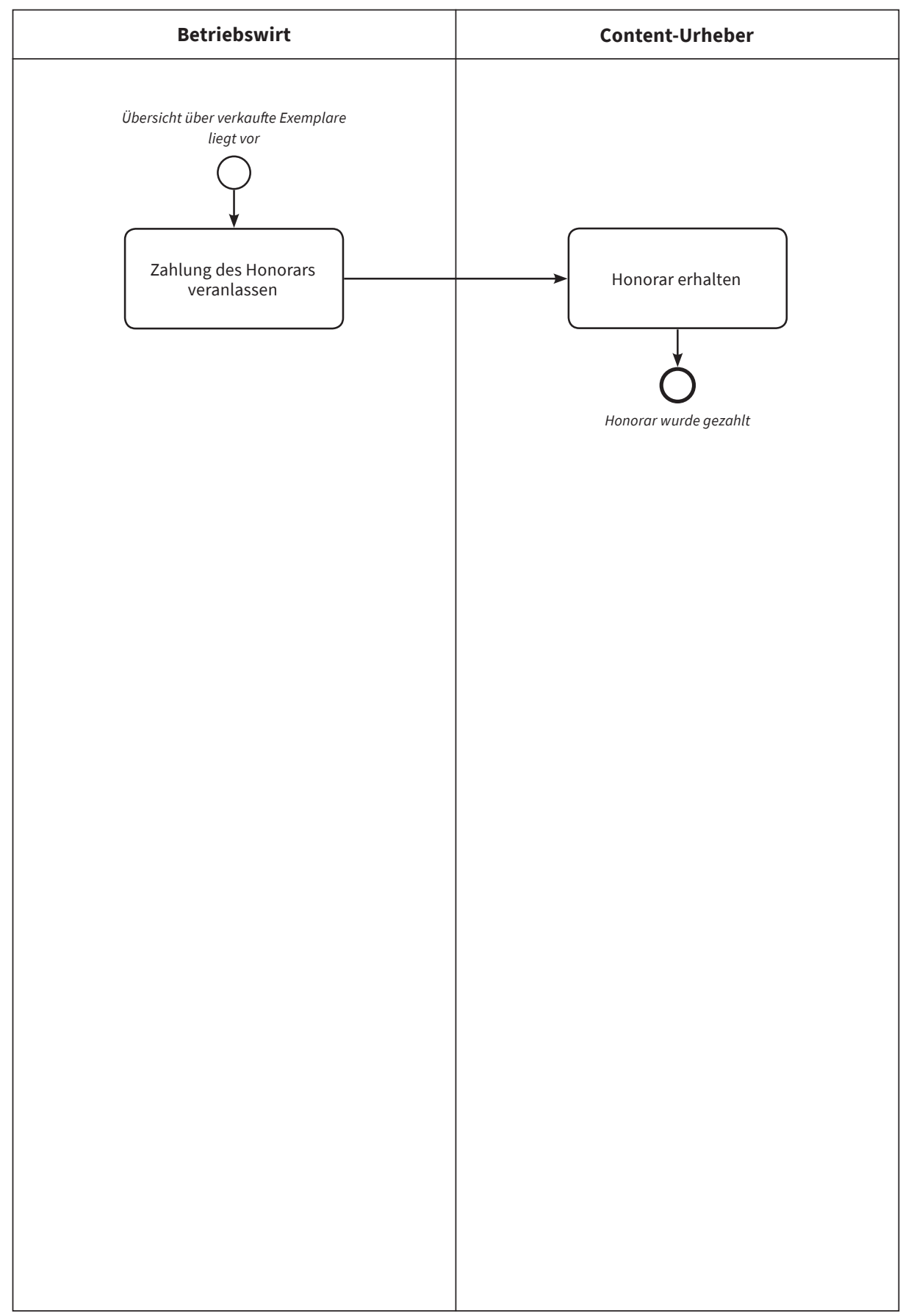





\section{Anwendung des Publikationsworkflow- Modells}

Bis hierhin wurde das Workflow-Modell mit seinen Elementen auf der Abstraktionsebene Prozessgruppe beschrieben. Im nächsten Schritt sollen der Fokus schärfer eingestellt und die Einzelprozesse diskutiert werden. Im Gegensatz zu den bisherigen Ausführungen werden diese aber nicht abstrakt, sondern praxisbezogen in Form einer Fallstudie vorgestellt. Damit soll ein Anspruch an das abstrakte Modell, dass sich aus diesem viele konkrete Anwendungsmodelle ableiten lassen, geprüft und verifiziert werden.

Für die Fallstudie agierte die Projektgruppe wie ein Hochschulverlag, d. h., alle Prozesse wurden konsequent, wie im Modell beschrieben, durchgeführt, wobei das Team alle hochschulverlagsinternen Rollen übernahm, während die weiteren Rollen von den jeweiligen Dienstleistern realitätsnah ausgeführt wurden. Die in der Fallstudie veröffentlichten Manuskripte sind allesamt reale Graduierungsarbeiten, welche durch Modellprozesse ausgewählt und bearbeitet wurden. Als einzige Randbedingung war gesetzt, dass sie unterschiedliche Komplexitätsgrade hinsichtlich ihrer Struktur aufweisen sollten, um verschiedene Workflow-Varianten evaluieren zu können. Durch die fakultäts- und damit fächerübergreifende Auswahl ergab sich diese Bedingung zwanglos.

So werden in diesem Kapitel einführend die Methode vorgestellt und nachfolgend die Durchführung der einzelnen Fälle detailliert und in konkretem Bezug auf den Hochschulverlag an der Hochschule für Technik, Wirtschaft und Kultur in Leipzig beschrieben. Die dabei erhobenen Kennzahlen zu Zeit-, Kosten- und Personalaufwand werden im zweiten Teil des Kapitels vorgestellt, analysiert und diskutiert. Sie stellen reale Ergebnisse für die in der Fallstudie vorgestellten Workflows dar und sind nicht direkt auf andere Rahmenbedingungen übertragbar. Trotzdem können sie einerseits Hinweise auf die Vielfalt der zu beachtenden Kennzahlen bieten und darüber hinaus Anhaltspunkte für die nötigen Investitionen bei der Gründung und dem Betrieb eigener OA-Hochschulverlage bieten.

\subsection{Methode}

Nachdem auf Grundlage von Recherchen und Expertenbefragungen ein WorkflowModell für die Publikation von OA-Monografien für akademische Institutionen entwickelt werden konnte, muss nun dessen Einsatz in der Praxis genauer perspektiviert werden. Wie in Kapitel 1 beschrieben, soll dies anhand ausgewählter Fallbeispiele - OA-Buchveröffentlichungen - durch das Projektteam (nachfolgend auch als Modellverlag bezeichnet) erfolgen. 
Zur Verwirklichung dessen findet hier die empirische Methode der Fallstudie Anwendung. Nach Eisenhardt liegt der Vorteil dieser Methode darin, dass sie in einem weiten Spektrum universell einsetzbar ist. ${ }^{85}$ Zudem können in der sogenannten vergleichenden Fallstudie (multiple-case design) „die gewonnenen Erkenntnisse durch Ähnlichkeiten und Unterschiede zwischen den Fällen kritisch beleuchtet werden". ${ }^{86}$ Mit diesen Eigenschaften ist die Methode der vergleichenden Fallstudie im Vergleich zu anderen besonders geeignet, um das WorkflowModell zu evaluieren und die Anwendbarkeit nachzuweisen. Zu diesem Zweck sollten fünf Graduierungs- oder Forschungsarbeiten unter Anwendung des WorkflowModells hergestellt und veröffentlicht werden. So galt es zunächst, geeignete Manuskripte aktueller Graduierungs- oder Forschungsarbeiten als Fall für die Durchführung der Studie auszuwählen. Nach Eisenhardt sollen die zu untersuchenden Fälle in einem Zusammenhang mit dem Forschungsziel stehen, sie dürfen „aber durchaus beliebig, wenngleich begründet ausgewählt werden, um bewusst bestimmte Typen von Fällen zu erfassen. [...] Für die vergleichende Fallstudie werden weitere Fälle so ausgewählt, dass sie den Rahmenbedingungen des oder der ersten analysierten Fälle entsprechen, sodass sie voraussichtlich die bisherigen Erkenntnisse bestätigen““ 87

\section{Fallstudiendesign: Auswahl der Manuskripte}

Für die Auswahl der Manuskripte wurden in Anlehnung an die Zielstellung für das Workflow-Modell (vgl. Kapitel 1) folgende Kriterien festgelegt:

Zunächst sollten die Manuskripte unterschiedliche Herausforderungen an die medienneutrale Aufbereitung stellen. Struktur, Layout und Inhalt haben Einfluss auf die Automatisierbarkeit der medienneutralen Aufbereitung von Manuskripten. In der Regel stellen Manuskripte aus den Geistes- und Sozialwissenschaften nur geringe Anforderungen an die Automatisierbarkeit, da sie in der Regel strukturell nur wenig komplex sind. Im Gegensatz dazu weisen Manuskripte aus den Naturund Ingenieurwissenschaften typischerweise eine mittlere bis hohe strukturelle Komplexität auf; entsprechend stellen sie höhere Anforderungen an die Automatisierbarkeit in einem XML-first-Workflow. Um diese Bandbreite abzubilden, wurden Manuskripte aus unterschiedlichen Fachbereichen für die Fallstudie ausgewählt.

Die Auswahl orientierte sich an den Fakultäten der HTWK Leipzig, die ein breites Spektrum an Fachgebieten abdecken. Zum Zeitpunkt der Auswahl ${ }^{88}$ gliederte sich die HTWK Leipzig in die Fakultäten:

85 vgl. Albers et al 2007, S. 35

86 vgl. Albers et al 2007, S. 36

87 Albers et al 2007, S. 37

88 Im Rahmen der Umsetzung des beschlossenen Hochschulentwicklungsplans wurden zum 01. April 2019 strukturelle Änderungen an der HTWK Leipzig vorgenommen, die u. a. die Neustrukturierung der Fakultäten betraf. Mehr dazu hier: https://www.htwk-leipzig.de/no_cache/hochschule/aktuelles/newsdetail/artikel/2133/ (Aufruf am 13.08.19; 10:15 Uhr) 
- Architektur und Sozialwissenschaften

- Bauwesen

- Elektrotechnik und Informationstechnik

- Informatik, Mathematik und Naturwissenschaften

- Maschinenbau und Energietechnik

- Medien

- Wirtschaftswissenschaft und Wirtschaftsingenieurwesen

Um die Auswahl der infrage kommenden Manuskripte noch weiter zu beschränken, wurde zugleich die Richtlinie für die Konzept-Annahme (vgl. Anhang A) wie folgt festgelegt:

- Die Arbeiten haben eine schriftliche Benotung von 1,0 bis 1,3 erhalten (sofern die Note weniger gut ist, werden auch Empfehlungen der Hochschulprofessoren berücksichtigt; etwa dann, wenn nur aufgrund von formalen Fehlern keine 1,0 gegeben werden konnte).

- Es sind aktuelle Arbeiten, die nicht älter als 3 Jahre sind, d. h., aus den Jahren 2016 bis 2019 stammen.

- Die Arbeiten haben keinen Sperrvermerk und können im OA veröffentlicht werden.

Unter Mitteilung dieser Vorgaben wurden, u. a. mit Hilfe der Hochschulbibliothek, die Professoren und Prüfungsämter ${ }^{89}$ der Fakultäten aufgerufen, Manuskripte einzureichen, die ihrer Meinung nach für die Publikation geeignet sind (entspricht Prozess I-Ka-200).

So konnten vier unterschiedlich komplexe Graduierungsarbeiten für die vergleichende Fallstudie ausgewählt werden. Darüber hinaus wurde beschlossen, die Publikation des hier vorliegenden Werks als fünften Fall in die Studie einzubeziehen.

Als Kriterium für die Gestaltung der zu untersuchenden Fälle wurde festgelegt, dass pro Fall unterschiedliche Rollenträger an der Umsetzung der Manuskripte beteiligt werden. Denn: Wie in Kapitel 2 erläutert, können XML-first-Workflows in Eigen- oder auch Fremdleistung umgesetzt werden. Durch dieses Vorgehen wird in der Gesamtheit der Fallstudien nicht nur das Konzept der Rollen praktisch demonstriert, sondern auch die Anpassungsfähigkeit des Workflow-Modells an unterschiedlich präferierte Workflow-Varianten. So wird durch die Fälle bspw. demonstriert, welche Prozesse geeignet sind, um Satz- und Druckdienstleister als Rollenträger in den Publikationsworkflow einzubinden. Im Sinne einer vergleichenden Fallstudie wurden für jeden Fall unterschiedliche Dienstleister beauftragt, um diese nach Zeit, Kosten und Qualität ihrer Leistungen vergleichen zu können.

89 Zu diesem Zeitpunkt waren die Prüfungsämter an der HTWK Leipzig dezentral organisiert, jede Fakultät hatte ein eigenes Prüfungsamt. 
Da zum Zeitpunkt der Drucklegung des vorliegenden Werks die Veröffentlichung von Fall 4 und selbstverständlich auch Fall 5 - das vorliegende Werk selbst - noch nicht abgeschlossen waren, werden diese Fälle der Vollständigkeit halber nachfolgend nur vorgestellt und erst in einer späteren Veröffentlichung im Detail beschrieben und ausgewertet.

In der vorliegenden Monografie werden die Fälle anonymisiert. Die in diesem Rahmen entstandenen Werke sind aber, wie es das Workflow-Modell vorsieht, elektronisch im OA und als gedruckte Produkte über den Buchhandel verfügbar. Die genauen bibliografischen Angaben zu den Werken sind im Anhang D zu finden.

Fall 1

Beim Manuskript des ersten Falles handelt es sich um eine Graduierungsarbeit zum Erwerb des Master of Arts im Studienfach Architektur der Fakultät Architektur und Sozialwissenschaften. Dem Fachgebiet sowie der Studienordnung dieses Studiengangs entsprechend unterscheidet sich diese Masterarbeit von Abschlussarbeiten aus natur- oder geisteswissenschaftlichen Studiengängen. Ein wesentlicher Teil des Studiums der Architektur ist der Erwerb der Fähigkeit, fachspezifische Probleme nach künstlerisch-wissenschaftlichen Methoden $\mathrm{zu}$ bearbeiten und unterschiedliche Fachaspekte, auch aus den Bereichen der Nachbardisziplinen, in die Problemlösung zu integrieren. ${ }^{90}$

Im Rahmen der Masterarbeit befasste sich der Autor (Content-Urheber) mit dem Leipziger Stadtbezirk Anger-Crottendorf. Konkret bearbeitet wurde die Problemstellung, dass in diesem Bezirk in einer alten, leerstehenden Feuerwache ein Nachbarschaftszentrum entstehen soll, die Stadt auf dem frei gewordenen Grundstück aber auch den Bau einer Schule plant. In der Arbeit des Content-Urhebers wird für diesen städtebaulichen Nutzungskonflikt ein Lösungsansatz vorgestellt.

Die Analyse des Manuskriptes zeigte, dass der Content-Urheber bei der Erstellung der Arbeit besonderen Wert auf die typografische Gestaltung gelegt hat. Diese spielt bei Graduierungsarbeiten, wie auch bei Forschungsarbeiten aus anderen Wissenschaftsdisziplinen, häufig eine eher untergeordnete Rolle. ${ }^{91} \mathrm{Zu}$ diesen typografischen Besonderheiten des Manuskriptes zählen vor allem:

- der großzügige Satzspiegel mit viel Weißraum

- der zweispaltige Satz

- die Verwendung einer Marginalspalte

- die Auszeichnung von Text in Schmuckfarbe

90 vgl. HTWK Leipzig 2018, S. 18

91 Es kann angenommen werden, dass bei wissenschaftlichen Monografien aus anderen Fachgebieten für den Rezipienten eher die Lesbarkeit und für den Wissenschaftsverlag darüber hinaus ein effizienter Satzspiegel im Vordergrund steht. 
Um den in der Arbeit entwickelten Lösungsansatz zu demonstrieren, wurden zudem detaillierte Zeichnungen und große Fotografien ergänzt, die zum Teil über Doppelseiten platziert sind. Beim Lesen der Arbeit fiel auf, dass der beschreibende Text kurzgehalten ist und der Fokus auf der Vermittlung des Problems und dessen Lösung liegt. Entsprechend sind die Formulierungen eher umgangssprachlich als wissenschaftlich und auch der Umfang der Arbeit ist mit 68 Manuskriptseiten eher gering.

Auf Grundlage dieser Analyse wurde entschieden, das Manuskript nicht medienneutral aufzubereiten, sondern lediglich die gedruckten Ausgabeformate Hard- und Softcover und die elektronische Ausgabe als ePDF herzustellen und zu veröffentlichen. Die Umsetzung in XML ist auf Grund der typografischen Besonderheiten und der großen Abbildungen nicht bzw. nur schwer automatisierbar. Zudem sind die Inhalte der Arbeit nicht geeignet, um auf einem kleineren als dem gewählten DIN A4-Format dargestellt zu werden, etwa auf dem Bildschirm eines Smartphones oder eines E-Book-Readers. Da der Content-Urheber die Gestaltung außerdem als ästhetisch und zweckdienlich empfand, wurde entschieden, das Manuskript nicht nach den Gestaltungsrichtlinien des Forschungsprojektes (vgl. Veröffentlichung) umzusetzen. Stattdessen sollten die Gestaltung von Inhalt und Cover sowie das Format des Manuskriptes beibehalten werden.

Der Content-Urheber hat somit bereits eine druckfähige Satzdatei geliefert, so dass Fall 1 die Workflow-Variante CRC-Workflow demonstriert.

\section{Fall 2}

Für die Konstruktion des zweiten Falles wurde eine Bachelorarbeit aus dem Studiengang Wirtschaftsingenieurwesen Produktions- und Energiewirtschaft der Fakultät Maschinenbau und Energietechnik ausgewählt. Diese Arbeit hatte der betreuende Hochschulprofessor zur Publikation empfohlen. Im Rahmen seiner Arbeit beschäftigte sich der Content-Urheber mit einer speziellen Problematik von funkbasierten Sensorsystemen und entwickelte einen Lösungsansatz hierfür.

Das Wirtschaftsingenieurwesen ist ein interdisziplinäres Fachgebiet, das aus ingenieur- und wirtschaftswissenschaftlichen Teilen besteht. Aus diesem Grund finden sich in der Arbeit mathematische Formeln, komplexe Tabellen und Quellcode. Darüber hinaus enthält das Manuskript 38 Abbildungen, ein Abbildungs-, Tabellen-, Abkürzungs- und Literaturverzeichnis sowie einen Anhang. Insgesamt sind weniger solcher Inhaltselemente in der Arbeit enthalten als im Manuskript des nachfolgend vorgestellten Fall 3. Dies ergibt sich auch aus dem geringeren Umfang von 105 Manuskriptseiten. Aufgrund dessen ist der Schwierigkeitsgrad für die medienneutrale Umsetzung im Vergleich zu Fall 3 geringer, so dass mit Fall 2 ein XML-first-Workflow mit einem Manuskript mit mittlerer Komplexität demonstriert werden konnte. Für die Umsetzung des Satzes (P-CiP-Prozesse) wurden gleich zwei Satzdienstleister beauftragt (XML-first-Workflow in Fremdleistung). Aus diesem Grund wird der Fall 2 an relevanten Stellen zwischen Fall 2.1 - Umsetzung mit Dienstleister B und Fall 2.2 - Umsetzung mit Dienstleister C unterschieden. Aus wel- 
chem Grund, gleich zwei Satzdienstleister für Fall 2 beauftragt wurden, wird in Kapitel 4.3.2 näher erläutert.

\section{Fall 3}

Beim Manuskript des dritten Falles handelt es sich um eine Graduierungsarbeit der Fakultät Bauwesen zur Erlangung des Master of Science im Studienfach Bauingenieurwesen. Der Verfasser hat sich in seiner Untersuchung mit einer speziellen Problematik innerhalb der Sanierung von Bestandsbauwerken aus Stahlbeton beschäftigt. Die Arbeit entwickelt einen vielversprechenden Ansatz, diese besonderen Schwierigkeiten zu umgehen. Das Fachgebiet Bauingenieurwesen wird grundsätzlich der Wissenschaftsdisziplin der Ingenieurwissenschaften zugeordnet, die sich durch eine hohe Interdisziplinarität auszeichnet. ${ }^{92}$ Diese Eigenschaft spiegelt sich auch das Manuskript des dritten Falles wider.

Im Inhaltsteil sind über 300 mathematische Formeln, aber auch vereinzelte Darstellungen von Quellcode zu finden. Es werden häufig Aufzählungen verwendet und das Manuskript enthält zudem 57 Abbildungen, die zum Teil komplexe, mathematische Zusammenhänge als Strichvorlage darstellen. Einige der 10 verwendeten Tabellen beinhalten mehr als 4 Spalten und/oder Zeilen und können somit als komplex bezeichnet werden. Zum hohen Umfang von 196 Manuskriptseiten tragen neben den Inhaltsseiten das Abkürzungs-, Abbildungs-, Tabellen-, Formel- und Literaturverzeichnis sowie der Anhang mit ergänzenden Forschungsdaten bei.

Aufgrund der eben beschriebenen formalen Besonderheiten des Manuskripts wies die Umsetzung von Fall 3 einen erhöhten Schwierigkeitsgrad auf, da gerade die Vielzahl der Formeln und komplexen Tabellen die automatisierte, medienneutrale Umsetzung vor Herausforderungen stellen. So wurde zunächst entschieden, dass der Großteil der Workflow-Rollen, insbesondere in den produktionsorientierten Prozessen, von Mitarbeitern des Forschungsprojekts eingenommen werden. Sie agieren als Mitarbeiter eines Hochschulverlages, die aufgrund ihrer Ausbildung zudem die Anforderungen erfüllen, die produktionsorientierten Prozesse selbstständig auszuführen. Das Outsourcing dieser Prozesse an einen Satzdienstleister oder an den Content-Urheber war in diesem Fall also nicht notwendig.

Da Hochschulen jedoch unterschiedliche Rahmenbedingungen für die eigenen OA-Verlage setzen und bislang kaum Stellen für ausgebildete Verlagshersteller schaffen, wird Fall 3 so konstruiert, dass parallel zur Umsetzung durch das Projektteam der Satz an einen Satzdienstleister ausgelagert wird. So können im direkten Vergleich zusätzliche Erkenntnisse zu Dauer und Kosten gewonnen werden. Aufgrund dessen wird auch für diesen Fall zwischen Fall 3.1 - eigene Umsetzung und Fall 3.2 - Umsetzung mit Dienstleister A unterschieden.

Für die eigenständige Umsetzung war es vorab im Sinne eines Null-Prozesses notwendig, ein Stylesheet-Template nach den selbst entwickelten Gestaltungsrichtlinien für die Transformation des Manuskriptes (Quelldokument) in das Zieldoku- 
ment (Ausgabeformate PDF, EPUB und MOBI) zu entwickeln. Das Manuskript von Fall 3 war hierfür besonders geeignet, da es - wie erwähnt - eine Vielzahl an unterschiedlichen Struktur-Elementen beinhaltet und die Entwicklung des Templates somit erleichterte. So ließ sich in kurzer Zeit ein universell einsetz- und dann nachnutzbares Template für die medienneutrale Umsetzung jedes Contents entwickeln. Daten zur Entwicklung des Templates wurden für die Fallstudienanalyse allerdings nicht erfasst.

\section{Fall 4}

Das gewählte Manuskript für Fall 4 ist eine Graduierungsarbeit der Fakultät Medien zur Erlangung des Master of Arts im Studienfach Verlags- und Handelsmanagement. Im Rahmen der Arbeit wurde eine Analyse der Mietangebote für E-Books vorgenommen, um konkrete Empfehlungen im Hinblick auf eine kundenorientierte Angebotsgestaltung geben zu können und Leistungen aufzudecken, für die potenziell eine Zahlungsbereitschaft aufseiten der Kunden, die solche Angebote grundsätzlich nutzen, besteht. Der Fokus der Arbeit liegt auf dem Massenmedium Buch und beleuchtet ökonomische Aspekte des Buchmarktes. Dementsprechend lässt sich die Arbeit den Sozialwissenschaften zuordnen. ${ }^{93}$ Wie die Mehrheit sozialwissenschaftlicher Forschungsarbeiten weist auch die Masterarbeit im Fall 4 eine vergleichsweise wenig komplexe Struktur auf. Das Manuskript umfasst 125 Seiten mit insgesamt 23 Abbildungen, 5 Tabellen (von denen 3 als komplex bezeichnet werden können), einem Anhang und einem Abbildungs-, Tabellen-, Abkürzungs- und Literaturverzeichnis. Auffällig ist, dass im Gegensatz zu den Manuskripten der anderen Fälle vom Content-Urheber Fußnoten zum Quellennachweis verwendet wurden. Zudem werden an einigen Stellen Sätze durch farbliche Hinterlegung und Umrandung (im Sinne einer Box) hervorgehoben. Da abgesehen von diesen Besonderheiten der Großteil des Manuskriptes aus einfachem Fließtext besteht, demonstriert der Fall 4 einen XML-first-Workflow mit einem Manuskript von einfacher Komplexität. Durch das Outsourcen des Satzprozesses (P-CiP-Prozesse) soll ebenfalls die Variante XML-first-Workflow in Fremdleistung abgebildet werden.

Die detaillierte Beschreibung und Analyse dieses Falles wird an anderer Stelle publiziert.

\section{Fall 5}

Wie bereits erwähnt, bildet Fall 5 die Herstellung und Veröffentlichung des vorliegenden Werkes ab. Es handelt sich hierbei um eine klassische Forschungsarbeit mit mehreren Autoren, die im Rahmen eines Forschungsprojektes an einer Hochschule entstanden ist.

Die Monografie wird ca. 350 Manuskriptseiten und eine Vielzahl von Grafiken und Tabellen enthalten. Dem Fachgebiet entsprechend sind weder mathematische 
noch chemischen Formeln oder Quellcode enthalten, stattdessen werden in Fußnoten Quellenhinweise und weitere Erläuterungen gegeben. Das Manuskript des vorliegenden Werks weist für die medienneutrale Umsetzung folglich eine einfache bis mittlere Komplexität auf. Es wird durch das Projektteam mit den zu Fall 3 entwickelten Stylesheets und mit Unterstützung eines studentischen Teams selbstständig umgesetzt. Die Realisierung demonstriert somit einen XML-first-Workflow in Eigenleistung.

Die detaillierte Beschreibung und Analyse dieses Falles wird an anderer Stelle publiziert.

\subsection{Dienstleisteranalyse}

Bereits während der Entwicklung des Workflow-Modells wurden Satz- und Druckdienstleister, Veröffentlichungsplattformen, (Metadaten-)Aggregatoren und Softwareanbieter analysiert und nach Auswahl der Manuskripte für die Fallstudie festgelegt, welche Prozesse des Publikationsworkflows an sie ausgelagert bzw. durch sie unterstützt ausgeführt werden. Dieses Vorgehen entspricht dem managementorientierten Prozess $M-P v-100$ : Produktionsweg festlegen und stellt den Beginn der Publikationsworkflows der Fälle dar. Die projektspezifische Qualitätsrichtlinie für diesen Prozess (Richtlinie für die Wahl des Produktionswegs, vgl. Anhang A) stellt daher lediglich die Bedingung, den Produktionsweg so zu wählen, dass er dem Zweck der Fallstudie dient. Für die Fallstudie wurde jedoch nur ein kleiner Teil der analysierten Dienstleister tatsächlich beauftragt. Nur für sie konnten zeitliche, finanzielle und qualitative Aspekte evaluiert werden, was die Grundlage für die Fallstudienanalyse in Kapitel 4.4 bildet. ${ }^{94}$

Neben der Vorstellung dieser Unternehmen sollen in diesem Kapitel auch die darüber hinaus analysierten Dienstleister präsentiert werden, um einen Überblick für andere Hochschulen und Institutionen zu entwickeln. Auch hier besteht wieder kein Anspruch auf Vollständigkeit.

\subsubsection{Satzdienstleister}

Für die Fälle 2.1, 2.2, 3.2 und 4 wurden 4 unterschiedliche Satzdienstleister mit der Durchführung der P-CiP-Prozesse beauftragt. Sie werden in Tabelle 4 anonymisiert vorgestellt. Die tatsächlich entstandenen Kosten für den Satz sind für die genannten Fälle - mit Ausnahme der Fälle 4 und 5 - im Kapitel 4.4.1.4 aufgeschlüsselt. Folgende Leistungen wurden bei den Satzdienstleistern angefragt:

94 Eine Ausnahme stellen hier die Fälle 4 und 5 dar. Zur Vollständigkeit werden die hierfür eingesetzten Dienstleister vorgestellt; die Daten zu Zeit, Kosten und Qualität sowie deren Analyse werden aber an anderer Stelle publiziert. 
- XML-first-Satz der eingereichten Manuskripte (Konvertierung vom Eingangsformat Word nach XML [BITS-Standard])

- Ausgabe der folgenden Ausgabeformate unter Anwendung der gelieferten Gestaltungsrichtlinien des Modellverlags: Print-PDF, ePDF, EPUB, MOBI

- keine Autorkorrekturen oder andere Änderungen am Inhalt

- keine Bildbearbeitung

- Grundlage sind: finales Manuskript als Word-Datei, druckreife Abbildungen als einzelne Dateien, Gestaltungsrichtlinien des Modellverlags

Tab. 4 Übersicht der beauftragten Satzdienstleister der Fallstudie

Dienstleister $\quad \begin{aligned} & \text { Anwendung in Kostenmodell } \\ & \text { Fallstudie }\end{aligned}$

\begin{tabular}{lll}
\hline $\begin{array}{l}\text { A } \\
\text { inländischer Publishing- } \\
\text { Dienstleister }\end{array}$ & Fall 3.2 & $\begin{array}{l}\text { Kosten pro Seite, Preisstaffelung nach } \\
\text { Komplexität und Format des Manuskriptes }\end{array}$ \\
\hline $\begin{array}{l}\text { B } \\
\text { ausländischer Publishing- } \\
\text { Dienstleister }\end{array}$ & Fall 2.1 & $\begin{array}{l}\text { Pauschalpreis auf Anfrage erhalten, } \\
\text { Preismodell nicht auf Website ersichtlich }\end{array}$ \\
\hline $\begin{array}{l}\text { C } \\
\text { ausländischer Outsourcing- } \\
\begin{array}{l}\text { Dienstleister } \\
\text { D }\end{array}\end{array}$ & Fall 2.2 & $\begin{array}{l}\text { Pauschalpreis für die Erstellung des Print- } \\
\text { PDFs, zusätzliche Kosten pro Seite für die } \\
\text { inländischer }\end{array}$ \\
$\begin{array}{l}\text { Satzdienstleister } \\
\text { elektronischen Formate }\end{array}$ \\
\hline
\end{tabular}

Dienstleister A ist ein inländischer Publishing-Dienstleister mit Niederlassung in Deutschland. Das Unternehmen besitzt nach dem Zusammenschluss mit einem Partnerunternehmen europaweite Standorte und bietet seinen Kunden (hauptsächlich Verlage) neben Satz- und Grafikdienstleistungen auch weitere Services an, wie bspw. Marktforschung und Unterstützung bei der Entwicklung von PublishingWorkflows. Einige dieser Leistungen führt der Dienstleister in Zusammenarbeit mit Partnerfirmen in Indien aus. Für die Anwendung im Fall 3.2 wurde mit der Niederlassung in Deutschland und dem Satzteam aus Indien zusammengearbeitet. Das Kostenmodell des Dienstleisters wurde transparent kommuniziert und umfasst Satzkosten pro Seite mit einer Preisstaffelung je nach Komplexität des Manuskriptes und je nach dem gewünschten Buchformat der zukünftigen Publikation.

Bei Dienstleister B handelt es sich um einen ausländischen Publishing-Dienstleister mit Sitz in Westeuropa, der Prepress- und Publishing-Services für die Verlagsbranche anbietet und hauptsächlich mit Verlagen aus Großbritannien, den USA und 
Europa zusammenarbeitet. Das Leistungsspektrum von Dienstleister B reicht von Satz- und Grafikdienstleistungen für gedruckte und elektronische Medien über Copy Editing, Übersetzungen, Projektmanagement bis hin zu Druckdienstleistungen. Im Vorfeld der Beauftragung war eine kostenfreie Umsetzung von Musterseiten nach den Gestaltungsrichtlinien des Forschungsprojektes für die qualitative Einschätzung der Umsetzung möglich. Bei den Kosten für die beauftragte Satzdienstleistung wurde nach Verhandlung ein Pauschalpreis vereinbart. Ein zugrundeliegendes Kostenmodell war nicht erkennbar.

Dienstleister $C$ ist ein ausländischer Dienstleister mit Sitz in Indien, der sich insbesondere auf Outsourcing-Services spezialisiert hat. Der vom Unternehmen gebotene Leistungsumfang ist breit gefächert und richtet sich nicht nur speziell an Verlage und Medienunternehmen. Es umfasst auch andere Branchen, wie bspw. den Einzelhandel, das Finanz- und Rechnungswesen, das Medizin- und Gesundheitswesen oder die Automobilbranche. Dienstleister $C$ bietet seinen Kunden neben Satz-, Grafik- und Publishing-Dienstleistungen für Medienprodukte u.a. auch Unterstützung bei Ausbau und Entwicklung von E-Commerce, Marketing, ContentManagement, Content-Writing und Vertrieb. Auch bei Dienstleister $C$ war eine kostenfreie Umsetzung von Musterseiten nach den Gestaltungsrichtlinien des Forschungsprojekts möglich. Bei der beauftragten Satzdienstleistung wurde ein Pauschalpreis für die Erzeugung einer druckfähigen PDF-Datei angeboten. Für die Ausgabe der elektronischen Formate (EPUB und MOBI) wurden zusätzliche Kosten pro Seite der gesetzten PDF-Datei aufgeführt.

Bei Dienstleister D handelt es sich um einen inländischen Satzdienstleister mit Spezialisierung auf automatische Satzproduktion in der Verlagsbranche. Das Unternehmen bietet Verlagen ein Tool für den selbstständigen Satz der Publikation mittels Word-Input. Dabei entwickelt der Dienstleister vorab die Templates nach den Gestaltungsvorgaben des Verlags. Anschließend kann der Verlag autonom WordDokumente hochladen, einen Umbruch erzeugen und Korrekturen ausführen. Neben dem eigenständigen Satz besteht für den Verlag auch die Möglichkeit, den Satz komplett durch den Dienstleister ausführen zu lassen. Der Leistungsumfang von Dienstleister $D$ beinhaltet die Erstellung von druck- und veröffentlichungsreifen Ausgabeformaten einer Buchpublikation.

Dienstleister D unterscheidet beim Geschäftsmodell zwischen Full-ServiceTypesetting, bei dem der Dienstleister den kompletten Satz übernimmt und Satzkosten pro Seite berechnet, und dem Self-Typesetting, wo dem Verlag im Rahmen eines Lizenzmodells lediglich die Werkzeuge zur Verfügung gestellt werden und dieser eigenständig die Satzdateien erstellt.

Im Vorfeld der Beauftragung der oben genannten Satzdienstleister wurden weitere Dienstleister recherchiert und angefragt. Aus den in Tabelle 5 aufgeführten Gründen kam es nicht zur Beauftragung dieser Dienstleister für die Fallstudie. 
Tab. 5 Übersicht der angefragten Satzdienstleister, die nicht für die Fallstudie beauftragt wurden

\section{Dienstleister}

\section{1}

Tool-Anbieter für kollaborativen Satz

02

inländischer Satz- und Publishing-Dienstleister

\section{3}

ausländischer Publishing-Dienstleister mit

Fokus auf OA-Publikationen

ausländischer Publishing-Dienstleister
aus

05

inländischer Druck-Dienstleister mit

Verlagsdienstleistungen

06

inländischer Selfpublishing-Dienstleister

07

ausländischer Satzdienstleister mit Fokus auf

mathematischen Satz im STM-Bereich

\section{Grund für Nichtbeauftragung}

Einschränkung der möglichen

Ausgabeformate

Kostenangebot überstieg das Projektbudget für die Fallstudie

kein Angebot erhalten (auch nicht nach

mehrmaliger Anfrage)

Angebot entsprach dem Projektbudget, aber

zugunsten von Dienstleister $A$ nicht

beauftragt

Kostenangebot überstieg das Projektbudget

der Fallstudie

keine OA-Veröffentlichungen im Modell

vorgesehen

kein Angebot auf Anfrage erhalten

\subsubsection{Druck- und Vertriebsdienstleister}

In allen Fällen der Studie wurden zur Durchführung der P-GP-Prozesse Druck- und Vertriebsdienstleister beauftragt.

Die Druckaufträge zur Herstellung der Autorenexemplare, der Pflichtexemplare für die DNB sowie der Verkaufsexemplare für den Buchhandel wurden in allen Fällen an denselben Dienstleister vergeben, um eine gleichbleibende Qualität für den Rezipienten sicherzustellen. Darüber hinaus wurden zum Zweck der vergleichenden Fallstudie und zur Datenerhebung für jeden Fall jeweils 3 weitere Druck- und Vertriebsdienstleister mit der Anfertigung von Musterexemplaren beauftragt. 
Tab. 6 Übersicht der beauftragten Druck- und Vertriebsdienstleister der Fallstudie

\begin{tabular}{|c|c|c|}
\hline Dienstleister & $\begin{array}{l}\text { Anwendung in } \\
\text { Fallstudie }\end{array}$ & Kostenmodell \\
\hline $\begin{array}{l}\text { E } \\
\text { ausländischer PoD-Dienstleister }\end{array}$ & Fall 1-5 & $\begin{array}{l}\text { Preiskalkulator als Offline-Anwendung } \\
\text { wird zur Verfügung gestellt, } \\
\text { Preisstaffelung pro Exemplar }\end{array}$ \\
\hline $\begin{array}{l}\text { F } \\
\text { inländische Buchdruckerei- } \\
\text { Gruppe }\end{array}$ & Fall 3 & $\begin{array}{l}\text { Preiskalkulator über Online-Account } \\
\text { verfügbar }\end{array}$ \\
\hline $\begin{array}{l}\text { G } \\
\text { inländischer Druck- und } \\
\text { Vertriebsdienstleister }\end{array}$ & Fall 2 & $\begin{array}{l}\text { Paulschalpreis pro Titel für die Nutzung } \\
\text { der Verlagssoftware, zusätzliche Kosten } \\
\text { für den Auflagendruck mit } \\
\text { Preisstaffelung pro Exemplar }\end{array}$ \\
\hline $\begin{array}{l}\mathbf{H} \\
\text { inländischer PoD- und } \\
\text { Selfpublishing-Dienstleister }\end{array}$ & Fall 1 und 4 & $\begin{array}{l}\text { Preiskalkulator frei im Internet } \\
\text { zugänglich und über Online-Account } \\
\text { verfügbar }\end{array}$ \\
\hline
\end{tabular}

Dienstleister E ist ein ausländischer PoD-Druckdienstleister mit Sitz in Westeuropa. Das Unternehmen ist spezialisiert auf die On-Demand-Produktion von Hard- und Softcover-Büchern, Magazinen und Zeitschriften im Digitaldruck ab einer Auflage von einem Exemplar. Weiterhin unterstützt das Unternehmen seine Kunden in logistischen Belangen, wie bspw. bei der Auslieferung der Druckprodukte in den Einzel- und Großhandel. Für die Kalkulation der zu erwartenden Kosten wurde ein Preiskalkulator als Offline-Anwendung zur Verfügung gestellt.

Dienstleister $F$ ist ein inländischer Druckdienstleister; mit mehreren Standorten in Deutschland gehört er zu einer internationalen Buchdruckerei-Gruppe. Als Druckdienstleister fertigt das Unternehmen für Buch- und Fachverlage sowie für öffentliche Auftraggeber Bücher, Kataloge und Kalender in niedrigen und hohen Auflagen. Außerdem bietet das Unternehmen Services zur Erstellung und Verbreitung von EBooks an. Die anfallenden Kosten können nach Freischaltung des Zugangs zum Kundenportal selbstständig online mit Hilfe eines Preiskalkulators ermittelt werden.

Bei Dienstleister $G$ handelt es sich um einen inländischen Druck- und Vertriebsdienstleister, dessen Leistungsspektrum sich speziell auf Publikationsdienstleistungen im Bereich Druck, Vertrieb, Projekt- und Datenmanagement für wissenschaftliche Publikationen von Hochschulverlagen konzentriert. Das Unternehmen bietet Hochschulverlagen eine Verlagssoftware, mit der ein Verlag seine Publikationen verwalten und steuern kann. In diesem Zusammenhang bietet Dienstleister $G$ weitere Dienstleistungen für die Publikation von Büchern an, wie bspw. eine technische Datenprüfung, die Produktion von Aushängern und Proofs, E-Book-Konver- 
tierung, ein Kalkulationstool, Grafik- und Druckdienstleistungen, sprachliches und technisches Copy Editing, ISBN-Vergabe und Plagiatscheck; außerdem Vertriebsdienstleistungen wie Pflichtbemusterung, Listung von Printausgaben im Barsortiment und Eintrag in die Kataloge aller relevanten Internetbuchhändler. Die anfallenden Kosten deckt ein Festpreis pro Publikation ab, in dem auch die Nutzung des gesamten Leistungsspektrums der Verlagssoftware enthalten ist. Zusätzliche Kosten fallen für den Auflagendruck der gedruckten Bücher an.

Dienstleister $H$ ist ein inländischer PoD- und Selfpublishing-Dienstleister mit Spezialisierung auf den On-Demand-Druck bei Verlags- und Self-Publishing-Buchtiteln. Über seine Druckdienstleistungen hinaus nutzen Verlage Dienstleister $H$ als Publikations- und Distributionspartner. Ein weiteres Angebot des Dienstleisters richtet sich speziell an Hochschulen, Universitäten und Institute, die wissenschaftliche Publikationen veröffentlichen möchten. Diesen Einrichtungen werden in verschiedenen Full-Service-Modellen Dienstleistungen speziell für wissenschaftliche Publikationen angeboten. Über die anfallenden Kosten können Verlage sich selbstständig mit Hilfe des Preiskalkulators im Kundenportal informieren.

Vor der Beauftragung der oben genannten Druck- und Vertriebsdienstleister wurden weitere recherchiert und angefragt. Tabelle 7 führt die Gründe auf, warum es in der Fallstudie jeweils nicht zur Auftragsvergabe an diese Druck- und Vertriebsdienstleister kam.

Tab. 7 Übersicht der angefragten Druck- und Vertriebsdienstleister, die nicht für die Fallstudie beauftragt wurden

\section{Dienstleister}

08

inländischer Prepress- und OnDemand-Druckdienstleister

\section{9}

inländischer Druckdienstleister mit

Fokus auf Nachhaltigkeit

\section{0}

inländischer Druckdienstleister

11

inländischer Druckdienstleister

\section{2}

inländischer Druck-Dienstleister mit Verlagsdienstleistungen

\section{Grund für Nichtbeauftragung}

spezifisches Buchformat nicht produzierbar und OnDemand-Produktion erst ab 20 Exemplaren möglich

On-Demand-Produktion ab Auflage 1 nur für Softcover möglich

Kostenangebot übersteigt das Projektbudget der Fallstudie (Anfrage für Fall 1 und 4)

On-Demand-Produktion ab Auflage 1 nicht möglich

wegen Annahmestopp von Neukunden keine Produktion möglich (Anmerkung: dieser Dienstleister wurde abseits der Fallstudie für den Druck eines Softund Hardcover-Blindbands zur Veranschaulichung der Reihengestaltung des Modellverlags beauftragt) 


\subsubsection{Veröffentlichungsplattformen für OA-Publikationen}

Wie bereits in Kapitel 1 erläutert, ist die digitale Verbreitung von OA-Büchern zwingend erforderlich, um diese sichtbar und auffindbar zu machen und somit eine schnelle Weitergabe und Volltext-Recherche zu ermöglichen. Daher wurden in Vorbereitung der Fallstudie Informationen zu geeigneten OA-Plattformen eingeholt, auf denen die elektronischen Ausgabeformate der Publikationen der Fallstudie neben der primären Veröffentlichungsplattform verbreitet werden können. Für die Publikationen der Fallstudie kamen dafür Plattformen in Frage, die die folgenden Punkte erfüllen:

- Plattformen, die bei der Auffindbarkeit und Sichtbarkeit der Produkte helfen

- Plattform, wo kostenlos oder preisgünstig OA-Bücher veröffentlicht werden können

- Plattformen mit einer guten Reputation in der OA-Community bzw. in der Wissenschaftscommunity im Allgemeinen oder in fachspezifischen Community

Diese Bedingungen wurden als projektspezifische Richtlinie für die Wahl weiterer Veröffentlichungsplattformen (vgl. Anhang A) festgelegt.

In Tabelle 8 sind die Plattformen vorgestellt, die dieser Richtlinie entsprechen und wo im Zuge des produktionsorientierten Prozesses P-EP-110 die elektronischen Ausgabeformate der Fallstudie tatsächlich hochgeladen wurden, sodass die Werke dort im OA zum Download zur Verfügung stehen. Bei einigen Plattformen war es zunächst notwendig, eine kostenpflichtige Mitgliedschaft abzuschließen (Null-Prozess: Mitgliedschaften schließen). Eine detaillierte Aufschlüsselung der hierdurch verursachten Kosten ist im Anhang D zu finden.

Tab. 8 Übersicht zu den OA-Veröffentlichungsplattformen der Fallstudie ${ }^{95}$

\begin{tabular}{lll} 
Plattform & Zugang & Kostenmodell \\
\hline OAPEN und & $-\quad$ auf individuelle Anfrage: Erhalt einer & Auswahl zwischen \\
DOAB & $\begin{array}{l}\text { Vereinbarung zur Regelung der } \\
\text { Leistungen von OAPEN und dem Verlag }\end{array}$ & $\begin{array}{l}\text { jährlicher Gebühr und } \\
\text { Gebühr pro Titel möglich } \\
\text { (vgl. Anhang D) }\end{array}$ \\
- & $\begin{array}{l}\text { Beschreibung des Peer-Review- } \\
\text { Verfahrens ist notwendig, um } \\
\text { sicherzustellen, dass es dem } \\
\text { akademischen Standard entspricht }\end{array}$ \\
& $-\begin{array}{l}\text { Publikation der Werke bei Open- } \\
\text { Content-Lizenz automatisch auf DOAB }\end{array}$ & \\
\hline
\end{tabular}




\begin{tabular}{|c|c|c|}
\hline Plattform & Zugang & Kostenmodell \\
\hline & $\begin{array}{l}\text { - Anmeldung und Veröffentlichung auf } \\
\text { der Plattform nur durch den Autor } \\
\text { selbst möglich }\end{array}$ & \\
\hline ScienceOpen & 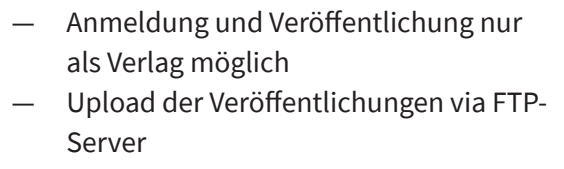 & $\begin{array}{l}\text { Einmalige } \\
\text { Einrichtungsgebühr und } \\
\text { Kosten pro Titel (vgl. } \\
\text { Anhang D) }\end{array}$ \\
\hline Qucosa & $\begin{array}{l}\text { - } \text { für Autoren, deren Institution/ } \\
\text { Universität/Hochschule Qucosa- } \\
\text { Mandant ist } \\
\text { - } \quad \text { Veröffentlichung der Publikation durch } \\
\text { Online-Eingabemaske }\end{array}$ & kostenfrei \\
\hline Unglue.it & - Accounterstellung auf der Plattform & kostenfrei \\
\hline Google Books & $\begin{array}{l}\text { - } \text { Anmeldung im Google Play Bücher- } \\
\text { Partnercenter notwendig } \\
\text { - } \\
\text { Nach Freischaltung individueller } \\
\text { Account für Veröffentlichung bei Google } \\
\text { Books }\end{array}$ & kostenfrei \\
\hline
\end{tabular}

OAPEN (Open Access Publishing in European Networks) ist einerseits eine HostingPlattform für wissenschaftliche Bücher, die von Verlagen als Repositorium benutzt werden kann, und andererseits ein Bibliothekskatalog für wissenschaftliche Bücher (mit Fokus auf den Bereich Geistes- und Sozialwissenschaften), die im Rahmen eines OA-Geschäftsmodells veröffentlicht werden. Darüber hinaus bietet OAPEN weitere Dienstleistungen für Verlage, Bibliotheken und Forschungsförderer an. ${ }^{96}$ Sofern die hier hochgeladenen Publikationen über eine Open-Content-Lizenz (wie Creative-Commons) verfügen, werden diese automatisch in den Katalog der Datenbank DOAB (Directory of Open Access Books) aufgenommen und gelistet.

Teil der Datenbank ist ein durchsuchbarer Index für peer-reviewed Monografien und Sammelwerke; verknüpft sind die Publikationen mit der Autorenwebsite oder der primären Publikationsplattform. Bereits 21.954 peer-reviewed Fachbücher und Kapitel von 323 Verlagen sind auf $D O A B$ zu finden. ${ }^{97}$

ResearchGate ist ein soziales Netzwerk und Datenbank für Forscher aus allen Bereichen der Wissenschaft. Zusätzlich kann die Plattform auch als Dokumentenserver für OA-Publikationen verwendet werden, da Autoren von wissenschaftlichen Wer- 
ken die Möglichkeit haben, ihr Werk selbstständig auf der Plattform zu veröffentlichen und somit die Sichtbarkeit ihrer Publikation zu erhöhen. Die Plattform ermöglicht ihren 15 Mio. registrierten Nutzern (in der Regel Forscher) einen Zugang zu über 130 Mio. Publikationen. ${ }^{98}$

ScienceOpen ist eine Hosting-Plattform zur Veröffentlichung, Verbreitung und Vermarktung von wissenschaftlichen Publikationen von Wissenschaftlern und wissenschaftlichen Institutionen. Anfangs noch auf die Publikation von Journals beschränkt, ermöglicht die Plattform nun auch das Hochladen von OA-Büchern. Inzwischen enthält die Datenbank von ScienceOpen bereits über 59 Mio. wissenschaftliche OA-Artikel von rund 25 Mio. Autoren. Die Plattform wird durch zahlreiche weitere Funktionen ergänzt, wie bspw. Such- und Entdeckungsfunktionen, ein offenes Peer-Review-Verfahren, die Nutzung von Altmetrics sowie Empfehlungsund Social-Sharing-Funktionen. ${ }^{99}$

Bei Qucosa handelt es sich um einen Dokumenten- und Publikationsserver, der für sächsische Institutionen und Bibliotheken zur kostenfreien Publikation und Archivierung wissenschaftlicher Werke zur Verfügung steht. Dabei garantiert die Veröffentlichung auf dem Repositorium eine weltweite Verfügbarkeit der Volltexte, eine langfristige Archivierung der Publikation, die Zitierfähigkeit durch eine konstante URL sowie als Nachweis in Katalogen, Verzeichnissen und Suchmaschinen. ${ }^{100}$

Unglue.it ist eine Plattform zur Bereitstellung von kostenfreien Büchern und Zeitschriften. Grundidee der Plattform ist das „Freikaufen“ von Werken vom Rechteinhaber durch Crowdfunding, damit E-Books schnell und kostenlos verfügbar sind. Dabei setzt der Rechteinhaber einen Preis für den Verkauf seiner Rechte fest; sobald diese Summe erreicht wurde, wird das E-Book mit einer CC-Lizenz kostenfrei zur Verfügung gestellt. ${ }^{101}$ Sofern die Publikation bereits über eine CC-Lizenz verfügt bzw. unter dieser Lizenz veröffentlicht wurde, kann kostenfrei ein Account erstellt werden, um die Werke auf der Plattform zu veröffentlichen. Über 10.000 EBooks sind bereits auf Unglue.it zu finden. ${ }^{102}$

Google Books ist eine Suchmaschine des Unternehmens Alphabet Inc., die die Volltextsuche in Millionen von Büchern ermöglicht. 2004 begann das Unternehmen mit der Digitalisierung von urheberrechtsfreien und urheberrechtlich geschützten Büchern. ${ }^{103}$

\footnotetext{
98 ResearchGate 2019

99 ScienceOpen (2) 2019

100 Qucosa 2019

101 Lesen.net 2019

102 vgl. Unglue.it 2019

103 vgl. Ryte 2019
} 
Verlagen ermöglicht die Plattform, Werke über das Google Play Bücher-Partnerprogramm im Google Play Store bzw. auf Google Books im OA zu veröffentlichen oder für einen selbst gewählten Preis zum Kauf anzubieten. Um Zugang zum Partner-Center-Account zu erhalten, muss lediglich ein Anmeldeformular ausgefüllt werden. ${ }^{104}$

\subsubsection{Metadaten-Aggregatoren}

Die Veröffentlichung der elektronischen Ausgabeformate von OA-Publikationen ist nur ein Weg, diese auffind- und sichtbar zu machen. Darüber hinaus gibt es verschiedene Anbieter, die Dienstleistungen zur Metadaten-Distribution von Publikationen anbieten, um bspw. die Titel bei Bibliotheken und Buchhändlern zu listen. Aus diesem Grund wurden für die Publikationen der Fallstudie vorab Mitgliedschaften mit den nachfolgenden Anbietern, hier als Metadaten-Aggregatoren bezeichnet, abgeschlossen (Null-Prozess: Mitgliedschaften schließen). Für den Vertrieb der gedruckten Publikationen der Fallstudie wurden außerdem mit den zwei bekanntesten Großbuchhändlern in Deutschland Konditionsvereinbarungen getroffen, die hier ebenfalls als Metadaten-Aggregatoren qualifiziert sind. Eine detaillierte Aufschlüsselung der hierfür angefallenen Kosten ist auch im Anhang D zu finden.

Für die Fallstudie wurde als Richtlinie für die Verbreitung von Metadaten für den entsprechenden Prozess M-MM-110 festgelegt (vgl. Anhang A), dass die Metadaten aller Publikationen der Fallstudie mindestens an die in Tabelle 9 genannten Metadaten-Aggregatoren übermittelt werden.

Tab. 9 Übersicht der Metadaten-Aggregatoren der Fallstudie ${ }^{105}$

\begin{tabular}{|c|c|c|c|}
\hline Aggregator & Zug & rang & Kostenmodell \\
\hline Crossref & - & $\begin{array}{l}\text { Mitgliedschaft muss } \\
\text { abgeschlossen werden }\end{array}$ & $\begin{array}{l}\text { jährliche Gebühr und Gebühr pro } \\
\text { DOI (vgl. Anhang D) }\end{array}$ \\
\hline $\begin{array}{l}\text { Verzeichnis } \\
\text { lieferbarer } \\
\text { Bücher (VLB - } \\
\text { MVB GmbH) }\end{array}$ & $\begin{array}{l}- \\
-\end{array}$ & $\begin{array}{l}\text { Mitgliedschaft muss } \\
\text { abgeschlossen werden } \\
\text { Kosten können je nach } \\
\text { Datenqualität der Titelmeldung } \\
\text { anfallen }\end{array}$ & $\begin{array}{l}\text { jährliche Gebühr und ggf. Gebühr } \\
\text { pro Titelmeldung (vgl. Anhang D) }\end{array}$ \\
\hline Newbooks & - & Vertragsschließung notwendig & $\begin{array}{l}\text { einmalige Einrichtungsgebühr und } \\
\text { jährliche Gebühr (vgl. Anhang D) }\end{array}$ \\
\hline KNV Zeitfracht & - & $\begin{array}{l}\text { Unterzeichnung von } \\
\text { Konditionsvereinbarungen }\end{array}$ & $\begin{array}{l}\text { kostenfrei, aber es muss je nach } \\
\text { Vereinbarung ein Handelsrabatt } \\
\text { von bis zu } 50 \text { \% gewährt werden }\end{array}$ \\
\hline
\end{tabular}




\begin{tabular}{lll} 
Aggregator & Zugang & Kostenmodell \\
\hline Libri & $-\quad$ Unterzeichnung von & kostenfrei, aber es muss je nach \\
& Konditionsvereinbarungen & Vereinbarung ein Handelsrabatt \\
& von bis zu 50\% gewährt werden \\
\hline
\end{tabular}

Crossref ist eine Non-Profit-Organisation, deren Ziel es ist, „Forschungsergebnisse einfach auffindbar, zitierbar, verlinkbar und bewertbar zu machen und die Kommunikation im Wissenschaftsbetrieb insgesamt zu verbessern. Hierzu stellt Crossref eine Reihe von Produkten und Services bereit [...]“. ${ }^{106}$ In erster Linie fungiert die Organisation als Registrierungsagentur für DOIs. ${ }^{107}$

Das VLB ist ein Katalog von Buchpublikationen der gesamten Buchbranche. Titeldaten werden hier allen Buchhändlern, Bibliotheken, Online-Plattformen und Webshops für Recherchen und Bestellungen zur Verfügung gestellt. Mit der Meldung von Buchtiteln im VLB können die Metadaten, u. a. im Branchenstandard ONIX, zur weiteren Distribution exportiert werden. Zudem werden alle Buchtitel des VLB automatisch für die Pflichtabgabe an die DNB erfasst. ${ }^{108}$

Newbooks ist ein Dienstleister zur Verbreitung von Metadaten zu wissenschaftlichen Buchpublikationen. Nach redaktioneller Prüfung und Klassifikation werden die Titeldaten der Verlagspublikationen in Approval Plans, Newsletter-Neuerscheinungsdienste, Literaturverwaltungsprogramme von Bibliotheken, Vorschlagslisten, Katalogdatenexporte und Web-Services aufgenommen. Darüber hinaus bietet der Aggregator noch weitere Services an, bspw. die automatisierte ONIX-Titelmeldung an Buchgroßhändler und Spezialshops, Klassifikation und Verschlagwortung, Titel-Monitoring, Novitätendienste, Web-Entwicklung und E-Procurement-Dienste für Buchhandlungen. ${ }^{109}$

Der Großbuchhändler KNV Zeitfracht ist auf die Bereiche Verlagsauslieferung, Barsortiment, Logistik und Transport spezialisiert. Von seinen Dienstleistungen profitieren neben Buchhandlungen, die zeitnah beliefert werden, v. a. Verlage von Services wie Verbreitung der Metadaten, Lagerung, Bestellannahme, Versand und Fakturierung. ${ }^{110}$

Die Dienstleistungen des Großbuchhändlers Libri sind auf das Barsortiment und die Logistik im Buchhandel fokussiert. Dabei lagert, kommissioniert und liefert

\footnotetext{
105 Stand Okt. 2019

106 Wikipedia (3) 2019

107 vgl. Wikipedia (3) 2019

108 vgl. Buchhandel.de 2019

109 Newbooks Solutions GmbH 2019

110 KNV Zeitfracht 2019
} 
Libri die Verlagspublikationen. Weiterhin bietet der Großbuchhändler Buchhandlungen verschiedene Bestell- und Logistiksysteme zur Optimierung der Sortimentsorganisation und -beschaffung an. ${ }^{111}$ Der Online-Händler Amazon bezieht bei Buchbestellungen aus dem Barsortiment von Libri und erstellt aus dessen Katalogen Einträge zum Produkt auf seiner Plattform.

\subsubsection{Dienstleister für Fach- und Sprachlektorat}

Im Rahmen der hier vorgestellten Fallstudie wurde lediglich im Fall 5 ein Sprachlektorat (Prozess P-Ca-110) beauftragt. Allerdings wurden zudem zwei vom Projektteam in englischer Sprache verfasste Dokumente von Fremdsprachenlektoren redigiert (American Journal Expert und proof-reading-service.com). Die hieraus gewonnenen Erkenntnisse sowie die der Analyse weiterer Anbieter für Fach- und Sprachlektorate werden in Tabelle 10 aufgelistet:

Tab. 10 Übersicht zu Dienstleister für Fach- und Sprachlektorat

\begin{tabular}{|c|c|c|}
\hline Dienstleister & Services (u. a.) & Kostenmodell \\
\hline Scribbr ${ }^{112}$ & $\begin{array}{l}\text { Lektorat, Korrektorat, } \\
\text { Erstellung von } \\
\text { Literaturverzeichnissen, } \\
\text { Plagiatsprüfung sowie } \\
\text { Wissensdatenbank }\end{array}$ & $\begin{array}{l}\text { _ Preiskalkulator auf Website } \\
\text { verfügbar } \\
\text { _ } \quad \text { Lektorat \& Korrektorat: } 0,014 € / \\
\text { Wort }\end{array}$ \\
\hline studi-lektor ${ }^{113}$ & $\begin{array}{l}\text { Fachlektorat, } \\
\text { Sprachlektorat, Lektorat } \\
\text { mit Software (Quickfix), } \\
\text { Coaching, Layout }\end{array}$ & 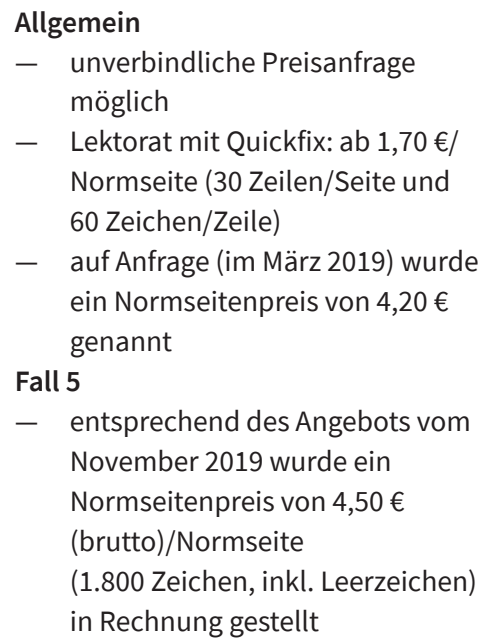 \\
\hline
\end{tabular}




\begin{tabular}{|c|c|c|c|}
\hline Dienstleister & Services (u. a.) & Kos & tenmodell \\
\hline $\operatorname{acadoo}^{114}$ & $\begin{array}{l}\text { wissenschaftliches } \\
\text { Lektorat }\end{array}$ & - & $\begin{array}{l}\text { Preis muss individuell angefragt } \\
\text { werden } \\
\text { auf Anfrage (im März 2019) wurde } \\
\text { ein Seitenpreis von } 15 € \text { genannt }\end{array}$ \\
\hline $\begin{array}{l}\text { Wissenschaftslektorat } \\
\text { Zimmermann }^{115}\end{array}$ & $\begin{array}{ll}\text { - } & \text { Korrektorat } \\
\text { - } & \text { Fachlektorat } \\
\text { - } & \text { Plagiatskontrolle } \\
\text { - } & \text { Textredaktion } \\
\text { - } & \text { Publikationslayout }\end{array}$ & $\begin{array}{l}- \\
-\end{array}$ & $\begin{array}{l}\text { Angebot muss individuell } \\
\text { angefragt werden } \\
\text { Korrektorat ab 2,80 €/Normseite } \\
\text { (1.500 Zeichen inkl. Leerzeichen) } \\
\text { zzgl. } 19 \% \text { MwSt. } \\
\text { stilistisches Lektorat ab 3,80 €/ } \\
\text { Normseite, zzgl. } 19 \% \text { MwSt. } \\
\text { Fachlektorat ab 4,80 €/ } \\
\text { Normseite, zzgl. } 19 \% \text { MwSt. }\end{array}$ \\
\hline Uni Korrektorat ${ }^{116}$ & $\begin{array}{ll}- & \text { Korrektorat } \\
- & \text { wissenschaftliches } \\
& \text { Lektorat }\end{array}$ & $\begin{array}{l}- \\
- \\
- \\
-\end{array}$ & $\begin{array}{l}\text { Probelektorat kostenlos möglich } \\
\text { Angebot muss individuell } \\
\text { angefragt werden } \\
\text { Korrektorat: } 2,00 € / \text { Normseite } \\
\text { (1.650 Zeichen inkl. Leerzeichen), } \\
\text { zzgl. } 19 \% \text { MwSt. } \\
\text { Lektorat: 3,50 €/Normseite, zzgl. } \\
19 \% \text { MwSt. }\end{array}$ \\
\hline 1a Studi ${ }^{117}$ & $\begin{array}{ll}\text { - } & \text { Lektorat } \\
- & \text { Plagiatsprüfung } \\
- & \text { Formatierung } \\
- & \text { Erstellen von } \\
& \text { Verzeichnissen }\end{array}$ & - & $\begin{array}{l}\text { Preisrechner auf Website } \\
\text { verfügbar } \\
\text { Unterschiedliche Preise für } \\
\text { Studierende und B2B/Privat } \\
\text { sowie Staffelung pro Seite - auf } \\
\text { Website ersichtlich } \\
\text { Buchlektorat } 60 \text { Seiten: } 287,28 €\end{array}$ \\
\hline Reindl Lektorat ${ }^{118}$ & $\begin{array}{ll}- & \text { Korrektorat } \\
- & \text { Lektorat }\end{array}$ & - & $\begin{array}{l}\text { Lektorat: } 6,00 € / \text { Normseite (ca. } \\
1.800 \text { Zeichen) } \\
\text { Wissenschaftliche Texte: } 50 € / h\end{array}$ \\
\hline Lektorat Frankfurt ${ }^{119}$ & $\begin{array}{ll}\text { - } & \text { Korrektorat } \\
\text { - } & \text { Basislektorat } \\
\text { - } & \text { Ganzlektorat } \\
\text { - } & \text { Werbekorrektorat }\end{array}$ & - & $\begin{array}{l}\text { Ermäßigung für Studenten } \\
\text { Korrektorat: 2,45 €/Normseite } \\
\text { (1.600 Zeichen inkl. Leerzeichen), } \\
\text { zzgl. } 19 \% \text { MwSt. }\end{array}$ \\
\hline
\end{tabular}

114 acadoo 2019

115 Wissenschaftslektorat Zimmermann 2019

116 Kuntscher 2019

117 1a-Studi 2019

118 Natali Reindl Lektorat 2019

119 Lektorat Frankfurt 2019 


\begin{tabular}{|c|c|c|c|c|}
\hline Dienstleister & Se & vices (u. a.) & Ko & tenmodell \\
\hline & & & $\begin{array}{l}- \\
-\end{array}$ & $\begin{array}{l}\text { Basislektorat: } 3,45 € / \text { Normseite } \\
\text { Ganzlektorat: } 3,85 € / \text { Normseite } \\
\text { Werbekorrektorat: } 2,65 € / \\
\text { Normseite }\end{array}$ \\
\hline $\begin{array}{l}\text { Akademisches } \\
\text { Lektorat }^{120}\end{array}$ & $\begin{array}{l}- \\
- \\
- \\
-\end{array}$ & $\begin{array}{l}\text { Korrektorat } \\
\text { Lektorat } \\
\text { wissenschaftliches } \\
\text { Lektorat } \\
\text { Plagiatsprüfung } \\
\text { Übersetzung }\end{array}$ & - & $\begin{array}{l}\text { unverbindliche Preisanfrage } \\
\text { möglich } \\
\text { Lektorat Masterarbeit: Lektorat } \\
\text { Fachbuch: 7,00 €/Normseite } \\
\text { (2.000 Zeichen, inkl. } \\
\text { Leerzeichen), zzgl. } 7 \% \text { MwSt. } \\
\text { Lektorat Dissertation: 9,00 €/ } \\
\text { Normseite, zzgl. } 7 \% \text { MwSt. } \\
\text { Lektorat Fachbuch: 5,00 €/ } \\
\text { Normseite, zzgl. } 7 \% \text { MwSt. }\end{array}$ \\
\hline Korrektorius $^{121}$ & $\begin{array}{l}- \\
- \\
-\end{array}$ & $\begin{array}{l}\text { Korrektorat } \\
\text { Lektorat } \\
\text { wissenschaftliches } \\
\text { Lektorat } \\
\text { Textredaktion }\end{array}$ & $\begin{array}{l}- \\
- \\
- \\
-\end{array}$ & $\begin{array}{l}\text { Angebot muss individuell } \\
\text { angefragt werden } \\
\text { Korrektur: } 4,00 € \text { netto/ } \\
\text { Normseite ( } 1.500 \text { Zeichen, inkl. } \\
\text { Leerzeichen) } \\
\text { Lektorat: } 5,00 € \text { netto/Normseite } \\
\text { Wissenschaftslektorat: } 7,50 € \\
\text { netto/Normseite }\end{array}$ \\
\hline $\begin{array}{l}\text { American Manuscript } \\
\text { Editors }^{122}\end{array}$ & $\begin{array}{l}- \\
- \\
-\end{array}$ & $\begin{array}{l}\text { General academic } \\
\text { Editing } \\
\text { Journal Manuscript } \\
\text { Editing } \\
\text { Dissertation and } \\
\text { Thesis editing }\end{array}$ & $\begin{array}{l}- \\
- \\
- \\
-\end{array}$ & $\begin{array}{l}\text { Preise auf Website ersichtlich } \\
\text { Standardpreis Journal } \\
\text { Manuscript Editing: } 0.045 \text { \$/Wort } \\
\text { Thesis/Dissertation Editing: } 0.025 \\
\text { \$/Wort } \\
\text { General Academic Editing: } 0.03 \$ / \\
\text { Wort }\end{array}$ \\
\hline $\begin{array}{l}\text { American Journal } \\
\text { Expert }^{123}\end{array}$ & $\begin{array}{l}- \\
- \\
-\end{array}$ & $\begin{array}{l}\text { englischsprachige } \\
\text { Redaktion } \\
\text { Übersetzung } \\
\text { Wissenschaftliche } \\
\text { Abbildungen/ } \\
\text { Illustrationen }\end{array}$ & $\begin{array}{l}- \\
- \\
- \\
-\end{array}$ & $\begin{array}{l}\text { Preis pro Seite über Kalkulator } \\
\text { auf Website berechenbar } \\
\text { Unterscheidung in Standard } \\
\text { Editing und Premium Editing } \\
\text { Standard Editing für } 500 \text { Wörter: } \\
75 \$ \\
\text { Premium Editing für } 500 \text { Wörter: } \\
240 \$\end{array}$ \\
\hline
\end{tabular}

120 Akademischeslektorat.com 2019

121 Lektorat Korrektorius 2019

122 Grammar Labs, LLC 2019

123 Research Square 2019 


\begin{tabular}{lll} 
Dienstleister & Services $($ u. a.) & Kostenmodell \\
\hline proof-reading- & - Korrektorat für & $-12.99 £ / 1.000$ Wörter oder \\
service.com ${ }^{124}$ & wissenschaftliche & -1.299 UK Pence Sterling pro Wort \\
& Texte von & - für 841 Wörter $=13.10 £$ (inkl. VAT \\
& Muttersprachlern & $20 \%)^{125}$ \\
& (Englisch) & \\
\hline
\end{tabular}

\subsubsection{Softwareanbieter}

Wissenschaftliche Arbeiten sollten auf ihre wissenschaftliche Qualität geprüft werden, bevor sie im Hochschulverlag publiziert werden; das sieht auch das hier entwickelte Workflow-Modell vor (vgl. Kapitel 3). Vor dem Peer-Review-Verfahren ist deshalb eine Plagiatsprüfung zu empfehlen (vgl. Prozess I-Qe-100). Hierfür offerieren verschiedene Anbieter Dienstleistungen bzw. Software, um die Plagiatsprüfung eigenständig im Verlag oder an der Hochschule vorzunehmen. Eine Anbieterauswahl wurde im Vorfeld der Fallstudie recherchiert; die Ergebnisse sind in der nachfolgenden Tabelle 11 zusammengefasst.

Für die Fälle der Studie war keine Plagiatsprüfung notwendig, da es sich um Graduierungsarbeiten handelt, für die eine (inhaltliche) Qualitätssicherung (I-QeProzesse) bereits vor der Durchführung des Publikationsworkflows erfolgte.

Tab. 11 Übersicht der recherchierten Anbieter von Plagiatssoftware

\begin{tabular}{|c|c|c|}
\hline Anbieter & Funktionsumfang & Kosten \\
\hline $\begin{array}{l}\text { iThenticate / } \\
\text { Turnitin }\end{array}$ & $\begin{array}{l}\text { - } \text { greift bei der Plagiatsprüfung auf } \\
\text { ein Datenbank-Volumen von } \\
60 \text { Mrd. Webseiten, } 49 \text { Mio. } \\
\text { wissenschaftliche Inhalte und } \\
105 \text { Mio. Nachrichtenseiten, } \\
\text { Magazine und Bücher zu } \\
-\quad \text { Erstellung eines Prüfberichts mit } \\
\text { Ähnlichkeitsfaktoren und } \\
\text { Inhaltsverfolgungen } \\
-\quad \text { ermöglicht die Integration von } \\
\text { Content Management Systemen } \\
\text { und Manuskript- } \\
\text { Verfolgungssystemen }\end{array}$ & $\begin{array}{l}\text { Preisstaffelung in Kostenpakete: }{ }^{127} \\
-\quad \text { Einzelpaket: } 100 \text { \$; zur } \\
\text { Einreichung eines einzigen } \\
\text { Manuskripts. Eine } \\
\text { Dokumentgutschrift wird für } \\
\text { die Vorlage von } 25.000 \\
\text { Wörtern und darunter } \\
\text { berechnet. Die Gutschriften } \\
\text { sind } 12 \text { Monate ab Kaufdatum } \\
\text { gültig. } \\
\text { 3 Pakete: } 300 \text { \$; zur } \\
\text { Einreichung mehrerer } \\
\text { Manuskripte oder eines }\end{array}$ \\
\hline
\end{tabular}

124 Proof-Reading-Service.com 2019

125 Der Preis ist der Rechnung an das Forschungsprojekt vom 16. Mai 2019 entnommen.

126 Turnitin, LLC. 2019

127 Turnitin, LLC. 2019 


\section{Anbieter Funktionsumfang Kosten}

\begin{tabular}{|c|c|c|c|}
\hline & & & $\begin{array}{l}\text { Manuskripts mit bis zu } 75.000 \\
\text { Wörtern. Die Gutschriften sind } \\
12 \text { Monate ab Kaufdatum } \\
\text { gültig. } \\
-\quad \text { Mengenrabatt für } \\
\text { Institutionen; auf Anfrage }\end{array}$ \\
\hline Scribbr & $\begin{array}{l}- \\
-\end{array}$ & $\begin{array}{l}\text { Kooperation mit Turnitin (gewährt } \\
\text { Zugriff auf die umfangreichen } \\
\text { Datenbank-Quellen von Turnitin) } \\
\text { vielsprachige Texterkennung } 128\end{array}$ & $\begin{array}{l}\text { Preisstaffelung nach Manuskript- } \\
\text { Umfang: }{ }^{129} \\
\text { - } \quad \text { Kleine Arbeit: } 14,95 € \text { (bis zu } \\
7.500 \text { Wörter) } \\
-\quad \text { Normale Arbeit: } 24,95 €(7.500 \\
\text { bis } 50.000 \text { Wörter) } \\
-\quad \text { Große Arbeit: } 34,95 €(50.000+ \\
\text { Wörter) }\end{array}$ \\
\hline Urkund & & $\begin{array}{l}\text { Abonnement enthält System zur } \\
\text { Plagiatsprüfung, Integrationsplan, } \\
\text { Informationsmaterial und } \\
\text { Handbücher, Training-Angebote } \\
\text { und Anwender-Support }\end{array}$ & $\begin{array}{l}\text { - } \text { Geschäftsmodell = } \\
\text { Abonnement (jährliche } \\
\text { Gebühr richtet sich nach } \\
\text { Anzahl der Vollzeit- } \\
\text { Studenten) }{ }^{131} \\
\text { - } \\
\text { Für einzelne Prüfung } 199 €^{132}\end{array}$ \\
\hline Copyscape & - & $\begin{array}{l}\text { Premium-Version bietet bei der } \\
\text { Plagiatsprüfung zusätzlich die } \\
\text { Überprüfung der Originalität durch } \\
\text { Kopieren und Einfügen, die } \\
\text { Stapelsuche, die private } \\
\text { Indexerstellung, die Fallverfolgung, } \\
\text { eine API und die WordPress- } \\
\text { Integration. }{ }^{133}\end{array}$ & $\begin{array}{ll}\text { _ } & \text { kostenlose Grundversion der } \\
& \text { Plagiatsprüfung } \\
\text { - } & \text { Variables Preissystem bei der } \\
& \text { Premium-Version: Nur } 0,03 € \\
\text { pro Suche nach den ersten } \\
200 \text { Wörtern }+0,01 € \text { pro } \\
\text { zusätzliche } 100 \text { Wörter }{ }^{134}\end{array}$ \\
\hline PlagAware & & $\begin{array}{l}\text { Einschränkung bei der } \\
\text { Plagiatsprüfung: sucht nur } \\
\text { Plagiate von Online-Texten und } \\
\text { hochschuleigenen Texten } \\
\text { bietet weitere Funktion, bspw. } \\
\text { Überwachung von Texten und }\end{array}$ & 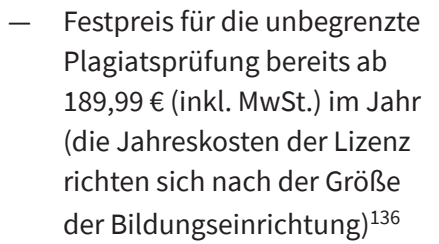 \\
\hline
\end{tabular}

128 Scribbr B.V. (2) 2019

129 Scribbr B.V. (2) 2019

130 Urkund 2019

131 Urkund 2019

132 Der Preis stammt von einem Angebot für das Forschungsprojekt vom 08.05.2019.

133 Indigo Stream Technologies 2019

134 Indigo Stream Technologies 2019 


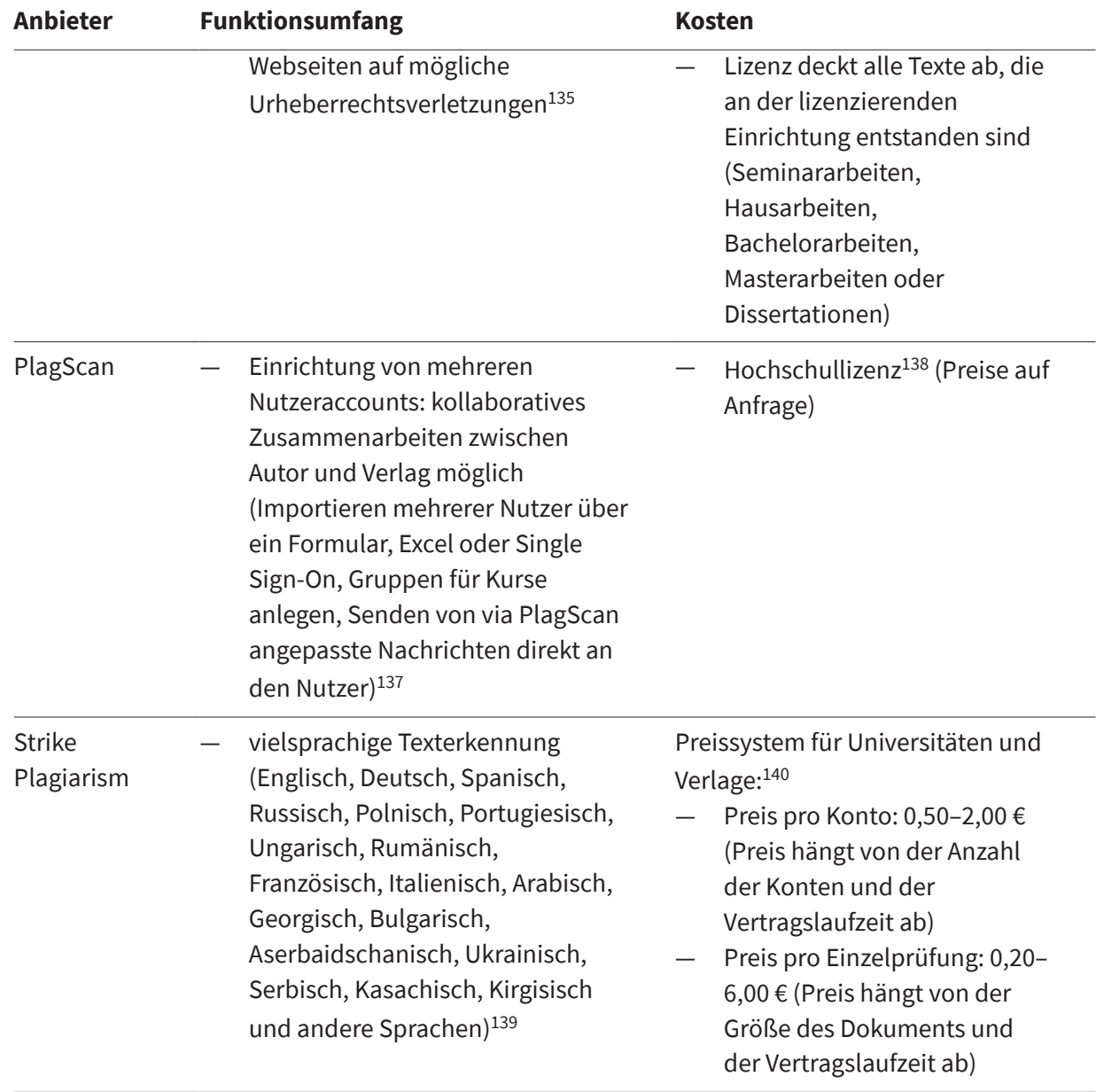

\section{Content-Management-Systeme}

Content-Management-Systeme (CM) können im Hochschulverlag unterstützend verwendet werden, um die Erstellung, Bearbeitung und Verwaltung von Content zentral zu managen. Für die Fallstudie wurde kein CM-Systeme verwendet, da aufgrund der geringen Anzahl an Publikationen und dem geringen verfügbaren Budget eine Anschaffung nicht realisierbar und zweckmäßig erschien. In Tabelle 12 werden einige der recherchierten Anbieter für CM-Systeme aufgeführt.

\footnotetext{
135 PlagAware (1) 2019

136 PlagAware (2) 2019

137 PlagScan (1) 2019

138 PlagScan (2) 2019

139 StrikePlagiarism.com (2) 2019

140 StrikePlagiarism.com (1) 2019
} 
Tab. 12 Übersicht zu Anbietern von Content-Management-Systemen

\begin{tabular}{|c|c|c|}
\hline Anbieter & Funktionsumfang & Kosten \\
\hline $\begin{array}{l}\text { Open } \\
\text { Monograph } \\
\text { Press }\end{array}$ & $\begin{array}{l}\text { - Software-Plattform zur Organisation des } \\
\text { Redaktionsverfahrens für wissenschaftliche } \\
\text { Publikationen im Buchformat } \\
\text { - } \text { unterstützt die Verwaltung von } \\
\text { Begutachtungsverfahren, Lektorat, Darstellung eines } \\
\text { Katalogs sowie die Herstellung und Publikation von } \\
\text { Büchern }\end{array}$ & kostenfrei \\
\hline xPublisher & $\begin{array}{l}\text { - Multichannel-Publishing im XML-Format } \\
\text { - } \text { Content- und Media-Asset-Management } \\
\text { - } \text { Workflow-Management } \\
\text { - } \\
\text { - } \\
\text { Printach- und Übersetzungsmanagement }\end{array}$ & $\begin{array}{l}\text { Preismodell } \\
\text { nicht } \\
\text { ersichtlich }\end{array}$ \\
\hline SiteFusion & $\begin{array}{ll}\text { - } & \text { medienneutrale Verwaltung von Content und } \\
& \text { Metadaten } \\
- & \text { Anbindung an XML-Editoren } \\
- & \text { Darstellung von Workflows } \\
- & \text { zentrale Verwaltung von Rechten, Rollen, Nutzer und } \\
& \text { Kontakten }^{143}\end{array}$ & $\begin{array}{l}\text { Preismodell } \\
\text { nicht } \\
\text { ersichtlich }\end{array}$ \\
\hline knkVerlag & $\begin{array}{l}\text { - Integration eines CRM-Systems } \\
- \text { Herstellung und Projektmanagement } \\
-\quad \text { Lektorat-, Manuskript- und Projektverwaltung } \\
-\quad \text { Programmplanung und -steuerung } \\
-\quad \text { Redaktions- und Beitragsverwaltung } \\
-\quad \text { Webshop-Anbindungen } \\
-\quad \text { Veranstaltungsmanagement }^{144}\end{array}$ & $\begin{array}{l}\text { Preismodell } \\
\text { nicht } \\
\text { ersichtlich }\end{array}$ \\
\hline klopotek & $\begin{array}{l}\text { - Produktplanungs- und Managementsystem für } \\
\text { interne wie externe Arbeitsprozesse } \\
\text { - } \quad \text { Titelmanagementsystem, Produktmarketing und } \\
\text { Herstellung } \\
\text { - } \quad \text { Lizenzgeschäft und Honorarabrechnung } \\
\text { - }\end{array}$ & $\begin{array}{l}\text { Preismodell } \\
\text { nicht } \\
\text { ersichtlich }\end{array}$ \\
\hline
\end{tabular}

\footnotetext{
141 Freie Universität Berlin 2019

142 Xpublisher GmbH 2019

143 SiteFusion GmbH 2019

144 knk Business Software AG 2019

145 Klopotek AG 2019
} 


\section{Customer-Relationship-Management-Systeme}

Mit Hilfe von Customer-Relationship-Management-Systemen (CRM) können Hochschulverlage Kontaktdaten von Autoren, Dienstleistern und anderen Stakeholdern erfassen und verwalten. Zudem bieten solche Systeme andere für den Verlag hilfreiche Funktionen, etwa Analyse- und Reporting-Tools oder Kalender- und Notizfunktionen. Zudem können sie Verlagshierarchien und Prozessabläufe abbilden.

Aufgrund des begrenzten Projektbudgets war es nicht möglich, ein CRM-System für die Fallstudie anzuschaffen. Ein großer Teil der Funktionen des Leistungsumfangs der recherchierten Anbieter wurde daher mit vorhandener Software der Hochschule (v. a. Microsoft Office, Excel und Outlook) und anderer kostenloser Tools, wie Trello, Slack und Google Drive, realisiert. Tabelle 13 sind die recherchierten Anbieter von CRM-Systemen, Funktionsumfang und Kosten zu entnehmen.

Tab. 13 Übersicht zu Anbietern von Customer-Relationship-Management-Systemen

\begin{tabular}{|c|c|c|c|}
\hline Anbieter & Fur & ktionsumfang & Kosten \\
\hline ZOHO CRM & $\begin{array}{l}- \\
- \\
- \\
-\end{array}$ & $\begin{array}{l}\text { zahlreiche Analyse-Tools } \\
\text { Darstellung von Prozessabläufen } \\
\text { möglich } \\
\text { umfangreiche Social-CRM- } \\
\text { Funktionen (E-Mail, Live-Chat, } \\
\text { Telefon, Social Media) } \\
\text { Integration von Unternehmen-Apps } \\
\text { (z. B. Slack, Microsoft Office, } \\
\text { Twitter) }^{146}\end{array}$ & $\begin{array}{l}\text { 4-stufiges Preismodell (jährliche } \\
\text { Abrechnung, steigender } \\
\text { Funktionsumfang): }{ }^{147} \\
\text { — Standard: } 12 € \text { pro Benutzer/ } \\
\text { Monat } \\
\text { - Professional: } 20 € \text { pro } \\
\text { Benutzer/Monat } \\
\text { - Enterprise: } 35 € \text { pro Benutzer/ } \\
\text { Monat } \\
\text { Ultimate-Edition: } 100 € \text { pro } \\
\text { Benutzer/Monat } \\
\text { kostenlose } 15 \text {-tätige } \\
\text { Testversion }\end{array}$ \\
\hline $\begin{array}{l}\text { Salesforce } \\
\text { CRM }\end{array}$ & $\begin{array}{l}- \\
- \\
- \\
-\end{array}$ & $\begin{array}{l}\text { hoch skalierbar } \\
\text { anpassbar an alle Bedürfnisse } \\
\text { online-basiert (daher von überall } \\
\text { aus erreichbar) } \\
\text { soziale Medien sind aus dem System } \\
\text { heraus nutzbar } \\
\text { Daten in der Cloud verschlüsselt }{ }^{148}\end{array}$ & $\begin{array}{l}\text { 4-stufiges Preismodell (jährliche } \\
\text { Abrechnung, steigender } \\
\text { Funktionsumfang): }{ }^{149} \\
\text { - Essentials: sofort } \\
\text { einsatzbereites CRM für bis zu } \\
5 \text { Anwender; } 25 € \text { pro } \\
\text { Benutzer/Monat } \\
\text { - Lightning Professional: } \\
\text { Komplettes CRM für Teams }\end{array}$ \\
\hline
\end{tabular}

146 Zoho Corporation Pvt. Ltd. (1) 2019

147 Zoho Corporation Pvt. Ltd. (1) 2019

148 salesforce.com Germany GmbH (1) 2019

149 salesforce.com Germany GmbH (2) 2019 


\begin{tabular}{|c|c|c|c|}
\hline Anbieter & Funktionsumfang & & ten \\
\hline & & - & $\begin{array}{l}\text { jeder Größe; } 75 € \text { pro } \\
\text { Benutzer/Monat } \\
\text { Enterprise: Flexibel } \\
\text { anpassbares CRM für den } \\
\text { Vertrieb } 150 € \text { pro Benutzer/ } \\
\text { Monat } \\
\text { Lightning Unlimited: } \\
\text { Unbeschränkte CRM- } \\
\text { Funktionen und Rund-um- } \\
\text { die-Uhr-Support } 300 € \text { pro } \\
\text { Benutzer/Monat } \\
\text { kostenlose 30-tätige } \\
\text { Testversion }\end{array}$ \\
\hline HubSpot & $\begin{array}{l}\text { - grundlegende Funktionen wie } \\
\text { Kontaktverwaltung, Kalender- und } \\
\text { E-Mail-Import, Anlegen von } \\
\text { „Deals“150 }\end{array}$ & - & $\begin{array}{l}\text { kostenfrei } \\
\text { Möglichkeit, } \\
\text { Funktionsumfang } \\
\text { kostenpflichtig zu } \\
\text { erweitern }^{151}\end{array}$ \\
\hline Bitrix 24 & 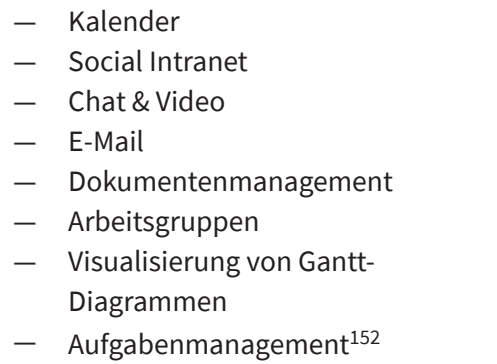 & - & $\begin{array}{l}\text { kostenfrei } \\
\text { Möglichkeit, } \\
\text { Funktionsumfang } \\
\text { kostenpflichtig zu } \\
\text { erweitern }^{153}\end{array}$ \\
\hline Vtiger CRM & $\begin{array}{ll}\text { - } & \text { Abbildung von } \\
& \text { Unternehmenshierarchien } \\
- & \text { Analyse- und Report-Tools } \\
- & \text { Kalender- und Notizfunktionen } \\
- & \text { alle Plug-ins und Add-ons sind } \\
& \text { ebenfalls Open Source }\end{array}$ & - & kostenfrei \\
\hline
\end{tabular}

150 HubSpot, Inc. (1) 2019

151 HubSpot, Inc. (2) 2019

152 Bitrix, Inc. (1) 2019

153 Bitrix, Inc. (2) 2019

154 Different Solutions GmbH 2019 


\subsection{Durchführung der Fallstudie}

Nachdem die in der Fallstudie umzusetzenden Manuskripte (Fälle), die beauftragten Dienstleister und Aggregatoren sowie die genutzten OA-Veröffentlichungsplattformen vorgestellt wurden, folgt nun die Dokumentation der Anwendung des Workflow-Modells auf die ausgewählten Fallbeispiele.

Hierfür wird entsprechend der Workflow-Prozesse beschrieben, wie die Fälle durchgeführt wurden und wer die entsprechenden Workflow-Rollen eingenommen hat. Die Reihenfolge der Beschreibung entspricht dem tatsächlichen zeitlichen Ablauf der Publikationsworkflows.

\subsubsection{Fall 1: CRC-Workflow}

\begin{tabular}{ll} 
Art des Content & Graduierungsarbeit - Masterarbeit \\
\hline Fakultät & Architektur und Sozialwissenschaften \\
\hline Studiengang & Architektur \\
\hline Titel & $\begin{array}{l}\text { Stadt als Schule. Nachbarschaftszentrum und Gemeinschaftsschule an } \\
\text { der alten Feuerwache in Anger-Crottendorf, Leipzig }\end{array}$ \\
\hline Workflow-Variante & CRC-Workflow
\end{tabular}

Prozess I-Ka-100 bis I-Ka-130

Der Content des Falls 1 wurde - wie in Kapitel 4.1 beschrieben - zum Zweck der vergleichenden Fallstudie nach bestimmten Kriterien ausgewählt. Daher wurde von vornherein ausgeschlossen, dass sich Content-Urheber oder-Lieferanten mit Publikationsanfragen an das Forschungsprojekt wenden können. Die Prozesse I$\mathrm{Ka}-100$ bis $\mathrm{I}-\mathrm{Ka}-130$ waren daher für keinen Fall der hier beschriebenen Studie relevant.

\section{I-Ka-200: Nach geeigneten Content-Urhebern recherchieren}

Wie in Kapitel 4.1 beschrieben, wurden für die Fälle der Studie geeignete ContentUrheber durch einen Aufruf an Vertreter der Fakultäten der HTWK Leipzig akquiriert, ihrer Meinung nach zur Publikation geeignete Manuskripte einzureichen. Zur Begrenzung der infrage kommenden Manuskripte fand die im Vorfeld festgelegte Richtlinie für die Konzept-Annahme (vgl. Anhang A) Anwendung.

Durch dieses Vorgehen ließ sich eine geeignete Arbeit aus dem Fachbereich Sozialwissenschaften finden. Allerdings fiel die Entscheidung letztlich gegen dieses Manuskript und zugunsten der Arbeit, die in der Fallstudie als Fall 4 verwirklicht wurde. Beide Manuskripte ähnelten sich hinsichtlich ihrer Komplexität, jedoch lag in Fall 4 die Zustimmung des Content- Urhebers zur Veröffentlichung bereits vor. 
Schließlich wurde aktiv bei Professoren des Fachbereichs Architektur nachgefragt, ob sie Abschlussarbeiten empfehlen können, die geeignet seien, um als Monografie publiziert zu werden. Daraufhin wurde die Graduierungsarbeit des Falls 1 empfohlen.

Beteiligte Rolle/n ausgeführt von

Content-Abnehmer Mitarbeiter der Hochschulbibliothek und Projektmitarbeiter 1

Content-Lieferant Mitarbeiter des Prüfungsamts und Professoren der Fakultät

\section{I-Ka-210: Content-Urheber anfragen}

Der Content-Urheber des Falls 1 wurde kurz darauf per E-Mail kontaktiert, über das Forschungsprojekt informiert und befragt, ob er Interesse an der Publikation seiner Arbeit im Rahmen der Fallstudie habe.

Beteiligte Rolle/n ausgeführt von

Content-Abnehmer Projektmitarbeiter 1

Content-Urheber Autor der Graduierungsarbeit von Fall 1

\section{I-Ka-220: Mit Content-Urheber über Publikation sprechen}

Der Content-Urheber bekundete Interesse an der Publikation seiner Abschlussarbeit. In einem Telefongespräch wurde der Inhalt der Arbeit besprochen und der Content-Urheber durch den Content-Abnehmer über den voraussichtlichen Publikationsablauf informiert.

Beteiligte Rolle/n

Content-Abnehmer

Content-Urheber ausgeführt von

$$
\text { Projektmitarbeiter } 1
$$

Autor der Graduierungsarbeit von Fall 1

\section{I-Cv-100: Content einfordern}

Nachdem das Einverständnis zur Publikation des Content-Urhebers vorlag, forderte der Content-Abnehmer die PDF-Datei der Graduierungsarbeit an, die der ContentUrheber - rund einen Monat nach Beginn der Suche nach für die Fallstudie geeignetem Content - per E-Mail übermittelte. 
Beteiligte Rolle/n ausgeführt von

Content-Abnehmer

Projektmitarbeiter 1

Content-Urheber

Autor der Graduierungsarbeit von Fall 1

\section{I-Cv-110: Content prüfen}

Beim darauffolgenden Treffen des Projektteams wurde das Manuskript zunächst gemeinsam analysiert und geprüft, ob es der folgenden Richtlinie für die ContentVereinnahmung (vgl. Anhang A) entspricht und somit tatsächlich als Fall für die Fallstudie in Frage kommt:

- Das Manuskript ist vollständig. Alle Kapitel sind dem Inhaltsverzeichnis entsprechend vorhanden.

- Der Umfang des Manuskripts ist ausreichend, um als gedrucktes Buch publiziert zu werden.

- Das Manuskript kann im OA veröffentlicht werden.

- Das Manuskript weist verschiedene Inhaltselemente auf (Abbildungen, Tabellen, mathematische Formeln, Quellcode etc.).

- Das Manuskript eignet sich, um eine bestimmte Workflow-Variante zu demonstrieren.

In Zuge dieser Prüfung wurde ermittelt, dass der Content dieses Falles als Workflow-Variante CRC-Workflow umgesetzt werden sollte (vgl. Kapitel 4.1). Da auch die anderen Aspekte der Richtlinie für die Content-Vereinnahmung (vgl. Anhang A) eingehalten wurden, konnte der Publikationsworkflow für Fall 1 fortgesetzt und der Produktionsweg festgelegt werden.

Darüber hinaus ergab die Analyse des Manuskriptes, dass es zitierte Abbildungen enthält, so dass der managementorientierte Prozess M-PVe-100: Rechte für Inhaltselemente klären bereits hier als notwendig erkannt wurde.

Beteiligte Rolle/n ausgeführt von

Content-Abnehmer Projektmitarbeiter 1 und 2 sowie Projektleiter 1 und 2

M-Pv-100: Produktionsweg festlegen

Die Entscheidung, den Content von Fall 1 als CRC-Workflow umzusetzen, hatte maßgebenden Einfluss auf die Gestaltung des Produktionswegs.

Zunächst erfolgte hiernach und aus weiteren, in Kapitel 4.1 beschriebenen Gründen keine Auszeichnung der Inhalte in XML. Stattdessen wurde entschieden, dass auf Basis des vom Content-Urheber eingereichten, finalen Manuskripts durch die Projektmitarbeiter des Modellverlags eine druckfähige PDF-Datei generiert wird, um Hard- und Softcover zu produzieren. Das Hardcover sollte in diesem Fall zusätzlich einen Schutzumschlag erhalten, so dass auch diese Ausstattungsvariante 
in der Fallstudie dargestellt ist. Die PDF-Datei sollte zudem zum ePDF für die Distribution im Internet aufbereitet werden. Das Ursprungsbuchformat DIN A4 sowie die typografische Gestaltung des Content-Urhebers wurden beibehalten. Das Outsourcen des Satzprozesses (CiP-Prozesse) war somit nicht notwendig. Die Druckund Vertriebsdienstleister, die Metadaten-Aggregatoren und die Veröffentlichungsplattformen wurden entsprechend der Darstellung in Kapitel 4.2 beauftragt.

Beteiligte Rolle/n

ausgeführt von

Content-Abnehmer

gesamtes Projektteam

\section{I-Cv-120: Content-Urheber beraten}

Da der Content des Falls 1 die Richtlinie für die Content-Vereinnahmung (vgl. Anhang A) erfüllt, ist es nicht notwendig, diesen Prozess durchzuführen.

\section{I-Cv-130: Content vereinnahmen \& definiert ablegen}

Der Content wurde mit der Entscheidung, diesen als Fall für die Fallstudie umzusetzen, vereinnahmt (vgl. Prozess I-Cv-110) und bereits im Zuge des Prozesses I$\mathrm{Cv}$-100 zentral in der Arbeitscloud des Modellverlags gespeichert.

Beteiligte Rolle/n

Content-Abnehmer ausgeführt von

Projektmitarbeiter 1

Prozesse I-Qe-100 bis I-Qe-140

Da es sich beim Content des Falles 1 um eine Graduierungsarbeit handelt, müssen die Prozesse I-Qe-100 bis Prozesse I-Qe-140 nicht durchgeführt werden.

Prozess I-Qe-200: Checkliste zu Content einfordern

Prozess I-Qe-210: Gutachten zu Content einfordern

Der Content von Fall 1 wurde bereits vor Beginn des Publikationsworkflows von zwei Hochschulprofessoren (Gutachter) entsprechend der Prüfungsordnung des Studienfachs Architektur (Master) der HTWK Leipzig ${ }^{155}$ auf seine wissenschaftliche Richtigkeit geprüft und in qualitativer Hinsicht bewertet.

Für die Prozesse I-Qe-200 und I-Qe-210 wurde vorab eine Checkliste zur Beurteilung der Publikationsfähigkeit entwickelt (vgl. Anhang A). Sie soll für den zu gründenden OA-Hochschulverlag der HTWK Leipzig als Musterdokument und Richtlinie für die Evaluation der Publikationsfähigkeit dienen. Diese Checkliste wurde per EMail an die Gutachter der Graduierungsarbeit von Fall 1 gesendet mit der Bitte, diese auszufüllen, zu unterschreiben und gemeinsam mit den bereits erstellten Gut- 
achten zur Arbeit bis zu einem bestimmten Termin an den Modellverlag zu übermitteln.

Beteiligte Rolle/n ausgeführt von

Gutachter zwei HTWK-Professoren des Fachgebiets; Erst- und Zweitgutachter der Graduierungsarbeit

Content-Abnehmer Projektmitarbeiter 1

\section{I-Ca-100: Gutachten bzw. Checklisten sichten}

Kurz darauf meldeten sich die Gutachter und baten darum, je nur eine gemeinsame Checkliste auszufüllen, da sie in ihrer Meinung zur Publikationsfähigkeit und der möglichen Verbesserungen für den Content von Fall 1 übereinstimmten.

Zudem wurde der Modellverlag informiert, dass die Prüfungsordnung des Studienfaches Architektur nicht vorschreibt, dass auch der Zweitgutachter ein Gutachten erstellen muss. Aus diesen Gründen erhielt Projektmitarbeiter 1 - in der Rolle des Content-Prüfers - nur ein Gutachten und eine Checkliste zum Content von Fall 1. Die Dokumente wurden entsprechend der folgenden projektspezifischen Richtlinie für die Publikationsannahme (vgl. Anhang A) gesichtet:

- Annahme: Die Gutachten und Checklisten stellen eindeutige Empfehlungen dar, das Werk in der vorliegenden Form zu publizieren.

- Überarbeitung des Content einfordern: Aus Gutachten und Checklisten gehen hervor, dass das Werk grundsätzlich publikationsfähig ist, aber noch weiteres Potenzial zur Überarbeitung besteht. Die Annahme des Werkes zur Publikation erfolgt somit nur unter Einhaltung bestimmter Bedingungen.

- Ablehnung: Aus den Gutachten und Checklisten geht entweder eindeutig hervor, dass das Werk in der vorliegenden Form nicht publikationsfähig ist oder dass die Richtlinie für die Content-Vereinnahmung (vgl. Anhang A) doch nicht erfüllt wurde.

In diesem Fall bestätigte das Gutachten eindeutig die Qualität der Graduierungsarbeit, so dass die Publikationseignung der Arbeit zunächst verifiziert war. Nichtsdestotrotz enthielt die Checkliste sehr ausführliche Empfehlungen zur Überarbeitung des Inhaltes. So u. a. die Empfehlung eine detaillierte Beschreibung der architektonischen Prinzipien sowie Abbildungsunterschriften zu ergänzen, ein Abbildungsverzeichnis zu erstellen, Kapitel zusammenzufassen und die Literaturangaben zu überarbeiten. Die Durchführung eines Fachkorrektorats oder/und Sprachlektorats wurde hier jedoch nicht gefordert. 


\section{I-Ca-110: Überarbeitung des Content einfordern}

Gemäß der Richtlinie für die Publikationsannahme (vgl. Anhang A) entschied der Content-Prüfer, den Content-Urheber um eine Überarbeitung des Contents entsprechend der Anmerkungen in der Checkliste zu bitten.

Hierzu wurde die Checkliste der Gutachter unverändert per E-Mail an den Content-Urheber weitergeleitet. Da die notwendigen Überarbeitungen umfangreich erschienen, wurde mit dem Content-Urheber eine Frist vereinbart, bis wann die Überarbeitung des Contents abgeschlossen sein sollte. Der Content-Urheber wurde aufgefordert, den überarbeiteten Content anschließend als offene Datei an den Content-Prüfer zu senden, da entsprechend der Festlegungen des Produktionswegs (vgl. Prozess $M-P v$-100) einige produktionsorientierte Prozesse durch den Modellverlag durchgeführt werden sollten.

\begin{tabular}{ll} 
Beteiligte Rolle/n & ausgeführt von \\
\hline Content-Prüfer & Projektmitarbeiter 1 \\
\hline Content-Urheber & Autor der Graduierungsarbeit von Fall 1
\end{tabular}

\section{M-PVe-100: Rechte für Inhaltselemente klären}

Während der Content-Urheber die vereinbarten Überarbeitungen durchführte, konnte bereits mit der Klärung der Rechte für die im Content enthaltenen Inhaltselemente begonnen werden. Hierfür analysierte Projektmitarbeiter 1 - in der Rolle des Content-Prüfers - die Arbeit erneut. Er dokumentierte in einer Tabelle den Quellenverweis der einzelnen Abbildungen, Grafiken oder Fotos und notierte, für welche der Rechteinhaber ermittelt und nach seiner Genehmigung zur Verwendung in der OA-Publikation angefragt werden muss. Da Abbildungsunterschriften mit dem zugehörigen Quellverweis zum Teil im ursprünglichen Content fehlten, war einiger Kommunikationsaufwand mit dem Content-Urheber nötig. Letztendlich konnten aber für alle Inhaltselemente die notwendigen Genehmigungen zur Publikation eingeholt werden. Dies war für 5 Abbildungen notwendig. Für ein Foto musste beim Stadtarchiv Leipzig die druckfähige Bilddatei erworben werden, was geringere Kosten verursachte (vgl. Kapitel 4.4.1). Eine zitierte Abbildung wurde in der überarbeiteten Fassung vom Content-Urheber entfernt.

Beteiligte Rolle/n ausgeführt von

\begin{tabular}{ll}
\hline Content-Prüfer & Projektmitarbeiter 1 \\
\hline Content-Urheber & Autor der Graduierungsarbeit von Fall 1
\end{tabular}

M-PVe-200: Vertragsabschluss \& die Buchvorankündigung vorbereiten

Im Vorfeld der Fallstudie wurde für den Prozess $M-P V e-200$ eine Checkliste für den Content-Urheber zur Vorbereitung des Vertragsabschlusses und für die Buchvoran- 
kündigung entwickelt. Diese soll dem zu gründenden OA-Hochschulverlag der HTWK Leipzig als Musterdokument und Richtlinie für den Vertragsabschluss und die Buchvorankündigung dienen. Sie ist im Anhang A zu finden.

Der Content-Abnehmer übermittelte die Checkliste per Mail an den ContentUrheber mit der Bitte, diese ausgefüllt und unterschrieben an den Modellverlag zurückzusenden.

Beteiligte Rolle/n ausgeführt von

Content-Abnehmer Projektmitarbeiter 1

Content-Urheber

Autor der Graduierungsarbeit von Fall 1

M-Pv-200: Stammdaten pflegen

Für die weitere Bearbeitung pflegte Projektmitarbeiter 1 - in der Rolle des Projektmanagers - die Stammdaten zum Content von Fall 1, indem er die ISBN für die Ausgabeformate Hardcover, Softcover und ePDF zuwies und dies entsprechend dem Musterdokument als Richtlinie für die Bildung von DOIs (vgl. Anhang A) die DOIs für das Produkt dokumentierte.

Beteiligte Rolle/n ausgeführt von

Content-Abnehmer

Projektmitarbeiter 1

\section{M-Pv-300: Produktionskosten klären}

Wie bereits in Kapitel 4.2 erläutert, wurden im Rahmen der Entwicklung des Workflow-Modells unterschiedliche Dienstleister für den Produktionsprozess recherchiert. Für den Fall 1 war bereits festgelegt worden, dass den Satzprozess $(P$ CiP-Prozesse) ausschließlich der und die Mitarbeiter des Modellverlags vornehmen. Somit mussten keine Satzdienstleister angefragt werden, um für Fall 1 Satzkosten zu klären. Im Gegensatz dazu wurden bereits vor dem Erhalt des überarbeiteten Contents Angebote für den Druck der Musterexemplare und der Vertrags- und Verkaufsauflage durch Projektmitarbeiter 1 - in der Rolle des Betriebswirts - eingeholt.

Die Druckkosten für die Vertrags- und Verkaufsauflage konnten mit dem Preiskalkulator von Dienstleister E ermittelt werden. Die Klärung der Druckkosten für die Produktion der Musterexemplare gestaltete sich etwas schwierig. Es wurde an dieser Stelle festgestellt, dass der vorab favorisierte Dienstleister $H$ nicht die Produktion eines Schutzumschlags für das Format DIN A4 anbietet. Daher wurden zwei weitere Dienstleister (Dienstleister F und Dienstleister 10) angefragt.

Leider konnte Dienstleister 10 nicht verbindlich mit der Produktion der Musterbände beauftragt werden, da er der Bitte nach Übersendung von Papiermustern nicht nachkam und schließlich kein Kontakt zu Dienstleister $10 \mathrm{mehr}$ hergestellt werden konnte. Das Angebot von Dienstleister F für die Produktion eines Hardco- 
vers mit Schutzumschlag wurde als zu hoch für das Budget, das für die Fallstudie zur Verfügung stand, eingeschätzt.

Aus diesem Grund wurde rund 2 Monate nach Beginn der Angebotssuche entschieden, die Musterbände doch von Dienstleister $H$ herstellen zu lassen, das Hardcover aber ohne Schutzumschlag. Dieser wurde zu Ansichtszwecken vom Modellverlag selbst erstellt. Die Kosten konnten für die Produktion der Musterbände durch Dienstleister $H$ mithilfe von dessen Online-Preiskalkulator ermittelt werden. Zur Kalkulation der Kosten der Vertrags- und Verkaufsauflage wurde das entsprechende Offline-Tool von Dienstleister E genutzt.

Darüber hinaus waren zu diesem Zeitpunkt bereits die Kosten für den Erwerb einer druckfähigen Bilddatei vom Stadtarchiv Leipzig (vgl. Prozess $M-P V e-100)$ und für die Vergabe der DOIs aus dem Prozess $M-P v$-300 bekannt (die konkreten Kosten werden in Kapitel 4.4.1 aufgeschlüsselt).

Beteiligte Rolle/n

Betriebswirt ausgeführt von

Projektmitarbeiter 1

\section{M-Pv-310: Soll-Kalkulation erstellen}

Da sich nun alle relevanten Produktionskosten beziffern ließen, konnte Projektmitarbeiter 1 - in der Rolle des Betriebswirts - eine Soll-Kalkulation für Fall 1 erstellen.

Beteiligte Rolle/n

Betriebswirt ausgeführt von

Projektmitarbeiter 1

\section{M-Pv-320: Ladenpreis für gedrucktes Produkt festlegen}

Auf Grundlage dieser Soll-Kalkulation ermittelte Projektmitarbeiter 1 mit Unterstützung der Projektleiter die Ladenpreise für Hard- und Softcover von Fall 1. Als Richtlinie wurde hierfür festgelegt, dass der Ladenpreis anteilig anhand der anfallenden betrieblichen Kosten aus der Soll-Kalkulation kalkuliert wird (progressive Kalkulation). So ist es möglich, den Ladenpreis so zu kalkulieren, dass nach Abverkauf der geplanten Auflagen über einen bestimmten Zeitraum alle Kosten gedeckt sind und ggf. ein kalkulierter Gewinn erwirtschaftet wird. Die Vorlage zur Kalkulation des Ladenpreises entsprechend der Richtlinie zur Preisstrategie ist im Anhang C zu finden.

Ausgehend von dieser Berechnung konnten rund 3 Monate nach Beginn des Prozesses die Ladenpreise für alle gedruckten Ausgabeformate der Fallstudie mit Hilfe einer Mischkalkulation final festgelegt werden. 


\section{I-Ca-120: Überarbeiteten Content überprüfen}

Nach rund 3 Monaten Bearbeitungszeit und zweimaliger Verlängerung der vereinbarten Frist übermittelte der Content-Urheber den überarbeiteten Content als PDFDatei sowie eine offene InDesign-Datei an den Content-Prüfer. Dieser überprüfte zeitnah, ob der Content-Urheber die Korrekturen wie vereinbart vorgenommen hatte. Dies traf zu, so dass keine weiteren Bearbeitungen gefordert wurden und die Bedingungen für den Prozess I-Ca-130 als erfüllt galten.

\begin{tabular}{ll} 
Beteiligte Rolle/n & ausgeführt von \\
\hline Content-Prüfer & Projektmitarbeiter 2 \\
\hline Content-Urheber & Autor der Graduierungsarbeit von Fall 1 \\
\hline
\end{tabular}

\section{I-Ca-130: Content annehmen}

Da der Content-Urheber die empfohlenen Korrekturhinweise zufriedenstellend umgesetzt hatte und auch die Klärung der Rechte für die Inhaltselemente abgeschlossen war, konnte der Content für den weiteren Publikationsworkflow angenommen werden. Der Content-Urheber wurde über diese Entscheidung informiert.

Beteiligte Rolle/n ausgeführt von

Content-Prüfer Projektmitarbeiter 2

Content-Urheber

Autor der Graduierungsarbeit von Fall 1

\section{I-Ca-150: Content ablehnen}

Die Ablehnung des Contents war in Fall 1 nicht notwendig.

\section{I-Ca-140: Content ablehnen}

Der Prozess I-Ca-140 erfolgte bereits im Zuge des vorherigen Prozesses I-Ca-130, konkret mit dem Ablegen des überarbeiteten Contents als PDF- und InDesign-Datei in der Arbeitscloud des Modellverlags.

Beteiligte Rolle/n ausgeführt von

\section{M-PVe-210: Publikationsvertrag schließen}

Nachdem der Content angenommen, außerdem die Checkliste für den Vertragsabschluss und die Buchvorankündigung ausgefüllt und unterschrieben vom ContentUrheber an den Content-Abnehmer gesendet worden war, konnte dieser den Publikationsvertrag vorbereiten und dem Content-Urheber auf dem Postweg sowie, zur Verkürzung der Bearbeitungszeit vorab auch per E-Mail, übersenden. Im Vorfeld 
des Publikationsworkflows wurde hierfür ein Muster-Publikationsvertrag als Richtlinie für die Vertragsunterzeichnung (vgl. Anhang A) erstellt. Ebenfalls erhielt der Content-Urheber eine zu unterschriebene Einverständniserklärung zur Publikation seines Werkes auf der OA-Veröffentlichungsplattform Qucosa (vgl. Kapitel 4.2.3). Der Content-Urheber wurde gebeten, Vertrag und Einverständniserklärung zu unterschreiben und zeitnah per E-Mail und ausgedruckt auf dem Postweg zurückzusenden.

\begin{tabular}{ll} 
Beteiligte Rolle/n & ausgeführt von \\
\hline Content-Abnehmer & Projektmitarbeiter 1 \\
\hline Content-Urheber & Autor der Graduierungsarbeit von Fall 1 \\
\hline
\end{tabular}

\section{P-CUe-100: Cover erstellen}

Da es sich bei Fall 1 um die Durchführung eines CRC-Workflows handelt, wurde von den Gestaltungsrichtlinien des Verlages abgewichen und das bereits gelieferte Cover des Content-Urhebers verwendet. Der Content-Urheber hat in diesem Fall die Rolle des Cover-Erstellers eingenommen.

Beteiligte Rolle/n

Cover-Ersteller ausgeführt von

Content-Urheber

\section{P-CUe-110: Umschlag erstellen}

Der Content-Urheber von Fall 1 hat lediglich das Cover (U1) zum Content erstellt. Da aber auch gedruckte Ausgabeformate hergestellt werden sollten, war es notwendig, dass Projektmitarbeiter 2 für diesen Prozess die Rolle des Cover-Erstellers einnahm und den Umschlag gestaltete. In diesem Fall wurde sich am Gestaltungsentwurf des Content-Urhebers für die Erstellung eines dazu passenden Umschlags orientiert. Hinsichtlich seiner Inhaltselemente (Abstract, Informationen zum Content-Urheber, ISBN-Strichcode, DOI- und QR-Codes und Logos) stimmt der Umschlag aber mit den Publikationen der anderen Fälle überein. Im Zuge der Covergestaltung erstellte Projektmitarbeiter 2 auch den Schutzumschlag nach den Gestaltungsrichtlinien des Modellverlags.

Beteiligte Rolle/n ausgeführt von

Cover-Ersteller

Projektmitarbeiter 2

\section{P-Ca-100: Fachkorrektorat beauftragen}

Da die Gutachter nicht explizit die Durchführung eines Fachkorrektorats für den Content von Fall 1 empfohlen hatten und diese Bedingung als einziges Kriterium 
für die Richtlinie für das Fachkorrektorat (vgl. Anhang A) formuliert wurde, ist der Prozess $P$-Ca-100 nicht durchgeführt wurden.

\section{P-Ca-110: Sprachlektorat beauftragen}

Diese projektspezifische Richtlinie zur Entscheidung, ob ein Manuskript ein Sprachlektorat benötigt, legt fest, dass für eine solche Überarbeitung mindestens einer der folgenden Punkte zutreffen muss:

- Das Manuskript wurde von einem Nicht-Muttersprachler geschrieben.

- Die Gutachter empfehlen die Durchführung eines Sprachlektorats am Manuskript.

- Aus den Gutachten bzw. Checklisten geht hervor, dass die Durchführung eines Sprachlektorats sinnvoll wäre.

Da keiner dieser Punkte auf den Content des Falls 1 zutrifft, wurde dieser Prozess nicht durchgeführt.

\section{P-Ca-120: Technisches Copy Editing durchführen}

Im Rahmen von Prozess $M-P v$ - 100 wurde festgelegt, dass das technische Copy Editing nicht der Content-Urheber, sondern der Modellverlag durchführt, da Projektmitarbeiter 2 die benötigten Kompetenzen hierfür besitzt. In der Rolle des Copy Editors bearbeitete er den Content nach der folgenden Richtlinie für das technische Copy Editing (vgl. Anhang A) und führte entsprechende Korrekturen in der InDesign-Datei aus:

- Überprüfung der Mikrotypografie (korrekte Anführungszeichen und Gedankenstriche, keine doppelten Leerzeichen etc.)

- Überprüfung der Druckqualität von Abbildungen, Grafiken, Fotos und Tabellen

- Überprüfung hinsichtlich der Konsistenz von Textelementen (wie Abbildungsunter- oder Tabellenüberbeschriftungen) sowie einer einheitlichen Überschriftennummerierung

Insbesondere nahmen die Überprüfung und Aufbereitung der Abbildungen und Fotos Zeit in Anspruch, da nicht alle in Druckqualität vom Content-Urheber geliefert wurden.

Beteiligte Rolle/n

Copy Editor ausgeführt von

Projektmitarbeiter 2

\section{P-CiP-100: Content-Eingangsformat in Produktionsformat überführen}

Nachdem die inhaltlichen sowie technischen Änderungen am Content abgeschlossen waren, konnte der Satzprozess (P-CiP-Prozesse) durchgeführt werden. Im Fall 1 ist das Content-Eingangsformat die offene Satzdatei im InDesign-Dateiformat. Da 
in diesem Fall davon abgesehen wurde, die Inhalte medienneutral aufzubereiten, war es, um die veröffentlichungsreife PDF-Datei zu generieren, nicht notwendig, die InDesign-Datei in ein anderes (Produktions-)Format zu überführen. Im Fall 1 ist das Content-Eingangsformat zugleich das Produktionsformat.

\section{P-CiP-110: Produktionsformat mit Metadaten anreichern}

Erforderlich war hingegen, das Produktionsformat mit Metadaten anzureichern. Nach vorheriger Festlegung wurde dieser Prozess nicht durch den Content-Urheber, sondern durch den Modellverlag vorgenommen.

Konkret bedeutete dies, dass Projektmitarbeiter 2 - in der Rolle des ContentUrhebers - den Schmutztitel und die Titelei als zusätzliche Seiten entsprechend der Gestaltungsrichtlinien des Modellverlags sowie die im Prozess $M-P v$-300 zugewiesenen DOIs im Produktionsformat (in der InDesign-Datei) ergänzte. Für die Distribution im Internet als ePDF pflegte er zudem Querverweise und Links ein.

Beteiligte Rolle/n ausgeführt von

Content-Umsetzer Projektmitarbeiter 2

\section{P-CiP-120: Qualität des Produktionsformats sichern}

In der Qualitätskontrolle des Produktionsformats kontrollierte der Content-Umsetzer v. a. die Paginierung des Contents in der Datei, da er im vorherigen Prozess Seiten ergänzt hatte. Anwendung fand hier die vorab entwickelte Checkliste als Richtlinie für die Produktfreigabe (vgl. Anhang A).

Beteiligte Rolle/n ausgeführt von

Content-Umsetzer Projektmitarbeiter 2

(1) P-CiP-130: Gewünschte Ausgabeformate generieren

Anschließend generierte der Content-Umsetzer aus der InDesign-Datei (Produktionsformat) eine PDF-Datei als Druckvorlage für die gedruckten Ausgabeformate sowie eine interaktive PDF-Datei, die als ePDF und elektronisches Ausgabeformat veröffentlicht werden sollte.

Beteiligte Rolle/n

Content-Umsetzer ausgeführt von

Projektmitarbeiter 2

\section{P-CiP-140: Gewünschte Ausgabeformate virtuell prüfen}

Nach Erstellung der gewünschten Ausgabeformate (Druck- und ePDF) übermittelte der Content-Umsetzer die Daten an Projektmitarbeiter 1, der in diesem Fall als Content-Prüfer, die Daten virtuell, d.h. am PC-Bildschirm, prüfte. Dabei nutzte der Content-Prüfer erneut die Checkliste für die Produktfreigabe (vgl. Anhang B). Der Con- 
tent-Prüfer markierte mittels der PDF-Kommentar-Funktion alles, was gemäß der Checkliste korrigiert werden muss.

Beteiligte Rolle/n

Content-Prüfer ausgeführt von

Projektmitarbeiter 1

\section{P-CiP-150: Korrekturen umsetzen}

Projektmitarbeiter 2 - in der Rolle des Content-Umsetzers - realisierte schließlich die vom Content-Prüfer angeforderten Korrekturen. Hierbei handelte es sich zum einen um wenige, typografische Feinarbeiten wie das Setzen von Überschriften als linksbündigen Satz statt als Blocksatz. Zum anderen wurden Korrekturen für das ePDF vorgenommen, etwa PDF-Lesezeichen für die einzelnen Kapitel ergänzt und Verlinkungen von einzelnen URLs berichtigt. Hierbei wurde festgestellt, dass drei der im Literaturverzeichnis genannten URLs nicht mehr existieren. Der ContentUrheber sollte entscheiden, wie mit diesen Quellenangaben zu verfahren ist.

Beteiligte Rolle/n

Content-Umsetzer ausgeführt von

Projektmitarbeiter 2

(2) P-CiP-130: Gewünschte Ausgabeformate generieren (Schleife)

Nachdem der Content-Umsetzer die genannten Korrekturen im Produktionsformat ausgeführt hatte, generierte er erneut eine PDF-Datei als Druckvorlage sowie eine interaktive PDF-Datei für das ePDF.

Beteiligte Rolle/n ausgeführt von

Content-Umsetzer

Projektmitarbeiter 2

P-CUe-120: Freigabe für Cover bzw. Umschlag einholen

P-CiP-160: Freigabe vom Content-Urheber einholen

Parallel zur Durchführung der bereits beschriebenen produktionsorientierten Prozesse erstellte Projektmitarbeiter 2 - in der Rolle des Cover-Erstellers - einen farbverbindlichen Proof für den Umschlag der gedruckten Ausgabeformate des Contents von Fall 1 und legte es dem Projektteam zur Freigabe vor. Diese vorgeschobene Freigabe wurde als wichtig angesehen, da der Umschlag von den Gestaltungsrichtlinien des Modellverlags abweicht, individuell gestaltet ist und hier somit größere Unsicherheiten bestehen als bei Covern/Umschlägen mit standardisiertem Layout. Das Team prüfte den Hardproof als Content-Prüfer und erteilte ohne Änderungsvorschläge die Freigabe. Für den Schutzumschlag erfolgte keine Freigabe, da es sich hierbei lediglich um ein Ansichtsexemplar handelt.

Somit übermittelte Projektmitarbeiter 2 - in der Rolle des Content-Umsetzers den Umschlagentwurf und - allerdings ohne weitere Überprüfung durch Projekt- 
mitarbeiter 1 (Content-Prüfer) - auch die Druckdatei und die interaktive PDF-Datei an den Content-Urheber mit der Bitte um Prüfung und ggf. Freigabe der Daten. Er erbat außerdem eine Mitteilung, wie mit den nicht mehr verfügbaren URLs im Literaturverzeichnis umgegangen werden soll.

Nach seiner Rückkehr aus dem Urlaub meldete sich der Content-Urheber mit der Freigabe für den Umschlag und Hinweisen zum Literaturverzeichnis zurück. Für die Inhaltsdaten erteilte er die vorläufige Freigabe; unter der Voraussetzung, dass die entsprechenden Korrekturen im Literaturverzeichnis umgesetzt werden.

Für Prozess P-CUe-120

Beteiligte Rolle/n ausgeführt von

\begin{tabular}{ll}
\hline Content-Ersteller & Projektmitarbeiter 2 \\
\hline Content-Prüfer & gesamtes Projektteam \\
\hline Content-Urheber & Autor der Graduierungsarbeit von Fall 1 \\
\hline
\end{tabular}

Für Prozess P-CiP-160

Beteiligte Rolle/n ausgeführt von

Content-Umsetzer Projektmitarbeiter 2

Content-Urheber Autor der Graduierungsarbeit von Fall 1

P-CUe-130: Korrekturen für Cover bzw. Umschlag umsetzen

Es wurden keine Änderungen eingefordert.

(2) P-CiP-150: Korrekturen umsetzen

(3) P-CiP-130: Gewünschte Publikationsformen generieren (Schleife)

Der Content-Umsetzer realisierte die vom Content-Urheber gewünschten Korrekturen im Produktionsformat und generierte erneut die Druckvorlage und die interaktive PDF-Datei für das ePDF.

Beteiligte Rolle/n

Content-Umsetzer ausgeführt von

Projektmitarbeiter 2

\section{P-CiP-170: Umschlag anpassen}

Nachdem die Korrekturen an den Inhaltsdaten abgeschlossen waren und somit der finale Umfang der gedruckten Ausgabeformate vorlag, konnte Projektmitarbeiter 2 als Cover-Ersteller die Umschlagsdatei entsprechend der Vorgaben der ausgewählten Druck- und Vertriebsdienstleisters anpassen. 
Beteiligte Rolle/n

Content-Ersteller ausgeführt von

Projektmitarbeiter 2

\section{P-CiP-180: Ausgabeformate zur Veröffentlichung freigeben}

Rund 6 Wochen nach dem Beginn des Satzprozesses (P-CiP-Prozesse) konnte Projektmitarbeiter 1 - in der Rolle des Content-Abnehmers - alle gewünschten Ausgabeformate zur Veröffentlichung freigeben.

Beteiligte Rolle/n

Content-Abnehmer ausgeführt von

Projektmitarbeiter 1

\section{P-GP-100: Druck auslösen}

Nachdem die Ausgabeformate zur Veröffentlichung freigegeben wurden, beauftragte Projektmitarbeiter 1 - in der Rolle des Content-Distributors - zunächst den Druck von je einem Testexemplar (Soft- und Hardcover) bei Dienstleister E. Die Testexemplare sollten Aufschluss darüber geben, ob das zur Verfügung stehende Inhaltspapier mit einer flächenbezogenen Masse von $115 \mathrm{~g} / \mathrm{m}^{2}$ für die gedruckten Produkte angemessen ist. Außerdem sollte vor Beauftragung der gesamten Auflage ein Eindruck von der druck- und buchbinderischen Qualität der Produkte von Dienstleister E gewonnen werden. Dies war im Fall 1 besonders wichtig, da die zahlreichen Abbildungen hier im Fokus des Contents stehen und somit eine gute Druckqualität verlangt war.

Beteiligte Rolle/n

Content-Distribution ausgeführt von

Projektmitarbeiter 1

\section{P-EP-100: Produkt auf primärer Veröffentlichungsplattform veröffentlichen}

In der Zwischenzeit bis zur Lieferung der Testexemplare der gedruckten Produkte von Fall 1 entschied das Projektteam, als primäre Veröffentlichungsplattform für die elektronischen Ausgabeformate aller Fälle eine eigene Verlagswebsite aufzubauen. Damit diese auch nach Abschluss des Projekts vom zu gründenden Hochschulverlag der HTWK Leipzig genutzt werden kann, erfolgte die Anlage als Micro Site und somit im Corporate Design der Hochschule. Darüber hinaus wurden weitere Anforderungen an die Verlagswebsite formuliert: So sollte es künftig möglich sein, die Website selbstständig zu pflegen und mit allen gewünschten Inhalten zu bestücken, außerdem, die elektronischen Ausgabeformate der Publikationen hier zum Download zur Verfügung zu stellen. Hierfür wurde die Richtlinie für die Wahl der primären OA-Veröffentlichungsplattform (vgl. Anhang A) formuliert.

Noch während der Wartezeit auf die gedruckten Produkte von Fall 1 konnte Projektmitarbeiter 1 als Content-Distributor das elektronische Ausgabeformat der 
Publikation, d. h. die ePDF-Datei, auf der neu erstellten Website oa-hochschulverlag.htwk-leipzig.de veröffentlichen.

Beteiligte Rolle/n ausgeführt von

Content-Distribution

Projektmitarbeiter 1

\section{M-MM-100: Metadatensatz erzeugen}

Nachdem die ePDF-Datei auf der primären Veröffentlichungsplattform veröffentlicht wurde, konnte der Metadatensatz durch Projektmitarbeiter 1 als MetadatenManager erstellt werden. Hierfür wurde im Vorfeld der Fallstudie als Richtlinie für die Erstellung von Metadaten (vgl. Anhang A) eine Checkliste erstellt, in der alle relevanten Informationen erfasst waren, die sich aus den Anforderungen der weiteren festgelegten OA-Veröffentlichungsplattformen und der Metadaten-Aggregatoren ergeben.

\section{M-MM-100: Metadatensatz distribuieren}

Entsprechend der Richtlinie für die Verbreitung von Metadaten aktiviert Projektmitarbeiter 1 als Metadaten-Manager zunächst die DOIs der Publikation des Falles 1, indem er diese bei Crossref mit der bestehenden URL der primären Veröffentlichungsplattform und weiteren Metadaten verknüpfte.

Beispiel: Hinter DOI http://doi.org/10.33968/9783966270069-00 verbirgt sich die URL https://oa-hochschulverlag.htwk-leipzig.de/katalog/wirtschaftsingenieurwesen/.

Anschließend konnte er die Metadaten in den Katalog des VLB für jedes Ausgabeformat einpflegen und den Metadatensatz als ONIX-Datei exportieren. Die ONIXDateien wurden zunächst nur an Newbooks übermittelt und rund 2 Monate später auch an KNV Zeitfracht zur weiteren Distribution bzw. Aufnahme in ihre Kataloge. Die Konditionsvereinbarungen mit Libri waren zum Abschluss der Fallstudie noch nicht getroffen worden, obwohl bereits 7 Monate vorher der Kontakt durch das Projektteam aufgenommen worden war.

Beteiligte Rolle/n

Metadaten-Manager

Metadaten-Aggregator ausgeführt von

Projektmitarbeiter 1

vgl. Kapitel 4.2.4 
M-MM-120: Weitere Aggregatoren beauftragen Metadaten zu distribuieren

Weitere Aggregatoren wurden im Rahmen der Fallstudie nicht mit der Distribution der Metadaten beauftragt.

\section{P-EP-110: Produkt auf weiteren Plattform veröffentlichen}

Entsprechend der Richtlinie für die Wahl weiterer Veröffentlichungsplattformen (vgl. Anhang A) hat Projektmitarbeiter 1 die elektronischen Ausgabeformate von Fall 1 auf den Plattformen $O A P E N$ bzw. DOAB veröffentlicht. Darüber hinaus lud er die Publikation auch bei Unglue.it, Google Books und ScienceOpen hoch und bat einen Mitarbeiter der Hochschulbibliothek um die Veröffentlichung auf Qucosa. Da nur Autoren auf ResearchGate Dokumente hochladen können, wurde der Content-Urheber des Falles 1 hierüber durch Projektmitarbeiter 1 informiert und gebeten, dies zu tun, um die Sichtbarkeit der Publikation zu erhöhen. Leider ist der ContentUrheber des Falles 1 zum Zeitpunkt der Drucklegung der vorliegenden Monografie dieser Bitte noch nicht nachgekommen.

Beteiligte Rolle/n ausgeführt von

Metadaten-Manager Projektmitarbeiter 1 und ein Mitarbeiter der Hochschulbibliothek

\section{P-GP-110: Produkt physisch prüfen}

Knapp 3 Wochen nach Beauftragung trafen die gedruckten Testexemplare vom Dienstleister $E$ ein. Diese wurden beim nächsten Projekttreffen gemeinsam im Team anhand der selbst entwickelten Checkliste als Richtlinie für gedruckte Produkte geprüft (vgl. Anhang A).

Es konnte festgestellt werden, dass die Qualität des von Dienstleister E gelieferten Produktes zufriedenstellend sowie das gewählte Inhaltspapier mit einer flächenbezogenen Masse von $115 \mathrm{~g} / \mathrm{m}^{2}$ passend waren. Zugleich wurde vermerkt, dass es einer umfassenderen Beratung des Content-Urhebers bei der Erstellung seiner Druckvorlage bedurft hätte, um insbesondere die Abbildungen im Inhalt noch besser in den Fokus zu rücken. Es wurde entschieden, dass lediglich die Hardcover-Version der Publikation in den Buchhandel gebracht wird, da die Broschur mit dem geringen Seitenumfang als nicht markttauglich erschien.

Beteiligte Rolle/n

Content-Distributor ausgeführt von

gesamtes Projektteam

Dienstleister $\mathrm{E}$

\section{P-GP-120: Gewünschte Auflage produzieren}

Da die Qualität der Leistungen von Dienstleister E keine Mängel aufwies, das Projektteam stattdessen die Druckvorlage als korrekturbedürftig einschätzte, wurde diese von Projektmitarbeiter 2 angepasst und Dienstleister $E$ anschließend mit dem 
Druck der gewünschten Auflage beauftragt. Die Exemplare trafen 3 Wochen nach Beauftragung ein.

Beteiligte Rolle/n

Content-Distributor

Drucker ausgeführt von

gesamtes Projektteam

Dienstleister $\mathrm{E}$

\section{M-Bb-100: Bestellung erhalten}

Knapp einen Monat nach der Distribution der Metadaten (Prozess $M-M M-110$ ) ging die erste Bestellung für die Publikation des Falles 1 ein. Die Bestellung wurde von Projektmitarbeiter 1 als Content-Distributor registriert, aber nicht sofort bearbeitet, da der im $V L B$ angegebene Erscheinungstermin noch in der Zukunft lag.

Beteiligte Rolle/n

Content-Distributor ausgeführt von

Projektmitarbeiter 1

Kunde

Buchhandlung in Norddeutschland

\section{M-Bb-110: Bestellung bearbeiten}

Kurz vor dem im VLB eingetragenen Erscheinungstermin der gedruckten Produkte von Fall 1 bearbeitete Projektmitarbeiter 1 die bis zu diesem Zeitpunkt eingegangenen 3 Bestellungen. Die bestellten Bücher wurden verpackt, mit Lieferscheinen versehen und Projektmitarbeiter 2 zum Versand übergeben. Zudem erfolgte die Dokumentation der Bestellungen in einer Bestellübersicht.

Beteiligte Rolle/n ausgeführt von

Content-Distributor Projektmitarbeiter 1

\section{M-Bb-120: Bestellung versenden}

Projektmitarbeiter 2 brachte die Pakete mit den bestellten Büchern anschließend zur zentralen Poststelle der HTWK Leipzig, damit diese an die entsprechenden Adressen versendet werden.

Beteiligte Rolle/n ausgeführt von

Content-Distributor Projektmitarbeiter 2

Betriebswirt Mitarbeiter des Dezernats Finanzen der HTWK Leipzig 
P-GP-130: Pflicht- und Vertragsexemplare versenden

Kurz nachdem die gesamte Verkaufs- und Vertragsauflage der gedruckten Produkte von Fall 1 im Modellverlag eingetroffen war, konnte der Content-Urheber per EMail informiert werden, dass seine Autorenexemplare, wie im Publikationsvertrag vereinbart, vorliegen. Projektmitarbeiter 1 - in der Rolle des Content-Distributors vereinbarte, dass der Content-Urheber die Exemplare vor Ort im Modellverlag abholt, was wenige Tage später erfolgte.

Zudem versendete Projektmitarbeiter 1 als Content-Archivar kurz nach dem im VLB angegeben Erscheinungstermin der gedruckten Produkte in Zusammenarbeit mit einem Studenten 2 Hardcover an die Medienabgabe der DNB, Standort Leipzig. Darüber hinaus meldete er die Netzpublikationen im Online-Portal der DNB und der Sächsischen Landesbibliothek - Staats- und Universitätsbibliothek (SLUB) Dresden. Hiermit wurde der Pflichtablieferungsverordnung (PflAV) bzw. dem Gesetz über die Deutsche Nationalbibliothek (DNBG) und das sächsische Gesetz über die Presse (SächsPresseG) nachgekommen. Dementsprechend wurde auch die projektspezifische Richtlinie für Pflichtexemplare (vgl. Anhang A) formuliert.

Beteiligte Rolle/n ausgeführt von

Content-Distributor Projektmitarbeiter 2

Content-Archivar Projektmitarbeiter 1 und ein Student bzw. DNB und SLUB Dresden

P-PFs-100: Für Langzeitarchivierung des Produktes sorgen

Die Abgabe zweier Hardcover-Exemplare an die DNB sicherte die Langzeitarchivierung des gedruckten Produktes des Falls 1. Durch die Meldung der elektronischen Ausgabeformate bei der DNB und SLUB Dresden ist auch für diese die Langzeitarchivierung sichergestellt.

Im Rahmen der Fallstudie konnten aufgrund des begrenzten Budgets leider keine weiteren Maßnahmen zur Langzeitarchivierung der Bücher vorgenommen werden, wie bspw. das Sichern der elektronischen Ausgabeformate auf einer Plattform, wie CLOCKSS oder Portico. Dementsprechend wurde auch die projektspezifische Richtlinie für die Wahl des Langzeitarchivs (vgl. Anhang A) formuliert.

Beteiligte Rolle/n

Content-Archivar ausgeführt von

DNB und SLUB Dresden

\section{M-Pv-400: Projektterminplan erstellen}

Da die Produktion und Publikation der Bücher Teil der Fallstudie im Rahmen des Forschungsprojektes waren, wurde weder ein Erscheinungstermin für die Veröffentlichung der Bücher vorab festgelegt noch ein Projektterminplan erstellt. Der Zeitraum für die Fallstudie war lediglich durch die Forschungsprojektplanung, die 
zur Beantragung des Projektes eingereicht werden musste, bzw. durch die Förderungsdauer terminiert.

\section{M-Pv-400: Projektterminplan erstellen}

Da im Rahmen der Fallstudie in erster Linie das Projektteam - bestehend aus 2 Projektleitern und 2 wissenschaftlichen Mitarbeitern mit insgesamt 1,88 Vollbeschäftigungsäquivalent - an den Publikationsworkflows beteiligt war, erfolgte die Durchführung der $M$-PVs-Prozesse direkt und niederschwellig, so dass diese hier zusammenfassend beschrieben werden sollen

Die Rolle des Controllers übernahm hierbei vor allem Projektmitarbeiter 1, der in regelmäßigen Abständen den aktuellen Stand der produktionsorientierten Prozesse (im Fall 1 Schwerpunkt der Arbeit) bei Projektmitarbeiter 2 erfragte, sich mit diesem austauschte und zu den Projekttreffen des gesamten Teams die Projektleiter über den aktuellen Stand informierte.

Daneben war aufgrund der Forschungsförderung die fortwährende Überprüfung der Kosten, der Abgleich dieser mit dem gegebenen Projektbudget sowie die Überprüfung von Rechnungen und Weiterleitung an den zuständigen Sachbearbeiter des Dezernats Finanzen der HTWK Leipzig, der das Forschungsprojekt betriebswirtschaftlich begleitete, essenziell (Prozess $M-P V s-160$ ).

Wie bereits zu Prozess $M-P v-400$ erwähnt, wurde kein Projektterminplan für Fall 1 erstellt. Ziel war es die Fallstudie, so schnell wie möglich umzusetzen und die entsprechenden Produkte zu veröffentlichen. Da der Zeitraum für die Produktion und Publikation der Bücher innerhalb der Projektdauer recht großzügig war, gab es diesbezüglich auch keine kritischen Situationen oder zeitliche Engpässe.

Die personellen Ressourcen waren, wie bereits oben beschrieben, ebenfalls durch die Anlage als Forschungsprojekt festgelegt. Da zum Ende der Fallstudie zudem eine studentische Projektgruppe aus dem Studiengang Buch-und Medienproduktion in den Publikationsworkflow involviert wurde, konnten die Verantwortlichkeiten für die Durchführung neu zugeteilt und die Mitarbeiter des Modellverlags entlastet werden.

Beteiligte Rolle/n ausgeführt von

Controller, Projektmanager, Betriebswirt Projektmitarbeiter 1

\section{Prozessgruppe M-OM: Operatives Marketing durchführen}

Aufgrund der begrenzten personellen Ressourcen des Projektteams und damit auch des Modellverlags wurde zum Ende der Fallstudie die bereits erwähnte studentische Projektgruppe mit der Verantwortlichkeit für die Prozesse des operativen Marketings betraut. Die Erstellung eines Marketing-Plans erfolgte zeitgleich mit der Inhaltserstellung des vorliegenden Buches, weshalb auf diese Prozesse an dieser Stelle nicht weiter eingegangen werden kann. 


\section{P-PFs-200: Ausgabeformate systematisiert ablegen}

\section{M-Pn-200: Verwaltungsdaten sichern}

Projektmitarbeiter 1 erstellte im Rahmen des Projektabschlusses zum Ende der Forschungsförderung zu Fall 1 einen Archivdatensatz mit allen relevanten Informationen zum Fall und legte sie in der hochschuleigenen Cloud-Infrastruktur ab. Dieser Datensatz enthält etwa alle Daten zu den Ausgabeformaten der Publikationen aller Fälle, Informationen zu den jeweiligen Content-Urhebern, Kommunikationsabläufe mit Dienstleistern und Protokolle. Dieses Vorgehen wurde für den zukünftigen Hochschulverlag als Richtlinie zur internen Archivierung (vgl. Anhang A) festgelegt.

Beteiligte Rolle/n ausgeführt von

Content-Archivar Projektmitarbeiter 1 bzw. hochschuleigenen Cloud-Infrastruktur

\section{M-Pn-200: Nach-Kalkulation erstellen}

Im Zuge der Kalkulation des Ladenpreises (Prozess $M-P v$-320) wurde festgelegt, dass erst ein Jahr nach der Veröffentlichung der gedruckten Produkte deren Wirtschaftlichkeit im Zuge einer Nach-Kalkulation geprüft werden soll. Dieser Zeitpunkt lag nicht mehr in der Projektlaufzeit, so dass dieser Prozess nicht näher beschrieben werden kann.

\section{M-Bb-200: Honorar zahlen}

Entsprechend des Publikationsvertrages aus Prozess M-PVe-210 hat der ContentUrheber keinen Anspruch auf Honorar, weswegen dieser Prozess nicht durchgeführt wurde.

\section{M-MM-200: Metadatensatz aktualisieren}

Zum Zeitpunkt der Fallstudie bzw. während der Projektlaufzeit war eine Aktualisierung des Metadatensatzes zu keinem Ausgabeformat der Publikation von Fall 1 notwendig. 


\subsubsection{Fall 2: XML-first-Workflow in Fremdleistung}

\begin{tabular}{ll} 
Art des Content & Graduierungsarbeit - Bachelorarbeit \\
\hline Fakultät & Maschinenbau und Energietechnik \\
\hline Studiengang & Wirtschaftsingenieurwesen Produktions- und Energiewirtschaft \\
\hline Titel & $\begin{array}{l}\text { Kommunikationstechnische Optimierung eines energieautarken } \\
\text { funkbasierten Sensorkonzepts }\end{array}$ \\
\hline Workflow-Variante & XML-first-Workflow in Fremdleistung
\end{tabular}

Prozess I-Ka-100 bis I-Ka-130

Prozesse wurden nicht durchgeführt (vgl. Kapitel 4.3.1).

\section{I-Ka-200: Nach geeigneten Content-Urhebern recherchieren}

Der Publikationsworkflow begann im Fall 2 analog zu Fall 1 damit, dass die Hochschulbibliothek im Namen des Modellverlags und entsprechend Prozess I-Ka-200 beim Dekan der (damaligen) Fakultät Maschinenbau und Energietechnik nachfragte, ob dieser Graduierungsarbeiten betreut hätte, die der Richtlinie für die KonzeptAnnahme (vgl. Anhang A) entsprechen und somit zur Publikation im Rahmen der Fallstudie infrage kommen.

Kurz darauf meldete sich der Dekan mit Informationen zu 4 Abschlussarbeiten (2 Master- und 2 Bachelorarbeiten) zurück, die zur Publikation geeignet hielt. Zwei der empfohlenen Arbeiten waren allerdings im Jahr 2015 erstellt worden und entsprachen somit nicht der Richtlinie für die Konzept-Annahme.

Beteiligte Rolle/n

Content-Abnehmer

Content-Lieferant ausgeführt von

Mitarbeiter der Hochschulbibliothek

Dekan der Fakultät

\section{I-Ka-210: Content-Urheber anfragen}

Projektmitarbeiter 1 nahm als Content-Abnehmer daraufhin per E-Mail Kontakt zu den zwei Content-Urhebern auf. Kurz darauf meldete sich der Content-Urheber einer empfohlenen Bachelorarbeit zurück, dass er diese gerne im Rahmen der Fallstudie veröffentlichen möchte. Der Content-Urheber der angefragten Masterarbeit meldete sich nicht zurück; seine Arbeit wurde daher als Fall für die Studie verworfen. 


\begin{tabular}{ll} 
Beteiligte Rolle/n & ausgeführt von \\
\hline Content-Abnehmer & Projektmitarbeiter 1 \\
\hline Content-Urheber & $\begin{array}{l}\text { Autor der vorgeschlagenen Bachelorarbeit, Autor der vorgeschlagenen } \\
\text { Masterarbeit }\end{array}$
\end{tabular}

\section{I-Ka-220: Mit Content-Urheber über Publikation sprechen}

\section{I-Cv-100: Content einfordern}

Der Content-Abnehmer übermittelte dem Content-Urheber, der bereit war, seine Arbeit zu publizieren, per E-Mail ein Informationsblatt, das u. a. die Ziele des Projektes, Informationen zur Bedeutung von OA sowie die weiteren Schritte des Publikationsworkflows aufführte (vgl. Anhang D) und bat ihn, den vollständigen Content als Word- und PDF-Datei (sofern vorhanden) zu übermitteln. Kurz darauf stellte der Content-Urheber die gewünschten Dateien bereit.

Beteiligte Rolle/n ausgeführt von

Content-Abnehmer Projektmitarbeiter 1

Content-Urheber Autor der vorgeschlagenen Bachelorarbeit

\section{I-Cv-110: Content prüfen}

Zum nächsten Treffen des Projektteams wurde die PDF-Datei des Contents gemeinsam analysiert und geprüft, ob sie der Richtlinie für die Content-Vereinnahmung (vgl. Anhang A) entspricht. Dies war der Fall, so dass der Prozess I-Cv-120 nicht notwendig war und der Content als Fall in die Fallstudie aufgenommen wurde.

In Zuge dessen wurde festgestellt, dass im Content zitierte Abbildungen enthalten sind, weshalb der managementorientierte Prozess $M-P V e-100$ als notwendig erkannt wurde.

Beteiligte Rolle/n ausgeführt von

Content-Abnehmer gesamtes Projektteam

\section{M-Pv-100: Produktionsweg festlegen}

Wie bereits im Kapitel 4.1 beschrieben, konnte im Verlauf des Prozesses I-Cv-110 bestätigt werden, dass der Content von Fall 2 medienneutral (XML-first) in allen gängigen Ausgabeformaten (Druck- und ePDF, EPUB und MOBI) umsetzbar ist. Der Satzprozess (P-CiP-Prozesse) sollte von einem Satzdienstleister ausgeführt werden. An dieser Stelle stand allerdings noch nicht fest, welcher Dienstleister beauftragt wird (mehr dazu bei Prozess $M$-Pv-300). Im Gegensatz dazu sollten die Druck- und Vertriebsdienstleister, die Metadaten-Aggregatoren und die Veröffentlichungsplattformen entsprechend der Darstellung in Kapitel 4.2 verpflichtet werden. 


\section{I-Cv-120: Content-Urheber beraten}

Da der Content des Falls 2 die Richtlinie für die Content-Vereinnahmung erfüllt (vgl. Anhang A), war es nicht notwendig, diesen Prozess durchzuführen.

\section{I-Cv-130: Content vereinnahmen \& definiert ablegen}

Der Content wurde mit der Entscheidung, diesen als Fall für die Fallstudie umzusetzen, vereinnahmt (vgl. Prozess I-Cv-110) und bereits im Zuge des Prozesses I$\mathrm{C} v$-100 zentral in der Arbeitscloud des Modellverlags gespeichert.

Beteiligte Rolle/n

Content-Abnehmer ausgeführt von

gesamtes Projektteam

\section{Prozesse I-Qe-100 bis I-Qe-140}

Da es sich beim Content des Falles 2 ebenfalls um eine Graduierungsarbeit handelt, mussten die Prozesse I-Qe-100 bis I-Qe-140 nicht durchgeführt werden.

\section{I-Qe-200: Checkliste zu Content einfordern}

I-Qe-210: Gutachten zu Content einfordern

Der Content des Falles 2 war zum Zeitpunkt der Fallstudie bereits von einem Hochschulprofessor (dem Dekan der Fakultät) und einem wissenschaftlichen Mitarbeiter der Fakultät entsprechend der Prüfungsordnung des Studienfachs geprüft und bewertet worden. ${ }^{156}$

Projektmitarbeiter 1 - in der Rolle des Content-Abnehmers - nahm mit den Gutachtern Kontakt auf, um die Checkliste für die Evaluation der Publikationsfähigkeit (vgl. Anhang B) zu übermitteln und die Gutachten zum Content einzufordern.

\begin{tabular}{ll} 
Beteiligte Rolle/n & ausgeführt von \\
\hline Gutachter & ein HTWK-Professor des Fachgebiets als Erstgutachter, ein \\
& wissenschaftlichen Mitarbeiter des Fachgebiets als Zweitgutachter der \\
& Graduierungsarbeit
\end{tabular}

Content-Abnehmer Projektmitarbeiter 1

\section{I-Ca-100: Gutachten bzw. Checklisten sichten}

Zum nächsten Projekttreffen wurden die Gutachten und die Checkliste gemeinsam vom Projektteam als Content-Prüfer nach der Richtlinie für die Publikationsannahme 
(vgl. Anhang A) gesichtet. Auch in diesem Fall ließen die Gutachten keinen Zweifel, dass der vorliegende Content zur Publikation geeignet ist.

In der Checkliste wurden wenige Hinweise zur Verbesserung des Contents gegeben, wie der Verweis auf die Primärquelle im Literaturverzeichnis, wo Wikipedia als Quelle angegeben wurde, oder die Überprüfung von Angaben in Tabellen. Allerdings gaben die Gutachter an, dass der sprachliche Ausdruck überarbeitet werden muss. Daher wurde die Arbeit stichprobenartig gelesen und schließlich entschieden, dass der Ausdruck für den Rahmen einer Bachelorarbeit angemessen ist und es keines Fach- bzw. Sprachlektorats bedarf.

Beteiligte Rolle/n ausgeführt von

Content-Prüfer gesamtes Projektteam

\section{I-Ca-110: Überarbeitung des Content einfordern}

Entsprechend der Richtlinie für die Publikationsannahme entschied das Projektteam, den Content-Urheber zu bitten, die genannten Korrekturempfehlungen aus der Checkliste in den Content einzuarbeiten, bevor weitere Prozesse des Publikationsworkflows durchgeführt werden konnten.

$\mathrm{Zu}$ diesem Zweck übermittelte Projektmitarbeiter 1 die gewünschten Änderungen an den Content-Urheber und bat ihn mitzuteilen, bis wann er die Korrekturen ausführen und die überarbeitete Datei zurücksenden kann. Zugleich wurde der Content-Urheber, alle verwendeten Abbildungen aus dem Manuskript als einzelne Datei an den Modellverlag zu liefern, da diese für den medienneutralen Satz benötigt würden.

Beteiligte Rolle/n ausgeführt von

Content-Prüfer Projektmitarbeiter 1

Content-Urheber Autor der Graduierungsarbeit von Fall 2

M-PVe-200: Vertragsabschluss \& die Buchvorankündigung vorbereiten

Gemeinsam mit der Aufforderung, den Content zu überarbeiten, übermittelte Projektmitarbeiter 1 auch die Checkliste für den Vertragsabschluss und die Buchvorankündigung (vgl. Anhang B) an den Content-Urheber mit der Bitte, diese ebenfalls bis zum vorgeschlagenen Termin auszufüllen.

\begin{tabular}{ll} 
Beteiligte Rolle/n & ausgeführt von \\
\hline Content-Abnehmer & Projektmitarbeiter 1 \\
\hline Content-Urheber & Autor der Graduierungsarbeit von Fall 2 \\
\hline
\end{tabular}




\section{I-Ca-120: Überarbeiteten Content überprüfen}

Nach ca. 2 Wochen und noch vor Ablauf der vereinbarten Frist übermittelte der Content-Urheber die überarbeitete Word-Datei des Contents sowie die ausgefüllte und unterschriebene Checkliste für den Vertragsabschluss und die Buchvorankündigung per E-Mail. Zudem schickte der Content-Urheber einen Teil der verwendeten Abbildungen im Manuskript als einzelne Dateien. Alle Abbildungen, die er nicht selbst erstellt, sondern aus anderen Quellen bezogen hatte, lagen ihm nicht mehr als einzelne Dateien vor.

Als Content-Prüfer nahm Projektmitarbeiter 1 die Prüfung des überarbeiteten Contents vor. Alle Korrekturempfehlungen wurden vom Content-Urheber umgesetzt, so dass keine weiteren Bearbeitungen von seiner Seite erforderlich waren; die Bedingung für den Prozess I-Ca-130 wurde als erfüllt betrachtet.

Beteiligte Rolle/n ausgeführt von

Content-Prüfer Projektmitarbeiter 1

Content-Urheber Autor der Graduierungsarbeit von Fall 2

\section{I-Ca-130: Content annehmen}

I-Ca-140: Content zur weiteren Bearbeitung zur Verfügung stellen

Auch wenn noch nicht alle Abbildungen vorhanden waren, wurde der Content formal nach der Überprüfung angenommen. Er wurde mit dem Ablegen der überarbeiteten Word-Datei durch Projektmitarbeiter 2 in der Arbeitscloud des Modellverlags zur weiteren Bearbeitung zur Verfügung gestellt.

Beteiligte Rolle/n ausgeführt von

Content-Prüfer Projektmitarbeiter 2

Content-Urheber

Autor der Graduierungsarbeit von Fall 2

\section{I-Ca-150: Content ablehnen}

Die Ablehnung des Contents war in Fall 2 nicht notwendig.

\section{M-PVe-100: Rechte für Inhaltselemente klären}

Wie im Fall 1, enthielt auch der Content des Falles 2 zitierte Abbildungen. Daher wurden durch Projektmitarbeiter 1 in einer Tabelle alle Abbildungen und die (vermeintlichen) Rechteinhaber erfasst. Der Content enthielt 15 zitierte Abbildungen, 2 Abbildungen, die den Verweis ,in Anlehnung an [Quelle]“ trugen und 28 Abbildungen, die der Content-Urheber selbst erstellt hatte. Daher wurden zunächst die Kontaktdaten aller Rechteinhaber der zitierten Abbildungen recherchiert und per E-Mail jeweils die Erkundigung eingeholt, ob die Abbildungen im Rahmen einer OA-Veröffentlichung unter der Lizenz CC BY-NC 4.0 verwendet werden dürfen. 
Nach ca. 4 Wochen lagen für alle angefragten Abbildungen die Genehmigungen zur Verwendung in der Veröffentlichung vor.

Es wurde entschieden, die 2 Abbildungen, die den Verweis ,in Anlehnung an [Quelle]“ trugen, durch Projektmitarbeiter 2, als Copy Editor nachzeichnen zu lassen, um rechtliche Schwierigkeiten zu vermeiden.

Beteiligte Rolle/n ausgeführt von

Content-Prüfer Projektmitarbeiter 1 und Projektleiter 1

\section{M-PVe-210: Publikationsvertrag schließen}

Da die Rechte für die im Content enthaltenen Abbildungen abschließend geklärt worden waren und bereits die ausgefüllte Checkliste für den Vertragsabschluss vorlag, konnte der Publikationsvertrag (vgl. Anhang B) vorbereitet werden. Die Unterzeichnung des Vertrages durch Content-Abnehmer und Content-Urheber erfolgte bei einem persönlichen Treffen. Bei diesem wurde auch eine Einverständniserklärung zur Veröffentlichung des Contents auf Qucosa vom Content-Urheber unterzeichnet.

Beteiligte Rolle/n ausgeführt von

Content-Prüfer Projektmitarbeiter 1

Content-Urheber

Autor der Graduierungsarbeit von Fall 2

\section{M-Pv-300: Stammdaten pflegen}

Nachdem der Publikationsvertrag geschlossen worden und somit sichergestellt war, dass der Content veröffentlicht werden darf, wies Projektmitarbeiter 1 als Projektmanager den 4 der 5 geplanten Ausgabeformate (Hardcover, Softcover, PDF, EPUB) eine ISBN zu und dokumentierte die DOIs für das Produkt nach der Richtlinie für die Bildung von DOIs (vgl. Anhang A).

Beteiligte Rolle/n ausgeführt von

Projektmanager Projektmitarbeiter 1

\section{M-Pv-300: Produktionskosten klären}

Obwohl der Produktionsweg (Prozess $M-P v$-100) bereits am Anfang des Publikationsworkflows von Fall 2 festgelegt worden war, erfolgte die Einholung von Angeboten für die Umsetzung des Satzprozesses (P-CiP-Prozesse) erst knapp 4 Monate später, nachdem der Publikationsvertrag geschlossen und die Stammdaten für den Content zugewiesen worden waren. 


\section{Satzdienstleistung}

Projektmitarbeiter 1 erfragte als Betriebswirt zunächst beim Dienstleister 02 die Kosten für den medienneutralen Satz (XML-first) des Contents nach den Gestaltungsrichtlinien des Modellverlags. Leider überstieg das Angebot das dafür vorgesehene Budget des Forschungsprojektes, weshalb es abgelehnt werden musste.

So wurde als Nächstes der inländische Publishing-Dienstleister A angefragt, dessen Angebot dem Budget entsprach, so dass dieser offiziell mit dem Satz des Contents von Fall 2 beauftragt wurde. Mit Zusendung der ersten Fahne durch Dienstleister $A$ an Projektmitarbeiter 1 stellte sich jedoch heraus, dass der Dienstleister versehentlich das Manuskript des Falles 3 bearbeitet hatte (für Fall 3 war zu zuvor ein Angebot für den Satz bei Dienstleister $A$ angefragt worden). Die erzielte Einigung sah vor, dass Dienstleister $A$ die Arbeiten am Manuskript des Falls 3 fortsetzt; auf Basis des Seitenpreises für den Content von Fall 2. Laut seinem Kostenmodell wäre der Seitenpreis für die Umsetzung von Fall 3 nämlich 1,25€ höher gewesen, auf Grund der höheren strukturellen Komplexität des Contents. Somit war es erneut notwendig, ein Angebot für die Satzprozesse des Falles 2 einzuholen. Projektmitarbeiter 1 recherchierte daraufhin verschiedene inländische und ausländische Satzdienstleister und fragte parallel die Dienstleister B, C und 07 an. Von Dienstleister 07 erhielt der Modellverlag, wie in Tabelle 5 beschrieben, bis zum Zeitpunkt der Drucklegung der vorliegenden Monografie kein Angebot.

Die zwei verbliebenen Satzdienstleister B und $C$ wurden zunächst um (kostenfreie) Arbeitsproben gebeten, damit sich das Projektteam von der Qualität der Arbeiten überzeugen kann. Die Arbeitsproben sahen vielversprechend aus, so dass um Erstellung eines verbindlichen Angebots gebeten wurde. Die Angebote unterschieden sich hinsichtlich des Preises enorm: Das Angebot von Dienstleister B führte Kosten in erwarteter Höhe auf, das Angebot von Dienstleister C lag weit darunter und war damit äußerst preiswert.

Da somit die Frage im Raum stand, inwieweit sich diese unterschiedlichen Preise in der Qualität der Dienstleistungen widerspiegeln, wurde gemeinsam im Team entschieden, beide Angebote anzunehmen. Dies führte zur Differenzierung des hier beschriebenen Falles 2 in Fall 2.1 - Umsetzung mit Dienstleister B und Fall 2.2 - Umsetzung mit Dienstleister $C$.

\section{Druckdienstleistung}

Im Vorfeld des Publikationsworkflows von Fall 2 wurde entschieden, dass Dienstleister $G$ mit der Herstellung der Musterexemplare beauftragt bzw. dessen Verlagssoftware verwendet wird. Die Kosten hierfür waren über ein Kalkulationstool der Verlagssoftware zu ermitteln.

Wie bei allen Publikationen der Fallstudie, sollte auch im Fall 2 Dienstleister E die Herstellung der Vertrags- und Verkaufsauflage übernehmen. Abermals wurden die Kosten mithilfe des vom Dienstleister zur Verfügung gestellten Offline-Preiskalkulationstools berechnet. 


\section{Weitere Kosten}

Darüber hinaus waren, wie im Fall 1, zu diesem Zeitpunkt bereits die Kosten für die Vergabe der DOIs aus dem Prozess $M-P v$-300 bekannt (die konkreten Kosten werden in Kapitel 4.4.1.4 aufgeschlüsselt).

Beteiligte Rolle/n

ausgeführt von

Betriebswirt

Projektmitarbeiter 1

\section{M-Pv-310: Soll-Kalkulation erstellen}

Nachdem die verbindlichen Angebote der gewählten Satzdienstleister B und C vorlagen, erstellte Projektmitarbeiter 1 - in der Rolle des Betriebswirts - die Soll-Kalkulation für die Publikationen des Falles 2 unter Einbeziehung der Druckkosten und Kosten für die DOIs.

Beteiligte Rolle/n

Betriebswirt ausgeführt von

Projektmitarbeiter 1

\section{M-Pv-320: Ladenpreis für gedrucktes Produkt festlegen}

Wie bereits im Kapitel 4.3.1 zu Fall 1 erwähnt, wurde auf Grundlage der Soll-Kalkulationen und mit der Kalkulationstabelle als Richtlinie zur Preisstrategie (vgl. Anhang A) frühzeitig damit begonnen, die Ladenpreise der gedruckten Produkte aller Fälle zu kalkulieren. Die finalen Ladenpreise für Soft- und Hardcover von Fall 2 ließen sich jedoch erst festlegen, nachdem die Ladenpreise für die gedruckten Produkte aller Fälle ermittelt worden waren (vgl. Kapitel 4.3.1).

Beteiligte Rolle/n

ausgeführt von

Betriebswirt

Projektleiter 1 und Projektmitarbeiter 1

\section{P-CUe-100: Cover erstellen}

\section{P-CUe-110: Umschlag erstellen}

Im Fall 2 lag Content vor, der im Reihendesign des Modellverlags veröffentlicht werden sollte, weshalb die Cover- und Umschlag-Gestaltungsrichtlinie (vgl. ScienceOpen) hier Anwendung fand. Daher passte Projektmitarbeiter 1 zunächst den Text für die U4 an (der vorgeschlagene Text vom Content-Urheber aus der Checkliste für den Vertragsabschluss und die Buchvorankündigung war zu lang für das Umschlagdesign) und sendete diesen zur Freigabe an Projektleiter 1. Nach Überarbeitung des Textes konnte Projektmitarbeiter 2 als Cover-Ersteller den Umschlag inkl. Cover für den Content erstellen. 
Beteiligte Rolle/ $n$

Cover-Ersteller

Content-Prüfer ausgeführt von

Projektmitarbeiter 1 und 2

Projektleiter 1

\section{P-CUe-120: Freigabe für Cover bzw. Umschlag einholen}

Nach Erstellung des Umschlags übermittelte Projektmitarbeiter 1 diesen zur Freigabe an den Content-Urheber, der ohne weitere Korrekturwünsche die Freigabe erteilte.

\begin{tabular}{ll} 
Beteiligte Rolle/n & ausgeführt von \\
\hline Cover-Ersteller & Projektmitarbeiter 1 \\
\hline Content-Urheber & Autor der Graduierungsarbeit von Fall 2 \\
\hline
\end{tabular}

P-CUe-130: Korrekturen für Cover bzw. Umschlag umsetzen

Die Korrektur von Cover bzw. Umschlag war in Fall 2 nicht notwendig.

\section{P-Ca-100: Fachkorrektorat beauftragen}

Wie bereits in der Darstellung von Prozess I-Ca-100 geschildert, wurde der Ausdruck der Arbeit als angemessen für eine Bachelorarbeit eingeschätzt, so dass die Beauftragung eines Fachkorrektorates in Fall 2 ausblieb.

\section{P-Ca-110: Sprachlektorat beauftragen}

Die Richtlinie für das Sprachlektorat (vgl. Anhang A) traf nicht auf den Content von Fall 2 zu, weshalb dieser Prozess nicht durchgeführt wurde.

\section{P-Ca-120: Technisches Copy Editing durchführen}

Bevor der Content zur weiteren Bearbeitung an die ausgewählten Satzdienstleister übermittelt werden konnte, war es notwendig, ihn entsprechend der Richtlinie für das technische Copy Editing (vgl. Anhang A) zu überprüfen bzw. vorzubereiten.

Dies übernahm Projektmitarbeiter 1 als Copy Editor; er ergänzte zudem die Titelei entsprechend der Gestaltungsrichtlinien des Modellverlags, generierte die fehlenden Abbildungen als einzelne Dateien (vgl. Prozess I-Ca-130) und stellte sie für die weitere Bearbeitung zur Verfügung.

Währenddessen zeichnete Projektmitarbeiter 2 ebenfalls als Copy Editor die 2 ausgewählten Abbildungen nach und prüfte anschließend die Druckqualität aller vorhandenen Abbildungen. Hierbei wurde festgestellt, dass einige Abbildungen nicht in ausreichender Qualität vorlagen, so dass Projektmitarbeiter 2 auch diese 5 weiteren Abbildungen nachzeichnete, um die Druckqualität zu garantieren. 
Beteiligte Rolle/n

Copy Editor ausgeführt von

Projektmitarbeiter 1 und 2

\section{P-CiP-100: Content-Eingangsformat in Produktionsformat überführen}

Nachdem die inhaltlichen und technischen Änderungen am Content abgeschlossen waren, konnte der Satzprozess (P-CiP-Prozesse) beginnen. Hierfür übermittelten die Projektmitarbeiter des Modellverlags die Word-Datei des Contents, die Gestaltungsrichtlinien und das InDesign-Template des Modellverlags in englischer Sprache (vgl. Anhang A) sowie die verwendeten Abbildungen als einzelne Dateien und Informationen zur Rechnungsadresse an die Dienstleister $B$ und $C$.

Für diesen Fall sollten durch die Dienstleister keine Vorgaben für die Barrierefreiheit beim EPUB (vgl. Richtlinie für die Projektumsetzung, Anhang A) beachtet werden. Wie genau dieser Prozess durch die Dienstleister ausgeführt wurde, kann hier nicht beschrieben werden.

Beteiligte Rolle/n

Copy Editor

Content-Umsetzer ausgeführt von

Projektmitarbeiter 1 und 2

Dienstleister B und C

\section{P-CiP-110: Produktionsformat mit Metadaten anreichern P-CiP-120: Qualität des Produktionsformats sichern}

Für diese Prozesse waren ebenfalls die Dienstleister $B$ und $C$ verantwortlich, so dass deren Durchführung hier nicht näher beschrieben werden kann.

Beteiligte Rolle/n

Content-Umsetzer ausgeführt von

Dienstleister B und C

\section{P-CiP-130: Gewünschte Ausgabeformate generieren}

Fall 2.1 - Umsetzung mit Dienstleister B

Dienstleister $B$ übermittelte 19 Arbeitstage nach der Beauftragung mit dem ersten PDF-Umbruch für die gedruckten Produkte auch die ersten Entwürfe für die EPUBund MOBI-Dateien an den Modellverlag.

\section{Fall 2.2 - Umsetzung mit Dienstleister C}

Dienstleister $C$ übermittelte 6 Arbeitstage nach der Beauftragung nur die erste PDFFahne für die gedruckten Produkte an den Modellverlag. Der erste EPUB- und MOBI-Entwurf wurde 11 Arbeitstage nach Beauftragung zusammen mit der dritten PDF-Fahne gesendet. 
Beteiligte Rolle/n

Content-Umsetzer

Content-Prüfer ausgeführt von

Dienstleister B und C

Projektmitarbeiter 1 und 2

\section{P-CiP-140: Gewünschte Ausgabeformate virtuell prüfen}

P-CiP-150: Korrekturen umsetzen

Um die übermittelten Ausgabeformate hinsichtlich der Richtlinie für die Produktfreigabe (vgl. Anhang A) zu überprüfen, teilten sich Projektmitarbeiter 1 und 2 die Arbeit des Content-Prüfers.

\section{Fall 2.2 - Umsetzung mit Dienstleister C}

Zunächst überprüfte Projektmitarbeiter 1 die erste PDF-Fahne von Dienstleister $C$ und übermittelte die Korrekturen an den zuständigen Mitarbeiter. Dieser übersendete nach 3 weiteren Arbeitstagen die Überarbeitung als zweite PDF-Fahne, die jedoch erneut korrekturbedürftig war. Weitere 8 Arbeitstage später übermittelte Dienstleister $C$ die dritte PDF-Fahne zusammen mit dem ersten EPUB- und MOBIEntwurf. Für die PDF-Datei war noch eine weitere Korrekturschleife notwendig, bevor die Leistung von Dienstleister $C$ aus Sicht von Projektmitarbeiter 1 den Qualitätsansprüchen des Modellverlags entsprach.

Zeitgleich prüfte Projektmitarbeiter 2 den EPUB- und MOBI-Entwurf und übermittelte seine Korrekturen. Bereits einen Arbeitstag später erhielt er den zweiten EPUB- und MOBI-Entwurf, den er in dieser Form freigeben konnte.

\section{Fall 2.1 - Umsetzung mit Dienstleister B}

Parallel dazu überprüfte Projektmitarbeiter 2 außerdem die Fahnen der Ausgabeformate von Dienstleister B. Es bedurfte lediglich 3 Korrekturschleifen, bis Projektmitarbeiter 2 die Fahnen aller Ausgabeformate von Fall 2 freigeben konnte.

Beteiligte Rolle/n ausgeführt von

\begin{tabular}{lll}
\hline & Fall 2.1 & Fall 2.2 \\
\hline Content-Umsetzer & Dienstleister B & Dienstleister C \\
\hline Content-Prüfer & Projektmitarbeiter 2 & Projektmitarbeiter 1 und 2 \\
\hline
\end{tabular}

\section{P-CiP-160: Freigabe vom Content-Urheber einholen}

Auch wenn für die Umsetzung des Contents in ein Produkt im Fall 2 zwei Satzdienstleister beauftragt wurden, sollten nur die Daten eines Dienstleisters im weiteren Publikationsworkflow Verwendung finden. Die Entscheidung fiel zugunsten der Daten des Dienstleisters B, da diese früher vorlagen und vom Projektteam als qualitativ besser eingeschätzt wurden. 
Aus diesem Grund übermittelte Projektmitarbeiter 2 - weiterhin als Content-Prüfer - die aus Verlagssicht finale PDF-Fahne ${ }^{157}$ an den Content-Urheber mit Bitte um Freigabe oder Anmerkungen. Der Content-Urheber prüfte die Datei sehr schnell und teilte lediglich eine Anmerkung mit, die der Content-Prüfer an den Content-Umsetzer ( Dienstleister B) übermittelte und, nach der Umsetzung dieser, um die Übermittlung der finalen Ausgabeformate bat. Noch am gleichen Arbeitstag erhielt der Content-Prüfer die überarbeiten Ausgabeformate von Dienstleister B. Eine Freigabe des Content-Urhebers für die PDF-Fahne musste kein weiteres Mal erfolgen; der ContentPrüfer kontrollierte nur, ob die gewünschte Korrektur umgesetzt wurde. Dies war der Fall.

Beteiligte Rolle/n ausgeführt von

\begin{tabular}{ll}
\hline Content-Prüfer & Projektmitarbeiter 2 \\
\hline Content-Umsetzer & Dienstleister B \\
\hline Content-Urheber & Autor der Graduierungsarbeit von Fall 2 \\
\hline
\end{tabular}

P-CiP-170: Umschlag anpassen

Nachdem somit die finalen Ausgabeformate der Publikation von Fall 2 vorlagen, passte Projektmitarbeiter 2 als Cover-Ersteller die Umschlagsdateien für die gedruckten Produkte (Hardcover und Softcover) entsprechend der Vorgaben des Druckdienstleisters E an. Für den Druckauftrag an Dienstleister $G$ war die Anpassung des Umschlags nicht notwendig, da Dienstleister $G$ dies selbst übernahm.

Beteiligte Rolle/n ausgeführt von

Content-Ersteller Projektmitarbeiter 2 und Dienstleister G

\section{P-CiP-180: Ausgabeformate zur Veröffentlichung freigeben}

Da nun alle notwendigen Daten zur Veröffentlichung der elektronischen und gedruckten Ausgabeformate der Publikation sowie die Freigabe des Content-Urhebers vorlagen, gab Projektmitarbeiter 2 - in der Rolle des Content-Abnehmers - diese zur Veröffentlichung frei.

Beteiligte Rolle/n ausgeführt von

Content-Abnehmer Projektmitarbeiter 2

157 Üblicherweise erhält der Content-Urheber nur die PDF-Datei seines Contents, und nicht die EBook-Formate zu Freigabe, da nicht immer ein Lesegerät zur Überprüfung vorhanden ist und außerdem häufig keine Kompetenzen beim Content-Urheber vorliegen, um diese effektive auf ihre Qualität zu überprüfen. Die Prüfung und Freigabe erfolgt durch den Content-Prüfer, in diesem Fall Projektmitarbeiter 2. 


\section{P-GP-100: Druck auslösen}

Im Anschluss an die Freigabe der Ausgabeformate der Publikation zur Veröffentlichung beauftragte Projektmitarbeiter 1 - in der Rolle des Content-Distributors rund 5 Monate nach Annahme des Contents, den Druck der Musterexemplare sowie der Verkaufs- und Verlagsauflage bei den ausgewählten Druck- und Vertriebsdienstleistern.

\section{Dienstleister $E$}

Leider konnte die von Dienstleister E produzierte Verkaufs- und Verlagsauflage zunächst nicht an den Modellverlag zugestellt werden, da der hierzu beauftragte Paketdienstleister nicht an ein Postfach, das der Modellverlag als Adresse angegeben hatte, liefert. Die Sendung ging zunächst an Dienstleister E zurück und musste erneut versendet werden. Aufgrund dessen trafen die Exemplare der Verkaufsund Verlagsauflage über 2 Monate nach Beauftragung beim Modellverlag ein.

\section{Dienstleister $G$}

Mit der Freigabe der Ausgabeformate der Publikation zur Veröffentlichung konnte Projektmitarbeiter 1 als Content-Distributor in der Verlagssoftware von Dienstleister $G$ das Projekt Fall 2 angelegen und die Druckdaten übermitteln. Nach der Prüfung der Druckdaten durch das Team des Dienstleisters erfolgte zunächst die Zusendung von Aushängern an den Modellverlag.

\section{P-EP-100: Produkt auf primärer Veröffentlichungsplattform veröffentlichen}

Noch bevor die gedruckten Produkte von Fall 2 im Modellverlag vorlagen, konnten die elektronischen Ausgabeformate der Publikation - rund 5 Monate nach Annahme des Contents - auf der Verlagswebsite oa-hochschulverlag.htwk-leipzig.de veröffentlicht werden. Dies wurde von Projektmitarbeiter 1 - in der Rolle des ContentDistributors - gemeinsam mit der Veröffentlichung der Ausgabeformate der Publikation von Fall 1 vorgenommen.

\section{M-MM-100: Metadatensatz erzeugen}

Zeitgleich mit der Durchführung von Fall 1 wurde für jedes Ausgabeformat der Publikation von Fall 2 die Checkliste für die Erstellung von Metadaten (vgl. Anhang B) ausgefüllt und somit der Metadatensatz zu den Titeln durch Projektmitarbeiter 1 als Metadaten-Manager erzeugt. 


\section{M-MM-110: Metadaten distribuieren}

Hierauf folgte, entsprechend der Richtlinie für die Verbreitung von Metadaten (vgl. Anhang A) und ebenfalls durch Projektmitarbeiter 1 als Metadaten-Manager die Verbreitung der Metadaten der Publikation von Fall 2 bei Crossref, VLB, Newbooks und KNV Zeitfracht.

Beteiligte Rolle/n

Metadaten-Manager

Metadaten-Aggregator ausgeführt von

Projektmitarbeiter 1

vgl. Kapitel 4.2.4

M-MM-120: Weitere Aggregatoren beauftragen Metadaten zu distribuieren

Weitere Aggregatoren wurden im Rahmen der Fallstudie nicht mit der Distribution der Metadaten beauftragt.

\section{P-EP-110: Produkt auf weiteren Plattformen veröffentlichen}

Nach der Richtlinie für die Wahl weiterer Veröffentlichungsplattformen (vgl. Anhang A) veröffentlichte der Metadaten-Manager des Modellverlags die elektronischen Ausgabeformate der Publikation von Fall 2 auf den Plattformen OAPEN und DOAB. Wie im Fall 1 wurde die Publikation darüber hinaus auf Unglue.it, Google Books, ScienceOpen und Qucosa veröffentlicht. Dem Content-Urheber von Fall 2 wurde außerdem vorgeschlagen, seine Publikation auf ResearchGate zu publizieren. Dem kam er nach, so dass die Publikation auch auf dieser Plattform zu finden ist.

\section{Beteiligte Rolle/n ausgeführt von}

Metadaten-Manager Projektmitarbeiter 1 und ein Mitarbeiter der Hochschulbibliothek

\section{P-GP-110: Produkt physisch prüfen}

Dienstleister $E$

In der Rolle des Content-Distributors begutachtete Projektmitarbeiter 2 die vom Dienstleister E gelieferten Exemplare der Auflage gemäß der Richtlinie für gedruckte Produkte (vgl. Anhang A). Er stellte fest, dass bei den Softcovern der Publikation Buchrückenelemente auf die U1 und U4 ragen. Dass dieser Fehler durch Mängel in der vom Modellverlag gelieferten Druckvorlage entstand, konnte ausgeschlossen werden, weshalb Projektmitarbeiter 2 die Softcover beim Dienstleister $E$ reklamierte und um Lieferung fehlerfreier Softcover bat. 


\section{Dienstleister $G$}

Rund 3 Wochen nach Freigabe der Druckdaten in der Verlagssoftware von Dienstleister $G$ wurden die Aushänger in Form von je einem vollständigen und gebundenen Soft- und Hardcover geliefert.

Erneut überprüfte Projektmitarbeiter 2 als Content-Distributor die Aushänger und konnte auch hier feststellen, dass bei den Softcovern der Publikation Buchrückenelemente auf die U1 und U4 ragen. Die Freigabe zur Veröffentlichung des Produktes bzw. zum Druck der gesamten Auflage wurde daher nur für die HardcoverVersion in der Verlagssoftware erteilt. Grundsätzlich wurde durch diese Erfahrungen entschieden, die Gestaltungsrichtlinien des Modellverlags für den Umschlag anzupassen.

Da Dienstleister $G$ lediglich mit der Produktion der Musterexemplare beauftragt und nicht beabsichtigt war, hier weitere Exemplare zu bestellen, fiel in Absprache mit dem Dienstleister die Entscheidung, den vereinbarten Testlauf der Verlagssoftware an dieser Stelle zu beenden. Ebenso wurde auf die Produktion eines „verbesserten“ Softcovers verzichtet. Andernfalls hätte an dieser Stelle Projektmitarbeiter 2 - in der Rolle des Cover-Erstellers - die Druckvorlage für den Umschlag des Softcovers anpassen und der Druckerei für den Druck der Auflage zur Verfügung stellen müssen.

Beteiligte Rolle/n ausgeführt von

Content-Distributor Projektmitarbeiter 2

Drucker Dienstleister E und Dienstleister G

\section{P-GP-120: Gewünschte Auflage produzieren}

Dienstleister $E$

Die Auflage des Hardcovers zu Fall 2 lag bereits vor. Die neu produzierten Softcover trafen in zufriedenstellender Qualität 3 Wochen nach der Reklamation im Modellverlag ein.

\section{P-GP-130: Pflicht- und Vertragsexemplare versenden}

Nachdem die Auflage zu Fall 2 vorlag, wurde der Content-Urheber hierüber von Projektmitarbeiter 1 als Content-Distributor informiert. Dieser erkundigte sich außerdem, ob die vereinbarten Autorenexemplare an die in der Checkliste für den Vertragsabschluss (vgl. Anhang B) angegebene Adresse gesendet werden sollen. Der Content-Urheber bat um den Versand an eine andere Adresse, da er sich zu diesem Zeitpunkt für längere Zeit im Ausland befand. Projektmitarbeiter 1 kümmert sich daraufhin um die Verpackung und den Versand der Autorenexemplare. 
Ebenso erfüllte er die Pflichtablieferung entsprechend der Richtlinie für Pflichtexemplare (vgl. Anhang A) und meldete zusätzlich die Netzpublikationen im OnlinePortal der DNB und der SLUB Dresden.

P-PFs-100: Für Langzeitarchivierung des Produktes sorgen

Für die Langzeitarchivierung des gedruckten Produktes des Falls 2 wurde durch die Abgabe der 2 Hardcover-Exemplare an die DNB gesorgt. Durch die Meldung der elektronischen Ausgabeformate bei der DNB und SLUB Dresden ist auch für diese die Langzeitarchivierung sichergestellt.

Beteiligte Rolle/n ausgeführt von

Content-Archivar

DNB und SLUB Dresden

M-Pv-400: Projektterminplan erstellen

Wie bei Fall 1 wurde auch für die Veröffentlichung der Publikation von Fall 2 vorab weder Erscheinungstermin festgelegt, noch den Dienstleistern eine konkrete Frist genannt, da dies kontraproduktiv für die Datenerhebung für die Fallstudienanalyse gewesen wäre. Der Prozess wurde daher nicht durchgeführt.

Prozessgruppe M-PVs: Projektverlauf sichern

Dieser Prozess erfolgte durchgängig wie für Fall 1 beschrieben.

Prozessgruppe M-OM: Operatives Marketing durchführen

Die Prozesse der M-OM-Gruppe wurde - wie für Fall 1 - durch eine studentische Projektgruppe durchgeführt.

P-PFs-200: Ausgabeformate systematisiert ablegen

M-Pn-100: Verwaltungsdaten sichern

Diese Prozesse erfolgten - wie für Fall 1 beschrieben - für alle Fälle der Studie zeitgleich.

M-Pn-200: Nach-Kalkulation erstellen

Dieser Prozess konnte vor dem Ende der Fallstudie noch nicht durchgeführt werden (vgl. Fall 1).

\section{M-Bb-200: Honorar zahlen}

Entsprechend der Vereinbarungen im Publikationsvertrag aus Prozess $M-P V e-210$ hat auch der Content-Urheber des Falls 2 keinen Anspruch auf ein Honorar, weshalb dieser Prozess nicht durchgeführt wurde. 
M-MM-200: Metadatensatz aktualisieren

Zum Zeitpunkt der Fallstudie bzw. während der Projektlaufzeit war eine Aktualisierung des Metadatensatzes zu keinem Ausgabeformat der Publikation von Fall 2 notwendig.

\subsubsection{Fall 3: XML-first-Workflow in Fremd- und Eigenleistung}

Prozess I-Ka-100 bis I-Ka-130

Diese Prozesse wurden nicht durchgeführt (vgl. Kapitel 4.3.1 und Kapitel 4.3.2).

I-Ka-200: Nach geeigneten Content-Urhebern recherchieren

Wie in den vorherigen Fällen begann auch der Publikationsworkflow des Falles 3 mit der Content-Recherche: Die Hochschulbibliothek bat im Namen des Modellverlags und im Sinne des Prozesses I-Ka-200 das Prüfungsamt der Fakultät Bauwesen um Information ihrer Professoren darüber, dass Graduierungsarbeiten für eine Fallstudie entsprechend der Richtlinie für die Konzept-Annahme (vgl. Anhang A) gesucht werden. Daraufhin meldeten sich zeitnah insgesamt vier Professoren der Fakultät mit Vorschlägen zurück. Eine der empfohlenen Arbeit enthielt allerdings einen Sperrvermerk und war somit nicht zur Publikation geeignet.

Beteiligte Rolle/n ausgeführt von

Content-Abnehmer Mitarbeiter der Hochschulbibliothek und Projektmitarbeiter 1

Content-Lieferant Professoren der Fakultät

I-Ka-210: Content-Urheber anfragen

I-Ka-220: Mit Content-Urheber über Publikation sprechen

I-Cv-100: Content einfordern

Projektmitarbeiter 1 - in der Rolle des Content-Abnehmers - nahm kurz darauf per E-Mail Kontakt zu allen drei Content-Urhebern der genannten Graduierungsarbeiten auf. In kurzen persönlichen Gesprächen bekundeten alle Content-Urheber Interesse an der Publikation ihrer Masterarbeit im Rahmen einer Fallstudie und stimmten zu, zunächst die PDF-Dateien ihrer Arbeiten zur weiteren Begutachtung zur Verfügung zu stellen.

Beteiligte Rolle/n ausgeführt von

Content-Abnehmer Projektmitarbeiter 1

Content-Urheber drei Autoren der vorgeschlagenen Masterarbeit 


\section{I-Cv-110: Content prüfen}

Gemeinsam im Team wurden die Manuskripte aller drei in Frage kommenden Content-Urheber analysiert und geprüft, ob sie der Richtlinie für die Content-Vereinnahmung (vgl. Anhang A) entsprechen. Dies traf auf alle drei Arbeiten zu. Aus den in Kapitel 4.1 genannten Gründen fiel die Wahl letztlich auf die Arbeit des hier beschriebenen Falls.

Im Zuge dieses Prozesses wurde ebenfalls festgestellt, dass der Content zitierte Abbildungen enthält, so dass der managementorientierte Prozess M-PVe-100 bereits an dieser Stelle als notwendig erkannt wurde.

Beteiligte Rolle/n ausgeführt von

Content-Abnehmer

gesamtes Projektteam

\section{M-Pv-100: Produktionsweg festlegen}

Wie bereits im Kapitel 4.1 beschrieben, war im Zuge des Prozesses I-Cv-110 festzustellen, dass der Content von Fall 3 medienneutral (XML-first) in allen gängigen Ausgabeformaten (Druck- und ePDF, EPUB und MOBI) umgesetzt werden kann. Der Satzprozess (P-CiP-Prozesse) sollte einerseits von einem Satzdienstleister ausgeführt werden und parallel dazu durch Mitarbeiter des Modellverlags, um vergleichbare Daten für die Fallstudienanalyse erheben zu können. Aufgrund dessen wird in Fall 3 an den relevanten Stellen zwischen Fall 3.1 - eigene Umsetzung und Fall 3.2 - Umsetzung mit Dienstleister A unterschieden.

$\mathrm{Zu}$ diesem Zeitpunkt stand noch nicht fest, welcher Dienstleister beauftragt wird (mehr dazu im Prozess $M-P v$-300). Hingegen sollten die Druck- und Vertriebsdienstleister, die Metadaten-Aggregatoren und die Veröffentlichungsplattformen entsprechend Kapitel 4.2.3 und Kapitel 4.2.4 verpflichtet werden.

Beteiligte Rolle/n ausgeführt von

Content-Abnehmer gesamtes Projektteam

\section{I-Cv-120: Content-Urheber beraten}

Da der Content des Falls 3 die Richtlinie für die Content-Vereinnahmung (vgl. Anhang A) erfüllt, ist es nicht notwendig, diesen Prozess durchzuführen.

\section{I-Cv-130: Content vereinnahmen und definiert ablegen}

Der Content wurde mit der Entscheidung, diesen als Fall für die Fallstudie umzusetzen, vereinnahmt (vgl. Prozess I-Cv-110) und bereits im Zuge des Prozesses I$\mathrm{Cv}$-100 zentral in der Arbeitscloud des Modellverlags gespeichert. 


\section{Prozesse I-Qe-100 bis I-Qe-140}

Da es sich beim Content des Falles 3 ebenfalls um eine Graduierungsarbeit handelt, mussten die Prozesse I-Qe-100 bis I-Qe-140 nicht durchgeführt werden.

\section{I-Qe-200: Checkliste zu Content einfordern}

\section{I-Qe-210: Gutachten zu Content einfordern}

Auch die Masterarbeit des Falles 3 war zum Zeitpunkt der Fallstudie bereits von zwei Hochschulprofessoren der Fakultät als Erst- und Zweitgutachter entsprechend der Prüfungsordnung des Studienfachs geprüft und bewertet worden. ${ }^{158}$

Projektmitarbeiter 1 - in der Rolle des Content-Abnehmers - nahm zu den Gutachtern Kontakt auf, um die Checkliste zur Evaluation der Publikationsfähigkeit (vgl. Anhang B) zu übermitteln und die Gutachten zum Content anzufordern. Kurz daraufhin meldete sich der Zweitgutachter mit der Information, dass von ihm kein Gutachten vorläge, da dies in diesem Studienfach nicht vorgesehen sei. Seine Anmerkungen zur Qualität der Arbeit seien im Gutachten des Erstgutachters berücksichtigt und er hätte auch keine weiteren Anmerkungen, weshalb er darauf verzichtet, die Checkliste auszufüllen.

Kurz nach Ablauf der gesetzten Frist erhielt der Content-Abnehmer daher lediglich das Gutachten sowie die ausgefüllte und unterschriebene Checkliste des Erstgutachters.

Beteiligte Rolle/n ausgeführt von

Gutachter zwei HTWK-Professoren des Fachgebiets als Erst- und Zweitgutachter

Content-Abnehmer Projektmitarbeiter 1

\section{I-Ca-100: Gutachten bzw. Checklisten sichten}

Anschließend erfolgte die Sichtung des Gutachtens und der Checkliste zum Content von Fall 3 nach der Richtlinie für die Publikationsannahme (vgl. Anhang A). Dies wurde von den Projektmitarbeitern 1 und 2 gemeinsam vorgenommen.

Auch hier bestätigte das Gutachten, dass die Arbeit zur Publikation geeignet ist. Die Checkliste enthielt nur wenige Anmerkungen zur Verbesserung. Die Durchführung eines Fachkorrektorats oder/und Sprachlektorats wurde nicht gefordert.

Beteiligte Rolle/n

Content-Prüfer ausgeführt von

Projektmitarbeiter 1 und 2 


\section{I-Ca-110: Überarbeitung des Content einfordern}

Da aus der Checkliste des Gutachters zwar die grundsätzliche Publikationseignung, zugleich aber auch das Überarbeitungspotenzial der Arbeit hervorging, war die Bedingung für den Prozess I-Ca-110 erfüllt.

Aus diesem Grund übermittelte Projektmitarbeiter 1 - in der Rolle des Content-Prüfers - die Anmerkungen aus der Checkliste an den Content-Urheber. Er bat diesen, den Content entsprechend zu überarbeiten und anschließend als offene Word-Datei zusammen mit den verwendeten Abbildungen als einzelne Dateien an den Modellverlag zu übermitteln.

Beteiligte Rolle/n ausgeführt von

\begin{tabular}{ll}
\hline Content-Prüfer & Projektmitarbeiter 1 \\
\hline Content-Urheber & Autor der Graduierungsarbeit von Fall 3 \\
\hline
\end{tabular}

M-PVe-200: Vertragsabschluss \& die Buchvorankündigung vorbereiten

Gemeinsam mit der Aufforderung zur Überarbeitung des Contents übermittelte Projektmitarbeiter 1 auch die Checkliste für den Vertragsabschluss und die Buchvorankündigung (vgl. Anhang B) an den Content-Urheber mit der Bitte, diese ebenfalls bis zur vorgeschlagenen Termin auszufüllen.

Beteiligte Rolle/n

ausgeführt von

Content-Abnehmer

Projektmitarbeiter 1

Content-Urheber

Autor der Graduierungsarbeit von Fall 3

\section{I-Ca-120: Überarbeiteten Content überprüfen}

Knapp einen Monat nach der Übermittlung der Verbesserungsvorschläge, also innerhalb der vereinbarten Frist, sendete der Content-Urheber den überarbeiteten Content als Word- und PDF-Datei sowie die Checkliste für den Vertragsabschluss und die Buchvorankündigung (vgl. Anhang B) an den Modellverlag.

Allerdings konnte der Content-Urheber die verwendeten Abbildungen nur teilweise als einzelne Dateien liefern, da er einige Abbildungen mit einem speziellen Programm zur Erstellung technischer Zeichnungen angefertigt, diese als PDFDatei exportiert und den Screenshot der Abbildung direkt in das Word-Dokument eingefügt hatte, ohne die Screenshots einzeln abzuspeichern.

Zugleich fiel auf, dass bei einigen Abbildungen die Abbildungsunterschrift fehlte. Der Content-Urheber wurde gebeten, diese nachzureichen, was schließlich erst rund 2 Monate nach der Aufforderung geschah. 
Beteiligte Rolle/n

Content-Prüfer

Content-Urheber ausgeführt von

Projektmitarbeiter 1

Autor der Graduierungsarbeit von Fall 3

\section{M-PVe-100: Rechte für Inhaltselemente klären}

Wie zum Prozess I-Cv-110 festgestellt, enthielt der Content des Falls 3 insgesamt 80 Abbildungen. Projektmitarbeiter 1 erstellte daher - in der Rolle des Content-Prüfers - auf Grundlage der Angaben aus der Checkliste für den Vertragsabschluss eine Übersicht, welche Abbildungen vom Content-Urheber und welche in Anlehnung an andere Abbildungen erstellt worden waren. Außerdem listete er auf, welche Abbildungen zitiert waren.

Es wurde festgestellt, dass der Content-Urheber den Großteil der Abbildungen selbst erstellt hatte und diese somit ohne weitere Prüfung im OA veröffentlicht werden dürfen. Die Abbildungen mit dem Vermerk ,in Anlehnung an“ waren Darstellungen von Forschungsdaten, die nicht die ausreichende Schöpfungshöhe erreichen, um schützenswert durch das Urheberrecht zu sein, weshalb sie ebenfalls im OA ohne Genehmigung der Rechteinhaber der Originalquelle veröffentlicht werden dürfen.

Für die 4 zitierten Abbildungen wurden im Gegensatz dazu die Rechteinhaber recherchiert und angefragt, ob die Genehmigung erteilt wird, die Abbildung im Rahmen einer Veröffentlichung im OA unter der CC-Lizenz BY-NC 4.0 zu verwenden.

Bereits eine Woche später lagen von allen angefragten Rechteinhabern die Genehmigungen zur Veröffentlichung vor, so dass dieser Prozess abgeschlossen werden konnte.

Beteiligte Rolle/n

Content-Prüfer ausgeführt von

Projektmitarbeiter 1

\section{I-Ca-130: Content annehmen}

I-Ca-140: Content zur Bearbeitung zur Verfügung stellen

Nachdem der Content-Prüfer neben dem überarbeiteten Content auch die fehlenden Abbildungsunterschriften erhalten hatte und bereits die Rechte für die Inhaltselemente im Content geklärt worden waren, konnte der Content für den weiteren Publikationsworkflow angenommen werden. Der Content-Urheber wurden über diese Entscheidung informiert und der überarbeitete Content zentral in der Arbeitscloud des Modellverlags gespeichert. 
Beteiligte Rolle/n

Content-Prüfer

Content-Urheber ausgeführt von

Projektmitarbeiter 1

\section{I-Ca-150: Content ablehnen}

Die Ablehnung des Contents war in Fall 3 nicht notwendig.

\section{M-PVe-210: Publikationsvertrag schließen}

Nachdem der Content offiziell für die Veröffentlichung angenommen und zur weiteren Bearbeitung zur Verfügung gestellt worden war, übermittelte Projektmitarbeiter 1 - in der Rolle des Content-Abnehmers - den bereits vom Projektleiter 1 unterschriebenen Publikationsvertrag (vgl. Anhang B) als Scan per E-Mail an den Content-Urheber. Nach ca. 3 Wochen und einigen Nachfragen sendete dieser den Vertrag ebenfalls unterschrieben an den Content-Abnehmer zurück. Zu einem späteren Zeitpunkt unterzeichnete der Content-Urheber auch noch die Einverständniserklärung für die Veröffentlichung des Contents auf Qucosa.

Beteiligte Rolle/n ausgeführt von

Content-Abnehmer

Content-Urheber

\section{Projektmitarbeiter 1}

Autor der Graduierungsarbeit von Fall 3

\section{M-Pv-200: Stammdaten pflegen}

Nachdem der Publikationsvertrag geschlossen und somit sichergestellt war, dass der Content veröffentlicht werden darf, wies Projektmitarbeiter 1, als Projektmanager, den 4 der 5 geplanten Ausgabeformaten der Publikation (Hardcover, Softcover, PDF, EPUB) eine ISBN zu.

Für die Vergabe der DOIs wurde in diesem Fall entschieden, nicht nur kapitelweise, sondern auch pro Abbildung und Tabelle DOIs zu bilden und zu vergeben, um auch diese Möglichkeit aufzuzeigen (vgl. Richtlinie für die Bildung von DOIs, Anhang A).

Beteiligte Rolle/n

Projektmanager ausgeführt von

Projektmitarbeiter 1

\section{M-Pv-300: Produktionskosten klären}

Wie bereits bei Prozess $M-P v-100$ sowie in Kapitel 4.1 beschrieben, war geplant, den Satzprozess (P-CiP-Prozesse) u. a. von einem Satzdienstleister (vgl. Fall 3.2) ausführen zu lassen. Hierfür mussten zunächst die Produktionskosten geklärt werden. 


\section{Satzdienstleistung}

Auch für die Umsetzung des Contents des Fall 3.2 wurde zunächst Dienstleister 02 angefragt. Nach einigen Nachfragen und einem persönlichen Gespräch mit dem Lead Content Engineer von Dienstleister 02 wurde entschieden, dass der Einrichtungsaufwand für die Erstellung eines Stylesheet-Templates zur medienneutralen Umsetzung des Contents nach den eigenen Gestaltungsrichtlinien zu kostenintensiv für den Modellverlag ist. Aus diesem Grund wurde Dienstleister 02 nicht mit der Umsetzung des Contents von Fall 3.2 beauftragt. Anschließend wurde die gleiche Anfrage parallel an Dienstleister 04 und $A$ gestellt.

Während das Angebot von Dienstleister 04 noch auf sich warten ließ, konnte festgestellt werden, dass auch die vom Dienstleister A aufgerufene Einrichtungsgebühr für die Erstellung eines Stylesheet-Templates nach den Gestaltungsrichtlinien des Modellverlags das Projektbudget übersteigen würde. Es wurde die Einigung erzielt, dass stattdessen ein bereits vorhandenes Template eines Wissenschaftsverlags, der bereits zahlreiche Bücher mit Dienstleister $A$ umgesetzt hat, verwendet wird (selbstverständlich mit dem Einverständnis dieses Verlages). Dies schien ein guter Kompromiss für die Fallstudie zu sein, da auf diesem Weg die Leistungen von Dienstleister A getestet werden können. Da ohnehin geplant war, den Content parallel dazu eigenständig im Modellverlag medienneutral aufzubereiten, würde die Publikation des Falls 3 jedoch nach den eigenen Gestaltungsrichtlinien veröffentlicht werden können.

Aufgrund des Umfangs und der Komplexität des Contents von Fall 3 überstieg das Angebot letztlich doch das Projektbudget. Daher wurde beschlossen, stattdessen mit diesem Dienstleister den Content von Fall 2 umzusetzen (vgl. Kapitel 4.3.2).

Mit Zusendung der ersten Fahne - vermeintlich zu Fall 2 - stellte sich jedoch heraus, dass der Dienstleister versehentlich doch den Content von Fall 3 bearbeitet hatte. Wie bereits in Kapitel 4.3.2 erläutert, konnte die Einigung erzielt werden, dass Dienstleister A die Arbeiten am Content von Fall 3 fortsetzt; auf Basis des Seitenpreises für den Content von Fall 2. Laut seinem Kostenmodell wäre der Seitenpreis für die Umsetzung von Fall 3 nämlich 1,25€ höher gewesen, auf Grund der höheren strukturellen Komplexität des Contents und somit nicht mehr finanzierbar. So wurde letztlich nicht Dienstleister 04 für die Umsetzung der P-CiP-Prozesse eingesetzt (Fall 3.2), sondern Dienstleister A.

\section{Druckdienstleistung}

Neben den Satzkosten für den Fall 3.2 mussten die Druckkosten von Fall 3 ermittelt werden, sowohl für die Musterexemplare als auch für die Vertrags- und Verkaufsauflage. Im Vorfeld des Publikationsworkflows von Fall 3 wurde entschieden, Dienstleister $F$ mit der Herstellung der Musterexemplare zu beauftragen. Wie in den anderen Fällen sollte Dienstleister E die Herstellung der Vertrags- und Verkaufsauflage übernehmen. Erneut wurden die Kosten mit Hilfe des im Online-Account zur Verfügung stehenden Preiskalkulators von Dienstleister F bzw. dem Offline-Preiskalkulationstools von Dienstleister E ermittelt. 
Beteiligte Rolle/n

Betriebswirt ausgeführt von

Projektmitarbeiter 1

\section{M-Pv-310: Soll-Kalkulation erstellen}

Als das Angebot von Dienstleister A vorlag, erstellte Projektmitarbeiter 1 für die Fälle 3.1 und 3.2 die Soll-Kalkulation unter Einbeziehung der Druckkosten. Hinzu kamen die Kosten für die Vergabe der DOIs pro Kapitel, Abbildung und Tabelle. Diese Kosten wurden lediglich dem Fall 3.1 zugeordnet.

Beteiligte Rolle/n ausgeführt von

Betriebswirt

Projektmitarbeiter 1

M-Pv-320: Ladenpreis für gedrucktes Produkt festlegen

Wie bereits erwähnt, konnte auf Grundlage der Soll-Kalkulationen und mit der Kalkulationstabelle als Richtlinie zur Preisstrategie (vgl. Anhang A) frühzeitig damit begonnen werden, für die gedruckten Produkte aller Fälle die Ladenpreise zu kalkulieren. Die finalen Ladenpreise für Soft- und Hardcover von Fall 3 ließen sich jedoch erst festlegen, nachdem die Ladenpreise für die gedruckten Produkte alle Fälle ermittelt worden waren (vgl. Kapitel 4.3.1 und Kapitel 4.3.2).

Beteiligte Rolle/n ausgeführt von

Betriebswirt $\quad$ Projektleiter 1 und Projektmitarbeiter 1

P-CUe-100: Cover erstellen

P-CUe-110: Umschlag erstellen

Auch bei Fall 3 handelte es sich um Content, der im Reihendesign des Modellverlags, also entsprechend der Cover- und Umschlag-Gestaltungsrichtlinie (vgl. Anhang A), veröffentlicht werden sollte.

$\mathrm{Zu}$ diesem Zweck sendete Projektmitarbeiter 1 zunächst die vom ContentUrheber zusammen mit der Checkliste für den Vertragsabschluss und die Buchvorankündigung (vgl. Anhang B) übermittelte Kurzbeschreibung des Inhalts sowie die biografischen Angaben des Autors an Projektleiter 1, da Projektmitarbeiter 1 erst wenig Erfahrungen bei der Formulierung von U4-Texten hatte. Projektleiter 1 schlug Änderungen vor, die dementsprechend eingearbeitet wurden.

Auf dieser Grundlage erstellte Projektmitarbeiter 2 als Cover-Ersteller den Entwurf zu Cover und Umschlag. Hierbei wurde festgestellt, dass die bereits überarbeitete Kurzbeschreibung des Contents zu lang für das Design des Umschlags war. Ebenso fehlte noch ein Autorenfoto. Daher wurde der Content-Urheber gebeten, die Kurzbeschreibung auf eine maximale Zeichenanzahl zu kürzen und das Foto zu übermitteln. 
Beteiligte Rolle/n

Cover-Ersteller

Projektleiter 1 ausgeführt von

Projektmitarbeiter 1 und 2

Projektleiter 1

\section{P-CUe-120: Freigabe für Cover bzw. Umschlag einholen}

Nachdem der Content-Urheber wenige Tage später die geforderten Dokumente lieferte, erstellte der Cover-Ersteller einen neuen Entwurf und sendete ihn zur Freigabe an den Content-Urheber. Eine Woche später gab der Content-Urheber Cover und Umschlag ohne weitere Korrekturwünsche frei.

Beteiligte Rolle/n

Cover-Ersteller

Content-Urheber ausgeführt von

Projektmitarbeiter 2

Autor der Graduierungsarbeit von Fall 3

P-CUe-130: Korrekturen für Cover bzw. Umschlag umsetzen

Dieser Prozess war im Fall 3 nicht notwendig.

\section{P-Ca-100: Fachkorrektorat beauftragen}

P-Ca-110: Sprachlektorat beauftragen

Wie bereits zu Prozess I-Ca-100 beschrieben, waren die Punkte der jeweiligen Richtlinien für das Fachkorrektorat und das Sprachlektorat (vgl. Anhang A) nicht erfüllt, so dass diese Prozesse nicht durchgeführt werden mussten.

\section{P-Ca-120: Technisches Copy Editing durchführen}

Bevor der Content zur weiteren Bearbeitung freigegeben werden konnte, war es notwendig, ihn entsprechend der Richtlinie für das technische Copy Editing (vgl. Anhang A) zu überprüfen bzw. vorzubereiten.

Dies übernahm Projektmitarbeiter 1 als Copy Editor. Er ergänzte die fehlenden Abbildungsunterschriften im Content und erstellte die Titelei entsprechend der Gestaltungsrichtlinien des Modellverlags.

Projektmitarbeiter 2, ebenfalls als Copy Editor, generierte inzwischen die fehlenden Abbildungen als einzelne Dateien (vgl. Prozess I-Ca-130) und überprüfte die Druckqualität aller Abbildungen. Bei einigen wurde zur Verbesserung der Druckqualität eine Bildbearbeitung, etwa Farbkonvertierung, Scharfzeichnen und Beschneiden, vorgenommen, jedoch musste keine der Abbildungen vollständig nachgezeichnet werden. Nach Abschluss dieser Schritte lag der inhaltlich finale Content für die weitere Bearbeitung vor. 
Beteiligte Rolle/n

Copy Editor ausgeführt von

Projektmitarbeiter 1 und 2

\section{P-CiP-100: Content-Eingangsformat in Produktionsformat überführen}

Wie bei Prozess $M-P v$-300 beschrieben, wurde der medienneutrale Satz des Contents von Fall 3 einerseits durch Dienstleister A (Fall 3.2) und parallel vom Modellverlag (Fall 3.1) vorgenommen.

\section{Fall 3.2}

Die Umsetzung durch Dienstleister A wurde rund 6 Monate nach Beginn des Publikationsworkflows durch Projektmitarbeiter 1 offiziell beauftragt. Hierfür erhielt er von Projektmitarbeiter 2 als Copy Editor die inhaltlich finale Word-Datei (ContentEingangsformat), die Gestaltungsrichtlinien und das InDesign-Template des Modellverlags in englischer Sprache (vgl. Anhang A), außerdem die verwendeten Abbildungen als einzelne Dateien und Informationen zur Rechnungsadresse. Wie genau Dienstleister A anschließend das Content-Eingangsformat in das Produktionsformat überführte, kann nicht beschrieben werden.

\section{Fall 3.1}

Rund 3 Monate zuvor erfolgte bereits die eigenständige Umsetzung durch Mitarbeiter des Modellverlags. Hierfür überführte eine wissenschaftliche Hilfskraft als Content-Umsetzer den Content aus der Word-Datei in eine XML-Datei, die nach BITSStandard (DTD Version 2.0) strukturiert war. Anschließend wurde der Content in der XML-Datei entsprechend der projektspezifischen Richtlinie für die Produktumsetzung (vgl. Anhang A) händisch getaggt.

Beteiligte Rolle/n

ausgeführt von

\section{Fall 3.1}

Fall 3.2

Copy Editor Projektmitarbeiter 2

Content-Umsetzer wissenschaftliche Hilfskraft

Dienstleister A

\section{P-CiP-110: Produktionsformat mit Metadaten anreichern}

\section{Fall 3.1}

Nach Überführung des Contents in die XML-Datei wurden von der wissenschaftlichen Hilfskraft außerdem verschiedene Metadaten, wie die DOIs, Lizenz-Informationen, Schlagwörter u. a. in der Datei ergänzt (vgl. Richtlinie für die Projektumsetzung, Anhang A). Wie dieser Prozess bei Dienstleister A genau durchgeführt wurde, kann nicht beschrieben werden, da hierzu keine Erkenntnisse vorliegen. 
Beteiligte Rolle/n ausgeführt von

\begin{tabular}{lll}
\hline & Fall 3.1 & Fall 3.2 \\
\hline Content-Umsetzer & wissenschaftliche Hilfskraft & Dienstleister A \\
\hline
\end{tabular}

\section{P-CiP-120: Qualität des Produktionsformats sichern}

\section{Fall 3.1}

Nach ca. einem Monat waren die ersten Arbeiten an der XML-Datei abgeschlossen, so dass ein Umbruch des Contents in Form einer PDF-Datei erstellt werden konnte. Projektmitarbeiter 2 überprüfte, ob die definierten Gestaltungsrichtlinien im selbst entwickelten XML-Template korrekt umgesetzt waren und ggf. Anpassungen für den Content von Fall 3 vorgenommen werden mussten. Seine Anmerkungen übermittelte er an die wissenschaftliche Hilfskraft; diese passte die XML-Instanz entsprechend an. In diesem Fall sollten erstmals und einmalig in der Fallstudie Teile der Vorgaben für die Barrierefreiheit beim EPUB (vgl. Richtlinie für die Produktumsetzung, Anhang A) berücksichtigt werden. Einschränkend wurde jedoch entschiedenen, die folgenden Punkte der Richtlinie nicht umzusetzen:

\section{Abbildungen}

- Verzicht auf Darstellung von Textinhalten mittels Bildern (z. B. bei Tabellen oder Formeln)

- sinnvolle Alternativtexte verfassen (max. 80 Zeichen) und mit @alt angeben einfach Bildunterschrift übernehmen, da es sonst zu Dopplungen kommen kann

- zusätzlich ausführliche Bildbeschreibung bei komplexeren Grafiken (z. B. Diagrammen), die entweder im Fließtext eingebaut oder ausgelagert und mit $<\mathrm{a}>$ oder <longdesc > verlinkt werden können

\section{Mathematische Formeln}

- nicht als Bild einbinden, sondern MathML nutzen

- evtl. Alternativtexte verwenden

Grund hierfür war, dass für die Umsetzung der Punkte hinsichtlich der Abbildungen der Content-Urheber zusätzlichen Inhalt hätte liefern müssen, was eine längere Verzögerung der Fallstudie verursacht hätte. Die Umsetzung der mathematischen Formeln als durchsuchbare Textbausteine und nicht als Abbildungen hätte zudem eine hohe Einarbeitungszeit für die wissenschaftliche Hilfskraft und Projektmitarbeiter 2 bedeutet, die ebenfalls im zeitlichen Rahmen der Fallstudie nicht möglich war. Zudem ist eine solche Umsetzung bei über 300 mathematischen Formeln eine große potenzielle Fehlerquelle; der Umgang damit hätte ebenso zusätzliche zeitliche und personelle Ressourcen erfordert, die nicht zur Verfügung standen. 


\section{Fall 3.2}

Die parallele Durchführung des Prozesses durch Dienstleister A kann nicht näher beschrieben werden.

\begin{tabular}{lll} 
Beteiligte Rolle/n & ausgeführt von & \\
\hline & Fall 3.1 & Fall 3.2 \\
\hline Content-Umsetzer & wissenschaftliche Hilfskraft & Dienstleister A \\
\hline Content-Prüfer/ Barrierefreiheit-Beauftragte & Projektmitarbeiter 2 & $/$
\end{tabular}

\section{P-CiP-130: Gewünschte Ausgabeformate generieren}

\section{Fall 3.1}

Die Arbeiten an der XML-Instanz dauerten rund 4 Monate. Erst danach konnte Projektmitarbeiter 2 die erste PDF-Fahne generieren und an den Content-Prüfer, in diesem Fall Projektmitarbeiter 1, übermitteln. Aufgrund der benötigten Zeit für die Einrichtung der Produktionsumgebung konnte Projektmitarbeiter 2 den ersten EPUB/MOBI-Entwurf nicht bis zum Abschluss der Fallstudie an den Content-Prüfer senden. Dieser Prozess war zum Zeitpunkt der Drucklegung dieses Buches somit noch nicht vollständig abgeschlossen.

\section{Fall 3.2}

15 Arbeitstage nach der Beauftragung übermittelte Dienstleister A die erste PDFFahne für die gedruckten Produkte an den Modellverlag. Den ersten EPUB- und MOBI-Entwurf lieferte Dienstleister A erst nach der Freigabe der PDF-Fahne durch den Content-Prüfer, d. h. insgesamt 27 Arbeitstage nach der Beauftragung.

Beteiligte Rolle/n ausgeführt von

\begin{tabular}{lll}
\hline & Fall 3.1 & Fall 3.2 \\
\hline Content-Umsetzer & $\begin{array}{l}\text { wissenschaftliche Hilfskraft und } \\
\text { Projektmitarbeiter 2 }\end{array}$ & Dienstleister A \\
\hline Content-Prüfer & (voraussichtl.) Projektmitarbeiter 1 & Projektmitarbeiter 1 und 2 \\
\hline
\end{tabular}

\section{P-CiP-140: Gewünschte Ausgabeformate virtuell prüfen P-CiP-150: Korrekturen umsetzen}

\section{Fall 3.1}

Projektmitarbeiter 1 überprüfte als Content-Prüfer zunächst die erste PDF-Fahne des Contents von Fall 3.1 anhand der Checkliste zur Produktfreigabe (vgl. Anhang B) und übermittelte die Anmerkungen an Projektmitarbeiter 2 als Content-Umsetzer. Dieser arbeitete die Korrekturen in die XML-Datei ein, generierte eine zweite PDFFahne und stellte diese dem Content-Prüfer zur erneuten Prüfung zur Verfügung. Es folgten 2 weitere Korrekturschleifen dieser Art. 
Da die EPUB/MOBI-Dateien des Contents von Fall 3.1 zum Abschluss der hier beschriebenen Fallstudie noch nicht vorlagen, wurden die Prozesse P-CiP-140 und P-CiP-150 nicht durchgeführt.

\section{Fall 3.2}

Wie im vorherigen Prozess beschrieben, erhielt Projektmitarbeiter 1 zunächst nur die erste PDF-Fahne des Contents von Fall 3.2 von Dienstleister A. Er kontrollierte diese ebenfalls nach der Checkliste für die Produktfreigabe (vgl. Anhang B). Seine Korrekturen übermittelte er an den zuständigen Mitarbeiter von Dienstleister $A$ und erhielt darauf die zweite PDF-Fahne. Auch hier durchlief das Material insgesamt 3 Korrekturschleifen, bis der Content-Prüfer mit der PDF-Datei zufrieden war und seine Freigabe erteilte. Erst daraufhin generierte Dienstleister A die EPUB- und MOBIEntwürfe und übermittelte diese 2 Wochen später an den Modellverlag. Projektmitarbeiter 2 nahm diesmal die Rolle des Content-Prüfers ein und kontrollierte die Daten mit Hilfe der Checkliste für die Produktfreigabe. Auch hier waren es insgesamt 3 Korrekturschleifen nötig, bis Projektmitarbeiter 2 als Content-Prüfer keine Beanstandungen an den EPUB- und MOBI-Entwürfen feststellte.

Beteiligte Rolle/n ausgeführt von

\begin{tabular}{lll}
\hline & Fall 3.1 & Fall 3.2 \\
\hline Content-Umsetzer & Projektmitarbeiter 2 & Dienstleister A \\
\hline Content-Prüfer & Projektmitarbeiter 1 & Projektmitarbeiter 1 und 2 \\
\hline
\end{tabular}

\section{P-CiP-160: Freigabe vom Content-Urheber einholen}

\section{Fall 3.1}

Nach der internen Freigabe der PDF-Fahne von Fall 3.1 durch Projektmitarbeiter 1 rund 2 Monate nach Beginn der Korrekturphase, konnte Projektmitarbeiter 2 als Content-Umsetzer diese zur Freigabe an den Content-Urheber senden. Dieser teilte daraufhin noch einige Korrekturwünsche mit. Nach weiteren 2 Korrekturschleifen erteilte der Content-Urheber die Freigabe für die Druckvorlage.

Der Prozess P-CiP-160 konnte für die EPUB- und MOBI-Entwürfe - wie in den vorherigen Prozessen beschrieben - vor dem Abschluss der Fallstudie noch nicht durchgeführt werden.

\section{Fall 3.2}

Wie bereits erläutert, sollten die Ausgabeformate der Publikation aus der eigenen Umsetzung (Fall 3.1) veröffentlicht werden. Die Freigabe der Ausgabeformate von Dienstleister A durch den Content-Urheber war somit nicht notwendig. 
Beteiligte Rolle/n ausgeführt von

\begin{tabular}{lll}
\hline & Fall 3.1 & Fall 3.2 \\
\hline Content-Umsetzer & Projektmitarbeiter 2 & Dienstleister A \\
\hline Content-Urheber & Autor der Graduierungsarbeit von Fall 3 & $/$ \\
\hline
\end{tabular}

\section{P-CiP-170: Umschlag anpassen}

Nachdem die Freigabe der Ausgabeformate für die Publikation Fall 3 vorlag, konnte Projektmitarbeiter 2 den Umschlag entsprechend der Vorgaben der Druckdienstleister $E$ und $F$ auf den finalen Seitenumfang anpassen und die druckfähigen Umschlagsdateien für Hard- und Softcover erstellen.

Beteiligte Rolle/n

Cover-Ersteller ausgeführt von

Projektmitarbeiter 2

\section{M-MM-100: Metadatensatz erzeugen}

Nachdem alle relevanten Metadaten zum Fall 3, wie Stammdaten, Seitenumfang der gedruckten Produkte und die Ladenpreise, vorlagen, konnte die Checkliste für die Erstellung von Metadaten (vgl. Anhang B) zu Fall 3 vom Metadaten-Manager ausgefüllt werden.

Beteiligte Rolle/n

Metadaten-Manager ausgeführt von

\section{Projektmitarbeiter 1}

\section{M-MM-110: Metadaten distribuieren}

Zeitnah zur Erstellung des Metadatensatzes erfolgte die Distribution dieser entsprechend der Richtlinie für die Verbreitung von Metadaten (vgl. Anhang A). Zur Verstetigung des Modellverlags in einen OA-Hochschulverlag der HTWK Leipzig führte ein Student als Metadaten-Manager unter Anleitung von Projektmitarbeiter 1 diesen Prozess durch.

Beteiligte Rolle/n ausgeführt von

Metadaten-Manager Student

\section{M-MM-120: Weitere Aggregatoren beauftragen Metadaten zu distribuieren}

Weitere Aggregatoren wurden im Rahmen der Fallstudie nicht mit der Distribution der Metadaten beauftragt. 


\section{P-GP-100: Druck auslösen}

Da die Freigabe für die Druckvorlage für Fall 3.1 bereits vorlag, konnte der Druck der Musterexemplare und Vertrags- und Verkaufsauflage durch Projektmitarbeiter 1 in der Rolle des Content-Distributors rund 10 Monate nach Annahme des Contents bei den ausgewählten Druck- und Vertriebsdienstleistern beauftragt werden.

\section{Dienstleister E}

Dienstleister $E$ wurde, wie für alle Fälle der Studie, mit Druck und Lieferung der Vertrags- und Verkaufsauflage beauftragt. Bereits nach 8 Arbeitstagen lagen die Exemplare im Modellverlag vor.

\section{Dienstleister F}

Für die Herstellung der gedruckten Musterexemplare für Fall 3.1 wurde Dienstleister $F$ beauftragt. An dieser Stelle fiel die Entscheidung, zu Ansichtszwecken auch ein Musterexemplar (Softcover) mit den Inhaltsdaten aus Fall 3.2 von Dienstleister F produzieren zu lassen. Aufgrund der hohen Auftragsauslastung konnte Dienstleister $F$ den gewünschten Liefertermin (10 Werktage) für alle 3 Exemplare nicht einhalten und lieferte die Produkte erst nach 15 Werktagen. Dienstleister F informierte den Content-Distributor hierüber rechtzeitig, konkret 4 Werktage vor dem gewünschten Liefertermin.

Beteiligte Rolle/n

Content-Distributor ausgeführt von

Projektmitarbeiter 1

\section{P-GP-110: Produkt physisch prüfen}

P-CiP-180: Ausgabeformate zur Veröffentlichung freigeben

Nach Erhalt der Produkte von Dienstleister $E$ und $F$ wurden diese anhand der Richtlinie für gedruckte Produkte (vgl. Anhang A) von Projektmitarbeiter 2 als Content-Distributor geprüft. Es wurden keine Mängel festgestellt, so dass die Ware final angenommen und die gedruckten Produkte bereits zur Veröffentlichung freigegeben werden konnten.

\begin{tabular}{ll} 
Beteiligte Rolle/n & ausgeführt von \\
\hline Content-Distributor & Projektmitarbeiter 2 \\
\hline Content-Abnehmer & Projektmitarbeiter 2 \\
\hline Drucker & Dienstleister E und $F$ \\
\hline
\end{tabular}

P-GP-130: Pflicht- und Vertragsexemplare versenden

Nachdem die Exemplare für die Vertrags- und Verkaufsauflage von Dienstleister E vorlagen, versendete Projektmitarbeiter 1 in der Rolle des Content-Distributors zeitnah die Pflichtexemplare (2 Hardcover) an die DNB. Da die elektronischen Aus- 
gabeformate dieses Falls nicht vor dem Abschluss der hier beschriebenen Fallstudie vorlagen, konnten die Netzpublikationen dementsprechend noch nicht an die DNB und SLUB Dresden übermittelt werden.

Zeitgleich mit dem Versand der gedruckten Exemplare an die DNB konnten aber die Vertrags-, bzw. Autorenexemplare an den Content-Urheber übergeben werden. Projektmitarbeiter 1 informierte ihn zunächst per E-Mail über das Vorliegen der Vertragsauflage und vereinbarte einen Termin, da der Content-Urheber seine Autorenexemplare persönlich abholen wollte. Dies geschah 5 Werktage nach der Kontaktaufnahme.

Beteiligte Rolle/n ausgeführt von

Content-Distributor Projektmitarbeiter 1

Content-Archivar Projektmitarbeiter 1 bzw. DNB

P-PFs-100: Für Langzeitarchivierung des Produktes sorgen

Die Langzeitarchivierung der gedruckten Produkte des Falls 3.1 wurde durch die Abgabe der 2 Hardcover-Exemplare an die DNB sichergestellt. Die Meldung der elektronischen Ausgabeformate, die in Kürze folgt, gewährleistet die Langzeitarchivierung auch dieser.

Beteiligte Rolle/n ausgeführt von

Content-Archivar DNB und SLUB Dresden

M-Pv-400: Projektterminplan erstellen

Dieser Prozess wurde nicht durchgeführt; vgl. Fall 2.

Prozessgruppe M-PVs: Projektverlauf sichern

Die Sicherung des Projektverlaufs erfolgte durchgängig; vgl. Fall 1 und 2.

Prozessgruppe M-OM: Operatives Marketing durchführen

Das operative Marketing führte eine studentische Projektgruppe durch; vgl. Fall 1 und 2.

P-PFs-200: Ausgabeformate systematisiert ablegen

M-Pn-100: Verwaltungsdaten sichern

Beide Prozesse erfolgten für alle Studien zeitgleich; vgl. Fall 1 und 2.

M-Pn-200: Nach-Kalkulation erstellen

Dieser Prozess konnte vor dem Ende der Fallstudie noch nicht durchgeführt werden; vgl. Fall 1 und 2. 
M-Bb-200: Honorar zahlen

Entsprechend des Publikationsvertrages aus Prozess $M-P V e-210$ hat auch der Content-Urheber des Falls 3 keinen Anspruch auf ein Honorar, weshalb dieser Prozess nicht durchgeführt wurde.

\section{M-MM-200: Metadatensatz aktualisieren}

Zum Zeitpunkt der Fallstudie bzw. während der Projektlaufzeit war eine Aktualisierung des Metadatensatzes zu keinem Ausgabeformat der Publikation von Fall 3 notwendig.

\subsection{Fallstudienanalyse}

Nachdem in den vorangegangenen Kapiteln die Methode der vergleichenden Fallstudie, die Ergebnisse der Recherche zu Dienstleistern und die konkrete Durchführung des Workflow-Modells durch die vorgestellten Fälle erläutert wurden, erfolgt an dieser Stelle die Fallstudienanalyse. Während der Durchführung der vorgestellten Fälle wurden hierzu Daten erhoben: Zu den Workflow-Rollen, zum Ablauf der Publikationsworkflows, zum zeitlichen Arbeitsaufwand, zu den Kosten für das Beauftragen von Dienstleistungen (im weiteren Herstellungskosten genannt) und zur Qualität der Leistungen der in diesem Rahmen beauftragten Satz- und Druckund Vertriebsdienstleister. Diese Daten werden nachfolgend präsentiert. Im Kapitel 4.4.2 folgt die Analyse dieser Datenerhebung und im Kapitel 4.4.3 die Einordnung der Ergebnisse, um allgemeine Schlussfolgerungen für die Publikation von OA-Büchern in einem Hochschulverlag treffen zu können.

\subsubsection{Datenerhebung zur Fallstudie}

\subsubsection{1 Übersicht zu den Workflow-Rollen}




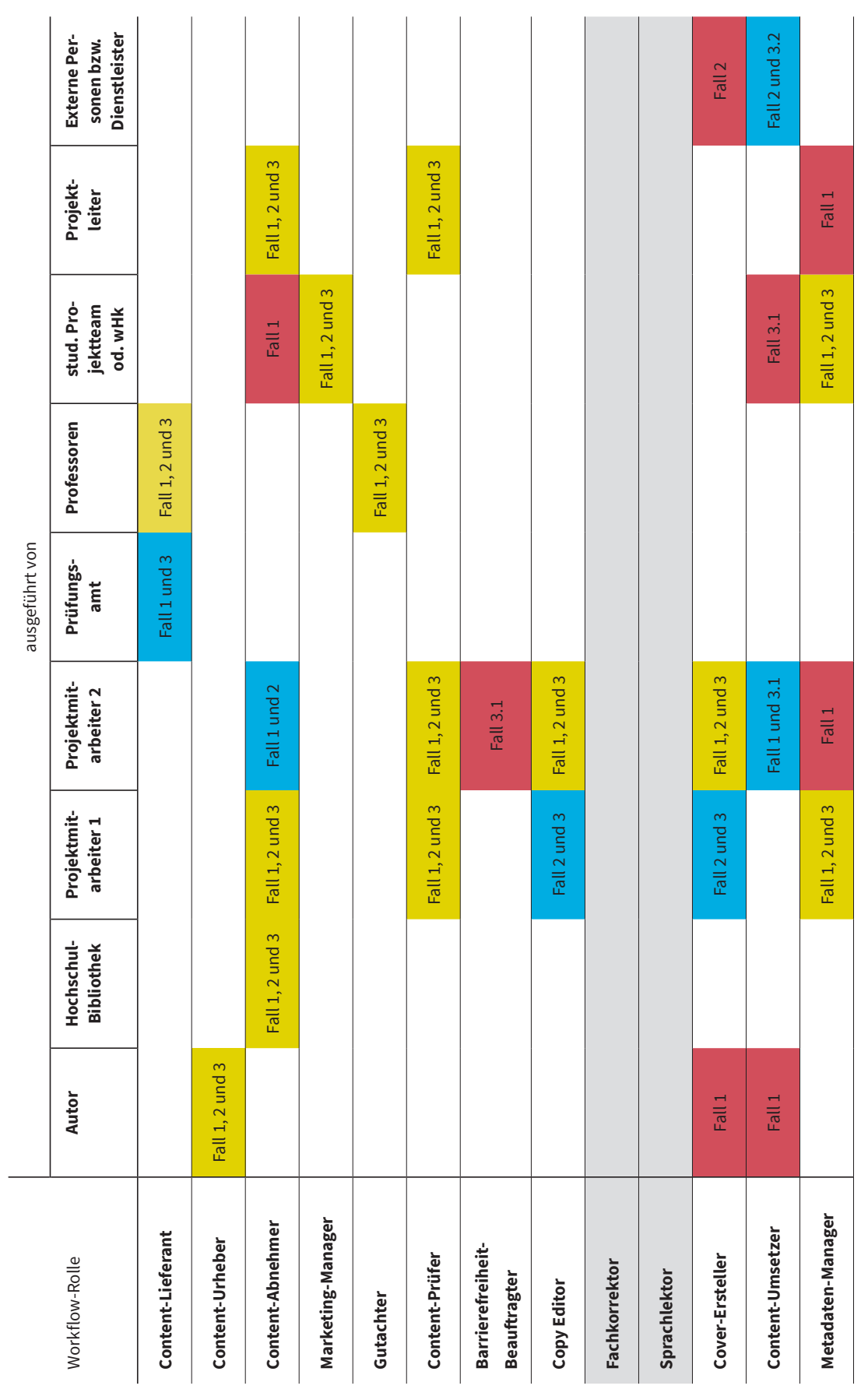

Abb. 12 Übersicht zur Ausführung der Workflow-Rollen in der Fallstudie 


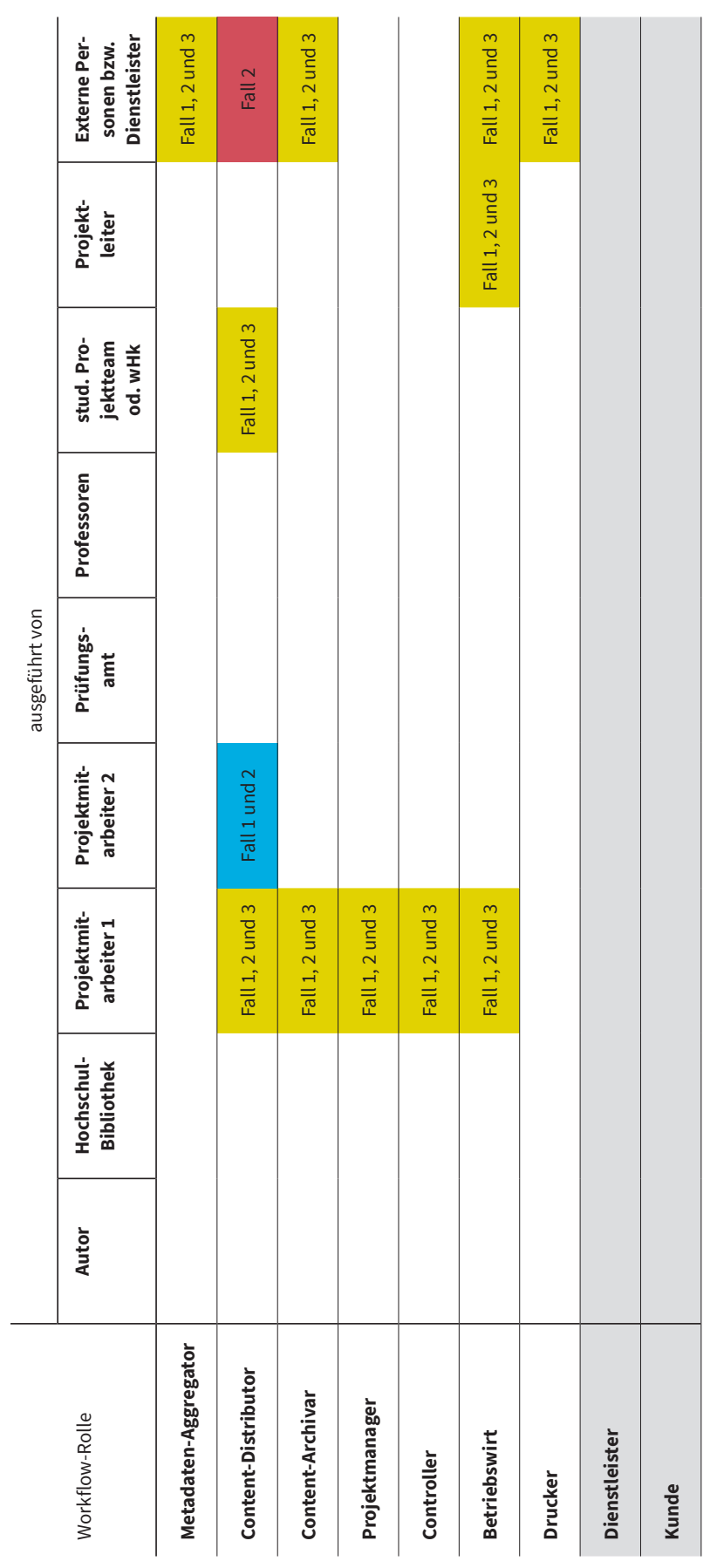


Die Abbildung 12 zeigt auf, welche realen Personen bzw. Dienstleister und Institutionen die Rollen des Workflow-Modells in der Fallstudie eingenommen haben. Farblich hervorgehoben sind hierbei 3 unterschiedliche Möglichkeiten:

- Gelb: Die Workflow-Rolle wurde in allen 3 Fällen von der bzw. den gleichen Person/en eingenommen.

- Rot: Die Workflow-Rolle wurde nur in einem der Fälle von der markierten Person eingenommen.

- Blau: Die Workflow-Rolle wurde in 2 Fällen von der bzw. den gleichen Person/en eingenommen.

Zu beachten ist, dass die Rollen Fachkorrektor und Sprachlektor für keinen der hier beschriebenen und analysierten Fälle relevant waren und daher in der Tabelle ausgegraut sind. Im Gegensatz dazu waren die Rollen Dienstleister und Kunde zwar im Rahmen der $M$-Bb-Prozesse relevant, jedoch tragen diese kaum zur Evaluation des Workflow-Modells bei, so dass sie in der Tabelle ebenfalls ausgegraut dargestellt sind.

\subsubsection{Zeitlicher Ablauf der Publikationsworkflows}

In diesem Kapitel wird der Ablauf der Publikationsworkflows der Fälle durch Gantt-Diagramme illustriert. Hierdurch soll effektiv der zeitliche Ablauf der Prozesse dargestellt werden. Jeder tatsächlich im Fall durchgeführte Prozess ist durch einen Balken abgebildet und der jeweiligen Arbeitswoche (AW) zugeordnet, in der er durchgeführt wurde. Die Länge der Balken gibt an, ob die Durchführung des Prozesses 1 oder 2 Arbeitswochen oder mehr Zeit benötigte. Sind im Gantt-Diagramm Prozesse untereinander aufgeführt, bedeutet dies, dass sie parallel bzw. an 2 aufeinanderfolgenden Tagen durchgeführt wurden. Auf die Angaben der tatsächlichen Start- und Enddaten der Prozesse wurde zu Gunsten der Übertragbarkeit verzichtet. 


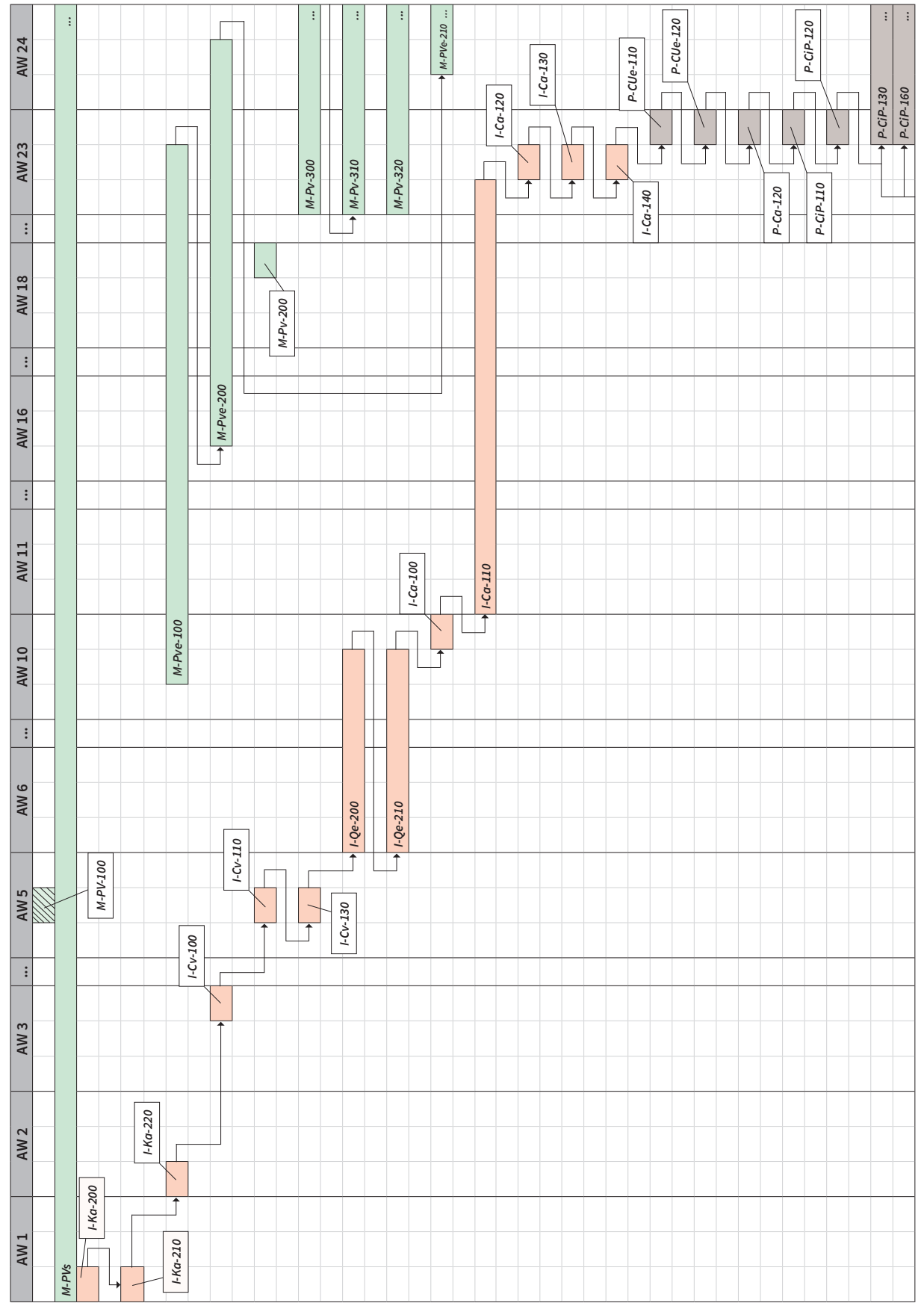

Abb. 13 Gantt-Diagramm zum zeitlichen Ablauf von Fall 1 (S. 1/2) 


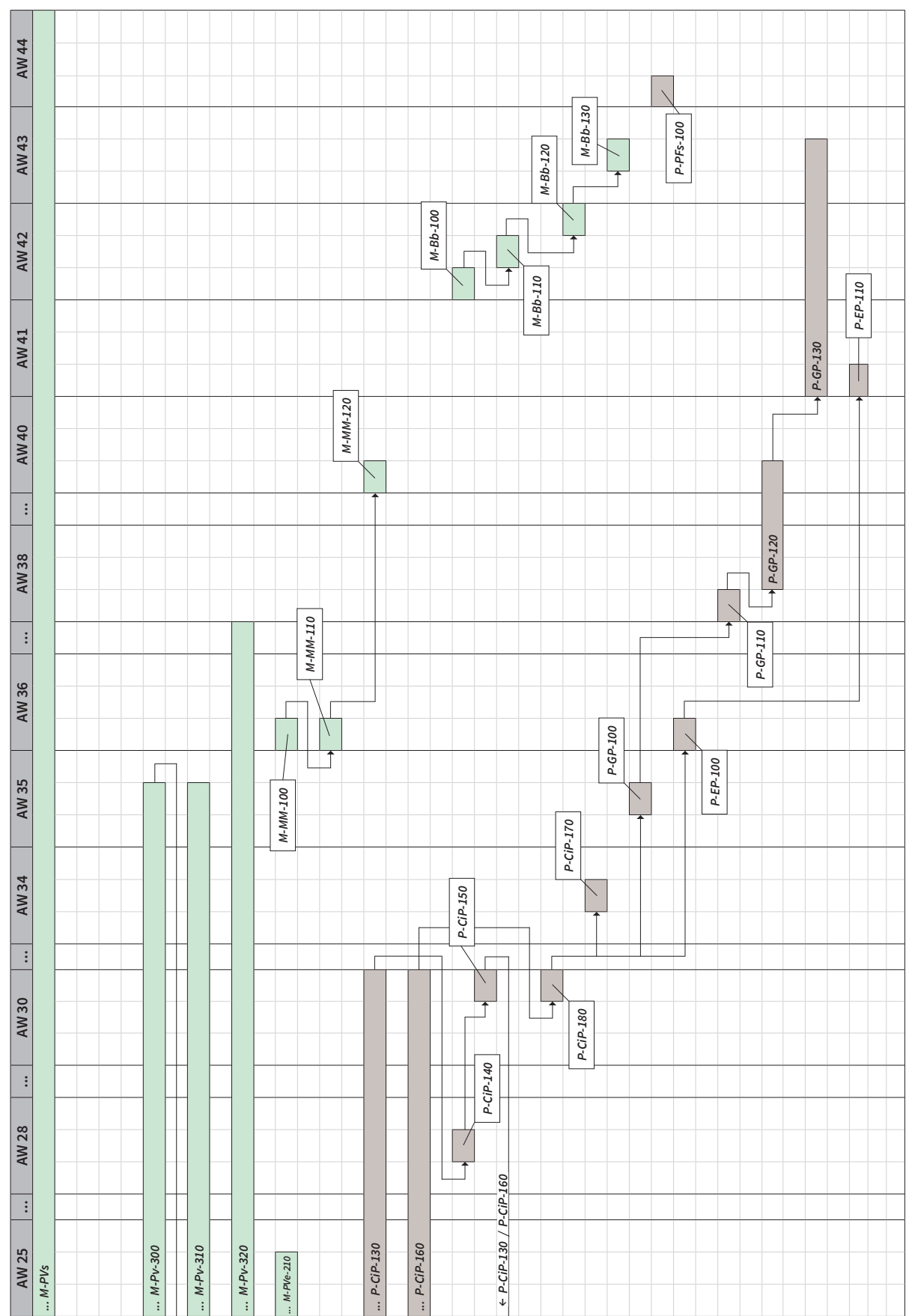

Abb. 14 Gantt-Diagramm zum zeitlichen Ablauf von Fall 1 (S. 2/2) 


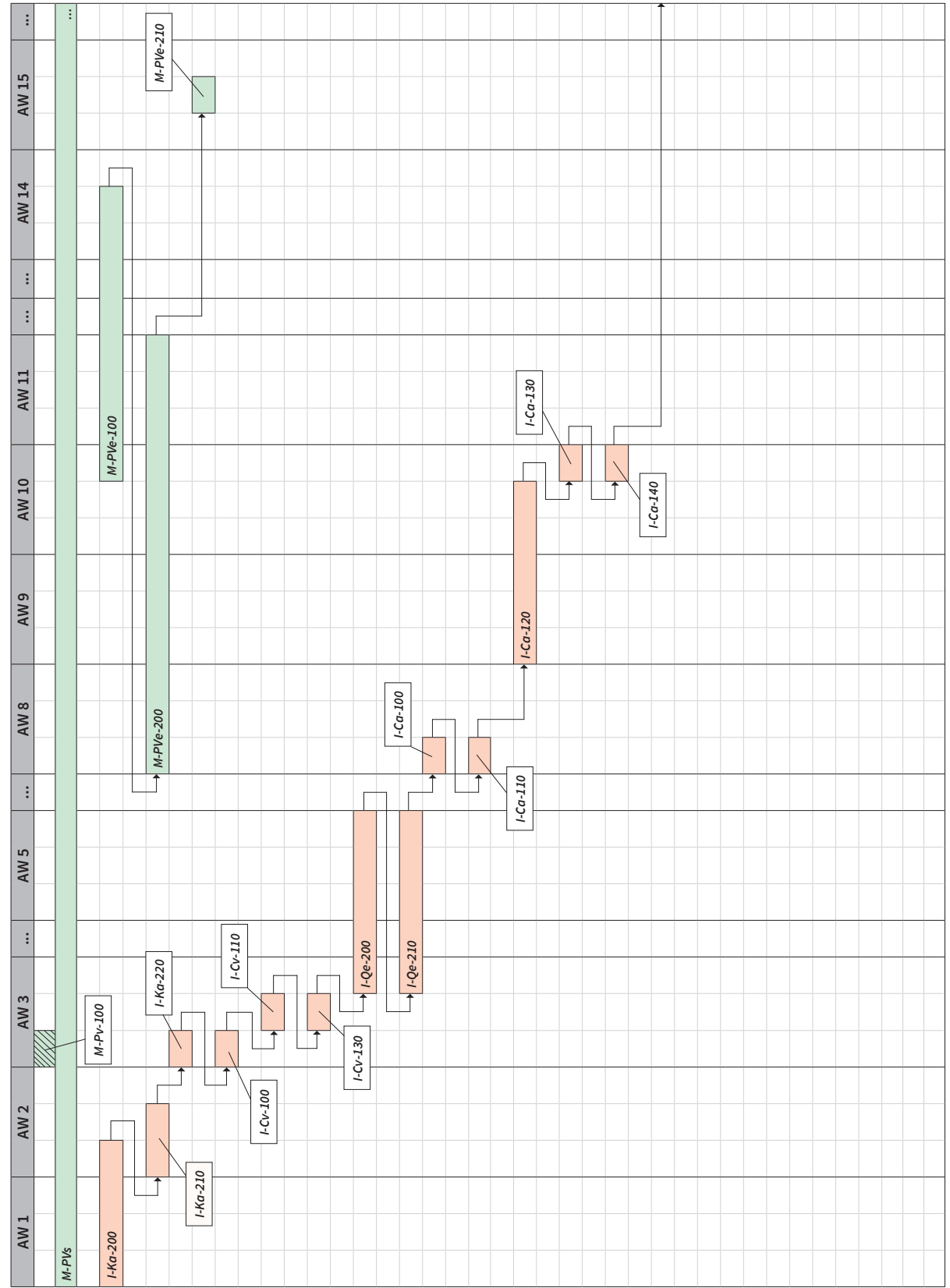

Abb. 15 Gantt-Diagramm zum zeitlichen Ablauf von Fall 2 (S. 1/3) 


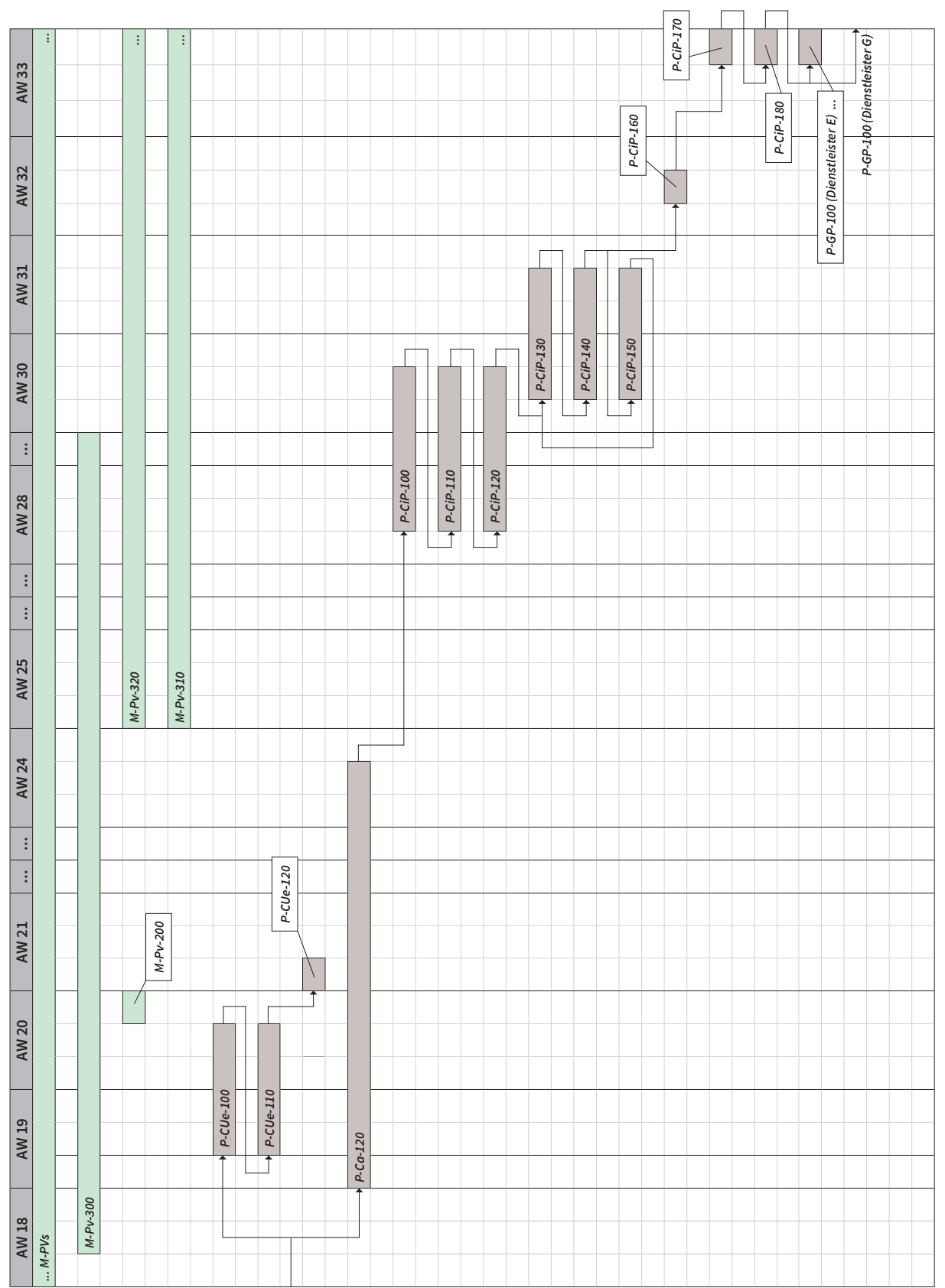

Abb. 16 Gantt-Diagramm zum zeitlichen Ablauf von Fall 2 (S. 2/3) 


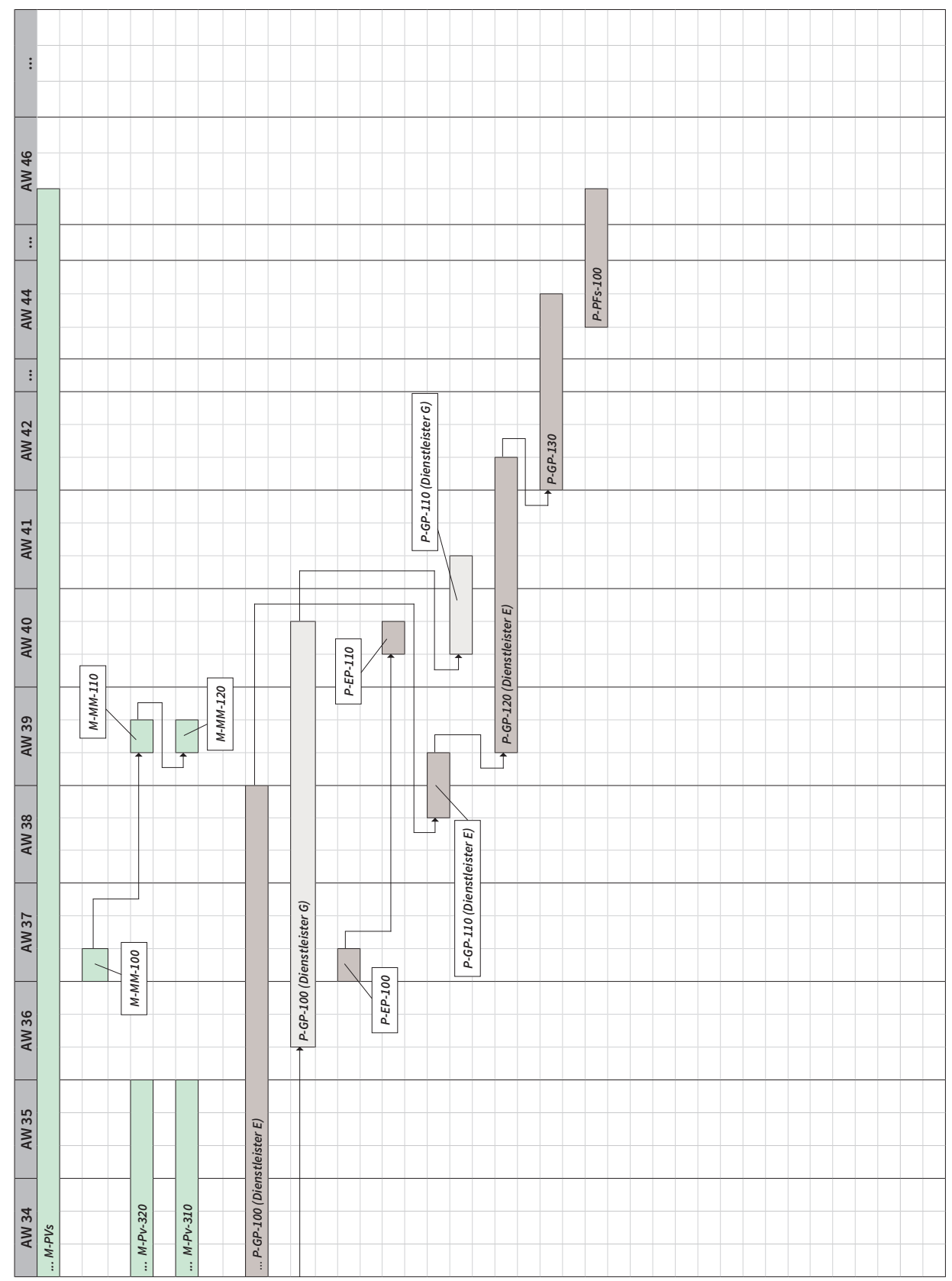

Abb. 17 Gantt-Diagramm zum zeitlichen Ablauf von Fall 2 (S. 3/3) 


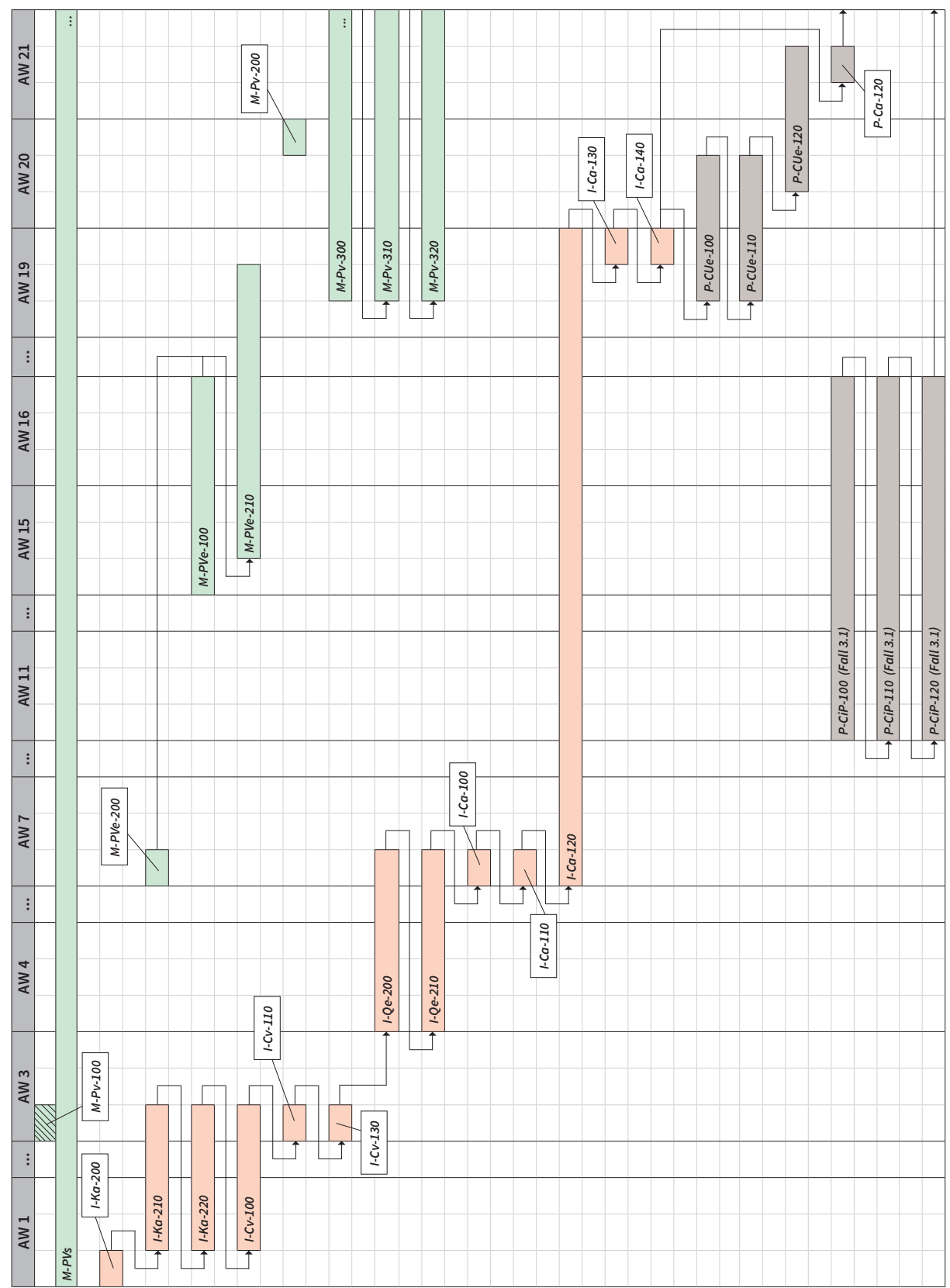

Abb. 18 Gantt-Diagramm zum zeitlichen Ablauf von Fall 3 (S. 1/3) 


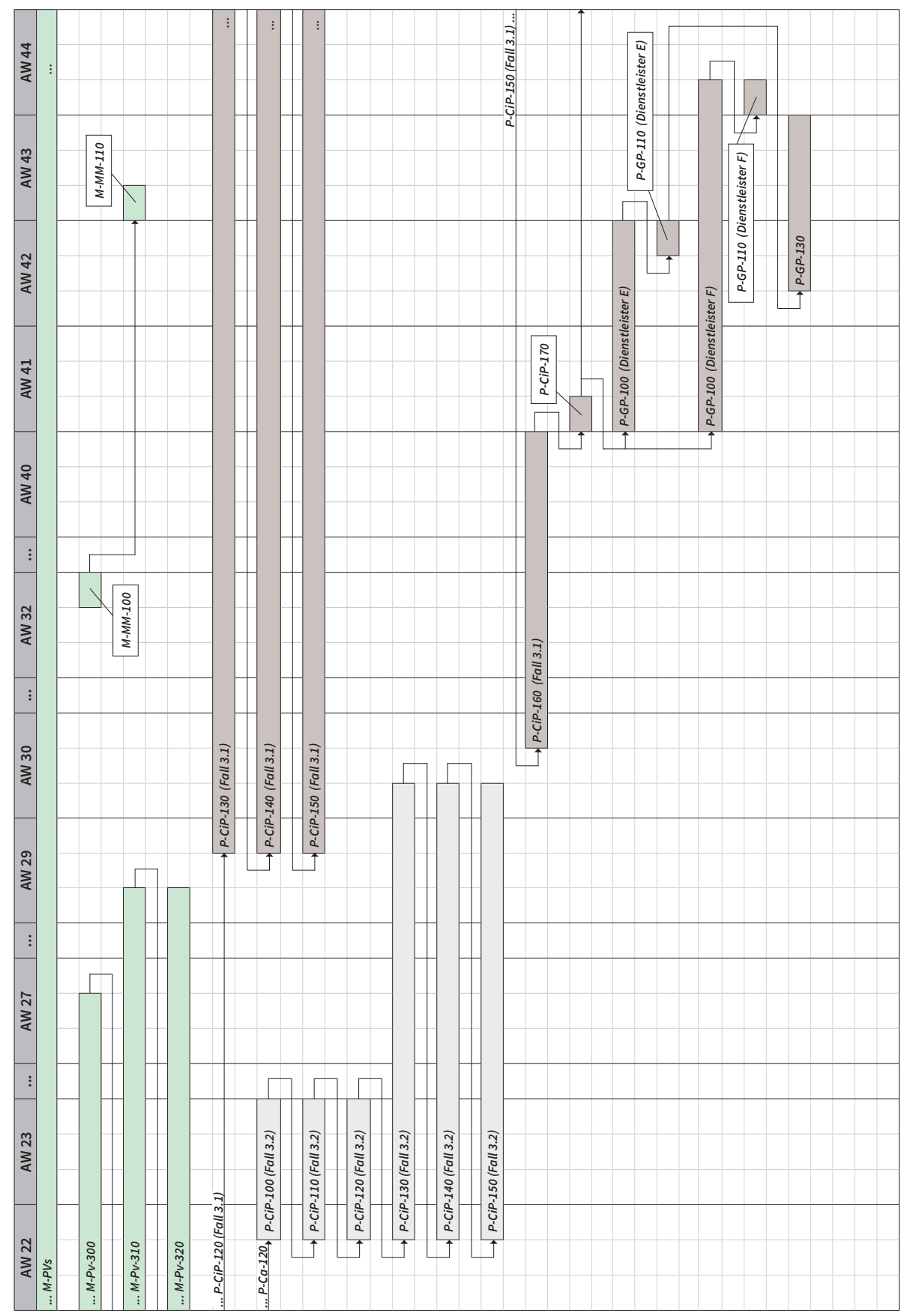

Abb. 19 Gantt-Diagramm zum zeitlichen Ablauf von Fall 3 (S. 2/3) 


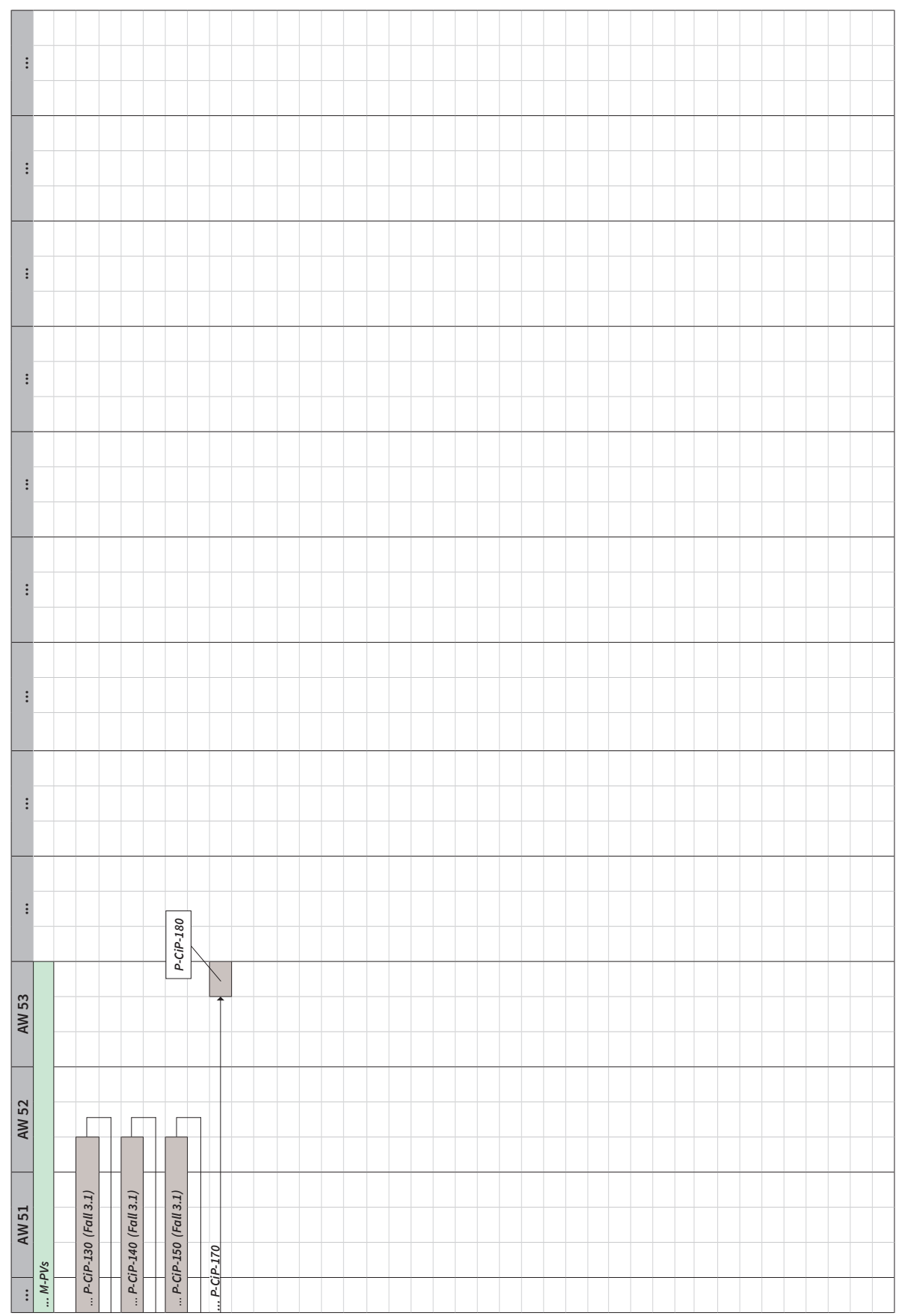

Abb. 20 Gantt-Diagramm zum zeitlichen Ablauf von Fall 3 (S. 3/3) 


\subsubsection{Arbeitsaufwand der Publikationsworkflows}

Für die Fallstudienanalyse wurde der zeitliche Arbeitsaufwand jedes Prozesses der Fälle in Minuten erfasst. D. h., die Mitarbeiter des Modellverlags haben dokumentiert, wie viel Zeit (nachfolgend als interner Arbeitsaufwand bezeichnet) sie für die Durchführung des jeweiligen Prozesses benötigten. Die Dauer bis zum Abschluss der einzelnen Prozesse ist den Gantt-Diagrammen im vorherigen Kapitel zu entnehmen. Zu beachten ist hierbei, dass Daten nur erfasst werden konnten, wenn ein Mitglied des Modellverlags die entsprechende Workflowrolle einnahm. Der zeitliche Arbeitsaufwand für Prozesse, die von Dienstleistern durchgeführt wurden, konnte nicht erfasst werden. Dies betrifft v. a. die P-CiP-Prozesse und Prozess PGP-120. Allerdings ist auch in diesem Zusammenhang an verschiedenen Stellen Aufwand für die Projektmitarbeiter entstanden, etwa durch die Kommunikation mit den Dienstleistern und um deren Leistungen sicherzustellen. Der entsprechende interne Arbeitsaufwand wurde erhoben und diesen Prozessen zugeordnet.

Tabelle 14 gibt einen Überblick für den internen Arbeitsaufwand pro Prozessgruppe. Die detaillierte Aufschlüsselung des Arbeitsaufwands pro Prozess ist im Anhang D zu finden. Zur besseren Darstellung und als Grundlage der nachfolgenden Datenanalyse wurden die Daten aus der Tabelle außerdem noch in Form eines Netzdiagrammes (vgl. Abbildung 21) aufbereitet.

In Tabelle 14 erfolgt für die P-CiP-Prozesse die Differenzierung des Arbeitsaufwandes in die Fälle 2.1, 2.2, 3.1 und 3.2, da - wie beschrieben - die Satzprozesse der Monografien der Fälle 2 und 3 auf zweifache Weise durchgeführt wurden. Zudem umfasst die hier dargestellte Datenerhebung die Prozessgruppen $M-P V s, M$ $O M$ und $M-P n$ nicht, da zum Zeitpunkt der Drucklegung des vorliegenden Werkes die entsprechenden Prozesse noch nicht durchgeführt oder keine Daten hierfür erhoben wurden (vgl. Kapitel 4.3). Für den Fall 3.1 können für die Prozesse PCiP-130 bis P-CiP-150, P-CiP-180, P-EP-100, P-EP-110 und P-PFs-100 ebenfalls keine Daten präsentiert werden, da zum Zeitpunkt der Drucklegung des hier vorliegenden Werkes diese Prozesse noch nicht abschließend vom Projektteam durchgeführt waren. 
Tab. 14 Arbeitsaufwand der Publikationsworkflows pro Prozessgruppe

\begin{tabular}{|c|c|c|c|c|c|c|c|}
\hline & \multirow[b]{2}{*}{$\begin{array}{l}\text { Prozessgruppe } \\
(\mathrm{Pg})\end{array}$} & \multicolumn{5}{|c|}{ Interner Arbeitsaufwand (min) } & \multirow{2}{*}{$\begin{array}{l}\text { Summe } \\
\text { pro Pg }\end{array}$} \\
\hline & & $\begin{array}{c}\text { F } 1 \\
\text { CRC- } \\
\text { Workflow }\end{array}$ & $\begin{array}{c}\text { F } 2.1 \\
\text { Dienst- } \\
\text { leister B }\end{array}$ & $\begin{array}{c}\text { F } 2.2 \\
\text { Dienst- } \\
\text { leister C }\end{array}$ & $\begin{array}{c}\text { F } \mathbf{3 . 1} \\
\text { eigene } \\
\text { Umsetzung }\end{array}$ & $\begin{array}{c}\text { F } 3.2 \\
\text { Dienst- } \\
\text { leister A }\end{array}$ & \\
\hline I-Ka & Konzept auswählen & 57 & \multicolumn{2}{|c|}{10} & \multicolumn{2}{|c|}{20} & 87 \\
\hline $\mathrm{I}-\mathrm{CV}$ & Content vereinnahmen & 26 & \multicolumn{2}{|c|}{8} & \multicolumn{2}{|c|}{17} & 51 \\
\hline I-Qe & $\begin{array}{l}\text { Inhaltliche Qualität des } \\
\text { Contents evaluieren }\end{array}$ & 15 & \multicolumn{2}{|c|}{10} & \multicolumn{2}{|c|}{10} & 35 \\
\hline $\mathrm{I}-\mathrm{Ca}$ & Content annehmen & 97 & \multicolumn{2}{|c|}{29} & \multicolumn{2}{|c|}{29} & 155 \\
\hline $\mathrm{P}-\mathrm{Ca}$ & Content aufbereiten & 45 & \multicolumn{2}{|c|}{225} & \multicolumn{2}{|c|}{85} & 355 \\
\hline P-CUe & $\begin{array}{l}\text { Cover bzw. Umschlag } \\
\text { erstellen }\end{array}$ & 110 & \multicolumn{2}{|c|}{44} & \multicolumn{2}{|c|}{56} & 210 \\
\hline P-CiP & $\begin{array}{l}\text { Content in Produkt } \\
\text { umsetzen }\end{array}$ & 240 & 235 & 387 & 2.298 & 750 & 3.910 \\
\hline P-GP & $\begin{array}{l}\text { Gedrucktes Produkt } \\
\text { produzieren }\end{array}$ & 34 & \multicolumn{2}{|c|}{207} & \multicolumn{2}{|c|}{110} & 351 \\
\hline P-EP & $\begin{array}{l}\text { Elektronisches Produkt } \\
\text { veröffentlichen }\end{array}$ & 39 & \multicolumn{2}{|c|}{35} & \multicolumn{2}{|c|}{-} & 74 \\
\hline P-PFs & Produktform sichern & 10 & \multicolumn{2}{|c|}{14} & \multicolumn{2}{|c|}{-} & 24 \\
\hline M-PVe & $\begin{array}{l}\text { Publikationsvertrag } \\
\text { erstellen }\end{array}$ & 161 & \multicolumn{2}{|c|}{208} & \multicolumn{2}{|c|}{41} & 410 \\
\hline M-P-Pv & Produktion vorbereiten & 105 & \multicolumn{2}{|c|}{193} & \multicolumn{2}{|c|}{80} & 378 \\
\hline M-MM & $\begin{array}{l}\text { Metadaten-Manage- } \\
\text { ment durchführen }\end{array}$ & 79 & \multicolumn{2}{|c|}{119} & \multicolumn{2}{|c|}{142} & 340 \\
\hline $\mathrm{M}-\mathrm{Bb}$ & Bestellungen bedienen & 43 & \multicolumn{2}{|c|}{0} & \multicolumn{2}{|c|}{-} & 43 \\
\hline \multicolumn{2}{|c|}{ SUMME (min) } & 1.058 & 1.367 & 1.519 & 2.888 & 1.340 & 6.423 \\
\hline \multicolumn{2}{|c|}{ SUMME (h) } & 18 & 23 & 25 & 48 & 22 & 136 \\
\hline
\end{tabular}




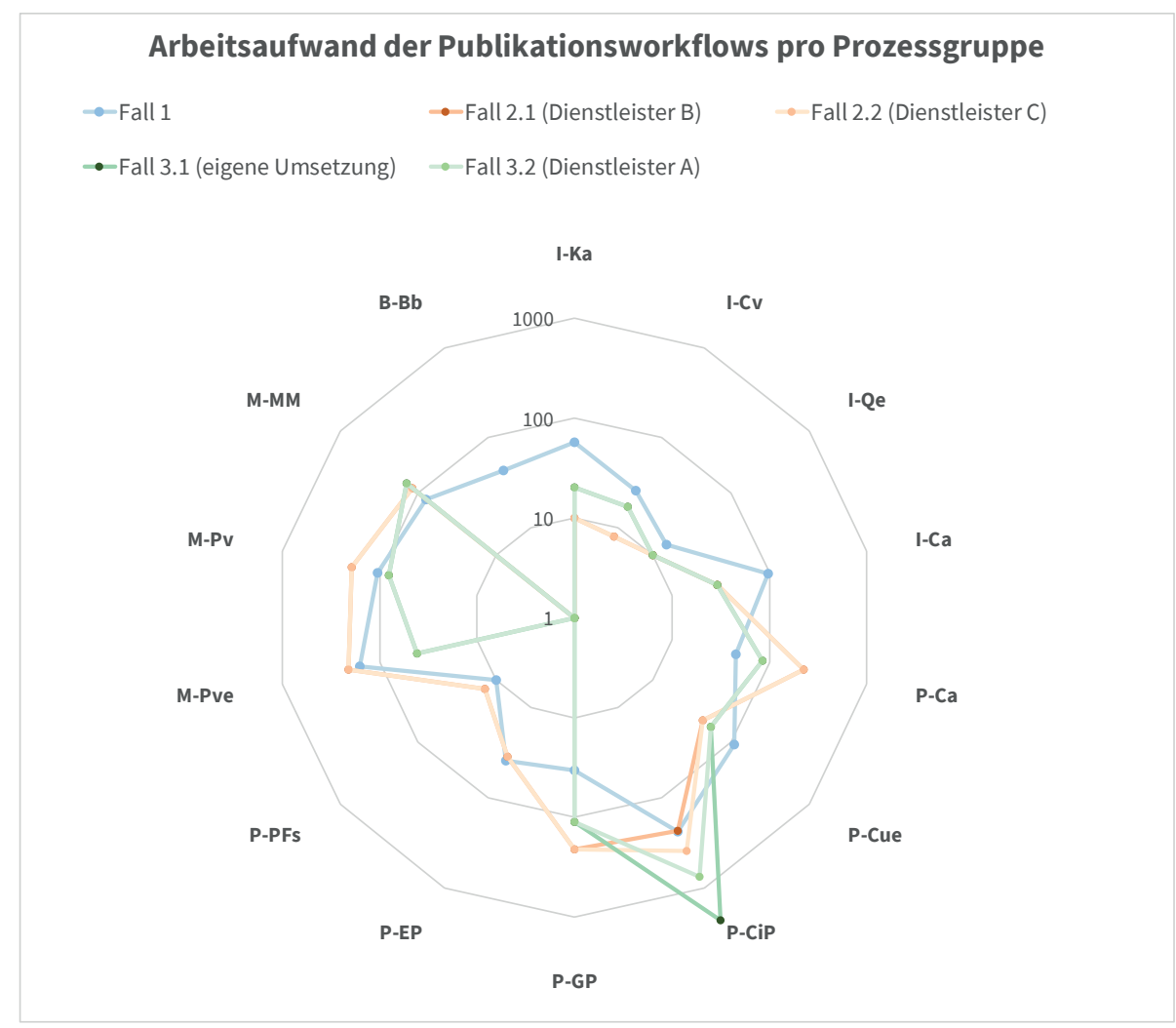

Abb. 21 Netzdiagramm zum Arbeitsaufwand der Publikationsworkflows

\subsubsection{Kostenaufwand der Publikationsworkflows}

An dieser Stelle erfolgt die Datenerhebung zum finanziellen Aufwand, respektive der Herstellungskosten. Hierfür werden in Tabelle 15 die Gesamtkosten pro Fall aufgezeigt, die sich aus den in den Tabellen 16 bis 19 aufgeführten Kosten für die $P$-CiP- und P-GP-Prozesse sowie aus den Prozessen $M-P V e-100$ und $M-P v-300$ ergeben. Herstellungskosten werden lediglich für den Fall aufgeführt, für den sie tatsächlich angefallen sind. Wie bereits in Kapitel 4.2 erwähnt, sind neben den Herstellungskosten auch Kosten für Mitgliedschaften angefallen, die zur Durchführung der Fallstudie abgeschlossen werden mussten. Da sie - im Sinne eines Null-Prozesses - die Durchführung der Publikationsworkflows erst ermöglichten, aber keinem Prozess des Workflow-Modells direkt zugeordnet werden können, sind diese Kosten im Anhang D aufgeschlüsselt. 
Tab. 15 Gesamt-Kostenaufwand der Publikationsworkflows

\begin{tabular}{cccccc} 
& \multicolumn{7}{c}{ Herstellungskosten $(\boldsymbol{\epsilon})$} \\
\cline { 2 - 6 } & $\begin{array}{c}\text { F 1 } \\
\text { CRC- } \\
\text { Workflow }\end{array}$ & $\begin{array}{c}\text { F 2.1 } \\
\text { Dienst- } \\
\text { leister B }\end{array}$ & $\begin{array}{c}\text { F 2.2 } \\
\text { Dienst- } \\
\text { leister C }\end{array}$ & $\begin{array}{c}\text { F 3.1 } \\
\text { eigene } \\
\text { Umsetzung }\end{array}$ & $\begin{array}{c}\text { F 3.2 } \\
\text { Dienst- } \\
\text { leister A }\end{array}$ \\
\hline SUMME & $\mathbf{1 6 8 , 9 2}$ & $\mathbf{8 1 1 , 9 0}$ & $\mathbf{1 3 9 , 1 0}$ & $\mathbf{1 9 6 , 4 5}$ & $\mathbf{1 . 2 9 0 , 9 9}$
\end{tabular}

Tab. 16 Kostenaufwand der Publikationsworkflows für die Prozessgruppe P-CiP

\begin{tabular}{lccccc} 
& \multicolumn{5}{c}{ Kosten $(\boldsymbol{(})$} \\
\cline { 2 - 6 } $\begin{array}{l}\text { K-[P-CiP]: } \\
\begin{array}{l}\text { Content in Produkt } \\
\text { umsetzen }\end{array}\end{array}$ & $\begin{array}{c}\text { F 1 } \\
\text { CRC- } \\
\text { Workflow }\end{array}$ & $\begin{array}{c}\text { F 2.1 } \\
\text { Dienst- } \\
\text { leister B }\end{array}$ & $\begin{array}{c}\text { F 2.2 } \\
\text { Dienst- } \\
\text { leister C }\end{array}$ & $\begin{array}{c}\text { F 3.1 } \\
\text { eigene } \\
\text { Umsetzung }\end{array}$ & $\begin{array}{c}\text { F 3.2 } \\
\text { Dienst- } \\
\text { leister A }\end{array}$ \\
\hline Dienstleister A & - & - & - & - & $1.278,00$ \\
\hline Dienstleister B & - & 650,00 & - & - & - \\
\hline Dienstleister C & - & - & 139,10 & - & - \\
\hline SUMmE & $\mathbf{0 , 0 0}$ & $\mathbf{6 5 0 , 0 0}$ & $\mathbf{1 3 9 , 1 0}$ & $\mathbf{0 , 0 0}$ & $\mathbf{1 . 2 7 8 , 0 0}$
\end{tabular}

Tab. 17 Kostenaufwand der Publikationsworkflows für die Prozessgruppe P-GP

\begin{tabular}{|c|c|c|c|c|c|}
\hline \multirow[b]{2}{*}{$\begin{array}{l}\text { K-[P-GP]: Gedrucktes } \\
\text { Produkt produzieren }\end{array}$} & \multicolumn{5}{|c|}{ Kosten $(€)$} \\
\hline & $\begin{array}{c}\text { F } 1 \\
\text { CRC- } \\
\text { Workflow }\end{array}$ & $\begin{array}{c}\text { F } 2.1 \\
\text { Dienst- } \\
\text { leister B }\end{array}$ & $\begin{array}{c}\text { F } 2.2 \\
\text { Dienst- } \\
\text { leister C }\end{array}$ & $\begin{array}{c}\text { F } \mathbf{3 . 1} \\
\text { eigene } \\
\text { Umsetzung }\end{array}$ & $\begin{array}{c}\text { F } 3.2 \\
\text { Dienst- } \\
\text { leister A }\end{array}$ \\
\hline Dienstleister E & 133,45 & 114,64 & - & 153,51 & - \\
\hline Dienstleister F & - & - & - & 33,38 & 12,99 \\
\hline Dienstleister G & - & 43,60 & - & - & - \\
\hline Dienstleister H & 26,25 & - & - & - & - \\
\hline SUMME & 159,70 & 158,24 & 0,00 & 186,89 & 12,99 \\
\hline
\end{tabular}


Tab. 18 Kostenaufwand der Publikationsworkflows für Prozess M-PVe-100

\begin{tabular}{lccccc} 
& \multicolumn{5}{c}{ Kosten $(\boldsymbol{\xi})$} \\
\cline { 2 - 5 } $\begin{array}{l}\text { K-[M-PVe-100]: } \\
\begin{array}{l}\text { Publikationsvertrag } \\
\text { erstellen }\end{array}\end{array}$ & $\begin{array}{c}\text { F 1 } \\
\text { CRC- } \\
\text { Workflow }\end{array}$ & $\begin{array}{c}\text { F 2.1 } \\
\text { Dienst- } \\
\text { leister B }\end{array}$ & $\begin{array}{c}\text { F 2.2 } \\
\text { Dienst- } \\
\text { leister C }\end{array}$ & $\begin{array}{c}\text { F 3.1 } \\
\text { eigene } \\
\text { Umsetzung }\end{array}$ & $\begin{array}{c}\text { F 3.2 } \\
\text { Dienst- } \\
\text { leister A }\end{array}$ \\
\hline $\begin{array}{l}\text { Rechte für } \\
\text { Inhaltselemente klären }\end{array}$ & $\mathbf{6 , 0 0}$ & - & - & - & -
\end{tabular}

Tab. 19 Kostenaufwand der Publikationsworkflows für Prozess $M-P v$-200

\begin{tabular}{|c|c|c|c|c|c|}
\hline \multirow[b]{2}{*}{$\begin{array}{l}\text { K-[M-Pv-200]: } \\
\text { Stammdaten pflegen }\end{array}$} & \multicolumn{5}{|c|}{ Kosten $(€)$} \\
\hline & $\begin{array}{c}\text { F } 1 \\
\text { CRC- } \\
\text { Workflow }\end{array}$ & $\begin{array}{c}\text { F } 2.1 \\
\text { Dienst- } \\
\text { leister B }\end{array}$ & $\begin{array}{c}\text { F } 2.2 \\
\text { Dienst- } \\
\text { leister C }\end{array}$ & $\begin{array}{c}\text { F } \mathbf{3 . 1} \\
\text { eigene } \\
\text { Umsetzung }\end{array}$ & $\begin{array}{c}\text { F } 3.2 \\
\text { Dienst- } \\
\text { leister A }\end{array}$ \\
\hline Stammdaten pflegen & 3,22 & 3,66 & - & 9,56 & - \\
\hline
\end{tabular}

\subsubsection{Personalkosten}

Die Personalkosten sind wie in vielen Branchen auch in der Verlagsbranche eine wichtige Kostenart und Grundlage für die Ressourcenplanung. Daher ist es auch für einen OA-Hochschulverlag sinnvoll, zu ermitteln, welche Personalkosten pro Arbeitsstunde eines Verlagsmitarbeiters anfallen. ${ }^{159}$

Aufgrund dessen werden an dieser Stelle beispielhaft die Personalkosten pro Fall der Studie ermittelt, indem die erhobenen Daten zum internen Arbeitsaufwand mit den fiktiven Personalkosten einer Vollzeitstelle in einem Hochschulverlag verrechnet werden. Da eine Stelle im Hochschulverlag typischerweise von einem Bibliothekar oder Verlagshersteller eingenommen wird, findet hier die branchentypische Eingruppierung dieser Berufe in die Entgeltgruppe E9a nach Tarifvertrag für den öffentlichen Dienst der Länder Anwendung. Die wöchentliche Arbeitszeit einer Vollzeitstelle wird bei einer 5-Tage-Woche mit 40 Stunden beziffert. Die Tabelle 20 schlüsselt die Berechnung der Plankapazität auf: 
Tab. 20 Berechnung der Plankapazität einer Vollzeitstelle (40 h)

\begin{tabular}{lll} 
& Tage/Jahr & Stunden/Jahr \\
\hline Kalendertage & 365 & -104 \\
\hline Samstage und Sonntage (5-Tage-Woche) & -11 & 2.000 \\
\hline Feiertage & $250^{160}$ & \\
\hline Arbeitsplatzkapazität & -30 & \\
\hline Urlaub & -5 & \\
\hline Krankheit (Durchschnitt) & -2 & 1.704 \\
\hline bezahlte Arbeitsverhinderung (z. B. Versammlungen, & & \\
Weiterbildung) & 213 & \\
\hline Mannkapazität & 0 & $1.704(\div 12=142$ h/Monat) \\
\hline Überstunden & 0 & 213 \\
\hline Springer / Aushilfen & & \\
\hline Plankapazität & & \\
\hline
\end{tabular}

Berechnung des Stundensatz einer Vollzeitstelle: ${ }^{161}$

$2900 €$ brutto $\times 23 \%$ (AG Versicherungen) $\div 142$ Arbeitsstunden/Monat $=\mathbf{2 5 , 1 2} € / \mathbf{h}$

Die Werte in Tabelle 21 ergeben sich aus der Multiplikation des in Tabelle 14 aufgeführten internen Arbeitsaufwands pro Prozessgruppe mit den Personalkosten pro Stunde einer Vollzeitstelle.

160 Entspricht den Arbeitstagen im Freistaat Sachsen im Jahr 2019

161 Das Gehalt entspricht der branchentypischen Einordnung einer Vollzeitstelle zur Betreuung eines Hochschulverlags in die Entgeltgruppe E9a nach TV-L 2019 (gültig vom 01.01.2019 bis 31.12.2019) 
Tab. 21 Beispielhafte Berechnung der anfallenden Personalkosten einer Vollzeitstelle $(40 \mathrm{~h})$

\begin{tabular}{|c|c|c|c|c|c|c|}
\hline & & \multicolumn{5}{|c|}{ Personalkosten $(€)$} \\
\hline & & $\begin{array}{c}\text { F } 1 \\
\text { CRC- } \\
\text { Workflow }\end{array}$ & $\begin{array}{c}\text { F } 2.1 \\
\text { Dienst- } \\
\text { leister B }\end{array}$ & $\begin{array}{c}\text { F } 2.2 \\
\text { Dienst- } \\
\text { leister C }\end{array}$ & $\begin{array}{c}\text { F } 3.1 \\
\text { eigene } \\
\text { Umsetzung }\end{array}$ & $\begin{array}{c}\text { F } 3.2 \\
\text { Dienst- } \\
\text { leister A }\end{array}$ \\
\hline I-Ka & Konzept auswählen & 23,86 & \multicolumn{2}{|c|}{4,19} & \multicolumn{2}{|c|}{8,37} \\
\hline $\mathrm{I}-\mathrm{CV}$ & Content vereinnahmen & 9,63 & \multicolumn{2}{|c|}{3,35} & \multicolumn{2}{|c|}{7,12} \\
\hline I-Qe & $\begin{array}{l}\text { Inhaltliche Qualität des Cont- } \\
\text { ents evaluieren }\end{array}$ & 6,28 & \multicolumn{2}{|c|}{4,19} & \multicolumn{2}{|c|}{4,19} \\
\hline $\mathrm{I}-\mathrm{Ca}$ & Content annehmen & 40,61 & \multicolumn{2}{|c|}{12,14} & \multicolumn{2}{|c|}{12,14} \\
\hline $\mathrm{P}-\mathrm{Ca}$ & Content aufbereiten & 18,84 & \multicolumn{2}{|c|}{106,76} & \multicolumn{2}{|c|}{35,59} \\
\hline P-CUe & Cover bzw. Umschlag erstellen & 46,05 & \multicolumn{2}{|c|}{18,42} & \multicolumn{2}{|c|}{23,45} \\
\hline P-CiP & $\begin{array}{l}\text { Content in Produkt } \\
\text { umsetzen }\end{array}$ & 87,92 & 98,39 & 162,02 & 962,10 & 314,00 \\
\hline P-GP & $\begin{array}{l}\text { Gedrucktes Produkt } \\
\text { produzieren }\end{array}$ & 14,23 & \multicolumn{2}{|c|}{86,66} & \multicolumn{2}{|c|}{46,05} \\
\hline P-EP & $\begin{array}{l}\text { Elektronisches Produkt } \\
\text { veröffentlichen }\end{array}$ & 16,33 & \multicolumn{2}{|c|}{14,65} & \multicolumn{2}{|c|}{0,00} \\
\hline P-PFs & Produktform sichern & 4,19 & \multicolumn{2}{|c|}{5,86} & \multicolumn{2}{|c|}{0,00} \\
\hline M-PVe & Publikationsvertrag erstellen & 67,41 & \multicolumn{2}{|c|}{87,08} & \multicolumn{2}{|c|}{17,17} \\
\hline M-P-Pv & Produktion vorbereiten & 43,96 & \multicolumn{2}{|c|}{80,80} & \multicolumn{2}{|c|}{33,49} \\
\hline M-MM & $\begin{array}{l}\text { Metadaten-Management } \\
\text { durchführen }\end{array}$ & 33,07 & \multicolumn{2}{|c|}{49,82} & \multicolumn{2}{|c|}{59,45} \\
\hline $\mathrm{M}-\mathrm{Bb}$ & Bestellungen bedienen & 18,00 & \multicolumn{2}{|c|}{0,00} & \multicolumn{2}{|c|}{0,00} \\
\hline SUMME & & 442,95 & 572,32 & 635,95 & $1.209,11$ & 561,01 \\
\hline
\end{tabular}

\subsubsection{Analyse der Datenerhebung}

Nach Eisenhardt existieren in der Literatur keine Verfahrensweisen für „die richtige“ Analyse einer Fallstudie, so dass deren Umsetzung letztendlich dem Forscher überlassen bleibt. Für die vorliegende Monografie wurde daher das Ziel gesetzt, das Datenmaterial aus Kapitel 4.4.1 zu verdichten, indem es strukturiert, paraphrasiert und in den Kontext der Beschaffenheit der Fallstudie gesetzt wird. ${ }^{162}$ Die Ana- 
lyse der Daten stellt die Grundlage für die anschließende Diskussion der Ergebnisse im Kapitel 4.4.3 dar.

\subsubsection{Analyse der Workflow-Rollen}

Abbildung 12 ist zu entnehmen, dass 15 der insgesamt 20 als relevant zu betrachtenden Rollen ${ }^{163}$ in allen 3 Fällen von der bzw. den gleichen Person/en eingenommen wurden. Auffällig ist hierbei, dass die Rolle des Content-Prüfers in allen 3 Fällen von 3 Personen - Projektmitarbeiter 1, Projektmitarbeiter 2 und den Projektleitern - ausgeführt wurden. Abweichungen hingegen gab es bei den Rollen Content-Lieferant, Content-Abnehmer, Copy Editor, Cover-Ersteller, Content-Umsetzer, Metadaten-Manager und Content-Distributor, die je nach Fall von verschiedenen Personen eingenommen wurden. Insbesondere die Rollen des Content-Abnehmers, Cover-Erstellers, Content-Umsetzers und Content- Distributors weisen große Abweichungen zwischen den Fällen auf.

Im Fall 1 wurde die Rolle des Content-Abnehmers von 5 verschiedenen Personen übernommen, im Fall 2 von 4 und im Fall 3 lediglich von 3 verschiedene Personen. Anderes gilt für den Cover-Ersteller: In allen 3 Fällen wurde diese Rolle von Projektmitarbeiter 2, aber in den Fällen 1 und 2 zusätzlich auch vom Autor (Fall 1), Projektmitarbeiter 1 und externen Personen bzw. Dienstleister (Fall 2) eingenommen. Ähnliches gilt für den Content-Distributor: Diese Rolle wurde für die gesamte Fallstudie vom Projektmitarbeiter 1 und einem studentischen Projektteam, außerdem für Fall 2 zusätzlich von Projektmitarbeiter 2 und externen Personen bzw. einem Dienstleister verantwortet. Innerhalb der Fallstudie wurde die Rolle des Content-Umsetzers ebenfalls für den Fall 1 zwischen dem Autor und Projektmitarbeiter 2 und für Fall 3.1 zwischen Projektmitarbeiter 2 und einer wissenschaftlichen Hilfskraft (studentisches Projektteam oder wHk) aufgeteilt, während in Fall 3.2 Projektmitarbeiter 2 und ein Dienstleister als Content-Umsetzer agierten. Darüber hinaus war nur in Fall 3.1, die Rolle des Barrierefreiheit-Beauftragten relevant.

\subsubsection{Analyse des zeitlichen Ablaufs}

Den Gantt-Diagrammen ist die jeweilige Dauer der Publikationsworkflows der Fallstudie zu entnehmen. Tabelle 22 gibt die Dauer der Fälle in Arbeitswochen an.

162 vgl. Albers et al. 2007, S. 57

163 Ausgenommen sind die 4 ausgegrauten Rollen: Fachkorrektor, Sprachlektor, Dienstleister und Kunde. 


\begin{tabular}{ccccccc}
\multicolumn{7}{c}{ Dauer der Publikationsworkflows in Arbeitswochen } \\
\cline { 2 - 6 } & $\begin{array}{c}\text { F 1 } \\
\text { CRC- } \\
\text { Workflow }\end{array}$ & $\begin{array}{c}\text { F 2.1 } \\
\text { Dienst- } \\
\text { leister B }\end{array}$ & $\begin{array}{c}\text { F 2.2 } \\
\text { Dienst- } \\
\text { leister C }\end{array}$ & $\begin{array}{c}\text { F 3.1 } \\
\text { eigene } \\
\text { Umsetzung }\end{array}$ & $\begin{array}{c}\text { F 3.2 } \\
\text { Dienst- } \\
\text { leister A }\end{array}$ \\
\hline SUMME & $\mathbf{4 3}$ & $\mathbf{4 3}$ & $\mathbf{3 0}$ & über 53 & $\mathbf{3 1}$
\end{tabular}

Hierbei fällt auf, dass Fall 3.1 deutlich am längsten dauerte. Zu beachten ist, dass wie in Kapitel 4.4.1.1 beschrieben, für diesen Fall die Prozesse P-CiP-130 bis $P$ CiP-150, P-CiP-180, P-EP-100, P-EP-110 und P-PFs-100 noch nicht abschließend durchgeführt waren. Im Gegensatz dazu hat die Umsetzung (einschließlich der gerade genannten Prozesse) für die Fälle 1 und 2.1 auf den ersten Blick 10 Arbeitswochen weniger gedauert. Fall 2.2 und 3.2 weisen nur rund 30 Arbeitswochen auf, da - bedingt durch das Fallstudiendesign (vgl. Kapitel 4.3.2 und 4.3.3) - der letzte abgeschlossene Prozess P-CiP-140 darstellt.

Somit lassen sich die Ergebnisse aus Tabelle 22 nur schwer vergleichen. Zudem muss Folgendes beachtet werden: Alle Fälle der Studie wurden gleichzeitig begonnen, sodass aus Effizienzgründen viele Prozesse des Workflows für die Fälle gemeinsam durchgeführt wurden. Dies führte zu Wartezeiten, die bei sequenzieller Durchführung der Fälle nicht aufgetreten wären. Weitere Wartezeiten hat außerdem die Tatsache verursacht, dass die Umsetzung der Fallstudie nur einen Teil der Arbeitsaufgaben und somit auch Arbeitszeit des Projektteams ausmachte. An manchen Stellen wurde die Durchführung der Fallstudie weniger hoch priorisiert und somit einige Tage ausgesetzt. Dies wirkt sich im Besonderen auf Fall 3.1 aus. Zu diesem Zeitpunkt lassen sich diese Wartezeiten nicht mehr im Detail nachvollziehen, so dass die Gantt-Diagramme keine bereinigte Darstellung des zeitlichen Ablaufs der Fälle enthalten und deshalb nur eine bedingte Aussagekraft zur tatsächlichen Dauer besitzen. Sie vermitteln v. a. einen Eindruck, welche Prozesse in den Publikationsworkflows der Fälle aufeinander folgten. Deshalb findet auch keine weitere Analyse der Gantt-Diagramme statt.

\subsubsection{Analyse des Arbeitsaufwands}

Die Erhebung des internen Arbeitsaufwands zeigt, wie viel Zeit in die Herstellung und Veröffentlichung der Publikationen der Fallstudie investiert wurde. Anhand der Darstellung in Tabelle 14 wird deutlich, dass für Fall 3.1 mit rund 48 Stunden der höchste interne Arbeitsaufwand in der gesamten Fallstudie entstanden ist, obwohl hierzu weniger Daten zur Analyse vorlagen als für die anderen Fälle (vgl. Kapitel 4.4.1.3). Im Gegensatz dazu hat die Umsetzung des gleichen Produktes mit Dienstleister A (Fall 3.2) rund 26 Arbeitsstunden weniger in Anspruch genommen. Mit nur rund 4 Stunden weniger wurde Fall 1 durchgeführt, der in der gesamten Fallstudie am wenigsten internen Arbeitsaufwand erzeugte. Zwischen den Fällen 
2.1 und 2.2 besteht lediglich ein Unterschied von rund 2 Stunden, der als vernachlässigbar angesehen werden kann. Sie befinden sich im Mittelfeld hinsichtlich des Ergebnisses zum Arbeitsaufwand der Fallstudie.

Nachfolgend werden die Daten nach Prozessgruppen detailliert analysiert. Hierbei wird mit der Prozessgruppe begonnen, die prozentual den größten zeitlichen Aufwand verursacht hat (vgl. Abbildung 22).

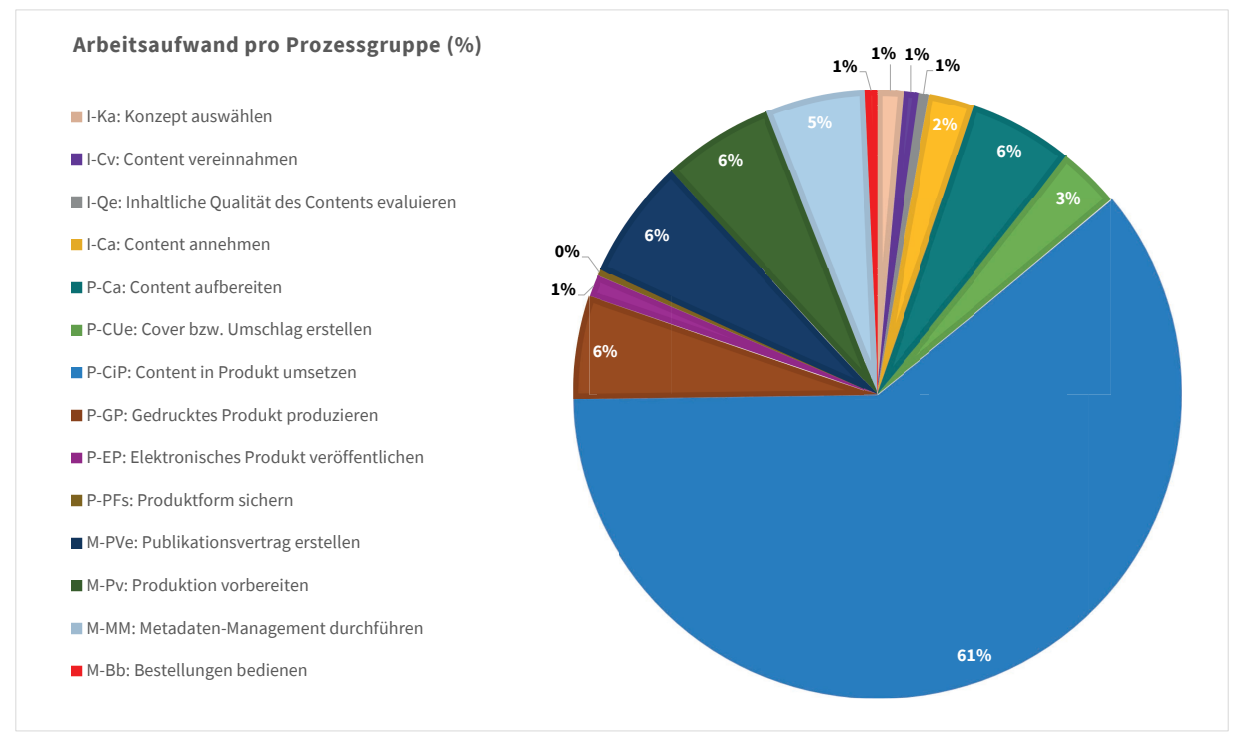

Abb. 22 Prozentualer Verteilung des Arbeitsaufwands der Publikationsworkflows pro Prozessgruppe

\section{P-CiP: Content in Produkt umsetzen}

Die Durchführung der P-CiP-Prozesse (Satzprozess) nahm mit $61 \%$ den größten Anteil des internen Arbeitsaufwandes in der Fallstudie ein. Auffällig ist, dass die Umsetzung dieser Prozessgruppe in Fall 3.1 mindestens dreimal so viel Zeit in Anspruch genommen hat wie in allen anderen Fällen der Studie. Dies ist auch der Grund, warum auf diese Prozessgruppe in der Gesamtheit der größten Anteil des Arbeitsaufwandes entfällt. ${ }^{164}$

Im Gegensatz dazu steht die Umsetzung des gleichen Falles mit Dienstleister A (Fall 3.2): Zwar nimmt auch hier die Durchführung der P-CiP-Prozesse den größten Anteil am Gesamtarbeitsaufwand ein (56 \%), aber der interne Arbeitsaufwand war wesentlich geringer als für Fall 3.1. Dieses Ergebnis ist nicht überraschend und wird in Kapitel 4.4.3 diskutiert.

164 Der interne Arbeitsaufwand für die P-CiP-Prozesse im Fall 3.1 macht rund 80 \% des Gesamtaufwandes dieses Falles aus. 
Interessanter ist hier der Vergleich der Daten von Fall 2.1, 2.2 und 3.2. Aus Tabelle 14 geht hervor, dass die Durchführung des Satzprozesses (P-CiP-Prozesse) für Fall 3.2 bis zu 8 Stunden ${ }^{165}$ mehr internen Arbeitsaufwand verursacht hat als die Fälle 2.1 und 2.2. Die detaillierte Aufschlüsselung der Daten zu dieser Prozessgruppe (vgl. Tabelle 75, Anhang D) zeigt, dass hierfür die Ergebnisse von Prozess P-CiP-140 ursächlich sind. Für Fall 3.2 hat dieser Prozess mit Abstand den höchsten Arbeitsaufwand im Modellverlag verursacht. Als ausschlaggebend hierfür werden die über 300 mathematischen Formeln im Content von Fall 3 gesehen. Im Unterschied zu Fall 3 sind im Content von Fall 2 nur wenige mathematische Formeln enthalten, was den Aufwand für den Dienstleister mindert. Darüber hinaus wurden, anders als im Fall 3.1, die Formeln vom Dienstleister A medienneutral und nicht als Abbildungen umgesetzt. Dies stellt eine erhebliche Fehlerquelle dar, so dass die Fahnen besonders intensiv von den Projektmitarbeitern kontrolliert werden mussten. Dies und die auch dadurch verursachten mehrmaligen Korrekturschleifen können als Grund für den höheren Zeitaufwand für die P-CiP-Prozesse des Falles 3.2 angesehen werden.

Werden im Gegensatz dazu Fall 2.1 und 2.2 verglichen, fällt auf, dass die Durchführung der P-CiP-Prozesse für Fall 2.23 Arbeitsstunden ${ }^{166}$ länger dauerte als für Fall 2.1. Dies deckt sich mit der qualitativen Analyse der Dienstleister aus Kapitel 4.4.2.6.

Des Weiteren wird ersichtlich, dass die Daten zum internen Arbeitsaufwand für diese Prozessgruppe von Fall 1 und Fall 2.1 nur eine geringe Differenz aufweisen. Obwohl es sich bei Fall 1 um einen CRC-Workflow handelt, zeigt Tabelle 75 (vgl. Anhang D), dass jeder Prozess der Prozessgruppe im Fall 1 internen Aufwand verursacht hat, im Gegensatz zu Fall 2.1. Dies gilt insbesondere für die Sicherung der Qualität des Produktionsformats (Prozess P-CiP-110), für den im Fall 190 Min. und im Fall 2.1 gar kein interner Arbeitsaufwand im Modellverlag anfiel, da für diesen Prozess alleine Dienstleister B verantwortlich war.

\section{M-PVe: Publikationsvertrag erstellen}

Die Durchführung der $M$-PVe-Prozesse macht einen prozentualen Anteil von $6 \%$ am internen Arbeitsaufwand der Fallstudie aus. Der Grund hierfür erschließt sich aus Tabelle 79 (vgl. Anhang D). Hier wird deutlich, dass der größte Anteil des Arbeitsaufwands für diese Prozessgruppe durch die Durchführung des Prozesses $M$ $P V e-100$ verursacht wird. Insbesondere für Fall 2 hat das Klären der Rechte für die Inhaltselemente internen Arbeitsaufwand bewirkt, da innerhalb der Fallstudie in diesem Fall die meisten zitierten Abbildungen vom Content-Urheber verwendet wurden (vgl. Kapitel 4.3.2).

165 vgl. Tab. 75: $750-387=363 \mathrm{~min} \div 60=6,05 \mathrm{~h}$ und $750-235=515 \mathrm{~min} \div 60=8,58 \mathrm{~h}$

166 vgl. Tab. $75: 387-235=152 \mathrm{~min} \div 60=2,53 \mathrm{~h}$ 


\section{P-GP: Gedrucktes Produkt produzieren}

Die Koordination des Druckprozesses (P-GP-Prozesse) machte rund $5 \%$ des gesamten internen Arbeitsaufwandes der Fallstudie aus. Werden die erhobenen Daten für die Prozesse genauer betrachtet (vgl. Tabelle 76, Anhang D), wird auch hier deutlich, dass für Fall 2 der höchste interne Arbeitsaufwand angefallen ist. Dieses Ergebnis lässt sich zunächst darauf zurückführen, dass alle Ausgabeformate der Publikation von Fall 2 als erste veröffentlichungsreif vorlagen. Die P-GP-Prozesse wurden somit erstmals für Fall 2 durchgeführt, was ein Grund für den höheren Wert des internen Arbeitsaufwands sein kann. Ausschlaggebend in diesem Zusammenhang ist außerdem, dass für Fall 2 erstmals über die Verlagssoftware von Dienstleister $G$ ein Druckauftrag erstellt wurde: Projektmitarbeiter 1 hatte zu diesem Zeitpunkt erst wenige Kenntnisse im Umgang mit der Software, so dass sein Arbeitsaufwand hier noch entsprechend höher war. Darüber hinaus musste - wie in Kapitel 4.3.2 beschrieben - die Verkaufs- und Verlagsauflage der SoftcoverExemplare von Fall 2 reklamiert werden. Hieraus lässt sich der höhere, interne Arbeitsaufwand für den Prozess P-GP-110 bzw. P-GP-120 erklären.

\section{P-Ca: Content aufbereiten}

Abbildung 22 zeigt, dass die Durchführung vom technischen Copy Editing (Prozess $P$-Ca-120) Ursache für $6 \%$ des Gesamtarbeitsaufwands der Fallstudie war. Werden die Ergebnisse für diese Prozessgruppe im Detail betrachtet (vgl. Tabelle 73, Anhang D), fällt auf, dass sich dies vor allem aus den Daten für Fall 2 ergibt. Die Durchführung des Prozesses P-Ca-120 hat für Fall 2 durchschnittlich 2,6 Stunden ${ }^{167}$ mehr internen Arbeitsaufwand verursacht als für Fall 1 und 3. Dies hängt mit dem oben erläuterten Prozess $M-P V e-100$ zusammen und lässt sich darauf zurückführen, dass für Fall 2 Abbildungen durch Projektmitarbeiter 2 nachgezeichnet und dadurch zeitliche Ressourcen gebunden wurden.

Der erhöhte Arbeitsaufwand für die Fälle 2 und 3 lässt sich darüber hinaus auch dadurch begründen, dass - wie in den Kapiteln 4.3.2 und 4.3.3 beschrieben die jeweiligen Content-Urheber nur teilweise die verwendeten Abbildungen im Content als einzelne Datei liefern konnten. Die fehlenden Abbildungen wurden durch die Projektmitarbeiter aus dem Manuskript erzeugt. Der entsprechende Arbeitsaufwand wurde ebenfalls dem Prozess $\mathrm{P}$-Ca-120 zugeordnet.

\section{M-Pv: Produktion vorbereiten}

Mit einem Anteil von $6 \%$ an der Summe des gesamten internen Arbeitsaufwands der Fallstudie ist die Durchführung der $M$-Pv-Prozesse zu beziffern. Ein Großteil davon entfällt auf Fall 2. Grund hierfür ist, dass für diesen Fall zur Klärung der Produktionskosten (Prozess $M$-Pv-110) insgesamt 5 Satzdienstleister angefragt wurden. Im Gegensatz dazu mussten für Fall 3.2 lediglich 3 Satzdienstleister vor der offiziel- 
len Beauftragung kontaktiert werden. Dies führt zum erhöhten Arbeitsaufwand für die Prozessgruppe für Fall 2 (vgl. Tabelle 80, Anhang D).

\section{M-MM: Metadaten-Management durchführen}

Die Erstellung und Distribution der Metadaten zu den Publikationen der Fälle machte $5 \%$ des gesamten Arbeitsaufwandes in der Studie aus. Hierbei hat die Durchführung der entsprechenden Prozesse im Fall 3 die meiste Zeit benötigt. Im Vergleich hat Fall 2.2 3 Minuten und Fall 1 sogar 63 Min. weniger Arbeitsaufwand verursacht. Dieses Ergebnis lässt sich damit begründen, dass der Prozess $M$ MM-110 an einen Studierenden ausgelagert wurde. Im Gegensatz zu Projektmitarbeiter 1 hatte dieser keine Erfahrung im Umgang mit der Distribution von Metadaten. Auf Grund dieser Einarbeitungszeit fällt der Arbeitsaufwand für die Prozessgruppe $M-M M$ im Fall 3 entsprechend höher aus.

\section{P-CUe: Cover bzw. Umschlag erstellen}

$3 \%$ des Gesamtarbeitsaufwandes beanspruchte die Durchführung der P-CUe-Prozesse im Modellverlag. Insbesondere die Erstellung des Umschlags für Fall 1 hat internen Arbeitsaufwand verursacht, da hierbei von den Gestaltungsrichtlinien des Modellverlags abgewichen und zunächst über das Design des Umschlags im Team beraten wurde, bevor Cover und Umschlag erstellt werden konnten. Es kann also festgestellt werden, dass allein aufgrund der Tatsache, dass für den Umschlag für Fall 1 kein Template vorlag, im Vergleich zu den Fällen 2 und 3, bei denen das Reihenlayout angewendet wurde, eine Stunde ${ }^{168}$ mehr für seine Erstellung aufgewendet werden mussten.

\section{I-Ca: Content annehmen}

Tabelle 14 ist zu entnehmen, dass, im Gegensatz zu Fall 2 und 3, insbesondere für Fall 1 interner Arbeitsaufwand für die Durchführung der I-Ca-Prozesse angefallen ist. Werden die Daten im Detail (vgl. Tabelle 72, Anhang D) betrachtet, kann festgestellt werden, dass sich dieses Ergebnis vor allem durch den internen Arbeitsaufwand für die Prozesse I-Ca-100 und I-Ca-120 ergibt. Wie in Kapitel 4.3.1 beschrieben, wurden durch den Content-Urheber umfangreiche Überarbeitungen am Content vorgenommen, welche auch entsprechend genau durch die Projektmitarbeiter des Modellverlags geprüft werden mussten. Der hieraus resultierende, im Vergleich zu Fall 2 und 3 höhere Arbeitsaufwand für diesen Prozess, lässt sich mit durchschnittlich 68 Min. ${ }^{169}$ beziffern. Die Sichtung der Gutachten bzw. Checkliste hat für Fall 1 ebenfalls einen doppelt so hohen internen Arbeitsaufwand verursacht wie für Fall 2 und 3. Ein Grund hierfür ist die umfangreiche Checkliste, die vergleichsweise detailliert vom Gutachter von Fall 1 ausgefüllt worden ist und ent-

168 vgl. Tab. 74: Fall 2 und $3=44+56=100 \div 2=50 \mathrm{~min}-110=60 \mathrm{~min}$

169 vgl. Tab. 72: Durchschnitt für T-[I-Ca-120] für Fall 2 und $3=11 \mathrm{~min}-65=54 \mathrm{~min}$ 
sprechend intensiv von den Projektmitarbeitern des Modellverlags geprüft werden musste.

\section{I-Ka: Konzept auswählen}

Auf die Inhaltsakquise, also die Durchführung der I-Ka-Prozesse, entfiel in der Fallstudie lediglich ein Anteil von weniger als $1 \%$ des Arbeitsaufwandes. Als Grund hierfür kann gelten, dass Prozess I-Ka-200 im Fall 2 und 3 an Mitarbeiter der Hochschulbibliothek ausgelagert wurde und somit kein interner Arbeitsaufwand für den Modellverlag anfiel. Im Gegensatz dazu übernahmen die Projektmitarbeiter im Fall 1 in diesem Prozess eine stärkere Rolle. Dies und die Tatsache, dass 2 Fachbereiche der Fakultät angefragt werden mussten (vgl. Kapitel 4.3.1), führte zu einem höheren internen Arbeitsaufwand in dieser Prozessgruppe für Fall 1.

\section{I-Cv: Content vereinnahmen}

Wie aus Kapitel 4.3 hervorgeht, stehen die Prozesse der Gruppe I-Ka und I-Ca in enger Verbindung und wurden teilweise in einem Schritt durchgeführt (insbesondere die Prozesse I-Ka-220 und I-Cv-100). Die Prüfung des Contents nach der Richtlinie für die Content-Vereinnahmung (vgl. Anhang A) hat für den Content von Fall 1 und 3 aus den folgenden Gründen mehr internen Arbeitsaufwand verursacht als für Fall 2:

Wie bereits beschrieben, stellte sich im Zuge dieses Prozesses für den Fall 1 heraus, dass dieser nicht medienneutral umgesetzt werden soll, sondern als CRCWorkflow. Da diese Entscheidung von den anderen Fällen der Studie abweicht, war hier eine etwas längere Beratung im Projektteam notwendig. Einfluss auf den Arbeitsaufwand für diesen Prozess im Fall 3 hatte außerdem die Tatsache, dass zu diesem Zeitpunkt noch 3 Manuskripte zur Auswahl standen. Dementsprechend war auch hier mehr Zeit für die Prüfung der Manuskripte nach der Richtlinie für die Content-Vereinnahmung notwendig. Da es nicht nötig war, den Prozess I-Cv-120 durchzuführen, ist hierfür auch kein interner Arbeitsaufwand entstanden.

\section{I-Qe: Inhaltliche Qualität des Contents evaluieren}

Anhand der Tabelle 14 kann festgestellt werden, dass für die Durchführung der IQe-Prozesse durchschnittlich nur rund $12 \mathrm{Min}$. benötigt wurden und diese somit nur einen Bruchteil des Gesamtarbeitsaufwands ausmachten (1\%). Grund hierfür ist v. a. die Tatsache, dass lediglich 2 Prozesse zur Qualitätssicherung des Contents durchgeführt werden mussten, da es sich beim Content aller 3 Fälle um Graduierungsarbeiten handelte.

\section{P-EP: Elektronisches Produkt veröffentlichen}

Die Veröffentlichung der elektronischen Ausgabeformate der Fälle 1 und 2 hat lediglich im Durchschnitt 37 Min. Arbeitsaufwand verursacht und macht somit einen Anteil von $1 \%$ des Gesamtaufwandes der Fallstudie aus. Es ist anzunehmen, dass die Veröffentlichung der Publikation von Fall 3 ebenfalls nicht mehr als 
30 Min. Zeit einnehmen wird. Der Anteil für diesen Prozess in der Gesamtbetrachtung würde sich somit nur marginal auf $2 \%$ erhöhen.

\section{P-PFs: Produktform sichern}

Zum Zeitpunkt der Drucklegung dieses Werkes wurde für Fall 1 und 2 lediglich der Prozess P-PFs-100 der Prozessgruppe PFs durchgeführt. Es lässt sich vermuten, dass die Durchführung von Prozess P-PFs-200 ebenfalls nur wenige Minuten einnehmen wird. Auch für Fall 3 kann angenommen werden, dass die Durchführung dieser Prozessgruppe nicht viel mehr Arbeitszeit benötigen wird als die anderen Fälle.

\subsubsection{Analyse des Kostenaufwands}

Der Tabelle 15 ist die Summe der Herstellungskosten für die Fälle der Studie zu entnehmen. Sie zeigt auf, dass für die Durchführung des Falls 3.2 mit Dienstleister A die höchsten Herstellungskosten innerhalb der Fallstudie angefallen sind. Im Gegensatz dazu hat die eigene Umsetzung des gleichen Falles (Fall 3.1) 1.094,45€ weniger Kosten verursacht. Auf Fall 3.2 folgt die Umsetzung des Falls 2.1 mit Dienstleister B. Ähnlich preiswert, wie Fall 3.1 war die Umsetzung der Fälle 1 und 2.2; so ist letzter auch der kostenmäßig günstigste Fall der Studie. Aus der Beschreibung der Fälle in Kapitel 4.3 lassen sich bereits die Gründe schlussfolgern. Aufschluss darüber gibt ebenso die detaillierte Betrachtung der Kosten pro Prozessgruppe. Wie im vorherigen Kapitel, wird hierbei mit der Prozessgruppe begonnen, die den größten prozentualen Anteil an der Gesamtsumme der Herstellungskosten in der Fallstudie verursacht hat. Dies ist Abbildung $23 \mathrm{zu}$ entnehmen. 


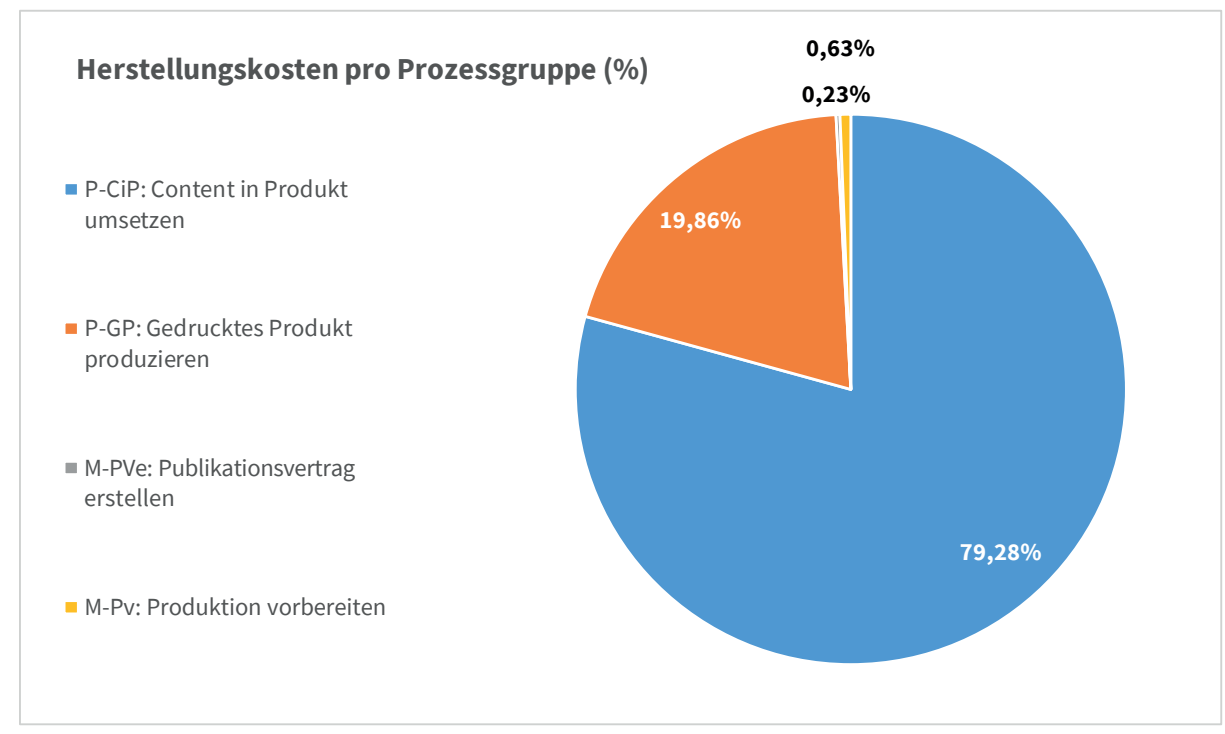

Abb. 23 Prozentuale Verteilung der Gesamtherstellungskosten der Publikationsworkflows

\section{P-CiP: Content in Produkt umsetzen}

Den größten Anteil an den Gesamtkosten der Fallstudie hatten die Kosten für die Beauftragung von Satzdienstleistern zur Durchführung der P-CiP-Prozesse. Daher fallen für diese Prozessgruppe auch keine Kosten für die Fälle 1 und 3.1 an. Bei Fall 1 handelt es sich, wie bereits mehrfach erwähnt, um einen CRC-Workflow. Der Content-Urheber erstellte selbst die Druckvorlage und übermittelte sie kostenfrei an den Hochschulverlag. Im Fall 3.1 wurde die Prozessgruppe P-CiP ausnahmslos durch Mitarbeiter des Modellverlages durchgeführt. Somit sind hier lediglich Personal-, aber keine Herstellungskosten angefallen. Im Gegensatz dazu wurden einige der PCiP-Prozesse in den Fällen 2.1, 2.2 und 3.2 an Satzdienstleister ausgelagert. Es zeigt sich, dass für den Fall 3.2 die höchsten Satzkosten und für Fall 2.2 die geringsten Kosten angefallen sind. Die Umsetzung von Fall 2.1 hat rund die Hälfte weniger gekostet als die von Fall 3.2.

Um die angefallenen Kosten für den Satz sinnvoll vergleichen zu können, ist es notwendig, die aufgerufenen Preise pro umgesetzter Seite zu berücksichtigen. Hierbei ist es sogar von Vorteil, dass Dienstleister A den Seitenpreis auf Grundlage des Contents von Fall 2 angesetzt hat, da somit die von den Satzdienstleistern unterschiedlichen Seitenpreise für ein und dasselbe Manuskript ersichtlich werden. In Tabelle 23 sind die Kosten pro umgesetzter Seite, also auf Basis des finalen Seitenumfangs der PDF-Datei, ersichtlich. 
Tab. 23 Kosten pro Seite

\begin{tabular}{lllll} 
Fall & Finaler Seitenumfang & Dienstleister & Gesamtsatzkosten $(\boldsymbol{€})$ & Kosten pro Seite $(\boldsymbol{€})$ \\
\hline 2.1 & 98 & B & 650,00 & 6,63 \\
\hline 2.2 & 98 & C & 139,10 & 1,42 \\
\hline 3.2 & 284 & A & 1278,00 & 4,50 \\
\hline
\end{tabular}

Anhand des Seitenpreises zeigt sich noch deutlicher, dass Dienstleister B der teuerste und Dienstleister $C$ der preiswerteste Satzdienstleister in der Fallstudie war. Erstaunlich ist der erhebliche Unterschied zwischen den aufgerufenen Seitenpreisen von Dienstleister $C$ und denen der Dienstleister $A$ und $B$, die für die Umsetzung des gleichen Manuskriptes einen vier- bis sechsfach höheren Seitenpreis ansetzten. Inwieweit sich der geringe Preis von Dienstleister $C$ auf die Qualität der Leistungen und weiterer Kriterien auswirkt, wird im Kapitel 4.4.2.6 diskutiert.

$\mathrm{Zu}$ beachten ist hierbei, dass, hätte Dienstleister A tatsächlich den Content von Fall 2 zum vereinbarten Seitenpreis von 4,50€ umgesetzt, die Gesamtsatzkosten lediglich bei $441 €^{170}$ liegen würden. Anderseits wäre die Umsetzung des Satzprozesses von Fall 3 der teuerste in der Fallstudie gewesen, wenn der eigentliche Seitenpreis (aufgrund der nach dem Kostenmodell von Dienstleister A vorgesehenen Einstufung des Contents als „komplex“) von 5,75€ berechnet worden wäre ${ }^{171}$. Leider liegen für Dienstleister B keine Informationen zum Kostenmodell vor, sodass keine Aussage getroffen werden kann, wie viel teurer die Umsetzung eines komplexen Werkes (wie von Fall 3) zu einem weniger komplexen Werk (wie von Fall 2) bei diesem Dienstleister wäre.

\section{P-GP: Gedrucktes Produkt produzieren}

Die Druckkosten (P-GP-Prozesse) machen rund $20 \%$ der Herstellungskosten der Fallstudie aus. Die höchsten Kosten sind hierbei für den Fall 3.1 angefallen. Für rund $28 €$ weniger wurden Exemplare des Falls 1 und des Falls 2.1 produziert. Für Fall 3.2 sind lediglich rund $13 €$ und für Fall 2.2 keine Druckkosten angefallen.

Dies hängt mit der Vorgehensweise der Fallstudie zusammen. Wie bereits in den Kapiteln 4.3.1 und 4.3.2 erläutert, wurden für die Fälle 1 und 2.1 sowohl Musterexemplare als auch Exemplare für die Verlags- und Verkaufsauflage produziert. Dies gilt ebenso für Fall 3.1. Zu Ansichtszwecken wurde darüber hinaus ein Musterexemplar mit der Druckvorlage des Fall 3.2 gedruckt, wodurch Kosten von rund $13 €$ entstanden sind. Die Druckvorlage aus dem Fall 2.2 wurde nicht weiterverwendet, so dass hier keine Druckkosten vermerkt sind.

170 Finaler Seitenumfang von Fall 2: $98^{*} 4,50 €($ Seitenpreis $)=441 €$

171 Finaler Seitenumfang von Fall 3: $284^{\star} 5,75 €($ Seitenpreis $)=1.633 €$ 
In Tabelle 15 sind die Gesamtdruckkosten pro Fall bzw. pro Druckdienstleister abgebildet. Für die Vergleichbarkeit sind diese Kosten in Relation mit der beauftragen Auflage zu setzen, um die Kosten pro Exemplar ermitteln und vergleichen zu können. Dies bildet die Tabelle $24 \mathrm{ab}$.

Tab. 24 Kosten pro Buch

Fall Dienstleister Gesamtkosten (€) Auflage

Kosten pro Buch $(€)$

\begin{tabular}{lllll}
\hline 1 & $E$ & 133,45 & 7 Softcover (SC), 9 Hardcover (HC) & 8,34 \\
\cline { 2 - 5 } & $H$ & 26,25 & 1 SC & 26,25 \\
\hline 2 & $E$ & 114,64 & $12 \mathrm{SC}, 8 \mathrm{HC}$ & 5,73 \\
\cline { 2 - 5 } & $\mathrm{G}$ & 43,60 & $1 \mathrm{SC}, 1 \mathrm{HC}$ & 21,80 \\
\hline 3 & $\mathrm{E}$ & 153,51 & $12 \mathrm{SC}, 11 \mathrm{HC}$ & 6,67 \\
\cline { 2 - 5 } & $\mathrm{F}$ & 46,37 & $2 \mathrm{SC}, 1 \mathrm{HC}$ & 15,45 \\
\hline
\end{tabular}

Aus der Tabelle 24 geht hervor, dass Dienstleister $H$ die höchsten Kosten pro Buch innerhalb der Fallstudie verursacht hat, während die Produktion der Verkaufs- und Verlagsauflage für Fall 2 bei Dienstleister E zum günstigsten Preis realisiert wurde.

\section{Musterexemplare}

Werden lediglich die Kosten pro Exemplar für die Herstellung der Musterexemplare der Fallstudie betrachtet (also von Dienstleister $H, G$ und $F$ ) zeigt sich, dass auch hier Dienstleister $H$ am teuersten, aber die Musterbücher für Fall 3 bei Dienstleister $F$ am preiswertesten waren.

\section{Verkaufs- und Verlagsauflage}

Die Verkaufs- und Verlagsauflage aller 3 Fälle der Studie wurden bei Dienstleister E beauftragt. Es zeigt sich, dass hierbei Fall 1 die höchsten und Fall 2 die geringsten Kosten pro Exemplar verursacht hat. Dies lässt sich auf Folgendes zurückführen:

Üblicherweise nehmen die Druckkosten pro Buch ab, umso höher die Auflage ist. Für Fall 1 wurde bei Dienstleister E die geringste Verkaufs- und Verlagsauflage beauftragt. Hierdurch ergeben sich bereits höhere Durchschnittskosten pro Buch. Darüber hinaus enthält das Produkt von Fall 1 ausnahmslos und über den gesamten Seitenumfang (68 Seiten) hinweg Farbseiten, was die Druckkosten in die Höhe treibt. Außerdem wurde das Produkt von Fall 1 in einem größeren Format (DIN A4) produziert als die Exemplare von Fall 2 und 3. Dies kann ebenso zu einem höheren Preis für die Produktion pro Buch bei der Druckerei führen und ursächlich für die höheren Kosten sein.

Die Kosten pro Buch waren im Fall 2 am geringsten. Zwar wurden in beiden Fällen insgesamt 20 Bücher produziert, aber diese hatten in Fall 2 mit 94 Seiten einen deutlich geringeren Seitenumfang als das Produkt von Fall 3. Hier wirkte 
sich der deutlich geringere Seitenumfang von Fall 2 - immerhin ein Unterschied von 190 Seiten - positiv auf die Kosten pro Buch aus, während sie für Fall 3 einen Kostentreiber darstellen.

Grundsätzlich betrachtet lässt sich also feststellen, dass die Leistungen von Dienstleister $E$ durchschnittlich ca. $26 \%$ preiswerter pro Buch ${ }^{172}$ sind, als die der anderen in der Fallstudie beauftragten Druckdienstleister. Inwieweit sich dies auf die Qualität und weitere Kriterien auswirkt, wird in Kapitel 4.4.2.6 analysiert.

\section{M-PVe: Publikationsvertrag erstellen}

Dem Prozess $M-P V e-100$ werden lediglich für den Fall 1 Kosten zugeordnet. Diese waren notwendig, um für eine Abbildung aus dem Stadtarchiv Leipzig die Genehmigung zur Veröffentlichung und die druckfähige Abbildungsdatei zu erhalten. Aufgrund des geringen Preises sind diese Kosten nicht ausschlaggebend für die Gesamtkosten von Fall 1, sollten aber durchaus in einer Soll-Kalkulation für ein ähnliches Buchprojekt berücksichtigt werden.

\section{M-Pv: Produktion vorbereiten}

Prozess $M-P v-200$ werden die Kosten für die Vergabe der DOIs zugeordnet. Hierfür wurde im Vorfeld die Mitgliedschaft bei Crossref abgeschlossen (die hierfür angefallenen Kosten sind Tabelle 84, Anhang D zu entnehmen). Die Summe der in Tabelle 19 aufgelisteten Kosten ergibt sich aus der Anzahl der für das Produkt benötigten bzw. gewünschten DOIs. Wie bereits in den Kapiteln 4.3.1 und 4.3.2 beschrieben, wurden für die Publikationen der Fälle 1 und 2 lediglich kapitelweise DOIs verteilt. Die Publikation aus Fall 3.1 erhielt darüber hinaus auch für Tabellen und Abbildungen DOIs. Aufgrund dessen sind die Kosten für den Prozess $M-P v-200$ für Fall 3.1 am höchsten:

Tab. 25 Kosten für Prozess $M-P v-200$

\begin{tabular}{lllll} 
DOI für & Buchtitel & Kapitel & Component (Abb./Tab.) & Gesamtkosten (€) \\
\hline F 1 & 1 & $0,25 \$(0,22 €)$ & $0,06 \$(0,05 €)$ & \\
\hline F 2.1 & 1 & 6 & 0 & 3,22 \\
\hline F 3.1 & 1 & 8 & 0 & 3,66 \\
\hline
\end{tabular}

172 Durchschnittliche Druckkosten pro Buch von Dienstleister E: $7 €$ (aufgerundet); Durchschnittliche Druckkosten pro Buch von Dienstleister F, G und $H$ : $24 €$ (aufgerundet) und unter der Voraussetzung, dass es zwischen der Beauftragung von einem bis zu zu 25 Exemplaren keine Rabattierung gibt. 


\subsubsection{Analyse der Personalkosten}

Werden die Ergebnisse zur beispielhaften Berechnung der Personalkosten betrachtet (vgl. Kapitel 4.4.1.5), ist erkennbar, dass für Fall 1 die geringsten und für Fall 3.1 die höchsten Kosten für einen Vollzeitmitarbeiter nach der branchentypischen Eingruppierung eines Bibliothekars oder Verlagsherstellers in die Entgeltgruppe E9a nach TV-L angefallen wären. Vor den bisherigen Ergebnissen verwundert dies nicht, da sich die Personalkosten auf Grundlage des angefallenen internen Arbeitsaufwands der Fälle ergeben. Wie Tabelle 26 zu entnehmen ist, hätten die Fälle 2.1, 2.2 und 3.2 durchschnittlich $590 €$ Personalkosten verursacht und befinden sich somit im Mittelfeld.

Interessanter wird es, wenn die errechneten, beispielhaften Personalkosten zunächst mit den tatsächlich angefallenen Herstellungskosten aus der Fallstudie (vgl. Kapitel 4.4.1.4) summiert werden:

Tab. 26 Summe der Personalkosten der Publikationsworkflows

\begin{tabular}{lccccc} 
& \multicolumn{5}{c}{ Kosten $(\boldsymbol{€})$} \\
\cline { 2 - 6 } & $\begin{array}{c}\text { F 1 } \\
\text { CRC- } \\
\text { Workflow }\end{array}$ & $\begin{array}{c}\text { F 2.1 } \\
\text { Dienst- } \\
\text { leister B }\end{array}$ & $\begin{array}{c}\text { F 2.2 } \\
\text { Dienst- } \\
\text { leister C }\end{array}$ & $\begin{array}{c}\text { F 3.1 } \\
\text { eigene } \\
\text { Umsetzung }\end{array}$ & $\begin{array}{c}\text { F 3.2 } \\
\text { Dienst- } \\
\text { leister A }\end{array}$ \\
\hline Summe Personalkosten & 442,95 & 572,32 & 635,95 & $1.209,11$ & 561,01 \\
\hline Summe Herstellungskosten & 168,92 & 811,90 & 139,10 & 196,45 & $1.290,99$ \\
\hline (Zwischen-)SUMmE & $\mathbf{6 1 1 , 8 7}$ & $\mathbf{1 . 3 8 4 , 2 2}$ & $\mathbf{7 7 5 , 0 5}$ & $\mathbf{1 . 4 0 5 , 5 6}$ & $\mathbf{1 . 8 5 2 , 0 0}$ \\
\hline
\end{tabular}

Hieraus ergibt sich, dass Fall 1 der günstigste Fall der Fallstudie wäre, da hier nicht nur die die geringsten Personalkosten, sondern auch die geringsten Herstellungskosten anfallen. Im Gegensatz dazu verursacht Fall 3.2 nach dieser Berechnung die höchsten Kosten. Zwar würden lediglich rund $120 €$ mehr Personalkosten für Fall 3.2 als für Fall 1 entstehen, jedoch haben die erheblich höheren Herstellungskosten Einfluss auf das Ergebnis. Die eigene Umsetzung des gleichen Manuskriptes im Fall 3.1 verursacht nach dieser Erhebung $447 €$ weniger Kosten als Fall 3.2. Hierbei muss jedoch beachtet werden, dass zum Zeitpunkt der Drucklegung des hier vorliegenden Werkes einige Prozesse (vgl. Kapitel 4.3.3) für Fall 3.1 noch nicht durchgeführt worden waren. Die fehlenden Daten zum internen Arbeitsaufwand werden vom Projektteam auf 185 Min., also rund 3 Arbeitsstunden, geschätzt. Dies würde eine Erhöhung der Personalkosten von rund $77 €$ für Fall 3.1 bedeuten; und somit in der Summe nicht die Gesamtkosten (Personalkosten und Herstellungskosten) von Fall 3.2 übersteigen.

Erstaunlich ist hierbei, dass für Fall 3.1 zwar doppelt so hohe Personalkosten anfallen würden, als für Fall 2.1. Auf Grund der geringen Herstellungskosten unterscheiden sich die Fälle in der Gesamtsumme jedoch nur marginal. Andershe- 
rum verhält es sich mit dem Fall 2.2. Aus Tabelle 26 geht hervor, dass dieser Fall rund $64 €$ mehr Personalkosten aufruft als Fall 2.1; unter Einbeziehung der Herstellungskosten fallen für Fall 2.2 in der Summe allerdings nur Kosten von $775 €$ an, und damit fast die Hälfte weniger als für Fall 2.1. Bei der Betrachtung dieser Ergebnisse muss berücksichtigt werden, dass für Fall 2.2 keine und Fall 3.2 nur sehr geringe Druckkosten beglichen werden mussten. Um diese Fälle mit den anderen vergleichen zu können, ist daher die Annahme sinnvoll, dass auch für diese Fälle Druckkosten in Höhe ihrer Pendants (Fall 2.1 und Fall 3.1) angefallen sind. Dies führt zu den Ergebnissen in Tabelle 27:

Tab. 27 Summe der Personalkosten, inkl. Druckkosten für Fall 2.2 und 3.2

\begin{tabular}{|c|c|c|c|c|c|}
\hline & \multicolumn{5}{|c|}{ Kosten $(€)$} \\
\hline & $\begin{array}{c}\text { F 1 } \\
\text { CRC- } \\
\text { Workflow }\end{array}$ & $\begin{array}{c}\text { F } 2.1 \\
\text { Dienst- } \\
\text { leister B }\end{array}$ & $\begin{array}{c}\text { F } 2.2 \\
\text { Dienst- } \\
\text { leister C }\end{array}$ & $\begin{array}{c}\text { F } \mathbf{3 . 1} \\
\text { eigene } \\
\text { Umsetzung }\end{array}$ & $\begin{array}{r}\text { F } 3.2 \\
\text { Dienst- } \\
\text { leister A }\end{array}$ \\
\hline Zwischensumme & 611,87 & 1384,22 & 775,05 & $1.405,56$ & $1.852,00$ \\
\hline + zzgl. Druckkosten & - & - & 158,24 & - & 186,89 \\
\hline SUMME & 611,87 & $1.384,22$ & 933,29 & $1.405,56$ & $2.038,8 \mathrm{~s}$ \\
\hline
\end{tabular}

Wie zu erwarten, ergibt sich, dass, wenn für Fall 3.2 die gleichen Druckkosten wie für Fall 3.1 angefallen wären, dieser weiterhin der teuerste Fall der Studie gewesen wäre; allerdings mit einer höheren Differenz zu Fall 3.1. Auch zuzüglich Druckkosten bleibt Fall 2.2 der zweitpreiswerteste Fall der Fallstudie.

Welche Schlüsse dieses Ergebnis für das Betreiben eines OA-Hochschulverlages zulässt, wird im Kapitel 4.4.3 diskutiert.

\subsubsection{Analyse der Dienstleister}

Die Daten der Fallstudie und ihre Analyse aus den vorherigen Kapiteln liefern bereits einen umfassenden Überblick über die personellen, zeitlichen und finanziellen Aufwendungen, die für das Betreiben eines OA-Hochschulverlages notwendig werden können. Jedoch gilt es, die Größen Zeit und Kosten immer auch im Hinblick auf die gewünschte Qualität zu betrachten. Aus diesem Grund erfolgt an dieser Stelle eine qualitative Einschätzung der innerhalb der Fallstudie beauftragten Dienstleister. Diese wird anschließend für den Vergleich hinsichtlich Zeit und Kosten operationalisiert und die Ergebnisse als Netzdiagramme dargestellt.

\section{Satzdienstleister}

Für die Analyse der Satzdienstleister wurden die Zieldimensionen Zeit, Kosten und Qualität zunächst qualitativ nach den folgenden Definitionen evaluiert: 


\section{Kriterium: Qualität}

Die Qualität setzt sich aus den Teilkriterien Produktqualität, Korrektur- und Kommunikationsaufwand zusammen: Das Qualitätskriterium Produktqualität spiegelt die Einschätzung zur Qualität der ersten PDF-Fahne und die Umsetzung der Gestaltungsvorgaben wider. Im Rahmen des Qualitätskriteriums Korrekturaufwand wird der entstandene Aufwand zur Überprüfung der Fahnen auf Richtigkeit oder zur Überprüfung der Umsetzung von Korrekturen, auch anhand der Anzahl der benötigten Korrekturschleifen und Korrekturen, bewertet. Das Qualitätskriterium Kommunikationsaufwand bewertet den entstandenen Kommunikationsaufwand mit den Dienstleistern vor der Beauftragung und während der Durchführung des Satzes.

\section{Kriterium: Zeit}

Die Bewertung der Zieldimension Zeit erfolgt anhand der benötigten Dauer in Arbeitstagen ab der Beauftragung des Dienstleisters bis zur Lieferung der beauftragten Leistungen und der benötigten Zeit zur Umsetzung von Korrekturen.

\section{Kriterium: Kosten}

Die Bewertung der Zieldimension Kosten erfolgt anhand der in Rechnung gestellten Gesamtkosten für die Leistung der Satzdienstleister (vgl. Kapitel 4.4.1.4).

Tab. 28 Qualitative Analyse von Dienstleister A

A inländischer Publishing-Dienstleister

\begin{tabular}{|c|c|c|}
\hline \multirow[t]{7}{*}{ Produktqualität } & \multicolumn{2}{|c|}{ Qualität der ersten PDF-Fahne: } \\
\hline & \multirow{3}{*}{\multicolumn{2}{|c|}{$\begin{array}{l}\text { - vermehrt mikrotypografische Fehler } \\
\text { - vereinzelt systematische Fehler } \\
\text { Umsetzung der Gestaltungsvorgaben: }\end{array}$}} \\
\hline & & \\
\hline & & \\
\hline & \multicolumn{2}{|c|}{ Satz nach vorhandenem Template eines anderen } \\
\hline & \multicolumn{2}{|c|}{ Wissenschaftsverlags, ${ }^{173}$ daher ist eine Einschätzung zur } \\
\hline & \multicolumn{2}{|c|}{ Umsetzung nur bedingt möglich } \\
\hline \multirow[t]{6}{*}{ Korrekturaufwand } & \multirow{2}{*}{\multicolumn{2}{|c|}{$\begin{array}{l}\text { Beschreibung Ablauf: Zunächst erfolgte die Lieferung der PDF- } \\
\text { Fahne und erst nach anschließender Freigabe die der EPUB- } \\
\text { und MOBI-Dateien. }\end{array}$}} \\
\hline & & \\
\hline & Anzahl Korrekturschleifen & Anzahl der Korrekturen \\
\hline & 1. PDF-Fahne & 160 \\
\hline & 2. PDF-Fahne & 82 \\
\hline & 3. PDF-Fahne & 0 (final) \\
\hline
\end{tabular}

173 Somit konnten hohen Einrichtungskosten für ein Template nach den Gestaltungsrichtlinien des Modellverlags eingespart werden. 
A inländischer Publishing-Dienstleister

\begin{tabular}{lll}
\hline & \multicolumn{1}{l}{ 1. EPUB/MOBI-Entwurf } & 10 \\
\cline { 2 - 3 } & 2. EPUB/MOBI-Entwurf & \multicolumn{1}{c}{3} \\
\cline { 2 - 3 } & 3. EPUB/MOBI-Entwurf & 0 (final) \\
\hline Kommunikationsaufwand & - & Beratung/Angebotsanfrage, Beauftragung und \\
& & Kommunikation während der Korrekturschleifen via E-Mail \\
& - & fester Ansprechpartner stand zur Verfügung \\
& - & erhöhter Kommunikationsbedarf vor Beauftragung nötig \\
& & (Abstimmung von Kosten und Leistungsumfang) \\
& - & letztendlich Angebotserstellung auf Grundlage eines \\
\hline Zeit & anderen Manuskriptes \\
\hline Kosten & 53 Arbeitstage \\
\hline
\end{tabular}

Tab. 29 Qualitative Analyse von Dienstleister B

B ausländischer Publishing-Dienstleister

\section{Produktqualität}

\section{Qualität der ersten PDF-Fahne:}

- sorgfältige und saubere Umsetzung, mikrotypografisch gut gesetzt

- Korrekturen bezogen sich größtenteils auf Änderungen der Gestaltungsrichtlinie

Umsetzung der Gestaltungsvorgaben:

- dem Dienstleister wurde ein InDesign-Template zur Verfügung gestellt

- die Erstellung von Musterseiten nach Gestaltungsvorgaben vor der Beauftragung war möglich

- sehr gute Umsetzung der Vorgaben, sehr wenige Abweichungen festgestellt

\section{Korrekturaufwand} Ausgabeformate gleichzeitig. Die PDF-Fahnen-Erstellung erfolgte mit InDesign, die offenen Daten wurden mitgeliefert.

\begin{tabular}{ll}
\hline Anzahl Korrekturschleifen & Anzahl der Korrekturen \\
\hline $\begin{array}{l}\text { 1. PDF-Fahne / EPUB/MOBI- } \\
\text { Entwurf }\end{array}$ & 54 \\
\hline $\begin{array}{l}\text { 2. PDF-Fahne / EPUB/MOBI- } \\
\text { Entwurf }\end{array}$ & 8
\end{tabular}


B ausländischer Publishing-Dienstleister

\begin{tabular}{ll}
\hline & $\begin{array}{l}\text { 3. PDF-Fahne / EPUB/MOBI- } \\
\text { Entwurf }\end{array}$ \\
\hline Kommunikationsaufwand & - Beratung/Angebotsanfrage, Beauftragung und \\
& Kommunikation während der Korrekturschleifen via E-Mail \\
& - fester Ansprechpartner stand zur Verfügung \\
& - explizite Nachfragen seitens des Dienstleisters vor \\
& Satzbeginn (Understanding Document) \\
& - berechtigte und gute Nachfragen und Hinweise während \\
& der Korrekturschleifen \\
\hline Zeit & immer zeitnahe Antwort erhalten \\
\hline Kosten & 22 Arbeitstage \\
\hline
\end{tabular}

Tab. 30 Qualitative Analyse von Dienstleister C

C ausländischer Outsourcing-Dienstleister

Produktqualität $\quad$ Qualität der ersten PDF-Fahne:
$-\quad$ viele mikrotypografische (vermeidbare) Fehler
$-\quad$ systematische Fehler
$-\quad$ einige offensichtliche Fehler (selbst auf der Titelseite)
$-\quad$ teilweise ungewöhnliche Bildplatzierung
Umsetzung der Gestaltungsvorgaben:
$-\quad$ dem Dienstleister wurde ein InDesign-Template zur
$\quad$ Verfügung gestellt
$-\quad$ die Erstellung von Musterseiten nach
$\quad$ Gestaltungsvorgaben vor der Beauftragung war
$\quad$ möglich
insg. gute Umsetzung, jedoch Nacharbeitung bei
und Formeln)
Entwurfs erfolgte erst mit der dritten PDF-Fahne. Die PDFFahnen-Erstellung erfolgte mit InDesign, die offenen Daten wurden mitgeliefert.

\begin{tabular}{ll}
\hline Anzahl Korrekturschleifen & Anzahl der Korrekturen \\
\hline 1. PDF-Fahne & 89 \\
\hline 2. PDF-Fahne & 37
\end{tabular}


C ausländischer Outsourcing-Dienstleister

\begin{tabular}{ll}
\hline & $\begin{array}{l}\text { 3. PDF-Fahne / 1. EPUB/MOBI- } \\
\text { Entwurf }\end{array}$ \\
\cline { 2 - 2 } & $\begin{array}{l}\text { 4. PDF-Fahne / 2. EPUB/MOBI- } \\
\text { Entwurf }\end{array}$ \\
\hline Kommunikationsaufwand & - Beratung/Angebotsanfrage, Beauftragung und \\
& Kommunikation während der Korrekturschleifen via E- \\
& - Mail \\
& $-\quad$ wester Ansprechpartner stand zur Verfügung \\
\hline Zeit & 20 Arbeitstage \\
\hline Kosten & $139,10 €$
\end{tabular}

An dieser Stelle erfolgt die Operationalisierung der vorgestellten Einschätzungen zu den definierten Kriterien, indem für jedes Kriterium eine Bewertung von 1 bis 5 abgegeben wird. Hieraus ergibt sich das entsprechende Netzdiagramm (vgl. Abbildung 24). Eine Diskussion der Ergebnisse findet in Kapitel 4.4.3 statt.

Tab. 31 Bewertungsskala der beauftragten Satzdienstleister für die Fallstudie ${ }^{174}$

\begin{tabular}{llll} 
Kriterium & Dienstleister A & Dienstleister B & Dienstleister C \\
\hline Qualität (durchschnittlich) & 3 & 4,66 & 3,33 \\
\hline$-\quad$ Produktqualität & 3 & 5 & 2 \\
\hline$-\quad$ Korrekturaufwand & 3 & 4 & 3 \\
\hline$-\quad$ Kommunikationsaufwand & 3 & 5 & 5 \\
\hline Zeit & 2 & 4 & 5 \\
\hline Kosten & 4 & 3 & 5 \\
\hline
\end{tabular}

1741 - schlecht / 2 - nicht zufriedenstellend / 3 - ausreichend / 4 - gut / 5 - sehr gut 


\section{Qualitative Analyse zu den Satzdienstleistern}

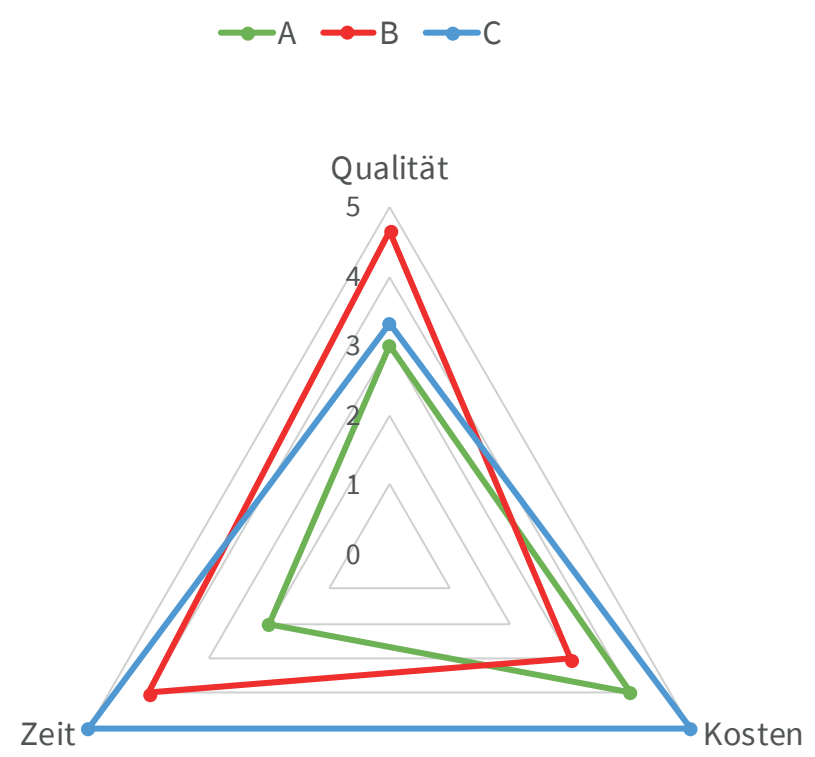

Abb. 24 Netzdiagramm zur qualitativen Analyse der Satzdienstleister

\section{Druck- und Vertriebsdienstleister}

Für die Analyse der Druck- und Vertriebsdienstleister wurden die Zieldimensionen Zeit, Kosten und Qualität qualitativ nach den folgenden Definitionen evaluiert:

\section{Kriterium: Qualität}

Die Qualität setzt sich aus den Teilkriterien Produkt- und Prozessqualität zusammen: Das Qualitätskriterium Produktqualität spiegelt die Einschätzung der druckund buchbinderischen Qualität der gelieferten Exemplare wider. Das Qualitätskriterium Prozessqualität spiegelt das allgemeine Handling und den damit verbundenen Aufwand zum Auslösen des Druckauftrags, zur Bereitstellung der Druckdaten sowie zur Auftragsüberwachung wider. 


\section{Kriterium: Zeit}

Die Bewertung der Zieldimension Zeit erfolgt anhand der Dauer von der Druckbeauftragung bis zur Lieferung und Annahme der in Auftrag gegebenen Bücher.

Kriterium: Kosten

Die Bewertung der Zieldimension Kosten erfolgt anhand der ermittelten Kosten pro Buch (vgl. Tabelle 24, Kapitel 4.4.2.4).

Tab. 32 Qualitative Analyse von Dienstleister E

E ausländischer PoD-Dienstleister

\begin{tabular}{|c|c|}
\hline Produktqualität & 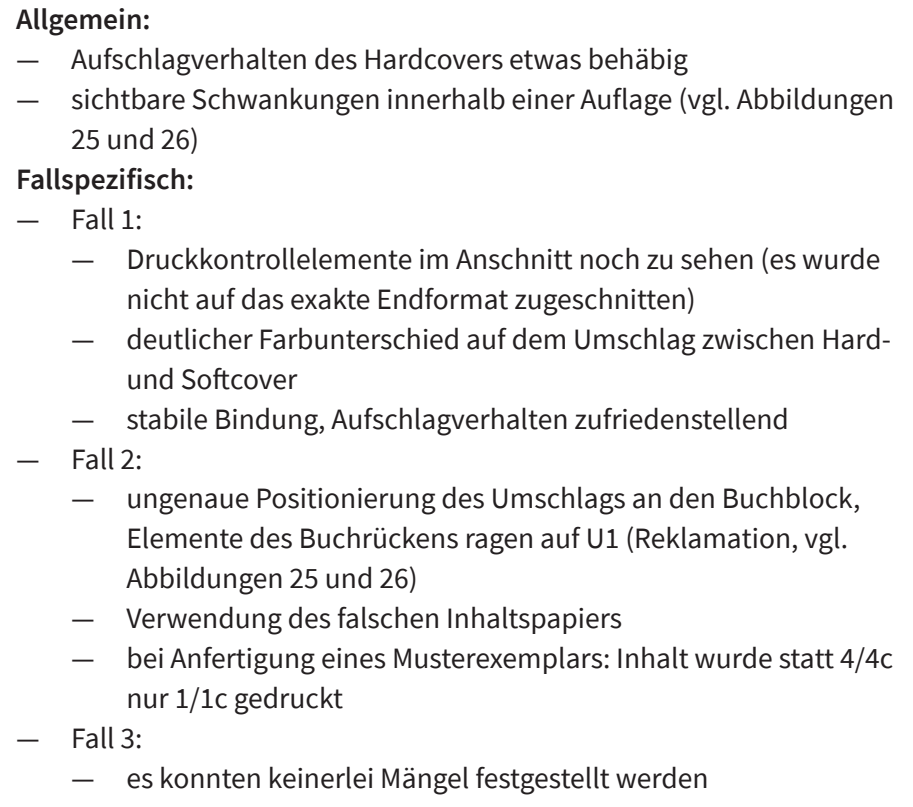 \\
\hline Prozessqualität & $\begin{array}{ll}\text { - } & \text { Auslösung des Druckauftrags, Auftragsbestätigung und sonstige } \\
& \text { Kommunikation per E-Mail } \\
- & \text { fester Ansprechpartner stand zur Verfügung } \\
- & \text { sehr hoher Kommunikationsbedarf notwendig } \\
- & \text { Antwort hat häufig etwas länger gedauert } \\
- & \text { ungenügende Beratung bei kritischem Buchrücken (Reklamation bei } \\
& \text { Fall } 2 \text { als Folge) }\end{array}$ \\
\hline Zeit & $\begin{array}{l}\text { - Fall 1: } 11 \text { Arbeitstage } \\
\text { - Fall 2: } \\
\quad-\quad \text { 1. Lieferung: } 26 \text { Arbeitstage (Reklamation) } \\
\quad \text { - 2. Lieferung: } 15 \text { Arbeitstage } \\
\text { - Fall 3: } 7 \text { Arbeitstage }\end{array}$ \\
\hline
\end{tabular}

Kosten pro Buch Durchschnitt: rund $7 €^{175}$ 


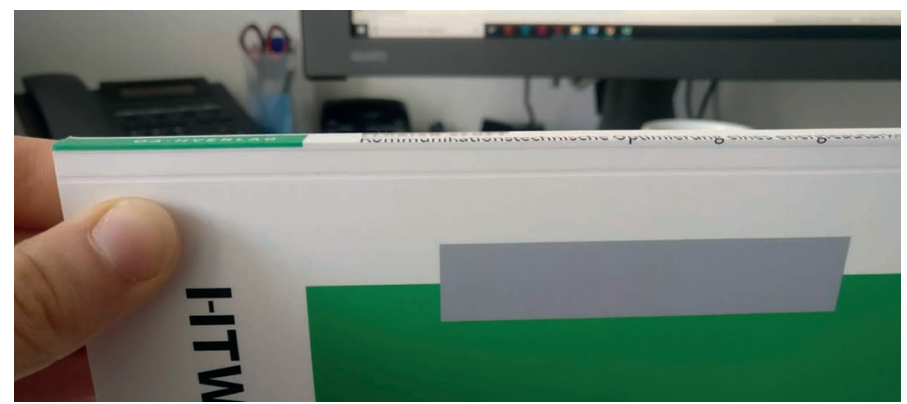

Abb. 25 Reklamation: Elemente des Buchrückens ragen auf die U1 des Buches (Fall 2)
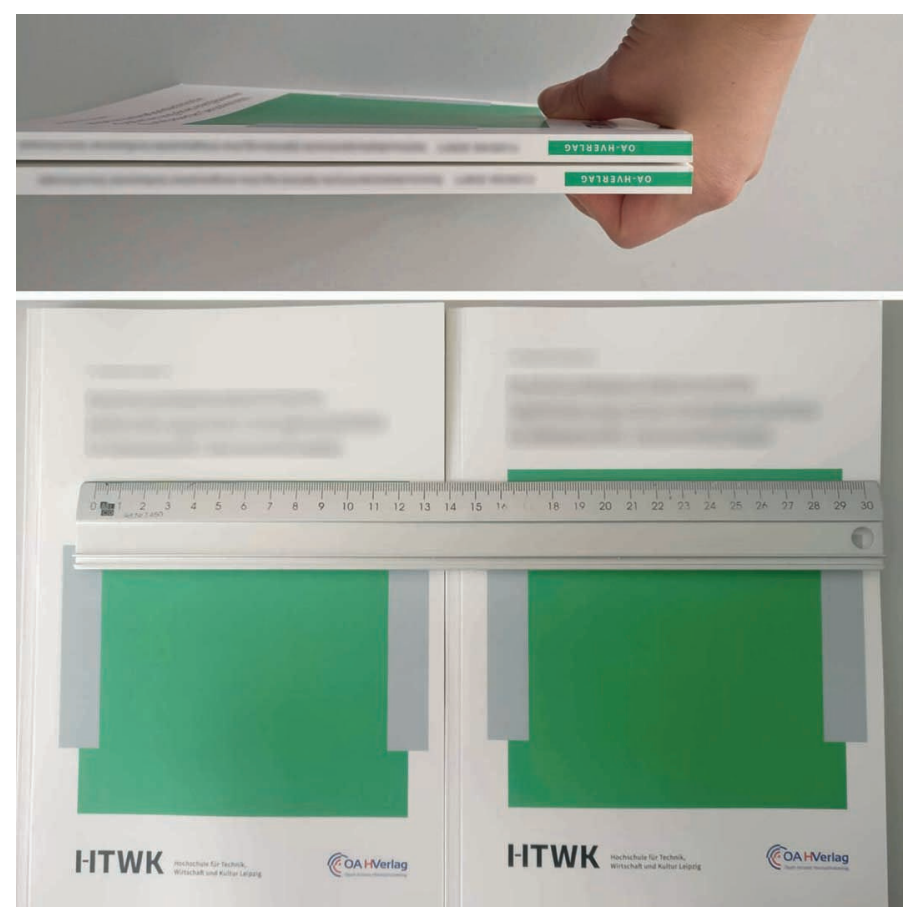

Abb. 26 Sichtbare Schwankungen bei der Positionierung des Umschlags innerhalb einer Auflage (Fall 2) 
Tab. 33 Qualitative Analyse von Dienstleister F

F inländische Buchdruckerei-Gruppe (Fall 3)

\begin{tabular}{ll}
\hline Produktqualität & - exakte Positionierung des Umschlags (Buchrücken sitzt mittig) \\
& - gute Druckbildschärfe \\
& - stabile Bindung, gutes Aufschlagverhalten \\
\hline Prozessqualität & - Nutzung des Web-Interface erst nach Freischaltung eines \\
& Kundenberaters möglich \\
- & wenig Kommunikationsbedarf notwendig \\
- & hoher Automatisierungsgrad und Usability \\
- & Druckdaten- und Auftragsmanagement über Web-Interface (Anlegen \\
& von Buchprojekten, Auslösung des Druckauftrags, Überwachung des \\
& Auftrags- und Lieferstatus, Umschlagbemaßungsvorgaben) \\
- & Softproof als Qualitätssicherung der Druckdaten \\
& Interface vorhanden) \\
- & Support per Mail erreichbar; rasche Antwortzeiten \\
\hline Zeit & 19 Arbeitstage \\
\hline Kosten pro Buch & $15,45 €$ \\
\hline
\end{tabular}

Tab. 34 Qualitative Analyse von Dienstleister G

G inländischer Druck- und Vertriebsdienstleister (Fall 2)

\begin{tabular}{|c|c|c|}
\hline Produktqualität & $\begin{array}{l}- \\
- \\
-\end{array}$ & $\begin{array}{l}\text { gute Druckbildschärfe } \\
\text { Softcover: nicht ganz exakte Positionierung des Umschlags, dadurch } \\
\text { Probleme beim schmalen Buchrücken } \\
\text { Hardcover: Vorsatzpapier nicht komplett verklebt }\end{array}$ \\
\hline Prozessqualität & $\begin{array}{l}- \\
- \\
- \\
- \\
-\end{array}$ & $\begin{array}{l}\text { Druckdaten- und Auftragsmanagement über eine Online- } \\
\text { Verlagssoftware (Anlegen von Buchprojekten, Auslösung des } \\
\text { Druckauftrags, Überwachung des Auftrags- und Lieferstatus, } \\
\text { Umschlagbemaßungsvorgaben) } \\
\text { Qualitätssicherung der Druckdaten durch Dienstleister und Beratung } \\
\text { des Kunden } \\
\text { Kommunikation über Kommunikationsmodul jederzeit möglich } \\
\text { rasche Antwortzeiten } \\
\text { die Herstellung eines Aushänger vor Produktion der Auflage ist } \\
\text { inbegriffen und wird automatisch nach Freigabe der Druckdaten } \\
\text { ausgelöst }\end{array}$ \\
\hline Zeit & \multicolumn{2}{|c|}{23 Arbeitstage } \\
\hline Kosten pro Buch & \multicolumn{2}{|c|}{$21,80 €$} \\
\hline
\end{tabular}


Tab. 35 Qualitative Analyse von Dienstleister H

H inländischer PoD- und Selfpublishing-Dienstleister (Fall 1)

\begin{tabular}{ll}
\hline Produktqualität & - stabile Bindung, gutes Aufschlagverhalten \\
& - teilweise streifige Farbabbildungen \\
\hline Prozessqualität & - hoher Automatisierungsgrad und Usability \\
& $-\quad$ Druckdaten- und Auftragsmanagement über Web-Interface (Anlegen \\
& $\quad$ Aun Buchprojekten, Auslösung des Druckauftrags, Überwachung des \\
& - Qualitätssicherung der Druckdaten beim Hochladen im Web-Interface \\
& $-\quad$ kaum Kommunikationsbedarf notwendig (Hilfestellungen im Web- \\
& - Interface vorhanden) \\
& Support per Mail erreichbar \\
\hline Zeit & 8 Arbeitstage \\
\hline Kosten pro Buch & $26,25 €$ \\
\hline
\end{tabular}

An dieser Stelle erfolgt die Operationalisierung der vorgestellten Einschätzungen zu den definierten Kriterien, indem für jedes Kriterium eine Bewertung von 1 bis 5 vergeben wird. Hieraus ergibt sich das entsprechende Netzdiagramm (Abbildung 27 ). Eine Diskussion der Ergebnisse findet in Kapitel 4.4.3 statt.

Tab. 36 Bewertungsskala der beauftragten Druck- und Vertriebsdienstleister für die Fallstudie $^{176}$

Kriterium

Dienstleister E Dienstleister F Dienstleister G Dienstleister H

\begin{tabular}{lllll}
\hline Qualität (durchschnittlich) & 2 & 5 & 4,5 & 4,5 \\
\hline$-\quad$ Produktqualität & 3 & 5 & 4 & 4 \\
\hline$-\quad$ Prozessqualität & 1 & 5 & 5 & 5 \\
\hline Zeit & 3 & 3 & 2 & 5 \\
\hline Kosten & 5 & 4 & 3 & 3 \\
\hline
\end{tabular}

1761 - schlecht / 2 - nicht zufriedenstellend / 3 - ausreichend / 4 - gut / 5 - sehr gut 


\section{Qualitative Analyse zu den \\ Druck- und Vertriebsdienstleistern}

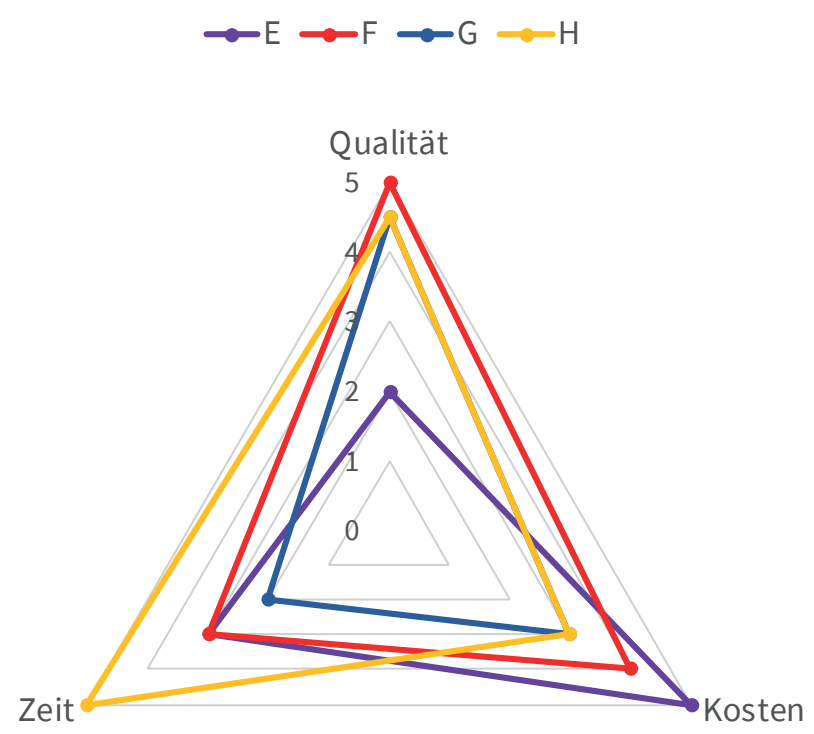

Abb. 27 Netzdiagramm zur qualitativen Analyse der Druck- und Vertriebsdienstleister

\subsubsection{Diskussion der Ergebnisse}

Die in den vorherigen Kapiteln präsentierten und analysierten Daten der Fallstudie lassen vielfältige Schlussfolgerungen zu. Einige ausgewählte Erkenntnisse sollen an dieser Stelle weiter diskutiert werden.

Zunächst konnte die Analyse der Workflow-Rollen aufzeigen, dass das hier vorgestellte Rollen-Modell idealtypisch beschrieben ist und somit auf unterschiedliche Gegebenheiten beim Publizieren von OA-Monografien angewendet werden kann. Die wichtigste Erkenntnis hieraus ist somit, dass die Rollen des Modells immer gleich sind, jedoch von unterschiedlichen Personen ausgeführt werden können. Je nachdem, welche Kompetenzen die jeweilige Person besitzt, kann die Rolle und somit der entsprechende Prozess mehr oder weniger effizient ausgefüllt bzw. durchgeführt werden. Dies gilt insbesondere für die Rolle des Content-Umset- 
zers, die in der Fallstudie je nach Workflow-Variante durch einen Mitarbeiter des Modellverlags oder Dienstleister eingenommen wurde. Auffällig ist außerdem, dass in allen 3 Fällen die Rolle des Content-Prüfers von jeweils 3 Personen - dem Projektmitarbeiter 1, Projektmitarbeiter 2 und den Projektleitern - eingenommen wurde. Zurückführen lässt sich dies auf die Komplexität des Anforderungsprofils, so dass hier 3 Personen notwendig waren, um die zugeordneten Prozesse effektiv auszuführen. Gleiches gilt für die Rolle des Betriebswirts; auch hier hatten in allen Fällen 3 Personen die Rolle eingenommen. Aus dieser Feststellung können Handlungs- und Gestaltungsoptionen für zukünftige Stellen abgeleitet werden.

Des Weiteren beinhalten die Ergebnisse der Datenanalyse zu den Zieldimensionen Zeit, Kosten und Qualität das Potenzial, aus verschiedenen Blickwinkeln betrachtet zu werden. Die Auswertung zeigte, dass die Umsetzung des Falles 1 mit insgesamt 17 Arbeitsstunden den kleinsten zeitlichen Aufwand im Modellverlag verursachte. Darüber hinaus sind für diesen Fall auch die geringsten Herstellungskosten entstanden und er würde die wenigsten Personalkosten verursachen (vgl. Tabelle 26).

Dieses Ergebnis ist nicht überraschend, da mit Fall 1 ein CRC-Workflow durchgeführt wurde. Der Autor bzw. Content-Urheber lieferte in diesem Fall ein publikationsfertiges Manuskript an den Verlag, so dass einige Prozesse zum Satz von Manuskript bzw. Cover (P-CiP-Prozesse und Prozess P-CUe-100) nicht (mehr) erforderlich waren. In der P-CiP-Prozessgruppe, welche in allen anderen Fällen der Studie den größten Anteil des internen Arbeitsaufwands verursachte, fielen weder Kosten für Satzdienstleistungen noch nennenswerter interner Arbeitsaufwand für die Projektmitarbeiter an.

Allerdings hatte dieses Vorgehen Auswirkungen auf die Qualität der Publikation von Fall 1. Nach Erhalt der gedruckten Produkte wurde festgestellt, dass insbesondere die enthaltenen Abbildungen und Fotografien nicht ausreichend für den Druck aufbereitet waren. Dieser Mangel kann tatsächlich auf die Druckvorlage zurückgeführt werden, da er sowohl im Musterbuch von Dienstleister $H$ als auch im Auflagendruck von Dienstleister E auffiel. Im Rahmen des Prozesses I-Ca-120 hätte der Content-Urheber besser hierzu beraten werden müssen, um die Qualität der Druckvorlage zu verbessern. Andererseits hätte dies einen höheren Arbeitsaufwand für die Projektmitarbeiter verursacht. Hier muss also die Zieldimension Zeit im Hinblick auf die Qualität kritisch betrachtet werden. Des Weiteren kann aus diesen Ergebnissen abgeleitet werden, dass sich für die Durchführung eines CRCWorkflows die sorgfältige Erstellung von Templates und Autoren-Guidelines oder Checklisten empfiehlt, um die Qualität der Druckvorlagen von vornherein sicherzustellen.

Auch, wenn Fall 1 aufgrund der oben genannten Punkte nur bedingt mit den Ergebnissen der anderen Fälle vergleichbar ist, überrascht es, dass die Fälle 2.1, 2.2 und 3.2 nur einen minimal größeren internen Arbeitsaufwand, als Fall 1, verursacht haben. Obwohl die Komplexität und der Seitenumfang der Publikationen der Fälle 2 und 3 um einiges höher waren, hat die gesamte Umsetzung der Publikationen - einschließlich dem medienneutralen Satz des Manuskriptes durch Satz- 
dienstleister - kaum mehr Zeit in Anspruch genommen als Fall 1. Der Grund hierfür ist sicherlich, dass sich der interne Arbeitsaufwand hinsichtlich der Satzprozesse (P-CiP-Prozesse) zwischen CRC-Workflow und XML-first-Workflow mit Dienstleistern kaum unterscheidet (vgl. Kapitel 4.4.2.3). Zwar erspart die Durchführung des Satzes des Manuskriptes durch den Content-Urheber oder einen Dienstleister den Projektmitarbeitern Arbeitsaufwand im Modellverlag, jedoch sind sie weiterhin dafür verantwortlich, die Qualität der erstellten Ausgabeformate sicherzustellen (Prozess P-CiP-140). Je nachdem, wie sorgfältig dies erfolgt ist, entsteht Arbeitsaufwand für die Kommunikation der Korrekturen im Modellverlag.

Das Ergebnis verdeutlicht, dass die Umsetzung eines CRC-Workflows zwar Kosten, aber kaum Arbeitszeit im Vergleich zur Umsetzung eines medienneutralen Workflows mit Dienstleistern spart, sofern im Vorfeld keine strikten Vorgaben für den Content-Urheber für den CRC-Workflow formuliert wurden.

Im Gegensatz zu den Ergebnissen der Erhebung des internen Arbeitsaufwands unterscheiden sich jedoch die Herstellungskosten - insbesondere die Kosten für die Satzdienstleistungen (P-CiP-Prozesse) der Fälle 2.1, 2.2 und 3.2 - deutlich. Inwieweit Preisunterschiede eine Auswirkung auf die Qualität der Leistungen haben, kann auf Grundlage der Ergebnisse aus Kapitel 4.4.2.6 diskutiert werden.

Die Abbildung 24 zeigt, dass zwar durchaus Abstriche in der Leistungsqualität von Dienstleister $C$ festzustellen sind, diese sich aber dennoch auf annähernd gleichem Niveau wie die Leistung von Dienstleister Abefindet. Dienstleister A benötigte die meiste Zeit, bevor die Ausgabeformate der Publikation durch die Mitarbeiter des Modellverlags freigegeben werden konnten. Besonders kritisch zu betrachten ist dies angesichts der Tatsache, dass Dienstleister $A$ ein vorhandenes Gestaltungstemplate für den medienneutralen Satz im Rahmen von Fall 3.2 verwenden konnte. Indes mussten Dienstleister $B$ und $C$ vor dem Satzprozess erst noch die Gestaltungsvorgaben des Modellverlags erarbeiten. Der von Dienstleister A geringer angesetzte Seitenpreis - im Vergleich zu Dienstleister B - wiegt dies nur marginal auf.

Das Ergebnis für Dienstleister A kann auch darauf zurückgeführt werden, dass dieser zunächst nur die PDF-Fahnen der Publikation zur Überprüfung an die Projektmitarbeiter des Modellverlags sendete, bevor die EPUB- und MOBI-Ausgaben folgten. Somit fanden im Fall 3.2 3 Korrekturschleifen für die PDF-Fahnen und anschließend 3 weitere Korrekturschleifen für die EPUB-und MOBI-Dateien statt.

Dieser Ablauf unterscheidet sich vom Vorgehen des Dienstleisters B, weshalb dieser in der Bewertung für die Zieldimension Zeit erheblich besser abschneidet. Dienstleister B übermittelte in einem Schritt die PDF-Fahne sowie die EPUB-und MOBI-Dateien zur Überprüfung an die Projektmitarbeiter des Modellverlags; so waren vor der Veröffentlichung lediglich 3 Korrekturschleifen notwendig. Aufgrund der höheren Herstellungskosten befindet sich Dienstleister B hinter Dienstleister C, im Mittelfeld dieser Einschätzung.

Es kann somit festgestellt werden, dass zumindest die Ergebnisse dieser Fallstudie die allgemeine Annahme, dass teurere (Satz-)Dienstleister auch eine bessere Qualität und im Umkehrschluss preiswerte Dienstleister nur mangelhafte Qualität 
liefern, nicht bestätigen. Diese Aussage ist natürlich nur bedingt belastbar, da innerhalb der Fallstudie nur Daten für eine nicht-repräsentative Anzahl von Satzdienstleistern erhoben wurden. Ebenfalls bleibt die Frage unbeantwortet, ob Dienstleister $C$ die Qualität seiner Leistungen auch für die Umsetzung von komplexeren Werken als der Publikation von Fall 2 halten kann.

Ein weiteres unerwartetes Ergebnis zeigen die Daten des Falls 3.1, insbesondere im Vergleich zu Fall 3.2, aber auch im Vergleich zu Fall 2.1. Es wird deutlich, dass Fall 2.1 und 3.1 fast gleich hohe Kosten verursachen, wenn in beiden Fällen der Publikationsworkflow vollständig durchlaufen und die Personalkosten mit einbezogen werden würden. Dies ist erstaunlich, da die Bearbeitung von Fall 3.1 mit mehr als doppelt so viel Arbeitsaufwand verbunden war wie Fall 2.1. Dies ist auf die Art der Durchführung (eigene Umsetzung des Satzes in Fall 3.1 und Umsetzung des Satzes mit Dienstleister B in Fall 2.1) zurückzuführen und muss entsprechend interpretiert werden.

Den größten zeitlichen Aufwand in Fall 3.1 hat die Auszeichnung der Inhalte in XML (P-CiP-Prozesse) durch (in erster Linie) eine wissenschaftliche Hilfskraft des Modellverlags verursacht. Diese war zwar durchaus kompetent genug, um diese Arbeit effektiv umzusetzen, aber sicherlich nicht so routiniert und damit effizient wie ein ausgebildeter, hauptberuflicher Setzer. Im Gegensatz zu einem Satzdienstleistungsunternehmen, in dem ggf. mehrere Mitarbeiter kontinuierlich am Satz eines Manuskriptes arbeiten, stand für Fall 3.1 lediglich die wissenschaftliche Hilfskraft zur Verfügung, die diese Aufgabe als Nebentätigkeit zum Studium ausübte. Einen Teil des hohen Arbeitsaufwands für die Durchführung der P-CiP-Prozesse für Fall 3.1 bedingen also sicherlich die notwendige Einarbeitungszeit und Lernkurve dieser wissenschaftlichen Hilfskraft als Content-Umsetzer.

Relativiert wird dies durch den Vergleich mit Fall 3.2. Die für die P-CiP- Prozesse angefallenen 750 Min. im Fall 3.2 sind ausnahmslos für die Kommunikation mit Dienstleister $A$ und die Überprüfung seiner Leistung aufgewendet wurden. Diese qualitätssichernden Handlungen sind in den gemessenen 2308 Min. Arbeitsaufwand von Fall 3.1 inbegriffen.

Daraus kann geschlussfolgert werden, dass der hohe Zeitaufwand für Fall 3.1 gerechtfertigt ist. Der Vergleich legt außerdem nah, dass lediglich zwei Drittel des Aufwandes von Fall 3.2 für die Durchführung der P-CiP-Prozesse notwendig sind, um diese selbständig im Hochschulverlag auszuführen (unter der Voraussetzung, dass entsprechend kompetente Mitarbeiter verfügbar sind).

Allerdings müssen hierbei zwei weitere Punkte beachtet werden: Zunächst ist deutlich zu machen, dass für die elektronischen Ausgabeformate von Fall 3.1 die mathematischen Formeln als Abbildungen eingefügt und nicht in XML ausgezeichnet wurden. Dies bedeutet, dass sie u. a. nicht durchsuchbar sind, was als Qualitätseinschränkung für die Nutzung des E-Books angesehen werden kann. Der Satzdienstleister $A$ war technisch versierter und konnte die Formeln in XML, vermutlich halbautomatisch, auszeichnen. Hieraus kann geschlossen werden, dass es sich für sehr komplexe Werke, wie das Manuskript von Fall 3, durchaus anbietet, den Satzprozess (P-CiP-Prozesse) an einen Satzdienstleister auszulagern, auch wenn dies 
höhere bzw. überhaupt Herstellungskosten verursacht. Im Umkehrschluss kann aber ebenso davon ausgegangen werden, dass der interne Arbeitsaufwand und somit mögliche Personalkosten für die eigene medienneutrale Umsetzung weit geringer ausfallen können, wenn es sich um ein weniger komplexes, eher textlastiges Werk handelt. Mit Qualitätseinbußen ist dann auch nicht zu rechnen.

Grundsätzlich darf an dieser Stelle nicht vernachlässigt werden, dass die Daten zu den Leistungen der Satzdienstleister von der erstmaligen Zusammenarbeit erhoben wurden. Es ist davon auszugehen, dass sich insbesondere die Zieldimensionen Zeit und Qualität verbessern, je mehr Projekte mit einem Dienstleister durchgeführt werden.

Letztendlich zeigen die Ergebnisse der Satzdienstleister nur, dass es große preisliche Unterschiede für die Ausführung von Satzdienstleistungen gibt, die nicht zwangsläufig Rückschlüsse auf deren Qualität zulassen.

Ein gänzlich anderes Ergebnis liefert die qualitative Einschätzung der in der Fallstudie beauftragten Druckdienstleister. Auch hierfür wurden zur besseren Darstellung die qualitative Analyse operationalisiert (vgl. Tabelle 36) und die Ergebnisse als Netzdiagramm (vgl. Abbildung 27) abgebildet.

Erkennbar ist, dass der preiswerteste Druckdienstleister - Dienstleister E - die geringste Qualität innerhalb der Fallstudie lieferte. Dahingegen konnten die teuersten Dienstleister - Dienstleister $G$ und $H$ - mit der Qualität ihrer Leistungen überzeugen. Insbesondere Dienstleister $H$ ist hervorzuheben, da er innerhalb der Fallstudie am schnellsten die Produkte lieferte. Dienstleister F sticht mit einer sehr guten Qualität und den niedrigsten Kosten nach Dienstleister E innerhalb der Fallstudie hervor.

Hierbei sollten die Ergebnisse von Dienstleister $E$ nicht zu kritisch betrachtet werden. Denn auch, wenn die Einschätzung von dessen Produkt- und Prozessqualität im Vergleich zu den anderen Dienstleistern schlechter ausfällt, ist sie durchaus marktüblich und somit annehmbar. Es stellt sich allerdings die Frage, wie gerade in diesem Punkt Alleinstellungsmerkmale geschaffen werden können, die es Universitäts- und Hochschulverlagen ermöglichen, neue Zielgruppen zu erschließen und sich gegenüber dem Leistungsportfolio der klassischen Fachverlage positiv abzuheben. In der Wahrnehmung der primären Zielgruppe von Hochschulverlagen - Wissenschaftler und akademisches Fachpublikum als Autoren und Leser stellt die Qualität der gedruckten Produkte eine kontrovers diskutierte Frage mit zum Teil diametral entgegengesetzten Standpunkten dar. Dies trifft vor allem zu, wenn es sich um Graduierungsarbeiten handelt, die in der gedruckten Fassung auch eine gewisse Repräsentationsfunktion haben. In den Geistes- und Sozialwissenschaften, aber auch in den Rechtswissenschaften ist diese Bedeutung relevanter als in den Natur- und Lebenswissenschaften, auch, wenn gerade die Arbeiten dieser Disziplinen von der Mehrheit der Nutzer OA mittels elektronischer Ausgabeformate rezipiert werden. Bei eher bildlastigen Monografien, wie Kunstbüchern oder Ausstellungskatalogen, sollte der Fokus in jedem Fall auf dem gedruckten Werk und somit auf dessen Qualität liegen. 
OA-Hochschulverlage sollten daher entsprechend ihrer Gegebenheiten, aber auch nach den zu veröffentlichen Produkten abwägen, wie sie die Zieldimensionen Zeit, Qualität und Kosten priorisieren, um entscheiden zu können, mit welchen Dienstleistern sie zusammenarbeiten. Gerade für sie kann es sinnvoll sein, vor allem die Wirtschaftlichkeit des Publikationsworkflows in den Fokus zu stellen. 


\section{Schlussbetrachtung}

Das Ziel unseres Projektes war die Analyse, Entwicklung und der Aufbau eines OAHochschulverlages für Fachbücher. Dieser sollte als Modellverlag zunächst an der HTWK Leipzig etabliert werden, um im Weiteren ausgehend von einem Transferkonzept auf andere Hochschulen und Universitäten übertragen werden zu können kann. Zugleich sollte die Forschungsfrage beantwortet werde, ob es möglich ist, hierfür ein Workflow-Modell zu entwickeln, das idealtypisch (allgemeingültig) durchführbar, personaleffizient und nachhaltig ist. Im Zuge der Durchführung Projektes ließ sich bestätigen, dass die Beschreibung und Entwicklung des gesuchten Workflows prinzipiell durchführbar ist: Drei Publikationsworkflows wurden durch die Veröffentlichung vorhandener Forschungsarbeiten entsprechend des entwickelten Modells in gedruckter Form und digital als OA erfolgreich realisiert. Dabei zeigte sich früh, dass der größte Teil aller hierfür relevanten Prozesse bereits durch das Modell abgedeckt und realistisch durchführbar ist. Durch die vom Forschungsteam geleistete praktische Arbeit an den Publikationen konnte das Workflow-Modell kontinuierlich evaluiert und somit angepasst werden. $\mathrm{Zu}$ diesen Anpassungen zählten u. a. das Ergänzen (in geringem Maß) und Zusammenfassen von Prozessen sowie die Entwicklung und Verbesserung der Qualitätsrichtlinien. Erst durch die eigenständige Durchführung bestimmter Prozesse war es zum Teil möglich, geeignete Checklisten für diese zu entwickeln, die so gestaltet sind, dass sie auch für die Realisierung anderer Buchprojekte nachgenutzt werden können.

Außerdem konnte die universelle Durchführbarkeit des Workflows nachgewiesen werden, was insbesondere zwei Faktoren belegen: Aus der Analyse der Workflow-Rollen lässt sich ableiten, dass die definierten Rollen immer gleich sind, jedoch von unterschiedlichen Personen ausgeführt werden können. Je nachdem, welche Kompetenzen die durchführende Person besitzt, kann die Rolle und somit der zugeordnete Prozess mehr oder weniger effizient abgewickelt werden. In den untersuchten Workflow-Varianten wurden innerhalb der Fallstudie die Grenzfälle abgebildet, in denen ein OA-Hochschulverlag neue Werke publizieren kann: vom CRC-Workflow ohne medienneutrale Aufbereitung, über die XML-first-Produktion mit Dienstleistern bis hin zur eigenen, autarken XML-first-Produktion. Alle drei Grenzfälle konnten erfolgreich durchgeführt werden, was die Vermutung nahelegt, dass das entwickelte Workflow-Modell auf verschiedene Gegebenheiten flexibel anpassbar ist.

Neben zahlreichen überraschenden oder auch erwarteten Ergebnissen lassen sich aus dem Projekt drei Tendenzen ableiten: Ein Offshore-Outsourcing bestimmter personal- und technologieintensiver Dienstleistungen, z. B. nach Asien, wie es inzwischen die meisten mittleren und großen Fachverlage durchführen, bewährt sich in summa nicht unbedingt für kleinere Verlagsstrukturen, zu denen auch Uni- 
versitäts- oder Hochschulverlage zählen. Der interne Betreuungsaufwand für Projektmanagement und Qualitätssicherung (über)kompensiert in Form zu erbringender Arbeitszeit (und den damit real verbundenen Kosten) die etwaigen Ersparnisse in Form von günstigeren Preisen für bestimmte ausgelagerte Dienstleistungen. Zudem erfordert ein Offshore-Outsourcing eine stärkere Standardisierung der zu veröffentlichenden Werke, wie es in einem Hochschulverlag oder auch einem kleinen oder mittelgroßen Wissenschaftsverlag mit einer großen Bandbreite an fachlichen Disziplinen kaum umsetzbar wäre. Auch ließ sich die These bestätigen, dass eine kamerafertige Lieferung des druckfähigen Werkes durch die Autoren (CRCPrinzip) nicht zu einer nennenswerten Einsparung von Arbeitszeit im Verlag führt, sondern lediglich die externen Kosten für den Satz des Manuskriptes wegfallen. Im ungünstigsten Fall erhöht sich sogar der interne Betreuungsaufwand, wenn bspw. Abbildungen im Vorfeld falsch oder uneinheitlich formatiert wurden, formale Fehler im Text nachträglich korrigiert oder Inkonsistenzen ausgeglichen werden müssen. Auch die medienneutrale Aufbereitung ist in solchen Fällen im Nachhinein, in einem XML-last-Workflow, nur durch internen Mehr- und damit Kostenaufwand möglich.

Schließlich zeigte bereits die Untersuchung im Vorfeld, dass es große preisliche Unterschiede zwischen den Wettbewerbern für Satzdienstleistungen gibt, die jedoch nicht zwangsläufig einen Schluss auf deren Qualität zulassen. Eine sorgfältige Auswahl und Erprobung verschiedener Dienstleister gerade in diesem Bereich der Wertschöpfungskette lässt sich daher als wichtiges Fazit für andere Hochschulen herausstellen. Besondere Ansprüche wurden innerhalb des Projektes an die Nachhaltigkeit gestellt, im Sinne eines kosten- und personaleffizienten Workflows. Dies wurde vor allem dadurch erreicht, dass eine Bottom-Up-Kalkulation des Ladenpreises für die gedruckten Werke durchgeführt wurde. Als Anforderung wurde hierfür festgelegt, dass der Ladenpreis anteilig anhand der anfallenden betrieblichen Kosten aus der Soll-Kalkulation ermittelt wird (progressive Kalkulation). Das ermöglicht, den Ladenpreis so zu kalkulieren, dass nach Abverkauf der geplanten Auflagen über einen bestimmten Zeitraum alle Kosten gedeckt sind und auch ein kalkulierter Gewinn erwirtschaftet werden kann. Diese Kalkulation ist so dokumentiert und in Form einer Excel-Tabelle hinterlegt worden, dass im Rahmen des Transfers andere Institutionen und Hochschulen mit ihren individuellen Kosten und Ressourcen rechnen können, um ihren eigenen Hochschulverlag ebenfalls in der gezeigten Weise nachhaltig ausrichten zu können.

Im Anschluss an das Projekt erscheint es somit als naheliegende Aufgabenstellung, den hier entwickelten Publikationsworkflow in einem neu zu gründenden Hochschulverlag an der HTWK Leipzig zu etablieren und zu verstetigen. Dies sollte mittels Anbindung des Hochschulverlages an die Hochschulbibliothek erfolgen, so dass auch nach dem Ausscheiden der befristet beschäftigten Projektmitarbeiter nach Laufzeitende des Projektes weitere Publikationen durch den Verlag mittels des beschriebenen Workflow-Modells realisiert werden können. Zudem wäre es sinnvoll, die praktische Umsetzung des Modells an der HTWK Leipzig innerhalb eines Zeitraums von etwa drei Jahren Controlling-seitig zu begleiten und dafür 
wirtschaftliche Kenngrößen zu entwickeln, die einen direkten Vergleich mit dem bisherigen Einsatz von personellen und finanziellen Ressourcen zur Publikation von Monografien erlauben. Die Analyse der gewonnenen Daten böte über den Zweck der im Rahmen des Forschungsprojektes durchgeführten Fallstudie hinaus die Möglichkeit, die Durchführung der Publikationsworkflows der Fallstudie zu evaluieren und somit die gezeigten Verbesserungspotenziale in dem an der HTWK Leipzig neu zu gründenden Hochschulverlag umzusetzen. Weiterhin können diese Daten als allgemeingültige Indikatoren für den Aufwand der Gründung und des Betreibens eigener OA-Hochschulverlage für andere akademische Institutionen außerhalb der HTWK Leipzig genutzt werden. Wichtig ist hierbei die Erkenntnis, dass die Zieldimensionen Zeit, Kosten und Qualität stets in Konkurrenz zueinanderstehen. Je nach Rahmenbedingungen und strategischer Zielstellung der betreibenden Institution müssen sie unterschiedlich priorisiert werden. Hiernach entscheidet sich dann individuell angepasst, aber auf konkreten quantitativen Daten basierend, welche Workflow-Variante für den eigenen OA-Hochschulverlag als am ehesten geeignet erscheint.

Die Einführung eines medienneutralen, kosten- und personaleffizienten OAHochschulverlags an der HTWK Leipzig soll daher modellhaft auch für andere Hochschulstandorte aufgefasst werden können. Im Projekt wird auch explizit auf die Zielstellung eines Know-how-Transfers hingewiesen, welcher es Hochschulen und Universitäten außerhalb der HTWK Leipzig erlauben soll, den neu entwickelten Workflow selbst einzuführen und umzusetzen. Diese Einführung im Rahmen dieses Transfers sollte, zumindest für einen Zeitraum von sechs bis zwölf Monaten, fallweise individuell begleitet werden, um organisatorische oder technische Anlaufschwierigkeiten auf ein Mindestmaß zu reduzieren. Diese qualitätssichernde Maßnahme stellt eine wichtige Anforderung an ein Change-Management allgemein dar. Andernfalls besteht die Gefahr - gerade an öffentlichen Institutionen und Einrichtungen -, dass eine effiziente und zielführende Einführung des Workflows nicht in dem zeitlichen Horizont möglich ist, der wünschenswert und grundsätzlich möglich wäre.

Grundsätzlich wurde das Konzept für einen OA-Hochschulverlag in dem vorliegenden Workflow so entwickelt, dass der Transfer des Modellverlages auf andere Hochschulen unter bestimmten Rahmenbedingungen ad hoc zunächst ohne zusätzliche Ressourcen flexibel möglich ist. Das vorliegende Handbuch dient daher in dieser Form als Transferkonzept mit konkreten Handlungsanweisungen für andere Hochschulen und Universitäten, die einen OA-Hochschulverlag etablieren wollen. Die erfolgreiche Umsetzung ist in dieser Form einmalig und wurde in Deutschland so noch nicht (nachhaltig) erreicht, da bisher keine ganzheitliche Analyse des Konzeptes eines Hochschulverlages erfolgt ist. Neben dieser unmittelbaren Auswirkung lässt die erfolgreiche Umsetzung des Vorhabens weitere, mittelbare Effekte durch die Schaffung einer leicht zugänglichen Infrastruktur und eines transparenten Workflows für OA-Publikationen erkennen. So ist davon auszugehen, dass Forscher aus den wissenschaftlichen Disziplinen, in denen die Publikation von Forschungsergebnissen als Monografie oder Sammelband in Buchform 
überwiegt, nach Einführung des Workflows an ihrer Hochschule oder Universität künftig eher von der Möglichkeit Gebrauch machen werden, OA zu publizieren, da die bisherigen Hürden in Form von (sehr) hohen Kosten oder eines (derzeit technisch) begrenzten Mehrwertes von Fachbüchern als OA-Veröffentlichung entfallen. Daneben stärkt das in diesem Projekt vorgestellte Konzept nach einem sukzessiven Transfer auf andere Hochschulen auch die Sichtbarkeit und Akzeptanz von OA als anerkannte und von Kollegen und Mitarbeitern dieser Einrichtungen unterstützte Form des offenen und freien Zugangs zu wissenschaftlichen Inhalten und Informationen. Diese Folgeeffekte wären in einem weiteren Projekt zu untersuchen und wissenschaftlich auszuwerten. Weiterhin wäre es zukünftig noch wünschenswert, ggfs. mit einem Drittanbieter eine technische Lösung zu entwickeln, die zur Indizierung von OA-Büchern oder einzelne Kapitel sowie für deren systematische, automatische Verknüpfung mit einer interdisziplinären Fachartikel-Datenbank geeignet ist. Letzteres würde für die Erhöhung der Sichtbarkeit und Auffindbarkeit der OA-Bücher sowohl im Sinne der Autoren, als auch der Rezipienten einen erheblichen Mehrwert darstellen. 


\section{Literaturverzeichnis}

1a-Studi (2019): Kostenbeispiele für Korrekturlesen \& Lektorat. URL: https://www.1a-studi.de/ korrekturlesen-lektorat-preise (Aufruf am 08.11.2019)

acadoo (2019): Wissenschaftliches Lektorat. URL: https://wissenschaftlicheslektorat.com/ (Aufruf am 08.11.2019)

Akademischeslektorat.com (2019): Wissenschaftliches Lektorat für Dissertation. URL: http:// www.akademischeslektorat.com/wissenschaftliches-lektorat-fuer-dissertation/ (Aufruf am 08.11.2019)

Albers, S. et al. (2007): Methodik der empirischen Forschung. Gabler Verlag, 2. Auflage, Wiesbaden. ISBN: 9783834904690

Allweyer, T. (2015): BPMN 2.0 - Business Process Model and Notation: Einführung in den Standard für die Geschäftsprozessmodellierung. BoD - Books on Demand, Norderstedt. ISBN: 9783738626711

Arbeitsgemeinschaft der Universitätsverlage (2019): Arbeitsgemeinschaft Universitätsverlage. Qualitätsstandards für Open-Access-Monografien und -Sammelbände. DOI: http://doi.org/10.5281/ zenodo.3562239

Bauer, K. (2019): Der Controller - Welche Hard- und Soft-Skills sollte er besitzen? URL: http:// www.maxcontrolling.de/blog/418-controller-faehigkeiten-soft-skills/ (Aufruf am 29.07.2019; 12:02 Uhr)

Berliner BPM-Offensive (2019): BPMNPoster. URL: http://www.bpmb.de/index.php/BPMNPoster (Aufruf am 15.01.2019)

Berliner Werkstatt Herstellung (2007): Standardprozesse der Herstellung: Berliner Werkstatt Herstellung - Ergebnisse 2007. Klopotek \& Partner, Berlin. URL: https://www.amazon.de/Standardprozesse-Herstellung-Berliner-Werkstatt-Ergebnisse/dp/3000227601

Bitrix, Inc. (1) (2019): Kommunikation. URL: https://www.bitrix24.de/tools/ (Aufruf am 05.11.2019)

Bitrix, Inc. (2) (2019): Preis für Cloud. URL: https://www.bitrix24.de/prices/ (Aufruf am 05.11.2019)

Börsenverein des Deutschen Buchhandels e.V. et al. (2018): Checkliste zur Barrierefreiheit von EPUB3-eBooks. Börsenverein des Deutschen Buchhandels e.V., IG Digital, Peergroup Produktion, Frankfurt. URL: https://www.igdigital.de/checkliste-barrierefreiheit-ebooks-v2/ (letzter Aufruf am 22.01.2020; 15:30 Uhr)

Buchhandel.de (2019): Über buchhandel.de und das VLB. URL: https://www.buchhandel.de/ueberdas-vlb (Aufruf am 30.10.2019)

Budapest Open Access Initiative (2019): German Translation Zehn Jahre nach der Open-Access-Initiative von Budapest: Den Standard auf "Offen" setzen. URL: https://www.budapestopenaccessinitiative.org/boai-10-translations/german-translation (Aufruf am 30.11. 2019)

Chan, L. (2004): Supporting and Enhancing Scholarship in the Digital Age: The Role of Open Access Institutional Repository. Canadian Journal of Communication 29 (3). DOI: https://doi.org/ $10.22230 /$ cjc. $2004 \mathrm{v} 29 \mathrm{n} 3 \mathrm{a} 1455$

Creative Commons (2019): CC Licenses and Examples. URL: https://creativecommons.org/shareyour-work/licensing-examples/ (Aufruf am 30.11. 2019) 
Crossref (2019): About. URL: https://www.crossref.org/ (Aufruf am 16.11.2019; 11:55 Uhr)

Deutsche Zentralbibliothek für Medizin (ZB MED) - Informationszentrum Lebenswissenschaften (2019): Peer Review: Warum ist es wichtig? URL: https://www.publisso.de/open-access-beraten/faqs/peer-review/ (Aufruf am 02.08.2019)

Different Solutions GmbH (2019): CRM Software für Profis. URL: https://www.vtiger.global/de/funktionen/ (Aufruf am 05.11.2019)

DOAB (2019): About. URL: http://www.doabooks.org (Aufruf am 16.11.2019; 14:51 Uhr)

Douglas, K. (1990): The Serials Crisis: Adjusting to Change. The Serials Librarian 18 (1-2): 111-21

Ernst, Th. (2015): Vom Urheber zur Crowd, vom Werk zur Version, vom Schutz zur Öffnung? Kollaboratives Schreiben und Bewerten in den Digital Humanities. In: Grenzen und Möglichkeiten der Digital Humanities. Hrsg.: C. Baum, Th. Stäcker: Sonderband der Zeitschrift für digitale Geisteswissenschaften 1, DOI: 10.17175/sb001_021

Ferwerda, E.; Snijder R.; Janneke, R.; Janneke, A. (2013): OAPEN-NL - A project exploring Open Access monograph publishing in the Netherlands, Final Report. URL: http://www.oapen.nl/ images/attachments/article/58/OAPEN-NL-final-report.pdf (letzter Aufruf am 21.01.2020; 13:31 Uhr)

Ferwerda, E.; Pinter F.; Stern, N. (2017): A landscape study on open access and monographs: Policies, funding and publishing in eight European countries. DOI: https://doi.org/10.5281/zenodo.815932

Fisher, J. H. (2008): Scholarly Publishing Re-Invented: Real Costs and Real Freedoms. The Journal of Electronic Publishing: JEP 11 (2). DOI: https://doi.org/10.3998/3336451.0011.204

Freie Universität Berlin (2019): Open Monograph Press. URL: https://www.cedis.fu-berlin.de/ services/e-publishing/e-books/omp/index.html (Aufruf am 04.12.2019; 16:20 Uhr)

Google (2019): Wie funktioniert der Verkauf von Büchern bei Google Play? URL: https:// support.google.com/books/partner/answer/1079107?hl=de (Aufruf am 08.11.2019; 14:46 Uhr)

Grammar Labs, LLC (2019): General Academic Editing Service. URL: https://americanmanuscripteditors.com/general_academic_editing.aspx (Aufruf am 08.11.2019)

Grimme, S.; Taylor, M.; Elliott, M. A.; Holland, C.; Potter, P.; Watkinson, C. (2016): The State of Open Monographs. DOI: https://doi.org/10.6084/m9.figshare.8197625 (Aufruf am 30.11. 2019)

Grossmann, A.; Brembs, B. (n.d.): Assessing the Size of the Affordability Problem in Scholarly Publishing. DOI: https://doi.org/10.7287/peerj.preprints.27809v1

Gründerszene (2019): Reporting. URL: https://www.gruenderszene.de/lexikon/begriffe/reporting? interstitial (Aufruf am 09.08.2019; 12:09 Uhr)

Hamann, H.; Hürlimann, D. (Hrsg.) (2018): Open Access in den Rechtswissenschaften. Tagung Frankfurt 2018. Paneldiskussion „Die Perspektive der Verlage“ in: Hanjo Hamann, Daniel Hürlimann (Hrsg.), Open Access in der Rechtswissenschaft, Sonderheft "Rechtswissenschaft". DOI: https://doi.org/10.5771/9783748903659

Harnad, S.; Brody, T.; F. Vallières; Carr, L.; Hitchcock, S.; Gingras, Y.; Oppenheim, C.; Stamerjohanns, H.; Hilf, E. R. (2004): The Green and the Gold Roads to Open Access. Nature Publishing Group. URL: https://www.nature.com/nature/focus/accessdebate/21.html

Hesse/Schrader (Hrsg.) (2019): Definition Soft Skills / Hard Skills. URL: https://www.berufsstrategie.de/bewerbung-karriere-soft-skills/soft-skills-definition-hard-skills.php (Aufruf am 12.12.2019; 15:40 Uhr) 
Hömberg, W. (2011): Lektor im Buchverlag: repräsentative Studie über einen unbekannten Kommunikationsberuf. Hömberg, W. \& Pypke, S., Konstanz, 2. Auflage 2011. ISBN: 9783867643382

Houghton, J.W. (2001): Crisis and Transition: The Economics of Scholarly Communication. Learned Publishing: Journal of the Association of Learned and Professional Society Publishers 14 (3): $167-76$

HTWK Leipzig (2018): Studienordnung für den Masterstudiengang Architektur an der Hochschule für Technik, Wirtschaft und Kultur Leipzig (StudO-ARM). URL: https://fas.htwk-leipzig.de/fileadmin/portal/htwk/studieren/1_unsere_studiengaenge/3_studien_pruefungsordnungen/ 2018-07-18_-_StudO_ARM_2018_onlinefassung.pdf (Aufruf am 19.07.2019; 14:35 Uhr)

HTWK Leipzig (1) (2019): Architektur. Studieninhalt. URL: https://fas.htwk-leipzig.de/architektur/ studiengaenge/architektur-master/ (Aufruf am 23.08.2019; 12:51 Uhr)

HTWK Leipzig (2) (2019): Wirtschaftsingenieurwesen Produktions- und Energiewirtschaft Bachelor. URL: https://www.htwk-leipzig.de/studieren/studiengaenge/auslaufende-studiengaenge/ wirtschaftsingenieurwesen-produktions-und-energiewirtschaft/ (Aufruf am 27.09.2019; 14:08 Uhr)

HTWK Leipzig (3) (2019): Bauingenieurwesen. URL: https://fb.htwk-leipzig.de/de/studium/studiengaenge/master-bauingenieurwesen/ (Aufruf am 25.10.2019; 14:45 Uhr)

HTWK Leipzig (4) (2019): Forschungsgrundsätze. URL: https://www.htwk-leipzig.de/forschen/ forschungsgrundsaetze/ (Aufruf am 11.11.2019; 13:18 Uhr)

HubSpot, Inc. (1) (2019): HubSpot CRM für die Vertriebsleitung. URL: https://www.hubspot.de/ products/crm (Aufruf am 05.11.2019)

HubSpot, Inc. (2) (2019): Marketing Hub. URL: https://www.hubspot.de/pricing/marketing?selectedPackage=professional\&edition=starter\&term=monthly (Aufruf am 05.11.2019)

Indigo Stream Technologies (2019): Copyscape Premium. The web's most powerful plagiarism search. URL: https://www.copyscape.com/premium.php (Aufruf am 29.10.2019)

Jacob, P. (2018): The Optimum Production Workflow of Open Access Books with Focus on Impact and Cost Structure. Bachelorarbeit. Hochschule für Technik, Wirtschaft und Kultur Leipzig, Fachbereich Medien, Bachelorstudiengang Buch- und Medienproduktion, Leipzig

Jahn, N.; Tullney, M. (2016): Data and Code Used from: A Study of Institutional Spending on Open Access Publication Fees in Germany. DOI: 10.4119/unibi/2905588

Jones, E. A.; Courant, P. N. (2014): Monographic Purchasing Trends in Academic Libraries: Did the "Serials Crisis' Really Destroy the University Press?” Journal of Scholarly Publishing. DOI: https://doi.org/10.3138/jsp.46.1.003

Journal of Pharmacology \& Pharmacotherapeutics (2010): Uniform requirements for manuscripts submitted to biomedical journals: Writing and editing for biomedical publication. Journal of Pharmacology \& Pharmacotherapeutics 1, (1) 42

Klopotek AG (2019): Produktübersicht. URL: https://www.klopotek.de/de/software/classic-line/ uebersicht.html (Aufruf am 05.11.2019)

knk Business Software AG (2019): knkVerlag für Buchverlage. URL: https://knkverlagssoftware.com/knk-business-software-ag-knkverlag/publikums-und-medienverlage/ (Aufruf am 05.11.2019)

KNV Zeitfracht (2019): KNV Zeitfracht Barsortiment. URL: http://www.knv-zeitfracht.de/barsortiment/ (Aufruf am 04.11.2019) 
Kuntscher, F. (2019): Preise für Korrektorat und Lektorat. URL: https://www.uni-korrektorat.de/ lektorat-preise/ (Aufruf am 08.11.2019)

lektorat.de (2019): Das Fachlektorat. URL: http://www.lektorat.de/Lektorat/Fachlektorat (Aufruf am 12.08.2019; 13:51 Uhr)

Lektorat Frankfurt (2019): Professionelles Lektorat zu fairen Konditionen. URL: https://www.lektorat-frankfurt.org/preise.html (Aufruf am 08.11.2019)

Lektorat Korrektorius (2019): Preise Lektorat Korrektorius. URL: https://www.korrektorius.de/preise-lektorat/ (Aufruf am 08.11.2019)

Lesen.net (2019): Startup unglue.it: Vom Buchbefreier zum Buchverkäufer. URL: https:// www.lesen.net/ebook-news/startup-unglue-it-vom-buchbefreier-zum-buchverkaeufer-10234/ (Aufruf am 30.10.2019)

Libri GmbH (2019): About. URL: https://www.libri.de/de/ (Aufruf am 04.11.2019)

Lucid Software Inc. (2019): Symbole und Notation von BPMN-Diagrammen. URL: https:// www.lucidchart.com/pages/de/symbole-und-notation-BPMN\#discovery__top (Aufruf am 13.11.2019; 11:22 Uhr)

Luhmann, N. et al. (2008): Einführung in die Systemtheorie. Carl-Auer-Verlag, Heidelberg. ISBN: 9783896704597

manager magazin (2019): Zehn Anforderungen an Projektmanager. URL: https://www.managermagazin.de/fotostrecke/fotostrecke-50532-10.html (Aufruf am 29.07.2019; 11:58 Uhr)

Maron, N.; Schmelzinger, K.; Mulhern, C.; Rossman, D. (2016): The Costs of Publishing Monographs: Toward a Transparent Methodology.” The Journal of Electronic Publishing. DOI: https://doi.org/10.3998/3336451.0019.103

Max-Planck-Gesellschaft (2019): Berlin Declaration on Open Access to Knowledge in the Sciences and Humanities. URL: https://openaccess.mpg.de/Berliner-Erklaerung (Aufruf am 16.11.2019; 19:47 Uhr)

McKiernan, E. C. et al. (2016): How open science helps researchers succeed. PubMed. DOI: 10.7554/eLife. 16800

Natali Reindl Lektorat (2019): Kosten und Ablauf. URL: http://www.reindl-lektorat.de/kosten-ablauf.html (Aufruf am 08.11.2019)

Newbooks Solutions GmbH (2019): About. URL: https://www.newbooks-solutions.com/de/ index.html\#about-us (Aufruf am 30.10.2019)

Nordhoff, S. (2018): Cookbook for Open Access books. Language Science Press, Berlin, DOI: 10.5281/zenodo. 1286925

NY Book Editors (2019): What's the Difference Between Copyediting and Proofreading? URL: https://nybookeditors.com/2016/05/whats-the-difference-between-copyediting-and-proofreading/ (Aufruf am 29.07.2019; 11:45 Uhr)

OAPEN (2019): Welcome to OAPEN. online library and publication platform. URL: https:// www.oapen.org/home (letzter Aufruf am 22.01.2020; 09:50 Uhr)

Ojennus, P. (2017): Open access and the humanities - The case of classics journals. Library Resources \& Technical Services 61(2), 81-92

Olijhoek, T. et al. (2015): Criteria for open access and publishing. ScienceOpen Research, DOI: 10.14293/S2199-1006.1.SOR-EDU.AMHUHV.v1 
OAPEN (2019): Welcome to OAPEN. URL: http://www.oapen.org/home (Aufruf am 16.11.2019; 11:33 Uhr)

Publishers Communication Group (2015): Open Access Monographs - Summary Report of Online Survey. Publishers Communication Group. URL: http://www.pcgplus.com/wp-content/uploads/2013/03/Open-Access-Monographs.pdf (letzter Aufruf am 22.01.2020; 09:07 Uhr)

PlagAware (1) (2019): Über PlagAware. URL: https://www.plagaware.com/de/info (Aufruf am 29.10.2019)

PlagAware (2) (2019): Leistungsmerkmale der Lizenzmodelle von PlagAware. URL: https:// www.plagaware.com/de/modelle-uebersicht (Aufruf am 29.10.2019)

PlagScan (1) (2019): Organisationsverwaltung. URL: https://www.plagscan.com/de/organisationsverwaltung (Aufruf am 29.10.2019)

PlagScan (2) (2019): Preise für Hochschulen. URL: https://www.plagscan.com/de/preise-fuer-hochschulen (Aufruf am 29.10.2019)

Proof-Reading-Service.com (2019): Prices. URL: https://www.proof-reading-service.com/en/prices/ (Aufruf am 08.11.2019)

PubMed Central (2019): About. URL: https://www.ncbi.nlm.nih.gov/pmc/ (Aufruf am 16.11.2019; 11:21 Uhr)

Qucosa (2019): Willkommen auf Qucosa ${ }^{\circledR}$, dem sächsischen Dokumenten- und Publikationsserver. URL: http://www.qucosa.de/startseite/ (Aufruf am 29.10.2019)

Reimus.net GmbH (2019): Vollständige Ermittlung von Personalkosten. URL: https://www.controllingportal.de/Fachinfo/Kostenrechnung/Vollstaendige-Ermittlung-von-Personalkosten.html (Aufruf am 26.11.2019; 09:50 Uhr)

ResearchGate (2019): About. URL: https://www.researchgate.net/about (Aufruf am 29.10.2019)

Research Square (2019): Pricing. URL: https://www.aje.com/pricing/\#!/pricing (Aufruf am 08.11.2019)

Rose-Wiles, L. M. (2011): The High Cost of Science Journals: A Case Study and Discussion. Journal of Electronic Resources Librarianship. DOI: https://doi.org/10.1080/1941126x.2011.601225

Ross-Hellauer, T. et al. (2017): What is open peer review? A systematic review. F1000Research 6, 588

Ryte (2019): Google Books. URL: https://de.ryte.com/wiki/Google_Books (Aufruf am 08.11.2019; 14:46 Uhr)

salesforce.com Germany GmbH (1) (2019): Was ist Salesforce? URL: https:// www.salesforce.com/de/cro/what-is-salesforce/?r=https\%3A\%2F\%2Fwww.salesforce.com \%2Fde\%2Fproducts\%2Fwhat-is-salesforce\%2F\&d=cta-body-promo-72 (Aufruf am 05.11.2019)

salesforce.com Germany GmbH (2) (2019): Sales Cloud - Preise. URL: https:// www.salesforce.com/de/editions-pricing/sales-cloud/ (Aufruf am 05.11.2019)

Schüller-Zwierlein, A. (2019): Übersicht über die Universitätsverlage in Deutschland (Stand: Herbst 2019). Nicht veröffentlicht; private Mitteilung

Schönstedt, E.; Breyer-Mayländer, T. (2010): Der Buchverlag: Geschichte, Aufbau, Wirtschaftsprinzipien, Kalkulation und Marketing. Springer-Verlag GmbH Deutschland, 3. Auflage, Stuttgart. ISBN: 9783476022585 
Schrader, A. (2019): Follow-Up-Stakeholder-Workshop - Open-Access-Publikationsworkflow für Bücher in Leipzig. URL: https://fim.htwk-leipzig.de/forschung/forschungsthemen/open-access-hochschulverlag/detailseite-oa-hs-news/artikel/2154/ (Aufruf am 03.12.2019)

Schweizerischer Nationalfonds (SNF) (2018): About. URL: http://www.snf.ch/de/Seiten/default.aspx (Aufruf am 15.7.2018; 10:55 Uhr)

ScienceOpen (1) (2019): ScienceOpen Collections. URL: https://about.scienceopen.com/collections/ (Aufruf am 06.12.2019; 13:51 Uhr)

ScienceOpen (2) (2019): ScienceOpen: An interactive discovery environment. URL: https:// about.scienceopen.com/ (Aufruf am 30.10.2019)

Scribbr B.V. (1) (2019): Lektorat \& Korrekturlesen. URL: https://www.scribbr.de/lektorat-korrekturlesen/deutsche-abschlussarbeit/ (Aufruf am 08.11.2019)

Scribbr B.V. (2) (2019): Gib Plagiat keine Chance. URL: https://www.scribbr.de/plagiatspruefung/ (Aufruf am 29.10.2019)

SiteFusion GmbH (2019): Best-of-Breed: moderne Software-Entwicklung. URL: https://www.sitefusion.de/produkt/ (Aufruf am 05.11.2019)

Snijder, R. (2013): A higher impact for open access monographs: disseminating through OAPEN and DOAB at AUP. Insights 26, 55-59

Snijder, R. (2014): The Influence of Open Access on Monograph Sales. Logos. DOI: https://doi.org/ $10.1163 / 1878-4712-11112047$

Solomon, D.; Björk, B. (2016): Article processing charges for open access publication. PeerJ 4:e2264. DOI: $10.7717 /$ peerj.2264

Sperr Jr., E. V. (2006): Libraries and the Future of Scholarly Communication. Molecular Cancer 5 (November): 58.

StrikePlagiarism.com (1) (2019): Our services and prices. URL: https://strikeplagiarism.com/en/ pricing\%20and\%20services.html (Aufruf am 29.10.2019)

StrikePlagiarism.com (2) (2019): About us. URL: https://strikeplagiarism.com/en/ (Aufruf am 29.10.2019)

studi-lektor.de (2019): Wir prüfen Ihre Bachelorarbeit. URL: https://studi-lektor.de/lektorat/bachelorarbeit-lektorat.html (Aufruf am 08.11.2019)

Suber, P. (2012): Open Access. The MIT Press, Boston, ISBN: 9780262517638

Tananbaum, G. (2003): Of Wolves and and Boys: The Scholarly Communication Crisis. Learned Publishing: Journal of the Association of Learned and Professional Society Publishers 16 (4): 285-89

Taubert, N.; Schön, K. (2015): Online-consultation "scientific publication system": documentation and main results. ScienceOpen Research, DOI: 10.14293/S2199-1006.1.SOR-SOCSCI.AE2GYG.v1

Tennant, J. et al. (2016): The academic, economic and societal impacts of Open Access. An evidence-based re-view. F1000Research 5, 632

The Library of Congress (2019): BITS (Book Interchange Tag Suite), version 2.0. URL: https:// www.loc.gov/preservation/digital/formats/fdd/fdd000453.shtml (Aufruf am 17.11.2019; 11:20 Uhr)

Transcript (2019): Publikationsanfrage: Monographie. URL: https://www.transcript-verlag.de/ publikationsanfrage-monographie (Aufruf am 30.07.2019; 09:06 Uhr) 
Turnitin, LLC. (2019): Find the Package Right for You and Publish With Confidence. URL: http:// www.ithenticate.com/products (Aufruf am 29.10.2019)

Unglue.it (2019): Frequently Asked Questions. URL: https:/unglue.it/faq/ (Aufruf am 08.11.2019; 14:29 Uhr)

Urkund (2019): Plagiate an Ihrer Universität lassen sich verhindern. URL: https:// www.urkund.com/de/services/higher-education/ (Aufruf am 29.10.2019)

von Foerster, H. (1993): Wissen und Gewissen. Versuch einer Brücke. Suhrkamp, Frankfurt am Main. ISBN: 3518284762; 9783518284766

Ware, M.; Mabe, M. (2015): The STM Report, 4th edition. International Association of Scientific, Technical and Medical Publishers, Den Haag, URL: https://www.stm-assoc.org/ 2015_02_20_STM_Report_2015.pdf (letzter Aufruf am 21.01.2020; 13:22 Uhr)

Wicherts, J. M. (2016): Peer Review Quality and Transparency of the Peer-Review Process in Open Access and Subscription Journals. PLoS ONE 11(1) e0147913, DOI: 10.1371/journal.pone. 0147913

Wikipedia (1) (2019): Ingenieurwissenschaften. URL: https://de.wikipedia.org/wiki/Ingenieurwissenschaften (Aufruf am 13.08.2019; 11:19 Uhr)

Wikipedia (2) (2019): Medienwissenschaft. URL: https://de.wikipedia.org/wiki/Medienwissenschaft (Aufruf am 16.08.2019; 14:22 Uhr)

Wikipedia (3) (2019): Crossref. URL: https://de.wikipedia.org/wiki/Crossref\#cite_note-4 (Aufruf: 05.12.2019; 13:28 Uhr)

Wissenschaftslektorat Zimmermann (2019): Honorarübersicht - unsere Leistungen. URL: https:// lektorat-zimmermann.de/fee.php (Aufruf am 08.11.2019)

Xpublisher GmbH (2019): Features. URL: https://www.xpublisher.com/de/produkt/uebersicht-29 (Aufruf am 04.11.2019)

Zoho Corporation Pvt. Ltd. (1) (2019): Funktionen, die den- verkauf erleichtern. URL: https:// www.zoho.com/de/crm/features.html (Aufruf am 05.11.2019)

Zoho Corporation Pvt. Ltd. (2) (2019): 15-tägige kostenlose Testversion. Keine Kreditkarte erforderlich. URL: https://www.zoho.com/de/crm/zohocrm-pricing.html (Aufruf am 05.11.2019) 

Anhang 



\section{Anhang A \\ Qualitätsrichtlinien}

Es besteht kein Anspruch auf Vollständigkeit.

\section{A.1 Qualitätsrichtlinien für inhaltsorientierte Prozesse}

Tab. 37 Richtlinie für die Konzept-Annahme

I-Ka-110: Publikationsanfrage sichten

I-Ka-200: Nach geeigneten Content-Urhebern recherchieren

\begin{tabular}{|c|c|c|}
\hline projektspezifisch: & $\begin{array}{l}\checkmark \\
\checkmark\end{array}$ & $\begin{array}{l}\text { Die Arbeiten haben eine schriftliche Benotung von 1,0 bis 1,3 erhalten } \\
\text { (sofern die Note weniger gut ist, werden auch Empfehlungen der } \\
\text { Hochschulprofessoren berücksichtigt; etwa dann, wenn nur } \\
\text { aufgrund von formalen Fehlern keine 1,0 gegeben werden konnte). } \\
\text { Es sind aktuelle Arbeiten, die nicht älter als drei Jahre sind, d. h., aus } \\
\text { den Jahren } 2016 \text { bis } 2019 \text { stammen. } \\
\text { Die Arbeiten haben keinen Sperrvermerk und können im OA } \\
\text { veröffentlicht werden. }\end{array}$ \\
\hline weitere: & $\begin{array}{l}\checkmark \\
\checkmark \\
\checkmark\end{array}$ & $\begin{array}{l}\text { Das Manuskript passt ins Verlagsprogramm. } \\
\text { Die Forschungsfrage stellt ein Alleinstellungsmerkmal dar (weist } \\
\text { Originalität auf). } \\
\text { Note der Abschlussarbeit } \\
\text { Die Arbeit wurde ausgezeichnet. } \\
\text { Das Manuskript liegt im geforderten Format vor (Word, LaTex, } \\
\text { InDesign oder PDF). } \\
\text { Es wurde das ganze Manuskript (Arbeitsfassung) eingereicht oder - } \\
\text { falls noch nicht vorliegend - ein aussagefähiges Konzept (Exposé, } \\
\text { Inhalt/Gliederung, vorhandenes Textmaterial). } \\
\text { Es wurden die gewünschten persönlichen Daten eingereicht, sowie } \\
\text { Angaben zum Umfang (Gesamtzeichenzahl, inkl. Leerzeichen) und } \\
\text { zur Bebilderung (Anzahl der geplanten Fotos/Diagramme/Tabellen) } \\
\text { gemacht. }{ }^{177}\end{array}$ \\
\hline
\end{tabular}

177 vgl. Transcript 2019 
Tab. 38 Richtlinie für die Content-Vereinnahmung

I-Cv-110: Content prüfen

\begin{tabular}{|c|c|c|}
\hline projektspezifisch: & $\begin{array}{l}\checkmark \\
\checkmark \\
\checkmark \\
\checkmark \\
\checkmark\end{array}$ & $\begin{array}{l}\text { Das Manuskript ist vollständig. Alle Kapitel sind entsprechend des } \\
\text { Inhaltsverzeichnisses vorhanden. } \\
\text { Der Umfang des Manuskripts ist ausreichend, um als gedrucktes Buch } \\
\text { publiziert zu werden. } \\
\text { Der Inhalt kann im OA veröffentlicht werden. } \\
\text { Das Manuskript weist verschiedene Inhaltselemente auf } \\
\text { (Abbildungen, Tabellen, math. Formeln, Quellcode etc.). } \\
\text { Das Manuskript ist geeignet, um eine bestimmte Workflow-Variante } \\
\text { zu demonstrieren. }\end{array}$ \\
\hline weitere: & $\begin{array}{l}\checkmark \\
\checkmark \\
\checkmark \\
\checkmark \\
\checkmark \\
\checkmark \\
\checkmark\end{array}$ & $\begin{array}{l}\text { Das Manuskript bzw. das Konzept passt tatsächlich in das } \\
\text { Verlagsprogramm. } \\
\text { Das Manuskript weist mit der Forschungsfrage tatsächlich ein } \\
\text { Alleinstellungsmerkmal auf. } \\
\text { Das vorgegebene Gestaltungstemplate wurde korrekt angewandt. } \\
\text { Alle Abbildungen liegen in Druckqualität vor. } \\
\text { Alle Abbildungen sind als einzelne Dateien vorhanden bzw. der } \\
\text { Content-Urheber kann diese nachreichen. } \\
\text { Die Rechte aller Inhaltselemente sind geklärt. } \\
\text { Das Manuskript enthält ein Stichwortregister oder andere } \\
\text { gewünschte Inhalte. } \\
\text { Es wurden die Vorgaben der DZB zur Wahrung der Barrierefreiheit für } \\
\text { E-Books eingehalten: } \\
\text { - Das Manuskript enthält Alternativtexte für Abbildungen und } \\
\quad \text { Grafiken. } \\
\text { - Für komplexere Grafiken, z. B. Diagramme oder schematische } \\
\text { Darstellungen, wurden ausführliche Beschreibungen verfasst. } \\
\text { - Informationen werden nicht nur über visuelle Merkmale wie } \\
\quad \text { Farben, Formen oder Positionen vermittelt (es sollte nicht } \\
\text { heißen „,der rote Balken im Diagramm zeigt ...“ oder „der } \\
\quad \text { Infokasten mit der grünen Umrandung erklärt ...“). }\end{array}$ \\
\hline
\end{tabular}


Tab. 39 Richtlinie für die Plagiatsprüfung

I-Qe-100: Plagiatsprüfung durchführen

projektspezifisch: $\quad \checkmark \quad$ Annahme: Es liegt kein Fehlverhalten entsprechend der Regeln guter wissenschaftliche Praxis der HTWK Leipzig vor. ${ }^{178}$

$\checkmark \quad$ Ablehnung: Es liegt Fehlverhalten entsprechend der Regeln guter wissenschaftliche Praxis der HTWK Leipzig vor.

weitere: $\quad \checkmark \quad$ Regeln guter wissenschaftlicher Praxis der eigenen Institution

Tab. 40 Richtlinie für die Wahl des Peer-Review-Verfahrens

I-Qe-110: Peer-Review-Verfahren auswählen

\begin{tabular}{lll}
\hline projektspezifisch: & $\checkmark$ & noch keine definiert \\
\hline weitere: & $\checkmark$ & institutionelle Vorgaben \\
& $\checkmark$ & Verfügbarkeit von Gutachtern \\
& $\checkmark$ & Vorgaben der Gutachter \\
\hline
\end{tabular}

Tab. 41 Richtlinie für die Evaluation der Publikationsfähigkeit

I-Qe-200: Checkliste zu Content einfordern

\begin{tabular}{lll}
\hline projektspezifisch: & $\checkmark$ & Checkliste für die Publikationsfähigkeit des Werkes (vgl. Anhang B) \\
\hline weitere: & $\checkmark$ & Regeln guter wissenschaftlicher Praxis der eigenen Institution \\
& $\checkmark$ & Das Manuskript bzw. das Konzept passt tatsächlich in das \\
& $\checkmark$ & Verlagsprogramm. \\
& & Das Manuskript weist mit der Forschungsfrage tatsächlich ein \\
& Alleinstellungsmerkmal auf.
\end{tabular}


Tab. 42 Richtlinie für die Publikationsannahme

I-Ca-100: Gutachten bzw. Checkliste sichten

\begin{tabular}{|c|c|c|}
\hline projektspezifisch: & $\checkmark$ & $\begin{array}{l}\text { Annahme: Die Gutachten und Checklisten stellen eindeutige } \\
\text { Empfehlungen dar, den Content in der vorliegenden Form zu } \\
\text { publizieren. } \\
\text { Überarbeitung des Content einfordern: Aus Gutachten und } \\
\text { Checklisten gehen hervor, dass der Content grundsätzlich } \\
\text { publikationsfähig ist, aber noch weiteres Potenzial zur Überarbeitung } \\
\text { besteht. Die Annahme des Contents zur Publikation erfolgt somit nur } \\
\text { unter Einhaltung bestimmter Bedingungen. } \\
\text { Ablehnung: Aus den Gutachten und Checklisten gehen entweder } \\
\text { eindeutig hervor, dass der Content in der vorliegenden Form nicht } \\
\text { publikationsfähig ist, oder dass die Richtlinie für die Content- } \\
\text { Vereinahmung doch nicht erfüllt wurde. }\end{array}$ \\
\hline weitere: & $\checkmark$ & Annahme: Prestigeträchtiger Content \\
\hline
\end{tabular}

\section{A.2 Qualitätsrichtlinien für produktionsorientierte Prozesse}

Tab. 43 Richtlinie für das Fachkorrektorat

P-Ca-100: Fachkorrektorat beauftragen

\begin{tabular}{lll}
\hline projektspezifisch: & $\checkmark$ & Gutachter haben Fachkorrektorat empfohlen \\
\hline weitere: & $\checkmark$ & Content-Urheber oder -Lieferant haben Fachkorrektorat empfohlen \\
& $\checkmark$ & institutionelle Vorgaben \\
\hline
\end{tabular}

Tab. 44 Richtlinie für das Sprachlektorat

P-Ca-110: Sprachlektorat beauftragen

\begin{tabular}{|c|c|c|}
\hline projektspezifisch: & $\begin{array}{l}\checkmark \\
\checkmark \\
\checkmark\end{array}$ & $\begin{array}{l}\text { Der Content wurde von einem Nicht-Muttersprachler geschrieben. } \\
\text { Die Gutachter empfehlen die Durchführung eines Sprachlektorats am } \\
\text { Content. } \\
\text { Aus den Gutachten bzw. Checklisten geht hervor, dass die } \\
\text { Durchführung eines Sprachlektorats sinnvoll wäre. }\end{array}$ \\
\hline weitere: & $\checkmark$ & $\begin{array}{l}\text { Content-Urheber oder -Lieferant empfehlen ein sprachliches Copy } \\
\text { Editing } \\
\text { institutionelle Vorgaben }\end{array}$ \\
\hline
\end{tabular}


Tab. 45 Richtlinie für das technische Copy Editing

P-Ca-120: Technisches Copy Editing durchführen

\begin{tabular}{lll}
\hline projektspezifisch: & $\checkmark$ & $\begin{array}{l}\text { Überprüfung der Mikrotypografie (korrekte Anführungszeichen und } \\
\text { Gedankenstriche, keine doppelten Leerzeichen, und ähnliches) }\end{array}$ \\
& $\checkmark$ & $\begin{array}{l}\text { Überprüfung der Druckqualität von Abbildungen, Grafiken und Fotos } \\
\text { sowie Tabellen }\end{array}$ \\
$\checkmark$ & $\begin{array}{l}\text { Überprüfung hinsichtlich der Konsistenz von Textelementen, wie } \\
\text { Abbildungs- oder Tabellenüber/unterschriften und hinsichtlich einer } \\
\text { einheitlichen Überschriftennummerierung }\end{array}$ \\
weitere: & $\checkmark$ & Anspruch des Hochschulverlages an Mikrotypografie
\end{tabular}

Tab. 46 Cover- und Umschlag-Gestaltungsrichtlinie

P-CUe-100: Cover erstellen

P-CUe-110: Umschlag erstellen
projektspezifisch: $\quad \checkmark \quad$ vgl. Style guide (veröffentlicht unter http://doi.org/10.14293/ S1111.000/000002.v1)
$\checkmark \quad$ außer für Content von Fall 1: ästhetisch ansprechend und zum Inhalt passend

weitere:

Für Cover:

$\checkmark$ ästhetisch ansprechend

$\checkmark$ bestimmte Wiedererkennungsmerkmale des Hochschulverlages sind abgebildet

$\checkmark$ eine Fotografie muss enthalten sein

$\checkmark$ nur in schwarz/weiß, duplex oder farbig (Prozessfarben)

Für Umschlag:
$\checkmark$ Hinweise zur Reihe
$\checkmark$ Werbung
$\checkmark$ Abstract
$\checkmark$ Kurzbiografie zum Autor
$\checkmark$ Titel des Werkes
$\checkmark$ Autorenname
$\checkmark$ Hochschullogo 
Tab. 47 Richtlinie für die Produktfreigabe

P-CUe-120: Freigabe für Cover bzw. Umschlag einholen

P-CiP-120: Qualität des Produktionsformats sichern

P-CiP-140: Gewünschte Ausgabeformate virtuell prüfen

projektspezifisch: $\quad \checkmark \quad$ Richtlinie für die Produktfreigabe (vgl. Anhang B)

weitere: $\quad \checkmark$ Prüfrichtlinien für HTML oder andere Ausgabeformate

Tab. 48 Richtlinie für die Produktumsetzung

P-CiP-100: Content-Eingangsformat in Produktionsformat überführen

P-CiP-110: Produktionsformat mit Metadaten anreichern

P-CiP-120: Qualität des Produktionsformats sichern

\begin{tabular}{lll}
\hline projektspezifisch: & $\checkmark$ & Richtlinie zur Qualitätssicherung des Produktionsformats (wird an \\
& & anderer Stelle veröffentlicht) \\
& $\checkmark$ & Richtlinie für die Barrierefreiheit beim EPUB (vgl. Anhang B) \\
& $\checkmark$ & Style guide des Forschungsprojektes \\
\hline weitere: & $\checkmark$ & institutionelle Vorgaben \\
& $\checkmark$ & grundsätzliche Richtlinien zur Einhaltung anderer DTD-Standards \\
& $\checkmark$ & Vorgaben der DZB zur Wahrung der Barrierefreiheit \\
& $\checkmark$ & institutionelle Vorgaben zur Wahrung der Barrierefreiheit \\
& $\checkmark$ & Richtlinie zur Überprüfung von CRC-Dokumenten
\end{tabular}

Tab. 49 Richtlinie für gedruckte Produkte

P-GP-110: Produkt physisch prüfen

P-CiP-180: Ausgabeformate zur Veröffentlichung freigeben

\begin{tabular}{lll}
\hline projektspezifisch: & $\checkmark$ & Richtlinie für gedruckte Produkte (vgl. Anhang B) \\
\hline weitere: & $\checkmark$ & institutionelle Vorgaben
\end{tabular}


Tab. 50 Richtlinie für Pflichtexemplare

P-GP-130: Pflicht- und Vertragsexemplare versenden

\begin{tabular}{lll}
\hline projektspezifisch: & $\checkmark$ & $\begin{array}{l}\text { gesetzliche Pflichtexemplarregelung (Pflichtablieferungsverordnung } \\
(\text { PflAV)) bzw. das Gesetz über die Deutsche Nationalbibliothek }\end{array}$ \\
& $\checkmark \begin{array}{l}\text { (DNBG) } \\
\text { gesetzliche Pflichtexemplarregelung des sächsischen Gesetzes über } \\
\text { die Presse (SächsPresseG) }\end{array}$ \\
& $\checkmark \quad \begin{array}{l}\text { gesetzliche Pflichtexemplarregelungen anderer Bundesländer oder } \\
\text { Staaten } \\
\text { weitere: }\end{array}$ \\
& $\checkmark$ & institutionelle Vorgaben \\
\hline
\end{tabular}

Tab. 51 Richtlinie für die Wahl der primären OA-Veröffentlichungsplattform

P-EP-100: Produkt auf primärer Veröffentlichungsplattform veröffentlichen

\begin{tabular}{|c|c|}
\hline projektspezifisch: & 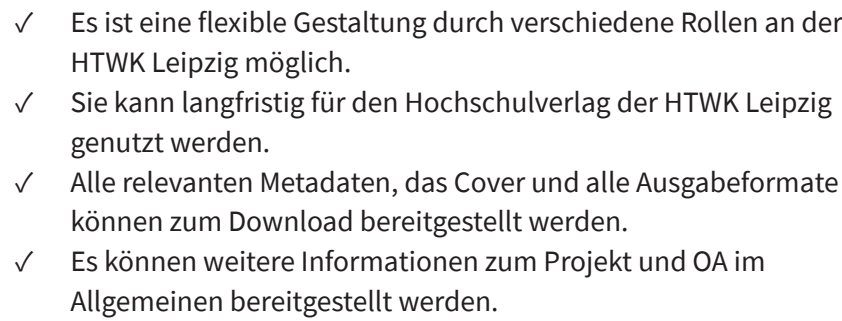 \\
\hline weitere: & $\begin{array}{ll}\checkmark & \text { institutionelle Vorgaben } \\
\checkmark & \text { Verfügbarkeit, Zugriff }\end{array}$ \\
\hline
\end{tabular}

Tab. 52 Richtlinie für die Wahl weiterer Veröffentlichungsplattformen

P-EP-110: Produkt auf weiteren Plattformen veröffentlichen

\begin{tabular}{lll}
\hline projektspezifisch: & $\checkmark$ & Plattformen, die bei der Auffindbarkeit und Sichtbarkeit der Produkte \\
& helfen \\
& $\checkmark$ & Plattform, wo kostenlos oder preisgünstig OA-Bücher veröffentlicht \\
& werden können \\
& $\checkmark$ & Plattformen mit einer guten Reputation in der OA-Community bzw. in \\
& der Wissenschaftscommunity im Allgemeinen oder in \\
& fachspezifischen Community \\
& $\checkmark$ & mind. OAPEN/DOAB \\
weitere: & $\checkmark$ & institutionelle Vorgaben \\
& $\checkmark$ & Verfügbarkeit, Zugriff
\end{tabular}


Tab. 53 Richtlinie für die Wahl des Langzeitarchivs

P-PFs-100: Für Langzeitarchivierung des Produktes sorgen

\begin{tabular}{lll}
\hline projektspezifisch: & $\checkmark$ & Verfügbarkeit, Zugriff \\
& $\checkmark$ & Finanzierbarkeit im Rahmen des Projektes \\
\hline weitere: & $\checkmark$ & institutionelle Vorgaben \\
& $\checkmark$ & Finanzierbarkeit durch den Hochschulverlag
\end{tabular}

\section{A.3 Qualitätsrichtlinien für managementorientierte Prozesse}

Tab. 54 Richtlinie für den Vertragsabschluss \& die Buchvorankündigung

M-PVe-200: Vertragsabschluss \& Buchvorankündigung vorbereiten

projektspezifisch: $\quad \checkmark \quad$ Autoren-Checkliste für die benötigten Informationen zum

Vertragsabschluss (vgl. Anhang B)

weitere: $\quad \checkmark \quad$ weitere wichtige Informationen zum Content oder Content-Urheber

Tab. 55 Richtlinie für die Vertragsunterzeichnung

M-PVe-210: Publikationsvertrag schließen

\begin{tabular}{lll}
\hline projektspezifisch: & $\checkmark$ & Mustervertrag für Hochschulpublikationen (vgl. Anhang B) \\
& $\checkmark$ & Einverständniserklärung für Qucosa \\
\hline weitere: & $\checkmark$ & eigener Mustervertrag \\
& $\checkmark$ & eigene Einverständniserklärung für Publikationsserver
\end{tabular}

Tab. 56 Richtlinie für die Wahl des Produktionswegs

M-Pv-100: Produktionsweg festlegen

\begin{tabular}{lll}
\hline projektspezifisch: & $\checkmark$ & muss dem Zweck der Fallstudie/dem Projekt dienen \\
\hline weitere: & $\checkmark$ & medienneutrale Umsetzbarkeit des Contents ist möglich \\
& $\checkmark$ & Wunsch des Content-Urhebers oder -Lieferanten \\
& $\checkmark$ & Finanzierung \\
& $\checkmark$ & Partnerschaften mit Dienstleistern \\
& $\checkmark$ & Promotionsordnung oder andere institutionelle Vorgaben \\
\hline
\end{tabular}


Tab. 57 Richtlinie für die Bildung von DOIs

M-Pv-200: Stammdaten pflegen

\begin{tabular}{lll}
\hline projektspezifisch: & $\checkmark$ & Richtlinie für die Bildung von DOIs (vgl. Anhang B) \\
\hline weitere: & $\checkmark$ & eigene Richtlinie \\
& $\checkmark$ & Budget
\end{tabular}

Tab. 58 Richtlinie zur Preisstrategie

M-Pv-320: Ladenpreis für gedrucktes Produkt festlegen

projektspezifisch: $\quad \checkmark \quad$ Ladenpreis-Kalkulation (vgl. Anhang C)

weitere: $\quad \checkmark \quad$ institutionelle Vorgaben, wie Festlegung der maximalen Preishöhe

Tab. 59 Richtlinie für die Erstellung von Metadaten

M-MM-100: Metadatensatz erzeugen

\begin{tabular}{lll}
\hline projektspezifisch: & $\checkmark$ & Richtlinie für die Erstellung von Metadaten (vgl. Anhang B) \\
\hline weitere: & $\checkmark$ & $\begin{array}{l}\text { eigener Qualitätsanspruch an Metadaten bzw. internes } \\
\text { Metadatenformat }\end{array}$
\end{tabular}

Tab. 60 Richtlinie für die Verbreitung von Metadaten

M-MM-100: Metadaten distribuieren

\begin{tabular}{ll}
\hline projektspezifisch: & Die Metadaten aller Ausgabeformate der Publikationen sollen mind. an \\
& die in Kapitel 4.2.4 genannten Metadaten-Aggregatoren übermittelt \\
& werden: \\
& $\checkmark \quad$ Crossref \\
& $\checkmark \quad$ VLB \\
& $\checkmark \quad$ Newbooks \\
& $\checkmark \quad$ KNV Zeitfracht \\
& $\checkmark \quad$ Libri \\
\hline weitere: & $\checkmark \quad$ institutionelle Vorgaben \\
& $\checkmark \quad$ Repositorien/Fachrepositorien \\
\hline
\end{tabular}


Tab. 61 Richtlinie zur internen Archivierung

M-Pn-100: Verwaltungsdaten sichern

P-PFs-200: Ausgabeformate systematisiert ablegen

projektspezifisch: $\quad \checkmark$ Vorgaben der Hochschulbibliothek zur Gewährleistung des Wissentransfers

weitere: $\quad \checkmark$ Ordner-bzw. Cloudstruktur

$\checkmark$ institutionelle Vorgaben 


\section{Anhang B \\ Checklisten}




\section{Checkliste}

\section{für die Publikationsfähigkeit des Werkes}

Bitte senden Sie uns die ausgefüllte Checkliste elektronisch

an redaktion@oa-hverlag.de und zusätzlich ausgedruckt und unterschrieben zu.

Gutachter/-in:

Kontakt: Wie können wir Sie bei Nachfragen oder Unklarheiten erreichen? (Tel./E-Mail)

Angaben zur Publikation

\begin{tabular}{c|c|c|}
\hline Autor/-in: & eingereicht am: \\
\hline
\end{tabular}

$\begin{array}{r}\hline \begin{array}{c}\text { Titel und Untertitel der } \\ \text { Veröffentlichung: }\end{array} \\ \hline \text { Hochschule: } \\ \hline \text { Fakultät (Kürzel)*: } \\ \hline \text { Content-Typ: } \square \text { Graduierungsschrift } \square \text { BA } \square \text { MA } \square \text { Sammelband } \square \text { Monografie } \square \text { Konferenzband } \\ \hline\end{array}$

*AS - Architektur und Sozialwissenschaften / WW - Wirtschaftswissenschaften und Wirtschaftsingenieurwesen / ING - Ingenieurwissenschaften / DIT - Digitale Transformation / B - Bauwesen / IM - Informatik und Medien

\section{Inhaltliche Anmerkungen}

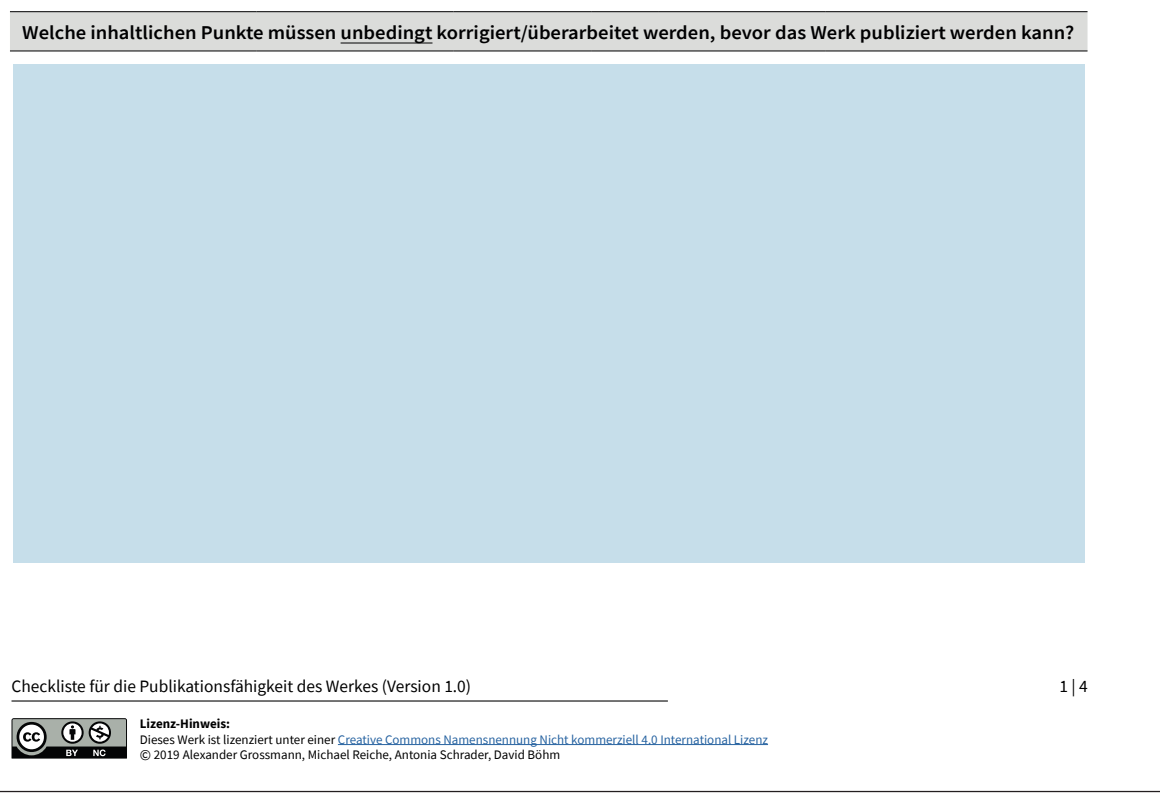

Abb. 28 Checkliste für die Publikationsfähigkeit des Werkes (S. 1/4) 


\section{Checkliste}

für die Publikationsfähigkeit des Werkes

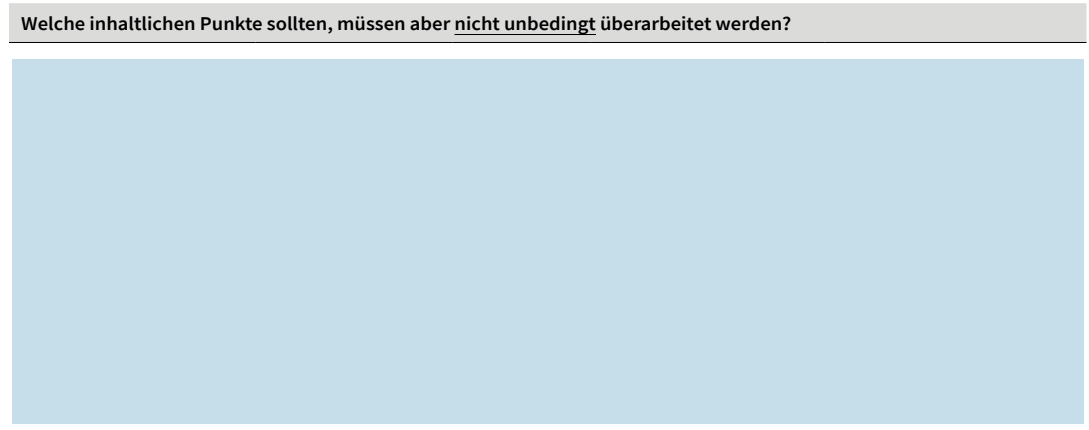

Sollten zusätzliche Verzeichnisse vom Autor/-in erstellt werden und wenn ja, welche? z.B. Abbildungs- oder Tabellenverzeichnis / Abkürzungsverzeichnis / Register

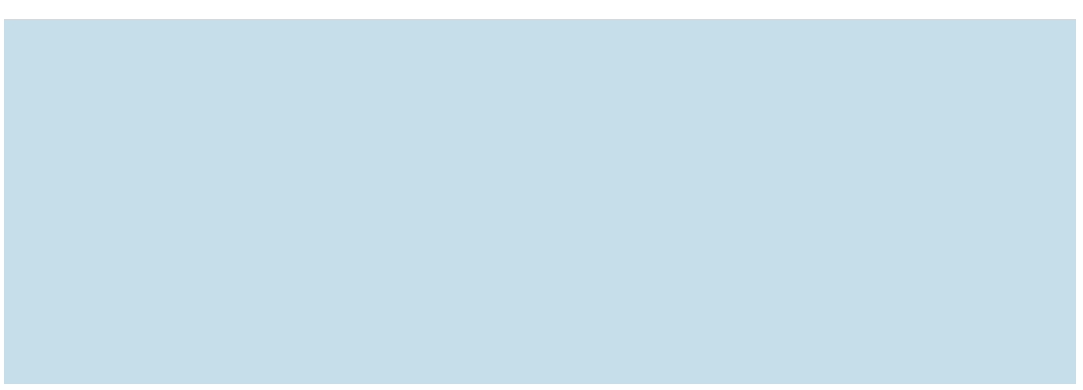

Sind die Anhänge/Forschungsdaten vollständig? Wenn nein, was fehlt?

Abb. 29 Checkliste für die Publikationsfähigkeit des Werkes (S. 2/4) 


\section{Checkliste}

für die Publikationsfähigkeit des Werkes

d.h. entspricht die angegebene Literatur dem Stand der Forschung, wird fachrelevante Literaturgenannt?

\section{Abbildungen / Grafiken}

Sind Abbildungen/Grafiken und/oder Tabellen klar und eindeutig dargestellt und beschriftet?

$\square$ Ja $\square$ Nein, folgende Abbildungen/

Grafiken und/oder Tabellen

müssen überarbeitet werden:

\section{Formale Anmerkungen}

Benötigt das Werk eine inhaltliche Überarbeitung durch einen Fachexperten (Fachkorrektorat)?

$\square$ Nein $\square$ Ja, aus diesen

Abb. 30 Checkliste für die Publikationsfähigkeit des Werkes (S. 3/4) 


\section{Checkliste}

für die Publikationsfähigkeit des Werkes

\section{CAHNerlag \\ Open Access Hochschulverlag}

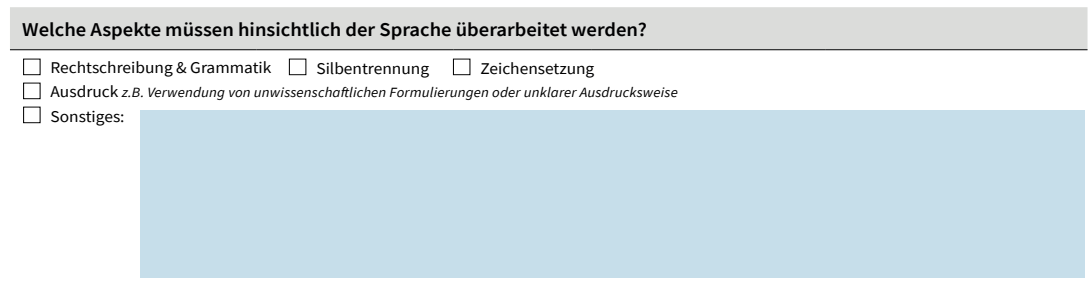

Welche Aspekte müssen zu Gunsten einer einheitlichen Verwendung überarbeitet werden?

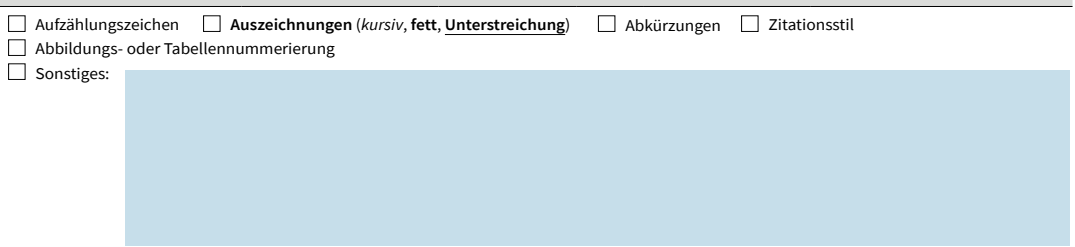

Gibt es systematische Fehler?

$\square$ Nein $\square$ Ja: $\square$ Falsche Paginierung $\square$ Falsche Fußnotenzählung $\square$ uneinheitlicher Zitationsstil

$\square$ Sonstiges:

\section{Nutzung des Gutachtens}

Ich bin damit einverstanden, dass meine Identität als Gutachter/-in dem Verfasser/-in der Arbeit sowie der Öffentlichkeit offenbart wird.

\section{$\square$ Ja $\quad \square$ Nein}

Ich bin damit einverstanden, dass das von mir erstellte Gutachten zum oben genannten Titel vollständig oder in Teilaspekten veröffentlicht wird (Open Peer Review).

$\square$ Ja $\square$ Nein

Ich bin damit einverstanden, dass das von mir erstellte Gutachten zum oben genannten Titel, falls dieses vollständig oder in Teilaspekten veröffentlicht wird, eine DOI zur besseren Auffindbarkeit erhält.
$\square \mathrm{Ja}$
$\square$ Nein

Datum

Unterschrift Gutachter/-in

Checkliste für die Publikationsfähigkeit des Werkes (Version 1.0)

Lizenz-Hinweis:
Dieses Werk ist lizenziert unter einer Creative Commons Namensnennung Nicht kommerziell 4.0 International Lizenz

(c) (i) (5) (c) 2019 Alexander Grossmann, Michael Reiche, Antonia Schrader, David Böhm

Abb. 31 Checkliste für die Publikationsfähigkeit des Werkes (S. 4/4) 


\section{Richtlinie}

für die Produktfreigabe

Buchprojekt:

\section{Richtlinie grundsätzlich für alle Ausgabeformate}

\begin{tabular}{cl}
\hline Inhalt & \\
\hline$\quad$ Der Inhalt wurde mit dem Original-Manuskript des Content-Urhebers abgeglichen und ist vollständig. \\
\hline$\quad$ Die Gestaltungsrichtlinien sind korrekt angewandt. \\
$\square \quad \begin{array}{l}\text { Die Seitenzahlen im Register und im Inhalts-, Abbildungs- und Tabellenverzeichnis sind geprüft und } \\
\text { korrekt. }\end{array}$ \\
\hline Die Seitenreihenfolge entspricht der definierten Seitenreihenfolge in den Gestaltungsrichtlinien. \\
\hline$\quad$ Abbildungen und Tabellen sind sinnvoll platziert (dem Kontext entsprechend). \\
\hline Dabellen sind sinnvoll und entsprechen dem Manuskript des Content-Urhebers umgesetzt. \\
\hline Die Bildung, Vergabe und Positionierung der DOIs entspricht der Richtlinie zur Bildung von DOIs. \\
\hline Impressum \\
\hline Im QR-Code ist die richtige DOI des Werkes hinterlegt. Er lässt sich korrekt einscannen.
\end{tabular}

\section{Richtlinie speziell für das Ausgabeformat Print}

Inhalt

\begin{tabular}{cl}
\hline Format und Seite \\
\hline$\square \quad$ Die Druckvorlage liegt im richtigen Buchformat (entsprechend der Gestaltungsrichtlinien) vor. \\
\hline$\quad$ Der Satzspiegel wurde eingehalten und ist registerhaltig. \\
\hline$\quad$ Die Seitenzahl der Druckvorlage ist durch vier teilbar. \\
\hline Farben \\
\hline$\square \quad$ Die Druckvorlage beinhaltet keine RGB-, Vollton- oder Schmuckfarben. \\
\hline$\quad$ Ein Gesamtfarbauftrag von 300\% wird nicht überschritten. \\
\hline Als CMYK-Farbprofil ist standardmäßig das Profil ISOcoated_V2 (Fogra39) hinterlegt. \\
\hline FlW-Abbildungen sind als Graustufen (nur in Farbe Schwarz) angelegt. \\
\hline Fließt, QR-Codes und das HTWK-Logo ist in 100\% Schwarz angelegt. \\
\hline
\end{tabular}

Abb. 32 Richtlinie für die Produktfreigabe (S. 1/4) 


\section{Richtlinie}

\section{für die Produktfreigabe}

\section{CAHNerlag \\ Open Access Hochschulverlag}

\begin{tabular}{|c|c|}
\hline \multicolumn{2}{|c|}{ Schriften } \\
\hline & Alle Glyphen sind in Unicode kodiert. \\
\hline & Es befindet sich kein einfarbiger Text kleiner als 5 pt in der Druckvorlage. \\
\hline & Es befindet sich kein farbiger Text kleiner als 8 pt in der Druckvorlage. \\
\hline & Schriften sind in der Druckvorlage eingebettet (Standard für PDF/X-1a und PDF/X-3). \\
\hline \multicolumn{2}{|c|}{ Bilder und Auflösung } \\
\hline & Die Bildauflösung aller Farb- und Graustufenabbildungen weist mind. 300 dpi auf. \\
\hline & Die Bildauflösung aller Strichabbildungen weist mind. 1200 dpi auf. \\
\hline & Bei im Anschnitt platzierten Bildern weist die Druckvorlage einen Beschnitt von 3 mm auf. \\
\hline \multicolumn{2}{|c|}{ Sonstiges } \\
\hline & $\begin{array}{l}\text { Mikrotypografie: Zeilen, bspw. im Inhaltsverzeichnis, brechen sinnvoll um, es gibt keine Hurenkinder } \\
\text { oder Schusterjungen, es wurden die korrekten Gylphen für Anführungszeichen und Gedankenstriche } \\
\text { verwendet, es gibt keinen Einzug nach einer Leerzeile, u.a. }\end{array}$ \\
\hline & Es wurden keine Transparenzen in der Druckvorlage verwendet. \\
\hline & Um wegbrechende Haarlinien im Druck zu vermeiden, wurde eine Strichstärke von mind. 0,28 pt eingehalten. \\
\hline$\square$ & Die Druckvorlage liegt als PDF Version PDF/X-1:2001 vor. \\
\hline
\end{tabular}

Umschlag

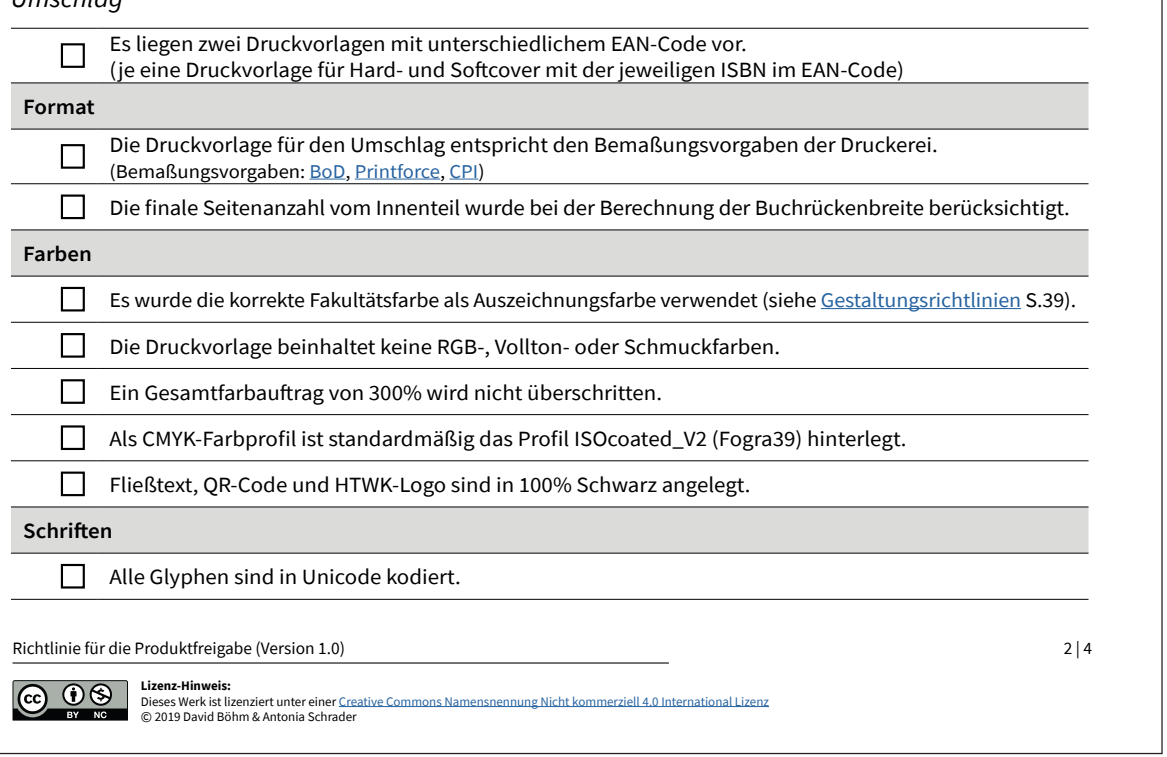

Abb. 33 Richtlinie für die Produktfreigabe (S. 2/4) 


\section{Richtlinie}

für die Produktfreigabe

Schriften sind in der Druckvorlage eingebettet (Standard für PDF/X-1a und PDF/X-3).

\begin{tabular}{l}
\hline Autorenfoto \\
\hline Die Bildauflösung des Autorenfotos weist mind. 300 dpi auf. \\
\hline QR-Code und EAN \\
\hline Im QR-Code ist die richtige DOI des Werkes hinterlegt. Er lässt sich korrekt einscannen. \\
\hline Der EAN-13 Code wurde mit der korrekten ISBN des jeweiligen Ausgabeformats erstellt. \\
\hline Sonstiges Der Buchrückentitel wurde an die Buchrückenbreite angepasst (siehe Gestaltungsrichtlinien S.40). \\
\hline Die Gestaltungsrichtlinien (Abstände, Größen, Layout) für den Umschlag wurden eingehalten. \\
$\square$ Die Druckvorlage liegt als PDF Version PDF/X-1:2001 vor.
\end{tabular}

Druckfreigabe Inhalt und Umschlag

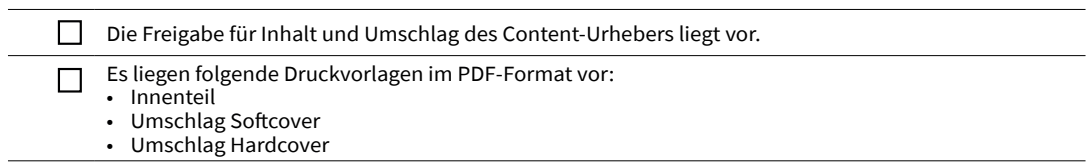

\section{Richtlinie speziell für das Ausgabeformat E-PDF}

\begin{tabular}{|c|c|}
\hline \multicolumn{2}{|c|}{ Verlinkungen } \\
\hline & Die Einträge im Inhalts-, Abbildungs- und Tabellenverzeichnis sind verlinkt. \\
\hline & Interne Querverweise im Inhalt sind verlinkt. \\
\hline & DOIs und Hyperlinks sind verlinkt und führen zum korrekten Ziel. \\
\hline \multicolumn{2}{|c|}{ Dateigröße } \\
\hline & $\begin{array}{l}\text { Das E-PDF wurde unter Beachtung der Bildqualität komprimiert (sollte kleiner als die Druck-PDF-Datei } \\
\text { sein). }\end{array}$ \\
\hline
\end{tabular}

Abb. 34 Richtlinie für die Produktfreigabe (S. 3/4) 


\section{Richtlinie}

für die Produktfreigabe

\section{Richtlinien für das Ausgabeformat ePUB/MOBI}

\begin{tabular}{|c|c|}
\hline \multicolumn{2}{|l|}{ Inhalt } \\
\hline & Alle Inhalte sind vorhanden und werden in der richtigen Reihenfolge angesprungen/angezeigt. \\
\hline & Alle Metadaten werden korrekt angezeigt. \\
\hline \multicolumn{2}{|c|}{ Usability } \\
\hline & Der Reader springt beim ersten Öffnen an die korrekte Stelle im Titel. \\
\hline \multicolumn{2}{|l|}{ Layout } \\
\hline & Das Cover wird auf dem Lesegerät und in der Bibliothek des Lesegeräts korrekt anzeigt. \\
\hline & Der TOC wird korrekt angezeigt. \\
\hline & Die Darstellung des Readers entspricht dem erwarteten Layout aufgrund des vorgegebenen CSS. \\
\hline & Das Layout verhält sich bei Schriftgrößen-Änderungen korrekt. \\
\hline & Eingebettete Fonts (Verlagsschrift Source Sans) werden korrekt angezeigt bzw. im Font-Menü verwendet. \\
\hline & Farbe im Layout: Alle farbigen Elemente sind auf elnk-Displays gut lesbar. \\
\hline & Die Glyphen von Sonderzeichen werden korrekt dargestellt. \\
\hline \multicolumn{2}{|c|}{ Tabellen } \\
\hline & $\begin{array}{l}\text { Die Spaltendarstellung und Linierungen sind korrekt umgesetzt. } \\
\text { Es laufen keine Inhalte aus dem Display heraus. }\end{array}$ \\
\hline \multicolumn{2}{|l|}{ Bilder } \\
\hline & Alle Abbildungen sind vorhanden. \\
\hline & Alle Abbildungen werden korrekt und in ausreichender Qualität angezeigt, auch auf elnk-Lesegeräten. \\
\hline \multicolumn{2}{|l|}{ Links } \\
\hline & Stichproben: Die internen und externen Verlinkungen sind korrekt. \\
\hline
\end{tabular}

Abb. 35 Richtlinie für die Produktfreigabe (S. 4/4) 


\section{Richtlinie}

für die Barrierefreiheit beim ePUB

Buchprojekt:

\begin{tabular}{|c|c|}
\hline \multicolumn{2}{|c|}{ Struktur } \\
\hline & Verwendung des EPUB3-Formates (bietet mehr Accessibility-Features als EPUB2) \\
\hline & $\begin{array}{l}\text { Semantische Strukturierung mithilfe von HTML5 } \\
\rightarrow \text { bietet spezielle Tags für bestimmte Inhalte (z. B. Zitate <blockquote>, Abschnitte <section>, ver- } \\
\text { schiedene Listentypen }<\text { ul }>,<0 l>,<d l>)\end{array}$ \\
\hline & $\begin{array}{l}\text { Weitere Inhalte mit Attribut @epub:type auszeichnen } \\
\rightarrow \text { Attributwerte dem Structural Semantics Vocabulary (EPUB3-Wortschatz für gängige Buchinhalte) } \\
\text { entnehmen }\end{array}$ \\
\hline & $\begin{array}{l}\text { Semantik und Hierarchie müssen aus Datenstruktur hervorgehen } \\
\rightarrow \text { darauf achten, dass z. B. Überschriftenhierarchien korrekt angewendet werden }\end{array}$ \\
\hline & $\begin{array}{l}\text { Logische, linear korrekte Lesereihenfolge einhalten } \\
\rightarrow \text { auch über mehrere Dokumente hinweg - hierfür dient das Element <spine> im Package-Dokument }\end{array}$ \\
\hline & Verschiedene Navigationsmöglichkeiten anbieten \\
\hline & $\square$ Inhaltsverzeichnis (TOC) bietet gesamte Struktur ab \\
\hline & $\square$ Orientierungspunkte (Landmarks) für wesentliche Bestandteile des Buches setzen \\
\hline & $\square$ Seitenlisten (Pagelists) als Kennzeichnung für Seitenwechsel \\
\hline & $\square$ Weitere Verzeichnisse (Abbildungs-, Tabellenverzeichnisse etc.) \\
\hline & $\begin{array}{l}\text { Aussagekräftige Metadaten über Zugänglichkeit für Sprachnavigation } \\
\rightarrow \text { Orientierung an ONIX-Daten Liste } 196 \text { oder schema.org Accessibility Metadata }\end{array}$ \\
\hline & $\begin{array}{l}\text { Seitenzahlen aus Print-Quelle übernehmen } \\
\rightarrow \text { trifft nur bei XML-/EPUB-Last zu }\end{array}$ \\
\hline
\end{tabular}

Abb. 36 Richtlinie für die Barrierefreiheit beim EPUB (S. 1/2) 


\section{Richtlinie}

für die Barrierefreiheit beim ePUB

\begin{tabular}{|c|c|}
\hline \multicolumn{2}{|l|}{ Inhalt } \\
\hline & $\begin{array}{l}\text { Unterscheidung in primäre und sekundäre Inhalte } \\
\rightarrow \text { sekundäre Inhalte sind z.B. Fußnoten, Marginalien etc. }\end{array}$ \\
\hline & Dokumentsprachen hinterlegen \\
\hline & $\square$ Hauptsprache in Metadaten der Publikation: <dc:language> \\
\hline & $\square$ Angabe der Dokumentsprache zu Beginn jedes neuen Inhaltsdokumentes mit @xml:lang \\
\hline & $\square$ Sprachwechsel innerhalb des Textes kennzeichnen \\
\hline & Abbildungen \\
\hline & $\square$ Verzicht auf Darstellung von Textinhalten mittels Bilder (z.B. bei Tabellen oder Formeln) \\
\hline & $\begin{array}{l}\square \text { SVG-Format verwenden, da es barrierefreier ist } \rightarrow z \text {. B. wird Text innerhalb von Grafiken als Text } \\
\text { ausgelesen und somit Screenreadern zugänglich gemacht }\end{array}$ \\
\hline & $\begin{array}{l}\square \text { Grafiken ohne inhaltsrelevante Informationen (Schmuckgrafiken) kennzeichnen } \rightarrow \text { leeres @alt } \\
\text { sowie @role="presentation“ }\end{array}$ \\
\hline & $\square$ Sinnvolle Alternativtexte verfassen (max. 80 Zeichen) und mit @alt angeben \\
\hline & $\rightarrow$ nicht einfach Bildunterschrift übernehmen, da es sonst zu Dopplungen kommen kann \\
\hline & $\begin{array}{l}\square \text { Zusätzlich ausführliche Bildbeschreibung bei komplexeren Grafiken (z. B. Diagrammen), die ent- } \\
\text { weder im Fließtext eingebaut werden können oder ausgelagert und mit <a> oder <longdesc> verlinkt }\end{array}$ \\
\hline & Mathematische Formeln \\
\hline & $\begin{array}{l}\square \text { nicht als Bild einbinden, sondern MathML nutzen } \\
\square \text { evtl. Alternativtexte verwenden }\end{array}$ \\
\hline & Multimediale Inhalte \\
\hline & $\begin{array}{l}\square \text { dazugehörige HTML5-Elemente nutzen und testen, ob Lese-Software Player bereitstellt } \\
\square \text { Fallback-Option: Video-/Audiodeskriptionen }\end{array}$ \\
\hline & Interaktive Inhalte mit ARIA-Vokabular auszeichnen \\
\hline \multicolumn{2}{|c|}{ Gestaltung } \\
\hline & $\begin{array}{l}\text { Typografische Auszeichnungen im Inhalt vermeiden } \\
\rightarrow \text { stattdessen auf semantische Hervorhebung setzen (z. B. }<\text { em }>\text { statt }<\text { i }>\text { oder }<\text { strong }>\text { statt }<\text { b }> \\
\rightarrow \text { beeinflusst Aussprache des Screenreaders im Gegensatz zu typografischer Auszeichnung }\end{array}$ \\
\hline${ }^{-}$ & $\begin{array}{l}\text { Veränderbares Layout } \\
\rightarrow \text { Schriftgröße, -art, Zeilenabstand, Hintergrundfarbe, Ränder, Abstände, sofern Reader-Applikation } \\
\text { Funktionen bereitstellt }\end{array}$ \\
\hline
\end{tabular}




\section{Richtlinie}

für gedruckte Produkte

Open Access Hochschulverlag

Buchprojekt:

Inhalt

$\square$ Die Inhaltsseiten wurden vollständig und richtig ausgeschossen in der richten Reihenfolge gebunden.

Stichprobe: Die gedruckten Abbildungen liegen in guter Druckqualität vor.

Bindung

Es liegt eine stabile Binding mit festen Buchseiten und gutem Aufschlagverhalten vor.

Ausstattung

Die Papiersorte bzw. -veredelung für Inhalts- und Umschlagspapier stimmt mit den Angaben im Druckauftrag überein.

Die Farbigkeit von Inhalt und Umschlag stimmt mit den Angaben im Druckauftrag überein.

Der gelieferte Buchtyp (Hardcover/Softcover) stimmt mit den Angaben im Druckauftrag überein.

Buchrücken

Der Titel und der Verlagsname sitzen passgenau zentriert auf dem Buchrücken.

Es ragen keine Buchrückenelemente auf die U1 und U4 des Buchs.

Umschlag

Die grauen „Tape“-Gestaltungselemente auf der U1 haben den gleichen Abstand zur Buchkante.

Anmerkungen:

Richtlinie für gedruckte Produkte (Version 1.0)

Lizenz-Hinweis:

Dieses Werk ist lizenziert unter einer Creative Commons Namensnennung Nicht kommerziell 4.0 International Lizenz

(c) 2019 David Böhm \& Antonia Schrader

Abb. 38 Richtlinie für gedruckte Produkte 


\section{Autoren-Checkliste}

\section{für die benötigten Informationen zum Vertragsabschluss}

\section{COAHVerlag}

Bitte senden Sie uns die ausgefüllte Checkliste elektronisch

an redaktion@oa-hverlag.de und zusätzlich ausgedruckt und unterschrieben zu.

AUTORENFOTO: Bitte liefern Sie uns ebenfalls ein Autorenfoto (vorzugsweise farbig, mind. 300 dpi Auflösung) für den Umschlag Ihres gedruckten Werkes.

\begin{tabular}{c|c}
\hline Allgemeine Angaben zum Autor/-in & \\
\hline Name, Vorname: & akadem. Titel: \\
\hline Wohnadresse: & PLZ, Wohnort: \\
\hline E-Mail-Adresse: & Telefon: \\
\hline Institutsadresse: & \\
\hline
\end{tabular}

Kurzvita:

(300-400 Zeichen mit Leerzeichen)

ORCID ID ${ }^{1}$ :

${ }^{[1]}$ (optional) Wir empfehlen Ihnen die Registrierung bei ORCID. Die Open Researcher and Contributor ID (ORCID) ist ein eindeutiger Autorenidentifikator. Er ermöglicht die Zuordnung einer wissenschaftlichen Arbeit zu einer Person, unabhängig von der Häufigkeit des Namens, Namensänderungen, Umlauten, karrierebedingten Wechsel der Einrichtungen. Sie können die ORCID kostenlos über www.orcid.org/ register beantragen.

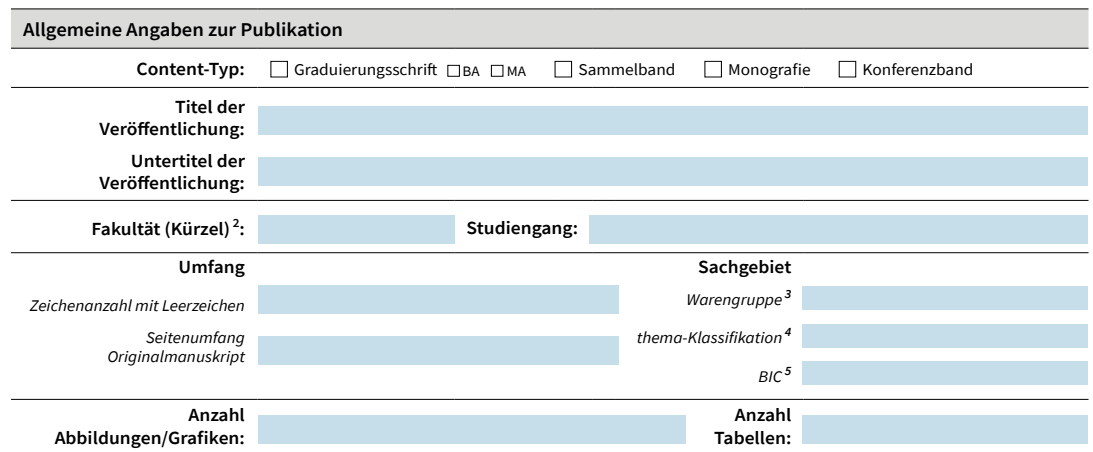

${ }^{[2]}$ AS - Architektur und Sozialwissenschaften / WW - Wirtschaftswissenschaften und Wirtschaftsingenieurwesen / ING - Ingenieurwissenschaften /

DIT - Digitale Transformation / B - Bauwesen / IM - Informatik und Medien

${ }^{[3]}$ Informationen zur Warengruppe finden Sie hier: https://www.boersenverein.de/sixcms/media.php/976/wgs2012.pdf

${ }^{[4]}$ Informationen zur thema-Klassifikation finden Sie hier: https://ns.editeur.org/thema/de

${ }^{[5]}$ Informationen zur BIC finden Sie hier: https://ns.editeur.org/bic_categories/114

Autoren-Checkliste für die benötigten Informationen zum Vertragsabschluss (Version 1.4)

Abb. 39 Autoren-Checkliste für die benötigten Informationen zum Vertragsabschluss (S. $1 / 4)$ 


\section{Autoren-Checkliste}

für die benötigten Informationen zum Vertragsabschluss

\section{Kurzbeschreibung Inhalt für Umschlag (U4)}

Beschreiben Sie in wenigen Sätzen, wovon Ihr Werk handelt, sodass auch das Interesse von Lesern geweckt wird, die
keine spezielle Expertise auf dem Sachgebiet besitzen.
keine spezielle Expertise auf dem Sachgebiet besitzen.

\section{Abstract}

Geben Sie eine prägnante Inhaltsangabe (max. 720 Zeichen mit Leerzeichen) ohne Interpretation und Wertung Ihrer wissenschaftlichen Arbeit in deutscher und englischer Sprache wieder.

deutsch:

englisch:

Abb. 40 Autoren-Checkliste für die benötigten Informationen zum Vertragsabschluss (S. 2/4) 


\section{Autoren-Checkliste}

für die benötigten Informationen zum Vertragsabschluss

THOAHNerlag

\section{Keywords}

Geben Sie mindestens 10 Schlagwörter (einzelne Wörter) in deutscher und englischer Sprache zur Indizierung in Katalogen, Datenbanken und Suchmaschinen für die optimale Sichtbarkeit und Auffindbarkeit des Werkes an.

deutsch: englisch:

\section{Marketing}

In welchen Punkten grenzt sich der Titel von anderen Titeln aus diesem Sachgebiet ab? Gibt es ein Alleinstellungsmerkmal?

Abb. 41 Autoren-Checkliste für die benötigten Informationen zum Vertragsabschluss (S. $3 / 4)$ 


\section{Autoren-Checkliste}

für die benötigten Informationen zum Vertragsabschluss

\section{Rechtliche Anmerkungen}

Bitte beachten Sie, dass Ihr Werk Open Access veröffentlicht wird. Sämtliche verwendeten Abbildungen, deren Urheber nicht Sie selber sind, müssen mit einer Open-Access-Lizenz vom Urheber versehen sein, sonst können diese nicht ohne rechtliche Probleme verwendet werden. Die Klärung von ungeklärten Text- und Bildvorlagenrechten übernimmt der Hochschulverlag.

Die im vorliegenden Manuskript enthaltenen Text- und/oder Bildvorlagen verletzen keine Rechte Dritter. Ich verfüge über die erforderlichen urheberrechtlichen Nutzungsrechte und es wurde weder ganz noch teilweise eine der Einräumung von Rechten widersprechende Verfügung getroffen.

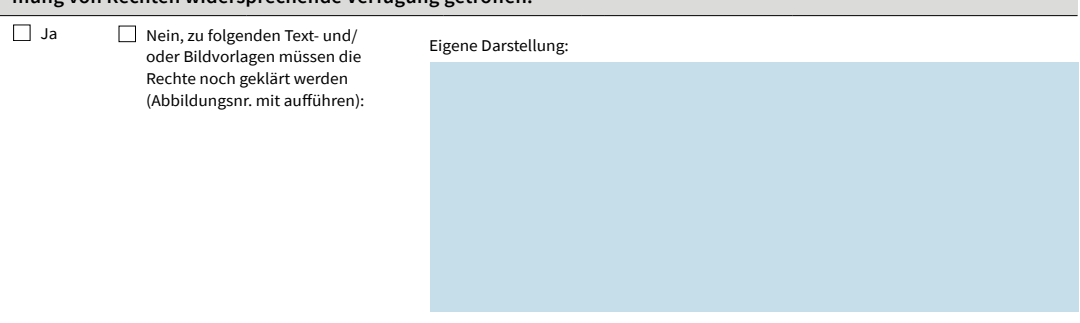

Zitierte Abbildungen (Angabe zur Quelle mit aufführen):

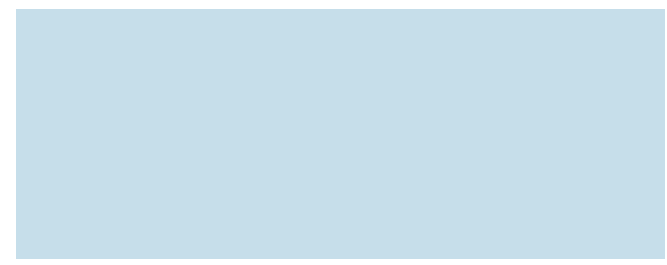

Abbildungen in Anlehnung an (Angabe zur Quelle mit aufführen):

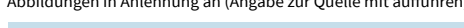

Abb. 42 Autoren-Checkliste für die benötigten Informationen zum Vertragsabschluss (S. 4/4) 


\title{
Publikationsvertrag
}

\author{
zwischen dem Forschungsprojekt, Open-Access-Hochschulverlag' \\ (nachstehend vereinfachend: Hochschulverlag) \\ vertreten durch Herr/Frau \\ HTWK Leipzig \\ und \\ Herr/Frau \\ (nachstehend vereinfachend: Verfasser)
}

\section{(roAHVerlag \\ Open Access Hochschulverlag}

\section{$\S 1$ Vertragsgegenstand}

Gegenstand des Vertrages ist folgendes Werk des Verfassers:

Titel des Werkes:

Grad-verleihende Institution:

Fakultät / Studiengang:

Reihe:

Herausgeber der Reihe:

\section{§ 2 Einräumung von Nutzungsrechten}

Die Urheberrechte am Werk liegen beim Verfasser (Rechteinhaber). Der Rechteinhaber räumt dem Hochschulverlag hiermit die folgenden Nutzungsrechte ein:

(1) das einfache, unentgeltliche, nicht-ausschließliche, räumlich und zeitlich unbeschränkte Nutzungsrecht zur Vervielfältigung und Verbreitung in gedruckter Form als Buch- und Taschenbuchausgabe (Hardcover \& Softcover);

(2) das einfache, unentgeltliche, nicht-ausschließliche, räumlich und zeitlich unbeschränkte Nutzungsrecht zur elektronischen Publikation im Internet im Gold Open Access* (Zur Definition von Gold Open Access siehe hier: www.open-access.net/informationen-zu-open-access/open-access-strategien/);

Das Recht wird zum Zweck der Langzeitarchivierung und öffentlichen Zugänglichmachung auch anderen Bibliotheken, Archiven und Aggregatoren eingeräumt (z. B. OAPEN, DNB, Google Books, Fachrepositorien), es gilt für alle bekannten Nutzungsarten;

Abb. 43 Mustervertrag für Hochschulpublikationen (S. 1/4) 
(3) das Recht die Metadaten zum Nachweis der Publikation in einschlägigen bibliographischen Datenbanken unter der Lizenz CCO (www.creativecommons.org/publicdomain/zero/1.0/) zu verbreiten.

\section{§ 3 Rechteübertragung an die Allgemeinheit}

Der Verfasser (Inhaber des Urheberrechts) ist einverstanden, dass die Publikation veröffentlicht wird unter:

CC BY 4.0 I Creative Commons Namensnennung 4.0 International Lizenz www.creativecommons.org/licenses/by/4.0/deed.de

oder

CC BY-NC 4.0 I Creative Commons Namensnennung Nicht kommerzielle 4.0 International Lizenz www.creativecommons.org/licenses/by-nc/4.0/deed.de

Die Urheberrechte des Verfassers bleiben durch die Pflicht zur Nennung des Verfassernamens gewahrt. Die Nennung des Verfassers erfolgt in allen Nutzungsarten als @ Vorname, Nachname (Verfasser), Jahreszahl. Nähere Informationen: www.creativecommons.org/licenses/

\section{§ 4 Rechtliche Unbedenklichkeit und Haftung}

(1) Der Verfasser versichert, alleiniger Inhaber aller Rechte am vorliegenden Werk zu sein. Insbesondere steht er dafür ein, dass das Werk sowie die in ihm enthaltenen fremden Text- und/oder Bildvorlagen keine Rechte Dritter verletzen, er befugt ist, über die zur Durchführung dieses Vertrages erforderlichen urheberrechtlichen Nutzungsrechte zu verfügen, und dass er bisher weder ganz noch teilweise eine der Einräumung von Rechten widersprechende Verfügung getroffen hat. In dem Werk reproduzierte oder verwendete Grafiken, Fotografien, Zeichnungen oder Tabellen dritter Urheber unterliegen der CC-BY- oder CC-0-Lizenz.

(2) Kommen dem Verfasser an seiner Befugnis Zweifel, so kommt der Publikationsvertrag mit dem Hochschulverlag solange nicht zustande, bis das Rechtshindernis oder die Unklarheit innerhalb einer angemessenen Frist vom Verfasser beseitigt wurde.

(3) Der Verfasser stellt den Hochschulverlag von Schadenersatzansprüchen gleich welcher Art frei, die er aufgrund von Verletzungen von Schutzrechten Dritter zu vertreten hat. Der Verfasser ist verantwortlich für den Inhalt des von ihm veröffentlichten Werkes. Für Störungen des Datennetzes sowie eventuelle Veränderungen der Daten während der Datenfernübertragung übernimmt der Hochschulverlag keine Haftung.

\section{§ 5 Mehrere Autoren}

(1) Haben mehrere Autoren gemeinsam ein Werk verfasst, so sind sie Miturheber des Werkes.

(2) Die Veröffentlichung des Werks kann daher nur mit Einwilligung und Unterschrift aller Autoren erfolgen. 


\section{ToAHVerlag \\ Open Access Hochschulverlag}

(3) Bei Sammelwerken ist folgende gesonderte Erklärung des Herausgebers über die Rechteinräumung der Autoren erforderlich:

Ich versichere, dass die Autoren mir die für diese Übertragung notwendigen Rechte eingeräumt haben, auch zur Online-Veröffentlichung. Die Autoren haben mir als Herausgeber bestätigt, dass sie ganz allein berechtigt sind, über das Urheberrecht an ihrem jeweiligen Beitrag zu verfügen. Sie stehen dafür ein, dass weder die Rechte Dritter noch schutzrechtliche Bestimmungen verletzt werden, insbesondere bei im jeweiligen Werk enthaltenen Abbildungen (Fotos, Grafikelemente).

Datum

Unterschrift

\section{$\S 6$ Rechte und Pflichten des Verfassers}

(1) Der Verfasser verpflichtet sich, dem Hochschulverlag ein vollständiges und satzfertiges Manuskript des Werkes in digitaler Form zu überlassen. Dieses wird zum Zweck der Dokumentation und Archivierung vom Hochschulverlag gespeichert.

(2) Der Verfasser wird zur Vervollständigung oder Illustration benötigte fremde Text- und/oder Bildvorlagen beschaffen und die erforderlichen Nutzungsrechte oder Zustimmungen Dritter auf eigene Gefahr und Kosten einholen.

(3) Das Originalmanuskript des Werkes sowie die durch den Verfasser beschafften Text- und/oder Bildvorlagen gehen nicht in das Eigentum des Hochschulverlages über.

(4) Das Werk muss nach Art und Zweck dem Vereinbarten und dem anerkannten fachlichen Standard des behandelten Gebiets oder Themas Rechnung tragen. Zur Beurteilung darf der Hochschulverlag Fachberater hinzuziehen.

\section{§ 7 Rechte und Pflichten des Hochschulverlages}

(1) Der Hochschulverlag legt die formale Gestaltung und Ausstattung des Werkes fest. Hierzu zählt u. a. die Gestaltung des Umschlags inkl. des Covers, der Innentitelseiten und des Impressums.

(2) Die Vorkorrekturen des Satzes des Werkes erfolgen durch den Hochschulverlag, einen Satzdienstleister oder eine Druckerei.

(3) Der Hochschulverlag legt fest, in welchen Ausgabeformaten (elektronische und/oder gedruckte) das Werk produziert und veröffentlicht wird. 


\section{Cisoatrerlag}

(4) Der Hochschulverlag übernimmt alle ihm notwendig erscheinenden Schritte, um das Werk im Internet, sowie im Buchhandel auffindbar und sichtbar zu machen. Hierzu zählen u. a. die Vergabe von ISBNs und DOIs, die Eintragung in das Verzeichnis lieferbarer Bücher (VIB) und die Eintragung in einschlägige bibliothekarische und buchhändlerische Datenbanken.

(5) Der Hochschulverlag ist berechtigt, den Ladenpreis für die gedruckten Werke zu bestimmen und ihn bei Bedarf zu erhöhen oder zu ermäßigen.

\section{$\S 8$ Kosten und Honorar}

(1) Der Hochschulverlag verpflichtet sich die Kosten für die Erstellung und Veröffentlichung des Werkes (in dem vom Hochschulverlag ausgewählten Ausgabeformaten) im Rahmen der Pilotphase des Forschungsprojektes vollständig zu tragen.

(2) Da der Hochschulverlag mit der Veröffentlichung des Werkes und den damit in Zusammenhang stehenden Dienstleistungen keine wirtschaftlichen Interessen verfolgt, erhält der Verfasser keine Vergütung und ggf. bestehende Erlöse durch den Verkauf der gedruckten Werke werden nicht an ihn ausgeschüttet. Dem Verfasser obliegt es, seine Publikation bei der Verwertungsgesellschaft WORT (www.vgwort.de) anzumelden.

\section{$\S 9$ Auflage, Frei- und Pflichtexemplare}

(1) Der Hochschulverlag organisiert die Pflichtlieferung an die maßgeblichen Bibliotheken.

(2) Der Verfasser erhält nach Erscheinen des Buches gedruckte Exemplare als

\section{$\S 10$ Kündigung}

(1) Dieser Vertrag kann von beiden Vertragspartnern jederzeit gekündigt werden. Die Rechtseinräumung des Verfassers an den Hochschulverlag für die Online-Publikation bleibt von der Kündigung unberührt; im Übrigen gilt § 314 BGB.

\section{\$11 Schlussbestimmungen}

(1) Endet der Vertrag ganz oder teilweise gleich aus welchem Rechtsgrund, bleibt das Forschungsprojekt unbeschadet weiterer Rechte - zum Abverkauf der vorhandenen Auflage sowie zur Weiterführung hinsichtlich des Werkes bestehender Verträge mit Dritten berechtigt.

(2) Änderungen und Ergänzungen dieses Vertrages bedürfen der Schriftform. Es gilt das Recht der Bundesrepublik Deutschland. Ergänzend finden die Regelungen des Urheber- und Verlagsrechts Anwendung.

(3) Sollten einzelne Bestimmungen dieses Vertrages rechtsunwirksam sein oder werden, wird die Gültigkeit des übrigen Vertragsinhaltes hiervon nicht berührt. Anstelle der unwirksamen Bestimmung tritt eine ihr auf rechtlich zulässige Weise wirtschaftlich möglichst nahekommende Regelung. 


\section{HTWK

\section{Einverständniserklärung für das elektronische Publizieren auf dem Publikationsserver der HTWK Leipzig}

Hochschulbibliothek HTWK Leipzig

Gustav-Freytag-Straße 40

04277 Leipzig

Name Autor/Autorin

oder sonstiger Rechteinhaber:

Adresse:

Telefonnr:

Geburtsdatum*:

( ${ }^{*}$ Diese Angabe wird ausschließlich zur Anlage eines eindeutigen Personennormdatensatzes in der Gemeinsamen Normdatei verwendet.)

E-Mail-Adresse:

Titel des Werkes:

\section{Einverständniserklärung}

(1) Ich gestatte der Hochschulbibliothek der HTWK Leipzig unwiderruflich, die elektronische Version des o.g. Dokumentes dauerhaft im Volltext zu archivieren, zu pflegen sowie öffentlich auf einem von der SLUB Dresden (Qucosa) betriebenen Server zur freien Benutzung im Internet bereitzustellen. Die Hochschulbibliothek der HTWK Leipzig ist berechtigt, das Dokument einschließlich der von mir gelieferten, das Dokument beschreibenden Daten (Metadaten) zu gleichen Bedingungen an andere Bibliotheken, Archive und Open-Access-Portale weiterzu-

Abb. 47 Einverständniserklärung für das elektronische Publizieren auf dem Publikationsserver der HTWK Leipzig (S. 1/2) 
geben, insbesondere an die Deutsche Nationalbibliothek und DFG-Sondersammelgebietsbibliotheken. Mir bleibt es freigestellt, über das Werk auch anderweitig zu verfügen, solange damit keine Einschränkung der der Hochschulbibliothek der HTWK Leipzig in dieser Erklärung eingeräumten Rechte verbunden ist.

(2) Ich bin berechtigt, über die Nutzungsrechte an diesem Dokument zu verfügen und versichere, dass mit der Veröffentlichung keine Rechte Dritter verletzt werden. In Zweifelsfällen oder bei Entstehen vermeintlicher oder tatsächlicher Rechtshindernisse setze ich die Hochschulbibliothek der HTWK Leipzig hiervon unverzüglich in Kenntnis. Ich stelle die Hochschulbibliothek der HTWK Leipzig von allen Ansprüchen frei, die Dritte ggf. auf Grund ihnen zustehender Rechte gegen sie erheben.

(3) Ich prüfe die Metadaten, die die Hochschulbibliothek der HTWK Leipzig gegebenenfalls ändern und vervollständigen kann, und die Netzversion des Dokumentes nach der Bearbeitung durch die Hochschulbibliothek der HTWK Leipzig auf Vollständigkeit, Authentizität und Lesbarkeit.

(4) Die Hochschulbibliothek der HTWK Leipzig ist berechtigt, gegebenenfalls nötige Datenänderungen vorzunehmen, wenn die technische Entwicklung es erfordert (z. B. eine Migration in andere Datenformate zum Zweck einer fachgerechten digitalen Langzeitarchivierung). Inhaltliche Veränderungen dürfen dabei nicht vorgenommen werden.

(5) Die Angabe des Geburtsdatums und -orts erfolgt auf freiwilliger Basis. Ich bin mit der Aufnahme dieser Daten in die Gemeinsame Normdatei (GND) und der Veröffentlichung über das Internet einverstanden.

Ort, Datum

Unterschrift Autor/Autorin

Abb. 48 Einverständniserklärung für das elektronische Publizieren auf dem Publikationsserver der HTWK Leipzig (S. 2/2) 


\section{Richtlinie}

für die Bildung von DOIs

\section{DoI-Vergabe}

DOI-Präfix OA-HVerlag: 10.33968

Der DOI für Bücher wird aus der e-ISBN (PDF) gebildet: https://doi.org/\{DOIprefix\}/\{elSBN_pdf\} (z.B.: https://doi.org/10.33968/9783110303568-00)

Suffix für Abbildungen: $9783110303568-00-A-00$

Suffix für Tabellen: $9783110303568-00-T-00$

\section{DOI-Granularität}

Eine DOI wird für das Werk an sich, für alle Kapitel erster Ordnung und für Abbildungen und Tabellen vergeben.

\section{DOI-Positionierung}

Die DOI für das Gesamtwerk findet sich im Impressum. Die DOI wird für alle Kapitel erster Ordnung in der Fußzeile der ersten Seite eingefügt. Der Abstand zwischen unterer Seitenkante und DOI beträgt 15,7 mm / 17,3 mm. Bei Abbildungen und Tabellen wird die DOI in der Abbildungsunterschrift bzw. Tabellenüberschrift integriert.

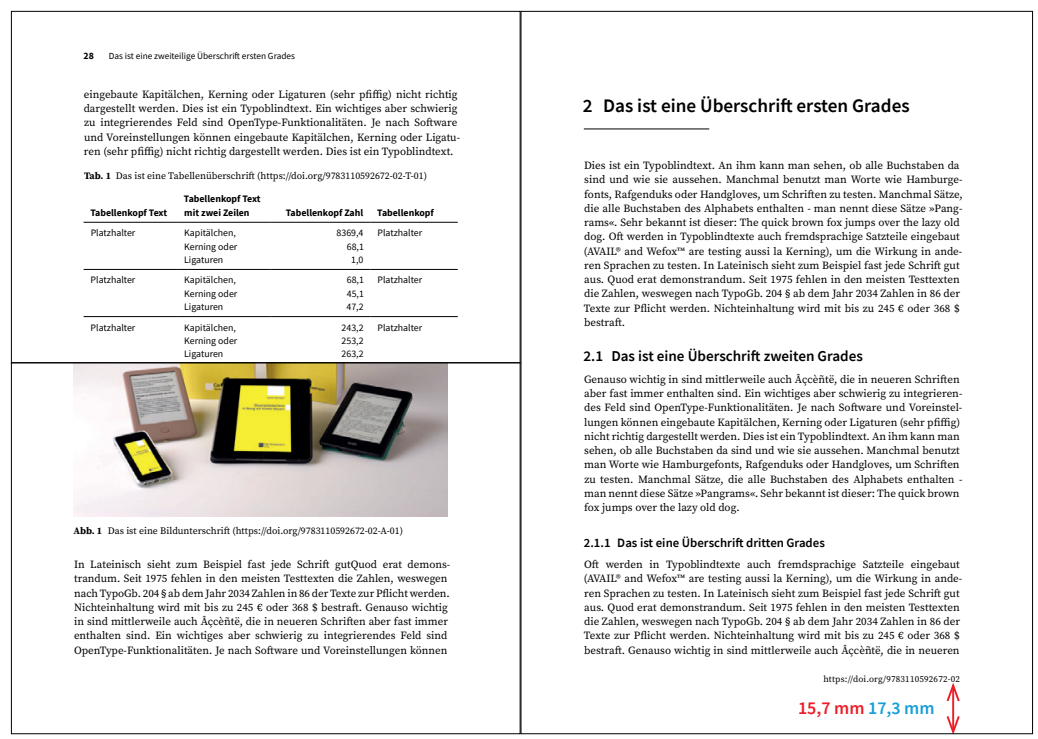

Format 1: $15,5 \times 22 \mathrm{~cm}$ | Format 2: $17 \times 24 \mathrm{~cm}$

Richtlinie für die Bildung von DOIs (Version 1.0)

Abb. 49 Richtlinie für die Bildung von DOIs 


\section{Richtlinie}

für die Erstellung von Metadaten

\begin{tabular}{|c|c|c|}
\hline \multicolumn{2}{|l|}{ Verlag / Publisher } & \\
\hline Produktform / Book type & $\square$ Hardcover $\square$ Softcover $\square$ ePUB/MOBI $\square$ E-PDF & \\
\hline \multicolumn{3}{|l|}{ Titel } \\
\hline \multicolumn{3}{|l|}{ Untertitel } \\
\hline \multicolumn{3}{|l|}{$\begin{array}{l}\text { Produktsprache } \\
\text { (nach } \underline{\text { ISO 639-1) }}\end{array}$} \\
\hline \multicolumn{3}{|l|}{$\begin{array}{l}\text { Name der Hochschule } \\
\text { (falls Hochschulschrift) }\end{array}$} \\
\hline Art der Hochschulschrift & $\square$ Bachelorarbeit $\square$ Masterarbeit $\square$ Dissertation $\quad \square$ Forschungsarbeit & \\
\hline \multicolumn{3}{|l|}{$\begin{array}{r}\text { Veröffentlichungsjahr } \\
\text { der Hochschulschrift }\end{array}$} \\
\hline \multicolumn{3}{|l|}{ ISBN-13 } \\
\hline \multicolumn{3}{|l|}{ DOI(s) } \\
\hline Urheberart & $\square$ Autor $\square$ Herausgeber $\square$ Übersetzer & \\
\hline \multicolumn{3}{|l|}{$\begin{array}{r}\text { Name, Vorname } \\
\text { (Urheber) }\end{array}$} \\
\hline \multicolumn{3}{|l|}{$\begin{array}{r}\text { Biographische Angaben } \\
\text { (Urheber) }\end{array}$} \\
\hline \multicolumn{3}{|l|}{ Preis in $€$} \\
\hline \multicolumn{3}{|l|}{ Auflage } \\
\hline Art der Auflage & $\square$ Neuausgabe $\square$ Nachdruck & \\
\hline \multicolumn{3}{|l|}{ Publikationsort } \\
\hline \multicolumn{3}{|l|}{ Erscheinungsland } \\
\hline Erscheinungstermin & Online: & \\
\hline Lieferbarkeitsstatus & $\square$ Noch nicht lieferbar $\square$ lieferbar & \\
\hline Publikationsstatus & $\square$ Noch nicht erschienen $\square$ erschienen & \\
\hline Umfang & insgesamt: & \\
\hline \multicolumn{3}{|l|}{ Anzahl der Abbildungen } \\
\hline \multicolumn{2}{|c|}{ Richtlinie für die Erstellung von Metadaten (Version 1.0) } & $1 \mid 2$ \\
\hline \multicolumn{3}{|c|}{$\begin{array}{l}\text { Lizenz-Hinweis: } \\
\text { Dieses Werk ist lizenziert unter einer Creative Commons Namensnennung Nicht kommerziell } 4.0 \text { International Lizenz } \\
\text { (C) } 2019 \text { Antonia Schrader, David Böhm }\end{array}$} \\
\hline
\end{tabular}

Abb. 50 Richtlinie für die Erstellung von Metadaten (S. 1/2) 


\section{Richtlinie}

für die Erstellung von Metadaten

\section{CAHVerlag}

Open Access Hochschulverlag

\begin{tabular}{|c|c|}
\hline Breite / Höhe & $\square \mathrm{B} 17 \mathrm{~cm} / \mathrm{H} 24 \mathrm{~cm} \quad \square \mathrm{B} 15,5 \mathrm{~cm} / \mathrm{H} 22 \mathrm{~cm} \square \mathrm{B} 21 \mathrm{~cm} / \mathrm{H} 29,7 \mathrm{~cm}$ \\
\hline $\begin{array}{l}\text { Warengruppe-Index } \\
\text { (mehr Informationen hier) }\end{array}$ & $\begin{array}{l}\square 1 \text { Hardcover, Softcover } \square 2 \text { Taschenbuch } \square 3 \text { Zeitschrift, Loseblatt-Ausgabe } \\
\square 4 \text { DVD, Video } \square 5 \text { Audio-CD, Kasette } \square 6 \text { CD-ROM, DVD-ROM } \\
\square 7 \text { Kalender } \square 8 \text { Karten, Globen } \square 9 \text { Nonbooks, PBS }\end{array}$ \\
\hline $\begin{array}{r}\text { Warengruppe } \\
\text { (mehr Informationen hier) }\end{array}$ & \\
\hline $\begin{array}{l}\text { thema-Klassifikation } \\
\text { (mehr Informationen hier) }\end{array}$ & \\
\hline $\begin{array}{r}\text { BIC } \\
\text { (mehr Informationen hier) }\end{array}$ & \\
\hline $\begin{array}{l}\text { Schlagwörter } \\
\text { (einzeln-deu) }\end{array}$ & \\
\hline $\begin{array}{r}\text { Keywords } \\
\text { (einzeln-eng) }\end{array}$ & \\
\hline Kundengruppe & $\square$ Fachpublikum/Wissenschaftler $\square$ Andere \\
\hline $\begin{array}{r}\text { Kurzbeschreibung } \\
(d e u)\end{array}$ & \\
\hline
\end{tabular}

$\begin{array}{r}\begin{array}{r}\text { Abstract } \\ \text { (eng) }\end{array} \\ \hline \text { Produktverweise } \\ \text { CC-Lizenz } \\ \hline \text { Förderer / Funder } \quad \square \text { CC BY-SA } \square \text { CC BY-ND } \square \text { CC BY-NC } \square \text { CC BY-NC-SA } \square \text { CC BY-NC-ND } \\ \hline \text { Main-Website / URL } \\ \hline \text { ORCID-Autor }\end{array}$

Richtlinie für die Erstellung von Metadaten (Version 1.0)

Abb. 51 Richtlinie für die Erstellung von Metadaten (S. 2/2) 



\section{Anhang C \\ Ladenpreis-Kalkulation}

\section{C.1 Richtlinie für die progressive Ladenpreis-Kalkulation}

Mit Hilfe einer progressiven Kalkulation kann der Ladenpreis errechnet werden, den ein Buch rechnerisch haben müsste, wenn alle Kosten gedeckt sein sollen und zusätzlich ein Plangewinn erwirtschaftet werden soll. ${ }^{179} \mathrm{Im}$ Rahmen der Fallstudie wurden die Ladenpreise für Hard- und Softcover für die beschriebenen OA-BuchVeröffentlichungen auf Grundlage einer progressiven Kalkulation und anschließender Misch-Kalkulation festgelegt. Abbildung 52 und die Erläuterungen in Tabelle 62 verdeutlichen die Vorgehensweise. Die Tabellen 65-67 stellen die tatsächliche Ladenpreis-Kalkulation dar, die auf Grundlage der Soll-Kalkulation erstellt wurde. Die dargestellten Kosten weichen daher von den tatsächlichen Kosten aus Kapitel 4.4 ab.

\section{Herstellungskosten \\ + Verlags-Overhead-Kosten \\ + Vertriebskosten \\ $=$ Selbstkosten \\ + kalkulierter Gewinn \\ = Gesamtaufwand}

Abb. 52 Schema für eine progressive (Ladenpreis)-Kalkulation 
Tab. 62 Erläuterung der Ladenpreis-Kalkulation in der Fallstudie

\begin{tabular}{|c|c|c|}
\hline \multicolumn{2}{|l|}{ Herstellungskosten } & \multirow{2}{*}{$\begin{array}{l}\text { Beschreibung } \\
\text { Die Kosten sind der individuellen Soll-Kalkulation zu } \\
\text { entnehmen. }\end{array}$} \\
\hline & Satz & \\
\hline & Grafik & \\
\hline & Korrektur/Lektorat & \\
\hline & DOls & \\
\hline & Druck & $\begin{array}{l}\text { Im Rahmen der Untersuchung ergeben sich die } \\
\text { Gesamt-Druckkosten aus: (Druckkosten/Hardcover für } \\
\text { die Vertrags- und Verkaufsauflage } \times \text { geplanter Auflage) + } \\
\text { Druckkosten (gesamt) für die Musterexemplare }\end{array}$ \\
\hline $\begin{array}{l}\text { Herstellungskosten } \\
\text { gesamt }\end{array}$ & & Summe der Herstellungskosten \\
\hline \multirow{3}{*}{$\begin{array}{l}\text { Verlags-Over- } \\
\text { head-Kosten }\end{array}$} & Personal & \multirow{3}{*}{$\begin{array}{l}\text { Üblicherweise müssen die Verlags-Overhead-Kosten } \\
\text { nicht gesondert durch deinen Hochschulverlag gedeckt } \\
\text { werden und werden somit in der Kalkulation mit } 0,00 € \\
\text { beziffert. }\end{array}$} \\
\hline & Raummiete & \\
\hline & Technik & \\
\hline
\end{tabular}

\begin{tabular}{lll}
$\begin{array}{l}\text { Verlags-Over- } \\
\text { head-Kosten gesamt }\end{array}$ & Summer der Verlags-Overhead-Kosten \\
\hline Vertriebskosten & $\begin{array}{l}\text { Auslieferungskosten } \\
\text { (Beschaffung+Versand) }\end{array}$ & $\begin{array}{l}\text { Die Summe der Auslieferungskosten ergibt sich aus: } \\
\text { (Auslieferungskosten (Beschaffung + Versand) } \\
\text { Verpackung + Porto Bücher- und Warensendung) } \\
\text { geplanter Auflage }\end{array}$ \\
\cline { 2 - 3 } & Lagerkosten & $\begin{array}{l}\text { Üblicherweise fallen in einem Hochschulverlag keine } \\
\text { Kosten für die Lagerung an. }\end{array}$ \\
\hline Vertriebskosten & $\begin{array}{l}\text { Summe der Vertriebskosten } \\
\text { gesamt }\end{array}$ & $\begin{array}{l}\text { Die Summe der Selbstkosten ergibt sich aus: Herstellungs- } \\
\text { kosten gesamt + Verlags-Overhead-Kosten gesamt + } \\
\text { Vertriebskosten gesamt }\end{array}$ \\
\hline kalkulierter Gewinn & $\begin{array}{l}\text { Fallstudie: } 10 \% \\
\text { Der erwartete Gewinn pro Buch ist individuell } \\
\text { festzulegen. }\end{array}$ \\
\hline Gesamtaufwand & Selbstkosten + kalkulierter Gewinn \\
\hline
\end{tabular}




\begin{tabular}{|c|c|c|}
\hline \multirow[t]{2}{*}{ Netto-Ladenpreis } & & Gesamtaufwand/geplante Auflage \\
\hline & Umsatzsteuer & $\begin{array}{l}7 \% \text { vom Netto-Ladenpreis (für Print und digital, seit } \\
01.01 .2020 \text { ) }\end{array}$ \\
\hline \multirow{3}{*}{$\begin{array}{l}\text { Brutto-Ladenpreis } \\
\text { (Direktverkauf) }\end{array}$} & & Netto-Ladenpreis + Umsatzsteuer \\
\hline & Handelsrabatte & $50 \%$ vom Brutto-Ladenpreis (Direktverkauf) \\
\hline & Vertreterprovision & $\begin{array}{l}\text { Üblicherweise fallen für einen Hochschulverlag keine } \\
\text { Vertreterprovisionen an. }\end{array}$ \\
\hline $\begin{array}{l}\text { Brutto-Ladenpreis } \\
\text { (Handel) }\end{array}$ & & $\begin{array}{l}\text { Brutto-Ladenpreis (Direktverkauf) + Handelsrabatte } \\
(50 \%)+\text { Vertreterprovision }(0 \%)\end{array}$ \\
\hline
\end{tabular}

\section{C.2 Ladenpreis-Kalkulation der Fallstudie}

Tab. 63 Erwartete Herstellungskosten der Publikationsworkflows

\begin{tabular}{|c|c|c|c|}
\hline \multirow[b]{2}{*}{ Herstellungskosten } & \multicolumn{3}{|c|}{ Kosten $(€)$} \\
\hline & $\mathbf{F} 1$ & $\mathbf{F} 2$ & $\mathbf{F} 3$ \\
\hline Satzkosten (gesamt) & 0,00 & 789,10 & 1278,00 \\
\hline Grafikerkosten (gesamt) & 6,00 & 0,00 & 0,00 \\
\hline Korrektur/Lektorat & 0,00 & 0,00 & 0,00 \\
\hline DOls & 3,22 & 3,66 & 9,56 \\
\hline $\begin{array}{l}\text { Druckkosten pro Hardcover für } \\
\text { die Vertrags- und Verkaufsauflage }\end{array}$ & 9,23 & 8,05 & 8,61 \\
\hline $\begin{array}{l}\text { Druckkosten pro Softcover für } \\
\text { die Vertrags- und Verkaufsauflage }\end{array}$ & 6,00 & 5,09 & 5,45 \\
\hline Druckkosten (gesamt) für die Musterexemplare & 63,70 & 43,60 & 33,38 \\
\hline
\end{tabular}


418 Anhang C Ladenpreis-Kalkulation

Tab. 64 Erwartete Vertriebskosten für die Ladenpreis-Kalkulation

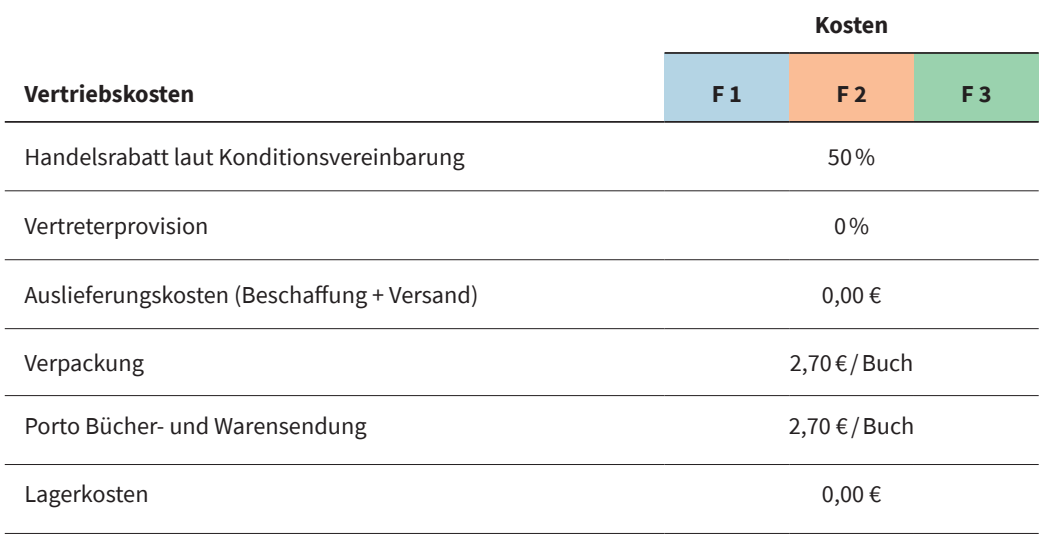


Tab. 65 Ladenpreis-Kalkulation für die Hardcover der Fallstudie

\begin{tabular}{|c|c|c|c|c|}
\hline \multirow[b]{2}{*}{ Herstellungskosten } & & \multicolumn{3}{|c|}{ Kosten $(€)$} \\
\hline & & F 1 & F 2 & F 3 \\
\hline & Satz & 0,00 & 789,10 & $1.278,00$ \\
\hline & Grafik & 6,00 & 0,00 & 0,00 \\
\hline & Korrektur/Lektorat & 0,00 & 0,00 & 0,00 \\
\hline & DOIs & 3,22 & 3,66 & 9,56 \\
\hline & Druck & 340,72 & 284,99 & 291,67 \\
\hline $\begin{array}{l}\text { Herstellungskosten } \\
\text { gesamt }\end{array}$ & & 349,94 & $1.077,75$ & $1.579,23$ \\
\hline \multirow{3}{*}{$\begin{array}{l}\text { Verlags-Over- } \\
\text { head-Kosten }\end{array}$} & Personal & 0,00 & 0,00 & 0,00 \\
\hline & Raummiete & 0,00 & 0,00 & 0,00 \\
\hline & Technik & 0,00 & 0,00 & 0,00 \\
\hline $\begin{array}{l}\text { Verlags-Over- } \\
\text { head-Kosten gesamt }\end{array}$ & & 0,00 & 0,00 & 0,00 \\
\hline \multirow[t]{2}{*}{ Vertriebskosten } & $\begin{array}{l}\text { Auslieferungskosten } \\
\text { (Beschaffung + Versand) }\end{array}$ & 147,00 & 147,00 & 147,00 \\
\hline & Lagerkosten & 0,00 & 0,00 & 0,00 \\
\hline $\begin{array}{l}\text { Vertriebskosten } \\
\text { gesamt }\end{array}$ & & 147,00 & 147,00 & 147,00 \\
\hline Selbstkosten & & 496,94 & $1.224,75$ & $1.726,23$ \\
\hline kalkulierter Gewinn & & 49,69 & 122,48 & 172,62 \\
\hline Gesamtaufwand & & 546,64 & $1.347,23$ & $1.898,85$ \\
\hline Geplante Auflage & & 30 & 30 & 30 \\
\hline \multirow[t]{2}{*}{ Netto-Ladenpreis } & & 18,22 & 44,91 & 63,30 \\
\hline & Umsatzsteuer $7 \%$ & 1,28 & 3,14 & 4,43 \\
\hline \multirow{3}{*}{$\begin{array}{l}\text { Brutto-Ladenpreis } \\
\text { (Direktverkauf) }\end{array}$} & & 19,50 & 48,05 & 67,73 \\
\hline & Handelsrabatte $50 \%$ & 19,50 & 48,05 & 67,73 \\
\hline & Vertreterprovision $0 \%$ & 0,00 & 0,00 & 0,00 \\
\hline $\begin{array}{l}\text { Brutto-Ladenpreis } \\
\text { (Handel) }\end{array}$ & & 38,99 & 96,10 & 135,45 \\
\hline
\end{tabular}


Tab. 66 Ladenpreis-Kalkulation für die Softcover der Fallstudie

\begin{tabular}{|c|c|c|c|c|}
\hline \multirow[b]{2}{*}{ Herstellungskosten } & & \multicolumn{3}{|c|}{ Kosten $(€)$} \\
\hline & & F 1 & F 2 & F 3 \\
\hline & Satz & 0,00 & 0,00 & 0,00 \\
\hline & Grafik & \multicolumn{3}{|c|}{$\begin{array}{l}\text { bereits in die Kalkulation für die Hardcover } \\
\text { eingegangen (First-Copy-Costs) }\end{array}$} \\
\hline & Korrektur/Lektorat & 0,00 & 0,00 & 0,00 \\
\hline & DOIs & \multicolumn{3}{|c|}{$\begin{array}{l}\text { bereits in die Kalkulation für die Hardcover } \\
\text { eingegangen (First-Copy-Costs) }\end{array}$} \\
\hline & Druck $^{*}$ & 6,00 & 5,09 & 5,45 \\
\hline $\begin{array}{l}\text { Herstellungskosten } \\
\text { gesamt }\end{array}$ & & 6,00 & 5,09 & 5,45 \\
\hline \multirow{3}{*}{$\begin{array}{l}\text { Verlags-Over- } \\
\text { head-Kosten }\end{array}$} & Personal & 0,00 & 0,00 & 0,00 \\
\hline & Raummiete & 0,00 & 0,00 & 0,00 \\
\hline & Technik & 0,00 & 0,00 & 0,00 \\
\hline $\begin{array}{l}\text { Verlags-Over- } \\
\text { head-Kosten gesamt }\end{array}$ & & 0,00 & 0,00 & 0,00 \\
\hline \multirow[t]{2}{*}{ Vertriebskosten } & $\begin{array}{l}\text { Auslieferungskosten } \\
\text { (Beschaffung+Versand) }\end{array}$ & 4,90 & 4,90 & 4,90 \\
\hline & Lagerkosten & 0,00 & 0,00 & 0,00 \\
\hline $\begin{array}{l}\text { Vertriebskosten } \\
\text { gesamt }\end{array}$ & & 4,90 & 4,90 & 4,90 \\
\hline Selbstkosten & & 10,90 & 9,99 & 10,35 \\
\hline kalkulierter Gewinn & & 1,09 & 1,00 & 1,03 \\
\hline Gesamtaufwand & & 11,99 & 10,99 & 11,38 \\
\hline Geplante Auflage & & 1 & 1 & 1 \\
\hline \multirow[t]{2}{*}{ Netto-Ladenpreis } & & 11,99 & 10,99 & 11,38 \\
\hline & Umsatzsteuer $7 \%$ & 0,84 & 0,77 & 0,80 \\
\hline \multirow{3}{*}{$\begin{array}{l}\text { Brutto-Ladenpreis } \\
\text { (Direktverkauf) }\end{array}$} & & 12,83 & 11,76 & 12,18 \\
\hline & Handelsrabatte $50 \%$ & 12,83 & 11,76 & 12,18 \\
\hline & Vertreterprovision $0 \%$ & 0,00 & 0,00 & 0,00 \\
\hline $\begin{array}{l}\text { Brutto-Ladenpreis } \\
\text { (Handel) }\end{array}$ & & 25,66 & 23,52 & 24,36 \\
\hline
\end{tabular}

* Die Summe ergibt sich aus: Druckkosten pro Softcover für die Vertrags- und Verkaufsauflage × geplanter Auflage 
Tab. 67 Ladenpreis-Kalkulation für den Fortdruck eines Hardcovers der Fallstudie

\begin{tabular}{|c|c|c|c|c|}
\hline \multirow[b]{2}{*}{ Herstellungskosten } & & \multicolumn{3}{|c|}{ Kosten $(€)$} \\
\hline & & F 1 & F 2 & $\mathbf{F} 3$ \\
\hline & Fort-Druck & 9,23 & 8,05 & 8,61 \\
\hline $\begin{array}{l}\text { Herstellungskosten } \\
\text { gesamt }\end{array}$ & & 9,23 & 8,05 & 8,61 \\
\hline \multirow{3}{*}{$\begin{array}{l}\text { Verlags-Over- } \\
\text { head-Kosten }\end{array}$} & Personal & 0,00 & 0,00 & 0,00 \\
\hline & Raummiete & 0,00 & 0,00 & 0,00 \\
\hline & Technik & 0,00 & 0,00 & 0,00 \\
\hline $\begin{array}{l}\text { Verlags-Over- } \\
\text { head-Kosten gesamt }\end{array}$ & & 0,00 & 0,00 & 0,00 \\
\hline \multirow[t]{2}{*}{ Vertriebskosten } & $\begin{array}{l}\text { Auslieferungskosten } \\
\text { (Beschaffung + Versand) }\end{array}$ & 4,90 & 4,90 & 4,90 \\
\hline & Lagerkosten & 0,00 & 0,00 & 0,00 \\
\hline $\begin{array}{l}\text { Vertriebskosten } \\
\text { gesamt }\end{array}$ & & 4,90 & 4,90 & 4,90 \\
\hline Selbstkosten & & 14,13 & 12,95 & 13,51 \\
\hline kalkulierter Gewinn & & 1,41 & 1,29 & 1,35 \\
\hline Gesamtaufwand & & 15,55 & 14,24 & 14,86 \\
\hline Geplante Auflage & & 1 & 1 & 1 \\
\hline \multirow[t]{2}{*}{ Netto-Ladenpreis } & & 15,55 & 14,24 & 14,86 \\
\hline & Umsatzsteuer $7 \%$ & 1,09 & 1 & 1,04 \\
\hline \multirow{3}{*}{$\begin{array}{l}\text { Brutto-Ladenpreis } \\
\text { (Direktverkauf) }\end{array}$} & & 16,64 & 15,24 & 15,90 \\
\hline & Handelsrabatte $50 \%$ & 16,64 & 15,24 & 15,90 \\
\hline & Vertreterprovision $0 \%$ & 0,00 & 0,00 & 0,00 \\
\hline $\begin{array}{l}\text { Brutto-Ladenpreis } \\
\text { (Handel) }\end{array}$ & & 33,27 & 30,48 & 31,80 \\
\hline
\end{tabular}




\section{C.3 Übersicht}

Nach Durchführung einer Misch-Kalkulation und unter Berücksichtigung von Schwellenpreisen wurden die in Tabelle 68 dargestellten Brutto-Ladenpreise (Handel) für die OA-Buchveröffentlichungen der Fallstudie festgelegt.

Tab. 68 Festgelegte Ladenpreise in der Fallstudie

\begin{tabular}{|c|c|c|c|c|c|c|}
\hline & \multicolumn{6}{|c|}{ Preise $(€)$} \\
\hline & \multicolumn{2}{|c|}{ F 1} & \multicolumn{2}{|c|}{ F 2} & \multicolumn{2}{|c|}{ F 3} \\
\hline & Hardcover & Softcover & Hardcover & Softcover & Hardcover & Softcover \\
\hline $\begin{array}{l}\text { Kalkulierter Brutto- } \\
\text { Ladenpreis (Handel) }\end{array}$ & 38,99 & 25,66 & 96,10 & 23,52 & 135,45 & 24,36 \\
\hline $\begin{array}{l}\text { Festgelegter Brutto- } \\
\text { Ladenpreis (Handel) }\end{array}$ & 49,00 & 29,00 & 49,00 & 29,00 & 79,00 & 49,00 \\
\hline
\end{tabular}




\section{Anhang D \\ Datenerhebung}

\section{D.1 Arbeitsaufwand der Publikationsworkflows je Prozess}

Tab. 69 Arbeitsaufwand der Publikationsworkflows der I-Ka-Prozesse

\begin{tabular}{lllll} 
& & \multicolumn{2}{c}{ Arbeitsaufwand (min) } \\
\cline { 3 - 4 } I-Ka: Konzept auswählen & F 1 & F 2 & F 3 \\
\hline T-[I-Ka-200] & Nach geeigneten Content-Urhebern recherchieren & 35 & 0 & 0 \\
\hline T-[I-Ka-210] & Content-Urhebern anfragen & 5 & 5 & 20 \\
\hline T-[I-Ka-220] & Mit Content-Urheber über Publikation sprechen & 17 & 5 \\
\hline SUMME & & $\mathbf{5 7}$ & $\mathbf{1 0}$ & $\mathbf{2 0}$
\end{tabular}

Tab. 70 Arbeitsaufwand der Publikationsworkflows der I-Cv-Prozesse

\begin{tabular}{llccc} 
& & \multicolumn{2}{c}{ Arbeitsaufwand (min) } \\
\cline { 3 - 4 } I-CV: Content vereinnahmen & F 1 & F 2 & F 3 \\
\hline T-[I-CV-100] & Content einfordern & 5 & - \\
\hline T-[I-CV-110] & Content prüfen & 15 & 7 & 15 \\
\hline T-[I-CV-120] & Content-Urheber beraten & 3 & 0 & 0 \\
\hline T-[I-CV-130] & Content vereinnahmen \& definiert ablegen & 3 & 1 & 2 \\
\hline SUMmE & & $\mathbf{2 6}$ & $\mathbf{8}$ & $\mathbf{1 7}$
\end{tabular}


Tab. 71 Arbeitsaufwand der Publikationsworkflows der I-Qe-Prozesse

\begin{tabular}{lllll} 
& & \multicolumn{2}{c}{ Arbeitsaufwand (min) } \\
\cline { 3 - 5 } I-Qe: Inhaltliche Qualität des Contents evaluieren & F 1 & F 2 & F 3 \\
\hline T-[I-Qe-200] & Checkliste zu Content einfordern & & 15 & 10 \\
\hline T-[I-Qe-210] & Gutachten zu Content einfordern & 15 & 10
\end{tabular}

Tab. 72 Arbeitsaufwand der Publikationsworkflows der I-Ca-Prozesse

\begin{tabular}{lllll} 
& & \multicolumn{2}{c}{ Arbeitsaufwand (min) } \\
\cline { 3 - 4 } I-Ca: Content anehmen & F 1 & F 2 & F 3 \\
\hline T-[I-Ca-100] & Gutachten bzw. Checkliste sichten & 20 & 10 & 10 \\
\hline T-[I-Ca-110] & Überarbeitung des Content einfordern & 9 & 5 & 2 \\
\hline T-[I-Ca-120] & Überarbeiteten Content überprüfen & 65 & 12 & 10 \\
\hline T-[I-Ca-130] & Content annehmen & - & - & 5 \\
\hline T-[I-Ca-140] & Content zur Bearbeitung zur Verfügung stellen & 3 & 2 & 2 \\
\hline SUMmE & & $\mathbf{9 7}$ & $\mathbf{2 9}$ & $\mathbf{2 9}$
\end{tabular}

Tab. 73 Arbeitsaufwand der Publikationsworkflows der P-Ca-Prozesse

\begin{tabular}{lllll} 
& & \multicolumn{3}{c}{ Arbeitsaufwand (min) } \\
\cline { 2 - 4 } P-Ca: Content aufbereiten & F 1 & F 2 & F 3 \\
\hline T-[P-Ca-120] Technisches Copy Editing durchführen & 45 & 225 & 85 \\
\hline SUMME & $\mathbf{4 5}$ & $\mathbf{2 2 5}$ & $\mathbf{8 5}$
\end{tabular}


Tab. 74 Arbeitsaufwand der Publikationsworkflows der P-CUe-Prozesse

\begin{tabular}{lllll} 
& & \multicolumn{2}{c}{ Arbeitsaufwand (min) } \\
\cline { 3 - 4 } P-CUe: Cover bzw. Umschlag erstellen & F 1 & F 2 & F 3 \\
\hline T-[P-CUe-100] & Cover erstellen & 0 & 0 & 15 \\
\hline T-[P-CUe-110] & Umschlag erstellen & 110 & 26 & 28 \\
\hline T-[P-CUe-120] & Freigabe für Cover bzw. Umschlag einholen & - & 13 & 13 \\
\hline T-[P-CUe-130] & Korrekturen für Cover bzw. Umschlag umsetzen & - & 5 & 0 \\
\hline SUMmE & & $\mathbf{1 1 0}$ & $\mathbf{4 4}$ & $\mathbf{5 6}$
\end{tabular}

Tab. 75 Arbeitsaufwand der Publikationsworkflows der P-CiP-Prozesse

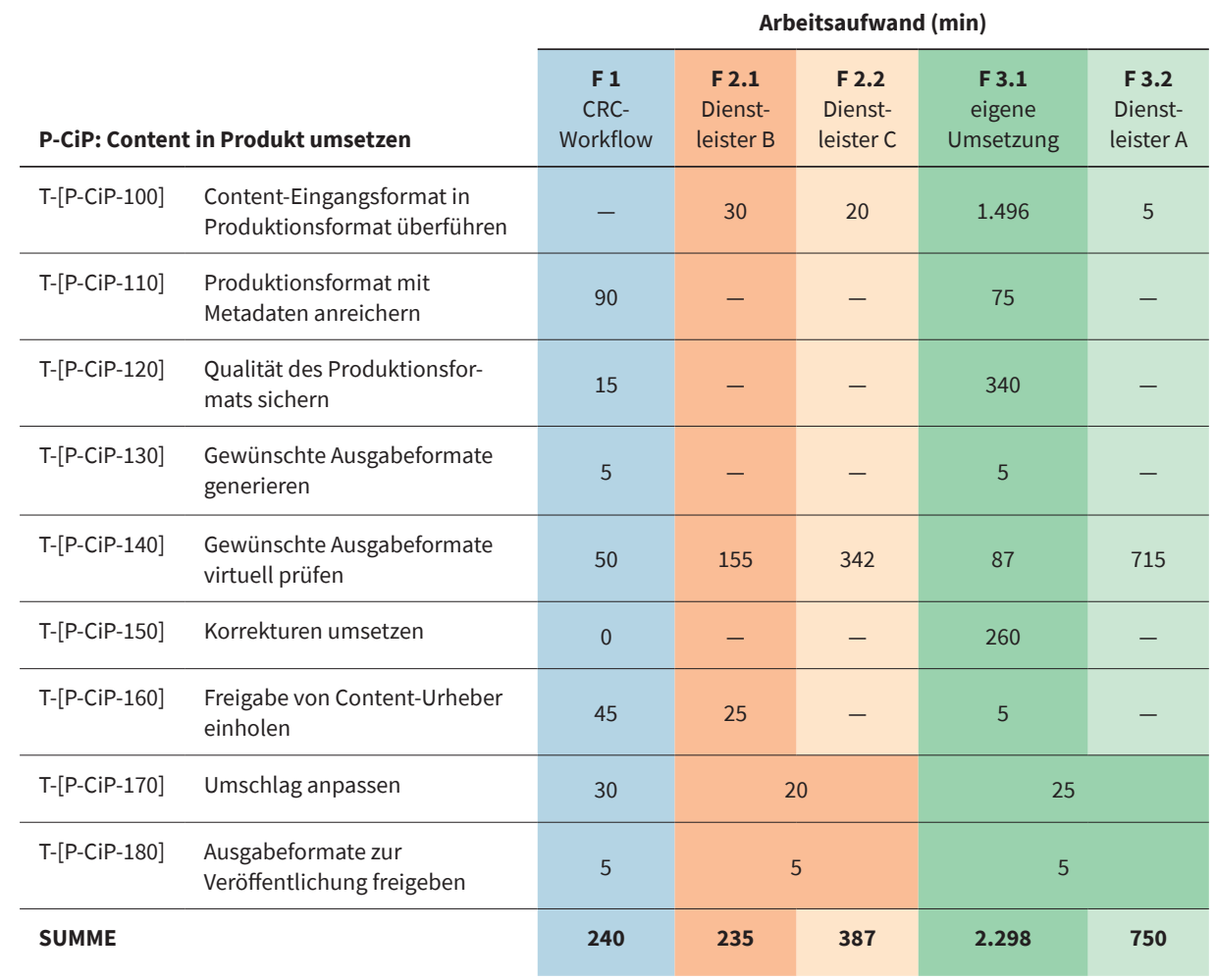


Tab. 76 Arbeitsaufwand der Publikationsworkflows der P-GP-Prozesse

\begin{tabular}{lllll} 
& & \multicolumn{2}{c}{ Arbeitsaufwand (min) } \\
\cline { 3 - 4 } P-GP: Gedrucktes Produkt produzieren & F 1 & F 2 & F 3 \\
\hline T-[P-GP-100] & Druck auslösen & 2 & 140 & 70 \\
\hline T-[P-GP-110] & Produkt physisch prüfen & 2 & 17 & 20 \\
\hline T-[P-GP-120] & Gewünschte Auflage produzieren & 15 & 10 & 0 \\
\hline T-[P-GP-130] & Pflicht- und Vertragsexemplare versenden & 15 & 40 & 20 \\
\hline SUMME & & $\mathbf{3 4}$ & $\mathbf{2 0 7}$ & $\mathbf{1 1 0}$
\end{tabular}

Tab. 77 Arbeitsaufwand der Publikationsworkflows der P-EP-Prozesse

\begin{tabular}{|c|c|c|c|c|}
\hline & & \multicolumn{3}{|c|}{ Arbeitsaufwand (min) } \\
\hline \multicolumn{2}{|c|}{ P-EP: Elektronisches Produkt produzieren } & F 1 & F 2 & $\mathbf{F} 3$ \\
\hline T-[P-EP-100] & $\begin{array}{l}\text { Produkt auf primärer Veröffentlichungsplattform } \\
\text { veröffentlichen }\end{array}$ & 5 & 5 & - \\
\hline$T-[P-E P-110]$ & Produkt auf weiteren Plattformen veröffentlichen & 34 & 30 & - \\
\hline SUMME & & 39 & 35 & - \\
\hline
\end{tabular}

Tab. 78 Arbeitsaufwand der Publikationsworkflows der P-PFs-Prozesse

\begin{tabular}{|c|c|c|c|c|}
\hline & & \multicolumn{3}{|c|}{ Arbeitsaufwand (min) } \\
\hline \multicolumn{2}{|c|}{ P-PFs: Produktform sichern } & F 1 & F 2 & $\mathbf{F} 3$ \\
\hline T-[P-PFs-100] & Für Langzeitarchivierung des Produktes sorgen & 10 & 14 & - \\
\hline SUMME & & 10 & 14 & - \\
\hline
\end{tabular}


Tab. 79 Arbeitsaufwand der Publikationsworkflows der P-PVe-Prozesse

\begin{tabular}{|c|c|c|c|c|}
\hline & & \multicolumn{3}{|c|}{ Arbeitsaufwand (min) } \\
\hline \multicolumn{2}{|c|}{ M-PVe: Publikationsvertrag erstellen } & F 1 & F 2 & $\mathbf{F} 3$ \\
\hline T-[M-PVe-100] & Rechte für Inhaltselemente klären & 133 & 162 & 25 \\
\hline T-[M-PVe-200] & Vertragsabschluss \& Buchvorankündigung vorbereiten & 11 & 36 & 8 \\
\hline T-[M-PVe-210] & Publikationsvertrag schließen & 17 & 10 & 8 \\
\hline SUMME & & 161 & 208 & 41 \\
\hline
\end{tabular}

Tab. 80 Arbeitsaufwand der Publikationsworkflows der $M$-Pv-Prozesse

\begin{tabular}{lllll} 
& & \multicolumn{2}{c}{ Arbeitsaufwand (min) } \\
\cline { 3 - 5 } M-Pv: Produktion vorbereiten & F 1 & F 2 & F 3 \\
\hline T-[M-PV-100] & Produktionsweg festlegen & 0 & 0 & 0 \\
\hline T-[M-PV-200] & Stammdaten pflegen & 5 & 3 & 15 \\
\hline T-[M-PV-300] & Produktionskosten klären & 60 & 150 & 25 \\
\hline T-[M-PV-310] & Soll-Kalkulation erstellen & 30 & 30 & 30 \\
\hline T-[M-PV-320] & Ladenpreis für gedrucktes Produkt festlegen & 10 & 10 & 10 \\
\hline SUMME & & $\mathbf{1 0 5}$ & $\mathbf{1 9 3}$ & $\mathbf{8 0}$
\end{tabular}

Tab. 81 Arbeitsaufwand der Publikationsworkflows der M-MM-Prozesse

\begin{tabular}{lllll} 
& & \multicolumn{2}{c}{ Arbeitsaufwand (min) } \\
\cline { 3 - 4 } M-MM: Metadaten-Management durchführen & F 1 & F 2 & F 3 \\
\hline T-[M-MM-100] & Metadatensatz erzeugen & 11 & 96 & 17 \\
\hline T-[M-MM-110] & Metadaten distribuieren & 52 & 6 & 125 \\
\hline T-[M-MM-120] & $\begin{array}{l}\text { Weitere Aggregatoren beauftragen Metadaten } \\
\text { zu distribuieren }\end{array}$ & 16 & 7 & - \\
\hline T-[M-MM-200] & Metadatensatz aktualisieren & - & 10 & - \\
\hline SUMME & & $\mathbf{7 9}$ & $\mathbf{1 1 9}$ & $\mathbf{1 4 2}$
\end{tabular}


Tab. 82 Arbeitsaufwand der Publikationsworkflows der $M$-Bb-Prozesse

\begin{tabular}{llll} 
& & \multicolumn{2}{c}{ Arbeitsaufwand (min) } \\
\cline { 3 - 4 } M-Bb: Bestellungen bedienen & F 1 & F 2 & F 3 \\
\hline T-[M-Bb-100] & Bestellung erhalten & 0 & - \\
\hline T-[M-Bb-110] & Bestellung bearbeiten & 25 & - \\
\hline T-[M-Bb-120] & Bestellung versenden & 3 & - \\
\hline T-[M-Bb-130] & Rechnung stellen & 16 & - \\
\hline SUMME & & 43 & $\mathbf{0}$ \\
\hline
\end{tabular}

\section{D.2 Kostenaufwand für Null-Prozesse}

Wie in Kapitel 4.4.1.3 erwähnt, sind Kosten im Vorfeld der Fallstudie entstanden, die deren Durchführung erst ermöglichten. Sie können einigen der in Kapitel 3.1.3 genannten Null-Prozessen zugeordnet werden, die abschließend in diesem Kapitel beschrieben werden. Zu beachten ist dabei, dass die in den Tabellen 83-86 aufgeführten Kosten, die tatsächlichen Kosten aus der Fallstudie wiedergeben und somit, je nach Vereinbarung abweichen können.

\section{Software beschaffen}

Tab. 83 Kostenaufwand des Null-Prozesses Software beschaffen

\begin{tabular}{|c|c|c|}
\hline Kostenart & Adobe Creative Cloud & Antenna House Formatter \\
\hline Anzahl der Lizenzen & Preis pro Lizenz (1 Nutzer) & Preis pro Lizenz (1 Nutzer) \\
\hline $\begin{array}{l}\text { Einmalige Kosten bzw. } \\
\text { Einrichtungsgebühr }\end{array}$ & keine & $\begin{array}{ll}- & 625 \$ \text { für Lizenz } \\
- & 250 \$ \text { für zusätzliche } \\
& \text { Wartungspauschale }\end{array}$ \\
\hline Jährliche Gebühr & $143,70 €$ & keine \\
\hline Anmerkung & $\begin{array}{l}\text { Stand 2018: Gebühren nach } \\
\text { Rahmenvereinbarung der } \\
\text { HTWK Leipzig; kann je } \\
\text { Einrichtung unterschiedlich } \\
\text { sein }\end{array}$ & $\begin{array}{l}\text { Stand 2019: Preis pro Lizenz, inkl. } \\
50 \text { \% Preisnachlass für } \\
\text { Bildungseinrichtungen }\end{array}$ \\
\hline
\end{tabular}




\section{Mitgliedschaften schließen}

Tab. 84 Kostenaufwand des Null-Prozesses Mitgliedschaften schließen (Stand Jan. 2020)

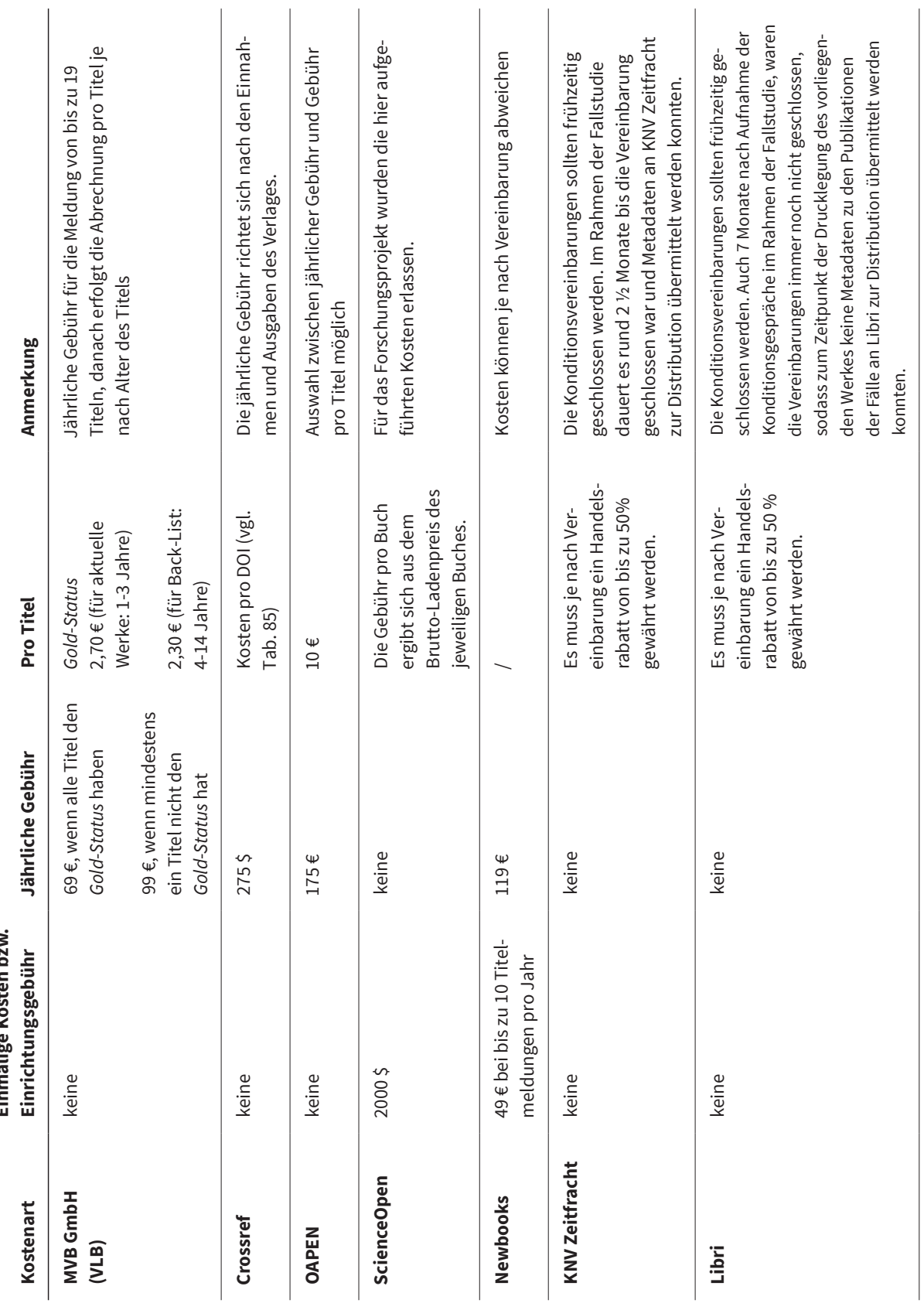




\section{ISBN-Stamm erwerben}

Tab. 85 Kostenaufwand des Null-Prozesses ISBN-Stamm erwerben (Stand Nov. 2019)

\begin{tabular}{|c|c|c|c|}
\hline $\begin{array}{l}\text { Kosten- } \\
\text { art }\end{array}$ & $\begin{array}{l}\text { Einmalige Kosten bzw. } \\
\text { Einrichtungsgebühr }\end{array}$ & Jährliche Gebühr & Anmerkung \\
\hline ISBN & $\begin{array}{l}\text { für } 1.000 \text { ISBNs } 260 € \text {, zzgl. } \\
\text { MwSt. }\end{array}$ & $\begin{array}{l}\text { Voraussetzung ist die } \\
\text { Mitgliedschaft bei MVB } \\
\text { GmbH (VLB) (vgl. } \\
\text { Tab. 84) }\end{array}$ & $\begin{array}{l}\text { Preisabstufungen je } \\
\text { nach Anzahl der ISBNs } \\
(1,10,100,1.000)\end{array}$ \\
\hline DOI & $\begin{array}{ll}\text { - } & \text { pro Buchtitel: } 1 \$ \\
\text { - } & \text { pro Kapitel: } 0.25 \$ \\
\text { - } & \text { pro Abbildungen/ } \\
& \text { Tabellen: } 0.06 \$\end{array}$ & $\begin{array}{l}\text { Voraussetzung ist die } \\
\text { Mitgliedschaft bei } \\
\text { Crossref (vgl. Tab. 84) }\end{array}$ & / \\
\hline
\end{tabular}

\section{Außendarstellung pflegen}

Tab. 86 Kostenaufwand des Null-Prozesses Außendarstellung pflegen (Stand 2019)

\begin{tabular}{|c|c|c|c|}
\hline Kostenart & $\begin{array}{l}\text { Einmalige } \\
\text { Kosten bzw. Ein- } \\
\text { richtungsgebühr }\end{array}$ & Jährliche Gebühr & Anmerkung \\
\hline $\begin{array}{l}\text { E-Mail- } \\
\text { Postfach } \\
\text { (Anbieter } \\
\text { Strato) }\end{array}$ & keine & $\begin{array}{ll}- & 1,00 € / \text { monatl. für das } \\
& \text { erste Jahr } \\
- & 3,00 € / \text { monatl. nach } 12 \\
& \text { Monaten }\end{array}$ & $\begin{array}{l}\text { Kosten für } 25 \text { Postfächer } \\
\text { mit je } 5 \text { GB } \\
\text { Speicherplatz und } 2 \text {.de- } \\
\text { Domains }\end{array}$ \\
\hline
\end{tabular}




\section{Anhang E \\ Weitere Dokumente}




\section{E.1 Informationsblatt für Autoren und Gutachter}

\section{Informationsblatt}

zur Publikation im Open-Access-Hochschulverlag

\section{Weiteres Vorgehen: Wie läuft die Publikation ab?}

Der Autor stimmt der Veröffentlichung seiner Arbeit und den damit einhergehenden

Prozessschritten zu.

Die Gutachter der Arbeit erhalten von uns, dem Projektteam OA-HVerlag, jeweils eine Checkliste, um anzugeben, ob aus ihrer Sicht noch Änderungen am Inhalt vorgenommen werden sollten, bevor die Arbeit publiziert werden kann. Zum Zweck der Qualitätssicherung stellen sie uns außerdem die Gutachten der Arbeit zur Verfügung.

Nachdem die Checklisten von den Gutachtern ausgefüllt wurden, informieren wir den Autor, ob von ihm Änderungen am Inhalt vorgenommen werden müssen, bevor die Arbeit publiziert werden kann.

Falls dies zutrifft, führt der Autor die vorgeschlagenen Änderungen am Inhalt aus.

Der Autor erhält von uns eine Checkliste, um relevante Angaben über den Inhalt zu machen, bevor der Publikationsvertrag geschlossen werden kann. Außerdem sendet er die offenen Daten der Arbeit, d.h. die Manuskriptdatei als Word-, InDesign- oder LaTex-Datei, an das Projektteam.

Sofern alle Formalitäten geklärt wurden, wird der Publikationsvertrag zwischen dem Autor und dem OA-HVerlag geschlossen.

Anschließend beginnt die Produktion des Inhaltes. Nähere Informationen hierzu erfolgen zeitnah.

Informationsblatt zur Publikation im Open-Access-Hochschulverlag 


\section{Informationsblatt}

\section{zur Publikation im Open-Access-Hochschulverlag}

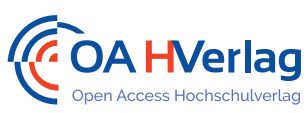

\section{Projektbeschreibung}

Im Forschungsprojekt Open-Access-Hochschulverlag (kurz OA-HVerlag) soll ein medienneutraler sowie kosten- und personaleffizienter Publikationsworkflow entwickelt werden, der es Hochschulen und Universitäten ermöglicht, Forschungsarbeiten und Graduierungsschriften in digitaler Form als Open Access und als gedrucktes Buch zu veröffentlichen.

Um diesen Workflow zu demonstrieren und zu evaluieren, ist es geplant, eine Pilotphase durchzuführen, zu dessen Zweck fünf sehr gute Abschluss- oder Forschungsarbeiten (je Fakultät eine Arbeit) von Mitgliedern/Absolventen der HTWK ausgewählt und publiziert werden.

Bis zum Laufzeitende des Projektes (April 2020) sollen gemeinsam mit der Hochschulbibliothek Strukturen etabliert werden, um langfristig einen Hochschulverlag an der HTWK Leipzig zu betreiben. Ausführlichere Informationen zum Projekt finden Sie auf unserer Forschungswebsite: www.htwk-leipzig.de/oa-hochschulverlag.

\section{Was bedeutet Open Access?}

„Ziel von Open Access ist es, wissenschaftliche Literatur und wissenschaftliche Materialien für alle Nutzerinnen und Nutzer frei zugänglich zu machen: kostenlos und möglichst frei von technischen und rechtlichen Barrieren. Ein wesentlicher Aspekt ist die Maximierung der Verbreitung wissenschaftlicher Information, wie in der Berliner Erklärung gefordert. Diese wurde 2003 formuliert und seither von namhaften Forschungsorganisationen und Universitäten (z.B. Deutsche Forschungsgemeinschaft (DFG), Schweizerischer Nationalfonds zur Förderung der wissenschaftlichen Forschung (SNF), Fonds zur Förderung der wissenschaftlichen Forschung (FWF), Rektorenkonferenz der Schweizer Universitäten (CRUS), Hochschulrektorenkonferenz (HRK), Max-PlanckGesellschaft (MPG), CERN) unterzeichnet.

Weithin anerkannte Gründe für Open Access sind neben dem unmittelbaren Zugang für alle Menschen und der bestmöglichen Aufmerksamkeit für die Ergebnisse wissenschaftlicher Forschung die Geschwindigkeit von Veröffentlichung und Zugriff, die (je nach Nutzungslizenzen) Möglichkeiten zur Weiternutzung und Aspekte von Transparenz und Qualitätssicherung." ${ }^{1}$

Open Access stellt somit eine wesentliche Voraussetzung für die heutige bzw. zukünftige wissenschaftliche Form der Kommunikation dar.

1 Quelle: http://open-access.net/informationen-zu-open-access/was-bedeutet-open-access/

(Dieses Werk ist lizenziert unter einer Creative Commons Namensnennung 4.0 International Lizenz)

Informationsblatt zur Publikation im Open-Access-Hochschulverlag 


\section{Informationsblatt}

\section{zur Publikation im Open-Access-Hochschulverlag}

\section{Die Vorteile}

Keine Kosten: Für den geplanten Publikationsprozess werden keine Kosten auf den Autor zu kommen. Das Projekt ist vom Bundesministerium für Bildung und Forschung (kurz BMBF) gefördert, worüber die Kosten für die Herstellung der fünf Abschlussarbeiten finanziert werden.

State-of-the-Art: Im Gegensatz zu vielen anderen, bereits bestehenden Universitätsverlagen werden die Arbeiten medienneutral publiziert. Das heißt, die Arbeiten werden in allen gängigen digitalen Medienformaten (PDF, Epub und Mobi) verbreitet. Außerdem wird es eine Hardcover- und Softcover-Version zu erwerben geben.

Qualität: Es ist uns ein großes Anliegen, die Arbeiten auf einem hohem, fachlichem Niveau zu publizieren. Aus diesem Grund sieht unserer Workflow vor, dass wir zur Qualitätssicherung des Inhaltes die Gutachten der Arbeiten einholen und den Gutachtern eine Checkliste zur Verfügung stellen, um zu prüfen, welche Änderungen am Inhalt noch getroffen werden können, bevor die Arbeit publiziert wird.

Offenheit: Im Sinn von Open Access werden die Arbeiten unter der weltweit anerkannten Creative-Common-Lizenz CC-BY 4.0 (Creative Commons Namensnennung 4.0

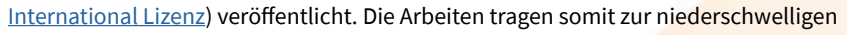
Verbreitung von wissenschaftlichen Erkenntnissen bei.

Sichtbarkeit: Besonders wichtig ist uns, dass die von uns publizierten Arbeiten bereits während der Pilotphase - gut auffindbar und sichtbar sind. Aus diesem Grund statten wir die Arbeiten mit einer ISBN und DOIs aus und tragen die Titel in alle gängigen Kataloge und Buchhandelverzeichnisse ein, wie VIB, DNB, OAPEN/DOAB, Google Books und weitere.

Distribution: Die gedruckte Version wird über Amazon, aber auch über den klassischen Buchhandel verfügbar sein.

\section{Kontakt}

Bei Fragen, Anregungen oder Kritik zum Projekt und/oder zum Publikationsablauf kontaktieren Sie bitte die verantwortlichen Projektmitarbeiter David Böhm und Antonia Schrader unter redaktion@oa-hverlag.de oder telefonisch unter Tel. +49 34130762870. 


\section{E.2 Bibliografische Angaben zu den Werken aus der Fallstudie}

\section{Beitrag zur Vorbemessung der Biegeverstärkung von Stahlbetonbauteilen mit Rechteckquerschnitt mittels aufgeklebten CFK-Lamellen}

Das Verstärken von Betonbauteilen mit aufgeklebten CFK-Lamellen unterliegt einer komplexen Bemessung. Im vorliegenden Werk wird beschrieben, wie die erforderliche Lamellenfläche anhand der Ansätze des Stahlbetonbaus, erweitert um die aufgeklebte Bewehrung, ermittelt wird. Es wurden Bemessungstafeln mit dimensionslosen Beiwerten entwickelt, die mit dem vereinfachten Nachweis der DAfStb-Richtlinie „Verstärken von Betonbauteilen mit geklebter Bewehrung“ die Vorbemessung einer solchen Biegeverstärkung ermöglichen. Zudem wird beschrieben, wie eine Excel-Arbeitsmappe konfiguriert werden kann, die den genaueren Nachweis der Lamellenkraftänderung am Zwischenrisselement sowie die iterative Bemessung der Biegetragfähigkeit enthält.

\section{CEVIN STIEHL}

Beitrag zur Vorbemessung der Biegeverstärkung von Stahlbetonbauteilen mit Rechteckquerschnitt mittels aufgeklebten CFK-Lamellen

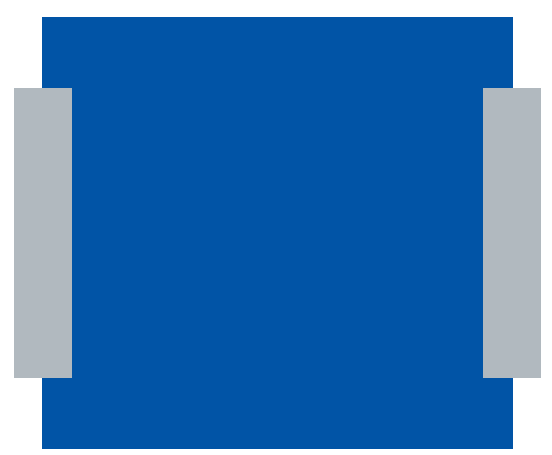

HITWK
Ladenpreis

$79 €$ - Hardcover

$49 €$ - Softcover

ISBN

978-3-96627-000-7 (Hardcover) 978-3-96627-001-4 (Softcover) 978-3-96627-002-1 (PDF) 978-3-96627-003-8 (EPUB/MOBI)

Autor Cevin Stiehl

Publikationstyp Masterarbeit

Grad-verleihende Institution HTWK Leipzig, Fakultät Bauwesen Umfang 252 Seiten

Abgabe Juli 2017

Sprache Deutsch

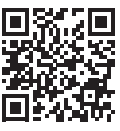

www.oa-hochschulverlag.htwk-leipzig.de 


\section{Kommunikationstechnische Optimierung eines energieautarken funkbasierten Sensorkonzepts}

Im Rahmen dieser Bachelorarbeit wurde ein bestehendes kabelloses Sensorkonzept hinsichtlich softwareseitiger Optimierungsmöglichkeiten untersucht. Es wurden zunächst die Anwendungsmöglichkeiten solcher Sensorsysteme in den bestehenden Instandhaltungsstrategien beschrieben. Anschließend erfolgte eine Analyse des bisherigen Sensorkonzepts, auf deren Basis mit der vorhandenen Hardware ein Alternativkonzept zur kommunikationstechnischen Optimierung realisiert wurde. Durch das Ersetzen des bisher verwendeten Anwendungsprotokolls HTTP durch das leichtgewichtige MQTT entstand ein alternatives Sensorkonzept. Zudem wurde ein Messkonzept zur Bestimmung der zur Datenübertragung benötigten Sendedauer entwickelt, welches einen Vergleich mit dem bestehenden Sensorkonzept ermöglicht. Es konnte somit eingeschätzt werden, inwieweit die Zielstellung erreicht wurde.

FLORIAN SENFT

Kommunikationstechnische Optimierung eines energieautarken funkbasierten Sensorkonzepts

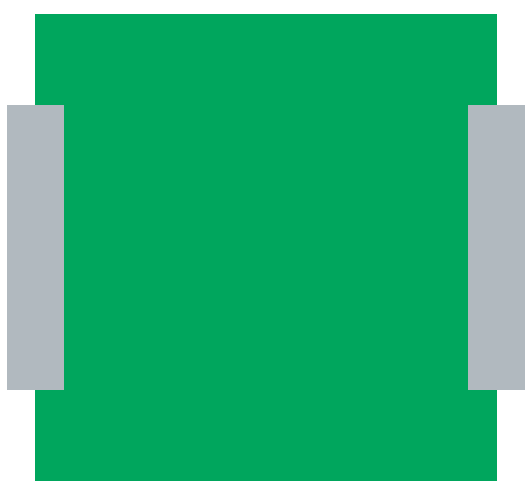

I-ITWK

\section{Ladenpreis}

$49 €$ - Hardcover

$29 €$ - Softcover

\section{ISBN}

978-3-96627-004-5 (Hardcover) 978-3-96627-005-2 (Softcover)

978-3-96627-006-9 (PDF)

978-3-96627-007-6 (EPUB/MOBI)

Autor Florian Senft

Publikationstyp Bachelorarbeit

Grad-verleihende Institution

HTWK Leipzig, Fakultät Maschinenbau

Umfang 96 Seiten

Abgabe Juni 2018

Sprache Deutsch

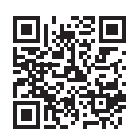

www.oa-hochschulverlag.htwk-leipzig.de 


\section{Analyse von Kundenaktivitäten und der Bereitstellungsqualität von Büchern aus Kundensicht}

Mit einer Untersuchung von Kundenkommentaren zu Mietangeboten für E-Books

Bücherlesen und -kaufen gehören bei vielen Deutschen nach wie vor zum Leben dazu. Durch den wiederholten Kontakt mit Büchern in verschiedenen Kontexten werden Handlungsweisen gelernt, Erwartungen gebildet und Bücher in andere Tätigkeiten im Alltag integriert. Anbieter, die Bücher bereitstellen, können sich diese Buchbeziehung zunutze machen, indem sie das Design und die Weiterentwicklung ihrer Angebote auf Erwartungen und Aktivitäten von Buchlesern abstimmen. Das vorliegende Werk stellt als eine Umsetzungshilfe das Konzept der Bereitstellungsqualität von Büchern vor und empfi ehlt die Analyse des Kundenverhaltens aus Sicht der Tätigkeitstheorie und der Customer-Dominant Logic. Zur empirischen Prüfung dient die Auswertung von Kundenkommentaren zweier Vermietungsangebote von E-Books.

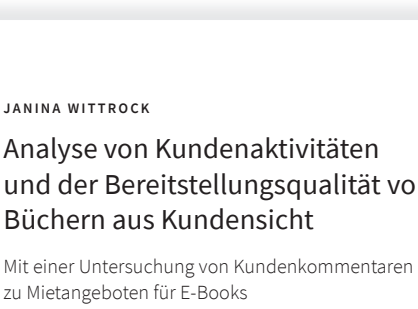

zu Mietangeboten für E-Books

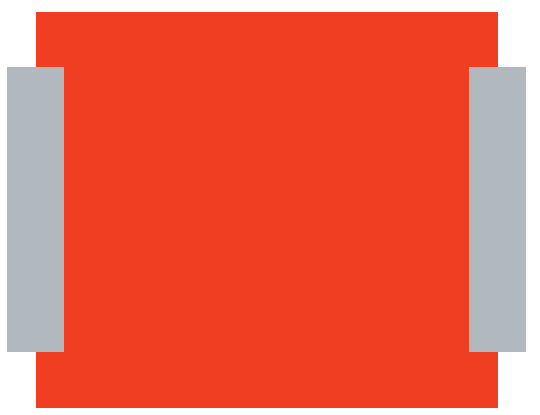

HITWK

CCOAHVerlag

\author{
Ladenpreis \\ $79 €$ - Hardcover \\ $49 €$ - Softcover
}

ISBN

978-3-96627-008-3 (Hardcover) 978-3-96627-009-0 (Softcover)

978-3-96627-010-6 (PDF)

978-3-96627-011-3 (EPUB/MOBI)

Autor Janina Wittrock

Publikationstyp Masterarbeit

Grad-verleihende Institution HTWK Leipzig, Fakultät Informatik und Medien

Umfang 160 Seiten

Abgabe September 2016

Sprache Deutsch

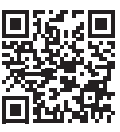

www.oa-hochschulverlag.htwk-leipzig.de 


\section{Stadt als Schule. Nachbarschaftszentrum und Gemeinschaftsschule an der alten Feuerwache in Anger-Crottendorf, Leipzig}

In der alten, leer gewordenen Feuerwache im Leipziger Osten soll ein Nachbarschaftszentrum entstehen. Gleichzeitig plant die Stadt auf dem frei gewordenen Grundstück eine Schule. Das vorliegende Werk stellt einen Lösungsansatz für diesen Nutzungskonflikt vor, in dem architektonisch, städtebaulich und pädagogisch Synergien geschaffen werden sollen. Nach dem Vorbild der FreinetPädagogik wurde eine Gemeinschaftsschule am Standort der alten Feuerwache in einem Neubau geplant, während das Nachbarschaftszentrum im Bestandsgebäude der alten Feuerwehr Platz findet. Durch einen gemeinsamen Hof, zusammen genutzte Werk- und Sportstätten sowie einen gemeinsamen Mittagstisch wird die Schule zur Stadt geöffnet und die Nachbarschaft zur Schule. So wird Stadt zur Schule.

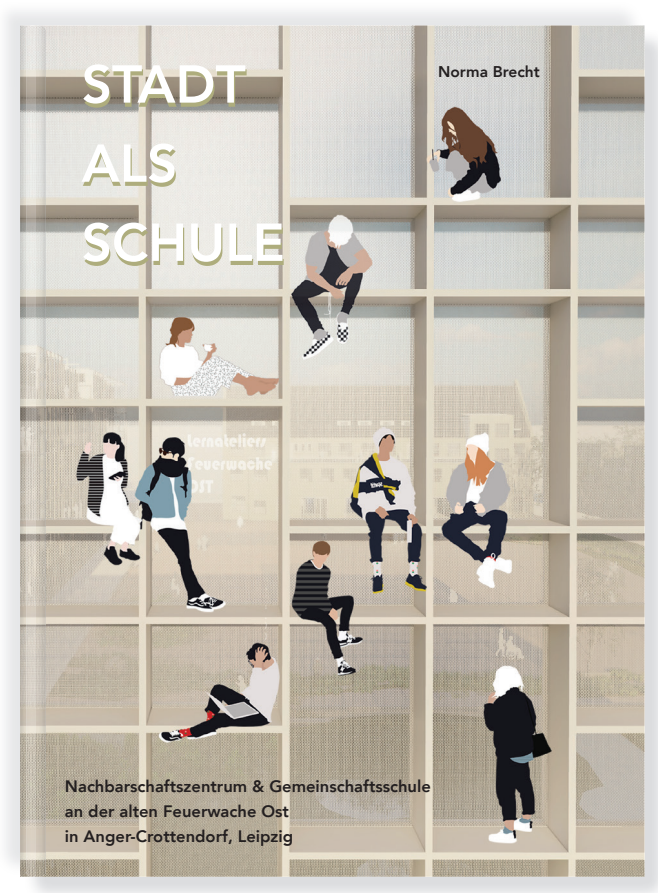

\author{
Ladenpreis \\ $49 €$ - Hardcover
}

ISBN

978-3-96627-012-0 (Hardcover) 978-3-96627-014-4 (PDF)

Autor Norma Brecht

Publikationstyp Masterarbeit

Grad-verleihende Institution HTWK Leipzig, Fakultät Architektur und Sozialwissenschaften

Umfang 68 Seiten

Abgabe Februar 2019

Sprache Deutsch

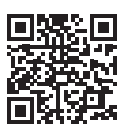

www.oa-hochschulverlag.htwk-leipzig.de 

